\title{
EUROPEAN \\ PERSPECTIVES \\ FOR PUBLIC \\ ADMINISTRATION
}

THE WAY FORWARD

Geert Bouckaert \& Werner Jann (eds)

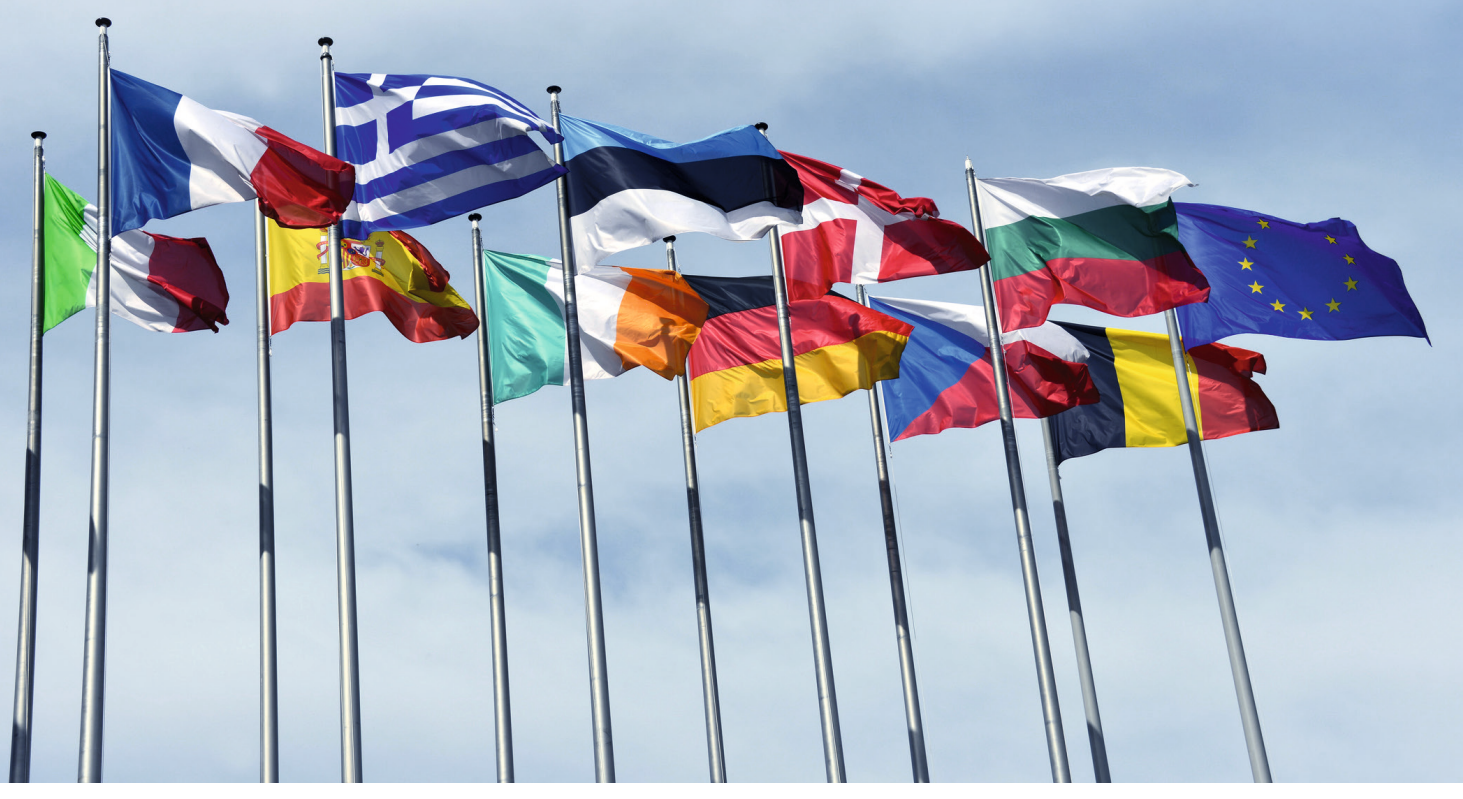

Leuven University Press 
European Perspectives for Public Administration 



\title{
European Perspectives for Public Administration
}

\author{
The Way Forward
}

\author{
Edited by
}

Geert Bouckaert and Werner Jann 


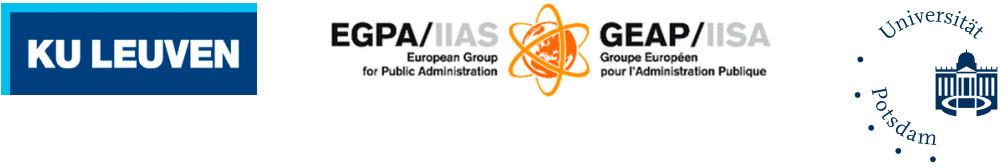

Published with the support of

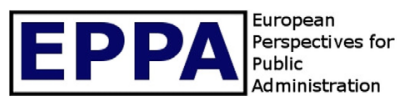

and

\section{Alexander von Humboldt \\ Stiftung/Foundation}

Published in 2020 by Leuven University Press / Presses Universitaires de Louvain / Universitaire Pers Leuven. Minderbroedersstraat 4, B-30oo Leuven (Belgium).

Selection and editorial matter (C) Geert Bouckaert and Werner Jann, 2020

Individual chapters (C) The respective authors, 2020

This book is published under a Creative Commons Attribution Non-Commercial

Non-Derivative 4.0 Licence.

\section{(1) (1) $\Theta \Theta$}

Further details about Creative Commons licences are available at

http://creativecommons.org/licenses/

Attribution should include the following information:

Geert Bouckaert, Werner Jann, eds, European Perspectives for Public Administration: The Way

Forward. Leuven, Leuven University Press. (CC BY-NC-ND 4.o)

ISBN 9789462702035 (Paperback)

ISBN 9789461663078 (ePDF)

ISBN 9789461663085 (ePUB)

https://doi.org/10.11116/9789461663078

$\mathrm{D} / 2020 / 1869 / 10$

NUR: 759

Layout: Crius Group

Cover design: Frederik Danko

Cover illustration: nmann77, (C) Fotolia.com

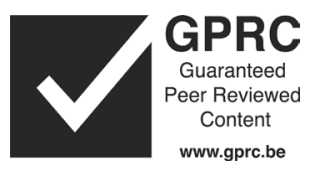


This book is dedicated to our friend and colleague Professor Christopher Pollitt (1946-2018). 



\section{Table of Contents}

Preface to the EPPA I Book

Acknowledgments

About the Authors

I Introduction

1 The EPPA Project

Geert Bouckaert and Werner Jann

2 The Survey: A Long-Distance Conversation about the Future of Public Administration in Europe

Jana Bertels, Geert Bouckaert, Werner Jann

II Public Administration and Futures

1 From Public Administration in Utopia to Utopia in Public Administration

Geert Bouckaert

2 Governing for the Future: Means, Ends and Disconnects Paul Joyce

3 Scenarios as Thought Experiments for Governance Meelis Kitsing

III Public Administration and Disciplines

1 Public Administration and Disciplines

Thurid Hustedt, Tiina Randma-Liiv and Riin Savi 
2 Public Administration and Law

Martin Burgi

3 Seeing Public Bureaucracies Like a Sociologist: (A Plea Towards) Reconnecting Sociology and Public Administration

Philippe Bezes

\section{Public Administration and Cultures}

1 Challenges in the Face of Diversities: Public Administration in Spain as an Example

Salvador Parrado

2 The New Diversity: Increasing Ethnic Heterogeneity and its Consequences for Public Governance

Mark Bovens, Roel Jennissen, Godfried Engbersen and

Meike Bokhorst

3 In Search of a Better Understanding of Cultural Diversity in European Public Administration Research and Practice, with a Focus on Religion and Language

Bogdana Neamtu

\section{Public Administration and Practices}

1 Shadowland: The Poorly-Mapped, Underdiscussed Yet Vital Interface Between Public Administration Research and Practice 249 Christopher Pollitt

2 Forms of Knowledge for the Practice of Public Administration 273 Edoardo Ongaro

3 Do we Practise What we Preach when we Teach (and Research) Public Administration?

Raffaella Saporito 


\section{Public Administration and Country Perspectives}

1 Public Administration in Estonia: A Search for Identity

Külli Sarapuu and Leno Saarniit

2 Public Administration in France: The Shadow and Light of a Revival

Jean-Michel Eymeri-Douzans

3 Public Administration in Germany: Precarious Present,

Promising Future?

Stefan Becker and Michael W. Bauer

4 Public Administration in Hungary: Emerging Dynamics in an Illiberal Democracy

György Hajnal

5 Public Administration in Italy

Denita Cepiku, Marco Meneguzzo

6 Public Administration in the Netherlands: State of the Field

Philip Marcel Karré, Martijn van der Steen, Zeger van der Wal, and Thomas Schillemans

7 Public Administration Research in Norway:

An Organisational and Institutional Approach to

Political Organisations

421

Per Lagreid

8 Public Administration in Portugal

Filipe Teles

\section{Lessons and next steps}

Lessons and Next Steps

Geert Bouckaert and Werner Jann 



\title{
Preface to the EPPA I Book
}

\author{
by Professor Jean-Michel Eymeri-Douzans (EGPA President, 2019) \\ and Professor Edoardo Ongaro (Past EGPA President 2013-2019)
}

Imagined by Geert Bouckaert, Past President of the International Institute of Administrative Sciences, IIAS, and his colleague and friend Werner Jann, the project baptised European Perspectives for Public Administration (EPPA) - of which this book embodies some of the key findings - undertakes to do something that learned societies rarely engage in. What the initiators have proposed to the epistemic community of scholars studying public institutions on our "old" European continent is to start a collective reflection upon the transformations of researching and teaching Public Administration, looking twenty years ahead from now. In doing so, Geert Bouckaert and Werner Jann have taken inspiration from a similar endeavour already existing on the other side of the Atlantic: the Minnowbrook Conferences. Convened every 20 years, at Syracuse University's Minnowbrook Conference Center, they bring together major scholars in Public Administration and management to discuss the state of the field and its future: the first meeting was held in 1968 under the high patronage of Dwight Waldo, Minnowbrook II in 1988, and Minnowbrook III in 2008. In a spirit of lively trans-Atlantic dialogue, Geert Bouckaert and Werner Jann have imagined that, also every twenty years, but in the "entre-deux" decade, starting from 2018, we, the Europeans, could have our own Minnowbrook exercise: that is precisely what the European Perspectives for Public Administration is all about!

EPPA can be seen as a sort of secularist and collective "spiritual exercise": we are asked to abandon our obsession with daily tasks and short-term duties, which are too often the vehicles for path-dependent and decreasing-returns thinking, and rather to take the move from longer-term possible or plausible futures, and then, from this unusual standpoint and perspective, reconsider how we research and how we teach public institutions. The EPPA exercise is a salutary effort to "think out of the (chronologic) box" to bring renewed lucidity and discernment to the very centre of our academic field, and revisit its foundations through embracing the challenges that confronting synchronic and diachronic variations 
inevitably brings with it. In particular, EPPA is a way of questioning the ways and means in which civilisations (including religions) and cultures (national, but also regional, or even institutional ones) have an influence on public administration, both as a practice (or "craft") and as an academic discipline, and what it means to develop research and teaching for an increasingly interdisciplinary field like Public Administration. We are so happy and proud that - thanks to the strong commitment and cordial leadership of Geert Bouckaert and Werner Jann, and also the excellent work of Jana Bertels - the EPPA I, which has so widely involved the participation of the EGPA community, has achieved its ambitious goals.

As a jewel of EGPA, the EPPA project happens to be a shining illustration of the progressive institutionalisation of EGPA in the European landscape as a major learned society, and an engine for the development of the study and the practice of public institutions and public policies worldwide. It is obvious that there are many functional, cultural and institutional good reasons for the existence of a European Group for Public Administration, a continentallevel form of governance of the organisation of research and teaching in the administrative sciences (Ongaro, 2019), between national associative forms and the global one (like the IIAS). The success of EPPA I is another testimony to this, as only a European dimension could have enabled the attainment of the results already achieved by it. The consolidation of EGPA as an institution and a leading learned society in the world has taken place thanks to the engagement of thousands of talented colleagues, through the chain of generations since the mid-1970s when EGPA was established (Bouckaert, van de Donk, 2010), and through the leadership of successive EGPA Presidents: the EPPA initiative, launched by two honorary EGPA Presidents, Werner Jann (2001-2004) and Geert Bouckaert (2004-2010), is another magnificently carved stone added to the solid walls of our common house.

The work of good craftsmen never ends. The EPPA I findings now being published here, it is not too early to start thinking in the perspective of EPPA II... for 2038: long live EPPA!

\section{References}

Bouckaert, G., \& van de Donk, W. (Eds.). (2010). The European Group for Public Administration (1975-2010): Perspectives for the Future. Brussels: Bruylant.

O'Leary, R., Van Slyke, D. M., \& Kim, S. (2010). The Future of Public Administration around the World: The Minnowbrook Perspective. Washington, DC: Georgetown University Press.

Ongaro, E. (Ed.). (2019). Public Administration in Europe: The Contribution of EGPA. London: Palgrave. 


\section{Acknowledgments}

This book would not have been possible without the amazing and quite unforeseen support of a great number of institutions and individuals. We are first of all both extremely grateful to the Alexander von Humboldt Foundation for the generous funding of this project through the Anneliese Maier Research Award, which allowed us to pursue unconventional questions and use even more unconventional ways to develop our agenda. Only a highly unusual and flexible research grant like this could make a project like this possible.

The project got under way when in September 2014 the Alexander von Humboldt Foundation awarded the Anneliese Maier Research Award to Geert Bouckaert. The (quite impressive) prize money, lasting for five years, stipulated that it should be used to strengthen Public Administration Teaching and Research in Germany, and that it should be undertaken in cooperation with a German university, but otherwise there were no strings attached. Geert Bouckaert choose the University of Potsdam and Werner Jann as his partners, and both of us decided that the money should be used not just for Germany, but for Public Administration in Europe in general.

Besides the Alexander von Humboldt Foundation we are therefore also very grateful to our two universities, in particular the Potsdam Centre for Policy and Management, as part of the Universität of Potsdam, and the KU Leuven Public Governance Institute, which supported this project right from the beginning, and especially Inge Vermeulen, who administered all the technical aspects of our project.

We are particularly grateful to the more than 150 participants of our four thematic seminars and the final conference at the University of Lausanne, which provided the necessary inspirations, debates and vital support for our project. We thank IDHEAP (Institut Des Hautes Etudes en Administrations Publiques) at the University of Lausanne and the CNFPT (Centre national de la fonction publique territorial, especially Benoit Cathala) in Strasbourg which, besides our two universities, hosted these events. The final conference in 2018, in Lausanne, gathered together the leadership of EGPA, Edoardo Ongaro, of NISPAcee, György Hajnal, and also the convenor of the latest Minnowbrook conference, Tina Nabatchi from the Maxwell School at Syracuse University. They all supported and encouraged this visionary project.

Last not least, we want to thank the contributors to this volume for their flexibility and willingness to answer our call for short, topical or even 
provocative essays, outside the usual academic comfort zone. The team at Leuven University Press did a great job in preparing this publication.

We are particularly grateful for the enthusiasm and support of the entire EGPA steering committee and its executive secretary Fabienne Maron. The decision of the consecutive EGPA presidents Edoardo Ongaro and JeanMichel Eymeri-Douzans to turn EPPA into a strategic EGPA project makes us proud and confident about the project's future. We are looking forward to the next milestones of this project in 2038 and 2058 , in dialogue with Minnowbrooks IV and V, in 2028 and 2048.

Finally, this whole project, the conferences and the publication would not have been possible without the exceptional and extraordinary support of Jana Bertels, who managed and navigated this obviously not always very easy programme (and its two leaders) with her unfailing good humour.

We dedicate this book to our friend and colleague the late Christopher Pollitt, who was a great inspiration during its conception and the early years. Unfortunately he cannot see the final result, nor tell us how to improve it.

We hope this book will inspire the European Public Administration community in its research and teaching strategies for the next twenty years.

Geert Bouckaert and Werner Jann

Leuven and Potsdam, September 2019. 


\section{About the Authors}

Michael W. Bauer is Jean Monnet Professor of Comparative Public Administration and Policy Analysis, German University of Administrative Sciences, Speyer.

Stefan Becker is Post-doctoral Fellow at the research unit of the Chair of Comparative Public Administration and Policy Analysis, German University of Administrative Sciences, Speyer.

Jana Bertels is Junior Researcher at the Faculty of Economics and Social Sciences, University of Potsdam.

Philippe Bezes is CNRS (Centre National de la Recherche Scientifique) Research Professor in the Centre d'Études européennes et de Politique comparée (CEE) at Sciences Po, Paris.

Meike Bokhorst is Senior Researcher at the Netherlands Scientific Council for Government Policy (WRR) in The Hague.

Geert Bouckaert is Professor at the Public Governance Institute at the Faculty of Social Sciences, KU Leuven. He is the former president of the International Institute of Administrative Sciences (IIAS) and of the European Group for Public Administration (EGPA).

Mark Bovens is Professor of Public Administration at the Utrecht University School of Governance and member of the Netherlands Scientific Council for Government Policy (WRR) in The Hague.

Martin Burgi is Professor of Public and European Law at the LudwigMaximilians-University in Munich as well as Director of the Public and European Law Research Centre for Public Procurement Law and Administrative Cooperation.

Denita Cepiku is Professor of Public Management at the Department of Management and Law in the Faculty of Economics at the University of Rome Tor Vergata. 
Godfried Engbersen is Professor of Sociology in the Department of Public Administration and Sociology at Erasmus University Rotterdam, and a member of the Netherlands Scientific Council for Government Policy (WRR).

Jean-Michel Eymeri-Douzans is Professor at the Institute of Political Studies of Toulouse. He is President of the European Group for Public Administration (EGPA).

György Hajnal is Professor at the Department of Public Policy and Management, Corvinus University of Budapest, and Research Chair at the Centre for Social Research of the Hungarian Academy of Sciences. He is the president of NISPAcee.

Thurid Hustedt is Professor of Public Administration and Management at the Hertie School, Berlin.

Werner Jann is Professor emeritus of Political Science, Administration and Organisation at the University of Potsdam and the former President of the European Group for Public Administration (EGPA).

Roel Jennissen is Senior Researcher at the Netherlands Scientific Council for Government Policy (WRR).

Paul Joyce is Associate Professor in the Institute of Local Government Studies (INLOGOV) at the University of Birmingham and Visiting Professor of Public Management at Leeds Beckett University (UK).

Philip Marcel Karré is Assistant Professor in Public Administration at Erasmus University Rotterdam.

Meelis Kitsing is Head of Research at the Foresight Centre and Professor at the Estonian Business School, Tallinn.

Per Lægreid is Professor at the Department of Administration and Organisation Theory at the University of Bergen. 
Marco Meneguzzo is Professor of Public Administration and Management at the Department of Management and Law in the Faculty of Economics at the University of Rome Tor Vergata, Italy, and Professor of public and non-profit management at the Faculty of Economics at the Università della Svizzera Italiana, Switzerland.

Bogdana Neamtu is Associate Professor in the Department of Public Administration and Management, Babeş-Bolyai University, Romania.

Edoardo Ongaro is Professor of Public Management at the Department of Public Leadership and Social Enterprise, the Open University, UK Fellow of the Academy of the Social Sciences and former president of the European Group for Public Administration (EGPA).

Salvador Parrado is Professor at the Department of Political and Administration Science at the National Distance Education University (UNED), Madrid.

Christopher Pollitt was Professor emeritus at the Public Governance Institute at the Faculty of Social Sciences, KU Leuven.

Tiina Randma-Liiv is Professor of Public Policy at the Ragnar Nurkse Department of Innovation and Governance, Tallinn University of Technology.

Raffaella Saporito is Associate Professor of Practice of Government, Health and Not for Profit at the SDA Bocconi School of Management, Milan.

Leno Saarniit is Lecturer and Programme Director of Bachelor studies at the Ragnar Nurkse Department of Innovation and Governance at Tallinn University of Technology.

Külli Sarapuu is Associate Professor at the Ragnar Nurkse Department of Innovation and Governance at Tallinn University of Technology.

Riin Savi is a Research Fellow at Ragnar Nurkse Institute of Innovation and Governance, Chair of Public Management and Policy, at Tallinn University of Technology. 
Thomas Schillemans is Professor of Public Administrations at Utrecht University and is co-dean at the Netherlands School of Public Administration (NSOB).

Filipe Teles is Assistant Professor in the Department of Social, Political and Territorial Sciences at the University of Aveiro.

Martijn van der Steen is an Endowed Professor in Public Administration at the Erasmus University Rotterdam and Associate Dean and Deputy Director at the Netherlands School of Public Administration (NSOB).

Zeger van der Wal is an Endowed Professor in Public Administration at Leiden University and Associate Professor at the Lee Kuan Yew School of Public Policy of the National University of Singapore (NUS). 
I

INTRODUCTION 



\title{
1 \\ The EPPA Project
}

\author{
Geert Bouckaert and Werner Jann
}

\section{Why EPPA?}

This book is a summary of a five-year project attempting to map and define "European Perspectives for Public Administration" (EPPA). The project got under way when, in September 2014, the Alexander von Humboldt Foundation awarded the Anneliese Maier Research Award to Geert Bouckaert. The (quite impressive) prize money was available for five years on condition that it would be used to strengthen Public Administration teaching and research in Germany, and that the project would be undertaken in cooperation with a German university. Geert Bouckaert chose Potsdam University and Werner Jann as his partners, and we both decided that the money should not be used just for Germany, but for Public Administration teaching and research in Europe. In hindsight, the main reason for our decision was probably the fact that both of us had been former presidents of the European Group of Public Administration (EGPA) and that, over the years, we have shared a keen interest in the future of PA in Europe. ${ }^{1}$

In the context of strengthening Public Administration in Europe, here understood to mean the undertaking of academic research and teaching, ${ }^{2}$ it was our principal aim from the beginning to start an informed debate about the most significant developments and problems in our field of study and research, and about a distinctively European way forward. From our many discussions with peers, colleagues and practitioners we knew that many of them shared our deep concerns about the present state and future of PA in their countries. Are we asking the right questions? Are we giving relevant answers? Do we attract first class students and researchers? Are we using appropriate methods and theories, and so on?

But just how justified and valid are these concerns? In order to give these debates a more solid grounding, we decided on a two-step approach. First, we carried out a predominantly quantitative survey among members and guests of EGPA (see next chapter), and to follow up the results we organised a 
series of four intensive seminars. As our time frame we chose the next twenty years. This is not too far ahead to become too speculative, but also far enough removed from our daily concerns. This book aims to summarise our main findings from these seminars and to sketch a way forward for PA in Europe. Above all, it aspires to spark ongoing discussion and commentary, which we hope will continue at the EGPA in the years to come.

In this introduction, our discussion proceeds through five steps. Following this brief outline, in the next section we deal shortly with the different names and concepts associated with Public Administration in recent decades, and how we interpret these diverse approaches. After that, we give an overview of some of the previous attempts to "take stock" of PA, both in Europe and the US and, based on these attempts, summarise our main aims and concerns, which we describe as the "four pillars." Finally, we explain how we have organised our project and subsequently this book. In the next chapter, we present a summary of the key results of our survey.

\section{$2 \quad$ Public Administration: what's in a name?}

Before we look at the different attempts to "take stock of" and survey our field of study and research, we need to clarify what the subject of our concern is. What do we mean by Public Administration? Obviously, our field of studies has had and still has different names. Public Administration (PA) is probably the oldest and carries, at least in some quarters, connotations of old-fashioned bureaucracy, formalism, a fixation with rules and dusty files. In the 1960s, public administration in the US became so unfashionable - "the backwater of the profession" - that a number of scholars, supported by the Ford Foundation, invented and defined a new field, and even new schools of Public Policy (PP). They were supposed to be more quantitative, more academic and more prestigious, and, at the same time, more concerned with outputs and outcomes than with structures and processes. About 20 years later, Public Management (PM) emerged as a new concept, again more modern, more inspired by the private sector, more dynamic and resource-oriented (see Jann 1991 for a brief narrative of these developments). But even this innovation did not last long - what innovation does? Public Governance (PG) has been the most recent addition, which is more network- and civil society-oriented, even more modern and up to date, and so on. These days we have schools, departments and degrees in PA, PP, PM and PG.

The obvious question following from this is, what's next? There is no end to history and we will certainly see new concepts, new schools, new 
questions and new theories emerging. But our project is not about predicting the next big theme or "fad," but rather preparing ourselves to be open to new questions and challenges, to be able to cope with and understand them, and eventually influence how we can better deal with future and as yet unknown developments and challenges.

So what's in a name? It is not difficult to identify scholars, papers and books that more or less clearly belong to one of these traditions. But what is their association, how do they relate to each other, and is it at all possible to define or even defend borders and distinctions? We will not be able to resolve this ongoing debate in this paper, with all its intricate connections to different disciplines and traditions, but we want to clarify how we have approached these confusions.

We start with Pollitt's observation of the alleged differences between Public Administration and Public Management (see Table 1), but want to add some more disciplinary and even national traditions, which as ideal types basically look like this:

1. Public Administration and Public Management belong to two different disciplines, one inspired and dominated by Political Science, the other by Generic Management. This we would call the Traditional Teutonic Type, still prevalent in most parts of Germany and continental Europe, where a professorship position in Public Management would usually never be filled by a social scientist, but by someone from Betriebswirtschaftslehre (BWL), a specific form of management teaching, which sees itself as a special subfield of Economics and is always located in Departments of Economics. Here, most of the old stereotypes put forward by Christopher Pollitt still exist.

Table 1 Alleged Differences of Character and Focus between Public Management and Public Administration (Pollitt 2016, 6)

PUBLIC ADMINISTRATION (PA)

Old-fashioned, traditional, introverted

Static - hierarchies and procedures

Focus on following rules - compliance and accountability

Focus on machinery of government
PUBLIC MANAGEMENT (PM)

Modern, outward-looking

Dynamic - leadership, innovation

Focus on managing resources - efficiency and performance

Focus on multi-stakeholder governance 
2. Another ideal type we would call the Traditional US Type. Here Public Administration is the more generic term, and Public Management is a subfield of the overall field. Professors of PA may be either social scientists or more traditional management scholars (but not usually economists), and the department may be called PA, PM, PP or PG, although the more generic term is $\mathrm{PA}$, which includes management as one obvious function of PA, but which is not identical to PA.

3. The third is the Northern European Type. This ideal type of PA is closely related not only to Political Science, but also to Organisation Theory (OT) in the March/Simon/Olsen tradition. Since management is seen, as a subfield of OT, usually established in modern business schools, there is no specific field of PM. PA is defined only by its material object, and management is an obvious function of public organisations.

4. This is also true for the fourth ideal type, which we would call the inclusive European Type. Here both PA and PM mean the same and there is no real theoretical or practical difference between them. This is the stance that Christopher Pollitt has taken for many years (most recently and distinctively in Pollitt 2016), whereby professors may be called one or the other, but the designation does not signify anything about disciplinary or theoretical background. In this tradition, many well-known professors of Public Management are typically social scientists by training and orientation.

Obviously, there are many more facets to these debates, amongst others the tradition of "administrative sciences," or how types are embedded in different faculties (see EGPA 2010). These ideal types have all kinds of combinations and subfields, which still cause a lot of confusion. But for our approach we have used the fourth ideal type. We do not neglect the differences between $\mathrm{PA}$ and PM in certain countries, but we use the more general term of Public Administration for our project and survey.

\section{Predecessors: on the shoulders of giants?}

Over the years there have been several repeated attempts to review the stateof-the-art and formulate its future directions, and this may even be a sign of a certain lack of maturity of the field. Some years ago, Christopher Pollitt even quipped that PA is suffering from some kind of "multiple personality 
disorder," a long-term chronic condition which in recent years has appeared to be getting slightly worse, but which does not appear to be having any serious effects on our ability to function in real-world contexts (Pollitt in this book).

But the cyclical need to reflect on the discipline and the field of research and teaching is obviously driven by a series of reasons and tensions, with underlying assumptions, theories and models, which appear to be unfit for addressing, let alone solving, emerging and re-emerging problems. These existentialist questions have driven scholars from different disciplines and fields of research again and again to organise reflective discussions within the community of Public Administration. From this point of view, it is useful to look briefly at some of these past initiatives.

\subsection{Europe and EGPA}

In Europe, a number of attempts have been made to map and bring the field into focus, the oldest and perhaps most significant of which is the "Bielefeld Project" (1985). In 1981-82, the Centre for Interdisciplinary Research at the University of Bielefeld hosted the meetings of a research group called "Guidance, Control, and Performance Evaluation in the Public Sector," which brought together participants from eight countries and a range of disciplines. The group's main focus was to "develop conceptual frameworks which help to understand the structured complexity of relationships within the public sector as a device to redefine situations for the actors concerned" (Kaufmann, 1986, p. 3). Even though there was a strong German presence, the aim was to be interdisciplinary and international. A key message that emerged from the discussions was that complexity needs to be taken into account, and that greater complexity requires increasingly complex theories and solutions. After looking at trends and issues and the shortcomings of current approaches, some of the problems such as "state vs. society," "performance" and quangos were redefined, and there was a special focus on "coordination." The conclusions were specialisation, new division of labour and coordination. These were interesting findings, but they did not really resonate very much in the field.

Other initiatives to "take stock" of the field of PA in Europe were undertaken by the European Group for Public Administration (EGPA) on the occasion of its $4 \mathrm{O}^{\text {th }}$ anniversary within the IIAS family (the International Institute of Administrative Sciences was founded in 1930), resulting in an anthropological book on the community of PA in Europe and European PA (Bouckaert and Van de Donk, 2010). Many concerns, which have since come up in our survey and in our seminars, were articulated, but still in a rather unsystematic way. The precarious relationship between the supply and 
demand of knowledge in PA was mentioned several times, together with the importance of training, as was the growing necessity of taking languages and different PA cultures into account. The problematic relationships between different disciplines were discussed, as was the unsatisfactory state of Public Law. The necessity for a more systematic institutional foundation for PA teaching and research was also a common concern. Finally, Pollitt recently published (2016) an enhanced and advanced "taking stock" of the field in which he looks at theories, methods and approaches, the community and its relationship with practice. He also identifies future trends such as fiscal austerity, technology, demography, climate and complex interactions.

Many of these themes were taken up in the most recent survey of Public Administration in Europe by EGPA (Ongaro (ed.), 2019), so there can be no doubt that the discussions within EGPA are the main inspiration and basis for our project. But there is another, more distant inspiration and motivation, which we have to acknowledge, because its focus on the relationship between overall societal and political developments and the development of PA, and therefore on the importance of values, is particularly relevant for our approach.

\subsection{Inspiration Minnowbrook}

Again, also in the US, there have been a number of attempts to survey the field. The Public Administration Review (PAR) published a "Symposium on the Future of Public Administration" (PAR, 2010), with a special section on "The Future of Public Administration as a Scholarly Field," and Raadschelders, as a long time editor of PAR, summarised these debates on several occasions (see Raadschelders, 2011). However, most of us actually felt that these contributions were overly concerned with intellectual traditions, methodology, epistemology and ontology, and insufficiently concerned with questions of relevance and values. On the occasion of its $75^{\text {th }}$ anniversary, the American Society for Public Administration (ASPA), published an overview of key topics with timelines and major "events" and trends (Guy and Rubin, 2015) and finally, at the $75^{\text {th }}$ anniversary of PAR, Bradley Wright (Wright, 2015) went back to the original controversy between Dahl and Waldo, and discussed how PA had done in Dahl's three critical areas, i.e. normative values, research methods and theories, and social and cultural settings.

This brings us back to the Minnowbrook Perspective, which we found highly instructive for our project. Minnowbrook is a small city in the Adirondack Mountains in Upstate New York, where Syracuse University has a conference centre. At this very location, the twenty-yearly milestone conferences in 1968,1988 and 2008 were organised to reflect on the future of PA from a US 
perspective. The "Minnowbrook" process was initially dialectic and based on small groups within a specific (USA) context. The process started in 1968 and the aim was "through the demonstration of a compelling manifest expertise, [to] assert an authority of legitimacy and thereby influence the course of the future inquiry and endeavour in Public Administration" (quoted in Marini, 1971, p. 7). Apart from the national and international political context, there were other elements that were significant at that time: several disciplines had "revolted," there was "new" sociology, "new" history, "new" political science; thus a combination of young intellectual revolts and confrontational politics, also at universities, added to "turbulent times" (Waldo, 1968).

Minnowbrook I (1968): The two major drivers for this first conference were that "neither the study nor the practice of Public Administration was responding in appropriate measures to mounting turbulence and critical problems" (Waldo, 1971:xiii). Second, there was a worry about the age of those producing research and teaching in PA, especially in 1968, where generation gaps and capacity for the future were at stake. All this sounds familiar today. The Minnowbrook I Perspective reflected and helped to catalyse a "new" Public Administration. It also helped to set new agendas for new topics and focused on adaptation, capacity and organisational development, normative and empirical theories, comparative Public Administration, policy-making and rationality; for example the failure of PPBS triggered debates about the inadequacy of traditional theories. Relevance and the "recovery of relevance" were key topics.

These debates were embedded in the specific context of the Vietnam War and the student revolts. Frederickson's conclusion of Minnowbrook I is coined in the term "New Public Administration." "The classic answer has always been the efficient, economical, and coordinated management of (the) services (...). The rationale for Public Administration is almost always better (more efficient or economical) management. (...) New Public Administration adds social equity to the classic objectives and rationale" (Frederickson, 1971, p. 311). This results in academic PA transcending the elements of POSDCORB (Planning, Organising, Staffing, Directing, Coordinating, Reporting and Budgeting) and shifting to become a "change agent." The Minnowbrook I perspective was, according to Marini (1971, p. 353), a shift in Public Administration to post-positivism, relevance, adaptation to turbulence, and use of new organisational forms with a client focus. However, what Minnowbrook I missed was the emerging economics revival focusing on public choice, and in its wake the new competition in public policy analysis.

Minnowbrook II (1988) was again driven by an awareness of a changed world with a PA not fit for that purpose. There was a new consciousness of the 
importance of the interdependence and interconnectedness of policy issues, private-public organisations, and nation-states, combined with a cultural diversity in a variety of forms (workforce, public, and the world in general). This pushed the participants to the conclusion that problems ultimately cannot be solved, but can only be ameliorated, and that the public administrator is a central actor taking on several new roles "beyond efficient and effective administrator, including facilitator, negotiator, and ameliorator" (Bailey and Mayer, 1992:viii). This again required "a new paradigm, a new view, a new orientation" (ibid.), and feminist views on and in Public Administration, for example.

Although Minnowbrook II built upon its original ideas and for some could be considered as a further development of New Public Administration, there were significant differences and concerns. As a consequence of interconnectedness "every solution creates its own new problems" (Bailey in White, 1992, p. 160). As a further consequence, "public administrators face a dynamic and fluid web of interconnected problems with a feeling of 'constrained hopefulness' about government's role in solving them" (Guy in White, ibid.). The 1988 version of Minnowbrook was thus influenced by post-modern thoughts: "Critiques of positivism, interconnected problems, and feelings of constrained hopefulness are manifestations of society's movement into the postmodern era, an era in which institutions such as science and government are losing their legitimacy. Stories about the power of science and government to solve problems are no longer universally believed" (White, 1992. p. 160). But what Minnowbrook II missed was the emerging New Public Management, and its ever-stronger belief in best practices and generic solutions.

Minnowbrook III (2008) combined critique from a historical perspective and new topics. A whole range of questions, which included "black public administration," PA in Asia, Global PA, the role of networks, and the impacts of markets, were discussed, and the main topic that surfaced was the impact of globalisation on the field of Public Administration. This included "increased studies in comparative public administration, more public policy research across international boundaries and the increased role of international organisations in governance" (O'Leary et al., 2010, p. 282). A second focus was on collaborative governance. This included two shifts, from "performance management to democratic performance governance" on the one hand (Moynihan, 2010), and government potentially not being the only or even not the most important actor on the other. "Governance forms and functions are evolving and manifest themselves and their connectedness to others through networks, contracts, and a range of information technology innovations. (...) Emerging from these governance changes is a field of public 
administration practice that is becoming more professionalised than in the past in terms of systems, processes, and tools. (...) This is one indication that while governance has become more global, diverse, and represented by complex governing arrangements and value, it also has departed from the long dominant norms embedded in Western notions of democratic governance" (O'Leary et al., 2010, pp. 284-285). It was even suggested that this could become the topic for Minnowbrook IV in 2028. The third major topic was the role of information technology.

Again, the most crucial topic was relevance. David Rosenbloom summarised five proactive steps for the field of Public Administration: aggregate knowledge in the sense of making it cumulative; maintain public administration's methodological and epistemological pluralism; maintain public administration's value diversity; continue to promote disciplinary boundary spanners; and monitor relevance (Rosenbloom in O'Leary et al., 2010, pp. 290-291). However, what Minnowbrook III missed was the global economic crisis and its impact on the public sector.

Finally, there was in 2018, outside the usual 20-year cycle, a meeting to commemorate "Minnowbrook at 50," under the heading of "Revisit the Administrative State" in a time of revolutions (Nabatchi and Carboni, 2019). The results are very much in the tradition of Minnowbrook, stressing relevance, the integration of Public Administration and its democratic foundations, but the debate seems to be less radical, more concerned about the profession and the field of PA, and less about how the practice of public administration in American government should change. Recommendations centre on how "compartmentalization, silos, and problematic institutional incentives" prevent relevance, that "unspecified or mistreated analytic levels in research undermine the accountability, generalisability, and scalability of findings, and ultimately hinder the field's ability to solve problems," that the cleavage between academic research and practitioner realities should be addressed through "an approach that aligns public problems and research through stakeholder engagement," and that the "links between democratic institutions and public administration" need to be strengthened. Even "overcoming the American centricity" becomes an issue. All this is very much in line with our findings and concerns, so there is obviously a common agenda. But Europe is not the US; we face different challenges, and that makes it even more necessary to develop a European agenda and to strengthen the European voice in Public Administration.

There are many aspects of the Minnowbrook approach which one could criticise. There is, for one, the sometimes rather parochial perspective. While Minnowbrook III was, for the first time, all about globalisation, comparison 
and collaboration, there were no significant inputs from international scholars and perceptions, and, in particular, no European voice. Minnowbrook is openly normative, but often it is not very clear where these norms come from and how they are legitimised. Furthermore, sometimes discussions do not very clearly distinguish between changes in the practice of public administration and the necessary academic and scientific developments in Public Administration to deal with all these challenges. But despite these shortcomings, some of which have been taken up in the 50 years conference, we are convinced that the Minnowbrook Perspective is an important inspiration for our project. From it we learn that we have to deal with developments outside Public Administration to understand necessary changes, we have to confront the questions of values, where they come from and what role they should play, and also that we have to deal quite explicitly with the future.

\section{$4 \quad$ Critical concerns: four pillars?}

When all these past efforts of "taking stock" and "substantial reflections" are analysed, we end up with an underlying set of critical common denominators, assumptions and expectations. Put rather crudely:

1. Public Administration research and teaching run too much behind facts and developments; however they should also be in front of the facts, they should not just be pushed by realities but also pull realities;

2. Public Administration is too much dominated by disciplinary boundaries and epistemological concerns; however, it should be much more taking several disciplines and approaches into account and result in an equilibrated approach;

3. Public Administration is thinking too much in causal terms; however, it should also, as a social science, think in teleological terms;

4. Public Administration is often pretending to be disconnected from time and space; however, it should actively and positively take context and culture into account;

5. Public Administration research aims to be relevant for practice; however, it should critically anticipate its future relevance for public administration and governance.

Even though these undertakings provide much inspiration, we were nonetheless convinced of the need for new inputs in this discussion and, furthermore, that we need a distinctively European view. Our basic question is how we, as researchers and teachers, will and should deal with the changing role of 
public administrations and the public sector in Europe. Our aims are to define the role of Public Administration (as an academic undertaking) in the universities and academic world of the future, to take alternative cultures and futures into account, to take multidisciplinarity seriously, and to strengthen the European voice in the world. We therefore distilled from our reading of the past discussions of our field four interrelated critical concerns, which we named our "four pillars":

1. Keeping an eye on the future: How should we deal with the future? Can we learn to trust utopias and distrust dystopias, and think beyond short-term problems and solutions while trying to be as realistic as possible at the same time?

2. Keeping an eye on the disciplines: What can we learn and how can we cooperate beyond the established disciplinary boundaries? What are the various roles of disciplines in PA, and how should we deal with them?

3. Keeping an eye around us: How can we take different cultures seriously avoiding a "one-size-fits-all" approach, while at the same time continuing to learn from each other?

4. Keeping an eye on practice: What can we teach and how can we help students to learn; what are the practical lessons of all this for teaching and research?

The first topic is thus about the role of the future, and different futures, in our research and teaching. What role do utopias and dystopias play in public administrations and Public Administration? How should we deal with them in academic teaching and research? Just to ignore them does not seem to be a very convincing option. The second topic is re-emphasising the role of established disciplines and of the existing and necessary contributions from economics, law, psychology, political science, history, anthropology and other relevant disciplines in the field of Public Administration. How can we establish new forms of cooperation and learning, and how can we avoid, or at least break down, academic silos and established misunderstandings? The third topic focuses on the fact that academic Public Administration has been for many years very Anglo-Saxon-oriented in the sense that it basically ignored contributions from public law and different national cultures. How can we change this parochial view? Finally, the fourth topic is about organising the accumulation and dissemination of knowledge. How can we strengthen comparative research and teaching, and how can we integrate different disciplines and scientific approaches into public administration practices? How can we make both our teaching and our findings more attractive not only for the practice of governments and governance, but also for students and academics? 
Why European perspectives? There is a difference between European Public Administration (EPA) and Public Administration in Europe (PAE), and it is necessary to differentiate between these two approaches. EPA takes the contingencies and features of Europe into account. It starts with the European specificities and moves on to the general and generic levels. PAE is more about applying general knowledge to the European sphere of public administration. Both approaches invite comparative research and learning from other practices.

There are specific problems facing Europe which need to be addressed, especially by European scholars. Studying the functioning of the European Institutions and their policies, and their interactions with Member Countries, is one of the most important areas where European Public Administration needs to increase its relevance and its capacity to be part of the solutions. At the same time, Europe is about ethno-linguistic and cultural diversity. There are 23 official languages in the European Union. To bring unity in diversity in the context of "requisite variety" becomes an important assignment for Public Administration.

The transformation of public administration systems across Europe is a combination of causality and path dependency as a push factor, but also, and to a greater extent, of a teleological drive as a pull factor. Defining this "telos" should be part of the role of Public Administration to develop possible futures. The European Union has moved from a chapter in foreign policy to a chapter in domestic policy and politics. Therefore, Public Administration also needs to move from Public Administration in its separate Member Countries to Public Administration in Europe, and ultimately to European Public Administration. This needs the broad umbrella of European Perspectives for Public Administration.

\section{Structuring our work: how did we proceed?}

The "four pillars," their main elements and essentials, emerged both from our interpretation of past discussions and reflections and from our survey, which we describe in detail in the next chapter. In order to deepen our understanding, to clarify and elucidate both relevant questions and possible answers, we first decided to organise four intensive seminars. We invited colleagues, both established and younger ones, from all parts of Europe, from different backgrounds and specialisations, and also from other disciplines, to get an outside view. 


\subsection{PA and futures (20-21 October 2016, Leuven)}

The aim of the first seminar - which took place at KU Leuven on the occasion of the Leuven city festival "5oo YEARS UTOPIA" dedicated to Thomas More's "Utopia," published in 1516 in Leuven - was therefore to discuss "possible futures" and bring "utopias" as one method back to social sciences, and especially to Public Administration. In five sessions on "general futures and innovations," "future cities, utopian architecture," "future citizens and diversity," "(big) data \& IoT" and "Ecotopia," we discussed approaches to envisage possible futures and future challenges for the public sector and, following on from that, for Public Administration as an academic field of study.

In the end, the seminar generated more questions than answers, but at least these were very salient and productive. As a starting point to follow up on the discussion about the future of the European Public Administration we captured seven "lessons learned," which are summarised below.

1. Problems with utopias? PA scholars are not accustomed to thinking about and with utopias because utopias have a strong normative connotation. In general, this way of thinking is hard to combine with traditional social sciences, but we need to find more relaxed ways to cope with utopias. "This is utopian" should not be the end of a debate, but a beginning.

2. Utopia by whom and for whom? Where do utopias come from - technocrats, visionary entrepreneurs, civil society? We have to be aware that there are many sources of utopias, and none of them is without its own problems. We should trust and distrust them all, but we should be especially aware of blindly trusting experts telling us how things will be.

3. PA is not a trendsetter? In its own view, PA too often lags behind the trends, only reacting to developments already happening. But why should that be the case? If the aim is to shape the future agenda - and we should not leave this to all kinds of other, often very unscientific and sometimes rather populistic discourses - then we have to engage more with the future, not less.

4. Different rationalities? As always, there are different ways of understanding not only the past, but also the future. So we should be aware of different utopias concerning how a legal, efficient, effective and legitimate public sector could, and should, look. But we should also be aware that perhaps there are hitherto other neglected rationalities.

5. Scale and scope? Should utopias be more local or more global? Should they be long-term oriented or should they be about our immediate future? There is no definitive answer. We need to be open to different provocations. 
6. Utopias for some, dystopias for others? Obviously we will not agree on utopias. While some may strive for a totally transparent and open society, for others this may be the road to serfdom. We have to live with deep and unresolvable contradictions and ambiguities. We have to accept indeterminable ambiguities as a fact of life.

7. Dangers of utopias? Yes, trying to realise utopias may create severe problems. We have ample experience of this in the last century. But it would be a strange lesson that we therefore should stop thinking about possible futures and how to achieve them.

In the end, while the participants agreed on very little else, everyone understood that for PA there is a vital challenge to organise and institutionalise teaching and research taking "futures" into account, and dealing with them systematically and critically.

\subsection{The many disciplines of PA (14-15 September 2017, Potsdam)}

The aim of our next seminar was to shed light on the relationship of different established social science disciplines with Public Administration, both as an academic field and as an object of teaching and research, in the light of recent developments, both in different disciplines and in the PA community. Starting with the more or less problematic linkages between the major disciplines that constitute the field of Public Administration, key questions were: How are disciplines such as political science, management, law, economics and others, interacting, sharing, collaborating (or not) with and within PA? Is it possible to achieve increasing and stronger synergies to produce relevant knowledge to understand the functioning of the field of public policies and administration? What can we learn and how can we cooperate across the established disciplinary boundaries?

Again, "lessons learned" can only be preliminary and simplified, and again were somewhat controversial.

1. PA is not a (traditional) discipline and should not strive to become one. It is a research platform or research field, a community of interest combining and using different disciplines and methods. This is not a weakness or a deficiency, but a strength and asset of our field. There are few social science research fields that are as interdisciplinary as PA. Despite trends towards an ever-stronger specialisation and "purification" of disciplines, interchanges and mutual learning between the many disciplines of PA have increased in recent years, and not declined. PA needs different and strong disciplines. 
2. But these overall findings are not true for all relevant disciplines and all countries. For example, PA and Political Science are brother and sister as well as opponents. The relationship is close, competitive and based on a certain division of labour, and in Europe PA is generally doing well in the countries where political science is strong. However, especially in the US, the relationship between PA and PolSc is not so friendly, to say the least, and it would seem Europe is imitating this behaviour. Here the more relaxed and productive European perspectives clearly need to be strengthened.

3. Also, the relationship between PA and Public Management is evolving, and in many countries there is now no distinction between PA and PM. Conversely, in some parts of Europe, public management is still treated as something different, is dominated by and usually placed in the traditional faculties of economics or business. It is necessary to change this blinkered approach.

4. The most problematic and challenging is the relationship between PA and Law which, in spite of a long common history in many European countries, live parallel lives hardly talking to one another, like the proverbial "ships passing in the night." Debates in many European countries on changes in administrative law are hardly associated with debates in administrative reforms and theories. PA and Law scholars and practitioners lead separate lives, but in solving practical problems they cannot avoid talking to one another. Therefore the way forward could be to concentrate on practical problem solving in common research projects, the more applied the easier to work together in an interdisciplinary way. This relationship needs to take into account the different context of "common law" countries and “administrative law” countries.

5. The relationship between PA and Public Policy Studies is the most ambivalent. Public administration and policy meet in all kinds of contexts. There is strong agreement that one cannot do Public Policy without PA and there are all kinds of common handbooks, etc., but at the same time silos exist between large conferences and journals. Generally, it is not clear why Public Policy and PA are so often seen as two areas since there are all kinds of common interests, scholars, theories and no obvious methodological differences.

6. That between PA and Sociology, especially organisational and institutional theory, is a rather recent re-established relationship, again in spite of a strong common heritage in bureaucratic theory and Max Weber. Also, here the institutional embeddedness at universities is crucial. Traditionally, organisational theory and "New Institutionalism," for example, 
is often located in Departments of Sociology, or sometimes moved to business and management schools, but it should have a stronger focus in PA programmes and research, i.e. we need more institutionalisation of sociological thinking in organisation and management research in PA.

7. Finally, PA needs to become more aware of new developments, especially in ICT technology, the Internet, clouds, and all the other developments associated with these upheavals. For the time being, PA is in many countries and programmes like a technology-free zone. There is a strong need to build technology and digital data competences in PA departments, and there is an even stronger need to include tech-rich social science thinking in PA.

Also here, the overall challenge is how to organise and institutionalise teaching and research in the future. The relationship between PA and its disciplines has up to now mainly taken shape from different clustering mechanisms, i.e. the clustering of interests and research problems vs. clustering via conferences, journals and associations. Journals especially shape and legitimise what we do not need to know. Journals create silos and make collaborative (interdisciplinary) research less likely. Therefore, we need more and more visible centres of research and teaching where scholars from different disciplines can interact, in both the development and implementation of common curricula and research projects.

\subsection{Cultures, diversity, and Public Administration (14-15 December 2017, Leuven)}

The aim of the third seminar was to discuss and investigate if, and how, cultures in European Public Administration are becoming even more diverse and relevant - and how PA is reacting, should react or could react. What are the defining characteristics and how and why do they change? The challenge is how to frame, discuss, map and improve our understanding of the relevance of different cultures and diversity, defined as languages, religions and ethnicities, and how this may and should, or could, affect the multidisciplinary field of PA in the future.

The seminar was organised around four thematic points. First, setting the scene: what is happening and going to happen? How can we map cultures and diversities, now and in the future, and present facts and figures on the shifts that are taking place? Second, theories and models in PA taking (or not taking) culture and diversity into account. How and to what extent is PA research positioning itself vis-à-vis languages, religions, ethnicities and 
(legal) traditions? Third, country clusters in research. Are clusters helpful; are they changing? What are the research strategies to explain differences based on types of clusters? Fourth, what are the implications for a PA research agenda - what kinds of new data, topics and methods are needed? What are the promising new directions for teaching and research?

Again, we present some preliminary and highly simplified first "lessons learned":

1. Cultural diversity is not - and traditionally has not been - an issue in PA in Europe, except in some very specialised areas. We have very little systematic empirical and theoretical knowledge about how language barriers and religious diversity, for example, have influenced PA in the past, despite the historical diversity of European demography, which is much more diverse, for example, than in the US. Maybe the strong influence from the US has also prevented a more diverse, European view of PA from taking place.

2. Migration will be one of the main challenges - also and especially for public administrations - of the coming decades. Migration is high on the political agenda and will stay there, but PA as academic teaching and research has hitherto widely ignored this topic. The diversity in Europe will continue to increase as a result of migration, and therefore conflicts will also increase. If men define situations as real, they are real in their consequences. And there is a lot at stake.

3. Also religion has been one hitherto widely neglected areas of PA. PA pretends to be neutral and secular, but it is not, at least not always. It has strong implications for everyday issues like education, health and food, which are close to practical public administration. There is no clear distinction between culture and religion, but there should be one. PA is a political vehicle for state actions and is neither neutral nor objective. Therefore, the interregional and interdisciplinary dialogue on religion and PA needs to be strengthened. This would involve new research agendas and questions. How should public policies deal, or not deal, with issues linked to religions, diversity and contemporary problems? Contemporary crisis management and religions - how should we deal with crises and the question of religious diversity in contemporary crises?

4. Language shapes thought, debate and hence also research. A key feature of public administration in Europe is language diversity and the enduring significance of the multitude of national languages beyond English both in the public and the academic debate. Language and language similarities reflect common historical roots and shape similarities, but also differences 
and misunderstandings in governance and administration. This has also been widely ignored in research and teaching. We need to study the theoretical and practical significance of language diversity for PA in Europe. An example would be to link language practices to classic PA topics: street-level bureaucracy and discretion, collaborative governance, innovation diffusion, political-administrative relations, public employee sorting and socialisation, effective policy instruments, and so on.

5. Country clusters (e.g. Nordic, Continental, Napoleonic, Anglo-Saxon, etc.) have until now very much influenced our (comparative) research and teaching of PA, but the question remains how much they can explain, and how much they help and are used in teaching PA. Country clusters may in the future become less relevant than intracountry differences in understanding and explaining behaviour and results.

6. The influence and relevance of new forms of culture and diversity on Human Resources Management and Organisation are still not very well understood and certainly not well researched. What is the role and impact of diversity and representation on public organisations? Some traditional PA concepts and theories deal with these issues, i.e. street level bureaucracy, representative bureaucracy, diversity management, etc., but very little genuine European research exists in these areas. How can we theoretically link diversity and representation in public organisations with the different dimensions of their performance? How can we address the "micro-macro" problem in diversity and representation research as we deal with mechanisms and effects at individual and collective levels? And what contextual factors will affect those mechanisms and effects?

Finally, also here PA research and teaching do not really even reflect the traditional diversity of Europe, let alone new and increased diversities and challenges. Some parts of Europe, even some larger countries in Southern and Eastern Europe, are very often beyond the reach of the mainstream European PA scholarships. The "European perspective" is therefore as of today very much influenced by the studies of a few core EU countries, which have very good established PA scholars and programmes. So again, the relevance of cultures and diversity in PA teaching and research is heavily dependent on the country's capacity for the institutionalisation of programmes. We need more outside challenges to our established convictions and assumptions, and this needs more and more diverse centres of teaching and research. 


\subsection{Public Administration and Practice (5-6 April 2018, CNFPT, Strasbourg)}

The aim of this final seminar was to shed light on the never easy relationship between the practice and the academic teaching and research of Public Administration. Our overall question was: when, how and why is Public Administration relevant, or perhaps even irrelevant in research, advice and teaching? The focus was on PA training and policy advice, and the organisation and institutionalisation of the interaction of research and advice and training. The latter involved asking what kind of schools, disciplines and programmes we have, and which we need for the future.

Again, some preliminary and pointed "lessons learned" from the seminar:

1. How can PA become more relevant in research, teaching and advice? Here, the somewhat surprising consensus was that problems might be more rooted in the supply rather than in the demand for PA knowledge and research. Traditional and rather well researched problems, for example co-ordination, are ever more present in the practice of public administration, but not enough relevant research reaches practitioners. On the other hand, communities, such as local governments, are much more open to practical interaction, i.e. in the form of "action research," than is usually assumed; however they are seldom approached and get few offers. The reason may be that academic PA offers the wrong incentives to researchers, especially young ones. The overriding currency here is articles in refereed journals, so practical relevant research is becoming more and more a luxury not everybody can afford.

2. Concerning the meaning and values of PA and public service, it was argued that normative concepts like values, goals and ethics are of central importance to practice and therefore have to be tackled more systematically in teaching and, following from that, also in research. At the same time, it was emphasised that practice and theory are certainly not opposites, as is so often naïvely assumed. There is, as we all should know, nothing as practical as a good theory, so theory should always enlighten practice. PA teaching, research and advice should therefore be much more concerned with the kind of knowledge they produce and what is demanded (enlightenment, problem-oriented extrapolation of existing knowledge, etc.).

3. What does all this mean for our teaching? Here it was argued, as so often, that context matters, so we should not look for generic models, but for specific cultures, traditions and needs. But there are some important caveats. For one, we should be aware of too much homogeneity among 
trainers and graduates. Programmes may become too uniform, so diversity matters. And again, institutions and infrastructure matter, PA teaching and research are most relevant in countries where there is a strong tradition of PA research and teaching at universities and, following from that, a strong tradition of hiring university graduates in PA for the public service. PA teaching has to create its own demand, not just sit there and wait for whatever kind of demand may come from either more or less well informed practitioners or pracademics.

4. How can and should research be transformed into practice? The obvious, but perhaps somewhat surprising, basic observation was that there are rather similar common reform agendas in most countries, i.e. the challenges of territorial reforms, the digital revolution and how to cope with that. At the same time, especially in these areas, the relationship between research and practice is weak. A lot of existing research is not used, and its existence is probably not even known to practitioners. One of the reasons may be that these areas are weak in theory. Only when robust theoretical assumptions are available can research more easily be turned into practice. Again, it may be a problem of supply. PA needs to concentrate more on synthesising research and making it more understandable, instead of using ever more resources in ever more specialised, but not very relevant, research questions. But, again, the incentives of academia may work strongly against that.

5. The result of all this is, again, that we need to put more resources into the organisation and institutionalisation of research centres and activities distributing and explaining research. We already have rich ecosystems of policy-oriented research centres, and many of these are also fairly prominent in public debates and in the media, but we lack comparable centres in PA. So the main challenge is not more and even more specialised and methodological advanced research, but the organisation of the interaction and "translation" of research.

For our PA schools and programmes, all this means that we should practise what we preach. We do not need more "managerial evangelists" who preach the latest sermon on salvation. We should be aware of superficial legitimacy through simple isomorphism and be more self-confident and assertive about our research findings and results. We need to offer programmes that are attractive to good students, which again means that they should also be attractive to future employers. All this boils down, again, to more resources and especially more attention to top-level centres of research and teaching. PA will not prosper if it consists of many more or less isolated researchers 
(however productive and innovative they may be) who work at the fringes of their different departments.

The rest of this book takes up, discusses and develops these preliminary findings. For each of the "four pillars" we asked both colleagues participating in the seminars and some new colleagues to reflect on our findings and to sketch out how, in their view, recent developments should be interpreted and, especially, how we should cope with these developments and future challenges. Since we have deliberately chosen to use the plural form "European Perspectives" for the future of Public Administration, and since we recognise the diversity and variety that exist across Europe, we decided that it would be highly desirable to have some specific "country visions" on the future of PA. In these country perspectives, we asked for the state of the field of PA in each country, including features of disciplinary dominance or diversity, culture, language (dominance of English?) and links and relevance to practice, efforts within the PA community to reflect on the future of the field, and elements of the futures of PA: priorities for research topics, changed settings, capacity changes (also in teaching programmes), new academic embeddedness and whatever our colleagues deemed important about their countries and the future of PA. We informed all our contributors that we were not expecting a typical academic paper, but rather a topical or even provocative essay on how they see the state-of-the-art and how it could or should develop in the years to come. Again, we have tried to be as diverse as possible, i.e. including both younger and established colleagues from all over Europe.

What we have learned from all this, and how in our view the future of PA in Europe should look, we try to summarise in the last chapter.

\section{Notes}

1. We are both extremely grateful to the Alexander von Humboldt Foundation for this generous funding, which allowed us to frame unconventional questions and use even more unconventional ways to develop our agenda. Only a highly unusual research grant like the Anneliese Maier Research Award could make a project like this possible.

2. In accordance with established practice we use capitals (Public Administration) when referring to the academic field of research and teaching, and "public administration" when referring to the practice of running governments and public organisations (see also Part 3 in this introduction). 


\section{References}

Bailey, M. T., \& Mayer, R. T. (eds). (1992). Public Management in an Interconnected World, Essays in the Minnowbrook Tradition. New York: Greenwood Press.

Bouckaert, G., \& Van de Donk, W. (eds). (2010). The European Group for Public Administration (1975-2010): Perspectives for the Future. Brussels: Bruylant.

Frederickson, H. G. (1971). Toward a New Public Administration. In F. Marini (ed.). Toward a New Public Administration: The Minnowbrook Perspective (pp. 309-331). Scranton, PA: Chandler Publishing.

Guy, M. E., \& Rubin, M. M. (2015). Public Administration Evolving. From Foundations to the Future. New York: Routledge.

Kaufmann, F. X. (1986). Introduction History of the Project and Background of the Problem. In F. X. Kaufmann, G. Majone \& V. Ostrom (eds). Guidance, Control and Evaluation in the Public Sector (pp. 3-24). Berlin: Walter de Gruyter.

Marini, F. (Ed.). (1971). Toward a New Public Administration, The Minnowbrook Perspective. Scranton, PA: Chandler Publishing.

Moynihan D. P. (2010). From Performance Management to Democratic Performance Governance. In R. O'Leary, D.M. Van Slyke, \& S. Kim (eds.). The Future of Public Administration around the World, The Minnowbrook Perspective (pp. 21-31). Washington, DC: Georgetown University Press.

Nabatchi, T., \& Carboni, J. L. (2019). Assessing the Past and Future of Public Administration: Reflections from the Minnowbrook at 50 Conference. Washington, DC: IBM Center for The Business of Government.

O'Leary, R., Van Slyke, D. M., \& Kim S. (eds). (2010). The Future of Public Administration around the World, The Minnowbrook Perspective. Washington, DC: Georgetown University Press.

Pollitt, C. (2016). Advanced Introduction to Public Management and Administration. Northampton: Edward Elgar Publishing.

Waldo, D. (ed.). (1968). Public Administration in a Time of Revolutions. Public Administration Review 28(4): 362-368.

Waldo, D. (ed.). (1971). Public Administration in a Time of Turbulence. Scranton, PA: Chandler Publishing.

White, J. D. (1992). Knowledge Development and Use in Public Administration: Views from Postpositivism, Poststructuralism, and Postmodernism. In M. T. Bailey, \& R. T. Mayer (eds). Public Management in an Interconnected World, Essays in the Minnowbrook Tradition (pp. 159-176). New York: Greenwood Press. 


\title{
2
}

\section{The Survey: A Long-Distance Conversation about the Future of Public Administration in Europe}

\author{
Jana Bertels, Geert Bouckaert, Werner Jann
}

\section{The EPPA survey}

As explained in the introduction, we started our own "soul-searching" exercise about the current state and future challenges of Public Administration by looking at what had been done by our predecessors, in both Europe and the US (see 1.3 in the previous chapter). But we soon found that we needed a more comprehensive and unconventional way to take stock of current concerns, expectations and new approaches. Starting with a large general debate at a conference did not seem to be very helpful, so we decided to start with a broad qualitative survey with mainly open questions.

The idea was to get an overview of the perceptions concerning the future challenges for Public Administration over the next 20 years from a wide range of scholars and teachers across Europe. As previously mentioned, 20 years seemed to be a good period, neither too far in the future nor too close to current difficulties, so the perspective we asked for was from 1995 to 2035. In contrast to other surveys conducted within the Public Administration community in Europe, which mainly focused on the relevance of journals, academic output and prestige, and on the relationship between the disciplines of Public Administration and targeting senior-level academics (see Curry et al., 2014 and the very detailed surveys in Germany carried out by Bauer and Becker, 2017ff ), our survey aimed to capture the varied perceptions held by senior and early-stage scholars on a wide range of future challenges, as expressed in their own words. The open-question design was intended to allow us to get a more comprehensive, fair indication of what our peers and colleagues define as their main concerns, and especially to find out their reactions, suggestions and solutions to future challenges, compared to the 
more standard procedure of closed, multiple-choice questions and answers. From the beginning, we were more interested in innovative ideas and honest opinions, rather than just the general views of the average PA scholar.

The first draft of a set of questions and questionnaire design was prepared at the beginning of 2015 and tested on a small sample of international colleagues in July 2015. After this pre-test, we decided to refine the questions and start each of our main areas of interest for future challenges: futures, disciplines, cultures and practice, with a clear thesis of our own, asking our respondents whether they agreed, and if not, why not. We ended up with 23 open questions, seven closed questions (yes/no or multiple choice with the option to add comments), and five additional questions about the personal background of each participant.

For our survey, we decided to target members of the European Group of Public Administration (EGPA) and participants who attended its 2015 Annual Conference in Toulouse, France. It was not easy to get an overview, or even decide through objective criteria, which respondents belonged to the population of researchers and teachers seriously engaged in Public Administration. Therefore we settled on the criteria of "self-selection." We assumed that scholars who joined EGPA and/or its Annual Conference were at least moderately interested in the field, its problems and future development, and might therefore be interested in answering our questions. Furthermore, EGPA has been the only platform for almost 45 years that enables colleagues from all over Europe to connect with each other. After selecting the sample questions, the survey was adapted as a web-based questionnaire. The technical implementation was outsourced to A\&O Consulting Oppermann, a small research company in Potsdam.

Following the EGPA Conference on 28 August 2015, the questionnaire was sent to about 500 contacts. After two reminders, 162 had started and 68 completed the survey. The majority of the respondents $(75 \%)$ were senior academics, teaching and researching PA at the level of professor (full and assistant), another $15 \%$ held a $\mathrm{PhD}$, and $10 \%$ were $\mathrm{PhD}$ candidates. About one-third had been in the field since 1995 or longer, but still, at least to some extent, we can draw conclusions about the perceptions of the future generation of Public Administration scholars and teachers. The academic backgrounds of respondents were quite diverse, with the majority coming from Political Science and Public Administration, 12\% from Management and about $10 \%$ from Law. Overall, they came from about ten different European countries.

The survey was obviously not representative, but invited participants to comment on a number of (more or less) controversial statements about the 
development of our field. It therefore allowed for a qualitative and discursive analysis of essential positions and contradictions. The raison d'être of our undertaking was to get a broad range of views and to map central concerns and perspectives as comprehensively as possible. Our aim was to start a discussion and not to summarise it, so we were therefore more interested in the diversity and variety of different views held and the major arguments for a changing field of Public Administration. Since we had received, as we had hoped, a large number of written answers and comments to our open questions (usually the length of a paragraph or more), our approach was more similar to a collection of semi-structured interviews than to a quantitative survey (see also Jansen 2011), and much more a conversation than a questionnaire.

To analyse and understand the wide-ranging collection of answers, ideas and concerns we used MAXQDA software for qualitative and mixed methods data analysis. The software helped us to organise and search relevant information in our data using a number of different, specifically developed codes and concepts, and we were thus able to retrieve much more contextual and supplementary information than would have been possible with simple quotations or word counts. We present these findings as a kind of "collage of quotes" that represent the amazing variety of views and concerns, and which should depict a unique picture of our field at the same time.

\section{The Results}

\subsection{The "grand" challenges for Public Administration as a scholarly field}

The first question we asked was a very general and somewhat suggestive one: will Public Administration as an academic field of research and teaching look very different in 2035 from how it was in 1995? The objective was to find out whether our colleagues expect major changes in our field over the next 20 years.

More than $85 \%$ of our respondents expected PA to change. The following is an example of a typical response:

\footnotetext{
"Much of what we accepted as collective wisdom in the 1960s was being challenged in the 1990s and again the views of the 80s and 90s are under increasing scrutiny. The field cannot possibly stagnate."
} 
However, $15 \%$ of our colleagues did not expect any major changes, and the reasons they gave are interesting and thought-provoking (we have put together quotations from different respondents to present the major arguments):

"Of course there will be differences (mainly in the eGov direction, transparency), but I am afraid that human character will not progress so much that we could talk about a very large difference. - Handling politics and production of complex services will not change qualitatively. - The object of what and how we seek to explain public management phenomena will not fundamentally change. - Contemporary western democracy has proven to be a durable institutional framework (...) And I don't see that being fundamentally challenged in Europe, consequently the context and values of PA won't either. Instead they will simply turn in circles: market, community, authority."1

Here an important caveat for change is hinted at, the conservative and stabilising effect of institutions, which sometimes gets lost in the dominating concern with administrative reforms, which has been the hallmark of academic PA, and especially Public Management for many years (Hood, 1991; Christensen \& Lægreid, 2005; Pollitt \& Bouckaert, 2017). It has been observed before that there has been for some time a lack of appreciation for the inertia of institutions, social structures and established ways of life, as well as an overly generous and optimistic view of the capacity of governments and/or leaders for change. But on the other hand, with the rise of populism and anti-liberal movements all over Europe, this is perhaps somewhat too optimistic. Maybe the world has already changed since 2015, when we conducted our survey.

One of our colleagues summed up this optimistic view and hinted at some of the changes, which we still have to expect and to deal with, and which are further developed and discussed in the following responses:

"I think the field will largely address the same fundamental and practical issues as we do today (and have done for over 100 years), but the availability of data will be better and the demands for scientific rigor will be higher."

\subsubsection{Challenges for PA research}

Our findings reveal that better and more data as well as more scientific rigour are among the main challenges that PA research will face over the next 20 years. As we had not expected, respondents expressed less concern about problems of theory or theoretical foundations, relationships with practice, new substantive challenges, even if all of them play a role, and more concern about the methodological foundations of our research. Typical comments were: 
"To make the field more methodological robust; a lot of research findings nowadays in EU PA are rather anecdotal than robust - many [of our] arguments lack extensive empirical support — methodological advancement (qualitative and quantitative) — produce credible causal evidence of our claims. Our main problem today is that very little public policy is shaped by insights from PA."

Looking more closely at methodological challenges - and opportunities - big data is mentioned most often:

"New European databases coming online are superior to anything else out there - information transparency of the public sector as basis for empirical research - to make better use of data which are produced by administrations and to cooperate with the public sector in this endeavour — collecting 'Big Data', that will in time be internationally comparable — to understand how to use internet and 'big data' for PA research."

Areas also mentioned in this regard, but much less frequently, were on the one hand the use of experiments, so here PA is also following mainstream social sciences:

"We need to make experiments our main tool of investigation in order to produce causal claims that in a credible way can inform public policy - We need to provide our students with a much strong ability to apply quantitative research designs and experimental methods."

and a classical concern to carry out more advanced and informed comparative research on the other hand:

"Finding a common base for comparative research — create conditions facilitating continued, high quality comparative work. - Take comparative PA research to a new level (away from country case studies to more advanced statistical methods) — Strengthening comparative researches, starting big research project that involves groups from different countries".

Another important issue raised was the long-standing problem of interdisciplinarity:

"The next big challenge is placing decision-making into more scientific frames, with the more interdisciplinary view — cross fertilization with 
other disciplines - developing PA (...) interdisciplinary and not (only) multidisciplinary, (which is) usually even asymmetric with one discipline too dominating, regardless of being policy analysis, law, management etc. - we (will) need to collaborate much more closely with other disciplines outside of social sciences in addressing issues of governance, especially as we start to acknowledge the complexity in addressing societal challenges (e.g. security, climate change and its social consequences, mobility, poverty, demographic changes, ...)".

The last comment lists the main challenges already addressed in our survey. Migration is very often mentioned, along with other major issues of great societal concern, such as climate change, demography, poverty and security, among others. Austerity was also mentioned, but not so frequently, and interestingly very often with concerns about less funding for universities, students and research, indicating a problem which our respondents have personally and directly experienced.

Overall, these results correspond with the findings of the COCOPS (Coordinating for Cohesion in the Public Sector of the Future) academic survey on European Public Administration indicating that, according to senior-level scholars, Public Administration should become more "internationally comparative" and more "interdisciplinary" (Curry et al., 2014, 28-29). Furthermore, our results show that the emphasis on newly developing social problems as challenges for PA research is about the same as the attention paid to more general theoretical concerns. Typical responses about the challenges were:

\begin{abstract}
"Strengthen the theoretical base - More emphasis should be given to theoretical and philosophical study to earn a more respectable status in basic research - The challenges are improving theory building and connecting the discipline to broader theories in sociology as well as political theory (e.g. rationalism, structuralism or post-foundationalism) - In terms of theory we must be more open to the broader social sciences - in particular cognitive and social psychology".
\end{abstract}

While most of these comments point towards greater interdisciplinarity, also in theoretical directions, others disagreed:

"We need to stop to import theories (...) from other fields, and place a greater emphasis on developing and refining PA theories, of both context and behaviour within and vis-à-vis public organizations". 
And there were also some relevant messages of warning:

"However, our (...) task as researcher is not getting carried away with fashionable topics. The last point can be done by testing theories, replication of research and to slow down the growth of new concepts (in other words: not coming up with a new concept but trying to embed our research in more broad theoretical streams)."

Finally, with about the same frequency, challenges concerning the relationship between research and practice were mentioned:

"Bringing practitioners and researchers together and make research relevant for practitioners - Dissemination [of research] into practice - Reinforcing the dialogue with practitioners".

Apart from these well-known claims, balancing "rigour and relevance" is seen by some of our colleagues as a much more demanding challenge. "Contrasting demands of the world of practice and academia" are identified, and keeping an "arm's length distance in respect to practical public administration, politics and business interests" is called for. Finally, the demands of the modern academic world may work against this ideal of practical relevance:

"Maintaining a tight coupling between research and practice and pursuing intellectual innovation is challenged by the incentive systems in the academic world (the need to publish to progress in academic career, and the need to repeat already expressed arguments in order to be published) pushing towards decoupling and conservatism."

To sum up, the main challenges for PA research identified by our respondents are made all the more surprising by the range of issues hardly mentioned, if at all, compared to the ones most often cited. Most common was the concern for more methodological awareness and rigour. Oflesser importance, but still quite frequently stated, were concerns about interdisciplinarity, theoretical foundations and practical relevance. By contrast, much less emphasis was placed on the classical concerns of Public Management, such as performance and efficiency. These were cited by some of our respondents, but even less than political concerns, such as democracy, participation and diversity. Although a number of our participants have a legal background, issues relating to public law and public administration were seldom mentioned. 


\subsubsection{Challenges for PA teaching}

We also asked our respondents about the main challenges PA teaching will face over the next 20 years. The most common concern here was the attractiveness of our programmes for potential and talented students on the one hand, and the attractiveness of the public sector and public management as promising career options on the other. The main concerns were, for example:

"The attractiveness of the discipline of PA for talented students - to attract talented and motivated persons to undertake a full training (MPA, PhD) - PA is often regarded as dusty. We need to place greater emphasis on its great relevance, especially with regard to solving the most hard-pressing topics facing EU societies nowadays. Why do we leave the debate about nudging to economists and psychologists, for example?”

At the same time:

"Degrees in public administration do not seem to be appealing for students, today. In some countries, a career in public administration is not considered as a good opportunity for different reasons: governments are cutting expenditures (recruitment is limited) and often public administration is de-legitimized (not dynamic, difficult careers, not meritocratic but bureaucratic, difficult to develop new ideas...)."

As one colleague summed it up, our main challenges therefore are:

"— to attract the brightest students to the field;

- to prepare students for the working life;

— to become better at convincing governments they should hire PA graduates".

All three challenges are interrelated. If we do not convince bright students to study PA, or if they do not gain practical knowledge, we will not be attractive for potential employers. However, if career opportunities in the public sector are slim or not seen as overly attractive, it becomes harder to attract bright students. This may help to explain some obvious differences in Europe. In countries where job opportunities in the public sector are attractive, such as in the Scandinavian countries, Public Administration as a field of study and research is attracting bright students and thriving. 
What can we do? We seem to be creating some of the problems for ourselves, which has something to do with our own incentive systems:

"The quality of teaching is under pressure with incentive systems rewarding research over teaching (no one makes career anymore by being a good lecturer) (....) [The] 'value' of teaching needs to be increased in comparison to other outputs (articles, research \& third party funds). (...) Otherwise quality of teaching may not be secure since there are no incentives to focus more on teaching".

Apart from this, there was generally a lack of consensus about which direction we should take. With regard to our curricula, the same challenges and cleavages which were discussed concerning research strategies were stressed again. While some participants argued that we need more interdisciplinarity and more methodological rigour, others saw the solution in stronger disciplinary self-reliance. And at the same time, while most agreed that our success in teaching depends on being relevant for both practitioners and future academics, it was not clear how to achieve this. Here are some contradictory suggestions: - "Getting insights on governance [from] other disciplines (...) as well as getting $(. .$.$) our own students to address other disciplines - We need$ better integration with the broader social sciences. We need to provide our students with a much stronger ability to apply quantitative research designs and experimental methods - Becoming methodologically more advanced while at the same time creating sufficient theoretical foundation (not merely data-driven)".

- "Public Administration Research and Teaching Network have to invest in (...) a new culture of Public Administration. I mean that Research and Teaching Network, universities and other teaching Institution, individuals, researchers and professors should focus less on abstract frameworks, methodology rigor, techniques - Teaching needs to spend more efforts on public governance, stakeholder interests and mediating forces in society; Teaching needs to give less focus in rationalistic maximizing performance tools".

- "The (...) challenge is to maintain [our] own specificity and identity because the interdisciplinary approach determines an evaporation of frameworks - Development of [a] distinctively EU public administration theory and methods of inquiry, rather than one that follows (normally) US models - defending [ourselves] as a unique area of research (and not only being part of political science, law, economics...) - showing the relevance of Public Administration to students". 
- "There is an increasing decline of public administration, public policy and public management teaching programmes (...) Students are opting for generic management degrees. The challenge for public administration is [to] enliven the discipline and engage with the practitioner communities on the relevance of public administration scholarship," but we have "a poor track-record of the field in showing its merits and influence."

Several participants stressed that we should be more concerned with "ethics, diversity, and social equity," but as one of our colleagues warned us:

"Public values (...) should be at the heart of our teaching. And we will find that we do not agree on what the core Public Values should be”.

"The main challenge is to establish the credibility of public administration teaching so that the numbers of civil servants and public managers taking university courses in public administration rises steadily year by year, and does so because the courses are seen to be worthwhile investments in the capabilities of the public sector in all European countries".

We can probably all agree on these concerns, but the question of how to achieve this is still elusive and has therefore marked the starting point for our seminar series on disciplines, futures, cultures and practice.

\subsection{Disciplines - fragmentation not necessarily a bad thing?}

Our first substantive questions centred on the many disciplines of Public Administration. We invited participants to comment on the following sweeping assertion:

In recent years, disciplinary barriers and silos (e.g. between political science, management, law, etc.) within Public Administration have become ever more imperative and impregnable and have intensified established misunderstandings in the field.

About $60 \%$ of our respondents tended to agree and this, of course, implies that about $40 \%$ disagreed. We received some interesting and relevant comments.

"PA is and always will be a multidisciplinary field, it is one of the major strengths of the field — the barriers between the different disciplines are shrinking - much of the field has developed positively by incorporating many relevant theories from economics, management, organisation theory, 
sociology etc. in recent years - there has been a convergence between disciplines, and PA is at the forefront here - there are a growing number of PA conferences to which people from Law, Management, Political Science now present work".

Our survey is not representative; therefore we cannot determine how many of our colleagues share this optimistic view, but obviously not everybody is dissatisfied. There is evidence that PA research and teaching have embraced and even enhanced mainstream social science theories and concepts in recent years, much more than it used to be the case (this is also the conclusion of a state-of-the-art report about Political Science and Public Administration in Germany: see Bogumil, Jann and Nullmeier, 2006). Maybe the situation in Europe is even more promising than elsewhere, as at least two of our respondents observe:

"while I agree [to the original assertion of more fragmentation], this is less so in Europe than in the US - certainly in the US public administration and political science operate as adversaries especially in the sub-field of public policy".

There can be no doubt that specialisation and, following from that, fragmentation are typical results of academic professionalisation and success: the more research and teaching the more specialisation. Our next questions thus asked how we should deal with these developments, and how we can enhance learning and collaboration across disciplines. First of all, nearly all of our respondents agreed that specialisation is necessary and even welcome:

"We cannot avoid specialization; we should rather avoid fragmentation - The pursuit of specialized and depth of knowledge is not the problem, but rather it is the presumption that other viewpoints are invalid that is the problem. Only when faculties accept the notion that both depth and breadth of knowledge are needed can we get past the emphases on silos of knowledge - we need both specialization and fragmentation, but we need also coordination and generalists".

But how do we achieve and strengthen breadth of knowledge, coordination and generalists? Here, the main answer is, not very surprising for students of organisations, institutionalisation: 
"In the structure and power distribution of the academy, we need strong institutions centred on PA (schools of PA, university departments, institutes, research centres), counteracting the trend (...) towards PA scholars being in a relatively marginalised position within academic loci dominated by disciplines (law, economics, political science), disciplines which are sources for PA research, but in themselves may tend to dominate and confine PA to a marginal field of application. It is very important to foster and grow a strong sense of community in scholars (and practitioners) of PA all over Europe."

Traditional "cross-disciplinary collaborations and joined enterprises - shared conferences, guest journal editorships drawn from other disciplines - providing cross-disciplinary publications and scholarly outlets to encourage works going beyond specialization and fragmentation" are mentioned several times, but this is probably not enough. We need to create "organizational incentives to multidisciplinary perspectives, e.g. through the creation of multidisciplinary research organisations with a focus on the public sector" and a "strong core."

Here again, practical concerns and teaching could form the nucleus of these kinds of centres:

\begin{abstract}
"The meeting point lies in teaching - The major instrument of institutionalisation of our discipline is education, study programmes. The main focus should be in the integrative role of Public Administration. Instead of multidisciplinary programmes where each discipline uses its own disciplinary autonomy without much collaboration between teachers, there should be more INTER-disciplinarity where the structure comes from Public Administration (e.g., strategy, HRM, performance, coordination, governance) but other disciplines (psychology, political science, economics, law) approach the theme from their point of view. In a way, a matrix structure of themes of Public Administration and contribution from other disciplines (...) Public Administration should lead the contribution coming from other disciplines because Public Administration is the owner of the programme."
\end{abstract}

Our next question focused on learning across established disciplinary boundaries. How can we create and enhance new forms of cooperation? Here two main approaches were proposed, and the major barriers that need to be overcome are "terminology and methodology." Taking terminology first: 
"Collaboration is hindered first by language and labels. We fail to see that similar (yet different) research is going on in other disciplines, in part because we label theories that are quite similar with different names. We miss the common threads and end up arguing about the label rather than the core ideas. - Concepts already used in other contexts are considered as new (...) simply because literature from another field of study was not considered - sometimes we use apparently similar concepts with different technical meanings and aims".

But there were also warning messages that we should "slow down the growth of new concepts (in other words, not come up with a new concept, but try to embed our research in more broad theoretical streams).” The other obstacle to collaboration and mutual learning ( 3.1 see above) arises from the different methods used:

"Give up on the concept of specific PA theories and methods and instead embrace a broad application of the methods and theories available to the social sciences. - Discussion about the methods used in (...) different fields and how they can complement each other. - Working across disciplines implies to work with multiple methods and theories. An important element in a strategy would be to enhance the quality of qualitative research - in order to have conversations about methods which are open for mutual learning."

Finally, obstacles that the academic system has created for itself, especially regarding academic publishing, are again mentioned a couple of times:

"A powerful force for segmentation however is the impact factor calculation in journals. Those with more narrow disciplinary scopes seem to have higher values. - Change the American dominated journal based assessment and reward system that predominates and public management is enslaved within - the myopic and entrenched peer system needs to come down - use reviewers from different sub-disciplines to ensure discussion between the sub-fields."

Additionally, we asked which disciplines are crucial for the research and teaching of Public Administration, and which disciplines need to play a more dominant role before 2035 (a normative question). The answers were not very surprising, but at least confirmed established "stakeholders." 
Table 1 Crucial Disciplines

\begin{tabular}{lllll}
\hline & \multicolumn{2}{c}{ Crucial Disciplines } & \multicolumn{2}{c}{ More Prominent in the Future } \\
\cline { 2 - 5 } & First ranking & Total ranking & First ranking & Total ranking \\
\hline 1 & Political Science & Political Science & Political Science & Management \\
2 & Management & Management & Management & Political Science \\
3 & Law & Sociology & Psychology & Sociology \\
4 & Sociology & Law & Sociology & Psychology \\
5 & Economics/ & Economics & Law & History \\
\hline
\end{tabular}

It should be noted that the differences between the two "top dogs" (political science and management science) are rather small, just as they also are between the "second tiers" (law, sociology and economics). "Also rans" were, amongst others, psychology, history and anthropology, which each were ranked several times, but only psychology and history made it into the list of the need for "more prominent disciplines" in the future. Particularly interesting is the fate of law, which is given even less relevance for the future. This is probably an artefact because our respondents come mainly from Northern and Anglo-Saxon Europe.

\subsection{Futures}

The next substantive questions in our survey were aimed at helping us identify future perspectives in the field of Public Administration and about how we, as a discipline, should react in facing the future. Again, we started this part of our survey questionnaire with a terse provocative statement:

Public Administration is stuck in short-term problem and solution thinking.

We need utopias and dystopias.

About half of the survey participants commented on our statement, and their responses can be roughly divided into two, with about half agreeing and half disagreeing. Examples of comments stressing the importance of integrating the future, utopia and dystopia in PA research were: 
"Utopias and dystopias allow to get out from this short-term thinking by having a broader perspective and enrich creativity. - Short-term problem solving is important, but we must not lose long-term perspectives. Dreams may be an engine to improve society, if they are well managed - (...) we need analytically informed research about futures".

But the second half of comments revealed how concerned some respondents are about a potential decline in the relevance of short-term problem solving and solution thinking in the field of Public Administration. Typical comments were:

"Short term is needed and fitting for today's society. We often do not act fast and short term enough - We need better theory and testing, not stories - Great theories are for the ivory tower. "

Even though our survey does not claim to be representative, these contradictory comments to our introductory statement hint at two dichotomous spectrums in PA research strategies. While some of our colleagues are convinced that short-term problem solving is important above all else, other PA scholars expressed the need for more long-term problem thinking.

To follow up on this introductory statement, respondents were asked whether more relevant short-term problem solving is needed. The answers can be categorised into three groups. The first group agreed with this statement and the importance of more short-term problem solving. Our respondents gave a number of different reasons.

"Yes - it enhances the perception that PA can actually improve the world and find solutions to actual problems. - yes, to increase the visibility of our discipline - Yes public policy is dominated by policy based evidence-making rather than evidence based policy making. It needs more relevant robust applied practice research as opposed to theoretically based high ranking journal centred research - We definitely need more mundane but rigorous empirical research on how things (tools, programs, reforms) work or not."

By contrast, the second group of answers highlighted the problems of shortterm problem thinking and the need for more long-term thinking:

\footnotetext{
"The major challenges in society will need more long-term thinking - (..) we too much emphasize short term problems and their solutions that in a long time are the very cause or root of the problems. - I think the job of public admin is not to contribute to instant problem solving, but to show
} 
how and why institutions matter for problem solving, how institutions and organizations work, how they shape problem solving and decision making (...)."

One colleague raised an important question about the need for and balance of short-term and long-term problem-solving strategies:

“(...) the crucial question is the relevance of our research for practitioners. What do we want? Do we want to be their consultants or do we want to explain their behaviour? Both directions have their advantages and disadvantages. Maybe we should also think about how we can bring them together."

This fundamental question leads to the third set of answers in relation to short-term and long-term problem solving. Most of our respondents were convinced that the ideal research strategy for PA involves a mix of both shortterm and long-term problem solving:

“(...) a good mix is necessary - We must be able to provide solutions to the problems faced by civil servants, but mostly help them redefine the frames of these problems to escape short-termism. But solutions would come from strong theory-building activity."

The combination of the two dichotomous approaches seems to be particularly important in dealing with crisis, as two respondents pointed out:

"There may be policy problems which require short-term problem solving
such as a crisis and there is a place for short term problem solving as not
every event is predictable. However, events which lead to a crisis are usually
complex and avoidable. A crisis usually demonstrates the lack of long term
problem solving. Long-term planning and attempting to address wicked
policy problems should be a priority, but may not be politically salient as
politicians want immediate solutions within election calendars - Crisis
management requests short-term problem solving and long-term solutions."

In the next stage of the survey process, we were interested in research instruments, which could be used to develop realistic visions for the future beyond short-term problem solving. Not surprisingly, the research instruments most often mentioned overlap to some extent with the major challenges facing 
PA until 2035, notably comparative research and interdisciplinary projects and collaborations:

"To create multi-group research projects that can activate comparative researches. - Cross-country and multidisciplinary research strategies to address long-term, wicked policy problems. - Discussing beyond disciplinary boundaries. - Interdisciplinary in topics, methods, profile of researches etc. \& international (cross-cultural) collaboration.”

In this vein, respondents also mentioned the necessity to combine practical and theoretical knowledge in interdisciplinary projects:

"To mix theoretical and practical aspects into interdisciplinary projects cross-disciplines collaborations with academia and practitioners.Combination of fundamental (and generic) research and cooperation/ inspiration between researchers and practitioners."

Besides comparative research and interdisciplinary collaborations, big data and in particular the use of big data in mixed methods research designs were frequently mentioned as a tool for creating realistic visions for the future:

"to learn how to use big data analytics - Collect big and complex data sets. - Ambitious mixed methods research (...). Mixed methods implies the use of rigorous observational data, including 'big data', experimental studies and advances qualitative methods, including ethnographic work - As much data as possible - discovery of patterns in data - predicting the future along non-stop monitoring for changes and adaptation - More mixed methodology combining quantitative series of data analysis and qualitative in depth understanding/interpretation.”

Apart from these two instruments, respondents again criticised the current publishing strategies used within the field of PA and suggested the following innovative approaches:

"First, a place to publish in an open form. Journals are outdated for this mission: academically indexed Youtube videos of researchers commenting, blog's, etc? - (...) rapid publication of shorter research pieces in PA journals". 
Others underlined the importance of philosophy for creating realistic visions for the future and overcoming short-term problem solving:

"foundational philosophical debate may stimulate long(er) - term thinking, and hence also research designs orientated to more fundamental issues (a drawback is reduced accessibility of research outputs so derived to 'standard' outlets of publication). — political philosophy - Theoretical and philosophical research could show the way for the overpowering role of administration in all social life (in all organisations and management/ leadership).”

With regard to teaching strategies for the futures of PA, comparative studies and interdisciplinary approaches were again most often cited. One of our respondents put forward the idea of a coherent and interdisciplinary concept of teaching:

"PA teaching should align itself with other disciplines, such as management, anthropology, sociology. Develop business and society modules to enhance the importance of PA in societal change processes. Work across disciplinary boundaries. Work with practitioners and policy makers to develop joint curriculum. Add value to society so have courses of greater relevance to societal problems. Predict future skills needs for public servants and align curricula according to these skills gaps. Work with HR Directors in PA agencies to determine what they need for the future."

Other issues raised included the integration of future topics and future scenarios in teaching strategies:

"Integrate 'future topics' (like the city of the future for instance) in current curriculums/seminars. Train students to look more ahead than backwards — add scenarios and make more interactive research with the students. More courses in (...) future studies - "Scenarios (look at a crystal ball)."

Furthermore, respondents stated that case-based teaching should still play a role in teaching futures:

"This could involve using case study type of teaching which engages students in design thinking; working on potential cases could be a strategy to facilitate this - Case-studies approaches. Best practices.” 
Conversely, case-based teaching and the use of best practices were also criticised:

"Case-based teaching and best practices are counterproductive to this, I think. Combining theoretical concepts and project work are preferred strategies."

Finally, a fairly controversial statement made by one of the respondents implies that teaching strategies are systematically and naturally biased by the interests of an "old generation" of teachers and therefore do not meet the needs of future scholars in the field of PA.

"The future matters for today's students more than it does to their teachers who have shorter futures! Those teachers have a selfish stake in the present state of knowledge which may inhibit their capacity to encourage more future-orientated work."

To conclude, the participants in our survey clearly stated that there is a need to improve both short-term and long-term problem and solution thinking. The main strategy identified to improve our discipline's fitness for the future was the rigorous application of an interdisciplinary, multidisciplinary and comparative perspective in research and teaching. Often cited, but not quite so often, were comments about integrating the issues of using big data into our research.

\subsection{Cultures}

The third section of our Internet-based qualitative survey was concerned with the interplay of different European traditions and cultures. We collected our colleagues' opinion on this pressing matter and specifically asked for their suggestions about how we, as the European PA community, should respond. Again, we started with a provocative statement:

Public Administration in Europe does not sufficiently take into account the many different cultures and traditions in Europe.

About two-thirds of our respondents agreed with this statement, but the remaining third gave interesting reasons for their disagreement. One comment raised the important issue that there is not a lack of recognition of cultural differences, but a lack of forward-looking EU-wide solution thinking instead: 
"I think different cultures are recognized. Moreover, there are EU-wide problems, which require EU-wide solutions (e.g. climate change). This needs EU-wide research whilst taking into account cultures and traditions."

According to some respondents, this situation results from shortcomings in our theories and methods.

"I think this is a result of the weaknesses of our theories and the failure to integrate context into the theories. - We talk about different cultures all the time. We lack a sufficient way to analyse differences, trends, convergence (...)".

When we asked how the European community of PA scholars could take the notion of different cultures seriously, avoid "one-size-fits-all" approaches and ensure mutual learning, most responded that comparative research is the strategy to embed and acknowledge cultural diversity in our studies: "comparisons are the key to appreciating diversity." Respondents asked to "take comparative PA research to a new level (away from country case studies to more advanced statistical methods)" and emphasised the need for the PA community to "be culturally sensitive and truly comparative."

This demand seems to be related to the challenge of "stopping the AngloSaxon domination and giving more attention to other parts of Europe":

"Some parts of Europe are totally beyond the scope of the mainstream European PA scholarships, e.g. especially some larger countries in Southern and Eastern Europe. The 'European perspective' is very much influenced by the studies of a few core EU countries, which have great PA scholars."

Another approach, often mentioned alongside the need for "real comparative work," is the requirement to extend "cross-national projects, programs and initiatives (...)" and to foster "contacts between researchers from different countries (...) in order to recognize different cultures and solutions" as well as "all forms of 'mobility', including long-term mobility of scholars in countries other than the home country (...)."

Again interdisciplinary work was raised as an issue, especially the need for "being open to introduce historians, anthropologists to the research teams." Respondents stressed the necessity to change the current PA curricula and pay "more attention to developments in Europe" and "international perspectives" as well as to "introduce intercultural awareness in our curricula." 
In the second stage, we asked more concretely for suggestions of how to embed cultural diversity in our research and teaching. Not surprisingly, our respondents again emphasised that this could not be achieved unless we carry out more comparative research. As regards "research," comparative projects/ research/work were often cited together with approaches to embed cultural diversity in PA teaching. In order to embed cultural diversity in our research, some of our respondents favoured using methodological approaches such as "cross-country experiments" and "in-depth interviews" as appropriate strategies. Additionally, others suggested the development of "specific cultural sensitive instruments/methodologies" as well as the awareness of the necessity "(...) to replicate findings made in one country in other countries and based on that, research on causes for differences in the replication."

Participation in exchange programmes was very often mentioned as a way of strengthening the nexus between cultural diversity and PA teaching. On the one hand, this implies "Erasmus-like programmes for teachers" as well as "more guest lectures," including "invitations to lecturers/speakers from different cultures." On the other hand, students should be encouraged by means of "Europe-wide excursions (...) to widen their scope." Furthermore, PA institutes should campaign to "recruit more diverse students."

To sum up, most comments in this part of the survey underlined the necessity to intensify comparative research, approaches and methods, interdisciplinarity and exchange programmes for scholars and teachers as well as for students as ways to promote cultural diversity in European PA.

\subsection{Practices}

In the last substantive part of the questionnaire we were interested in the relationship between PA research and teaching and practitioners in public administration as well as in the usefulness of our disciplinary output for European citizens.

Again, we started this section of the survey with a controversial statement:

Public Administration research and teaching do not deliver sufficient useful knowledge for practitioners and citizens.

Even though about $57 \%$ of our respondents tended to agree with our view, another $43 \%$ disagreed and provided some valuable input. Some respondents criticised our "utilitarian view of science": 
"Because the question is framed in an outdated fashion. Knowledge is not produced in a way that it can be packed and delivered to practitioners. It is produced by interaction. Maybe there is not enough interaction, or public administration is not good enough in facilitating interactions (...).”

Another respondent expressed concerns about the role of PA as a discipline:

"I am not sure that we should do this, we are not consultancy firms (...)."

By contrast, other respondents did not agree with our sceptical view about the usefulness of our research and emphasised that the PA community can deliver useful knowledge to practitioners

"We can always do better, but at least in my country the knowledge transfer has been improved a lot during the last years. - When there is at least some basic receptivity, in my experience PA knowledge is perceived as useful - though PA scholars are less prone to furnish policy advice than other scholarly communities."

As a follow-up to this introductory statement, we asked our survey participants about suitable strategies to improve the matching of supply and demand between PA research and teaching and practice and citizens. The most frequently mentioned tools for PA research were the improvement of dialogue and development of networks. The majority of respondents perceived these two approaches as being the most promising strategies:

"Improve dialogue - Bring well-educated graduates into practice and to increase the understanding of PA research in practice (...) - How can we develop communities of exchange $(. .$.$) - Both environments should$ not be as isolated as they currently often are. The dialogue and each other comprehension is basic, even if sometimes difficult. (...) - Establish communication (conferences, workshops ...) between researchers and practitioners and stress the necessity of knowing each other better. - Closer links with the EU Public Administration.”

What is more, some respondents stressed that practitioners should be actively involved in research projects:

"Include practitioners in different phases of the research (probably in the earlier phases - model construction) so that the theories/models then 
tested are already sensitive to specific issues found in practice. - Thinking about some research projects as being co-created by researchers and practitioners."

Additionally, some respondents again criticised the current publishing strategy in PA research. We, as the European PA community, should ask ourselves: “(...) do we publish in the right papers?" Additional suggestions for more effectively reaching practitioners and citizens were:

\begin{abstract}
"Publish results in practice-oriented journals - We need to value more articles close to practical problems. Theory should be more seen as a way to contribute to real problem solutions - Work more with writing newspaper articles, policy papers and giving speeches in order to disseminate our best research, and picking up relevant topics for on-going and future research. - twitter, write 'practitioner' articles, practitioner points in journals, more active attitude of journal editors in promoting research articles, for instance, I very much like the PAR approach.”
\end{abstract}

Also in the context of teaching, the most frequently mentioned approaches were PA dialogue and network:

\begin{abstract}
"Inviting them to participate and accept our students for internships, doing research on themes relevant to more communities. - Invite practitioners as guest lecturer for special topics that are challenging for practice. Encourage students for practitioner's internships."
\end{abstract}

In addition, respondents stressed that:

"Practitioners from policy communities and government should be involved or consulted in the design of programmes and research".

Furthermore, respondents stated that it should be an aim of PA teachers to "convince that public organisations need generalists, not only professionals (e.g. civil engineers, accountants) (...)" and that they should "connect and have dialogue with public managers about initial training for their new members." Finally, respondents stated that it should also be an aim for PA teachers to:
"(...) give our students the tools that are necessary both to solve practical problems and to understand their conceptual context. Just one side is not enough to find efficient solutions, unfortunately." 
To sum up, it seems that "dialogue" and "networks" are the ultimate strategy to break down the barriers between the PA research and practitioners as well as between scholars of PA and the European citizens.

\section{The survey as starting point for the EPPA seminars}

This qualitative survey aimed at setting out a "forward view" based on our colleagues' perceptions of the major challenges and opportunities facing PA, and their suggestions for possible ways forward for the development of PA in Europe over the next 20 years. The survey marked the starting point of the EPPA project's journey with a "collage" of opinions expressed by a wide variety of both senior-level PA scholars and PA scholars in the early stages of their career.

To conclude, our survey results on the main challenges for PA research and teaching identified by our respondents are made all the more surprising by the range of issues hardly mentioned, if at all, compared to the ones often cited. The overriding concern was the need for more methodological awareness and rigour. Of less significance, but still quite often mentioned, were concerns about interdisciplinarity, theoretical foundations and practical relevance. The attractiveness of the field was seen as the major concern for teaching.

As for the disciplines, there was awareness that multidisciplinarity is both an asset and a necessity, but it has to be organised and developed. A balance between sufficient specialisation and co-ordination of disciplines will be necessary to tackle complex problems and issues. Political Science and Management Sciences substantially shape the PA agenda, as they are the most prominent stakeholders. Clearly, big data, comparisons and theories are on the agenda to remain relevant for practice. Even though a number of our respondents have a background in law, issues relating to public law were hardly mentioned. This may be an artefact of the typical Northern-European bias of academic PA and our survey. However, the integration or reintegration of law into current PA discourses remains an open and ongoing concern of the field. As for the future, a pragmatic focus on short-term problems as a driver for research is part of staying relevant. However, the long-term and future-focused strategy is clearly recognised and is most likely to be developed. The participants in our survey clearly express the need to improve research approaches, short-term and long-term problem and solution thinking in coherent research strategies. Regarding cultures, there is a realisation that traditions and language need be taken much more into account in the future PA agenda. This also implies that a European voice should distinguish itself 
from the dominant Anglo-Saxon voice, which will ultimately have implications for the comparability of research itself. Finally, when it comes to practice, there is a major concern to stay relevant, even if there is still uncertainty about what strategies are needed to stay relevant. Generally, as the most promising way forward for PA, the establishment of truly interdisciplinary schools and institutes is identified. More specifically, the views of many scholars in our survey add to discussions stipulating that organisation theory should be taken seriously and more should be invested in institutionalisation. Also in $\mathrm{PA}$, instititutionalisation is the only way to achieve significance and remain relevant.

Overall, we were pleasantly surprised and reassured by the underlying optimism of our colleagues. The survey results thus delivered the basis of diagnosis and therapeutical treatments that shaped the agenda of our four thematic EPPA seminars on the futures, disciplines, cultures and practices of PA (see the summaries in Chapter 1.4), which continued the conversation already started in our survey. Clearly the field is under pressure and needs to reorganise and reassert itself, but as the following contributions building on these seminars will show, we have both the resources and the ideas to achieve that.

\section{Notes}

1. We corrected simple typos in the responses of our participants.

\section{References}

Bauer, M. W., \& Becker, S. (2017). Verwaltungswissenschaft in Deutschland: Relevanz und Reputation im Urteil der Fachvertreterinnen und Fachvertreter. Der moderne staat: Zeitschrift für Public Policy, Recht und Management 10(1): 31-48.

Bauer, M. W., \& Grande, E. (2018). Status und Perspektiven der politikwissenschaftlichen Verwaltungsforschung in Deutschland. In M.W. Bauer, \& E. Grande (eds.). Perspektiven der Verwaltungswissenschaft (pp. 9-38). Baden-Baden: Nomos.

Bauer, M. W., \& Becker, S. (2019). Public Administration in Germany: Problems and Potential of a Fragmented Community. International Journal of Public Administration 42(11): 950-960.

Bogumil, J., Jann, W., \& Nullmeier, F. (eds.). (2006). Politik und Verwaltung. Wiesbaden: Verlag für Sozialwissenschaften.

Bouckaert, G., \& Van de Donk, W. (eds.). (2010). The European Group for Public Administration (1975-2010): Perspectives for the Future. Brussels: Bruylant. 
Christensen, T., \& Lægreid, P. (2005). New Public Management: The Transformation of Ideas and Practice. Aldershot: Ashgate.

Curry, D., Van de Walle, S., \& Gadella, S. (2014). Public Administration as an academic discipline: Trend and changes in the COCOPS academic survey of European Public Administration scholars. COCOPS Report Work Package 8.

Jansen, H. (2011). The Logic of Qualitative Survey Research and its Position in the Field of Social Research Methods. Forum qualitative social research and its position in the Field of Social Research Methods 11(2): Art. 11, May 2010.

Pollitt, C., \& Bouckaert, G. (2017). Public Management Reform: A Comparative Analysis - Into The Age of Austerity (4th ed.). New York: Oxford University Press. 
PUBLIC ADMINISTRATION AND FUTURES 



\section{1 \\ From Public Administration in Utopia to Utopia in Public Administration}

Geert Bouckaert

Utopia is a necessary technique for waking up from reality (Abensour, 2012, p. 8). Carey's "Faber and Faber" provides an overview of a selection of 101 historical utopias (Carey, 1999). The challenge or, rather, the assignment is "facing the future again" (Toulmin, 1992, p. 203). According to Toulmin, there are two attitudes to the future: one of imagination, the other of nostalgia: "the task of defining realistic 'futuribles' is open only to those who are ready to adopt imaginative attitudes" (Toulmin, 1992, p. 203). In this chapter, these "futuribles" will be put in the context of public administration and public governance.

This chapter will first look at how realities influence utopias. In the second part, a (limited) historical view on public governance in Utopia will be sketched out. The third section will discuss the utopian dimension of current governance policies. Fourth, we discuss how utopias may influence future realities.

\section{$1 \quad$ From old and new realities to old and new utopias ${ }^{1}$}

Utopias are connected and embedded in their realities. Utopias belong to their realities. Since realities evolve, utopias evolve as well. Old utopias belong to old realities. Therefore, new realities require new utopias (Achten et al., 2016).

When revolutions are driven by utopias they result in excesses, which may deny the premises of these utopias. According to Micklethwait and Wooldridge (2014) the first revolution in the seventeenth century resulted in kings and queens building centrally administered states. Cromwell supported Henry VIII in this endeavour, and Thomas More was a witness to and an actor in this revolution. This centralised state evolved into a Hobbesian Leviathan. This system triggered a second revolution, exemplified by the French and the American revolutions, with a focus on meritocracy and accountable 
administrations, which subsequently in the third revolution evolved into the modern welfare state. This resulted in "big government", which triggered a fourth revolution based on ICT and management (Bouckaert and Crompvoets, 2016), and which, according to Micklethwait and Wooldridge, will determine a sustainable state with a future.

The Arab Spring also had a utopian ambition: "Democracy is a dream that can be fulfilled around the globe. (...) Tunisia's new constitution is a source of immense pride for all Tunisians" (Ghannouchi, 2014, p. 97, 99). This illustrates that there is an interaction between realities and utopias. Utopias challenge realities since they allow for a shared analysis, but also for shared imagined ideals, and ultimately they allow for a voluntarist appeal to contribute to change or even revolution.

Utopias evolve with their realities, and realities evolve with their utopias. Realities need utopias, just like utopias need their matching realities. H.G. Wells refers to this in the final sentence of his A Modern Utopia: "That is my all about Utopia, and about the desire and need for Utopia, and how that planet lies to the planet that bears the daily lives of men" (Wells, 1917, p. 357).

The German sociologist Norbert Elias calls this the historicity of utopias (Deluermoz, 2009; Elias, 2009). A specific reality triggers a specific utopia. More's Utopia corresponds to More's historical context. This is also the reason from the nineteenth century onwards why, next to the social and political utopias, technological utopias have surfaced matching the reality of a technological and industrial revolution. This makes utopias not just illusions, but real locations for revelation and observation of these realities.

However, especially since the twentieth century, the dark utopias, i.e. the dystopias, have taken over from the utopias. There has been a reverse from dream to nightmare, and from utopia to dystopia (Elias, 2009b, p. 116).

\section{Public Administration in Utopia}

If one is looking for what public administration looks like in "utopia", then three dimensions could be expected to emerge:

- the relationship between politics and citizens, or the leadership in utopia and its public sector;

- the organisation of a utopian society with its human beings and its public and private sphere;

- the content of public policies on major issues such as property and money, and the administration of concrete service delivery in policy fields such as health, education and security. 
Of course, one could also expect most of the issues that require public intervention to be solved in a utopian system. The needs are defined and under control, technology has resolved many practical problems, people behave differently and take the general interest into account. And therefore public policies have no objection, since managerial and governance issues are typical for real societies, not for utopian societies.

Nevertheless, most "utopias" do have substance on these three clusters of concern.

In More's Utopia of 1516 (More, 1980; see also Elias, 2009a, 2009c), the care for the poor and the weak is taken care of by collective services such as schools, hospitals and libraries. This implies big state control. There is contracting out of warfare. Society is a kind of abbey. There is no private property. There are also very developed work and study ethics, as in the ora et labora (pray and work) from the monasteries. Also, there is no freedom of expression or opinion, no freedom of religion, of travel, and of choice of profession. "There are fifty-four splendid big towns on the island, all with the same language, laws, customs, and institutions. They're all built on the same plan" (More, 1980, p. 70). The hierarchical structure of the governance is based on representation and (secret) elections from local governments. "The population is divided into groups of thirty households, each of which elects an official called a Styward every year. Styward is the old Utopian title - the modern one is District Controller. For every ten Stywards and the households they represent there is a Bencheater, or Senior District Controller. Each town has two hundred Stywards, who are responsible for electing the Mayor. They do so by secret ballot (...) The Mayor remains in office for life, unless he's suspected of wanting to establish a dictatorship" (Ibid., p. 74). All fifty-four cities elect three persons to the Aircastle, the parliament in the capital. There are not many laws and therefore all are expert.

Just as sleep may induce a dream or a nightmare, the exercise of creating the non-existent future may result in a utopia or a dystopia. In Bellamy's utopian novel Looking Backward (1887), Julian West falls into a deep hypnosis-induced sleep and awakes in the year 2000 in Boston, Massachusetts. "We have no wars now, and our governments no war powers, but in order to protect every citizen against hunger, cold and nakedness, and provide for all his physical and mental needs, the function is assumed of directing his industry for a term of years. (...) We have no parties or politicians, and as for demagoguery, and corruption, they are words having only an historical significance" (Bellamy, n.d., pp. 46-47). Government distributes everything: "A system of direct distribution from the national storehouses took the place of trade, and for this money was unnecessary" (Ibid., p. 65). In the power structure, there 
is an industrial army with a general-in-chief, who is the President of the United States, lieutenants-general and generals, etc. (Ibid., p. 141). There are hospitals, but no prisons, no lawyers and almost no legal cases. The only administration is the organisation of the industry. There are municipalities. They have important and extensive functions "in looking out for the public comfort and recreation, and the improvement and embellishment of the villages and cities. (...) Every town or city is conceded the right to retain, for its own public works, a certain proportion of the quota of labour its citizens contribute to the nation" (Ibid., p. 157).

However, there are also dystopias as described by Orwell, Huxley, Butler, among others. In Butler's Erewhon (the inverse of "nowhere") machines are not allowed, and there are Schools of "Unreason" (Butler, 1985). In most cases, the dystopian nightmare is about "mass utopia" with dictatorships and loss of personal freedoms, in East and West (Buck-Morss, 2002).

\section{Utopia in Public Administration}

The link with utopia is the mostly implicit, sometimes explicit, normative dimension of public administration. The purpose of studying and researching the scientific field of Public Administration is to improve its research object of public administration, which is the public sector and its activities for service delivery, and its policies. Public Administration is not just scientifically studying governance, public administration or public policy, it is also about trying to improve and promote "good" governance, public administration and public policy. As a consequence, there is a normative side in the field of study to Public Administration. The most extreme expansion of this normative side turns into a utopia.

At the beginning of the twentieth century, the scientific approach to administration and management, which was almost an engineering approach (Fayol, Taylor, Gilbreth), had a very mechanistic view of improvement and of an ideal of the man-machine model of interaction. These administrations, as utopian machine models in scientific public administration (classical mechanistic), evolved into models (Herbert Simon) which included limited rationality, where not every actor had all the information; where the purpose was not to aim for a maximum, but for an acceptable and satisfying optimum; and where there was an acceptance that the ideal solution was not possible.

Also the Weberian model, as an expression of authority, with a bureaucracy as the most procedural and rational way to rule, dominate and control, aimed 
at an administrative system with the general interest and a shared objective among all civil servants as an ideal or pure model.

The generalised rational choice model, which implies that individual objectives are predominant, is also found in the public sector sphere and has ultimately resulted in a dystopian model of public administration with private interests.

Finally, the economic neo-institutional model of principals and agents has been imported into the public sector. This has resulted in a shift from trusting harmony models to distrusting conflict models. Principals cannot trust agents, and agents cannot trust principals. Again, this is more an expression of a dystopian model than a utopian harmony model of shared objectives of trusting principals and agents.

Additionally, there is a growing agenda to move away from a utopian governance system (including democracy) to a dystopian governance without democracy. The debate on whether good governance includes democracy is ongoing (Fukuyama, 1992, 2004) and is even shifting to a position of good governance in non-democratic systems.

The whole debate on Public Value, the value of "public" and public values as operational variables is also increasingly being included in the normative context. It ultimately means a discussion on the meaning of "good" in "good governance" and "Public Value".

There is a broad agenda for discussing systemic utopian reforms such as "worldwide PA", which includes wicked problems, transborder policy issues, etc.; sustainable development (what is ultimately sustainable?); governing without government (autopoiesis, self-regulation, etc.); good governance (indicators); corruption-free systems; full participation of stakeholders (co-design, co-decide, co-implement; co-evaluate); full transparency (Transparency International Index); economic, efficient, and effective policies; trusting public administrations; and ethical governments and public sectors.

There are two cases demonstrating the global utopian ambition by major institutions, namely the World Bank and the UN (see also Bouckaert and Brans, 2019).

In this section, the two cases of the World Bank (Governance indicators), and of the UN (Millennium Development Goals (2000-2015) and the Sustainable Development Goals (2015-2030)) will be developed as an expression of evidence-based utopias in Boxes 1 and 2. 


\section{Box 1: Good Governance (The World Bank)}

The whole scientific agenda of "Good Governance" has a clear utopian dimension.

The following checklist is almost like a utopian to-do list.

1. The process by which governments are selected, monitored, and replaced:

- voice and accountability

- political stability and absence of violence/terrorism

2. The capacity of the government to effectively formulate and implement sound policies:

- government effectiveness

- regulatory quality

3. The respect of citizens and the state for the institutions that govern economic and social interactions

- rule of law

- control of corruption

In September 2015, the UN Millennium Development Goals (MDGs) were replaced with the UN Sustainable Development Goals (SGDs) (2015). The major difference is that the new goals are universal and involve all countries, including all their stakeholders.

The UN SDGs state: "In the goals and targets which we have agreed, we are setting out a supremely ambitious vision. We envisage a world free of poverty, hunger, disease and want. A world, for example, of safe and nutritious food; of affordable drinking water; of universal access to basic education; of physical, mental and social well-being. A world of universal respect for human rights and human dignity; of justice and equality; of respect for race and ethnicity; and of equal opportunity permitting the full realization of human potential while promoting shared prosperity. A world in which every woman and girl enjoys full gender equality and all barriers to their empowerment in our societies have been removed. A just, equitable, tolerant and inclusive world. And one in which humanity lives in complete harmony with nature."

\section{Box 2: 17 SDGs (Sustainable Development Goals, 2015-2030):}

Goal 1. End poverty in all its forms everywhere

Goal 2. End hunger, achieve food security and improved nutrition and promote sustainable agriculture

Goal 3. Ensure healthy lives and promote wellbeing for all at all ages

Goal 4. Ensure inclusive and equitable quality education and promote lifelong learning opportunities for all

Goal 5. Achieve gender equality and empower all women and girls

Goal 6. Ensure availability and sustainable management of water and sanitation for all

Goal 7. Ensure access to affordable, reliable, sustainable and modern energy for all 
Goal 8. Promote sustained, inclusive and sustainable economic growth, full and productive employment and decent work for all

Goal 9. Build resilient infrastructure, promote inclusive and sustainable industrialisation and foster innovation

Goal 10. Reduce inequality within and among countries

Goal 11. Make cities and human settlements inclusive, safe, resilient and sustainable

Goal 12. Ensure sustainable consumption and production patterns

Goal 13. Take urgent action to combat climate change and its impacts

Goal 14. Conserve and sustainably use the oceans, seas and marine resources for sustainable development

Goal 15. Protect, restore and promote sustainable use of terrestrial ecosystems, sustainably manage forests, combat desertification, and halt and reverse land degradation and halt biodiversity loss

Goal 16. Promote peaceful and inclusive societies for sustainable development, provide access to justice for all and build effective, accountable and inclusive institutions at all levels

Goal 17. Strengthen the means of implementation and revitalize the global partnership for sustainable development

These 17 SDGs are constructed around the five Ps: planet, people, prosperity, peace and partnerships. The "action verbs" have different degrees of ambition, and some are therefore "utopian" (end, achieve), and others much more pragmatic (strengthen, promote, reduce, ensure).

After studying the content of the policy goals, it is clear that many policies are related to utopias. Ecotopia wants a society with full respect for the ecological agenda, or at least a respect for the triple bottom line of economy, social and ecology. Ideal cities are about this dream of a perfect city that has been around for the history of humanity. The search for the ideal city has resulted in many utopias to include cities in their models.

Many Public Administration and Public Policy studies include objectives such as education for all, health for all, housing for all, and security for all, etc.

\section{"Planet" becomes explicit in the ecotopian agenda}

Environmental catastrophes and scenarios have triggered discourses to save the world (Achten et al., 2016, p. 11). They have also called for radical problem solving by experts (administrative rationalism), the people (democratic pragmatism), and markets (economic rationalism) (Dryzek, 1997). Moreover, they have resulted in ecotopias, such as that described in Callenbach's novel Ecotopia (1990) in which a New York Times-Post reporter, Will Weston, visits 
Ecotopia as the first outsider 20 years after it was founded, when northern California, Oregon and Washington seceded from the Union and created a new stable-state ecosystem. Ecotopia has the perfect balance between human beings and the environment. In his overview of ecological utopias, De Geus refers to the historical 'austerity' utopias, where it is common to exercise self-imposed constraints on consumption (More), to ultimately live a simple life (Thoreau) and with simple beauty (Morris). The contemporary versions are about ecological city gardens (Howard), green communities (Skinner), paradise islands (Huxley), and a "stable state society" (Callenbach). It is clear that ecological utopias have inspired environmental debates and the ideologies of green parties. One could probably also read Pope Francis' encyclical letter On Care for Our Common Home (Laudato Si), calling for a "global ecological conversion," "a new lifestyle," and "ecological citizenship" (Francis, 2015) as a utopian ambition that should be realised.

But how are utopias realising these policies? The UN Sustainable Development Goals seem utopian in their ambitions; however they take on the nature of long-term strategic thinking. First there is a specific date (2030) for attaining these objectives. However, the Joachimite Age of the Spirit has also been connected to a specific millennium where the years 1000 and 2000 had a specific appeal. Huxley also referred to the date "1984" and Bellamy in his Back to the Future, referred to the year 2000, which was far away from 1887. Third, the UN SDGs also set out a quite precise trajectory and roadmap with actors, indicators, stepping stones, and operational national plans. From this perspective, the SDGs form part of a signed document with an official and institutionalised status, and are not just an intuitive utopia-driven text. Nevertheless, these goals have a utopian connotation, and once they have been achieved they will be seen as a realised utopia.

Utopian SDGs will be achieved by moving to concrete principles of effective SDG governance through public administration. There are three specific "utopian" public administration-oriented, horizontal SDGs: 11, 16 and 17. All three SDGs are directly related to the field of Public Administration. SDG 11 aims at "making cities and human settlements inclusive, safe, resilient and sustainable." SDG 16 is about "promoting peaceful and inclusive societies or sustainable development, providing access to justice for all, and building effective, accountable, and inclusive institutions at all levels." The target for SDG 17 is "strengthening the means of implementation and revitalizing the global partnership for sustainable development."

All three SDGs are at the core of public administration for achieving public policy goals. CEPA, the UN/ECOSOC Committee of Expert of Public 
Administration, has developed principles of effective governance which are clustered around effectiveness (competence, sound policy making, collaboration), accountability (integrity, transparency, independent oversight), and inclusion (leaving no one behind, non-discrimination, participation, subsidiarity, intergenerational equity) (ECOSOC/CEPA, 2018). We need a utopian vision for public administration to realise these utopian policies, also for including much better public policy into public administration.

\section{From utopias to possible future realities: how to use "utopias" (or how not to use them)}

Even if "realised utopias" sound like an oxymoron, it is clear that a man has walked on the moon and that certain diseases have been eradicated. From this perspective, an old utopia is confronted and applied to a new reality. Utopia is "a uniquely effective form of politics. (...) elements of the utopia are gradually assimilated by the outside world, altering it in subtle but sometimes profound ways (...). Utopias are realized piecemeal, but realized they frequently are" (Neville-Sington et al., 1993, p. 255). Unfortunately, both Dystopias and Utopias have been realised. Achterhuis (1998, p. 303), for example, makes reference to Orwell's 1984 and "Big Brother" and to Foucault's Panopticum. From this point of view, utopias could either be at the beginning or at the end of a reality.

Utopias and dystopias have been useful for looking at the past better to know what is desirable and feasible. If it helps to understand the past better, does it also help to understand the future?

Even if for the last 500 years "utopianism has been one of mankind's principal navigational instruments," the great lesson of the twenty-first century has been, however, "that asking for a blueprint of the ideal society is asking for trouble" (Neville-Sington et al., 1993, p. 253, 254). Utopias are a "mental experiment," where the "imaginary procedure is used to test scientific ideas, not against the real world, but against each other, to reveal the connections between them and to seek out contradictions" (Neville-Sington et al., 1993, p. 255). Therefore, utopias are "useful as a tool of political thought," since they force us "to look at our unexamined assumptions, to explore those things which otherwise remain undisputed and undiscussed. (...) Utopia is the perfect vehicle (...), the field of opinion where they can influence social change" (Neville-Sington et al., 1993, p. 255).

There are "only the politics of fear and the politics of trust" (Gore, 2004, p. 792). Utopias could be a remedy against fear or could even contribute to 
an agenda of hope for the future (Elias, 2009b). However, utopia could also be mapping and developing this fear. According to dialectical reasoning, in a situation of catastrophic consequences and fear, utopias as a thesis become an antithesis, which is hopeful and reverses this fearful reality (Achterhuis, 1998, p. 94). This utopian antithesis, for example, could be the New Jerusalem. Joachim of Fiori (1135-1202) developed his "age of the spirit" and a revolutionary theology, which inspired several utopias including More's Utopia and Charles Péguy's "Cité Harmonieuse" (de Lubac, 1978). Cultural pessimism, which is about the end of history or mankind (Fukuyama, 1992, 2004) contributes to this. Obviously, utopias could also call for "resistance to civil government" to be able to create a new "Walden" (Thoreau, 1991).

Finally, utopias may contribute to an agenda of voluntarism and develop a strategy against fatalism. There are three situations where fatalism could emerge and weaken or even destroy pro-active voluntarism and utopias. There could be systemic determinism that explains why some systems will always be more successful than others. When "Guns, Germs, and Steel" determine the fates of human societies (Diamond, 1999), Western European and Asian societies will always dominate. When catastrophes frequently swipe civilisations they also affect the capacity to surmount them with utopias. The current research on catastrophes includes five characteristics: disaster, hazard, vulnerability, resilience and culture (Walter, 2008, p. 17). This is combined with the "risk" terminology in current policy and management research. Finally, when chaos overrules systems and "God plays dice", then again utopia as a perspective is under pressure (Stewart, 1997).

\section{Using "utopia" for social sciences}

For H.G. Wells, it is clear that utopias and science are related. In his "note to the reader", he clearly states: "I have inserted certain sections reflecting upon the established methods of sociological and economic science" (Wells, 1917, p. 7). And he continues: "It is good discipline for the Utopist to visit this world occasionally” (Wells, 1917, p. 350).

According to this logic, it is important to bring Utopias back to social sciences (topic, method, project) to develop "possible futures". For Elias, "utopia" becomes part of a social science research toolkit, just like a critique of sources, statistics, studies of trajectories, reconstructing networks, content analysis, reception studies, comparisons, etc. (Elias, 2009). Utopia as a social science technique allows you to look at potentials for realisation, gap analysis and possible futures. 
One of the key tensions for social sciences is the scientific challenge of different development dynamics and the levels of knowledge between the knowledge and handling of biotechnological and technological systems on the one hand (Isaacson, 2014) and the knowledge and handling of social/ societal mechanisms and systems on the other. This increasing distance and the diverging scientific dynamics create a real disequilibrium in a functional development of systems. This results in tension between realised technological utopias and unrealised social utopias. Technological realities increasingly challenge social utopias. As a result, technological progress triggers social dystopias.

Utopias also put more emphasis on the teleological rationality, rather than causality, for change patterns. They make path-dependency, bifurcations, constraints, backward mapping and effect/cause or objective/means logics more central. For Elias, this helps to re-evaluate the role of imagination in social sciences and opposes the fatalism of pragmatism in research and policies (Deluermoz, 2009, p. 29).

Ultimately, utopias help to discover the possible and desirable futures (Elias, 2009b, p. 216), but also the impossible and undesirable futures.

\section{Conclusion}

In this chapter we first looked at how realities influence utopias. The second part provided a (limited) historical view on public governance in Utopia. The third section discussed the utopian dimension of current governance policies. In the fourth part, we discussed how utopias may influence future realities. Finally, we conclude that utopias deserve to become part of our social sciences toolkit in general, and public administration in particular.

\section{Note}

1. Parts of this section are based on the introductory chapter of Achten et al. 2016. 


\section{References}

Achten, V., Bouckaert, G., \& Schokkaert, E. (eds). (2016). The Scholarly Quest for Utopia. Leuven: Leuven University Press.

Abensour, M. (2012). L'Utopie, Une nécessaire technique du réveil. Le Monde, October, Hors Série, Paris, 8-9.

Achterhuis, H. (1998). De Erfenis van de Utopie. Amsterdam: Ambo.

Bellamy, E. (n.d.). Looking Backward. London: William Reeves.

Bouckaert, G., \& Brans, M. (2019). The politics of bureaucracy in the face of different legal futures. The British Journal of Politics and International Relations 21(3): 530-540.

Bouckaert, G., \& Crompvoets, J. (2016). Utopian Public Governance: Cloudy, Cloudier, Cloudiest. In V. Achten, G. Bouckaert, \& E. Schokkaert (eds). The Scholarly Quest for Utopia. (pp. 158-171). Leuven: Leuven University Press.

Buck-Morss, S. (2002). Dreamworld and Catastrophe: The Passing of Mass Utopia in East and West. Cambridge, MA: MIT Press.

Butler, S. (1985). Erewhon. London: Penguin Classics.

Callenbach, E. (1990). Ecotopia: The Notebooks and Reports of William Weston, A Novel. New York: Bantam Books.

Carey, J. (ed.). (1999). The Faber Book of Utopias. London: Faber and Faber.

ECOSOC/CEPA. (2018). Principles of effective governance for sustainable development. ECOSOC, Official Records, Supplemnet No.24, E/2018/44-E/C.16/2018/8, para. 31.

De Geus, M. (1996). Ecologische utopieën, Ecotopia's en het milieudebat. Utrecht: Van Arkel. de Lubac, H. $(1978,1980)$. La postérité spirituelle de Joachim de Flore (tome I: de Joachim à Schelling; Tome II: de Saint-Simon à nos jours). Paris: Editions Lethielleux.

Deleurmoz, Q. (2009). Les utopies d'Elias. La longue durée et le possible. In N. Elias (ed.). L'Utopie (pp. 5-29). Paris: La découverte.

Deproost, P.-A., Nyns, C.-H., \& Vielle, C. (2015). Chemins d'Utopie, Thomas More à Louvain, 1516-2016. Louvain-la-Neuve: UCL Presses Universitaires de Louvain.

Diamond, J. (1999). Guns, Germs, and Steel: The Fates of Human Societies. New York: W.W. Norton \& Company.

Dryzek, J. S. (1997). The Politics of the Earth, Environmental Discourses. Oxford: Oxford University Press.

Elias, N. (2009a). La critique de l'Etat chez Thomas More. In N. Elias, L'Utopie (pp. 31-102). Paris: La découverte.

Elias, N. (2009b). A quoi servent les utopies scientifiques et littéraires pour l'avenir?. In N. Elias, L'Utopie (pp. 103-132). Paris: La découverte.

Elias, N. (2009c). Thomas More et l'utopie. In N. Elias, L'Utopie (pp. 133-150). Paris: La découverte. Francis (Holy Father). (2015). Laudatio Si, On care for Our Common Home. Vatican City: Holy See. Fukuyama, F. (1992). The End of History and the Last Man. New York: Free Press. 
Fukuyama, F. (2004). State Building: Governance and world order in the twenty-first century. Ithaca NY: Cornell University Press.

Gawthrop, L. C. (1998). Public Service and Democracy: Ethical Imperatives for the $21^{\text {st }}$ Century. New York and London: Chatham House Publishers.

Ghannouchi, R. (2014). The Tunisian Experience. Cairo Review of Global Affairs 13(Spring): 97-102.

Gore, A. (2004). The Politics of Fear. Social Research 71(4): 779-798.

Isaacson, W. (2014). Inventing the Future. Time Magazine, The Genius Issue, December 1-8: 54-82.

Kaufmann, D., Kraay, A., \& Mastruzzi M. (2010). The Worldwide Governance Indicators: Methodology and Analytical Issues. Policy Research Working Paper Nr. 5430. Washington, DC: World Bank.

Lawlor, R. (1991). Voices of the First Day: Awakening in the Aboriginal Dreamtime. Rochester: Inner Traditions.

Micklethwait, J., \& Wooldridge, A. (2014). The Fourth Revolution. London: Penguin Books.

More, T. (1980). Utopia. London: Penguin Classics.

Neville-Sington, P., \& Sington, D. (1993). Paradise Dreamed, How Utopian Thinkers have Changed the Modern World. London: Bloomsbury.

Stewart, I. (1997). Does God Play Dice?: The New Mathematics of Chaos. Harmondsworth: Penguin Books.

Suleiman, E. (2003). Dismantling Democratic States. Princeton, NJ: Princeton University Press. Theunissen, P., \& Cumps, L. (1992). De Reis van de Ontmoeting, Logboek van Christoffel Columbus (3 augustus 1492 - 15 maart 1493). Leuven: Acco.

Thompson, P., \& Zizek, S. (eds). (2013). The Privatization of Hope: Ernst Bloch and the Future of Utopia. Durham, NC, and London: Duke University Press.

Thoreau, H. D. (1991). Resistance to Civil Government. Edited by W. Rossi, T. Owen, New York and London: W.W.Norton \& Company.

Toulmin, S. (1990). Cosmopolis, The Hidden Agenda of Modernity. Chicago, IL: University Press of Chicago.

UN. (2015). Zero draft of the outcome document for the UN Summit to adopt the Post-2015 Development Agenda.

Walter, F. (2008). Catastrophes, Une Histoire Culturelle, XVI-XXI Siècle. Paris: Editions du Seuil. Wells, H. G. (1917). A Modern Utopia. London: Thomas Nelson and Sons.

http://www.worldbank.org/en/topic/poverty/overview. 



\section{2 \\ Governing for the Future: Means, Ends and Disconnects}

Paul Joyce

\section{Deciding on the ends to include in a utopian future is relatively easy}

Getting the political leaders from the world's countries to sign up to an agenda that is utopian (in the best sense of the word) is a major achievement. In 2015 the leaders of the governments of the world decided to commit their individual countries to delivering the 2030 Agenda for Sustainable Development.

In hindsight it is quite surprising how much global agreement there is on the things that really matter and what a much better world would look like. This chapter argues that the really difficult thing is not agreeing on what would be desirable, but judging what is possible and making sure it is delivered. In the public sector world of strategic management, the existence of a "possibility" is implied whenever an attempt is made to evaluate the feasibility of proposed actions or programmes. When public administrators selecting strategic action ensure that they factor in judgments of feasibility, the result is strategic plans that have significantly more impact.

In this chapter I will be briefly looking at how the future happens, the ambitious and utopian character of long-term strategic visions published by governments, populism in Europe, the need for realism in utopian thinking, techniques for addressing the future, and practical recommendations for academics working in the field of public administration.

\section{How Does the Future Happen?}

The future is not fixed and determined until it has happened. In the future, what was a possibility has become a reality, and at the same time some of the things that we thought were possible will have failed to materialise. We only know that something really was possible for sure when it has become a reality. 
As was said by a government minister in Ireland recently (Department of Housing, Planning, Community and Local Government, 2017, p. 2): "What will Ireland be like in 20 years' time? It's a fundamental question that no one can answer for sure...”

It has been said before: people make the future by the actions they take, and the actions they take have consequences for the future. Governments as well as people take actions, and they too have consequences for the future. The actions taken by governments are often a response to challenges or are attempts to deal with problems and issues. Nowadays governments put out vision statements and publish long-term strategies as frameworks for the actions they will take. The hallmark of a modern government following best practice guidance on public governance is to have long-term strategic priorities and a long-term strategic vision, and to pursue them through planned, coordinated and integrated action by civil servants and government entities, by mobilising stakeholders in society behind the long-term vision, and by engaging citizens and getting their support.

But it is well understood that there can be no guarantees that government action always works and always produces better futures. There are times, for example, when individual political leaders worry that the priorities and visions are wrong, that government action is not adequately coordinated and integrated, and that stakeholders and citizens appear to be withholding their involvement and support. In the last decade EU politicians have worried that Europe has had big strategic agendas, but their relationship to the public's concerns has not been obvious or clear enough to the public. With the rise of populism after the international crisis of 2007 to 2009 , criticisms have grown of the government as an institution (with government referred to as "the political establishment" or the "political class"). In such times the big long-term visions and strategic agendas of government may seem "utopian" in the sense of not having a chance of realisation.

\section{Ambitious ends}

Like all areas of human endeavour, thinking about the future involves thinking about ends and means. (See Figure 1.) We can find instances of government leaders taking time out of the busy whirl of day-to-day politics to think long-term about ends and means. Some of these instances go back 50 or more years. Units and think tanks have been set up, foresight programmes established, and strategic policy making capacity developed. Some initiatives were transitory. In the UK, for example, the Prime Minister's Strategy Unit 
was set up in 2002 as the main central government unit for future thinking but was closed in 2010 by Prime Minister Cameron. But since the late 1990s, for 20 years, the momentum towards long-termism and strategic thinking has been sustained.

Figure 1 Means and Ends

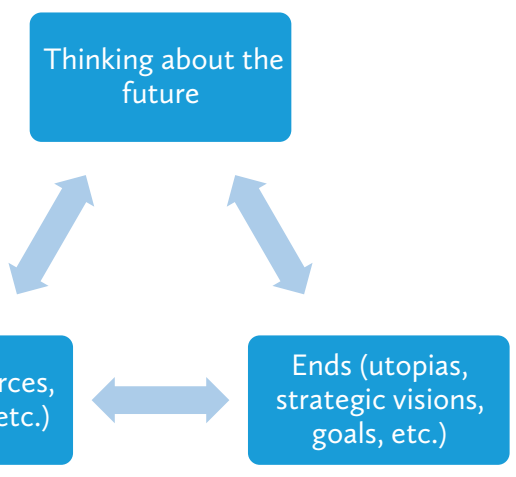

PA practitioners may have traditionally thought that it was important to conduct their work based on norms, rules, laws, procedures, etc., but increasingly from the late 1980s onwards they have been asked to pay more attention than in the past to means and ends (outcomes). One of the characteristic features of public governance today is the enormous ambitions to be found in government statements of desired ends. This is true within Europe and beyond Europe. A perfect example of this for the whole world is the vision contained in the 2015 United Nations resolution on transforming the world, that is, the 2030 Agenda for Sustainable Development.

The delivery of this avowedly transformative agenda was scheduled to be a long-term endeavour taking 15 years. In the vision for the 2030 Agenda it was imagined, among other things, that there could be an end to poverty, hunger, disease, want, fear and violence. According to the vision, all people would have access to quality education, health care, social protection, safe drinking water and sanitation. This list already may seem a long way towards a better world, but the vision contained much more. It saw a future of equal opportunity and full realisation of human potential. It saw a future world of justice, "A just, equitable, tolerant, open and socially inclusive world in which the needs of the most vulnerable are met" (United Nations General Assembly, 2015, p. 4/35). The world of 2030 was to be a world "in which development and the application of technology are climate-sensitive" and people lived in harmony with nature (ibid., 2015, p.4/35). And still this does not exhaust all 
the good things anticipated by the vision. It was a breathtaking and dazzling synopsis of a better world for all. The United Nations Secretary-General included in one of his speeches a powerful rallying call - that no one would be left behind - a phrase which has since appeared in other visionary statements.

Another example of the ambitious ends being placed in long-term government visions of the future is to be found in the 2017 draft of the National Planning Framework in Ireland. Three statements are picked out here to make the point about ambition. These statements offer a succinct set of concepts for a more desirable future, for a utopian future in Ireland:

"The vision for Ireland in 2040 is for a fair society with strong social cohesion and converging living standards throughout the country, in which all individuals, businesses, communities and regions have the opportunity to prosper." (Government of Ireland, 2017, p. 19).

"The vision for Ireland in 2040 is for the highest possible quality of life for our people and communities, underpinned by high quality, well managed built and natural environments." (Government of Ireland, 2017, p. 19).

\footnotetext{
"The vision for Ireland in 2040 is to commit to being the most successful, advanced, competitive and environmentally sustainable economy and society in Europe." (Government of Ireland, 2017, p. 22).
}

The second of the characteristic features of the present period is the weakness of the planning of means and the provision of means to deliver ambitious ends. In practice the planning of means may be vague, skimpy on details, and incomplete. It may fail to plan properly for the acquisition or mobilisation of all the resources needed. Some sign of this can be found in the well-known "planning fallacy" - the widespread experience that big government projects are delivered late and are delivered much over budget. One way or another, for one reason or another, the planning and provision of means is often simply inadequate. For example, in June 2019 Theresa May, just weeks before stepping down as the UK Prime Minister, announced a laudable and ambitious longterm goal, one that she was intending to be made into a legal commitment. This goal was for Britain to achieve net zero carbon emissions by 2050. She planned to act immediately, taking advantage of legislation going through Parliament, making an amendment to it using a statutory instrument, which avoided the need for a vote in Parliament. One criticism of her was that she had not ensured a full costing of the plan to deliver net zero carbon emissions (Walker et al., 2019). 
Another example might be the Europe 2020 strategy, which was authorised by the European Council in 2010 (Drumaux and Joyce, 2018). The desired ends of the strategy were clearly identified in a set of headline targets, but the institutional means for the governance of the long-term strategy proved to be insufficient. For example, the departments of the Commission from 2014 onwards were not sufficiently focused in their plans for the delivery of the headline targets; the targets were peripheral to the planned work of the departments. The overall integration of member states into the implementation of the strategy was insufficient; this was most explicit in the case of the UK which for a long time did not report on all the headline targets in its national reform programmes. In other words, the governance arrangements for the delivery of the strategy depended on the institutional relationships of the EU, and it might have been reasonably doubted from the start that these were right for delivering Europe 2020. In 2019, it was clear that delivery of two of the most important goals of the Europe 2020 strategy might not be achieved by the scheduled end of the strategy in 2020. These goals were expressed as targets for lifting people out of the risk of poverty and increasing the rate of $R \& D$ investment:

"challenges remain with regards to the target of lifting 20 million people out of the risk of poverty and social exclusion. (...) by the end of 2017 the number of people at the risk of poverty was only 4.2 million less than in 2008. (...) With the latest figures indicating a rate of $R \& D$ investment of slightly above $2 \%$ of GDP, the gap to the $3 \%$ target remains considerable and will not be closed by 2020." (Employment and Social Protection Committee, 2019, p. 8).

A disconnect between the ends to be realised and the means used to deliver the ends must undermine the success of public governance in addressing the future. There are signs that this disconnect is often recognised in practice. The international attention that has been paid by governments to the ideas of Michael Barber on how to become better at delivery is one such sign (Barber, 2015). Governments often appreciate that unless the right means are put in place the ends are just wishful thinking. Of course, the means are critical for efforts to implement and deliver priorities and long-term strategic visions in the right way. 


\section{$4 \quad$ Populism proves that the long-term strategic visions are failing?}

The relationship between government and the public in the processes of public governance in European societies has to be understood in concrete terms, meaning that how this relationship operates in practice depends on many conditions, including the existence of social cleavages, generational conflicts and the strength of populist sentiments in the general population. In recent years all of these conditions must have made themselves felt in terms of politics and public administration. But populism has offered the sharpest and most stinging assault on the credibility of Europe's democratic governments in recent years - and as a by-product cast doubts on governments' strategic ambitions and conceptions of utopian futures. In the case of Europe, for example, the ideal of a future of social inclusiveness and social solidarity was often implicitly, if not explicitly, challenged by the growing resistance of many citizens to the scale and effects of immigration. In fact, we might conclude that the rise of populism in Europe over the last decade could be taken as evidence that a new utopianism through the long-term strategic visions of governments has not been enough.

One obvious answer to populism would be to bring the public into the government's decision-making process. This is based on assuming that the public needs to see its main concerns written into the long-term visions and it has to perceive that the delivery of the visions has been effective. Opening up government to public participation might work - it might cause government's visionary ends to be more in line with public concerns and the means of delivery might be increased by virtue of additional public support. While this answer has its appeal, is there any evidence that it would work? Is much greater public engagement and public participation realistic?

This chapter may be guilty of offering a superficial impression of the populist "moment" in Europe, but the possibility of a rationality gap between public administrators and populist-minded members of the public seems very real. Furthermore, it is tempting to suggest that European public administration may be ill-equipped intellectually for operating effectively to close a rationality gap in circumstances where populism is strong.

The rationality gap may be described as follows. On one side is public administration that has been repeatedly told for years that best practice requires evidence-based decision making and evidence-based policy. It has also been told to embrace open government principles of transparency, accountability and participation, and therefore it should build a high trust relationship with the public. The result is that in seeking to be transparent and 
accountable public administrators should provide rationales and justifications to the public that involve explaining public services decisions and actions on the basis of analysis and evidence. On the other side is populism, which is energised by distrust of the establishment, and in some countries distrust has been accompanied by a populist rejection of experts and professionals, a manifestation of post-truth society, some would say. Public administration in trying to operate a system of rationality that formally endorses and formally practises evidence-based decision-making and policy will encounter criticism and cynicism from those citizens who are rejecting the official rationality of the establishment. In some countries an emphasis on evidence and analysis by public administration may hit a wall of public indifference to the official evidence and a preference for believing the words of populist leaders who are attacking the government.

If many members of the public are in a populist frame of mind, and if they are relying heavily on using beliefs and feelings as the main source of their understanding of public problems, how are they to be engaged with the work of government in evidence-based policy making? One response might be for public administration to "try harder" to be transparent and accountable using the language and arguments of evidence-based decisions and policy.

The alternative responses are political ones and belong to the world of the elected politicians rather than professional public administration as such. One response would be for "establishment" politicians to look for events and situations in which populist thinking is clearly running into difficulty and error - presumably seeking to identify events and situations that are providing a reality check on the ideas of populist politicians. Then the establishment politicians will have to try to use the authority of these events and situations to rebuild their credibility with the populist-minded members of the public. This is a problematic option for the same reason that the public administration response is problematic, that is, currently evidence and reasoning appear not to be very convincing to the members of the public attracted by populist rhetoric.

A second political response to populism is utopianism. It will need to be a bold utopianism - one with big visionary ideas. The assumption here is, of course, that utopian politicians are better placed to compete with populist politicians than, say, technocratic politicians. To differentiate themselves from populist politicians, however, the utopian politicians will also have to concern themselves with visionary ideas for how better futures can be delivered. This means being visionary about ends but also about means. They will have to be "practical idealists" - being boldly idealistic about the future (ends) and at the same time practical in their big ideas on delivery (means)! 
They would be making utopian promises about means as well as ends to capture the imagination of the public in a contest with populist promises.

To sum up, the specific nature of the populist challenge, combined with governments' commitments to open government and evidence-based policy, may be very difficult for public administration trying to sustain government credibility. The dilemma being experienced by politics and public administration may be one of trying to decide between focusing on finding more effective ways to communicate with populists in the public despite a rationality gap or focusing on making changes in government policy to satisfy the political demands of populist parties. At this point in the trajectory of populism in Europe, these suggestions about the challenges and options for public administration (and politics) seem pretty speculative.

\section{$5 \quad$ What is to be done?}

So far, this chapter has argued that utopian thinking is an important element of governments' long-termism and government strategic visions. Utopian thinking is flourishing to the extent that there are ambitious long-term strategic visions. But that is not enough. The acid test is the delivery of utopian thinking, and this depends on realism and on means.

There are some obvious responses to the need for more realism in terms of techniques available for use in public administration. But the answer cannot be solely in terms of techniques. Resources, the institutions of governance and the democratic basis of successful public governance all matter.

\section{Trend analysis}

Trend analysis is the analysis of evidence to identify trends. One reason for doing this analysis is to come up with predictions about future reality. It can be used to anticipate the future by the simple action of projecting past trends into the future. In the case of the European Union's economy, for example, it has been predicted that it will not be one of the three largest economies in the world by 2035; this prediction was based on analysing trends, which were trends in population and trends in emerging markets (European Parliamentary Research Service, 2018).

One limitation of this type of analysis is that trends may unexpectedly change. Even if attempts are made as part of a trend analysis to assess reasons for expecting future changes in trends, analysts can still be surprised by them. 
This is presumably why predictions that the European Union's economy might no longer be a top three economy in 2035 were couched in terms of it being a possibility, rather than a certainty. Uncertainty is not just a matter of uncertainty about whether the trend will continue as in the past; there are also other uncertainties that are produced by the effects of trends continuing as expected. Take the case of the trends leading to the predicted relative decline of the European Union's economy because of the phenomenal economic growth of China and India. It is expected that these two economies will carry greater weight in the future global economy. Their respective models for national economic and social development are different from Europe's model. As their models assume greater importance, it is uncertain how this will affect the European Union in terms of the consequences for the dynamics of the global economy. Will the most important conditions in the global economy move slightly away from market forces and nearer to government decision? Possibly.

Trend analysis can be used to think strategically about policy. Governments can ask the question: what are the implications for people's lives of this trend continuing? What strategies, policies, government support or public services might they need because of these trends? But in practice there may be difficulties in integrating work on trend analysis with strategies and policy making.

Another technique that does address the existence of uncertainty head on is scenario planning, a technique for formulating alternative scenarios of the future.

\section{Confronting uncertainty - scenarios framed by "axes of uncertainty"}

Scenario planning has partially replaced trend analysis and forecasting as a popular way of anticipating the future. It involves weaving together assumptions and a small number of "important" uncertainties. It is manageable as a technique only if there are just a small number of important uncertainties.

An example of a scenario planning exercise in the UK was one commissioned by David Triesman, a government minister in the Blair Government. He was the new minister at the Department for Innovation, Universities \& Skills, which had been formed in 2007. He apparently wanted to see scenario planning used in order to check the policies inherited from the merged departments and to create new ideas. During the first few months of the project, the scenario planning was scoped and support for the project sought. At a workshop "drivers for change" were identified. These became a 
list of 12 factors comprising: global balance of power; economic integration; layers of power; communities and communications; demographics and migration; values and beliefs; inequalities, research and innovation; climate change and resources. Four issues were drawn out of the list of 12 "drivers": globalisation, the development of the emerging economies, social values and resource constraints. All the issues featured in the eventual scenarios, but two of them - globalisation and social values - became the "axes of uncertainty." Each axis (variable) was dichotomised and two labels (values) were provided for it. Four scenarios were written with each using a label from each axis.

In my opinion greater thought needs to be given to the implications of the language of "drivers for change." It is a language fundamentally different from the language of cause and effect. "Drivers for change" conjure up memories of force field analysis in which equilibrium is the result of two sets of equally matched opposing forces. This is a "tug of war" analogy to explain the current status of things. Of course, in scenario analysis, it is just one set of "drivers" all pointing in the direction of the future. But, what precisely does it mean to say that the future is being created by drivers for change such as "inequalities" or "layers of power" or "climate change"? It is possible that this way of explaining movement to the future works intuitively but makes less sense as you consciously delve into its actual meaning. Does the use of "drivers" also cause us to gloss over how the scenarios have been formulated? And what about the issue of the validity of the scenarios generated by using drivers for change? Have there been any attempts to check out the "truth" of the scenarios by reviewing what did happen in the future, some years after the scenarios had been published? Such checking does not seem to get any extensive coverage in the technical literature on scenario planning.

It might be important for PA academics to consider whether or not scenarios are judged by most stakeholders in a very subjective way. It is possible that this subjective way is to say: do I find this scenario a coherent narrative? If scenario users do find the scenarios coherent, then they may gloss over the details of how they were formulated and end up having unwarranted confidence in the scenarios before them. It may be useful to note here that some people have advocated the use of scenario planning by claiming that using scenario planning creates higher states of alertness and readiness for change among its users. Given such a rationale the validity of the scenarios becomes unimportant.

Scenario planning can, in principle, be used to test the viability of a government's current long-term strategic visions. It is even possible to use scenarios to inspire strategic visions (by asking what vision might make sense in a given scenario). 


\section{Strategic intent, experimentation and learning}

One way out of thinking that the future has to be intellectually approached solely in terms of extrapolation of trends or in terms of scenarios is to recognise the way in which resource-based theories in strategic management can conceptualise foresight as strategic intent and then argue for experimentation and learning to create the means (find the path) by which strategic intent (the outcomes imagined) can be achieved.

This thinking can be applied by governments in Europe. Long-term strategic visions, and their utopian ends, can be delivered more effectively on the basis of learning and experimentation, with the learning providing a way of increasing the input of realism into visions and strategic plans. Learning can take place in advance of formulating long-term strategic visions - through benchmarking studies of governments in other countries. Individual governments can try learning from their own country's earlier attempts to implement strategic visions and plans. This may be easier than people appreciate, since there are certainly examples of strategic visions in Europe that had clear continuities with preceding strategic visions (e.g. the European Union's Lisbon strategy and the subsequent Europe 2020 strategy).

"Realism" can be progressively introduced through evaluation and learning that could, and I would say should, take place during the delivery of a strategic vision. The character of the processes of strategic management and of public policy can be important here: they can be endowed with an experimental character. If governments institute a culture of experimentation it should, in principle, create more propensity to learn from delivery. In this way, a dialectical process may be created in which possibilities are turned into real better futures.

This emphasis on monitoring and evaluation during delivery is far from a new idea. It has been around for a while. One example comes from the mid 1990s, which we might see as a "change-over" period, when the 198 os preoccupation with government efficiency gave way in the late 1990 s to an interest in government effectiveness. The example is the Strategic Management Initiative in Ireland. It was launched by the Taisoeach (prime minister) in 1994. Its aim was to improve efficiency. The Initiative required each government department to produce a statement of strategy by the end of 1996. According to Boyle and Fleming (2000, Part 2, 2.2) the framework for strategic management included monitoring and corrective action. The framework also envisaged that there would be adjustments of statements of strategy based on an ongoing review process and major reviews. The idea of monitoring and evaluation being important continues to this day; for example, they were strongly recommended to the Spanish government in order to improve public governance (OECD, 2016, p. 33). 


\section{Practical recommendations to the academics of the PA community}

Should the academic PA community be giving more importance to the future in its research and teaching? And what more should the community be doing than it is presently doing? Answers to these questions depends on the role prescribed for PA academics. They could be disinterested (unbiased) and impartial social scientists studying and analysing events and situations with the pure aim of understanding to satisfy their curiosity. In that case, it is entirely up to them what they find interesting and to what they attach importance. Given that democracy is such an important aspect of the culture and politics of Europe, academics could have the role of contributing to the democratic process by conducting academic inquiry to support public debate and discussion about government long-term visions, strategies and policies (defining policies as linked to strategies). In this case, the answer would be quite different: much more attention should be given by academics to the future in both their research and their teaching. Then again, the academics could have the role of advisers and consultants to government; they would offer their expertise for solving government's administrative problems, being ready to diagnose issues and draw attention to successful examples of government reform and social change in other countries, skilled in evaluating what government policies have worked well in the past and why, and knowledgeable about the monitoring and evaluation of the visions and strategies that are in the throes of implementation. In this case, the importance given by academics to the future will be a function of the clients' interest. Do governments want them to become more expert and more focused on the future?

Then there is an overlapping issue for the role of academics; should academics make it clear when they are expressing their personal values about what ends governments should have, what good futures look like, and how better futures should be pursued, making it crystal clear that they are not trying to be analytical and "objective"? Or can their personal values about what is good and bad be integrated into their "scientific" work analysing public governance and their professional teaching of vocational courses on public governance?

These are not novel ideas about the role of academics. However, it might be presumed that they are ideas that each new generation of PA academics has to consider afresh and about which it has the responsibility to produce its own point of view suitable for the circumstances of the day.

In this chapter it has been argued that public administration has become good at setting out ambitious ends (in utopian strategic visions of the future); the means and their use are now the weakness that needs attention 
in government work. What might be recommended as solutions, or partial solutions, to the problem? We can approach this using a technique for creative thinking: a morphological box. This is presented in Table 1.

Table 1 Morphological Box

\begin{tabular}{|c|c|c|c|}
\hline Dimension & 1 & II & III \\
\hline $\begin{array}{l}\text { Philosophical } \\
\text { framework }\end{array}$ & $\begin{array}{l}\text { The reality of the } \\
\text { world is one big } \\
\text { causal network that } \\
\text { stretches back into } \\
\text { the past and forward } \\
\text { into the future }\end{array}$ & $\begin{array}{l}\text { The future is a reality } \\
\text { largely constructed } \\
\text { through the process } \\
\text { of ideas becoming } \\
\text { real }\end{array}$ & $\begin{array}{l}\text { The future is inde- } \\
\text { terminate because of } \\
\text { the existence of free } \\
\text { will and because of } \\
\text { pluralism }\end{array}$ \\
\hline $\begin{array}{l}\text { Form of } \\
\text { anticipation of } \\
\text { the future }\end{array}$ & $\begin{array}{l}\text { Prediction [planning } \\
\text { is possible] }\end{array}$ & $\begin{array}{l}\text { Belief (including } \\
\text { religious faith) } \\
\text { [readiness is possible] }\end{array}$ & $\begin{array}{l}\text { Foresight } \\
\text { [intent is possible] }\end{array}$ \\
\hline $\begin{array}{l}\text { Knowledge } \\
\text { desired of ... }\end{array}$ & Future reality & Uncertainties & Possibilities \\
\hline $\begin{array}{l}\text { Input to future } \\
\text { thinking }\end{array}$ & $\begin{array}{l}\text { Realism (Condi- } \\
\text { tions and causal } \\
\text { relationships) }\end{array}$ & $\begin{array}{l}\text { "Drivers" } \\
\text { [Trends in economics, } \\
\text { values, etc.] }\end{array}$ & $\begin{array}{l}\text { Expectations and } \\
\text { values [cultural } \\
\text { ingredients] }\end{array}$ \\
\hline Techniques & Trend analysis & Scenario planning & Experiments \\
\hline $\begin{array}{l}\text { Definition of } \\
\text { utopia }\end{array}$ & $\begin{array}{l}\text { Fantasy (the future } \\
\text { will be what it will } \\
\text { be - irrespective of } \\
\text { our choices) }\end{array}$ & $\begin{array}{l}\text { No definition of a } \\
\text { utopia possible - clos- } \\
\text { est related concept } \\
\text { would be "best case } \\
\text { scenario" }\end{array}$ & $\begin{array}{l}\text { A long-term strategic } \\
\text { vision of a better } \\
\text { society that acts as a } \\
\text { target for choices and } \\
\text { actions }\end{array}$ \\
\hline $\begin{array}{l}\text { Use of future } \\
\text { thinking }\end{array}$ & $\begin{array}{l}\text { Technocratic - ex- } \\
\text { perts and politicians } \\
\text { use future thinking } \\
\text { better to meet the } \\
\text { needs of the public }\end{array}$ & $\begin{array}{l}\text { Managerial - for } \\
\text { wind-tunnelling } \\
\text { (checking robustness } \\
\text { of plans), preparation } \\
\text { for formulating } \\
\text { visions and goals }\end{array}$ & $\begin{array}{l}\text { Democratic - to sup- } \\
\text { port public debates } \\
\text { and arguments about } \\
\text { ends and means }\end{array}$ \\
\hline $\begin{array}{l}\text { Role description } \\
\text { for PA academics }\end{array}$ & $\begin{array}{l}\text { Disinterested } \\
\text { (unbiased) role - } \\
\text { possibly focused } \\
\text { on understanding } \\
\text { causal relation- } \\
\text { ships or process } \\
\text { models, etc.; possibly } \\
\text { author of long-range } \\
\text { predictions }\end{array}$ & $\begin{array}{l}\text { Academic adviser/ } \\
\text { consultant role - help } \\
\text { government with } \\
\text { problem solving and } \\
\text { decision-making so } \\
\text { that visions and goals } \\
\text { are delivered }\end{array}$ & $\begin{array}{l}\text { Evaluator role - } \\
\text { concerned with } \\
\text { theory and practice } \\
\text { relationship; priority } \\
\text { placed on describing } \\
\text { what happens in } \\
\text { practice and what the } \\
\text { consequences are }\end{array}$ \\
\hline
\end{tabular}


The point of view I am taking here is that the future is indeterminate and influenced by the presence of pluralism in society, that governments are right to develop strategic foresight and have a responsibility to make changes (short-term and long-term) towards better societies, and that academics in democratic societies do not have to be restricted to the role of disinterested and curiosity-driven observers. Such a point of view has implications for my recommendations that techniques for thinking about the future should get our attention, that democratic relationships are of major interest to us, and that the theory and practice of making decisions aligned to possibilities needs more work.

For academic researchers it is recommended that research into evaluation, learning and experimentation is made a higher priority. This is so that the theory and practice of delivering long-term strategic visions should be developed much further than it is. There is great variability in the effectiveness of monitoring and evaluation by government and this could, in Europe, be highly significant for government effectiveness and government capacity for reform. It might be appropriate, therefore, for academic research to investigate what is happening in governments and public administrations that have achieved the highest European standards in respect of the work of evaluating, learning and experimenting as part of the delivery of important strategic outcomes and policy objectives.

It might be doubted that this research topic is a high priority for academics in PA. After all, proposals for public management reforms, going back 30 or 40 years, have included the need for performance measurement and monitoring and evaluation. Despite all the exhortation to monitor, evaluate and learn, the amount of good quality research on these processes can easily be added to.

Secondly, for academic researchers there is a need to research and better understand the relationship between government and citizens in respect of the governance of long-term strategic visions of national governments. In a European context, democracy appears to be a critical condition for government effectiveness (Drumaux and Joyce, 2018). Public opinions on democracy, again in Europe, are closely linked to public confidence in governments, and thus can be seen as highly relevant to issues of government credibility. Arguably, academic researchers need to be especially concerned with inquiry into the public's engagement with government processes of creating longterm strategic visions and delivering them. The study of public engagement might include engagement through representative democracy (as complex as that may seem in the context of populism) but should definitely include engagement through consultation and participation mechanisms.

A third topic - or problem - for academic research is the phenomenon of public administrators "knowing what are actual possibilities" with respect 
to the future. A suitable research question might be: how do public administrators making decisions about the future decide what are actual future possibilities? This would involve distinguishing what is possible from what is impossible. As part of this research agenda, academic investigators could look into the theory and practice of deciding on the feasibility of a strategic course of action.

For academic teachers designing and delivering courses to public administrators the challenge is to give more thought and careful consideration to how they help students become more aware of the need to be forward-looking and how they support the students' learning of appropriate techniques and skills useful for the delivery of long-term utopian strategic visions and goals. The techniques learnt could include trend analysis, scenario planning and public policy experimentation.

If current public administration students on university courses are learning techniques for thinking about the future, they are very likely to be learning about trend analysis and scenario planning rather than about experimental methods to implement utopian strategic visions. Trend analysis tends to convey the assumption that the future can be predicted by extrapolation of existing trends. Even if the trends do continue into the future, there is a big leap from identifying a possible future trend to developing foresight about government action. Scenario planning normally makes future uncertainty the paramount feature of thinking about the future. What effect is scenario planning likely to have on the students' mind-sets and how they approach working in the public sector? Much more important, in my opinion, is for the students to learn experimental techniques and methods and to learn how to learn from them. Politicians may increasingly put a premium on administrators being willing and able to experiment with new pathways to desired futures, and being willing and able to do so in ways leading to learning, refinements and adjustments to government actions. Maybe there does not have to be a choice between trend analysis, scenario planning and experimental techniques and methods. But there should not be over-reliance on trend analysis and scenario planning. This is an important question for the academics of public administration to consider over the next five to ten years and to take into account when designing university courses.

Making the future an important part of courses may be uncomfortable for some academic teachers. Asking teachers to devote (more) time in courses to teaching, and assessment of, student knowledge of, and skills in, the anticipation of the future may take some of them out of their comfort zone. There could be a parallel drawn here with the teaching of ethics: many academics feel happier teaching students about ethics and quite nervous about teaching 
them to be ethical. We need to do more than teach students about the future; we need to enable students of public administration to be better at anticipating and managing the future. Accepting this particular recommendation may mean that teachers will experience some disruption to their current ideas of what is easy and possible to teach and assess.

Finally, we need to reject the idea that utopias are the result of fantasy thinking and cannot be delivered in practice. In fact, because of populism, we need utopian thinking more than ever. But it has to be a bold utopianism that addresses means as well as ends, with big but workable ideas on delivery. To adapt a statement of Michael Barber (2015, p. 64), a utopian long-term strategic vision without the right means and the right delivery is pure fantasy; the right means and the right delivery without a utopian vision may be government stuck in a rut or government that lacks purpose. In my view, public administration academics should be "engaged scholars." If we were to engage more with the challenge of supporting democratic involvement of the public in governments' long-term strategic visions and their delivery, and if we were to do more to support civil servants and public sector managers in learning to be effective in revising, refining and delivering visions and strategies, we could help increase the realism of utopias.

\section{References}

Barber, M. (2015). How to Run a Government: So that Citizens Benefit and Taxpayers Don't Go Crazy. London: Allen Lane.

Boyle, R., \& Fleming, S. (2000). The Role of Strategy Statements. Committee for Public Management Research Report No. 2. Dublin: Institute of Public Administration.

Department of Housing, Planning, Community and Local Government (Ireland). (2017). Ireland 2040: Our Plan - Issues and Choices. http://npf.ie/wp-content/uploads/2017/02/PositionPaper-Issues-and-Choices-Ireland-2040-web.pdf.

Drumaux, A., \& Joyce, P. (2018). Strategic Management for Public Governance in Europe. London: Palgrave Macmillan.

Employment and Social Protection Committee. (2019). European Semester: Horizontal note on employment and social policy aspects of Country Specific Recommendations: Policy debate. https:// data.consilium.europa.eu/doc/document/ST-9879-2019-INIT/en/pdf.

European Parliamentary Research Service. (2018). Global Trends to 2035 Economy and Society. Brussels: Global Trends Unit of the Directorate for Impact Assessment and European Added Value, within the Directorate-General for Parliamentary Research Services (DG EPRS) of the general secretariat of the European Parliament. 
Government of Ireland. (2017). 2040 Our Plan. Draft National Planning Framework. A Government of Ireland Policy. http://npf.ie/wp-content/uploads/2017/10/Ireland-2040-Our-Plan-DraftNPF.pdf.

OECD. (2016). OECD Public Governance Reviews: Spain 2016: Linking reform to results for the country and its regions. Paris: OECD Publishing.

United Nations General Assembly. (2015). Resolution: Transforming our world: the 2030 Agenda for Sustainable Development. http://www.un.org.

Walker, P., Mason, R., \& Carrington, D. (2019). Theresa May commits to net zero UK carbon emission by 2050. The Guardian, June 11, 2019. https://www.theguardian.com/environment/2019/ jun/11/theresa-may-commits-to-net-zero-uk-carbon-emissions-by-2050. 



\title{
3 \\ Scenarios as Thought Experiments for Governance
}

\author{
Meelis Kitsing
}

\section{$1 \quad$ Introduction}

A century ago, American poet Robert Frost published his famous poem "The Road Not Taken" which has the following lines "Two roads diverged in a wood, and I - / I took the one less traveled by, / And that has made all the difference" (Frost, 1916). Frost's poem highlights a fundamental dilemma in human affairs concerning the future. Our individual and collective choices lead us intentionally or unintentionally to take a certain path. This leads to intended and unintended consequences. There is vast social science literature trying to identify "critical junctures" which leads groups, governments and societies onto a particular path. This process is sometimes called "punctuated equilibrium." As Frost indicated in his poem and numerous social scientists have pointed out, it is often not possible to go back and choose another path because developments that unfold are "path-dependent" (Campbell, 2010).

However, it is still worth exploring not only roads that have been taken but also roads that are not taken; particularly as path-dependence can have many alternative interpretations and there is no reason to take one overly deterministic view. Furthermore, critical junctures which unfold path-dependent development are not necessarily rational affairs where different parties engage from what Rawls (1971) called "original position" but rather semi-rational and unintentional aggregation of various forces.

Hence, it is still worth asking the "what if..." question. What if a different path had been chosen? It is worth doing so in regard to history and to develop so-called alternative histories. Even more, it is worth doing so in regard to the future and to ask what kind of consequences might emerge if we were to follow a different path.

Some may dismiss outcomes of "what if..." questions as utopias. For them, the future is another present and present is another history. There is a linear 
logic which is often followed, and it is not easy to break free of the chains of path-dependence. Certainly, the approach of forecasting is one way to deal with the future and has its place in the great tradition of social science.

Nevertheless, we are dealing more and more with environments characterised by turbulence, uncertainty, novelty and ambiguity ("TUNA environments" as labelled by Ramirez and Wilkinson, 2016). In many fields, and particularly in medium- and long-term perspectives, forecasting has serious limitations. An alternative is to develop various narratives about the future. For some, it may be a qualitative exercise which may seem to lack the rigour of forecasting.

However, we have to keep in mind that the rigorous quantitative forecasts are based on qualitative assumptions. Most fundamentally, forecasters assume that present and past trends will extrapolate in the future. What if this assumption is wrong? Essentially, forecasts are based on a particular narrative about the future. Hence, it would make sense to develop a range of narratives about the future instead of relying on one narrative. In the end, we can always generate a quantitative visualisation based on particular qualitative assumptions for alternative scenarios - if the presentation of numbers is valued in itself.

However, it is impossible to do the opposite - to quantify our perspective of the future without making qualitative assumptions. Every quantification about the future is based on either explicit or implicit qualitative assumptions, i.e. narratives. Scenario planning often limits itself to alternative narratives and increasingly relies on design thinking to visualise these narratives about the future. However, it could also take one step further and quantify scenario narratives for those who prefer working with numbers.

In this essay I will explore the opportunities and limitations of asking "what if” questions about the future. I will discuss how utopias and scenarios may overlap in our attempts to think about the future. I will highlight key elements of five public governance scenarios developed by the Foresight Centre at the Estonian Parliament in order to show how the scenario planning process involves utopias and dystopias (Arenguseire Keskus, 2018).

\section{Scenarios as utopias}

Even if in everyday conversations some assessment of the present or view of the future is dismissed as "utopian," then actually utopias are everywhere and the most practical of people rely on utopias all the time. One way to think about utopias is that they are mental short-cuts in a world characterised by 
complexity and information overload. They are simplifications of reality assuming certain developments. We can find utopias in government decisions, policy documents and, as Paul Joyce points out, particularly in strategy documents (Joyce, 2019). Particularly, in this post-truth world characterised by social media echo chambers and political polarisation one person's reality is another's utopia.

This is well characterised by recent political developments in Estonia where, after recent elections, one liberal party decided to form a government with a conservative party and populist nationalist party. The liberal party could have formed a majority government with another liberal party, but since it received an insufficient percentage of votes to appoint a prime minister in such a coalition, it decided to favour conservatives and nationalists.

Critics of the new coalition saw the whole premise that it is possible to govern with the extreme nationalist party without harming the Estonian liberal democratic order as utopian. For them, the self-interest of one politician and his party in securing the prime minister's position trumped broader interests. Fans of Machiavellian realpolitik saw this outcome as natural. They argued that every politician would do the same in the same circumstances. A politician's self-interest is to have power and he should do everything in his power to maximise his self-interest - of course, within the constraints of liberal democracy.

So, here we have two competing narratives with the same variable: selfinterest. One narrative seems utopian to one side and another narrative utopian to the other side because of different values in interpreting the outcome. Interestingly enough, both narratives could be interpreted within a rational choice paradigm and within a game theoretic angle. This does not imply that a range of other interpretations from a non-rational choice perspective should not be explored. Quite the opposite. However, the point of this discussion is to demonstrate how "self-interest" in politics can have a multiple of meanings even within the same paradigm.

It reminds one of debates among rational choice scholars about voting. Mancur Olson once argued that voting is an irrational act from an individual rational perspective because one vote does not change the outcome and the costs of voting outweigh the benefits (Olson, 1965). Other scholars disagreed by pointing out that voters may maximise their utility by the simple act of voting, by deriving benefits from the process or voting as an act of rational ignorance where asymmetrical information may lead voters to believe that voting matters.

This advancement from basic universal rationality to more "bounded" (Simon, 1957; Cunlisk, 1996) or "adaptive" rationality (Mueller, 1986) allows us to highlight different meanings of "self-interest" in the behaviour of the 
Estonian prime minister. The first "self-interest as a power grab regardless of costs" school represents a primitive application of the first lessons of Microeconomics 101 to politics. In more advanced courses concerning collective action it is best represented by a one-shot prisoner's dilemma game where individual self-interested behaviour leads to a Nash equilibrium which represents a suboptimal outcome.

The second "self-interest balanced by broader interest" school represents more advanced rational choice scholarships which point out that self-interest cannot always be subjected to a narrow universal definition. Self-interest may be "enlightened." In game theoretical terms, the game that is being played is a coordination such as the battle of the sexes, where multiple optimal equilibria are possible due to the incentive of the compatibility of players. This implies that the context is fundamental and constraints on self-interest much more numerous because of both formal and informal rules, information asymmetry, positive transaction costs and uncertainty.

Certainly, the self-interest in politics can be tackled in a number of other ways, but the point of this example is to demonstrate how even such a simple concept can generate alternative narratives. For some they represent reality, but for others they are either utopian or dystopian. Narratives carry utopian elements. Scenarios are based on ideal types which by nature rely on utopias. This carries a fundamental value because it generates alternative utopias and we do not have to rely on just one.

Most importantly, scenarios allow us to conduct thought experiments. Experiments are not easy to carry out and are often impossible in social sciences and difficult in public administration, especially on a large scale.

Utopias and scenario-building overlap. Some scenario planners may argue that they develop alternative futures which are believable. However, this again raises the question of a perspective. Believability may depend on a particular perspective of an individual or a group. What is believable for some is not believable for others, particularly as science fiction may be an important source of idea generation for some scenario planners. Scenario planning may have more respectability and allow us to generate ideas in a more rigorous approach than simply fantasising about the future.

If we take recent scenarios developed by the Organisation for Economic Cooperation and Development (OECD) on digital transformation as an example, then some of them seem certainly more utopian than others (OECD, 2018). Their "Corporate Connectors" scenario is probably one of the least utopian as it foresees the increasing dominance of large private digital platforms. The "Platform Governments" scenario foresees the increasing importance of government or government-supported platforms, which is more likely in 
some parts of the world than others. The "iChoose" scenario emphasises the importance of privacy and individual rights to data control. However, the least likely scenario is "Artificial Invisible Hands," which represents the radical decentralisation of governance where nobody controls the data.

Another example is four scenarios on the future government published by the European Commission's Joint Research Centre (JRC) (Vesnic Alujevic, 2019). These scenarios to some extent overlap with the OECD's scenarios on digital transformation as digitalisation is a fundamental factor. Their "DIY Democracy" scenario entails limited availability of public services which are replaced by the strong co-creation of services by citizens. Digitalisation facilitates grassroots initiatives, but offline engagement at a local level remains important as well.

Their "Private Algocracy" scenario is characterised by the dominance of large private digital companies where citizens' interests are derived from their data profiles. The "Super Collaborative Government" scenario combines the rise of artificial intelligence (AI) with a citizen-centric government. Citizens can engage seamlessly in decision-making through digital platforms. The "Over-Regulatocracy" scenario visualises the nationalisation of leading digital platforms under democratic governments. However, citizens have difficulty obtaining rights and accessing good services because of bureaucratic overreach.

Technology-centricity in the JRC scenarios is certainly a limitation as institutional constraints and enablers are not fully explored. Even if some OECD and JRS scenarios may seem from our current perspective utopian or dystopian, we cannot dismiss them as impossible because the future remains uncertain. Obviously, utopias not only exist in scenarios but can be found everywhere.

\section{Governance scenarios}

In Estonia, we did not take a technology-centric approach to the co-creation of governance scenarios. Technology remains an important ingredient, but institutional variables are the key. We also did not follow fully the advice of the late Christopher Pollitt who pointed out that "Big models, such as NPM (...) often do not take one very far" (Pollitt, 2011). At the Foresight Centre at the Estonian Parliament with the assistance of many experts and scholars (their names are indicated in the acknowledgements) we generated five alternative governance scenarios (Arenguseire Keskus, 2018; Kitsing, 2018).

On the one hand, they rely on big models and, being ideal types, are utopian or dystopian depending on perspective. This creates a certain universality. In principle, the scenarios can be applied to other countries as well. However, the degree of utopian or dystopian elements depends on context. A decentralised 
governance scenario may seem most utopian in Estonia, but it is probably less - if not least - utopian in Switzerland.

The governance scenarios combine both external and internal factors which may or may not contribute to the realisation of specific scenarios. Fiscal pressures and tough budget constraints limit the range of possible scenarios. However, budget constraint can be both endogenous and exogenous. It can be the outcome of developments in the world economy, reduction in the inflow of structural funds of the European Union (Estonia still is and will remain for years to come a net recipient of EU structural funds), consequences of Brexit and a number of other developments that Estonian policy-makers do not control and influence.

At the same time, the budget constraint can be self-imposed and thus endogenous. Policy-makers with certain ideological leanings may become dominant in the policy sphere, and hence impose strict limits on public spending and reduce the number of government officials. The bottom line is that scenarios emerge as a result of endogenous and exogenous, as well as more and less objective and subjective, factors.

Furthermore, endogenous and exogenous drivers of change are constantly interacting. Hence, exogenous drivers will also impact on endogenously set priorities. As Pollitt (2011) points out, universal best governance models do not exist. The real-life developments will quite likely lead to a combination of various scenarios discussed below. However, the use of ideal types in the form of scenarios offers clarity and simplicity which contribute to the understanding of the interaction of key drivers and potential outcomes.

Five scenarios allow us to understand the interplay of different approaches to public sector governance and potential routes to realising different scenarios. Scenarios are specifically meant for policy-makers in order to broaden their horizons and generate useable, concrete policy solutions for advancing governance. Scenarios serve as a risk assessment tool as they identify potential bottlenecks in the implementation of policy. Hence, one of the central questions is which conditions facilitate certain breakthroughs in governance reforms.

In other words, scenarios are not an end in themselves but a tool for citizens, politicians, officials, experts, activists and other stakeholders for advancing public governance. Above, I argued that scenarios are like utopias. In essence, they are an advancement of governance through utopias.

The real value of scenarios depends on their use. Will scenarios contribute to a clearer strategy formation in public governance and will they help to generate new ideas for better governance? The fundamental goal is to make governance more agile, equitable and efficient. This implies that scenarios are normative. They are also provocative and utopian. However, all scenarios 
consist of costs and benefits. Whether the costs exceed the benefits or vice versa in the context of a specific scenario depends on perspective.

Certain current trends may also indicate that realisation of some scenarios is more probable in the future. Other scenarios are plausible but not probable. Nevertheless, it does not imply that the aim of the exercise is to predict the future. First, predicting or forecasting future developments, especially in the long run, has severe limitations, as has been discussed above. Hence, it is important to consider not only small variations, but fundamentally different developments which are exogenous. We do not know whether scenario A or scenario $B$ will be realised in the future. However, we can comprehend to some degree the implications of scenario $A$ and those of scenario B. Scenario planning as a method is about developing alternative, equal scenarios. Most importantly, public governance should be prepared for different developments.

Second, the realisation of a specific scenario or combination of scenarios depends on exogenous factors. A precondition for realising certain developments is priority-setting by policy-makers and mobilisation of resources for that purpose. Certainly, this is a necessary but not sufficient condition. Unintended consequences stemming from uncertainty may undermine even the best plans. The road to hell is paved with good intentions. Nevertheless, there are certain benefits for a pro-active approach to policy-making rather than a reactive or fatalist state of mind. It is about mental models which are prepared for the emergence of new external environments. Different scenarios should contribute to policy space which is more adoptive and adaptive to changes.

\subsection{Ad Hoc Governance}

This scenario combines tight budget constraint, centralised and fast decisionmaking processes. The budget constraint needs either to cut public sector spending because of external or internal developments or to have a dominant ideological position among decision-makers that public sector governance must be managed within limited financial resources. The scenario is characterised by top-down fast decision-making in order to overcome economic crises and to exploit emerging new opportunities. Budget constraint also implies the privatisation of public services in some areas, which indicates that a government does not have sufficient leverage to change the situation in every area.

Citizens may benefit from this scenario as long as government priorities match their own priorities. However, they are left out of the decision-making processes as to involve them would significantly slow down the procedure. Citizens have also to deal with uneven delivery of public services where some 
services advance rapidly while others do not get enough attention and deteriorate as a result of resource constraints. Dissatisfied numbers of citizens may grow as a result of suboptimal services and inappropriate government priorities. The scenario may become a self-fulfilling prophecy where dissatisfaction with the limited involvement of citizens feeds into a need to keep decision-making centralised as policy-makers are afraid of opening the so-called genie's bottle.

Since the budget imposes significant constraints, ministries and agencies will be consolidated and the number of ministers reduced. These processes will simplify decision-making. Cost-cutting also implies that the proportion of public sector employees will be reduced across the total workforce. However, as the government will continue supporting some areas on an ad hoc basis, public sector expenditure as a percentage of gross domestic product (GDP) may increase. Furthermore, it can be assumed that the central government will increase public sector debt to GDP ratio. Government budgeting will be made more results-driven.

The role of the legislative sector in setting the agenda for strategic priorities will be modest. Parliament will be an instrument of representative democracy rather than participatory democracy. The role of local governments will be reduced. The central government will try to reduce the number of local governments by exploiting fiscal incentives. The fiscal autonomy of local governments will be reduced. Local governments will become basically agents of central government, which is their main function - rather than representing the interests of the local population and getting them involved in decision-making processes. This governance framework implies that in principle it is easier to implement strategic projects in some areas as long as budget constraints allow that. For the delivery of public services, it implies uneven development where some areas are prioritised while others lack the necessary resources. On the one hand, ad hoc governance values experimentation with new services and its delivery methods. However, focus is constantly shifting from one priority to another, which challenges the implementation of new ideas.

Digitalisation is valued in this scenario because it allows cost-cutting and the starting of new projects. It facilitates improvements in service delivery and data collection for policy-making, as well as directing citizens to needed services and reacting to changing circumstances. Since the budget imposes significant constraints and decision-making is centralised, then an ad hoc governance scenario implies that most services are standardised and special circumstances are rarely considered. Standardisation implies so-called forced digitalisation where the use of digital services might be the only option. On an ad hoc basis, some areas will receive special attention and these pet projects will be developed differently. 
The government will prioritise the use of big data, but since the approach is not systematic many institutional barriers do not allow the benefits to be exploited. The use of open data does not attract sufficient systemic attention, which implies no improvement in comparison with other countries. The combination of data from different public and private sources is possible in some areas but not in others. The government does not see the whole picture in its data policy by focusing on some areas but ignoring others. The government's digital identity used in different services will increase, but unevenly. Various private and public sector digital identities will emerge, and many citizens will rely increasingly on private sector solutions.

\subsection{Night-watchman State}

This scenario combines strong budget constraint with centralised and calculative decision-making processes. The underlying aim is to reduce the role of the state in many areas and focus on the areas where state intervention and the provision of services are absolutely necessary. The government will cut expenditure, reduce the number of public sector employees and will privatise services. The scenario implies that a systemic framework will be created for governance of the public sector where the limited role of government intervention in the private sector and the lives of individuals is the key priority.

Citizens will have considerable freedom in directing their lives, but their opportunities to get involved in public sector decision-making processes are limited to elections. Access to public education and health will be limited. The scenario also implies that the government response to substantial changes in the external environment such as environmental, geopolitical and economic changes, will be limited because of narrow policy-making perspectives and small public administration capacity. At the same time, the dominant fiscal prudence may allow them to react properly to certain external economic shocks such as a global financial crisis.

Since severe budget constraints mean significant self-imposed fiscal constraints, ministries and agencies will be substantially consolidated and the number of public sector employees significantly cut. The government wants Estonia to have the lowest public sector expenditure as a percentage of GDP and the smallest proportion of public sector employees per total workforce. The government will keep the budget balanced and will furthermore reduce the already low public sector debt-to-GDP ratio.

The role of the prime minister will increase in this scenario. Responsibility for managing the public sector will be clear and simplified, which may imply greater trust. However, decision-making will be efficient in predictable 
circumstances but may face considerable delays and bottlenecks in unforeseen circumstances. The parliament does not play a substantial role in this scenario. Its budget will be cut and the number of members reduced by one-third. Furthermore, term limits will be imposed which will reduce the number of professional politicians in the parliament but may make decision-making more complicated in areas where political skills are required. The self-imposed budget constraint implies that the role of local governments and their fiscal autonomy will be reduced. Their number and employees will decrease.

The government in principle will not engage in large public sector projects because the risk-taking involved and management of such projects do not fit with the role of the minimalist state. Public services are standardised and characterised by universal basic services with no allowance for special requirements. Every citizen has its own public service account where they can see financial limits and options for service use. The government issues vouchers for education, social and healthcare services which can be used for both private and public providers. This implies that service delivery can vary significantly across geographical regions and socio-economic groups resulting from differences in wealth and social capital.

On the one hand, digitalisation is valued in this scenario because it enables cost-cutting and reduces bureaucracy. On other hand, several barriers will be created for digitalisation because of privacy and security concerns. The minimalist government is worried about data collection because it might increase government intervention in individual lives and the private sector.

As cost-cutting is a key driver of digitalisation, it would imply a high degree of standardisation and universal basic solutions. The lack of customised solutions which consider specific needs may lead to dissatisfied users. Both open data and big data use have not advanced sufficiently. Barriers stem from institutional factors as government is concerned about the misuse of data. The combination of different public and private sector databases is mired in complexity or the impossible. The use of a government-issued digital identity is limited because of privacy and security concerns. An increasing number of citizens will rely on private solutions, including those provided by global digital platforms from the United States and China.

\subsection{Entrepreneurial State}

This scenario combines, first, strong centralised decision-making with generous budget constraints. The flexibility of resources allows the government to invest more in service delivery as well as large projects, often in the form of Public Private Partnerships (PPP). The government will behave as a large 
enterprise by developing and investing in certain key areas. The government's mission is to increase economic development and improve the country's position in the international division of labour.

The risks involve over-investment of public funds in failed projects which will become so-called white elephants. Radical external shocks may impose severe budget constraints which, in turn, may mean the activation of an "Ad Hoc Governance" scenario instead of an entrepreneurial state. This scenario is also sensitive to changes in government as well as the quality and strategic agility of government's top management.

Since flexible budget constraints imply more public sector investments and spending, the proportion of public sector employees in the total workforce and public sector expenditure as a percentage of GDP will increase. The central government will borrow funds for its priority projects, which implies an increase in the public debt-to-GDP ratio as well as annual budget deficits.

The role of prime minister will increase and he will act as chief strategist in the government. Some ministries and agencies will be consolidated, while new agencies might be created for developing priority areas such as infrastructure projects. The involvement of different stake-holders and interest groups in the decision-making processes will be reduced because the government values fast processes. The role of the parliament will be secondary to that of the executive branch as the logical implications of the scenario do not support a long-term calculative approach with unlimited discussions. Some parliamentary commissions may become more important sources of legitimacy than the general assembly.

The top-down logic of the scenario also implies that the number of local governments and their fiscal autonomy will be reduced. An exception will be the governments of the two largest cities of Tallinn and Tartu with which the central government is interested in cooperation involving large-scale projects. This also implies that this scenario is very favourable for large-scale public investment projects such as a tunnel between Helsinki and Tallinn and a four-lane highway between the two largest cities. The scenario also enables increased spending on public service delivery, where priority areas such as education will receive most of the investment. As the development of services will remain uneven due to priorities, these differences may cause dissatisfaction among citizens.

Digitalisation plays a fundamental role in this scenario because it allows data to be collected, better services to be offered and increases anticipatory policy-making. As government spending is generous and fast decision-making is appreciated, digitalisation can occur rapidly in many areas. However, government priorities imply that some areas receive more funding than others, 
which will lead to uneven outcomes. Over-investment and misallocation of investment may also lead to failures in large-scale projects.

Big data and open data use is highly encouraged by breaking down so-called silos among agencies. Government designs policies for the combination of different public and private databases. The government's mission is not only to focus on domestic projects but to enhance digital data projects globally in order to understand trends and developments worldwide. This means active cooperation with international organisations, private and public sector actors.

One of the key priorities is to develop further Estonian government-issued digital identity by offering solutions globally. Government prioritises eresidency as a global digital platform as through this platform other Estonian public sector platforms can be diffused to other countries.

\subsection{Caretaker State}

This scenario combines budget constraint, centralised and analytical decisionmaking processes. Improved living standards and economic development mean increased demand for high-quality public services. The government aims to meet this demand by increasing social spending and employing more officials. The main mission of a government is to improve the wellbeing of its citizens. For these purposes, government intervenes in many areas of life, protects people from evils and ills, and regulates different economic and social activities.

Citizens benefit from good access to high-quality education and health care. At the same time, their ability to shape public governance is limited. Government intervention in private lives may create the feeling that citizens live in a police state. The focus on current issues regarding citizens' wellbeing may also imply that the government may lack the capacity to deal with large-scale strategic challenges, particularly in the external environment's top management.

Since flexible budget constraints imply more public sector investments and spending, the proportion of public sector employees in the total workforce and public sector expenditure as a percentage of GDP will increase. The central government will borrow funds for improving the wellbeing of citizens. This implies an increase in public debt-to-GDP ratio as well as annual budget deficits.

Governance will be centralised but analytical, and will focus on increasing legitimacy. The number of regulations will increase as the government tries to solve problems in every area. The assessment of the impacts of various laws and regulations, which keep government departments busy, will increase. The involvement of different stakeholders and interest groups in the decisionmaking processes will be increased at least formally, because the government 
values legitimacy. The role of the parliament will be an important source of legitimacy with detailed parliamentary discussions. However, key decisions will in fact be made by the executive branch.

The top-down logic of the scenario also implies that the number of local governments and their fiscal autonomy will be unchanged. However, their importance will be emphasised in political rhetoric. This also implies that the scenario is unfavourable for large-scale public investment projects because the government is concerned about the environmental impact and wellbeing of citizens affected by those projects. Analytical, calculative decision-making processes and an increase in regulations will also reduce the likelihood of such projects.

The scenario also allows for increased spending on public service delivery, where the focus is on improving both the quality of and access to services. As the development of services will even be due to a holistic approach, satisfaction among citizens will grow.

Digitalisation plays an important role in this scenario because it helps to collect data, offer better services, direct citizens towards better choices and enhance anticipatory policy-making. As government spending is generous and analytical decision-making is appreciated, digitalisation will occur evenly in different areas. However, technological lock-in and path-dependence may lead to difficulties in adopting solutions in some areas.

Big data use is encouraged by breaking down so-called silos among agencies. Government designs policies for the combination of different public databases. However, the government is reluctant to cooperate with the private sector in this field because of risks and security concerns. The government does not encourage open data projects for the same reason. Instead of offering public data to the private sector, the government will design incentives and regulations for ensuring access to private sector data.

The government's mission is to focus on domestic services and not globally to enhance digital data projects, which will carry unknown risks. This implies that one of the key priorities is to develop further Estonian government-issued digital identity for domestic users. E-residency as a global digital platform will be closed down because domestic online service delivery may suffer from new risks and the overcrowding of platforms.

\subsection{Networked Governance}

This scenario combines generous budget constraint with de-centralised and calculative decision-making processes. The government aims to get citizens involved in decision-making processes and public service delivery through 
co-creation. For these purposes, decisions are made in bottom-up fashion, closest to citizens and without unnecessary bureaucracy.

Citizens benefit from opportunities to get involved in policy-making as well as in service delivery if they wish. Their ability to shape public governance is visible and actual. At the same time, it offers more opportunities for active citizens than passive. Communities with stronger social capital may benefit more than areas with a limited ability to cooperate. Government spending may not be able to reduce the gap.

Since flexible budget constraints imply more public sector investments and spending, the proportion of public sector employees in the total workforce and public sector expenditure as a percentage of GDP will increase. The growth is unevenly distributed but comes primarily from local governments which will borrow funds. It implies an increase in public debt-to-GDP ratio as well as annual budget deficits.

Governance will be de-centralised but calculative, and will focus on increasing legitimacy and satisfaction among citizens. The involvement of different stakeholders and interest groups in the decision-making processes will increase considerably. The governance is pluralistic and diverse. Local governments and the parliament will considerably limit the powers of central government. In some areas, the power of "silos" is dominant, while other areas are characterised by loose networks which collaborate across different domains.

The role of the parliament will be an important source of legitimacy, and its role in strategic decision-making will be increased. As long as severe budget constraint prevails, it is possible to use more resources for improving the quality of decision-making by hiring experts and encouraging the wider public to participate.

The bottom-up logic of the scenario also implies that the number of local governments will not be reduced and their fiscal autonomy will grow. They will take over crucial functions of central governments and will become true local governments. This scenario implies that it is unfavourable for massive public investment projects because consensus is difficult to reach and different stakeholders have the ability to block these projects for various reasons.

The scenario also allows for increased spending on public service delivery. However, a bottom-up approach suggests different abilities to use these resources well. Some areas will be innovative while others will lag behind. As the development of services will be uneven due to a decentralised approach, satisfaction among citizens in some areas will grow, while in others it will be reduced.

Digitalisation plays an important role in this scenario because it allows for data collection, better services to be offered and involves citizens 
in policy-making. Combining government spending with decentralised decision-making, digitalisation will occur unevenly in different areas. Different governance models will emerge where some rely more on public sectors while others engage in the private sector and with volunteers.

In this scenario, a direct trade-off between efficiency and equity may not be present if the increasing number of digital platforms in governance allows for a greater use of co-creation of public services by citizens. It is based on the assumption that open government data are made available and their use is encouraged. Big data and open data use is highly encouraged as well as combining different public and private databases. However, many different models will emerge in their use. Digital identity and e-residence will be developed further by involving numerous stakeholders from the public and private sectors.

The following table summarises the key points concerning governance and the digitalisation of five scenarios.

Table 1 Summary of five governance scenarios and digitalisation

\begin{tabular}{|c|c|c|}
\hline Scenario & Governance & Digitalisation \\
\hline $\begin{array}{l}\text { Ad Hoc } \\
\text { Governance }\end{array}$ & $\begin{array}{l}\text { Centralised and fast decision-making under } \\
\text { strong budget constraints. Executive branch- } \\
\text { centric, reduced role for the parliament and } \\
\text { local governments. }\end{array}$ & $\begin{array}{l}\text { Uneven digitalisation. } \\
\text { Cost-cutting and } \\
\text { standardisation in most } \\
\text { areas. }\end{array}$ \\
\hline $\begin{array}{l}\text { Night- } \\
\text { watchman } \\
\text { State }\end{array}$ & $\begin{array}{l}\text { Centralised and calculative decision-making } \\
\text { under severe budget constraints. Executive } \\
\text { branch dominance, minimal role for the } \\
\text { parliament and local governments. }\end{array}$ & $\begin{array}{l}\text { Limited digitalisation } \\
\text { aimed at efficiency gains. } \\
\text { Privacy and security } \\
\text { concerns. }\end{array}$ \\
\hline $\begin{array}{l}\text { Entrepre- } \\
\text { neurial State }\end{array}$ & $\begin{array}{l}\text { Centralised and fast decision-making under } \\
\text { severe budget constraints. Executive branch } \\
\text { aims at strategic agility and acts as a corpora- } \\
\text { tion. Limited role for the parliament and local } \\
\text { governments. }\end{array}$ & $\begin{array}{l}\text { Strategically important } \\
\text { areas are the priority. } \\
\text { Internationalisation of } \\
\text { government platforms. }\end{array}$ \\
\hline $\begin{array}{l}\text { Caretaker } \\
\text { State }\end{array}$ & $\begin{array}{l}\text { Centralised and analytical decision-making } \\
\text { under severe budget constraints. Government } \\
\text { focuses on the welfare of all citizens. The } \\
\text { parliament and local governments play a } \\
\text { formally important role but not in reality. }\end{array}$ & $\begin{array}{l}\text { Holistic digitalisation } \\
\text { and quality of services } \\
\text { and preventive policies } \\
\text { through social analytics. }\end{array}$ \\
\hline $\begin{array}{l}\text { Networked } \\
\text { Governance }\end{array}$ & $\begin{array}{l}\text { Decentralised and analytical decision-making } \\
\text { under severe budget constraints. Executive } \\
\text { branch has limited role. The parliament, local } \\
\text { governments, communities and citizens play } \\
\text { an important role. }\end{array}$ & $\begin{array}{l}\text { Diverse digitalisation } \\
\text { with different models. } \\
\text { Co-creation of } \\
\text { services and many tools } \\
\text { for participation. }\end{array}$ \\
\hline
\end{tabular}

Source: Compiled by the author on the basis of Arenguseire Keskus, 2018. 


\section{Trade-offs in governance digitalisation}

Even though the scenarios were developed for the Estonian context they do offer some universally applicable trade-offs concerning the future of governance. The Estonian scenarios also overlap with the OECD's and JRC's scenarios to some extent - even though they are less technology-centric and emphasise institutional factors as key drivers. For instance, the Estonian "Entrepreneurial State" scenario has characteristics in common with the OECD's “Platform Governments." The JRC's "Over-Regulatocracy” scenario has many elements in common with the Estonian "Caretaker State" scenario. The dominance of private digital platforms as envisaged in the OECD's "Corporate Connectors" and in the JRC's "Private Algocracy" scenarios is most likely in the Estonian "Night-watchman State" scenario. The Estonian "Networked Governance" scenario has many elements in common with the JRC's "Super Collaborative Government" and the OECD's "Artificial Invisible Hands" scenarios.

The key difference is that both the OECD and JRC scenarios place much greater emphasis on digitalisation than the Estonian scenarios. It is completely understandable in the case of the OECD scenarios because these are digital transformation scenarios which also discuss some aspects of governance. However, the JRC's scenarios are government scenarios where technologycentricity may narrow down a range of possible and plausible alternatives. Particularly so because institutional constraints are not likely to be broken down in 10-15 years.

In this sense, the Estonian scenarios are more general and offer a wider range of alternatives. For instance, both the JRC's "DIY Democracy" and "Super Collaborative Government" scenarios could be sub-scenarios of "Networked Governance". The latter would work under severe budget constraint while the former would in the case of tight budget constraint.

Why did the Estonian scenarios focus more on institutional rather than technological drivers? In order to understand this it is important to understand trade-offs concerning digital governance. First, various initiatives of digital governance have been around for more than two decades. A vast body of policy and academic literature has emerged on how to plan and implement digital governance in different countries during this time, as digitalisation has a promising appeal for making governance more efficient, equitable and agile.

Yet the progress of digital governance has been slow and uneven. This is certainly so if different countries are compared. The United Nations EGovernment Survey demonstrates that there is a tremendous gap between 
countries in the implementation of government online, even though the World Wide Web has been around for almost 30 years (United Nations, 2016).

The digitalisation gap is also wide in comparing different aspects of digital governance within countries. While some services are highly digitalised, others are not. In general, digital service delivery has received more attention than online political participation. This can be explained by different the emphasis of alternative scenarios.

Second, technological advancements are often seen as revolutionary. In discussions of digitalisation and its impacts terms like "digital revolution" are used. In fact, many changes enabled by the use of digital technologies are evolutionary because of institutional constraints. However, the main point is that digitalisation of governance takes time in an evolutionary rather than revolutionary process. Often the building blocks for successful public sector digitalisation outcomes were laid decades ago.

For example, even seemingly revolutionary developments such as internet voting have evolved over time. Almost $50 \%$ of votes were submitted online in the last Estonian European Parliament elections in May 2019. However, internet voting was launched in 2005 when only $2 \%$ of votes were submitted online. This a typical story of the diffusion of innovations which was described by Everett Rogers already in 1962 where innovators are followed by early adopters and then the early majority in adopting new innovations (Rogers, 1962).

\subsection{Institutions}

So why is the process of the adoption of digital technologies so slow? The simple answer is because of institutions which have a strong impact on both demand and supply of technologies. Different scenarios imply variation in institutional arrangements.

Often digital governance is analysed from a perspective of (utopian, if you will) technology optimists, if not technology determinists. They tend to believe in certain dystopias or utopias that technology itself is sufficient for implementing changes. For instance, the internet may mean that the need for certain organisations may disappear. Instead of voting for members of parliament every four years, people could vote directly online for various legislative proposals. Instead of government statistical offices, people could directly use data generated by various online transactions.

However, technology is a necessary but not sufficient ingredient for the digitalisation of public sector governance. The diffusion of digital technologies depends on institutions and the changes to them. Institutions are both formal 
and informal rules of the game (North, 1990). Public sector governance is interdependent on formal institutions such as laws and regulations, as well as on informal institutions such as habits, norms, customs and values.

Furthermore, the diffusion of digital technologies takes time because the interaction of various institutions and their impact on digitalisation stems not from a single rule but from the sum of rules of the game and from the specific context in which these rules operate. Certainly, institutions are products of human actions, but that does not imply that they can all be changed overnight to increase digitalisation. Institutions affecting digitalisation, as in any other area, are complex, built through time and may have unanticipated developments. Therefore, institutional change is often gradual. This helps us to understand why digitalisation of public sector governance is an evolutionary process.

In addition to institutional complexity, digitalisation is affected by pathdependence. Digital government efforts in developed countries have faced challenges from so-called legacy information technology systems. Technology is changing rapidly, but governments cannot update their systems fast because they are dependent on old systems and lack the resources for a complete overhaul. At the same time, some emerging democracies such as Estonia in the 1990 s benefited from not having legacy systems, and this allowed them to start from scratch.

All of this implies that technology use and the digitalisation of governance follow different paths in different political, economic, social and cultural contexts. One limited but stylised way is to take a rational choice approach to show how through political institutions "winners and losers from the technology can translate their preferences into influence" (Milner, 2006). Losers from technology adoption may use political institutions to slow down the digitalisation. This begs the question about the role of decision-makers and to what extent they can impact on digitalisation in specific institutional contexts, as was highlighted by the five scenarios.

Often individual decision-makers and governments receive credit for successful outcomes in public sector digitalisation. Usually, their insights and strategies are seen as a reason for the success. At the same time, many grand government digitalisation projects have also failed and ended up creating so-called white elephants - costly projects that are no use to anyone.

On the basis of various digitalisation efforts it seems that governments have to find a certain balance between top-down decision-making and bottom-up entrepreneurial discovery processes in digitalisation. Relying more on bottom-up decision-making processes also facilitates a degree of entrepreneurial discovery in the public sector which is an important ingredient 
for the digitalisation of governance. Of course, it also carries risks such as rent-seeking. This means that public means are used for private benefits.

Nevertheless, policy entrepreneurs always operate in institutional contexts. In this sense the entrepreneurial discovery process and policy entrepreneurship are important, but the role of policy entrepreneurs is always a "soft" one and "institutional entrepreneurship is rarely a case of individual heroism" (Djelic, 2010).

\subsection{Co-creation}

Furthermore, institutions should not be seen only as constraints, but also as resources to be utilised by policy entrepreneurs. This is particularly important for inter-agency cooperation or the ability of government to work across so-called silos, and cooperation between the private and the public sectors. Without such cooperation there will some islands of excellence in digitalisation but general development will be uneven.

Greater cooperation also allows one to take advantage of digitalisation and benefit from network governance because "the future of government relies not simply on greater efficiency, but also on increasing capacity to work effectively across agency boundaries to gain traction on pressing, inherently cross-boundary challenges" (Fountain, 2016). This requires coordination and proper incentives for decision-makers at various levels, but not top-down management (Laegreid et al., 2015).

The tension between efficiency and equity in governance has been created by governments which have focused more on efficiency of service delivery rather than citizens' engagement in the broadest sense. Nevertheless, the emphasis on co-creation of public services and approaches such as government as a platform as well as participatory governance may reduce these tensions (Janssen and Estevez, 2013; Linders, 2012; Paulin, 2018).

Obviously, this depends on what kind of government platforms will become dominant, as discussed in various Estonian, JRC and OECD scenarios. Large centralised platforms are likely to be more efficiency driven, while decentralised platforms may be capable of enhancing both efficiency and equity. Top-down platforms focus on uniformity (Kenney and Zysman, 2016).

More decentralised platforms can take advantage of pluralism and facilitate what Ostrom (1972), called "co-production of public services" by observing that citizens' cooperation created more value for law enforcement services on the basis of policing in Los Angeles in the early 1970s. This insight corresponds well with the network-based governance scenario. In this scenario governance need not necessarily be conducted exclusively by governments. Private 
firms, associations of firms, non-governmental organisations (NGOs) and associations of NGOs all engage in it, often in association with governmental bodies, to create governance, sometimes without governmental authority. Most importantly, a direct trade-off between efficiency and equity may not be present if the increasing number of digital platforms in governance allows for greater use of co-creation of public services by citizens as envisaged in the networked governance scenario.

\section{$5 \quad$ Conclusion and implications}

Many experts and scholars emphasise certain trends such as the emergence of network governance and the death of New Public Management (Dunleavy et al., 2006). However, the future of governance is uncertain. Instead of emphasising one prediction or forecast on the basis of previous developments and current trends, it would be wise to think about it in terms of alternative scenarios.

Our current understanding may indicate that some of these scenarios are more or less likely depending on the specific economic, social and political context. However, our understanding of context and context itself can change. The scenario planning approach allows the breaking up of linear logic in thinking about the future and widening the view of potential futures of governance. In other words, scenarios can be used as thought experiments for exploring the future of governance.

This is a most important implication for public governance scholars. Scenarios often have a systemic approach for engaging in positivist theorising. Instead of relying on one theory, an issue can be explored from different theoretical angles. In many ways, the Estonian governance scenarios discussed in this chapter relied on different theoretical and empirical research which was operationalised in the Estonian context.

For those scholars who are interested in offering policy solutions, scenarios allow us to conduct thought experiments. Experiments in social sciences can be conducted usually on a small scale which itself creates biases and limitations. Large-scale experiments are costly - both directly and indirectly. Scenarios allow us to play out possible and plausible developments in our heads - however utopian or dystopian they may seem. If the desired approach is to offer policy recommendations from one angle, then scenarios at least allow us to stress-test how future-proof recommendations offered by scholars are. By asking "what if..." questions, it is possible to test the robustness of proposed policy solutions. 
As far as policy-makers, students and the broader public are concerned, scenarios are a good way to frame debates on governance and broaden horizons. However, the marketplace for scenarios is characterised by a mismatch between supply and demand. Supply is more important than demand because demand for scenarios is really a "derived demand," i.e. scenarios are not needed for their own sake. Many substitutes are available such as linear forecasts, single vision-based strategies or whatever ideas are cooked up in the echo chambers of the so-called post-truth era. Communication of scenarios is always more difficult than one-line policy suggestions or single number-based forecasts.

Firstly, this implies improved attention to the communication of scenarios. One approach is to combine scenario planning with design thinking. For instance, the Danish Design Centre created four scenarios for the future of health care where people can physically enter into the different futures in central Copenhagen by smelling, touching, hearing and feeling what they mean.

Secondly, it implies constant stakeholder engagement in research projects. If the aim is to help to improve policy, then policy-makers and other stakeholders have to be engaged in every step of the process. They have to be instrumental in deriving policy implications from scenarios.

Last but not least, scenarios and utopias about governance must play a central role in classrooms and lecture halls. Social science classes in particular rely on overly rationalistic approaches to thinking about the future. Scenarios should be part of a range of alternative ways of approaching the future. They would allow us to bring a certain playfulness and social interaction to the classroom as many different role-playing exercises can be carried out on the basis of scenarios.

\section{Acknowledgements}

The author would like to acknowledge the contribution of the following experts in the scenario planning workshops from March to May 2018: Tea Danilov (Director, Foresight Centre), Kai Härmand (Under-Secretary, Ministry of Justice), Nele Leosk (Researcher, European University Institute), Innar Liiv (Associate Professor, Taltech), Külli Sarapuu (Associate Professor, TalTech), Siim Sikkut (Under-Secretary, Ministry of Economic Affairs and Communications), Mihkel Solvak (Director of Skytte Institute, University of Tartu), Erik Terk (Professor, University of Tallinn) and Rauno Vinni (Head of Governance Programme, Praxis). Many thanks go to the members 
of the Estonian parliament and policy-makers who participated in various meetings and gave feedback on the scenarios. In addition, many thanks go to the Foresight Centre at the Estonian Parliament for financing the research stream on public governance and e-governance as well as for the support of the Foresight Centre's team.

\section{References}

Arenguseire, K. (2018). Riigivalitsemise ja E-riigi stsenaariumid. Tallinn.

Campbell, J. L. (2010). Institutional Reproduction and Change. In G. Morgan, J L. Campbell, C. Crouch, O. K. Pedersen, \& R. Whitley (eds). The Oxford Handbook of Comparative Institutional Analysis. Oxford: Oxford University Press.

Conlisk, J. (1996). Why Bounded Rationality? Journal of Economic Literature 34(2): 669-700.

Djelic, M.-L. (2010). Institutional Perspectives-Working Towards Coherence or Irreconcilable Diversity. In G. Morgan, J L. Campbell, C. Crouch, O. K. Pedersen, \& R. Whitley (eds). The Oxford Handbook of Comparative Institutional Analysis. Oxford: Oxford University Press.

Dunleavy, P., Margetts, H., Bastow, S., \& Tinkler, J. (2006). New Public Management Is Dead: Long Live Digital-Era Governance. Journal of Public Administration Research and Theory (J-PART) 16(3): 467-494.

Fountain, Jane. (2016). Building an Enterprise Government. Washington, DC: Partnership for Public Service and IBM Center for The Business of Government.

Frost, Robert. (1916). Mountain Interval. New York: Henry Holt and Company.

Janssen, M., \& Estevez, E. (2013). Lean Government and Platform-based Governance. Government Information Quarterly 30: 1-8.

Joyce, Paul. (2020). Governing for the Future: Means, Ends and Disconnections. In G. Bouckaert, \& W. Jann (eds). European Perspectives for Public Administration: The Way Forward. Leuven: Leuven University Press.

Kenney, M., \& Zysman, J. (2016). The Rise of Platform Economy. Issues in Science and Technology 32: 3.

Kitsing, M. (2018). Future of Public Sector Governance and Digitalisation. Some recommendations for the policy-makers, BSR Policy Briefing 9: 1-18.

Lægreid, P., Sarapuu, K., Rykkja, L. H., \& Randma-Liiv, T. (2015). New Coordination Challenges in the Welfare State. Public Management Review 17: 927-939.

Linders, D. (2012). From E-government to We-government: Defining a Typology for Citizen Coproduction in the Age of Social Media. Government Information Quarterly 29(4): 446-454.

Milner, H. (2006). The digital divide: The role of political institutions in technology diffusion. Comparative Political Studies 39(2): 176-99.

Mueller, Dennis. (1986). Rational egoism versus adaptive egoism as fundamental postulate fora descriptive theory of human behavior. Public Choice 51(1): 3-23. 
North, Douglass C. (1990). Institutions, institutional change and economic performance. Cambridge: Cambridge University Press.

OECD. (2018). OECD Scenarios for Digital Transformation. Paris: OECD.

Olson, M. (1965). The Logic of Collective Action: Public Goods and the Theory of Groups. Cambridge, MA: Harvard University Press.

Ostrom, E. (1972). Metropolitan reform: Propositions derived from two traditions. Social Science Quarterly 53(3): 474-493.

Paulin, A. (2018). Smart City Governance. Oxford: Elsevier.

Pollitt, C. (2011). 30 years of public management reform: Has there been a pattern?. http://blogs. worldbank.org/governance/node/884.

Ramirez, R., \& Wilkinson, A. (2016). Strategic Reframing: Oxford Scenario Planning Approach. Oxford: Oxford University Press.

Rawls, J. (1971). A Theory of Justice. Cambridge, MA: Belknap Press.

Rogers, E. (1962). The Diffusion of Innovations. New York: Free Press.

Simon, H.A. (1957). A Behavioral Model of Rational Choice. In Models of Man, Social and Rational: Mathematical Essays on Rational Human Behavior in a Social Setting. New York: Wiley.

United Nations. (2016). UN E-Government Survey 2016. E-Government in Support of Sustainable Development. New York: United Nations.

Vesnic-Alujevic, L., Stoermer, E., Rudkin, J.-E., Scapolo, F., \& Kimbell, L. (2019). The Future of Government 2030+: A Citizen-Centric Perspective on New Government Models. EUR 29664 EN. Luxembourg: Publications Office of the European Union. 

III

PUBLIC ADMINISTRATION AND DISCIPLINES 



\title{
1 \\ Public Administration and Disciplines
}

\author{
Thurid Hustedt, Tiina Randma-Liiv and Riin Savi
}

\section{$1 \quad$ Introduction}

Public administration is in many respects a chameleon: it changes its colour depending on its environment. In the real world, it advises politicians, helps to formulate policies and is crucial in implementation at different levels and across levels of government, i.e. there are local town halls, regional agencies, central government ministries and agencies and - as discovered only lately supranational bureaucracies such as the European Commission and the UN General Secretariat. Inside universities around the globe, Public Administration [sic!] (PA) refers to the academic research and teaching (and sometimes policy advice) that considers the real world 's public administrations as its object of study. There is considerable variation in the academic organisation of Public Administration, its intellectual traditions and disciplinary backgrounds. As a contribution to the EPPA project, this chapter looks into the varying colours of the chameleon to discuss the traditions and origins of PA in Europe. The chapter aims to contribute to the understanding of the disciplinary origins and links of PA as an academic endeavour.

Such "belly-button pieces" (Raadschelders, 2011, p. 917) have a long history in US American PA, but gained ground only more recently in Europe, at least across territorial borders (Pollitt, 2010). Reflecting on the state of the discipline has for long taken place only within national or regional scholarly communities, for example, in Continental Europe (Kickert, 2005; Sager et al., 2018; Wegrich, 2017). There are good reasons for these geographically focussed reflections because also within Europe there is considerable variety in terms of disciplinary backgrounds, dominant research perspectives, history and organisation of PA. With the increasing internationalisation of research and academic life more generally, the time has come to enhance and strengthen a European reflection of what PA is all about, where it comes from and what its ambitions are. 
Why should one bother to reflect on the disciplinary relations of European PA? Or is it just a self-centred academic observation of the chameleon moving through the ivory tower? While some may think so, there are good reasons to establish self-reflection on the state of PA as an exercise to take stock of the broader lines guiding research, teaching and advising in PA and their inherent biases and blind spots as stipulated by disciplinary roots and related theoretical, methodological and empirical approaches. This chapter aims to contribute to such an exercise through recapping how PA links to different academic disciplines and the variation thereof across Europe.

The chapter is structured as follows. The next section outlines the broad lines of development in PA in Europe to identify its core features as a field of study. After that, the chapter looks into the different disciplinary environments shaping the field's colours, such as Political Science, Public Policy, Law, Sociology, Management and ITC. The chapter concludes by discussing the "state of the field" that is often said to suffer from crisis these days.

\section{Public Administration as a field of study}

There is no shared understanding of what PA is - either across national contexts or within academia in general or in the practical realm. Assumingly, part of this variety of understandings relates to the considerable diversity in disciplinary backgrounds and intellectual traditions. There is a long-standing debate whether PA is an academic discipline or not which moreover varies across national contexts. As Christopher Pollitt once put it: "What unifies public administration is its subject - the state, the public sector, and the public realm - not its aims, theories, or methods” (Pollitt, 2010, p. 292). This "community of interest" (Pollitt, 2010, p. 292) developed very different self-understandings across the two sides of the Atlantic as well as within Europe (cf. Sager et al., 2018). Ever since the famous dispute between Herbert Simon and Dwight Waldo on the scientific potential of researching public administration, US American PA has developed a self-reflective tradition which created the aforementioned history of belly-button pieces. The selfunderstanding in the field is discussed in terms of a long-standing "identity crisis" (Raadschelders, 2011:, p. 917) seeking to validate themselves as either craft, art or science (Raadschelders, 2011). The US American PA tradition has always had strong ties to government practice and emphasises prescriptive studies and government advice. In organisational terms, PA is affiliated with Schools of Public Affairs or Public Policy and sometimes "on its own" in Schools of Public Administration or departments such as Harvard 's famous 
"John F. Kennedy School of Government." In Europe, PA is more diverse and followed very different historical paths. Possibly related to the lack of a PanEuropean reflection, this variety is accepted and perhaps even appreciated. Other than in the US, PA is not plagued by a Pan-European identity crisis, though the state and outlook of PA are critically discussed, for example, in Germany (Bauer and Becker, 2019). Across Europe, PA is moulded differently by traditional academic disciplines. For example, in some Central and Eastern European countries, PA has either a strong Economics and Management or legal flavour, whereas in Germany Public Law shaped (and for some still does) PA. Accordingly, also, academic organisation varies. While PA plays a key role in Political Science departments, for example in Denmark and Norway, it is affiliated to Economics departments in the Czech and Slovak Republics. Hajnal (2003), for example, clusters European PA in three families according to disciplinary dominance: policy/administration, legal and business. Though he uses these types to group educational programmes in PA, it is fair to assume that they also indicate the overall academic organisation of PA across the continent. Though one assumes that it is less intense than in the US, in Europe, too, PA is linked to government practice - and, as often argued, much more than, for example, in Political Science (Bauer, 2018). In many countries, these links are particularly evident when it comes to administrative reforms - and in particular in the 1980-1990s with the reform trend of the New Public Management (NPM). Though comparative studies show that these reform ideas were implemented very differently across countries, NPM was a dominant reform paradigm for at least a decade and kept both reformers and PA busy (Pollitt and Bockaert, 2017). Analysing NPM reforms was a key issue in PA publications, and in many countries leading academics served as reform advisors. A visit to the key conferences of US American and European PA, ASPA and EGPA respectively, neatly illustrates differences at first sight: Whereas the panels at ASPA's annual conferences are packed with both PA scholars and practitioners who also give talks and are explicitly addressed by the overall conference call, EGPA's annual conferences are predominantly academic events.

But what is more, as Michael Bauer points out, as a social science, the national societal, political and cultural context also shapes the chameleon's colour - not just academic tradition (Bauer 2018, p. 1049). In the US, PA emerged in a context in which the state was considered rather critical, and thus PA has always had a strong focus on interest groups, individuals and overall state-society-interactions. In contrast, in France and Germany, for example, two countries in which PA is largely influenced by Law, the central notion of the state in the social and political development of both countries 
is the starting point for any reflection on public administration, and thus also shapes PA (Sager et al., 2018, pp. 6-9). And yet another example is the more recent development in Central and Eastern Europe, where PA emerged within post-socialist transformation in an attempt to help create, sustain and manage newly democratic institutions (Randma-Liiv and Drechsler, 2017).

Yet, what unifies the US and European PA is the lack of a unifying paradigm, grand theory or methodology. Scholars apply multiple theories borrowed from other disciplines, use a variety of methods - and it is only more recently, if at all, that methodology and research design have gained more attention in PA, as reflected in some recent books (see van Thiel, 2014; James et al., 2017). Whether this increasing awareness of methodological approaches is an indication of PA slowly but steadily developing into a discipline, or just an implication of the increasing pressure to publish in peer-reviewed journals, in which reviewers emphasise methodological ambitions and transparency, is an open question.

Thus, the absence of clear contours and components of PA as an academic discipline, the lack of a unifying paradigm, its partly contested nature or status, its relations to government practice and its role in advising governments and administrative reformers often resonate in the conclusion that PA is not striving for theoretical and general knowledge and is hence not scientific or academic as such. It is rather striving for applicable knowledge for "optimizing public administration in the widest sense - i.e., making the state work as legitimate, fair, effectively and efficiently as possible" (Bauer, 2018, p. 1060). However, not least because the diversity of the PA "community of interest" also includes different types of scholars, one can clearly contest this view.

\section{Public Administration as a multidisciplinary field}

As a multidisciplinary field, PA has its roots in a number of social science disciplines, from which it borrows theories and methods. In a Pan-European survey as part of the COCOPS Project, PA scholars ranked Political Science as the most important discipline for PA, followed by Political Sociology and Business/Management, and then, on an equal footing, Economics and Law (Curry et al., 2014, p. 24).

In what follows this chapter briefly discusses the relationship of PA to Political Science, Public Policy, Law, Sociology, Management and ICT. However, a caveat needs to be in place. As national traditions and approaches vary (see above), such a discussion can only be cursory within the constraints of a book chapter. That is why the following sections focus on core traits in the 
relationship between PA and the respective fields and on their specific links and intersections.

\subsection{PA and Political Science: friend or foe?}

Political Science is often considered the mother discipline of PA. Not only do national traditions in the relevance and links of PA and Political Science vary, they can and do also change over time. In the abovementioned COCOPS survey, $30.2 \%$ respondents identified themselves as political scientists by education, followed by scholars with a genuine PA degree (Curry, 2014, p. 16). Almost a quarter of those PA scholars are affiliated to Political Science departments. Thus overall, Political Science serves as a key starting point for PA as a field of study and for individual careers.

Though PA has strong roots in Political Science and in many places the close links served for mutual inspiration, Michael Bauer describes this link as "close, competitive, and based on a certain division of labour" (Bauer, 2018, p. 1049). Like others, Bauer emphasises "important differences in the goals of PA and PS: the former is a multidisciplinary endeavour that focuses on applied research, while the latter aims to advance our theoretical knowledge in terms of basic research" (Bauer, 2018, p. 1049).

Yet, of course, Political Science is also a somewhat specialised discipline structured into various sub-disciplines such as international relations, political theory, comparative politics, political philosophy and electoral studies, and suffers from problems of fragmentation. Though there is no uniform Political Science, it is fair to say that across all disciplinary specialisations, political scientists share a genuine interest in political orders and their emergence, institutionalisation, change and implications for society and politics; in the distribution and exercise of power; and in the legitimacy of institutions, actors and processes in a political order.

If choosing from this delicate menu, PA scholars do obviously share research interests in the state and government as a core institution in any political order; in the institutional arrangements shaping the role of government and its capability for problem-solving and the overall functioning of government to address problems effectively. Moreover, PA also studies public service delivery, which is important in generating public trust in the ability of government to cope with citizens' needs, and hence to generate and sustain legitimacy.

Political Science and PA share genuine research interests which can be illustrated through a number of concepts used in both fields. In Political Science, the study of democracy, power and legitimacy is at the core of 
disciplinary scholarship: how does democracy work? How is power gained and maintained by political actors and exercised in government? How do political processes, institutions and actors generate or thwart legitimacy? Core questions such as those do have a counterpart in PA scholarship: what is the role of bureaucracy in democracy? Who is able to influence decisions in the government apparatus? How do accountability relations function in different contexts and for different actors? These are just some of the "sibling questions" posed in PA scholarship that actually reflect Political Science lenses. Thus, democracy, legitimacy and power serve as intersectional concepts, and both communities are interested in these shared concepts to be subsumed under the more abstract category of "the state" which (at least in Europe) guides much scholarly interest in the first place in both Political Science and PA. Yet, starting from an interest in researching the state, the two communities then followed different paths.

Thus, the relationship is close, but also competitive. Here, the US example is the most intriguing. With PA being a strong part of Political Science until shortly after the Second World War, it rapidly lost esteem and relevance in post-War Political Science. PA migrated out of Political Science departments, and in 1939 it formed its own association, the ASPA (see above). Yet, the drifting apart of Political Science and PA is not restricted to the US: for example, the Dutch case represents a clear process of differentiation in which Political Science was the strongest disciplinary influencer to initiate PA research and education, which, however, from the 1970s onwards differentiated into its own discipline with its own departments and educational programmes (Ongaro et al., 2018, p. 16). In the UK, for example, Political Science departments are home to PA scholars, but when public management reforms became the core concern of both research and practice, PA moved to business schools in many cases (Pollitt, 2016).

Today, there is exchange between Political Science and PA. However, the specialised nature of both places limits on this communication. Take, for example, research on coalition formation and coalition governance, which is a key strand of contemporary Political Science research and its research interest intersects closely with research on political-administrative relations, which is in turn a major research area in PA. Yet, there is a blatant lack of exchange or mutual recognition through citations or panels at conferences. However, PA scholars regularly present papers and organise panels at major Political Science conferences such as the biannual conference of the International Political Science Association (IPSA) or the General Conference of the European Consortium of Political Research (ECPR). Yet, while regularly present at the latter, PA does not have its own ECPR Standing Group. 
In general in Europe, PA does well in countries with a strong Political Science tradition. And even though relations could be stronger, and go back to the mutual inspiration of past times, they are still better and more constructive than in the US, where it is not unusual in Political Science to consider PA a foe, and vice versa. And there are good reasons for both fields to work on re-establishing closer links. Many PA scholars indeed follow the advice of the late Christopher Pollitt: "(...) it would be an unwise scholar who chose to ignore the political contexts within which management issues arose" (Pollitt, 2016, p. 5).

\subsection{PA and Public Policy: let us open the black box}

While Political Science focusses mainly on the input side of the political process, Public Policy is particularly interested in the output side - and PA has a place in between, with its interest in how administrative factors shape the formulation and implementation of public policy.

However, the relationship between PA and Public Policy studies is ambivalent. Though in the real world public administration and public policy are closely intertwined, this is somewhat different in academia - and, again, differs between countries. As mentioned, in the US PA is often organised in policy schools. In Europe, there is considerable variety. For example, in Germany both share a similar history of emergence in the late 1960s and early 1070s, during the heyday of the planning era. But, at least from the 1980 on onwards, the two developed somewhat differently and their relations are contested (Janning, 2006; Jann, 2009; Döhler, 2014; Wegrich, 2017; Töller, 2018). In particular, German PA scholars blame their policy analysis colleagues for ignoring the contribution of public organisations. In contrast, policy scholars argue that public policy analysis just developed somewhat differently in terms of theory development, research questions, designs and their relevance - and while PA is still closer to practice, policy analysis developed more into a purely academic exercise (Töller, 2018). Many German academics consider themselves PA scholars and policy analysists - but most often with an emphasis on either one. This close link is also reflected in the fact that the two form a joined "Policy Analysis and Public Administration" section within the German Association for Political Science (Deutsche Vereinigung für Politikwissenschaft, DVPW). Yet, Germany may be a particular case illustrating the stark contrast between the two.

Close links certainly do exist: there are many handbooks collecting works from both PA and policy studies, for example the Oxford Handbook of Classics in Public Policy and Administration (Balla et al., 2015). In terms of conference 
organisation and top journals, the two communities are rather separate, however, with permeable borders: at the International Conference of Public Policy, for example, established in 2013, there is a clear dominance of public policy panels, yet one also finds panels with a PA focus and colleagues from both sides present papers. While journals usually have a focus on either policy or administration, many publish articles from both communities - think for example of Public Administration, Policy Sciences, the Journal of European Public Policy and the Journal of Comparative Policy Analysis.

In terms of research topics and questions, Public Policy and PA have drifted apart over time, yet still have intersected interests: while for example Harold Lasswell envisaged policy sciences to create both "knowledge of and in policy" (Lasswell, 1971, p. 2), it is fair to conclude that the field developed towards the latter with a well-developed set of theories and concepts.

Many Public Policy scholars are now highly specialised experts in single policy domains such as environmental, labour or health policy. Public Policy studies have a traditional interest in the ability of policies to solve problems, and many PA scholars share this interest. In particular, the more recent re-discovery of various types of capacity to address problems effectively may serve as a vehicle to link research interests across the two communities (for example, Xun et al., 2018), as well as the renewed interest in policy design (see the contributions in Howlett and Mukherjee, 2017). In more general terms, PA and Public Policy scholars share an interest in understanding the outcome of policy processes to which PA could contribute a focus on administrative organisation and bureaucratic behaviour and their implications for policy.

\subsection{PA and Law: ships passing in the night}

The relationship between PA and Law is today shaped by mutual neglect across Europe. Yet, the two have a long common history in many European countries, particularly in the Napoleonic countries and in Germany (Kickert, 2005). In both France and Germany an "organic notion of the state" prevails, emphasising a close link between state and society that makes the two almost impossible to disentangle (Sager et al., 2018, p. 6). The state, however, is separate from politics, which may have contributed to making the state an object of distinct scholarly interest rooted in Public and Administrative Law. Both countries share similar traits: in both, access to the civil service was traditionally controlled by the legal profession; it was not until the post-war era that a separate PA community developed. Yet, while this has a socialscience orientation in Germany, it emanated from Administrative Law in France and is still shaped by legal thinking, i.e. a normative approach to 
administrative legality and legitimacy. In both countries the strong historical links continue to have an effect: the notion of the "Rechtsstaat" that became widely accepted beyond Germany emphasises that it is the law that shapes state action and intervention - and, in turn, jurisprudence may help if the state abuses its power. Yet, in France close links seem to persist, and the empirical approach to state behaviour is today taken by Public Policy scholars and sociologists. While the strong historical link to Law contributed to only a weak institutionalisation of a social science PA in Germany, the links have weakened over recent decades.

Today, PA scholars and Law scholars live separate lives in many European countries and only rarely communicate. Debates about change in Administrative Law are not related to discussions in PA about administrative reform and changing management practices. Though both communities do research on regulation, citizen participation or the involvement of private actors in public service delivery, to name just a few shared objects of interest, they hardly take notice of each other. Publication habits are separate. While the legal community predominantly publishes in national fora and peer review has remained alien to many, many PA scholars seek to publish in international peer-reviewed journals recognised outside their home countries. In a similar vein, the two communities approach research differently: much PA research today is empirically seeking to describe and explain distinct properties or implications of administrative organisation or behaviour, while research in Law is predominantly normative with the composition and nature of administrative norms as the core interest.

Thus, today's link between the two communities can well be characterised as ships passing in the night. If they meet, then they do so occasionally in discussions with practitioners that often need both legal and organisational, policy and management advice grounded in empirical data.

\subsection{PA and Sociology: towards disciplinary convergence?}

Sociologist Max Weber is considered by many to be a founding father of modern PA thinking with the notion of the ideal-type bureaucracy as part of his typology of authority, as well as his conceptualisation of politics and administration. The latter represents a distinct European approach to separate politics and administration to "take administration out of politics" (Overeem, 2012) and thus ensure mechanisms for the political control of bureaucracy. ${ }^{1}$ Yet, though one of the founding fathers has his home in what is now Sociology, links between Sociology and PA developed only more recently - at least outside France. $^{2}$ 
The advent of new institutional theory served to link PA and Sociology by importing its organisational theory institutionalist variant (see, for example, DiMaggio/Powell, 1991), sometimes called "sociological institutionalism" (Hall/Taylor, 1996), to PA scholarship. Before, organisation theory and PA developed in "mutual disregard" (Olsen, 1991) in many countries. This disregard may also be a result of academic organisation, because organisation studies migrated to Business School in many US universities in the 1960s, and other countries followed (Augier et al., 2005). Scandinavian and - particularly - Norwegian PA is an exception here: Johan Olsen and his cooperation with Stanford's James March strongly shaped Norwegian PA scholarship to develop into a "Scandinavian school of PA" (Jann, 2006, translation by the authors). The influx of new institutionalism in organisation theory to PA scholarship - also beyond Norway - provided for a theoretical framework sensitive to regulative, normative and cognitive properties of institutions which shape organisations, their structure, culture and processes (for example, Scott, 2014). With its broad notion of institutions, new institutionalism serves as a bridge between the institutional environment and organisations, which makes it particularly attractive to PA scholarship, for it allows the linking of administrative-organisational analysis with their political environments. These recent links offer more potential for shared research interests between PA and Sociology as regards the role of public organisations in society.

In addition, contemporary societies are confronted with various global challenges such as climate change, migration or digitalisation that are not only pressing policy problems, but moreover reflect social change, including changing social norms, behaviour and expectations towards governmental problem-solving capacities as well as public service delivery. At the same time, this social change creates new cleavages and social conflict. Thus, there are substantial intersections in the research interest of PA and Sociology with great potential for further collaboration.

\subsection{PA and Management: how important is context after all?}

Mixing the fields of Management and PA goes back as far as the 1930s. Then, almost a century ago, the private management principles of Taylor and Fayol were imported into the public sector by Gulick and Urwick through their wellknown POSDCORB acronym which introduced the stages of administrative process (Planning, Organising, Staffing, Directing, Coordinating, Reporting and Budgeting). In Europe, it was not until the 1960 s that managementbased approaches paved the way to PA, bringing with them an attention to organisation and management issues in the public sector (Rutgers, 2010, p. 1). 
In that period some PA scholars, struggling with the identity of PA, began searching for an alternative. They found it in "Management," called either "administrative science" or "Generic Management," which holds by and large that a specific culture, sector, institution or task has little consequence for efficient and effective management, and that "a body of knowledge" - statistics, economics, accounting, operation research and organisation is often needed in the field of PA.

The burst of management approaches into PA took place in the 1980s-1990s with the introduction of the NPM model. We can distinguish between NPM as a paradigm and NPM as a set of tools. At the higher level, NPM is a general theory or doctrine that the public sector can be improved by the importation of business concepts, techniques and values. At the more mundane level, NPM is a label for a bundle of partially connected, partially quite random tools and practices, such as performance-related pay, competitive tendering, Total Quality Management and the creation of autonomous agencies. The popularity of NPM, often strongly supported by international organisations, such as the OECD or the World Bank, flooded both academic PA as well as the public administration practice with a variety of management approaches and tools. This affected many traditional PA curricula (often dominated by the disciplines of Political Science and Law in Europe) where managementrelated courses were introduced, e.g. on Human Resource Management, Financial Management, Operations Management, Public Sector Marketing, and so on. Within the field of PA, new topics have been developed on the basis of Management concepts, such as Corporate Governance of SOEs; PPPs; Network Management; and Public Service Motivation. Moreover, NPM brought to the field of PA not only new management-related topics but also strong prescriptive elements and "reform recipes," as often practised in Business Administration and Management Schools. Although Continental European countries were less affected by NPM than their Anglo-Saxon counterparts (Pollitt and Bouckaert, 2017), the global wave of managerialism has left its footprint on any administration and the field of PA per se.

The relationship between PA and Public Management is constantly evolving. In some European countries there is by now almost no distinction between PA and PM, but in others Public Management is still treated as something different, usually placed in the traditional faculties of Economics and/or Business Administration. Although some PA scholars attend Management conferences such as those of the European Academy of Management (EURAM) or the European Group for Organisational Scholars (EGOS), there are hardly any Generic Management scholars presenting in PA conferences. Since the turn of the millennium, the emphasis on context surrounding any - public or 
private - organisation has become widely recognised (more in PA than in Management studies, though). The recognition that context matters (Pollitt, 2013) implies that not only are public organisations different from their private counterparts but there are also major differences within the public sector. Consequently, a management tool that fits one organisation does not necessarily work in another national, sectoral, institutional, legal or cultural context. This understanding challenges some "Generic Management" concepts and one-size-fits-all prescriptions. At the same time, there is great potential for analysing the political and legal dimensions of management tools in the public sector as well as mixing perspectives of Management and Sociology in the study of public sector organisations. Moreover, emphasis on management issues is likely to help to bridge the gap between PA academics and practitioners.

\subsection{PA and ICT: new kid on the block}

While the previously mentioned disciplines have been in and around PA scholarship for decades, the emergence of ICT is so recent that it sometimes still gets forgotten when addressing the chameleon. However, in the current era of data and digital transformation, ICT and PA cannot work without one another. Why should we care about teaching ICT in public administration schools? Because we live in a digital world (Ganapati and Reddic, 2016). Regrettably, ICT is still largely ignored by or isolated within many PA faculties and curricula. This is in sharp contrast with the e-government hype in (some) governments, and also in EU research funding schemes. The field of PA needs to be and become more aware of new technological developments, especially in ICT, the Internet, clouds and recent developments associated with social media, big data, cloud computing, crowd-sourcing and so on. For the time being, PA in many countries and programmes resembles a technology-free zone.

There is a strong need to build not only ICT but more generally technologybased competences in PA departments, and there is an even stronger need to include tech-rich social science thinking in PA. PA and technology meet in twofold realms: on the one hand, innovation through the public sectorgovernments as market makers, e.g. for green energy or biotechnology; on the other hand, innovation in the public sector ranging from digitalisation of data exchange to algorithms deciding whether to sack teachers. In addition, new experimental organisational forms (e.g. labs) and new emerging organisational cultures bring PA and ICT closer to each other. ICT and other technology fields come with different logics from classical PA. They all struggle with understanding and finding the best governance models, where PA can and must offer valuable insights (Lember et al., 2018). 
There are at least eight reasons why the leaders of the academic field of PA should care about including ICT in public administration education. First, ICT is a large force reshaping the public sector, to borrow Alasdair Roberts' (2014) wording. Second, it is already possible to detect changing PA patterns due to ICT development: new forms of citizen engagement, re-allocation of control and power in organisations, core-periphery task re-allocation, and so on. Third, it is argued that algorithmic governance will speed up the decision-making processes and magnifies the effects of policies and services (Eubanks, 2018), hence also potentially magnifying the negative effects of ill-designed services and inequalities. Algorithms control human action, but who controls algorithms? This is a question for political scientists and public administrators, not for the tech professionals. Fourth, the use of ICT in public organisations is rife with problems. Often the reasons e-government initiatives fail are not technical, but related to management, planning, coordination and oversight. Addressing such issues is the domain of public administrators, not technical experts. Fifth, public agencies are vast repositories of sensitive digital data that they collect to fulfil their missions. The information is often in the public domain, but there are privacy considerations with cyber-security being a major concern. These issues are not only technical but also organisational and managerial. Sixth, as the digital world evolves so too will the nature of the demands for preserving public service values. For example, in the ongoing debate about the benefits and problems of the sharing economy, public administration students can play a key role in maintaining the public interest (Ganapati and Reddic, 2016, pp. 156-157). Seventh, ICT provides new opportunities for understanding the public sector. For example, it offers tools for predictive governance which is likely to have an impact on how policies are designed and public services planned and provided. And, last but not least, eighth, a public administration curriculum without adequate ICT training would make its graduates unprepared for the modern workforce.

There is a pressing need to build ICT (and more generally technologybased) competences in PA departments, to work together with ICT people, to attend ICT conferences, to co-author with ICT professionals, and to re-think what PA knowledge and skills we teach and to whom. It is a growing challenge for PA as a discipline to analyse how to manage the collaboration of humans and machines/algorithms in public administration. It is crucial to expand public management skills when managing big data. These skills relate to ethics, technology, process innovations, organisational and institutional changes, and analytical skills (Mergel, 2016). While other related disciplines share a general social science approach with PA, the growth in importance of ICT offers completely new challenges to both PA academics and practitioners, as 
ICT professionals enter the field of public administration with very different background knowledge of democratic governance, different value systems and professional cultures.

\section{Conclusion: the state of Public Administration in Europe}

This chapter outlines the field of PA and how it relates to other academic fields. As a multidisciplinary field of study, PA entertains links to various academic disciplines, the most important of which are Political Science, Public Policy, Law, Sociology, Management and ICT. Yet, as a chameleon, PA adapts its specific colour to its environment and, thus, the scope and strength of the links between PA and the related fields vary both over time and across European countries. Overall, the field of PA is shaped by structures of academic organisation, as well as the setting up of conferences, journals and academic communities that direct the attention of PA scholars, tend to create silos of specialisation, and may hinder research across fields.

This chapter cursorily explores the varying colours of the PA chameleon through its multiple links and the different academic traditions of PA in Europe. While potentially biased through the limited cross-national insights and experiences of the authors, it remains fair to conclude that PA scholarship is not just diverse and rich in traditions, research foci and how it links to other fields. Moreover, PA in Europe has developed into a pan-European community of scholars who share a genuine interest in the scientific analysis of public organisations in their political environments. Practical relevance is often emphasised as a core objective or concern of PA scholarship and considered by many a defining property that sets PA apart, for example, from Political Science or Sociology. At the same time, the career paths of PA scholars take place at universities and their performance is assessed according to standard criteria in contemporary academia: the number and/or impact of peer-reviewed publications in top journals, success in acquiring third-party funding, teaching quality and - most recently - societal impact and outreach. Current career paths reflect neither a very strong practice orientation nor a scepticism or reluctance towards theory-building or methodological development.

As a social science species, the PA chameleon also changes its colour depending on its social and political context. In a time of increasing political polarisation and populism, when liberal democracy comes under attack, and in which anti-elitist, anti-government and often anti-democratic claims gain ground and undermine trust in the ability of the democratic state to serve the 
people and to solve pressing problems, close links across different fields within the social sciences are becoming crucial in responding to such fundamental challenges. Indeed, real-life problems do not follow disciplinary boundaries. The borders of academic disciplines are artefacts and many leading social scientists have crossed those borders throughout their careers - think of Herbert Simon and Elinor Ostrom. Addressing the real world `s problems is expected from all contemporary social sciences - they are increasingly incorporated into curricula and teaching methods (e.g. governance labs) and demanded by organisations funding research. Scholars are increasingly asked to justify the societal impact of their work, and PA scholars are expected to offer lessons as to "what works" in terms of organisation, policies or decisionmaking processes. Answering such questions requires cooperation across academic disciplines and fields of study, because the complexities of the real world 's administrations do not correspond to the boundaries of a single field. To put it differently, real-world public administration is not only about performance and efficiency, it is also about fairness and equality as well as legality, the rule of law and - ultimately - legitimacy. Yet, the abovementioned academic career incentives favour narrow specialisation within one field or discipline, by that further increasing specialisation and weakening links to neighbouring fields.

On a self-critical note, PA with its turn towards managerial models and a dominating focus on performance and efficiency in recent decades may have neglected issues of democratic governance and the role of executive government and public service delivery therein. As democratic governance is under pressure on a global level, there is a need better to integrate democratic governance in PA curricula and to build conceptual links to relate democracy and bureaucracy in research, teaching and executive education to each other. After all, public administration is a political institution operating under political constraints and in interaction with other political institutions, such as parliament and political parties. The study of public administration and its political context are inseparable, which is why there is an urgent need to bring democracy "back in" to the study of PA.

The field of PA is at a crossroads. On the one hand, the changing colours of the PA chameleon bring a risk of isolation and non-communication across its shades as one considers PA merely as a "vertical" department in the university hierarchy. On the other hand, PA in its varying colours can be considered a "horizontal" field, cross-cutting academic organisational structures and hence boundaries, which is particularly needed in a world of wicked problems and polarisation. Such a horizontal dispersal would not make the field of PA weaker but more visible and understandable in both 
the academic community and PA practice. An encompassing view on PA in its political, legal and social environments may help sustain or regain trust in democratic governance through countering conspiracy theory and other stereotypes on public sector misbehaviour and through shedding light on the internal dynamics of executive government and public service delivery.

The future debate on PA as a scholarly field should shift the current focus on PA as a borrower that has borrowed from other disciplines or has inherited bits and pieces from the old and mighty. PA without doubt has a very special role also as a lender itself - through integrating different disciplines and bridging them. As Yang (2018, p. 1) puts it, "public administration is a dynamic balance and an integrative science. Seeking dynamic balance and synthesis is the nature of public administration, it differentiates public administration from other disciplines, and stresses its status as an independent discipline. We must admit that this unique feature represents a specific advantage of public administration." Perhaps the time has come to move PA up in the academic "value chain." In an environment that explicitly favours multi- or interdisciplinarity, PA has an advantage by reason of its experience in collaborating across fields and disciplines. The PA academic community should not be shy (or even defensive) about it, but proudly share its more or less successful cross-disciplinary experiences with other academic communities and public administration practitioners.

\section{Notes}

1. In contrast, the Wilsonian US notion of the politics-administration dichotomy served to "take politics out of administration" in order to ensure the professional independence of administration.

2. In France, the study of Public Policy (and thus in part also PA) as institutionalised today in French universities is largely influenced by French sociologists such as Michel Crozier. This strong sociologist flavour sets French Public Policy studies and PA apart from those of other European countries.

\section{References}

Augier, M., March, J. G., \& Sullivan, B. N. (2005). Notes on the Evolution of a Research Community: Organization Studies in Anglophone North America, 1945-2000. Organization Sciences 18(1): 85-95. 
Balla, S., Lodge, M., \& Page, E. (eds). (2015). The Oxford Handbook of Classics in Public Policy and Administration. Oxford: Oxford University Press.

Bauer, M. (2018). Public Administration and Political Science. In E. Ongaro, \& S. Van Thiel (eds). The Palgrave Handbook of Public Administration and Management in Europe (pp. 1049-1065). London: Palgrave Macmillan.

Bauer, M., \& Becker, S. (2019). Public Administration in Germany: Problems and Potential of a Fragmented Community. International Journal of Public Administration 42(11): 950-960.

Chandler, J.A. (1991). Public administration: A discipline in decline. Teaching Public Administration 9(2): 39-45.

Curry, D., van de Walle, S., \& Gadella, S. (2014). Public administration as an academic discipline: Trends and changes in the COCOPS academic survey of European Public Administration scholars. COCOPS Report Work Package 8. http://www.cocops.eu/wp-content/uploads/2014/02/ COCOPS_PAasadiscipline_report_09.02.pdf.

DiMaggio, P., \& Powell, W. (1991). Introduction. In W. Powell, \& P. DiMaggio (eds). The New Institutionalism in Organizational Analysis (pp. 1-38). Chicago, IL: University of Chicago Press.

Döhler, M. (2014). Verwaltungswissenschaftliche Problemperspektiven in der Politikfeldanalyse. In K. Schubert, \& N. C. Bandelow (eds). Lehrbuch der Politikfeldanalyse. 3., aktualisierte und überarbeitete Auflage (pp. 75-94). Munich: Oldenbourg.

Eubanks, V. (2018). Algorithms Designed to Fight Poverty Can Actually Make It Worse. Scientific American 319(5): 68-71.

Ganapati, S., \& Reddick, C.G. (2016). Information Technology in Public Administration Education. Journal of Public Affairs Education 22(2): 155-160.

Hall, P.A., \& Taylor, R.C.R. (1996). Political Science and the Three New Institutionalisms. Political Studies 44(4): 936-957.

Hajnal, G. (2003). Diversity and convergence: A quantitative analysis of European public administration education programs. Journal of Public Affairs Education 9(4): 245-258.

Howlett, M., \& Mukherjee, I. (eds). (2018). Routledge Handbook on Policy Design. New York and London: Routledge.

James, O., Jilke, S., \& van Ryzin, G. G. (eds). (2017). Experiments in Public Management Research: Challenges and Contributions. Cambridge: Cambridge University Press.

Jann, W. (2006). Die skandinavische Schule der Verwaltungswissenschaft: Neo-Institutionalismus und die Renaissance der Bürokratie. In J. Bogumil, W. Jann, \& F. Nullmeier (eds). Politik und Verwaltung. PVS-Sonderheft 37 (pp. 121-148). Wiesbaden: VS Verlag.

Jann, W. (2009). Praktische Fragen und theoretische Antworten. 50 Jahre Policy: Analyse und Verwaltungsforschung. Politische Vierteljahresschrift 50(3): 476-505.

Janning, F. (2006). Koexistenz und Synergieeffekte? Über das Verhältnis zwischen PolicyForschung und Verwaltungswissenschaft. In J. Bogumil, W. Jann, \& F. Nullmeier (eds). Politik und Verwaltung. PVS-Sonderheft 37 (pp. 77-96). Wiesbaden: VS Verlag.

Kickert, W. (2005). Distinctiveness in the study of public management in Europe: A historicalinstitutional analysis of France, Germany and Italy. Public Management Review 7: 537-763. 
Lasswell, H. D. (1971). A pre-view of policy sciences. New York: Elsevier.

Lember, V., Kattel, R., \& Tõnurist, P. (2018). Technological Capacity in Public Sector: The Case of Estonia. International Review of Administrative Sciences 84(2): 214-230.

Mergel, I. (2016). Big Data in Public Affairs Education. Journal of Public Affairs Education 22(2): 231-248.

Ongaro, E., van Thiel, S., Massey, A., Pierre, J., \& Wollmann, H. (2018). Public Administration and Public Management Research in Europe: Traditions and Trends. In E. Ongaro, \& S. van Thiel (eds). The Palgrave Handbook of Public Administration and Management in Europe (pp. 11-39). London: Palgrave Macmillan.

Pollitt, C. (2010). Envisioning public administration as a scholarly field in 2020. Public Administration Review 70: 292-294.

Pollitt, C. (ed.). (2013). Context in Public Policy and Management. The Missing Link? Cheltenham: Edward Elgar.

Pollitt, C. (2016). Advanced Introduction to Public Management and Administration. Cheltenham: Edward Elgar.

Pollitt, C., \& Bouckaert, G. (2017). Public Management Reform: A Comparative Analysis. 4th ed., Oxford: Oxford University Press.

Randma-Liiv, T., \& Drechsler, W. (2017). Three decades, four phases. Public administration development in Central and Eastern Europe, 1989-2017. International Journal of Public Sector Management 30(6/7): 595-605.

Raadschelders, J.C.N. (2011). The study of public administration in the United States. Public Administration 89: 140-155.

Roberts, A. (2014). Large Forces: What's Missing In Public Administration. Create Spaces.

Sager, F., Rosser, C., Mavrot, C., \& Hurni, P. (2018). A Transatlantic History of Public Administration: Analyzing the USA, Germany and France. Cheltenham: Edward Elgar.

Scott, R. W. (2014). Institutions and Organizations. London: Sage Publications.

Toeller, A. E. (2018). Politikwissenschaftliche Verwaltungswissenschaft und Policyanalyse in Deutschland. Überlegungen zu einer komplizierten Beziehung. In M. Bauer, \& E. Grande (eds), Perspektiven der Verwaltungswissenschaft. Wiesbaden: Nomos.

van Thiel, S. (2014). Research Methods in Public Administration and Public Management. An Introduction. London: Routledge.

Wegrich, K. (2017). Ironie des Schicksals: Verwaltungswissenschaft im Zeitalter von Governance. dms - der moderne staat 10(1): 3-30.

Xun, W., Howlett, M., \& Ramesh, M. (eds). (2018). Policy Capacity and Governance. Assessing Governmental Competences and Capabilities in Theory and Practice. London: Palgrave Macmillan.

Yang, L. (2018). Public Administration as a Dynamic Balance and Integrative Science across Politics, Management, and Law: Rosenbloom's Framework and Chinese Experiences. The American Review of Public Administration 49(2): 1-19. 


\title{
2 \\ Public Administration and Law
}

\author{
Martin Burgi
}

\section{$1 \quad$ Introduction}

There are more than 20 administrative law systems in Europe and, as a consequence, researchers in various countries focus on a vast variety of different topics, take different approaches and use different research methods. However, these differences in administrative law itself and the corresponding differences in administrative law scholarship between continental Europe and common law countries are decreasing due to the permanent and enduring impact of European Union law. However, as a German administrative law scholar, when I speak of administrative law hereinafter I refer to German and European administrative law, including the "Europeanisation" of domestic administrative regimes and the interplay and cooperation of domestic and EU administrative bodies.

Traditionally, administrative law scholarship ("Verwaltungsrechtswissenschaft") in Germany followed a mere doctrinal approach, the so-called "juristic method" ("Juristische Methode") to administrative law. The central aim of this approach is the systematic ordering of legal concepts, and it places special emphasis on the systematisation and development of a system of concepts (Eifert, 2014, p. 204). From this perspective, administrative law scholarship is largely a hermeneutic science of systematisation and interpretation dedicated to a "strictly legal point of view" (Ernst, 2007, p. 15f.). Therefore, researchers who are committed to this doctrinal approach ("Rechtsdogmatik") describe, develop and advance the inner system of terms, institutions, principles and rules of administrative law (Brohm, 1972, p. 246) to reconstruct and shape the grammar, categories and concepts that legislators, judges and administrators use when designing or applying legal rules (Voßkuhle \& Wischmeyer, 2017, p. 85). Although Rechtsdogmatik is strongly related to the application of legal provisions, it goes beyond that and attempts to create a layer of legal constructs and styles of argument between legal provisions and their application in an 
individual case which ensures the operationalisation of the legal provisions as well as the coherence of the legal system (Eifert, 2014, p. 214).

The doctrinal approach to administrative law focuses strongly on rightsprotection through courts. Therefore, the perspective administrative law scholars take by adopting this approach is similar (but by no means identical) to that of administrative courts, focusing on citizens who seek legal protection against administrative agencies. Doctrinalists focus on the final administrative decision, the so-called "legal act" ("Rechtsakt"). This act is measured against constitutional and statutory standards. Administrative law is thus treated as a benchmark for assessing what the administration has done wrong (Hoffmann-Riem, 1993). Against this background, administrative law scholars collect, analyse and categorise legal acts primarily to support courts in keeping control of an ever more complex legal system, and thus to strengthen the rule of law (Voßkuhle \& Wischmeyer 2017, p. 87).

However, Verwaltungsrechtswissenschaft is not "just" about interpreting legal provisions, systematisation and helping courts to cope with an increasingly complex legal landscape. Administrative law scholarship also takes the administration itself into account and reflects that administrative agencies are not only applying the law but also producing legal provisions themselves.

Furthermore, it is worth stressing that administrative law scholarship is not just about a certain legal technique to resolve specific cases. Apart from individual cases, administrative law scholarship also deals with broader factual phenomena, areas and developments and incorporates impact orientation into its research efforts as well as theoretical, comparative and interdisciplinary insights. It is important to emphasise that introducing new research perspectives, methods and approaches does not mean abandoning the "juristic method." On the contrary, the doctrinal method remains at the core of administrative law scholarship, since new regulatory approaches must not obscure the important legal task of assessing the conformity of administrative action with constitutional and European law. In addition, however, administrative law scholarship must also be able and willing to take into account empirical contexts of law and findings of other disciplines.

In this article I will argue that administrative law scholarship should broaden its perspective and expand its methodological portfolio by approaching neighbouring disciplines, and in particular political scientists who work in the field of public administration. I relate primarily to political science as it is probably the closest discipline to administrative law scholarship, due to the inherently political nature of administrative law. Nevertheless, the following considerations also apply to cooperation with other disciplines dealing with public administration, as for example economics and sociology. From my 
point of view, cooperation via collaboration on concrete research topics in a problem-oriented way promises the highest scholarly output.

\section{Golden history}

The first steps away from the traditional approach of administrative law scholarship in Germany were taken in the 1960s and 1970 s when changes in society as a whole and growing scepticism towards traditional paradigms and concepts also influenced administrative law scholars. From now on practitioners, researchers as well as legislators showed an increasing interest in the empirical reality of law and, thus, aimed at scholarly exchange and cooperation with the social sciences and their protagonists. This general development in legal research meant for the field of public administration becoming a forum for different disciplines. However, the field of public administration should not be viewed and developed as a separate discipline beyond a research platform of interdisciplinary exchange because political science, legal scholarship, business administration, etc. will continue to have different perspectives on public administration (Burgi, 2017, p. 38, p. 42f.). Hence, administrative law scholarship is one important discipline dealing with public administration which does not lose its disciplinary autonomy by engaging on the public administration research platform. Vice versa, Public Administration is not limited to a legal perspective but includes among other disciplines administrative studies ("Verwaltungslehre") and administrative science ("Verwaltungswissenschaft").

In the early 1960 , the interdisciplinary perspective was promoted mainly by contacts to US research on public administration, organisational theory and management theory. Later, factors supporting this development can also be found in Germany. One important development was the establishment of specific institutions committed to research on public administration and administrative law. An important example is the establishment of the Research Institute for Public Administration at the (former) Hochschule für Verwaltungswissenschaften (today: German University of Administrative Sciences) in Speyer in 1976. Furthermore, this included new publications formats combining the perspectives of Public Administration and administrative law scholarship. Examples of such publication formats are the renowned German journal Die Verwaltung. Journal for Administrative Law and Administrative Science, which was founded by Ernst Forsthoff in 1968.

In a second period covering the 1980 os and early 1990 os, major political and social changes including the deepening of the European integration, 
continuing globalisation, German reunification, economic liberalisation and privatisation provided fresh impulses for interdisciplinary research within administrative law scholarship. This new "tide" of reform that spread across Europe and Germany called for a more realistic, impact-orientated task and purpose-based approach to administrative law. These demands made it necessary to take a greater account of social sciences and of economics in administrative law scholarship. Complementing the doctrinal method with an interdisciplinary and an empirical approach is one of the central elements of these scholarly reform efforts.

Specifically, the occupation with economics resulted in a "More Economic Approach" of administrative law scholarship comprising two major concepts. The first is New Public Management, rooted in earlier ideas of New Institutional Economics (e.g. the principal-agent theory), which aims at transferring approaches and experiences of the private sector to public administration. Strategies of New Public Management include - among others - taking a stronger output orientation (e.g. through controlling) and outsourcing those tasks (e.g. in the form of public-private partnerships) that are not core state responsibilities (Voßkuhle, 2012, \50).

The second major concept, called the "new steering model" "Neues Steuerungsmodell"), is not a separate approach but a more specific form of New Public Management that was developed by German municipalities in 1993 based on the administrative reform conducted by the city of Tilburg in the Netherlands (Voßkuhle, 2012, \$53). In contrast to New Public Management, the Neues Steuerungsmodell is characterised by focusing on output orientation but not so much on the other strategies of New Public Management and, thus, takes not a descriptive but an internal perspective. In Germany, the concept of "steering" (Steuerung) became very important and replaced the older concepts of "planning," "social engineering" and "rational politics" which had been popular in the 1960 and early 1970 (Voßkuhle \& Wischmeyer, 2017, p. 93).

In the subsequent third phase, which lasted from 1991 to 2006 , administrative law scholars reflected the developments of the previous decades. A group of administrative law scholars, headed by Wolfgang Hoffmann-Riem and Eberhard Schmidt-Aßmann, aimed for a new methodological re-orientation and organised itself through a series of conferences between 1991 and 2003 whose proceedings were published in ten volumes under the title "On the reform of administrative law" "Schriften zur Reform des Verwaltungsrecht") (Eifert, 2014, p. 206f.; Voßkuhle \& Wischmeyer, 2017, p. 89).

These reflective debates then resulted in the publishing of the three-volume handbook "Foundations of Administrative Law" "Grundlagen des Verwaltungsrechts"), whose second edition appeared in 2012 and 2013 and which 
summarises the current state of administrative law research. It gives a full account of German administrative law scholarship and complements analyses of legal acts, principles and institutions by insights from regulation theory, regulatory choice and implementation studies (Voßkuhle \& Wischmeyer, 2017, p. 92). Its editors Wolfgang Hoffmann-Riem, Eberhard Schmidt-Aßmann and Andreas Voßkuhle had the ambition to provide an impetus for the development of a new approach to administrative law scholarship, called "Neue Verwaltungsrechtswissenschaft" ("New Administrative Law Scholarship"). The common fundamental concern of the publishers and some of the contributors was a change of perspective in administrative law. Whereas citizens and the protection of their fundamental rights used to be the focus of doctrinal administrative law scholarship, now under the so-called regulatory approach the perspective of the legislator and the administration enacting regulations was increasingly taken up as well. From now on, German administrative law studies dealt more and more with the question of how political goals could (better) be implemented through legal provisions and their enforcement. The regulatory approach sees law as a means of behavioural regulation and as a medium for accomplishing context-appropriate tasks marked by questions of organisation and procedure. Overall, the Neue Verwaltungsrechtswissenschaft is much more interested in the impact and outcome of specific regulatory choices than traditional administrative law scholarship. A decisive factor for this change in perspective was the insight that the modern welfare state, with its ambition of social engineering through law, had led to a significant "legalisation" of social life. However, the actual effect of legal regulations was insufficient to achieve these aims. The discourses on legalisation, the change in the state's function and the lack of effectiveness of traditional legal tools called into question whether the traditional approach was still sufficient to meet the current challenges of public administration and administrative law (Eifert, 2014, p. 206f.; Voßkuhle \& Wischmeyer 2017, p. 95). Although this third phase had an emphatic interdisciplinary outset, unfortunately it was conducted only by administrative law scholars and almost completely lacked contributions from other disciplines.

\section{Silent present}

Despite previous efforts and proclamations to include empirical approaches and to align administrative law scholarship interdisciplinary, contemporary research on administrative law hardly ever incorporates research of political scientists on public administration and vice versa. Part of this problem is 
already inherent in the theory-building phase itself, since the abovementioned leading publication "Grundlagen des Verwaltungsrechts" takes only an administrative law scholarship view. Another example - this time from the Public Administration side - is Wolfgang Seibel's (2016) recent publication "Verwaltung verstehen," in which he only recurs to Hartmut Maurer's (2017) (now co-authored with Christian Waldhoff) "Allgemeines Verwaltungsrecht," a traditional German textbook on administrative law following the traditional doctrinal approach, but takes no notice of the "Grundlagen des Verwaltungsrechts" and with it also leaves out the "Neue Verwaltungsrechtswissenschaft" as a whole. Today, only a few administrative law scholars are interested in Public Administration and joint research projects are rarely conducted. In his article on Public Administration in the US, Bradley E. Wright (2011) makes a similar observation about the US debate.

\section{$4 \quad$ Towards a brighter future?}

\subsection{Promising developments}

Despite the current unsatisfactory situation regarding the implementation of the concepts of the "Neue Verwaltungsrechtswissenschaft," developments of recent years indicate that administrative law scholarship is indeed beginning to open up to interdisciplinarity. In the past few years, several profound books and articles have been published which focused on the fundamental principles and the theory of interdisciplinary cooperation. These important contributions show that the theoretical foundations for interdisciplinary cooperation of administrative law and Public Administration have already been laid. Important examples on which the actual implementation of such cooperation of both fields can be based are the establishment of the interdisciplinary journals "Journal of Law and Public Administration" and "European Journal of Law and Public Administration," which were established in 2015 and 2012 respectively.

As mentioned above, Public Administration is supposed to function as a "research platform" (Burgi, 2017, p. 42f.) for future interdisciplinary discourse on public administration between administrative law scholars, political scientists and researchers of other disciplines. Although Public Administration is no discipline in its own right, personal, institutional, publication-related communication contexts centred on "administration" as a shared research object make it easier to establish cooperation between the various disciplines. 
Concerning teaching programmes, it follows from the focus on an interdisciplinary research platform that in particular in elective course programmes of law faculties and in postgraduate and master programmes an interdisciplinary course should be added to the curriculum. So, for example, an elective programme at a law faculty concerning administrative law should include classes on "Public Administration" or on "Interdisciplinary Aspects of Administrative Law Scholarship” to its syllabus.

\subsection{Focus on concrete issues instead of "grand formulas"}

If one opts for an interdisciplinary research perspective, an equally conscious and transparent approach is required, especially with regard to the terminology used and the research questions formulated. In this respect, it was pointed out aptly that even the researched object is not identical from the point of view of different disciplines, but is also constituted by their perspectives, concepts and methods. This theoretical insight suggests that starting points for interdisciplinary research efforts should be actual phenomena and developments outside the realm of law. Examples of such "concrete issues" are "the role of local governments in developing local politics" or "forms of citizen participation in large-scale infrastructure projects," in contrast to abstract concepts such as, for example, "legitimation" or "participation." Jointly formulated research topics of this kind are concrete rather than abstract and they produce "micro" rather than "macro" knowledge. They are from the outset problem-solving oriented and their tailoring causes fewer difficulties, because the terms, concepts and perspectives are describable and manageable. In my opinion, such problem-oriented interdisciplinary research projects are the most promising. In contrast, the importance of common key terms and guiding principles (e.g. "responsibility," "governance" or "public service" ["Daseinsvorsorge"]) tends to be overrated. They are largely too abstract and are not necessarily mutually connective (see however for a special accentuation of the importance of these concepts Voßkuhle \& Wischmeyer, 2017, p. 96).

In what follows I will present several broad research topics that could be eligible for interdisciplinary collaboration and which are already discussed and debated in Public Administration (a). I will also suggest a number of contemporary issues of administrative law scholarship that should be considered for cooperation between public administration and administrative law scholars in the future (b). Finally, I would like to present two promising interdisciplinary research projects, in which I am involved and which are paradigmatic for the problem-oriented interdisciplinary research approach I advocate in this paper (c). 
a) An evaluation of German publications and conferences on public administration-related topics since 2010 shows that five research areas in particular are being worked on, which are all well suited for cooperation between administrative law and public administration scholars.

The first topic concerns the organisation of administration. The research in this field focuses on general aspects, but in some studies individual policy areas are also analysed in relation to organisational issues, such as the current organisation of migration and integration administration, e.g. the 2017 congress of the International Institute of Administrative Sciences (IIAS) on "Managing Migrations, Integration and Poverty." Special attention within the organisation-related research cluster is paid to the territorial and functional reforms carried out or intended in various countries as well as to the various phenomena of Public-Private Partnerships.

A second field of scholarship focuses on types and modes of administration and is therefore also organisation-related. Studies in this area deal with topics such as participation and the relationship between politics and administration. Furthermore, questions relating to ministerial administration and "legitimation" are repeatedly examined.

Another focus is on cooperation, coordination and governance issues. Here (again in a loose enumeration) the focus is on cooperative environmental policy, regulatory structures or governance. Almost as expected, the various aspects of digitalisation form a research priority within the branch of public administrative research dedicated to "public management," but aspects of ethics in public administration are also repeatedly discussed.

Another branch of Public Administration discusses the effectiveness and economic viability of administration, including benchmarking and evaluation. A fifth and final subject area lies in the area of the administration of the European Union and the various interactions of EU agencies with national administrative bodies. In this field, Public Administration could easily access the extensive administrative law scholarship on European administrative law and its impacts on the domestic administrative law systems.

b) In addition to the topics that have already been dealt with, there are many more contemporary issues that should be considered for cooperation between public administration and administrative law scholars in the future. From the perspective of an administrative scholar, in this section I use concrete examples to illustrate what kinds of questions and research arrangements could contribute to a fruitful interdisciplinary dialogue on public administration in its various dimensions. The selection of examples is purely subjective, i.e. primarily based on the scholarly interests of the author of this article. 
The aim is not to formulate concrete research projects or even to address them, but to demonstrate how to proceed in the interest of an accentuated interdisciplinary approach to Public Administration and administrative law.

A first example concerns commercial activities of the state on all levels of government, i.e. the phenomenon of the state acting as an entrepreneur. Apparent developments are the steady increase, diversification and, above all, internationalisation of such activities, in which the federal government, states ("Länder") and local communities engage in. The very interesting aspect of commercial activities of states concerns the fact that public entities, by participating in economic markets, blur the lines between their capacity as sovereigns and their role as market players. Angela Merkel, for example, when she is on a state visit to China, acts as German Chancellor, but also as a representative of state-owned Deutsche Bahn AG, a company that makes billion-dollar investments in China. This field of economic state activity is largely ignored by Public Administration research, as carried out by political scientists, not - of course - by public management scholars. However, it raises interesting questions the answers to which require competence in political science: what is the relationship between the political and the administrative spheres with respect to public commercial enterprises (organisational as well as personnel and financial)? What is the self-conception of the actors involved? What are the specific expectations of citizens/clients?

Another area, which promises a fruitful interdisciplinary dialogue concerns public contracts. Although administrative action through public contracts is a frequently used tool of administrative agencies, it is still scarcely researched, at least in Public Administration. Public contracts are increasingly being used and are becoming increasingly diversified. However, what is particularly worth mentioning is that they are utilised in more and more areas of administrative practice, and in recent years also in new fields such as youth welfare and the integration of immigrants. In these areas we are dealing with both individual fates and large sums of money. This raises new legal questions, concerning for example the competences to enter into contracts and the internal administrative control and sanction system. Interesting topics for Public Administration could relate, e.g., to the question of how administrative branches are organised in fields which are dominated by public contracts, the processes in which this takes place and what specific expertise to which personnel is required in these fields. This also includes the question of transparency versus secrecy.

Thirdly, the fields of administrative procedures and remedies, which have so far been relatively seldom dealt with jointly, offer a rich potential for interdisciplinary research projects. For example, new types of administrative procedures such as distribution mechanisms (e.g. awarding public contracts) 
and inner-administrative supervision and control call for empirical and analytical assessment from political scientists, who conduct research in the area of public administration. The same applies to questions of organisational structure of court systems and their procedural processes. Political science and public administration research in these fields could provide interesting impulses, which in turn could give rise to new legal questions or new answers to conventional questions.

c) The author of this paper tries to set a good example and has been involved in two major interdisciplinary research projects. Both projects are exemplary for the problem-oriented approach advocated here. For this reason, they will be presented in some detail below.

In the course of the first project, "Better administration of migration and integration policy" ("Bessere Verwaltung in der Migrations- and Integrationspolitik") on behalf of the Mercator foundation, an interdisciplinary research group, composed of political scientists Jörg Bogumil and Jonas Hafner of the University of Bochum and Sabine Kuhlmann and Moritz Heuberger from the University of Potsdam and legal scholars, namely Martin Burgi and Christoph Krönke from LMU Munich, developed recommendations for better administrative management of the challenges posed by the so-called "migration crisis."

The background of the study, which was published in 2018, is the significant immigration that Europe and especially the Federal Republic of Germany experienced in the years 2015 and 2016. During this time the administrative agencies on all federal levels showed flexibility and great improvisation skills. Nevertheless, there were numerous problems in the area of migration and integration management and policy. Since then, there has been intensive discussion about whether responsibilities should be changed in the sense of new legal regulations and whether tasks in the federal asylum and integration administration should be distributed differently. Against this backdrop, established administrative procedures and organisational structures are under scrutiny. The question arises: which federal level can most effectively solve which task in the field of asylum and integration administration? This concerns aspects such as the initial reception, processing of asylum applications and integration of refugees.

Our comprehensive interdisciplinary research project tries to answer the most important of the above questions. It is the result of a year-long systematic study of administrative structures at the federal, state and local levels. The different levels are examined with a focus on essential tasks in the field of asylum and integration, supplemented by the cross-sectional topics of data management 
and administrative litigation. On this basis, optimisation proposals for a better assignment and performance of tasks in the area of migration and integration are developed. The reorganisation proposals are oriented towards the goal of reducing unnecessary interfaces, avoiding overlaps, speeding up administrative procedures (without loss of quality) and, where necessary, strengthening cooperation).

In addition to the extensive evaluation of the research literature and existing materials, more than 70 expert interviews have been conducted at the Federal Office for Migration and Refugees (Bundesamt für Migration und Flüchtlinge = BAMF), the Federal Administrative Office (Bundesverwaltungsamt), federal police offices, various administrative courts, district and regional governments, state ministries, local immigration authorities, job centres, registration authorities, social services, integration centres and other local authorities, as well as with representatives of national local authority organisations. The study collects information from a total of ten Länder. This study also builds on a study published in 2017 (Bogumil, Hafner \& Kastilan, 2017) and extends, supplements, updates and deepens it. It provides an empirical political and administrative analysis and formulates recommendations for action. The jurisprudential parts provide a legal analysis of the possible legal implementation and the constitutional law framework of certain organisational or procedural proposals. In particular, the study takes up those questions from political and administrative analysis that have a particularly intensive legal and current relevance.

A second interdisciplinary research project in which I am involved is called RISE (Research and Innovative Staff Exchange) - the Project on "Local Government and the Changing Urban-Rural Interplay - LoGov." It is founded by the European Union and brings together eight European research institutions (inter alia EURAC research Bolzano, the Autonomous University of Madrid and the University of Warsaw) and nine non-European partners (inter alia the University of the Western Cape, Addis Ababa University and the National University of Singapore).

The project builds on the observation that the changing urban-rural dynamics bring enormous repercussions on daily lives and, as a consequence, on local governments. From an economic point of view, metropolitan regions are often places of great innovation and nodal points of a globalised economy, and as such have a reciprocal relationship with similar metropolitan regions that are both partners and competitors. However, metropolitan regions also have to bear many specific burdens such as environmental pollution, housing shortages, congestion, inequality and poverty, which are important issues for local government today. Local authorities in peripheral rural areas, on 
the other hand, face different challenges. Among the most urgent are the emigration of young people and the corresponding loss of workforce and tax revenues to guarantee public services, especially in view of the often aboveaverage age of the rural population. The specific nature of these problems in urban and rural areas means that local governments need tailored policy instruments to address these problems.

The "Local Government and the Changing Urban-Rural Interplay"project aims to form an international and intersectoral training and research network in order to provide best-fit practices for local governments to address the changing urban-rural interplay and manage its impacts. These practices are meant to cater to the specific needs of local governments and to be easily applicable. To achieve the projects goals LoGov adopts a five-pronged methodological approach, which consists of comparative case study research, a multilevel perspective, the identification of linkages between different local government areas, the inclusion of local policy-makers and especially a multiand interdisciplinary research approach that is facilitated by the consortium's expertise in different disciplines. The ambitious research activities require a pooling of complementary expertise, if the project is to provide a holistic picture of practices to address the impact of urban-rural differences on the major areas of local government.

In order to counter the undeniable risks of interdisciplinary research, for example with regard to the sometimes very different understanding of concepts and terminologies, the LoGov consortium was consciously limited to neighbouring disciplines with similar research concepts and terminologies. The consortium therefore includes experts from the fields of public law, political science, public administration and economics. Combined with LoGov's comparative approach, the inclusion of these four disciplines means that the project is based on the methods of comparative constitutional and administrative law, comparative political science, comparative public administration and comparative economics.

Another important feature of the cooperation between the various research institutions is the exchange of skills and knowledge. Mobility across national borders and sectoral boundaries permit LoGov Consortium efficiently to compare and share dissimilar practices that local governments worldwide have developed in response to similar problems and to widen the career perspectives of researchers by fostering the development of their skills and exposing them to new professional environments. 


\subsection{Promoting and supporting collaboration}

Finally, the personnel-institutional component of interdisciplinary work needs to be discussed. In particular, the obstacles arising from the personnel configuration of the various disciplines or from institutional circumstances have so far been rather underestimated. They must be addressed openly if interdisciplinary research projects are not to be left exclusively to incidental personal contacts.

The institutional starting point is characterised by the fact that the disciplines concerned are becoming more and more specialised. As a consequence, working capacities for interdisciplinary activities are becoming ever less. Against this background, interdisciplinary projects will be primarily pursued if they promise concrete findings.

The group of political scientists interested in public administration has always been rather small, and it has certainly not grown as a result of the attention paid to international publications and empirical-statistical methods. For most topics, the number of administrative law publications outweighs the number of political science papers by far.

However, it would also be rewarding for political scientists to make greater use of the expertise of public law scholarship and engage in interdisciplinary cooperation with administrative law scholars. From my perspective, the reflection and integration of legal knowledge and scholarship on public administration has in particular two benefits for scholars of other disciplines.

The first obvious advantage is that administrative law scholarship has the important function of informing other disciplines dealing with public administration. Administrative law scholarship describes, systematises and structures the legal framework of the legislative and executive facets of public administration. The structure of this legal framework can be extremely complex in times of rapidly advancing Europeanisation and internationalisation. Without a basic understanding of the underlying legal framework, research activities in other disciplines, in particular those aimed at policy recommendations, are in danger of losing any form of practical relevance from the outset.

A second advantage for other disciplines of taking an interest in administrative law scholarship lies in the fact that they can utilise the usually much greater practical orientation of jurisprudential research. Thus, administrative law scholarship often works on topics and addresses problems that emerge in other disciplines at a much later point in time. Against this background, the consultation of administrative law research can help to identify the real problems of the moment, which often require an interdisciplinary assessment 
for their solution. Examples of such topics concern the challenges of the housing crisis in urban centres for public administrations and the whole field of policing and security administration, which is intensively dealt with in administrative law scholarship, but hardly - with a few important exceptions (see especially Lange \& Wendekamp, 2018) - in political science.

Overall, it is necessary to maintain old and create new structures which can bring researchers, including young scholars, together. Opportunities for interdisciplinary exchange can be created, for example, through the establishment of joint discussion groups, the organisation of interdisciplinary conferences (such as the IIAS conferences) and the publication and cultivation of joint research journals, as for example Die Verwaltung and European Journal of Law and Public Administration. Finally, interdisciplinary research projects can be initiated or enabled by funding programmes of research foundations like the "Deutsche Forschungsgemeinschaft" (German Research Foundation) and the European Union.

Throughout, the focus should be on the search for specific research questions which can be addressed by way of flexible cooperation between administrative law and public administration scholars, with low thresholds, while institutional solutions such as the designation of interdisciplinary chairs or the merging of faculties are rather counterproductive in my opinion.

\section{Outlook}

The potential for cooperation between Public Administration and administrative law scholarship is far from exhausted. It is possible to answer the many interesting interdisciplinary research questions, either already on the table or still to be developed, without having to question one's own discipline. It is simply a question of an additional research perspective that promises a great benefit for all participating disciplines. Thus, interdisciplinary work is often more beneficial than expected and poses fewer difficulties than often feared. So let us get to work, because many problems await a first or better solution. 


\section{References}

Bogumil, J., Hafner, J., \& Kastilan, A. (2017). Verwaltungshandeln in der Flüchtlingspolitik Vollzugsprobleme und Optimierungsvorschläge für den Bereich der kommunalen Integration. Verwaltungsarchiv 108: 467-488.

Bogumil, J., Burgi, M., Kuhlmann, S., Hafner, J., Heuberger, M., \& Krönke, C. (2018). Bessere Verwaltung in der Migrations- und Integrationspolitik - Handlungsempfehlungen für Verwaltungen und Gesetzgebung im föderalen System. Baden-Baden: Nomos.

Brohm, W. (1972). Die Dogmatik des Verwaltungsrechts vor den Gegenwartsaufgaben in der Verwaltung. In Veröffentlichungen der Vereinigung der Deutschen Staatsrechtslehrer (vol. 30). Berlin: De Gruyter.

Burgi, M. (2017). Intradisziplinarität und Interdisziplinarität als Perspektiven der Verwaltungsrechtswissenschaft. In M. Burgi (ed.), Zur Lage der Verwaltungsrechtswissenschaft (pp. 33-62). Berlin: Duncker \& Humblot.

Eifert, M. (2014). Conceptualizing Administrative Law - Legal Protection versus Regulatory Approach. In H. Pünder, \& C. Waldhoff (eds). Debate in German Public Law. Oxford and Portland, OR: Hart Publishing.

Ernst, W. (2007). Gelehrtes Recht - Die Jurisprudenz aus der Sicht des Zivilrechtslehrers. In C. Engel, \& W. Schön (eds). Das Proprium der Rechtswissenschaft. Tübingen: Mohr-Siebeck.

Hoffmann-Riem, W. (1993). Verwaltungsrechtsreform. In W. Hoffmann-Riem, E. SchmidtAßmann, \& G. Folke Schuppert (eds). Reform des Allgemeinen Verwaltungsrechts. Baden-Baden: Nomos.

Hoffmann-Riem, W., Schmidt-Aßmann, E., \& Voßkuhle, A. (2012), Grundlagen des Verwaltungsrechts (vols. 1-3). 2nd ed., Munich: Beck.

Lange, H.-J., \& Wendekamm, M. (2018), Die Verwaltung der Sicherheit - Theorie und Praxis der Öffentlichen Sicherheitsverwaltung. Wiesbaden: Springer.

Maurer, H., \& Waldhoff, C. (2017). Allgemeines Verwaltungsrecht. 19th ed., Munich: C.H. Beck. Seibel, W. (2016). Verwaltung verstehen - Eine theoriegeschichtliche Einführung. Berlin: Suhrkamp. Voßkuhle, A. (2012). Neue Verwaltungsrechtswissenschaft. In W. Hoffmann-Riem, E. SchmidtAßmann, \& A. Voßkuhle (eds). Grundlagen des Verwaltungsrechts (vol. 1, 2nd ed.). Munich: C.H. Beck.

Voßkuhle, A., \& Wischmeyer, T. (2017). The "Neue Verwaltungsrechtswissenschaft" against the backdrop of traditional administrative law scholarship in Germany. In S. Rose-Ackerman, P. L. Lindseth, \& B. Emerson (eds). Comparative Administrative Law. 2nd ed. (pp. 85-101), Cheltenham: Edward Elgar Publishing.

Wright, B. E. (2011). Public Administration as an Interdisciplinary Field. Public Administration Review 71: 96-101. 



\title{
Seeing Public Bureaucracies Like a Sociologist: (A Plea Towards) Reconnecting Sociology and Public Administration
}

\author{
Philippe Bezes
}

Exploring the past and future relationships between sociology and Public Administration is both a classic question and a challenging task. On the one hand, it is too often forgotten that public bureaucracies have been first studied from various sociological points of view since the 1950s, leading to many classic contributions to the field. On the other hand, the development of many academic disciplines and the constitution of Public Administration as an autonomous field in many countries have led to strong dynamics of division of scientific labour as well as a parting of ways in the studies of public bureaucracies, among approaches coming from sociology, anthropology, political science, history, organisation studies and even public management. This specialisation has made dialogue across disciplines more difficult and rarer than it used to be, with damaging effects produced by fragmentation and atomisation. Besides, public administration as an academic field, recently ever more influenced by the growth of public management studies and their economic flavours, has made huge efforts in developing its own epistemological and methodological foundations, thus totally ignoring or forgetting the many lessons learned from scholars in sociology.

Of course, there are great variations across countries in the ways public administration has been studied, according to the strength of sociology and political science as a discipline in a given context and according to the existence or not of Public Administration as an autonomous subfield in each country. Thus, my contribution of this chapter does not come from nowhere. It comes from a French political scientist, initially trained in the "sociology of organisations" (a French school of thought wich locates itself somehow in between organisation theory, sociology and public policy) in a specific country, France, where Public Administration (under the label of 
"administrative science") was not institutionalised as a proper field despite several efforts in the 1960 s and early 1980 s (see Bezes, 2009, pp. 92-96) and where sociology is still quite strong. In this French context, sociology is vibrant, both as a discipline per se and in the study of bureaucracies through the outreach of many French sociologists (classic authors like Michel Crozier, Pierre Bourdieu and even Bruno Latour) or French philosophers like Michel Foucault, and through the influence of its paradigms.

Against the somehow marginalisation of sociology within the international scene of studies in Public Administration, this chapter then proposes first to acknowledge the diversity and richness of the past contributions sociology has made in the study of public bureaucracies. It does so by first emphasising the importance of three main broad traditions of research in sociology (1.), and then by identifying four significant lenses for viewing public bureaucracies like a sociologist (2.). In the last part of the chapter (3.), we defend the idea that the sociological eye is nowadays ever more needed, crucial and decisive in the contemporary context for several reasons: because of the massive transformations public administrations have experienced; because the social dimensions of these changes are many; because sociology can help in giving attention to several blind spots of the actual study of bureaucracies; because it can contribute to breaking routines and bringing new perspectives in the field.

\section{Remembering the Classics! Four (at least) broad traditions of research in sociology}

The contribution of sociology to the study of bureaucracies is both ancient and foundational. It is also quite remarkable that sociology brings such a large diversity of traditions from the micro (bureaucratic behaviours and organisations) to the macro (historical political sociology) and from exploring individuals, their social backgrounds and dispositions (interactionist and Bourdieusian perspectives) to the most material aspects of public bureaucracies (anthropology, science and technology studies, actor network theory). Table 1 below identifies these four traditions. We are well aware that this overview may be too simplistic, but we use it here as a first attempt to draft a global picture mapping how sociology has been influential in studying bureaucracies. 
Table 1 Four traditions of research in sociology studying bureaucracies

\begin{tabular}{|c|c|c|c|}
\hline Traditions & $\begin{array}{l}\text { Main } \\
\text { "classic" } \\
\text { authors }\end{array}$ & $\begin{array}{l}\text { Disciples and } \\
\text { successors }\end{array}$ & Main Focus \\
\hline $\begin{array}{l}\text { Sociology of } \\
\text { bureaucratic } \\
\text { behaviours and } \\
\text { organisations }\end{array}$ & $\begin{array}{l}\text { Merton, } \\
\text { Simon, Blau, } \\
\text { Gouldner, } \\
\text { Selznick, } \\
\text { Crozier }\end{array}$ & $\begin{array}{l}\text { Meier, Page, } \\
\text { Hood, Moynihan }\end{array}$ & $\begin{array}{l}\text { Rules and behaviours } \\
\text { Informal relations } \\
\text { Bureaucratic vicious circle } \\
\text { Expertise } \\
\text { Organisations }\end{array}$ \\
\hline $\begin{array}{l}\text { Historical and } \\
\text { comparative } \\
\text { historical } \\
\text { sociology of } \\
\text { bureaucracy- } \\
\text { making in time }\end{array}$ & $\begin{array}{l}\text { Weber, } \\
\text { Eisenstadt } \\
\text { \& Rokkan, } \\
\text { Bendix, } \\
\text { Moore }\end{array}$ & $\begin{array}{l}\text { Comparative his- } \\
\text { torical sociology } \\
\text { Skocpol, Tilly, } \\
\text { Mann, Shefter, } \\
\text { Silberman }\end{array}$ & $\begin{array}{l}\text { Historical processes and institutional } \\
\text { change over the long term and in } \\
\text { configurative perspective } \\
\text { Bureaucratisation/ } \\
\text { professionalisation } \\
\text { Public bureaucracies, social classes } \\
\text { and educational systems } \\
\text { Building of administrative capacities }\end{array}$ \\
\hline $\begin{array}{l}\text { Sociology of } \\
\text { bureaucracies as } \\
\text { individuals with } \\
\text { various socialis- } \\
\text { ing experiences } \\
\text { and playing } \\
\text { bureaucratic } \\
\text { roles }\end{array}$ & $\begin{array}{l}\text { Bourdieu, } \\
\text { Goffman }\end{array}$ & $\begin{array}{l}\text { Lipsky, } \\
\text { Maynard-Moody, } \\
\text { Musheno, } \\
\text { Dubois, } \\
\text { Oberfield, } \\
\text { Watkins-Hayes }\end{array}$ & $\begin{array}{l}\text { Bureaucracies as sets of everyday } \\
\text { roles } \\
\text { Bureaucracies' structures and } \\
\text { institutions, articulating public } \\
\text { agents as individuals with disposi- } \\
\text { tions and positions in bureaucracies, } \\
\text { resulting in the endorsements of } \\
\text { bureaucratic roles }\end{array}$ \\
\hline $\begin{array}{l}\text { Sociology and } \\
\text { anthropology of } \\
\text { bureaucracies } \\
\text { as cultural } \\
\text { and material } \\
\text { institutions }\end{array}$ & Foucault & $\begin{array}{l}\text { Herzfeld, Scott, } \\
\text { Riles, Rose \& } \\
\text { Miller, Gupta, } \\
\text { Hibou, Hull }\end{array}$ & $\begin{array}{l}\text { Construction and ordinary function- } \\
\text { ing of bureaucracies through } \\
\text { categories, norms, instruments and } \\
\text { representations } \\
\text { Ideological and material aspects } \\
\text { Everyday practices } \\
\text { Domination }\end{array}$ \\
\hline
\end{tabular}

It is first crucial to recall that sociology was the main source of the development of the empirical study of administrations with famous sociologists dedicating their early works to public bureaucracies. We refer here to the tradition of early organisational sociologists like Robert K. Merton (1940), Herbert A. Simon (1947), Peter M. Blau (1955), Alvin W. Gouldner (1954), Philip Selznick (1949) and, in France, Michel Crozier (1964). Their core focus was an exploration of the ordinary functioning of increasingly powerful (public and private) bureaucratic organisations in the 1950s and 1960s. Their approach challenged a view, (unfairly) associated with Max Weber, that was said to over-emphasise the formal and rational organisational 
structure of bureaucracy (Weber's "ideal type"). They were pleading, in contrast, that one should pay great attention to informal relations, unofficial norms, ordinary behaviours of bureaucrats as well as social actors. Their scales of analysis were deliberately micro-sociological, centred on individual behaviours and interactions in relation to bureaucratic rules. Their sociological perspectives place both rule compliance and expertise at the centre of understanding the functioning of public bureaucracies. Taking these two features into consideration is central to apprehending the distribution of power within administrations and sets the foundations for perspectives based on the discretionary power of public agents in general and street-level bureaucrats in particular. It is not surprising that rational choice perspectives such as John Brehm and Scott Gates's book Working, shirking and sabotage (1997) acknowledge the value of sociological and organisational studies on "bureaucratic discretion" before presenting rational choice models (public choice, transaction costs, principal-agent, delegation theory).

At the somehow opposite side of the continuum, given its macro-level perspective, lies the significant contribution of historical political sociology studying state-building processes, both in the long term and comparatively. The initial landmark here is the comparative historical sociology of Max Weber, but many authors from the 1950s to the 1980s, like Barrington Moore (1966), Shmuel N. Eisenstadt and Stein Rokkan (1973), Reinhard Bendix (1980), Charles Tilly (1990), Theda Skocpol (1985) and more recently Martin Shefter (1994), Michael Mann (2012) and Bernard Silberman (1993) developed their works in this tradition with specific focus on the building of bureaucracies. These perspectives have been labelled "the historical turn in sociology" (Smith, 1991; Adams et al., 2005). They explore under what cultural, structural and environmental conditions legal rational bureaucracy was likely to emerge. The dynamics of political ordering (elites, leaders) are central in these studies of processes of state formation and transformation. However, while remaining widely macro-sociological, these scholars very often paid great attention to the building of bureaucracies. Issues like centralisation (concentration of resources), bureaucratisation (the setting up of administrative capacities) and professionalisation (the formation of civil services with protections in the nineteenth century) are then examined in the long term. Quite significantly, they also connect these institutional processes to broad social dynamics like class conflicts, upward or backward mobility of social groups (nobles, bourgeoisie, clerics, etc.) and transformations of educational systems, but also changes in political 
systems (competition between political parties, etc.) and major policy changes (tax, war, etc.). Quite paradoxically, these perspectives have paved the way for the development of historical neo-institutionalism, but this theoretical perspective has been more central in political science than in sociology and, while claiming to "bring the state back in," they have been predominantly focused on welfare states and capitalisms, much more than on public bureaucracies.

A third tradition of research coming from sociology emphasises the importance of individuals and their dispositions on two complementary dimensions: on the one hand, on the process of socialisation through which individuals develop the knowledge, attitudes and behaviours necessary to become bureaucrats or, to put it differently, to play the role of bureaucrats; on the other hand, on the ordinary or "street-level" interactions between public agents and citizens-users-clienteles, these daily practices being viewed as the central arena in which public bureaucracies and the states are substantiated in people's lives and are routinely constructed as public organisations. While less immediately influential, this tradition can be rooted in the works of sociologists like the French Pierre Bourdieu (1994) and the American Erving Goffman (1978). Basically, summed up, Bourdieu's perspectives put strong emphasis on dispositional perspectives (the "habitus") emphasising the idea that individuals' extra-organisational backgrounds and experiences (educational, social, prior professional) have a major influence on how entrants develop and construct their roles as bureaucrats. In the Bourdieusian approach, prior and embodied socialisations shape the beliefs, understandings and scripts (public) agents use in action. Of course, this does not mean that individuals are not also socialised by and within public bureaucracies as institutions since public organisations impose categories, norms and routines on their members. However, the dispositional approach first favours the attitudes and experiences public agents bring with them into bureaucracies because they affect how they think and act and shape the making of their bureaucratic identities and roles. This perspective requires developing a detailed sociography of public servants, whatever their positions, and explores how inherited individual socialisations interact in action with institutional bureaucratic cultures. This perspective has been highly influential in the French context (for an overview see Eymeri \& Bouckaert, 2013). In addition, this sociological tradition is also echoed in the street-level bureaucracy literature where administrative encounters between front-line agents and users are central scenes in which rules, norms, values and scripts from both sides co-adjust. 
As is well-known, interactions on the ground here are the major focus on the dominant theorisation of a discretionary power (Lipsky, 1980) but also on a great range of roles to be endorsed and played by public agents in the context of various forms of interaction between bureaucrats and their users (Maynard-Moody \& Musheno, 2003). French authors have insisted on the influence of social dispositions and trajectories in the making of bureaucratic roles (Spire, 2005; Siblot, 2006; Dubois, 2010).

The fourth tradition of research comes both from sociology and from anthropology (Sharma \& Gupta, 2006, pp. 8-11). This perspective argues that viewing bureaucracies as autonomous institutions is itself a taken-for-granted reification produced by administrative categories, expertise and disciplinary practices that shape everyday understandings of what bureaucracies are and what they do. The boundaries between bureaucracies (or the state) and civil society and the vertical position of the former are seen as an effect of power. Political science as well as public management or Public Administration have participated in discursively constructing "the bureaucracy" as a distinct entity. Consequently, the task for anthropology or sociology is to counter evidence what is taken for granted, and becomes one of analysis through which processes public bureaucracies come to assume and legitimise their power, their representations as coherent and their role in societies as an instrument of domination. The study of bureaucracies then proposes to re-embed them within other institutional forms (the political sphere, the economy, civil society) and explore the multiple institutional and social networks through which they have been culturally constructed in different contexts. One then explores bureaucratisation or "stateisation" as social and political processes. From these results a great variety of approaches (studying categories, instruments or everyday practices), all bringing together the ideological and material aspects of the making and ordinary functioning of bureaucracies. Categories, for instance, are a key modality through which bureaucracy enacts and sociologists and anthropologists then explore how bureaucratic actors seek to construct, classify, standardise or measure the lines of social differences, categorising and stratifying people along the lines of race, nationality, class, gender, economic or social conditions, to name a few. Bureaucracies are examined in their power to name as well as define and order the contours of the social world (what Bourdieu calls the symbolic capital) but also in the images and representations of self they produce through diverse techniques (uniforms, organisational charts, statistics, public communications, etc.). Instruments are technologies of power and government that Foucault-inspired studies have favoured in 
order to analyse power and politics without being fooled by reified forms of bureaucracy. Scholars like James Ferguson (1994), James C. Scott (1998) and Miller and Rose (2008) provided examples of work on governing techniques or instruments with major effects on individuals. Through bureaucratisation, professionalisation or scientificisation, power becomes inextricably linked to the authority of expertise. The third operationalisation focuses on the routine practices of bureaucracies. Papers, written documents and all sorts of artefacts (Riles, 2006; Feldman, 2008; Gupta, 2012; Hull, 2012; Weller, 2018) as well as bureaucratic procedures are seen as the clue to understanding the micro-politics of bureaucratic work and exploring how bureaucracies operate in people's daily lives. The fundamental insight of this literature is that documents are not simply instruments of bureaucratic organisations, but rather are constitutive of bureaucratic rules, ideologies, knowledge, practices and subjectivities. A central theme in this literature is materiality.

\section{Seeing public bureaucracies like a sociologist: four points of view}

These four lines of inquiry remind us of the richness of traditions of sociological research on public bureaucracies. They show how diverse sociology may be in its ways of understanding and explaining how public bureaucracies develop and work, but also reflect the distinction in epistemologies of knowledge. Moreover, they reveal the great variety of empirical objects and angles, what we can describe as the diverse sociological ways of seeing public bureaucracies. Indeed, beyond authors and traditions, viewing bureaucracies like a sociologist results in specific ways of finding meaning in the observed bureaucratic life. The sociological eye leads to particular framing of research questions and interests as well as to value-specific issues sociologists deem central to address. In table 2 and this second section we would like briefly to present what we believe to be the main snapshots of the sociological eye. 
Table 2 Seeing public bureaucracies like a sociologist: four glances

\begin{tabular}{|c|c|c|}
\hline Approaches & Analytical Focus & Empirical focus \\
\hline $\begin{array}{l}\text { Studying people and } \\
\text { individuals }\end{array}$ & $\begin{array}{l}\text { Social, educational, gender, } \\
\text { ethnic background matter. }\end{array}$ & $\begin{array}{l}\text { Social trajectories } \\
\text { Dispositional perspectives } \\
\text { Representative bureaucracy } \\
\text { Discrimination } \\
\text { Gender perspective }\end{array}$ \\
\hline $\begin{array}{l}\text { Exploring orderings } \\
\text { other than political }\end{array}$ & $\begin{array}{l}\text { Beyond politicians vs top } \\
\text { bureaucrats } \\
\text { Professsional, hierarchical, } \\
\text { power-based and social dynam- } \\
\text { ics matter within bureaucracies }\end{array}$ & $\begin{array}{l}\text { Professions vs managers } \\
\text { Street-level bureaucracies and } \\
\text { their discretionary power } \\
\text { Ordinary encounters between } \\
\text { citizens and bureaucrats }\end{array}$ \\
\hline $\begin{array}{l}\text { A world of } \\
\text { instruments and } \\
\text { organisations in } \\
\text { context }\end{array}$ & $\begin{array}{l}\text { Critiques of top-down and } \\
\text { reified views of bureaucracies } \\
\text { Social and historical construc- } \\
\text { tions of instruments and } \\
\text { bureaucratic organisations } \\
\text { valuing contexts, uses and } \\
\text { practices }\end{array}$ & $\begin{array}{l}\text { Instruments in bureaucracies } \\
\text { and in contexts (records, statis- } \\
\text { tics, performance management, } \\
\text { rankings, market-based, etc.) } \\
\text { Organisational restructuring } \\
\text { and redesign of bureaucracies } \\
\text { "Boundary work" }\end{array}$ \\
\hline $\begin{array}{l}\text { Bureaucracies as } \\
\text { institutions }\end{array}$ & $\begin{array}{l}\text { Critique of efficiency } \rightarrow \\
\text { appropriateness } \\
\text { Diffusion of rationalised myths } \\
\text { and standards } \\
\text { (HNI). Power struggle between } \\
\text { competing actors and compet- } \\
\text { ing institutional logics } \\
\text { Institutional changes in } \\
\text { bureaucracies }\end{array}$ & $\begin{array}{l}\text { (SNI) Diffusion of organisational } \\
\text { formats (mergers, agency) } \\
\text { Institutional entrepreneurs in } \\
\text { organisational reforms } \\
\text { (HNI) Trajectories of institu- } \\
\text { tional changes in bureaucracies } \\
\text { The many actors of administra- } \\
\text { tive reform processes }\end{array}$ \\
\hline
\end{tabular}

\subsection{Bureaucracies are made of people}

Let us put it bluntly. A sociological eye pays central attention to the fact that bureaucracies are made of people, with social properties and trajectories having experienced complex socialisation processes. Their dispositions and diverse characteristics matter greatly in understanding how bureaucracies act and work. More generally, sociology expands the field of view of bureaucracies by exploring what happens in social settings (social trajectories, social groups, society) other than the classic formal institutions. From this follows a wide range of arguments and approaches. The dispositional perspective - analysing the "social body" of civil servants and their dispositions resulting from their social trajectories (Dubois, 2010) - is as important as the institutional perspective (March \& Olsen, 1989), considering how administrative institutions are 
socialising machines shaping how bureaucrats see themselves and imposing appropriateness. The dispositional approach has strong interest in the importance of social trajectories, but also in the gender identifications and sexual orientations of public agents. Within it, public professionals are seen as individuals under tensions between their various social embeddedness and their organisational belongings. Studies may emphasise the issue of gender, exploring the specific processes of feminisation of certain professional groups in bureaucracies, but they may also analyse the structuring biases of career management practices in the making of gender inequalities, particularly strong in the management of elites with the presence of "glass ceilings" and their discriminating effects (Marry et al., 2017). The questions raised by the "representative bureaucracy" literature are also in line with this approach. Changes in the representativeness of bureaucrats (whatever the dimensions, whether social, gender, ethnic, etc.) are likely to affect their behaviours and public policies. Moreover, this sociological eye also sees bureaucracies as open systems exposed to diverse societal demands such as mobilisations for equal opportunities or demands for a better representativeness of bureaucracies under different criteria.

\subsection{Exploring orderings other than political}

A second major sociological input rejects the dominant argument of political science that pays exclusive attention to the political ordering of public administrations with the idea that the government of bureaucracies relies only on political actors (politicians, political leaders, top bureaucrats). By contrast, the sociological eye looks closely into bureaucracy and identifies other forms of balance based on interactions, conflicts or zones of power, distinct from the mere dialogue between politicians and bureaucrats. This is why sociologists in various policy fields systematically explore the role played by professional groups within the state like police and justice agents, public bureaucrats working on hospital and healthcare policies, social workers, teachers, etc. Here, changes in public bureaucracies are linked to long-term evolutions within the occupational structures of these groups, like social and demographic transformations in recruitment and hierarchies, changes in the expertise they possess, in their collegial mode of organising or in the forms of control other actors exert on them. This is why so many scholars in sociology have addressed the issue of the effects of NPM reforms on these professional groups and the extent to which they alter their autonomy, identity and professional models (for a global view see Bezes, Demazière et al., 2012). From a sociological point of view, the ordering mechanism within bureaucracies relies 
much more on the power struggles between professions and managers than on relations to politicians. Indeed, the enduring effect of NPM reforms has been demonstrated by a redistribution of power among professional groups inside bureaucracies and through the institutionalisation of new functions and the social stratum of "managers" and management professionals (controllers, auditors, project or HRM managers, etc.). A possible new "class of managers" has been said to emerge, in charge of making and endorsing the strategy of the public organisations against "policy" professional groups (like doctors, teachers, social workers, etc.) and based on the use of control instruments. This generates the redefinition of stratifications and hierarchies and brings about new modes of regulation inside public bureaucracies.

Similarly, the street-level literature put its emphasis on micro-sociological equilibrium at the ground level, emerging in the face-to-face encounters between front-line public servants and users or entrenched in the discretionary power and autonomy of street-level bureaucrats.

\subsection{A world of instruments and organisations in contexts and in practices}

The third conventional glance from sociology is a critique of the assumption that takes bureaucracies as given, fixed and coherent entities, for instance easily characterised by supposedly well-designed national administration traditions. Instead, it shifts the focus from these idealised and rational views of public bureaucracies towards paying attention to the multiple mix of instruments and organisations that actually make their ordinary life. It seeks to understand how bureaucracies come into being, are produced through the uses of instruments and differentiated as organisations in contexts and particular settings, and what effects these constructions produce.

On the one hand, material devices in bureaucracies turn out to be essential for understanding how knowledge expertise is produced and related to power, thus constituting a crucial part for governing populations, territories and organisations and forming the inextricable savoir/pouvoir pairing in Michel Foucault's works. The diversity of instruments in bureaucracies is endless from the most classic forms (records, application and registration forms of all kind, maps, categories, rates, thresholds, etc.) to the governing technologies of the neo-managerial and neoliberal world with the variety of steering techniques (new accounting systems, benchmarking, evaluation, targets, indicators, rankings, contracts, etc.). While all these technologies have technical dimensions and relied on various forms of expertise at the 
heart of bureaucracies, they are also highly political and render effective the exertion of power and governing as well as having important moral and symbolic dimensions over people and groups. The focus on instruments is eminently sociological (Lascoumes \& Le Galès, 2007) and offers a very good marker of the "real" functioning of public bureaucracies beyond tradition or reform discourses and rhetoric. The sociological approach argues that understanding the true legacy of instruments, whatever their types, requires understanding the institutional, context-based and locally tailored roots of their entrenchment in national contexts and paying attention to real practices and uses. Beyond the presence or absence of an instrument, local institutional logics and contexts are crucial to explain the performativity of a tool because the interlinking between instruments, institutions and contexts, as well as the contact with other possible contradictory logics, can attenuate, hybridise or transform the effects of an instrument and its legacy and persistence.

On the other hand, scholars in sociology also deconstruct bureaucracies by breaking them into a multiple set of organisations (ministries/departments, agencies) and analyse in different contexts how individual and collective actors create, transform, redesign but also reproduce bureaucracies through repeated programmes of reorganisations.

First, the sociological eye, strongly influenced by organisation theory, puts emphasis on the main issue at stake in manipulating the administrative machine: acting upon the "division of labour" e.g. changing the distribution of tasks, roles and responsibilities within public organisations (Bezes \& Le Lidec, 2016). As argued by sociologists Durkheim and Weber, both of whom insisted upon the importance of stable and regular principles of specialisation in the making of bureaucracies, the division of labour constitutes a structuring dimension within the organisation of the state and of power. The act of dividing, differentiating, specialising or, on the contrary, integrating, merging, or uniting, constitute an instrument for the redistribution of power, functions and hierarchies, one that is central to the transformation of forms of state government. Second, the empirical focus for the sociological glance is placed on the so-called "boundary work," the delineation of organisational boundaries, in connection with another core activity within bureaucracies: jurisdictional struggles or "bureaucratic politics" as coined by Allison and Halperin (1972). The interest is in the process through which boundaries of public organisations are put under critique, de-legitimated and renegotiated, but the interest is also in uncovering the power gamesmanships and the conflicts at stake in these politics of reorganisation. Restructuring transforms the hierarchies and the distribution of power, both vertically (by creating 
additional hierarchical tiers, autonomous agencies or even new levels of administration in the case of decentralisation) and horizontally (by adding new organisational units at the same level).

In these processes, thirdly, actors are a crucial focus for sociologists. All theoretical thought on organisational and institutional change is confronted with what is often presented as a paradox: how and why, within dense and constrained institutional fields, do actors who are embedded in organisational structures and socialised into their routines find the motivation, resources, and capacities to take reorganisation forward? This raises questions regarding agency and entrepreneurs of change, with the intention of understanding through what resources, skills, positions and/or capacities these reformers manage to free themselves of the existing institutional order and become able to imagine alternative designs (Battilana, Leca \& Boxenbaum, 2009). The first task is therefore to understand which properties (positions, dispositions) favour their emergence with diverging arguments in the literature: are these actors above all "outsiders," on the margins of an institution and therefore all the more capable of freeing themselves from it; or, on the contrary, are they "insiders," actors who are central to the institution, and endowed with sufficient legitimacy to be able to propose an unprecedented vision of reform? For example, sociologist Neil Fligstein (2001) insists on the importance of these entrepreneurs' social skills to generate and to diffuse new ideas that will redefine problems and solutions, to take in hand the "demand for change," and to develop "reform narratives" capable of eliciting cooperation with and engendering conviction about reform. This raises the fundamental question of how public bureaucracies relate to their institutional environments.

Indeed, fourth, the sociological approach also pays attention to the fact that these reorganisations (either by agencification or by mergers) take place in particularly dense institutional fields, which they throw into turmoil. In rearranging ministerial departments and agencies, reforms call into question the institutional logics and cultures at work within bureaucracies - that is to say, the longstanding and well-anchored categories, meanings, practices and skills that have always been constitutive of them. Of course, public bureaucracies are not made in one piece and one may keep in mind that several institutional logics may be present, thus generating ambiguity and contradictions in organisations. Moreover, a complementary sociological instinct ought to pay attention not only to institutional entrepreneurs but also to the ways reorganisations construct, revise or reproduce the identities, narratives and values of public bureaucracies and their agents. 


\subsection{Bureaucracies as institutions}

The last sociological view emphasises bureaucracies as institutions, which may have several meanings. Let us stick to two of them. Both are rooted in the critique of the efficacy or efficiency of bureaucratic rules, a very often used and implicit assumption in many works in public administration or public management. This rational view of bureaucratisation considers that rules, instruments and formal structures are adopted in public bureaucracies because they are efficient devices. Bureaucratic rules are then very often seen as responses of politicians to the need of the state or the society in order to maintain stability, equilibrium or survival. The sociological perspective still focuses on rules, but does so by reorienting the argument in two distinct ways, corresponding to sociological neo-institutionalism and to historical sociology, echoed in the historical neo-institutionalism.

Within the sociological neo-institutionalist perspective (Powell, DiMaggio, Meyer, March, Olsen), bureaucratic rules and formal structures are regarded as "rationalised myths" that establish procedures, understandings and meanings and constitute collective beliefs, thereby creating legitimacy (Olsen, 2006). These norms of rationality are the causal vehicles of bureaucratisation (Meyer \& Rowan, 1977). They are proliferating globally, not because they are efficient but because they are legitimate. Within this approach, research has to refocus on analysing the creation and diffusion of institutional myths in organisational fields (DiMaggio \& Powell, 1983) since organisations are assumed to become more alike in their structural features due to the strength of isomorphic pressures developed in their field and in their environments. Tolbert and Zucker (1983), for instance, examined how many cities adopted civil service reforms from 1880 to 1930 in response to increasing normative pressures, while more recent scholars explore the institutionalisation of management knowledge, managerial instruments or organisational forms like agency or merger, that, once legitimated, become subject to processes of circulation and diffusion. Such work sees public organisations as being embedded in institutionalised fields composed of organisations of the same nature, structured by legitimate rules, norms and templates. Members of these organisations enter into contact with a great many equivalent institutions in the context of transnational or international associations and organisations, where norms and standards are enacted that exert major normative influences and mechanisms of homogenisation - what authors such as Paul DiMaggio and Walter W. Powell describe as "isomorphisms." Organisations tend to adopt the same organisational forms because they correspond to the expectations of conformity within the field, and, in turn, tend to reinforce their legitimacy. 
Understanding bureaucracies then requires paying attention to the "logic of appropriateness" (March \& Olsen, 1989) inside the organisation but also in institutional environments. In addition to isomorphism, this view results in another important concept and mechanism, the one of decoupling. Bureaucracies as institutions may often decouple their practices from their formal rules or adopted structures (developed to increase organisations' external legitimacy and to abide by international dominant standards). This means that these rules and structures may not result in corresponding practices and relations that are actually in place for effective action, production and coordinating their activities and which are often referred to as the "informal organisation” (Brunsson \& Olsen, 1993, p. 9; Meyer \& Rowan, 1977).

Within the lenses of historical sociology, clearly revitalised in the historical neo-institutionalist perspective, the adoption of NPM-oriented rules and structures could be viewed quite differently. From this socio-historical perspective, only rarely adopted in the literature (for two examples see SaintMartin, 2001; Bezes, 2009, 2017), the movement of repeated administrative reforms that has taken place in all Western countries since the 1960 s should be viewed as a long-term process of transformations of public bureaucracies, an equivalent of the long-term bureaucratisation of the nineteenth and first half of the twentieth centuries. Put differently, the institutionalisation of Weberian bureaucracies took over one and half centuries to stabilise, and took many power struggles of all kinds (Silberman, 1993). There is no reason why we should not adopt a similar long-term perspective to explain i) the gradual institutionalisation of administrative reform as a specific public policy in Western states, and ii) the long-term effects of transformations they produce on the rules and structures of inherited bureaucracies. Indeed, administrative reform policies have become a central item on the governmental reform agenda and the leitmotif of political executives, civil service elites, ministries and crosscutting structures within the state (particularly finance ministries) and experts of all kinds, including the major role of consultancy firms (Bezes, 2009). From the historical sociology perspective I adopted (Bezes, 2009), administrative reforms can be examined as repeated configurations in time where several interdependent groups of actors - experts, executive leaders and senior civil servants from key ministries in competition to lead administrative reforms (finances, civil service, Interior) - interact to gradually renegotiate and transform the main historical inherited institutions that characterised Weberian bureaucracies. Of course, this process cannot be described in monocausal, linear and homogenous terms. Each sequence of reform, in a given country, has its own specific issues and power struggles inside and outside the state. But viewed cumulatively in the longue durée as a trajectory 
unfolding over time, these repeated episodes institutionalise a substantial shift towards a new model of public administration (for instance, see Bezes \& Parrado, 2013). With this eye, more systematic comparative efforts should be made to understand the global picture of how and in what directions the national public bureaucracies have been transformed, of course by keeping in mind the idea that specific national institutional arrangements may influence differently the content and results of administrative reforms.

\section{Why bureaucracies should still be watched with sociological eyes in the future}

Western public administrations have been confronted with a massive number of transformations since the 1960 os with a sharp intensification in the last four decades. To conclude this chapter, we would like to emphasise the many reasons why sociological perspectives should be revitalised and still operationalised to understand the various changes affecting public bureaucracies. Again, we provide a comprehensive table (table 3 ) crossing the salient issues at stake in the contemporary mutations of bureaucracies with the benefits some specific research traditions in sociology would bring.

\section{Table 3 Challenging issues for the sociological eyes}

\section{New Challenges in Public Bureaucracies Sociological Likely Contributions}

Civil service reforms (limiting or dismantling institutional protection) and their transformative effects on public agents

Blurring of boundaries between the public and private spheres

A new world of instruments: performance management, marketisation and e-government
Who are the civil servants and public agents nowadays? What can we say about their social trajectories, dispositions, motivations, beliefs, role commitment?

What are the new social class conflicts at the heart of the transformations of civil services? What are the new interactions between bureaucracies and educational systems?

Explore the hybridisation as well as the process of tracing new boundaries and their effects.

Critique of taken-for-granted efficacy and efficiency

Explore the practices and uses of instruments in contexts and their effects

New material dimensions of bureaucracies Study the renewed technologised forms of street-level encounters between citizens and bureaucrats 
Let us first raise the fact that we know too little about the civil service dimension of public bureaucracies and the changes that have constantly affected it. Ultimately, outside the numerous studies on specific professions (police, teachers, health), there is a lack of research about the identities, social trajectories and beliefs of the "new" civil servants. These are fascinating issues where the contribution of sociology is decisive because they deal with the many ways in which social and educational dynamics, as well as dispositions, shape the main structures of public bureaucracies. How do interests and beliefs in joining and having careers in public administrations vary by class, gender, ethnicity or education? We now know too little about the reasons that lead individuals to become public servants today, their motivations and expectations, but also their social background and their perceptions of being recruited into the civil service in terms of social mobility. These are particularly important issues as the context is marked by several phenomena that affect the perceptions individuals have of how attractive a career and/or employment in public administrations might be. On one hand, deep social and economic dynamics have affected the training and employment systems. There has been a democratisation of higher education with sharp increases in the number of diplomas but also many joint dynamics likely to transform the conditions under which individuals perceive their entry into and careers within public administrations: increases in levels of qualifications but simultaneous (relative) decreases in the value attached to diplomas, degradation of the labour market, transformations in higher education, the collective defence of equal rights between men and women, etc. On the other hand, civil service systems have been considerably transformed under the effect of more or less visible dynamics. There have been processes of flexibilisation of the public services that have partially or more radically challenged (and even dismantled) strong inherited institutional protections (status, career guarantees, selection by competitive examination and promotion to seniority) (for overviews see Bach\& Bordogna, 2011; Meer, Raadschelders \& Toonen, 2015). Simultaneously, several changes are likely to transform the stratification of hierarchies, positions and jobs within public bureaucracies: a massive increase in skilled tasks associated with a rise in managers, a gradual opening up to outsiders of the administrative public labour market, the increasing percentage of non-permanent staff (contractual, temporary workers etc.), a diversification of public organisations from departments to agencies, public enterprises or third sector actors, etc. How do all these changes affect the life of agents within public administrations? Making sense of contemporary public bureaucracies requires us to examine institutions and belief systems that shape how individuals in bureaucracies view themselves and their roles 
as state agents. All these issues raise fundamental sociological questions with considerable effects on the very nature of the civil service system and its agents. They engage large, crucial and long-term historical dynamics where social classes, social stratification, elites, educational systems and bureaucracies interact, thus making the lessons of historical sociology even more vital.

A second massive transformation for public bureaucracies is the so-called "blurring" of boundaries between the public and the private spheres and the institutionalisation of hybrid and highly fragmented systems with "many hands" of diverse sorts, mixing central administrations, agencies, private or non-profit actors. This "state of many hands," as nicely coined by Kimberly J. Morgan and Ann Shola Orloff (2017), largely results from the delegation of responsibility for public programmes to non-state actors (private firms, non-profit-making organisations) or to remote organisations such as agencies. The boundaries between jurisdictions then become more open, since states subsidise private organisations to deliver public policies and public organisations collaborate more with many other organisations of diverse status, generating what some have called "network governance" (Rhodes, 1997), "delegated governance" (Morgan \& Campbell, 2011), "new public governance" (Osborne, 2010) or "open government" (Mulgan, 2014). Whatever the label, these transformations raise fundamental questions where sociology may contribute significantly. Its epistemological approach does not take for granted the distinction between the state, bureaucracies and society. At the opposite, it explores the relationships, interactions and flow of resources and power between these entities, the displacements along the lines of the public-private divide (and also the public and the non-profit-making) but also the processes of boundary making between these different organisations and the way their jurisdictions and identities are reconstructed. Here we see the interest sociologists have in not taking for granted the reified organisations but in analysing both the struggles around jurisdictions and the operations through which new, more or less porous boundaries are redesigned. Of course, these profound changes also challenge administrative capacity by eroding the power of bureaucracies, the monopoly of their expertise and their modes of control. They raised problems of steering and control as well as accountability and responsibility issues in all Western countries, although with national variations. But sociologists favour the thick description and understanding of what Elisabeth S. Clemens (2017) calls the "symbiotic state" e.g. the systematic interactions between domains of activity organised around different principles but now contributing to each other. All these issues nicely fit a sociological agenda where the study of bureaucracies is eminently "relational" and where the issue of autonomy remain critical. 
The third big transformation that suggests how important sociological approaches remain is related to the extraordinary explosion of instruments of all sorts within bureaucracies, under the pressures of performance management, marketisation of administrative activities and e-government. The contribution of the sociological approach and its trade mark is rooted in the close analysis of the social uses and practices of instruments as well as in the exploration of their effects. Let us take performance management as an example. Many empirical studies on the development of performance indicators systems (PIS) have identified new forms of bureaucratisation with parallel power relations or "vicious bureaucratic circles," echoing those identified by Blau, Gouldner and Crozier. Sociologist Evelyn Z. Brodkin (2011), for instance, uses Merton's concept of "goal displacement" and Lipsky's Street-Level Bureaucracy (1980) "coping mechanisms" to analyse the behaviours of US welfare street-level bureaucrats who develop diverse adaptive strategies to NPM techniques (achieving participation rates, caseload reduction target, etc). Hood (2011) and Moynihan (2008) insist on the need to explore the contrasting uses of PIS according to different organisational levels and positions (politicians and top officeholders, street-level bureaucrats, middle managers, actors outside state structures). A clear echo of the founders of the sociology of bureaucracy is the idea that PIS are not actually governed according to the formal rules of performance management theories or the expected effects of instruments, but much more by other parallel and "latent" structuring relationships. New classics in the field resort to a parallel explanatory system of relationships to understand the "real games" beneath targets and indicators. In Moynihan's The Dynamic of performance management (2008), the concept of ambiguity in organisational life is at the centre of the many social constructions and interrelations generated by PIS. In Hood's The Blame Game (2011), vicious circles and negative side effects produced by PIS are manifold and based on the explosion of "blame avoidance" strategies, understood as "a process of interaction among the players in these different worlds, as they combine or conflict and seek to pass the blame onto those in other worlds" (Hood, 2011, p. 22). These perspectives result again in the idea of decoupling: PIS are often said to be loosely coupled to the effective steering of public organisations and often phenomena of "dissociation" (which consists for professional groups in adopting PIS formally but without allowing them to affect their actual work (Dent et al., 2004)).

Similarly, the marketisation of public bureaucracies, extending "the rationality of the market to domains previously viewed as non-market and non-economic" (Kurunmäki et al., 2016, p. 397), is embodied in the worldwide diffusion of a long list of instruments like public-private partnerships, 
contracting out, competition through internal markets, the separation between purchaser and provider, user/customer choice, vouchers, individual incentivisation, etc. The exploration of these tools is clearly part of a research agenda in sociology that requires understanding the institutional, contextbased and locally tailored roots of their entrenchment in national and local contexts as well as paying attention to real practices. Along the same lines, the strong technology-driven dimension of the multi-scale development of e-government invites us to keep the sociological eye even more open. All the very distinct e-technologies (big data, open data, tracing and tracking tools, biometrical instruments, websites, platforms, etc.) carry with them strong and taken-for-granted assumptions about their efficacy, their efficiency and their capacity to solve all problems. Here, the lessons drawn from the branch of sociology studying science and technology (one field is labelled STS) are crucial ideas to keep in mind, since they do not stop re-inscribing technologies and knowledge in the web of social and political interactions they constitute. Like all tools, e-technologies must be studied by taking into consideration their political and social dynamics (power relationships, the ideas they carry, socio-technical controversies, policy effects, etc.) like any other social object as well as researching practices where knowledge of e-technologies is produced, used and diffused. Moreover, since sociology also has a great interest in investigating the relationship between state bureaucracies and populations whatever their conceptualisations (interactions and/ or domination), e-government revitalises and makes even more urgent the sociological view on the new technologised encounters at the front line.

\section{Conclusion}

It is beyond the objective, scope and reach of this chapter to explain historically the relationships between sociology and public administration both as an object of study (what the contribution of sociology to studying public bureaucracies has been) and as a discipline and a field (to what extent and in which national context sociology has or has not influenced Public Administration as a field). There are huge variations across countries regarding the place of Public Administration as an institutionalised academic field (sometimes central, sometimes simply absent) and the influence sociology has played in its development. Our general assumption is that the disconnection between Public Administration and sociology has been real and often strong, so that scholars in Public Administration do not very much look at and cite the articles and books still studying bureaucracies within the field of sociology. 
Yet, "bureaucracies" remain, directly or indirectly a much-studied object from various sub-disciplines in sociology (organisational theory, sociology of occupations, sociology of work/labour, elite theory, fiscal sociology, etc.) but not a proper sub-discipline in sociology. By using tables and playing with the ocular metaphor we have presented several angles of sight through which public bureaucracies have been investigated by sociologists in the past and we have defended their usefulness for the present and the future of Public Administration. Thus, this chapter can be read as a sketchmap reminding us of the value of sociological views but also as a plea for a reconnection.

\section{References}

Adams, J., Clemens, E. S., \& Orloff, A. S. (eds). (2005). Remaking Modernity: Politics, History, and Sociology. Durham, NC: Duke University Press.

Allison, G.T., \& Halperin, M.H. (1972). Bureaucratic Politics. A Paradigm and Some Policy Implications. World Politics, 24 (S1): 40-79.

Bach S., \& Bordogna, L. (2011). Varieties of New Public Management or Alternative Models? The Reform of Public Service Employment Relations in Industrialized Democracies. International Journal of Human Resource Management 22(11): 2281-2294

Battilana, J., Leca, B., \& Boxenbaum, E. (2009). Agency and Institutions: A Review of Institutional Entrepreneurship. Academy of Management Annals 3 (1): 65-107

Bendix, R. (1980). Kings or People: Power and the Mandate to Rule. Berkeley: University of California Press.

Bezes, P. (2009). Réinventer l'État. Les réformes de l'administration française (1962-2008), Paris, Presses universitaires de France.

Bezes, P. (2017). The Neo-Managerial Turn of Bureaucratic States: More Steering, More Devolution. In D. King \& P. Le Galès (eds). Reconfiguring European States in Crisis (pp 251-278). Oxford: Oxford University Press.

Bezes, P., \& Demazière, D. (eds). (2012). Introduction to Debate. New Public Management and Professionals in the Public Sector: What new Patterns beyond Opposition? Sociologie $d u$ Travail in English 54(S1): 1-52

Bezes, P., \& Le Lidec, P. (2016). The Politics of Organization. The New Divisions of Labor in State Bureaucracies. Revue française de science politique in English 66 (3): 407-433.

Bezes, P., \& Parrado, S. (2013). Trajectories of Administrative Reform: Institutions, Timing and Choices in France and Spain. West European Politics 36(1): 22-50.

Blau, P. M. (1955). The Dynamics of Bureaucracy: A Study of Interpersonal Relationship in Two Governmental Agencies, Chicago: Chicago University Press.

Bourdieu, P. (1994). Rethinking the State: Genesis and Structure of the Bureaucratic Field. Sociological Theory 12(1): 1-18. 
Bourdieu, P. (2014). On the State. Cambridge: Polity.

Brehm, J., \& Gates, S. (1997). Working, Shirking and Sabotage. Bureaucratic response to a Democratic Public. Ann Arbor, MI: The University of Michigan Press

Brodkin, E. Z. (2011). Policy Work: Street-Level Organizations Under New Managerialism, Journal of Public Administration Research and Theory 21 (S2): i253-i277

Brunsson, N., \& Olsen J. P. (1993). The Reforming Organization: Making Sense of Administrative Change. London: Routledge.

Clemens, E. S. (2017). Reconciling Equal Treatment with Respect for Individuality: Associations in the Symbiotic State. In K. J. Morgan, \& A. S. Orloff (eds). The Many Hands of the State: Theorizing Political Authority and Social Control (pp. 35-57). Cambridge: Cambridge University Press.

Crozier, M. (1964). The Bureaucratic Phenomenon. Chicago: Chicago University Press.

Dent, M., Chandler, J., \& Barry, J. (2004). Questioning the New Public Management. Ashgate: Aldershot.

DiMaggio, P.J., \& Powell, W. W. (1983). The Iron Cage Revisited: Institutionalized Isomorphism and Collective Rationality in Organizational Fields. American Sociological Review 48: 147-160.

Dubois, V. (2010). The Bureaucrat and the Poor: Encounters in French Welfare Offices. Burlington, VT: Ashgate (1st ed. in French, (1999) La vie au guichet. Paris: Economica).

Eisenstadt, S. N., \& Rokkan, S. (1973). Building States and Nations, Beverly Hills, CA: Sage.

Eymeri-Douzans, J.-M., \& Bouckaert, G. (eds) (2013). La France et ses administrations. Un état des savoirs. Bruxelles: Bruylant.

Feldman, I. (2007). Governing Gaza. Bureaucracy, Authority, and the Work of Rule, 1917-1967. Durham: Duke University Press.

Ferguson, J. (1994). The Anti-politics Machine:"Development", Depoliticization, and Bureaucratic Power in Lesotho. Minneapolis: University of Minnesota Press.

Fligstein, N. (2001). Social Skill and the Theory of Fields. Sociological Theory 19(2): 105-125

Foucault, M. (2009). Security, Territory, Population. Lectures at the Collège de France (1977-1978), New York: Palgrave McMillan.

Foucault, M. (2008). The Birth of Biopolitics: Lectures at the Collège de France, 1978-1979. New York: Palgrave McMillan.

Goffman, E. (1978). The Presentation of Self in Everyday Life. London: Harmondsworth.

Gouldner, A. W. (1954). Patterns of Industrial Bureaucracy. A Case Study of Modern Factory Administration. New York: The Free Press.

Gupta, A. (2012). Red Tape: Bureaucracy, Structural Violence, and Poverty in India. Durham: Duke University Press.

Herzfeld, M. (1993). The Social Production of Indifference. Chicago: University of Chicago Press. Hibou, B. (2015). The Bureaucratization of the World in the Neoliberal Era. London: Palgrave McMillan.

Hood C. (2011). The Blame Game. Spin, Bureaucracy and Self-Preservation in Government. Oxford: Princeton University Press. 
Hull, M. S. (2012). Government of Paper: The Materiality of Bureaucracy in Urban Pakistan. Berkeley: University of California Press.

Krause G. A., \& Meier K. (Eds). (2003). Politics, Policy and Organizations. Frontiers in the Scientific Study of Bureaucracy, Ann Arbor, The University of Michigan Press.

Kurunmäki, L., Mennicken, A., \& Miller P. (2016). Quantifying, Economising, and Marketising: Democratising the Social Sphere? Sociologie du Travail 58(4): 390-402.

Lascoumes, P. \& Le Galès, P. (2007). Understanding Public Policy through its Instruments: from the Nature of Instruments to the Sociology of Public Policy Instrumentation. Governance 20 (1): 1-21.

Lipsky, M. (1980). Street-level Bureaucracy. Dilemmas of the Individual in Public Services. New York: Russell Sage Foundation.

Mann, M. (2012). The Sources of Social Power: Volume 2. The Rise of Classes and Nation-states, 1760-1914. Cambridge: Cambridge University Press.

March, J. G., \& Simon, H. A. (1958), Organizations. New York: Wiley.

March, J G., \& Olsen J. P. (1989). Rediscovering Institutions: the Organizational Basis of Politics, New York: The Free Press.

Marry, C., Bereni, L., Jacquemart, A., Pochic, S., \& Revillard, A. (2017). Le plafond de verre et l'état. Paris: Armand Colin.

Maynard-Moody, S., \& Musheno M. (2003). Cops, Teachers, Counselors: Stories from the Front Lines of Public Service - Narratives of Street-level Judgement. Ann Arbor, MI: University of Michigan Press.

Merton, R. K. (1940). Bureaucratic structure and Personality. Social Forces 17: 560-68. (Reprinted in Robert K. Merton et al., (1952). Reader in Bureaucracy, Glencoe, IL: Free Press).

Meyer, J. W., \& Rowan, B. (1977). Institutionalized Organizations: Formal Structure as Myth and Ceremony. American Journal of Sociology 83(2): 340-363.

Miller, P., \& Rose, N. (2008). Governing the Present. Administering Economic, Social and Personal Life. Cambridge: Polity.

Moore, B. (1966). Social Origins of Dictatorship and Democracy. Lord and Peasant in the Making of the Modern World. Boston, Mass.: Beacon Press.

Morgan K. J., \& Campbell A. L. (2011). The Delegated Welfare State: Medicare, Markets, and the Governance of Social Policy. New York: Oxford University Press.

Morgan, K. J., \& Orloff, A. S. (Eds). (2017). The Many Hands of the State: Theorizing Political Authority and Social Control. Cambridge: Cambridge University Press.

Moynihan, D. P. (2008). The Dynamics of Performance Management: Constructing Information and Reform, Wahsington D.C.: Georgetown University Press.

Mulgan R. (2014). Making Open Government Work. Basingstoke: Palgrave Macmillan.

Oberfield, Z. W. (2014). Becoming Bureaucrats: Socialization at the Front Lines of Government Service. Philadelphia PA: University of Pennsylvania Press.

Olsen, J. P. (2006). Maybe It Is Time to Rediscover Bureaucracy. Journal of Public Administration Research and Theory 16(1): 1-24. 
Osborne, S. P. (2010). The New Public Governance. Emerging Perspectives on the Theory and Practice of Public Governance. London: Routledge.

Page, E. C. (2005). Policy Bureaucracy. Government with a Cast of Thousands, Oxford: Oxford University Press.

Page, E. C. (2012). Policy without Politicians: Bureaucratic Influence in Comparative Perspective, Oxford: Oxford University Press.

Rhodes R. A. W. (1997). Understanding Governance. Buckingham: Open University Press.

Riles, A. (2006). Documents: Artifacts of Modern Knowledge. Ann Arbour, MI: University of Michigan Press.

Saint-Martin, D. (2001). Building the New Managerialist State. Consultants and the Politics of Public Sector Reform in Comparative Perspective. Oxford: Oxford University Press.

Scott, J. C. (1998). Seeing Like a State: How Certain Schemes to Improve the Human Condition Have Failed. Yale: Yale University Press.

Selznick, P. (1949). TVA and the Grass Roots. Berkeley: University of California Press.

Sharma, A., \& Akhil G. (eds). (2009). The Anthropology of the State: a Reader. Malden, Mass., Blackwell.

Shefter, M. (1994). Political Parties and the State: The American Historical Experience. Princeton, Princeton University Press.

Silberman, B. S. (1993). Cages of Reason The Rise of the Rational State in France, Japan, The United States and Great-Britain, Chicago: Chicago University Press.

Siblot, Y. (2006). Faire valoir ses droits au quotidien, Les services publics dans les quartiers populaires. Paris: Presses de Sciences Po.

Simon, H. A. (1947). Administrative Behavior. A Study of Decision-Making Processes in Administrative Organizations. New York: The Free Press.

Skocpol, T. (1985). Bringing the State Back in. Strategies of Analysis in Current Research. In P. B.

Evans et al. (eds). Bringing the State Back in (pp. 3-43). Cambridge: Cambridge University Press. Smith, D. (1991). The Rise of Historical Sociology. Cambridge: Polity Press.

Spire, A. (2005). Étrangers à la carte. L'administration de l'immigration en France (1945-1975). Paris: Grasset.

Tilly, C. (1990). Coercion, Capital and European States, AD 990-1990. Oxford: Blackwell.

Tolbert, P. S., \& Zucker, L. G. (1983). Institutional Sources of Change in the Formal Structure of Organizations: The Diffusion of Civil Service Reform, 1880-1935. Administrative Science Quarterly 28: 22-39.

van der Meer, F. M., Raadschelders, J., \& Toonen, T., (Eds). (2015). Comparative Civil Service Systems in the 21st century. Basingstoke: Palgrave McMillan.

Watkins-Hayes, C. (2009). The New Welfare Bureaucrats. Entanglements of Race, Class and Policy Reform. Chicago: University Of Chicago Press.

Weller, J.-M. (2018). Fabriquer des actes d'État. Une ethnographie du travail bureaucratique. Paris: Economica. 

PUBLIC ADMINISTRATION AND CULTURES 



\title{
1
}

\section{Challenges in the Face of Diversities: Public Administration in Spain as an Example}

\author{
Salvador Parrado
}

The cultural context mediates between Public Administration (as a topic of study and academic approach) and the changing practices of public administration. This context is continuously evolving with features that are entrenched in the past, but also with more recent developments and shifts that change the course of this evolution. This chapter follows these interdependencies. It outlines the recent evolution of the Spanish state and society choosing relevant features for the study of public administration, and it connects the disciplinary work with the changing Spanish culture.

The study of Spanish public administration has been the subject of several reviews (Ballart, 2008; Subirats, 1999; Parrado, 1999; Baena, 1999). All of them focus on the most popular topics analysed by Public Administration (PA) scholars and on the evolution of the discipline. However, these works pay limited attention to the context and relevant cultural features in which Public Administration, as an academic branch of social science, is practised. A contextual view of Public Administration is offered by Ongaro (2010), Kickert (2007) and Parrado (2008) when assigning (or disputing) the label of the Napoleonic tradition to the Spanish state. This tradition, constituted of cultural and institutional elements, offers expectations as to how public sector organisations and their agents are likely to behave.

The aftermath of the Second World War witnessed the flourishing of democracy in many European countries. Spain, however, endured the dictatorship of Franco with an initial period of isolation and autarchy and, unlike other European countries, limited development of the Welfare State. During Franco's time, the state's agents and institutions displayed the core tenets of the Napoleonic state tradition. Furthermore, society was highly homogeneous in terms of language, ethnic origin and religion. The only official language was Spanish and other regional languages were banned 
from public life. Any meaningful political differentiation of the regions was suppressed. International immigration hardly existed, either from Latin American countries or from any other regions of the world. Tourism started to penetrate the coastal areas in the 1970s, but still the residence of European or Latin American nationals in Spain was a rarity.

Moreover, Spain has been traditionally Catholic. Catholicism was pervasive in Spain from the early years of Christianity and had a strong impact on society during the Middle Ages and the infamous episodes of the Inquisition. In more recent history, the government of the Second Spanish Republic suspended the 1851 Concordat with Rome to establish a laïc state. Franco recovered confessionalism with the signing of the 1953 Concordat. All these features were challenged after the transition to democracy, with a waning adhesion to religion by Spaniards and an increasing number of believers from other confessions.

The Spanish state and society have been subject to trends that constitute the focus of this chapter: the transition from a centralised state to a multilevel and more pluralistic polity, and the increase of diversities in all the areas mentioned above. In the same period, Spanish PA studies in identifying the new challenges and in becoming internationally competitive were limited in number. The chapter is structured in three sections. The first section assesses the recent transformation of the government and the administration employing the state tradition approach. A second section focuses on the increasing diversity of society and the limited echo that it has triggered in the scholarly PA tradition. The third section centres around the summary of PA work and the low-level competitive internationalisation of Spanish scholars, and offers a glimpse into the future of the field.

\section{One or several state traditions in Spain?}

The state tradition approach is a heuristic instrument that advances a configuration of the state and its relations with society. This tool is useful because a persuasive analysis of causal mechanisms of well-selected cases in one country can be exported to other countries with similar institutional features. Spain has been clustered with the "Napoleonic" tradition. Many of its traits were imported after the first Spanish Constitution of 1812, launched while the Napoleonic troops were gradually being expelled from Iberian lands. Some of these features already existed earlier on and the influence of the French administration only cemented them. The state evolution and its territorial reconfiguration have softened some of the most striking notes 
of this tradition. The question is how many of the so-called Napoleonic characteristics are still in place.

\subsection{State centralisation and the state-society relationship}

State centralisation took a long time to forge. During the unification of the kingdoms of Castilla and Aragón at the end of the sixteenth century and right after reconquering the peninsula from the Arabian domination, an absolutist state started to take shape. The political and administrative autonomy of Castilla and Aragon during the seventeenth century as well as the pluricentre system of corporations (local, regional, mercantilists and religious) that challenged state-level supra-administrative entities were abolished. Furthermore, the synodal system of councils that served the king was subject to hierarchical pressures in the eighteenth century with the introduction of state secretaries and a ministerial bureaucracy.

The 1812 Constitution of Cadiz, inspired by French revolutionary principles, set the roots of administrative law, while the 1834 Constitution inaugurated a more concrete and coherent administrative system according to the principles of the division of power and the hierarchical division of tasks. This Constitution confirmed the position of state secretary, created the council of ministers, granted under-secretaries the management of ministerial horizontal resources and structured departments in directions general. In the territory, each settlement was entitled to form a municipality, leading to more than 8,000 municipalities today. The judiciary also followed a pyramidal hierarchy in the territory with regional and provincial audiences and the Supreme Court at the highest level.

The state played a central role in integrating society through organic links thanks to its centralisation and the uniformity of law implementation in the territory via the provincial governors, similar to the French prefects. Moreover, the organic connection between state and society privileged corporatist interest mediation over pluralistic access to decision-making. Finally, the centrality of the state in its relations with society favoured service delivery by public sector institutions instead of private or non-profit-making organisations.

These features have started to erode in the last 40 years with the gradual devolution of political power to the regional authorities. The regions have their own parliaments and governments. The devolution of functions to politically autonomous regions and the negotiation of the regional constitutions have made the system more contractarian in nature since 2005. Although Spain has not formally become a federation, the system has acquired quasi-federal 
status with regions that enjoy asymmetrical powers. Not all competences (taxation, police forces, services linked to the language amongst others) are distributed evenly across the territory. This profound decentralisation significantly changed the prefectural system of civil governors and the link between the municipalities and the central state, now mediated by regional authorities.

The creation of several centres of power, including the European Union institutions, facilitated the influence of territorial elites and the pluralistic dimension in different policy fields, such as health, farming, fishing, agriculture, to name just a few. In these sectors, several stakeholders started to have access to the decision-making process at different levels of government. Alongside a more pluralistic influence in decision-making and a more diversified territorial structure, the use of short- and long-term contracting out to private firms has been of relevance and the private sector has increased its presence in areas such as health, education, social services and other basic local services. The state is still predominant, but the private and non-governmental sectors have entered with an impetus in the service delivery equation.

In sum, this Napoleonic tradition of (central) state has faded in the last four decades and the Spanish administrative tradition is assuming more of a contractarian and pluralistic nature; it is based on mixed service delivery and has quasi-federal status.

\subsection{Professionalisation, politicisation and the autonomous power of the civil service}

The differentiation of a career as a civil servant from the professional paths of politicians and private sector managers is a trait of the Napoleonic tradition. Public servants constitute a separate "caste" with specific features for entry, promotion and payment. Furthermore, in some countries like France and Spain there is an elitist and differentiated corps system.

Two civil service-related phenomena dominated the nineteenth century: "militarisation" and political control of the civil service. After a period of continuous wars and decolonisation of Spanish domains in Latin America, returning soldiers were given an administrative job, which "militarised" the administration and politics. The century witnessed several military uprisings and a succession of various Constitutions. The military culture was embedded in the administration and, for certain periods, administrative post-holders had to wear a uniform during duty hours (Jiménez-Asensio, 1989).

At the same time, encouraged by alternative political parties in government and military uprisings, the civil service was gradually being politicised through 
a full sweep "spoils system," which lasted until 1918. The bureaucracy was the patrimony of the government. The post, or rather the employment, and not the employee, was the subject of the transaction. The job was the "good" of "contractual exchange." The important role civil servants played in the frequent elections during the alternation between liberals and conservatives from 1875 to 1917 politicised the civil service further. Civil servants voted in groups and decorated their uniforms to make their choice, the governmental choice, more evident.

The 1918 Act culminated in a piecemeal reform process to professionalise the civil service. The reforms in the eighteenth century attempted to uniformise the whole civil service, but they failed due to opposition from the special corps, to whom the general rules did not apply. The 1918 Statute, enacted in response to the civil service demonstrations against the economic hardships of the time, introduced merit-based criteria for recruitment and buried the "spoils system." The twentieth-century dictators, Primo de Rivera (1923-1931) and Franco (1939-1975), purged critical voices from the civil service and temporarily abolished job security and tenure. Their replacement with people who were politically identified with the new regime did not always produce competent professionals (Nieto, 1986).

During Franco's dictatorship, the power of the corps grew substantially. Most political appointments of the executive were occupied by members of the most relevant corps. Bureaucrats also controlled the ceremonial legislative chambers established by Franco. Moreover, bureaucrats held a variety of positions in public and private enterprises at the same time (Baena, 1999). The Francoist state became synonymous with the bureaucratic state.

In Spain, the influence of the corps has declined over the last 30 years for a number of reasons. First, their influence on personnel management has been severely limited. Second, service delivery has been transferred to the regions and the municipalities, where the spirit of corps is almost non-existent. Third, at the local level, the number of labour contracts outnumbers the percentage of local officials. Furthermore, the number of corps members in the municipalities as agents from the centre is limited to a handful of civil servants in charge of legal and financial control. Hence, the influence of this Napoleonic feature has waned.

\subsection{Legalism}

In the Napoleonic tradition, administrative law constitutes a separate body that regulates public activities. The duties and rights of citizens vis-à-vis public administration play a more important role than managerial principles. 
Administrative law provides very detailed procedures for guaranteeing citizens' rights. Civil servants are more concerned with duly applying the law and the right use of inputs and guarantees attached to processes than about outputs and outcomes.

The legalistic nature of Spanish public administration poses challenges to the reforms of central Administration, as happened with the reforming plans of the late 1980 s and again in the mid-2010s. The last government reform in 2013 was steered by state lawyers. The policy documents hardly contained any form of thorough evidence-based review of the problems to be solved, and neither the plan nor the monitoring reports reflected the results to be achieved. In line with the predominance of the legal system, accountability in the Napoleonic tradition is mostly formal, legalistic, ex ante and not results-oriented.

However, legalism, although highly relevant, does not play the same role in all policy sectors. For instance, the biggest organisations at the central level, the Tax Agency and Social Security, have always been outside the scope of the overall reforms because they have implemented a results-oriented culture since the mid-1980s. Healthcare, devolved to the regions, displays strong results and managerial orientation in most autonomous communities. Some of them also have a choice system with private sector providers under the public system umbrella. Schools and universities are subject to choice and competition everywhere. In social services, there are myriad governmental and non-governmental providers, which compete for resources and must prove themselves through the impact they achieve. Some regional audit commissions are increasingly using effectiveness measurements to assess the performance of public sector entities. Local authorities have been under pressure to optimise their service delivery, in particular because of the economic and financial crisis. Managerialism is part of the culture of many municipalities. One cannot identify a clear "dejurification" process, but there are clear signs of increasing managerialism. Legalism is becoming reminiscent of horizontal and transversal policy areas, while the service delivery logic is increasingly subject to market and managerial forces.

In sum, as mentioned above, the Napoleonic administrative tradition seems to have rhetorical power rather than constituting a firm basis for homogeneously considering the legacy of Napoleon and categorising Spain as such. Many of the features of the Spanish administration of the nineteenth century that have forged the modern state were copied from France. Many of these features (prefect system, centralised system, the duality of central and local government, strong central administration with provincial delegations, and the relevance of the corps) have changed extensively with the devolution of powers to regional authorities. The use of codification and the status of administrative 
law still enjoy a privileged position in the system, but with increasing hybridisation of managerialism, which is affecting even highly autonomous professions such as doctors, professors, teachers and social workers.

\section{Social diversity and limited response from PA scholars}

For most of the twentieth century, Spanish society had been uniform and homogeneous in terms of population, language and religion. After the advent of democracy in 1978 , society became noticeably diversified in all these dimensions. This increasing diversification process, however, did not lead to a corresponding increase in the work of PA scholars. The transition of a homogeneous society to a heterogeneous one and the research attention paid to it by academia are outlined in this section.

Spanish society has become more diverse. This diversity, apart from challenging and enriching the mainstream culture, offers challenges and incentives for the academic community to assess and explain the state, its configuration, and the policies and services that it delivers. This section deals with religion, the Spanish languages, immigrant residents, irregular immigration and the increasing affluence of international tourists and the considerable impact this is having.

\subsection{From Catholicism to Agnosticism and Religious Variety}

A relevant cultural driver for diversity in the population is religion. Religion not only plays a role in the differentiated relationships between public authorities and citizens from different confessions. There are at least three elements that should be considered: the organisational impact that the structure of the relevant confessions may have on the state; the confessionalism versus laïcism of the state; and the way in which the academic community considers the diversity of believers.

Spain has traditionally been a Catholic confessional state, except for a 2o-year interlude in the twentieth century. Franco signed a confessional pact (1953 Concordat with the Church) in the hope of exiting the autarchic economic policy and the ostracism experienced during the first years of his dictatorship. Religious orders were granted a juridical status and the state committed to financially supporting the Church. Furthermore, the state introduced mandatory canonical marriages for all Catholics, and bestowed upon the Spanish Church censure rights, control over religious teaching in schools and the right to establish universities and schools. In return, Franco 
secured the right to appoint bishops. The Concordat offered his regime international legitimacy.

The Constitution of 1978 thwarted the confessionality of the state. The fundamental law proclaimed the spiritual freedom of citizens and the religious neutrality of the state. It laid down the nature of relationships with the Church in 1979, reducing its role. Since the introduction of the $42 / 2006 \mathrm{Act}$, funding of the Church has transitioned from direct budgetary appropriations to a competitive voluntary church fee system. Under this system, the Church receives funding from taxpayers (who can opt to donate to other non-governmental organisations) as a percentage of their income tax. From 2007 to 2015 , between $34 \%$ and $35 \%$ of taxpayers ticked the box in their tax declaration agreeing to support the Spanish Catholic Church (Conferencia Episcopal, 2017). This percentage is lower than the percentage of Spaniards who considered themselves Catholic during the same period, but higher than the proportion of believers who practised the religion on a regular basis. The number of Catholics in the population has shrunk from $77.6 \%$ in 2006 to $66.9 \%$ in 2019 , and the number of believers (from any religion) who are regular churchgoers (at least once a month) halved from $53.4 \%$ to $24.1 \%$ in the same period (Barometer CIS, January every year (2005-2019)).

After the 1978 Constitution, the Spanish Church lost its monopoly of indoctrinating pupils about religious matters. Notwithstanding, the Church has been able to monopolise the teaching of religion in schools under conservative governments. Socialist governments, however, have replaced "Religion" in the curriculum with "Citizenship." In any case, the Catholic Church has not lost complete control of education and social affairs. The Catholic Church has 2,447 non-university education centres in the Spanish territory, run as private institutions or in concert with the state (the state provides considerable funding) and 15 universities. It represents approximately $10 \%$ per cent of all private schools at the secondary educational level. Private education accounts for one-third of all education centres in Spain. Furthermore, the Church also plays a major role as provider of social assistance. It owns 307 centres for promoting employment, 6,298 centres for mitigating poverty, 201 centres for caring for immigrants, and 158 centres for minors (Conferencia Episcopal, 2017). This does not include the activities of Cáritas and Manos Unidas.

While Catholicism is on the decline, religious diversity is growing in Spain. Although the percentage of believers in religions other than Catholicism is insignificant, it went up from $1.7 \%$ to $3.1 \%$ between 2006 and 2019 . According to the latest census by UCIDE (2019), the number of Muslims in Spain is almost 2 million (4.3\% of the Spanish population in 2018), of whom $42.5 \%$ have Spanish nationality. The second largest Muslim group is made 
up of Moroccans. The number of Muslim religious communities was 1,626 in 2018. Since 1996, it has been possible to offer Islamic religion classes at school, but only 12 Autonomous Communities comply with the minimum legal requirements. As yet, no imams are employed in the military; however in 2006 Muslim prisoners obtained the right to receive religious assistance, and since 1992 there has been an agreement of religious assistance for Islam believers in hospitals. These broad agreements are only now materialising in some Autonomous Communities (UCIDE, 2019).

In sum, the Catholic Church has lost some of the privileges in its relationship with the state, but it still enjoys the benefits of public funding (compared to other religions) and plays a very active role in education and health. This is against a background of a waning interest in religion and a growing percentage of believers from other confessions, Islam being the most relevant one.

This interface between religion and the state at the individual and the organisational levels offers considerable scope for scholarly work in public administration. The evolution of the status of the Catholic religion in Spain and the gradual growth of other religions offer an avenue for research. The following non-exhaustive list of areas illustrates the potential for research. First, PA scholars could, for instance, analyse how the public funding of the Church impacts on resources management, tertiary economic activities, accountability and the fostering of an inclusive society. Second, the role of the Church in the welfare state is insufficiently understood. This role affects the impact of intervention in the state functions (including poverty alleviation and immigration) and also has an impact on school choice exercised by students and their families. Third, regarding the steady expansion of other religions, PA scholars could focus on the representativeness of the civil service and, in particular, of street-level bureaucrats. They could also analyse how different public sector organisations manage the interactions between the religious centres (both Catholic and non-Catholic) and how religious diversity is managed in hospitals, schools and prisons. Non-PA scholars in these areas have done a handful of studies, however the PA community does not seem to be taking an interest in an area that is expected to grow significantly over time.

\subsection{From the predominance of Spaniards to a plurality of nationals}

The Spanish population has become considerably diverse in the last 40 years and this demographic broadening is defying the traditional philosophy of public sector organisations. In the 1980 os and early $1990 \mathrm{os}$, immigrants represented $1 \%$ or less of the population. According to the Spanish National Institute, this 
increased to $12.8 \%$ (5.95 million) in 2019. 32\% of immigrants come from other EU Member States, such as Rumania, the United Kingdom, Italy, Bulgaria, Germany and France; 37.5\% were born in Latin America. The nationality that assumes the highest representation in Spain is Moroccan with over 750,000 citizens. The presence of these nationalities in the territory also depends on the economic activities they perform. EU nationals normally belong to the upper limits of the age range and come to Spain to enjoy their retirement, mostly in the coastal cities; other non-European and Latin American nationals are found in low-skilled jobs and Africans are employed in agriculture. In some municipalities, the proportion of non-nationals is considerable and has even created different social orders within the community.

Furthermore, the number of irregular migrants arriving on the Spanish coasts every year is high, with 50,962 arrivals in 2018 (221,000 since 2016), more than all the other European coastal countries combined (IOM, 2019), not including the humanitarian crisis connected with the Syrian war in 2015. Although not all irregular migrants stay in Spain, they rely on a complex network of different public and non-governmental organisations to deliver services and make decisions regarding their deportation to their country of origin, placing minors in special centres, or leaving them in legal limbo regarding refugee status. The response to this non-stop humanitarian crisis requires continuous coordination with public authorities, NGOs and businesses from different policy sectors and levels of government.

Finally, while international tourists provide enrichment to society, they increasingly bring more challenges for public sector organisations. The number of international tourists entering Spain has almost doubled from 48.5 million in 2001 to 82.7 million in 2018 (National Institute for Statistics). Most visitors come from the European Union, with high seasonal concentrations in particular geographical areas. Several studies have shown that tourists and their culture have contributed considerably to the enrichment of Spanish society. However, tourism represents a challenge for public sector organisations, such as the need to use artificial intelligence to understand tourism inflows, popular destinations, tourist behaviour and the flexible arrangements of services and policies to cope with concentrated demands. In peak season, the population in several coastal municipalities doubles or even triples. These fluctuations pose considerable challenges for local councils in the delivery of municipal services (waste collection, street cleaning, public safety, traffic monitoring, the regulation of tourist apartments, etc.). It also affects other regional services, such as health and intermunicipal transport.

Since the 200os, Spanish society has experienced increased diversity as a result of these three different types of population inflows (irregular 
immigration, increase in the number of residents from other countries and international tourism). Several international languages and cultures coexist in many municipalities. Public administrations are slowly adapting to this new situation, but the work of Spanish PA scholars is lagging behind. Legal scholars pay attention to the level of autonomy of local authorities, their capacity to deliver services, and their increasing needs for finance. The overburdened health services receive attention as regards spending, public and private provision and waiting lists. PA scholars, though, have remained mute about the specific challenges of population diversity concerning education, service delivery and the contracting of services for these communities, or the difficulties of delivering services under ever changing conditions. For instance, there are many issues related to the use of power and the legitimacy of the state machinery as regards immigrant residents when considering their educational achievement, capacity of real choice and interaction with the educational system. The PA scholarly community is missing from these debates.

\subsection{From the Spanish language as a national symbol to the Spanish languages and national identities}

The Spanish language (officially Castilian under the 1978 Constitution) coexists with Spain's other languages that are official in their respective regions, such as Catalan, Basque, Galician, Valencian and Aranese (spoken in the areas of Aragon and Catalonia). Their co-officiality is practised in one-quarter of the territory. Nevertheless, the daily use of official regional languages is declining according to statistical records. For instance, the Statistics Institute of Catalonia states that the percentage of citizens from the region using the Catalan language daily went down from $46 \%$ to $36 \%$ between 2003 and 2018, ${ }^{1}$ although the recent upsurges in peripheral nationalism may reverse this declining trend.

The co-official languages spoken in certain territories and their impact on national identities constitute a relevant area of study for PA scholars. The use of language has an impact on the organisation of bureaucracy and the judiciary, the representativeness of civil servants, their mobility, and on the way in which public authorities monitor respect for language rights. There are studies in some of these areas, but PA scholars are not well represented, and there are gaps where more research is required to understand how language shapes the bureaucratic interactions between the state and its citizens as well as different parts of the state.

The existence of several official languages has had an impact on the dissemination of administrative studies, but not on research; for instance, on 
how the co-existence of official languages impacts on bureaucracy and its interactions with the citizens. Most Spanish regions have an official school for public administration. Their main functions are linked to recruiting and training civil servants for the regional authorities, and publishing studies on territorial administrative affairs. Research plays a lesser role in these institutions. When it comes to publications, they typically host journals on public administration with a strong juridical perspective. Galicia and the Basque country each have a scientific journal on administrative matters; Catalonia has one on administrative law. These institutions publish all articles in Spanish and in the regional language and many of their publications are also published in both languages. In general, books are also published in two languages, which may be a burden for the authors. Although Spanish speakers can understand Catalan, Valencian and Galician (no chance of Basque), it is very rare to identify references in their publications that are cited in languages other than Spanish or English. Therefore, the publication of scientific work on PA in the regional languages may satisfy national identity issues and political goals, but it does not foster exchange in the Spanish scholarly community.

\section{The Spanish PA community and its internationalisation: present and future}

Spanish society and culture have become more plural and diverse and Spanish public administration displays characteristics that distance it from the uniform Napoleonic traits. The territorialisation of power has created a new administrative space at the regional level and in the intergovernmental arena. These parallel evolutions should be having an impact on PA scholars, but this does not seem to be the case.

The present shape of the PA scholarly community is owing to its evolution since its inception. The development of the study of Public Administration in Spain has followed a similar path to that in France and Germany. Police science and cameralism had a considerable impact in Spain during the second half of the eighteenth and at the beginning of the nineteenth centuries. French and German texts were translated into Spanish and were used by Spanish PA scholars. These disciplines preceded the research of specialists in administrative law, whose ascendancy grew after the Napoleonic invasion. Administrative lawyers displaced the police science and cameralistic approaches.

In parallel with the modernisation of the late 1950 and early 1960 s, the works of American authors such as Taylor, Gulick, Barnard and Elton Mayo were translated into Spanish, 20 to 40 years later than in the rest of Europe. 
They had a minor impact on Spanish administrative law scholars, who were reluctant to use private sector techniques for the public sector.

Political science, as a science of democracy, did not appear until the parliamentary period begun by the 1978 Constitution. Political science tried to detach itself from constitutional law, while administrative sciences aimed at departing from administrative law. In that period, political science was not interested in public administration. The major concerns were elections, voting behaviour, political parties and political representation. This is still the case today. Administrative science (singular), like "science administrative" (France) and "Verwaltungswissenschaft" (Germany), represented the Verwaltungslehre tradition that combined legal, political and sociological approaches to administrative affairs, but was more prescriptive than analytical and empirical.

PA as a discipline, in combination with either political science or administrative law, has grown in popularity at the universities. There was only one university offering political science (and administrative science studies) in the early 1980s. In 2019, the number of universities offering this combination has increased to 25. These departments have attracted European students thanks to the Erasmus grants, but in order to become a more relevant choice for international students, public administration studies should strive for international accreditation instead of remaining local.

The field has also been developed by the regional institutes of public administration (mentioned above) and by the National Institute of Public Administration (INAP) based in Madrid. The National Institute of Public Administration (INAP) has evolved in recent years and acquired two different dimensions. First, it has several nationally accredited master programmes. These professional (not academic) programmes target only civil servants. As a result, INAP became affiliated to one national university. This has helped to support the professional development of civil servants. Second, the institute has promoted research by giving grants on a competitive basis. The duration (one year), the amount (maximum $€_{15}, 000$ ) and the open nature of the call for applications restricts the capacity to reach high impact results, but still a considerable number of books have been produced following this route. The number of internationally recognised journal articles in this endeavour remains limited.

This section does not deal with the major research topics of the Spanish PA community. The work of Subirats (1999), albeit outdated, is still relevant in offering the major trends of Spanish PA scholarship 20 years later - Subirats offered a review of the research in the area of civil service and administrative organisations (this interest has waned since the 2000s) - along with incipient studies on 
public management (this area has grown, although not significantly, thanks to the limited interest of business administration scholars and economists) and the analysis of public policies (with a certain upsurge in particular sectors, although not always procured by PA scholars). Astonishingly, the transformation of the state, the loss of its Napoleonic nature through the territorialisation of power and the creation of autonomous entities at the regional level, with new interactions between different levels of government, has triggered minimal interest from PA scholars on administrative interactions and new structures.

Language is also a vehicle of expression in an international world, where scientific fields are dominated by the English language, although Spanish is one of the three most commonly spoken languages in the world. The English language constitutes a challenge when writing about a public sector system embedded in a culture and, especially, in a particular language. Both institutions and administrative (managerial) practices are difficult to translate for different reasons. However, the underlying reasons for the barriers to communicating research effectively have less to do with the language in use, but more with the absence of a theoretical body and a scientific language that facilitate exchange.

If we take Spanish as an example, the conversion of terms from Spanish into English and vice versa has raised two challenges. First, like other languages and systems, it is difficult to find the right equivalent for Spanish institutions in the English or American system. The same institutions in very similar systems, such as in Spain, France and Italy, do not possess the same features. Ministerial cabinets, grand corps, the council of state and prefects (or equivalent labels), for instance, are similarly named in the native language and have Napoleonic roots in all these countries, but their nature and their contribution to the system are substantially different. Furthermore, even within the same language and perhaps contrary to expectations, Latin American countries and Spain use different words for the same concept, or the same words for different concepts. On many occasions, scholars and practitioners are divided by the same language.

The barriers to stop institutional concepts from travelling among different languages have to do with the absence of proper analytical tools and theoretical frameworks that help to transfer knowledge on idiosyncratic institutions. Different political science approaches might cope with this problem by giving priority to theoretically oriented concepts over the particular institutions of a country. In this regard, political science could have an edge in PA if the use of theories became more prominent. When PA texts written in Spanish are analysed, there is still a dearth of theoretical categories that can travel across countries. 
A second challenge is that English management jargon has little appeal for practitioners. In most cases, the adoption of English terms (but with Spanish spellings), or translations in the academic arena, has not crystallised in the praxis, unlike in Latin American countries where English terms are used by practitioners and academics alike. In a civil service dominated by legal experts, management jargon has not really penetrated Spanish public administration. A translation of many English terms (not all), such as governance, steering, nudging, benchmarking, inputs, outputs and outcomes, would be found among Spanish academics, but would not receive wide support and use among practitioners. Furthermore, the number of civil servants who can interact in English is still relatively low, despite the Europeanisation process.

Sharing the Spanish language on both sides of the Atlantic has not helped the development of the PA community as had been anticipated. The influence of Spanish scholars and the Spanish system in Latin American institutions is smaller than expected. Unlike in Spain, the Napoleonic imprint does not seem to have been important in Latin America, as the arrival of the Napoleonic influence in the Iberian Peninsula took place while most colonies were obtaining their independence from the Spanish Empire. As anecdotal evidence, research carried out in 72 regulatory agencies in 18 Latin American countries (Salvador and Parrado, 2011) showed that none of them regarded their Spanish counterparts as role models. Joint projects on PA between Latin American scholars and Spaniards are rare, although exchange between them has flourished recently. In addition to the annual conferences and other activities organised traditionally by CLAD (Latin American Centre for Administration and Development), founded in 1972, and the association's publication outlets, there are two recently created networks with contributions from both sides of the Atlantic: GIGAPP (Research Group on Government, Administration and Public Policies), with annual conferences since 2015, and Novagob, which is an ecosystem for public innovation for Latin America, Spain and Portugal. The latter has a strong presence in social media and is fostering a wealth of mutual collaborations.

To sum up, the configuration of public administration in Spain, or in other words its culture and institutions, has changed considerably due to a number of factors that include democratisation, the territorialisation of power, substantial population inflows, the expansion of the welfare state, and the diversity of society and religion. Some of these factors (regionalisation of power, for instance) have had a great impact on the erosion of the Napoleonic features of 
the state tradition, but also piecemeal reform processes in individual agencies and sectors with high user demand have softened the legalistic approach of Spanish bureaucracy.

The diversity in society in terms of language, religion and people from different countries with temporary or permanent residency, regular or irregular status, has had, and still has, differentiated impacts on the geography. Unfortunately, PA scholars have not dealt sufficiently with the challenges that these pressures are exerting at all governmental and administrative levels and on organisations. Furthermore, little research has been done on the interactions between governments at different levels, despite the fact that these relationships have a considerable impact on issues related to equitable treatment across the territory. International organisations like the OECD have recently criticised the central government's reform programme for not being able to structure a more consensual and coordinated reform approach that also includes the autonomous communities. Subirats (1999) tied the flourishing of the study of public administration to the democratisation process. This chapter agrees with this perspective. However, his optimistic view that the upsurge in PA studies in the first 20 years of democracy is a predictor of a productive future is not fully shared. PA scholars still need to cover more fields and become more internationally competitive than hitherto. Still, there are elements that underscore the potential that PA and non-PA scholars have to improve the analytical capacity of the field. Non-PA scholars are taking an interest in the intersection between management, and economics and public administration. Electronic government, artificial intelligence and similar topics are eliciting the interest of a specific and active community. Thanks to several associations (CLAD, NovaGob, GIGAP), the ties with Latin American scholars are becoming more fruitful. Institutes of public administration are showing increasing interest in research, although this area requires more funding. These exchanges are likely to have a long-term impact on PA research in Spain and will hopefully promote these studies in a more competitive international environment.

\section{Note}

1. https://www.idescat.cat/indicadors $/ ? \mathrm{id}=$ anuals $\& \mathrm{n}=10364 \&$ lang $=\mathrm{es} \& \mathrm{col}=1$ 


\section{References}

Baena, M. (1990). Education and research in administrative science in Spain. International Review of Administrative Sciences 56(3): 521-535.

Baena, M. (1999). Élites y conjuntos de poder en España, 1939-1992: un estudio cuantitativo sobre parlamento, gobierno y administración y gran empresa. Madrid: Tecnos.

Ballart, X. (2008). The study of public management in Spain. In W. Kickert (Ed.). The Study of Public Management in Europe and the US: A Competitive Analysis of National Distinctiveness (pp. 189-207). London: Routledge.

Conferencia Episcopal. (2017). Memoria Anual de Actividades de la Iglesia Católica en España Año 2016. https://www.conferenciaepiscopal.es/wp-content/uploads/2018/06/Memoriaactividades-Iglesia-Catolica-2016.pdf

Kickert, W. (2007). Public management reforms in countries with a Napoleonic state model: France, Italy and Spain. In C. Pollitt, S. Van Thiel, \& V. Homburg (Eds), New public management in Europe (pp. 26-51). London: Palgrave Macmillan.

March, J. G., \& Olsen, J P. (1983). The new institutionalism: Organizational factors in political life. American political science review 78(3): 734-749.

Ongaro, E. (2010). The Napoleonic administrative tradition and public management reform in France, Greece, Italy, Portugal and Spain. In M. Painter, \& B.G. Peters (Eds). Tradition and public administration (pp. 174-190). London: Palgrave Macmillan.

Parrado, Salvador. (1999). The History of Spanish Administration: Some Notes from an Administrative Science Point of View. In E. V. Heyen (Ed.). Jahrbuch für Europäische Verwaltungsgeschichte (pp. 337-353). Baden-Baden, Nomos Verlagsgesellschaft.

Parrado, S., \& Salvador, M. (2011). The institutionalization of meritocracy in Latin American regulatory agencies. International Review of Administrative Sciences 77(4): 687-712.

Subirats, Joan. (1999). Public Administration and administrative science in Spain. In W. J. Kickert, \& R.J. Stillman (Eds). The modern state and its study: New administrative sciences in a changing Europe and the United States (pp. 137-158). Cheltenham: Edward Elgar.

UCIDE (Unión de Comunidades Islámicas de España). (2019). Estudio demográfico de la población musulmana. http://observatorio.hispanomuslim.es/estademograf.pdf. 



\title{
2
}

\section{The New Diversity: Increasing Ethnic Heterogeneity and its Consequences for Public Governance}

\author{
Mark Bovens, Roel Jennissen, Godfried Engbersen and \\ Meike Bokhorst ${ }^{1}$
}

\section{$1 \quad$ Public governance and the new diversity}

Across Western Europe immigration patterns are changing rapidly. In the second half of the twentieth century, large groups of immigrants came from a limited number of countries. Many migrants came from former colonies, such as India, Pakistan and the West Indies to the UK, Algeria and West Africa to France, and Suriname and the former Netherlands Antilles to the Netherlands. Large groups also came as labour migrants to Western Europe, in particular from Turkey and the Maghreb. This we call the "traditional diversity."

In the twenty-first century, smaller groups are coming from a very large variety of countries. Migrants come from all over the globe to Western Europe, from EU countries, such as Poland, Bulgaria and Germany, from Syria, Eritrea and Iran, but also from the former Soviet republics, India and China. Many come to work, others come as refugees, or as students. This we call the "new diversity."

The traditional migrant groups were relatively homogeneous in socioeconomic terms. Many were low skilled, had low levels of literacy, and came from rural areas outside Europe. The "new" migration is much more heterogeneous. Among them are well educated professionals, with high incomes and urban life styles, but also low skilled labour migrants who work in rural areas, or semi-literate refugees from Sub-Saharan Africa. Some come to stay, but large percentages leave within five years.

This "new diversity" can be observed across Western Europe (Castles, De Haas, \& Miller, 2014). Steven Vertovec (2007, p. 1024) has called it "superdiversity": the "new, small and scattered, multiple-origin, transnationally 
connected, socio-economically differentiated and legally stratified" nature of international migration. This super-diversity is a new chapter in the history of migration to Western Europe, with new challenges for social cohesion and public governance.

In this paper we will use the case of the Netherlands to illustrate this transformation of migration and society. The Netherlands is fairly representative of Western Europe. It experienced post-colonial immigration in the 1950s, 1960 s and 1970s, as did Belgium, France, the UK, Spain and Portugal. It saw large waves of labour and family migration in the 1960s, 1970s and 1980s, as did Belgium, Luxemburg, France, Germany, Switzerland and Austria. ${ }^{2}$ And from the 1990s onwards it has experienced a wave of post-industrial migration, consisting of refugees, intra-EU migrants, and highly skilled workers, as have Germany, Sweden, the UK, Switzerland and Austria (White, 1993; Jennissen, Van Wissen, \& Van der Gaag, 2006). Some Western European countries, such as the UK, France, Belgium, Luxemburg, Sweden and Switzerland, are probably even more diverse than the Netherlands. Others, such as Ireland, Finland, Norway, Italy, Spain and Greece, are less diverse. An advantage of the Netherlands is also that very large and accurate data sets are available on the ethnic composition of neighbourhoods, municipalities and regions.

Firstly, we will provide more empirical details about this new diversity and show that this diversity differs greatly across the country. Some municipalities are characterised by the traditional forms of diversity, others by "superdiversity," and some are only slightly affected by migration. This means that a "one size fits all" policy approach does not make much sense. Secondly, we found that higher levels of ethnic heterogeneity coincide with lower levels of social cohesion. In the final part of the paper, we will present an agenda for public governance and public administration.

\section{Increasing ethnic diversity in the population}

In the Netherlands, the proportion of residents with a migration background ${ }^{3}$ in the population has risen considerably in the last few decades - from $\mathbf{9 . 2 \%}$ in 1972 to $23.1 \%$ in 2019 . Moreover, the group of residents with a migration background is becoming increasingly diverse. These days, only one-third of migrants living in the Netherlands belong to the traditional migrant communities, while the remaining two-thirds come from a wide range of other countries of origin. In 2017, the migrants living in the Netherlands hailed from 222 different countries of origin. ${ }^{4}$ 
Interestingly enough, people are often unaware of this increased ethnic diversity. Policy documents and studies on immigration and integration continue to focus on traditional post-colonial and migrant worker communities, i.e. on citizens with a Surinamese, Moroccan or Turkish background. A few groups of refugees and Polish labour migrants may occasionally also receive a share of the attention. Furthermore, a rather rough distinction between "Western" and "non-Western" migrants is often made in policy documents.

This traditional perspective on migration and integration does not fit the empirical reality of the twenty-first century. If we keep seeing migration through 1970 s migrant-worker glasses or through post-colonial glasses, we will see only migrants from the traditional countries of origin and from poorly defined categories in which many migrants are lumped together, such as "from Western countries" and "from non-Western countries." This is shown in the circle on the left in Figure 1. Once we let go of our traditional frameworks and look at things as they are now, we will see a multitude of new groups, as shown in the circle on the right in Figure 1.

Figure 1 Traditional and new perspectives on diversity among inhabitants
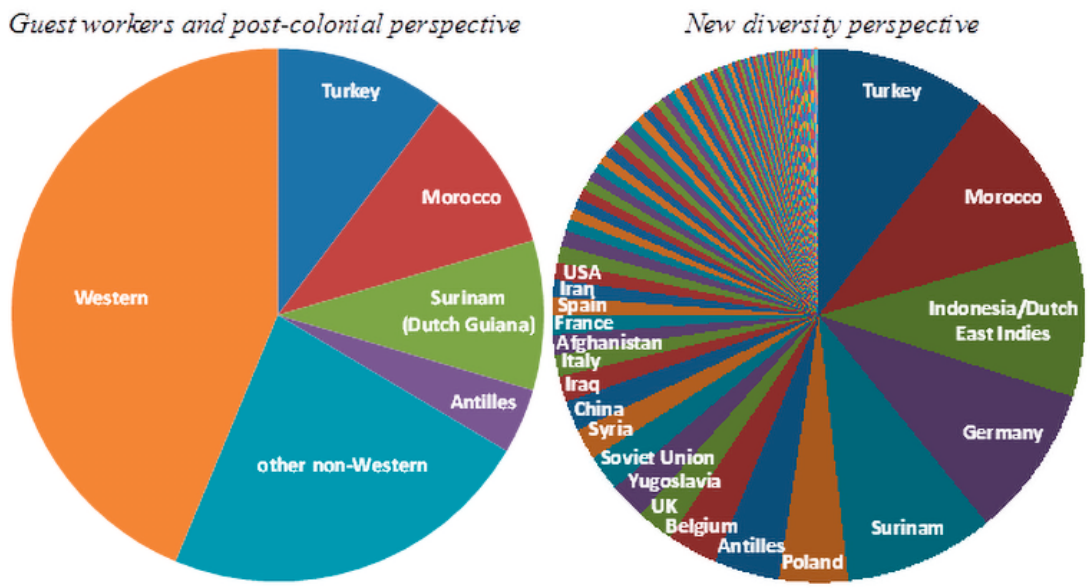

(c) WRR 2018 | Source: Statistics Netherlands

By acknowledging that today's migration situation is much more diverse, we can do greater justice to the wide ethnic diversity of migrants living in society. The traditional countries of origin are no longer in the top 15 countries with the most significant positive net immigration rates (more immigrants than emigrants). During the 2007-2016 period, in the Netherlands, this top 15 was as follows: Poles, Syrians, people from the former Soviet Union, 
Bulgarians, Chinese, Indians, Romanians, Italians, Germans, Somalis, Eritreans, Spaniards, Hungarians, Greeks and Iranians (see Figure 2). As a matter of fact, the traditional groups had negative migration rates - they had more emigrants than immigrants.

Figure 2 Top 15 countries supplying the greatest number of migrants (net migration), the Netherlands, 2007-2016

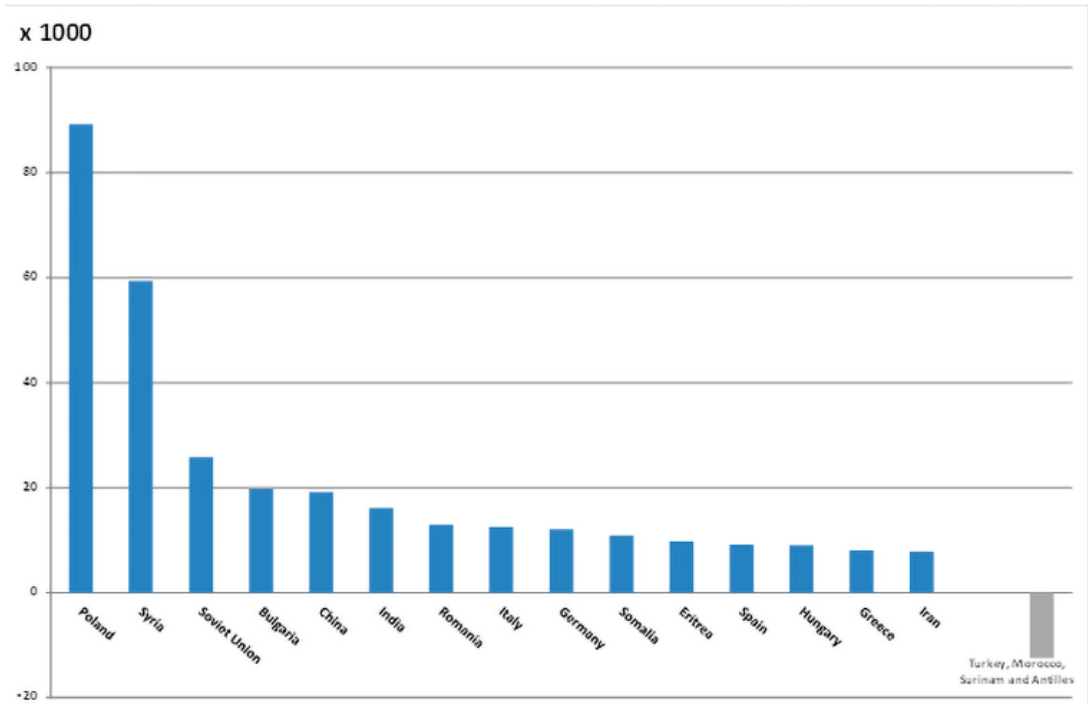

(c) WRR 2018 | Source: Statistics Netherlands

Another reason why the Netherlands is becoming increasingly ethnically diverse is that birth rates tend to be higher for residents with a migration background than for the native population. At the same time, mortality rates for residents with a migration background tend to be lower, since migrants tend to be younger than the average resident. After a generation, the birth and mortality rates of migrant communities tend to conform to those of the country in which they have settled. However, we are still a long way from reaching that point. This means that Dutch society will continue to grow more diverse over the next few decades, even if we were to completely ban immigration starting from today.

This sustained increase in the ethnic diversity rate is reflected in Statistics Netherlands' population projection. Figure 3 shows that the proportion of representatives of the four traditional emigration countries in the Dutch population will continue to increase slightly until the mid-twenty-first century, 
after which it will stop growing. The projection mainly shows that the share of the highly diverse group of people with a non-European/non-AngloSaxon background will continue to increase significantly. Starting from the 2040s, this group will outnumber the traditional "big four": citizens with a Surinamese, Moroccan, Turkish and Antillean background. The diverse group of people with a European or Anglo-Saxon migration background will continue to represent a larger share of the population as well.

Figure 3 Share of people in the Netherlands with a migration background, 1996-2060

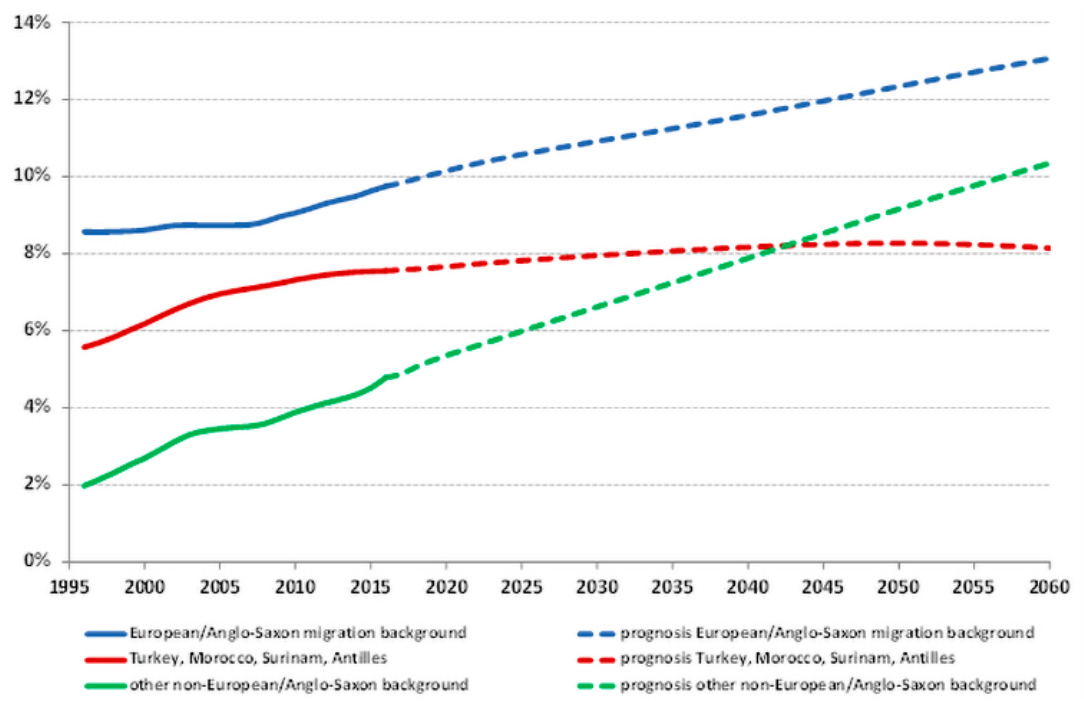

(c) WRR 2018 | Source: Statistics Netherlands

\section{$3 \quad$ Large variety between and within municipalities}

We calculated how ethnically diverse Dutch municipalities and neighbourhoods are. We started by subdividing all the residents of the Netherlands into 18 groups, reflecting 18 different ethnic backgrounds. Needless to say, the largest of these groups consists of "native Dutch" people. This group comprises nearly $80 \%$ of Dutch society. In addition, we distinguished 17 other groups based on country of origin, e.g. Turkey, Morocco, Anglo-Saxon countries, Arab countries, Sub-Saharan Africa, South Asia, etc. We then calculated a diversity index for all Dutch neighbourhoods, municipalities and regions, the so-called Herfindahl-Hirschman Index (HHI). This is expressed as a number between $o$ and 1 that indicates the likelihood that two random sample people 
from a given area belong to groups that have different ethnic backgrounds. The higher the index the greater this likelihood. In other words, a low HHI denotes great homogeneity, whereas a high $\mathrm{HHI}$ denotes heterogeneity. The mean HHI for the Netherlands is 0.38 . However, as Figure 4 shows, the index considerably differs from municipality to municipality.

Figure 4 Herfindahl-Hirschman Index of Dutch municipalities, 1 January 2015

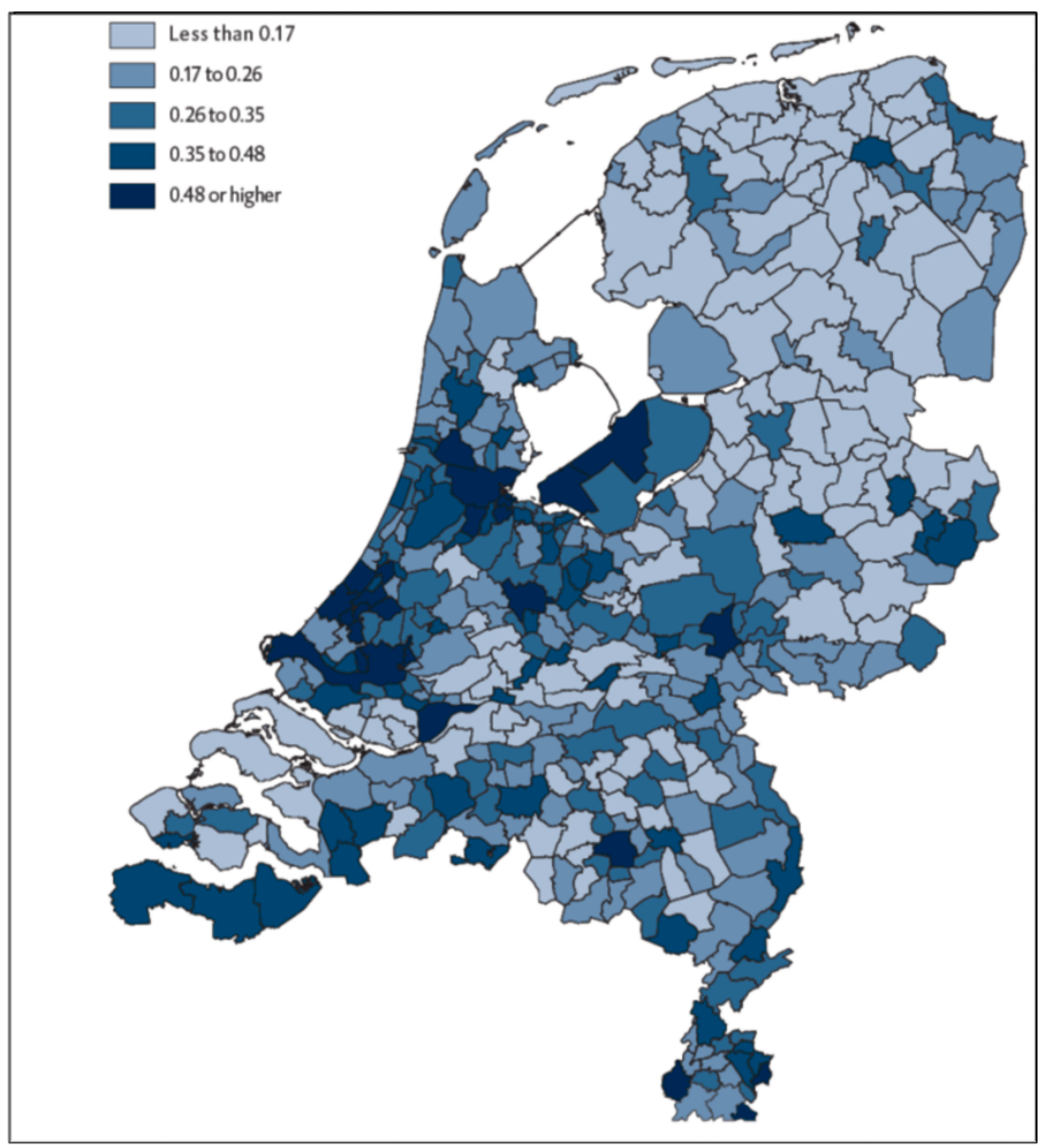

(C) WRR 2018 | Source: Statistics Netherlands

Two-thirds of all Dutch citizens live in a municipality where the odds of two residents belonging to different ethnic groups are approximately one in three or higher. In the Netherlands' three largest cities, Amsterdam, Rotterdam and The Hague, the odds are the highest, more than two in three. In other 
words, a large degree of ethnic diversity is an everyday reality for many people living in the Dutch society. However, the nature of this diversity varies wildly between municipalities. We distinguish eight categories of municipalities, in addition to the "average Dutch municipality" (see figure 5):

- Majority-minority cities (Amsterdam, Rotterdam, The Hague): in these very diverse big cities, the majority of residents will have a migration background, and they will hail from many different countries.

- Suburbs (e.g. Capelle, Diemen, Rijswijk): these towns are highly diverse as well, although the majority of people still have a native Dutch background.

- Large provincial municipalities (e.g. Utrecht, Eindhoven, Arnhem): these large cities are also highly ethnically diverse, but native Dutch people are in a much larger proportion here than they are in the three big cities and their suburbs.

- Medium-sized municipalities with a sizeable community of migrants from one particular background (e.g. Gouda, Almelo, Delfzijl): these towns are characterised by the fact that they have a large migrant community from one specific non-European/non-Anglo-Saxon background. This is typically due to these towns having recruited immigrant workers from one particular country, or to many people from the former Netherlands Antilles having settled there.

- Expat municipalities (e.g. Amstelveen, Wassenaar, Voorschoten): expat communities are very diverse in terms of ethnic backgrounds, with residents coming from all over the world. However, they tend to have relatively few residents from Turkish, Moroccan, Surinamese or Antillean backgrounds.

- Horticultural municipalities (e.g. Westland, Zeewolde, Horst aan de Maas): towns with a lively horticultural industry have a relatively high diversity rate due to the large proportion of people with a Polish or, to a lesser extent, Bulgarian background.

- Border municipalities (e.g. Vaals, Kerkrade, Terneuzen): in these towns, the high degree of diversity is mainly caused by people with a German or Belgian background.

- Homogeneous municipalities (e.g. Urk, Staphorst, Grootegast): in these rural municipalities, the overwhelming majority of residents - over $90 \%$ - have a native Dutch background. 
Figure 5 Variety between municipalities in the Netherlands

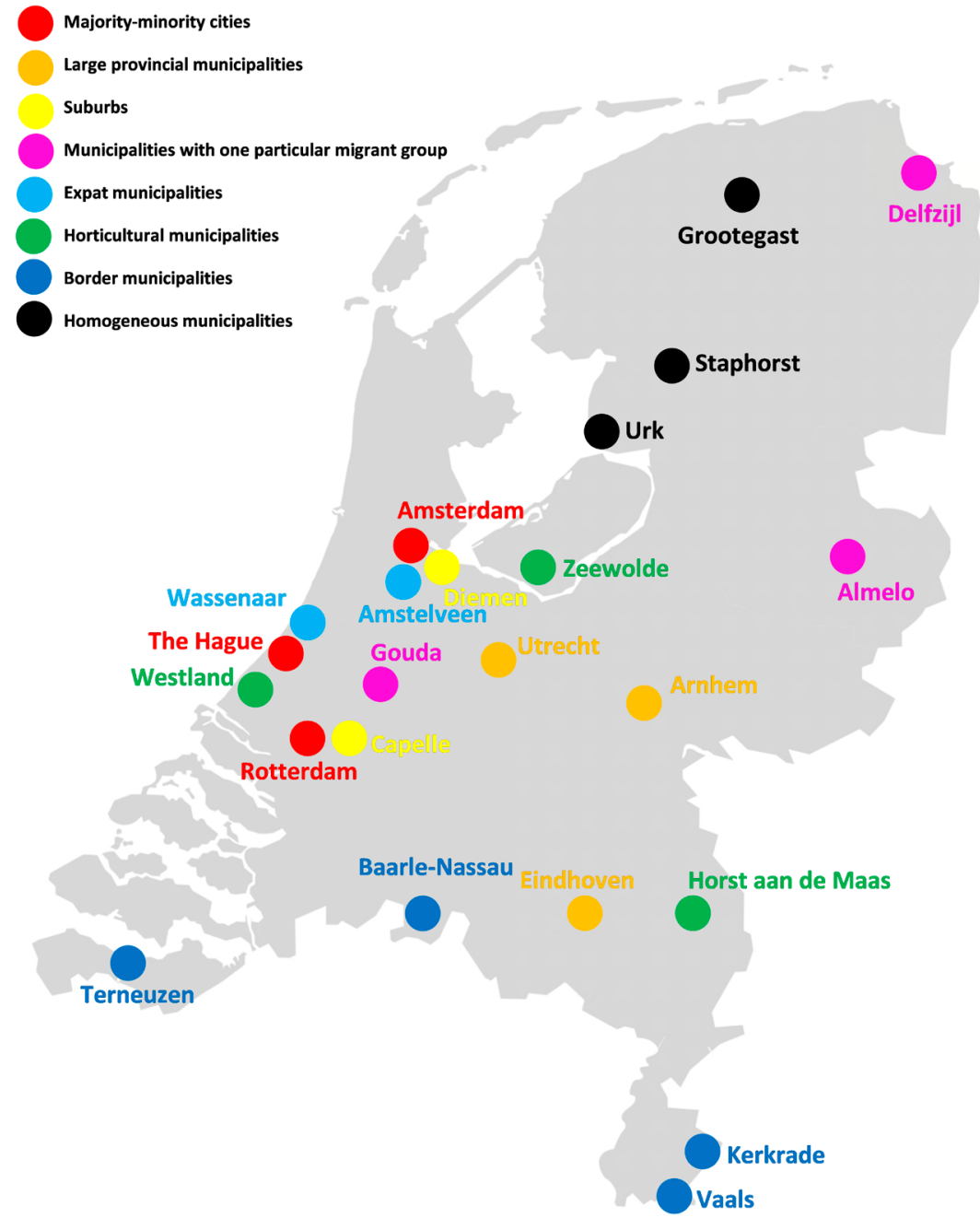

We also conducted an analysis of ethnic diversity on the neighbourhood level. In the three big cities, there are clear differences between the various neighbourhoods. For example in The Hague, the diversity is extremely high in traditional migrant neighbourhoods such as Transvaal, Schilderswijk, Laak and Spoorwijk. The probability of two random residents of these neighbourhoods belonging to the same ethnic group is less than $20 \%$. Neighbourhoods such as Zorgvliet are also highly ethnically diverse, but in a different way. This green upper-class neighbourhood has many residents with a variety of 
different European and Anglo-Saxon backgrounds. Other neighbourhoods, such as Duindorp, on the other hand, have an HHI which is below the national average and consist mainly of native Dutch residents.

\section{Consequences for cohesion}

The rise of this new diversity is good news for those who worry about increasing social and political polarisation along ethnic lines in society. As heterogeneity increases, the chances of overlapping cleavages diminish. In the traditional diversity of the late twentieth century, there were a few, albeit large and relatively homogeneous, ethnic groups in society. This entails a risk of polarisation along ethnic lines, particularly when the cleavage between "natives" and immigrants overlaps with socio-economic, geographical, religious, or linguistic divides. These overlapping cleavages may give way to pillarisation and political conflict, which require major political efforts to accommodate, as has been the case in the past in Belgium, the Netherlands, Northern Ireland, the former Yugoslavia and Lebanon.

However, the increasing diversity in country of origin, socio-economic status, education, religion, and language among the "new" migrants makes it less likely that overlapping cleavages will emerge at the level of society. For example, one finds a large plurality of religions among the new migrants Catholics from Poland, Sunni and Alevi Muslims from Syria, Copts from Eritrea, Greek and Russian orthodox from the Balkans and the former Soviet republics, Hindus from India, and Buddhists from China, to mention just a few. Also, the "new" diversity has prevented the rise of a competing national language, as has happened in the US with Spanish, and in Estonia with Russian. Migrants and their children speak a large variety of languages at home, but in school, in shops, or at work the lingua franca is Dutch or, in some instances, English. And, as we saw, migrants have been dispersed over the country. There are no ethnically homogenous migrant neighbourhoods, let alone regions. Due to this new diversity, there is even not one single neighbourhood in the Netherlands that has a majority of one single ethnic group - with the exception of native Dutch. ${ }^{5}$

We also find a wide socio-economic variety among the new immigrants. Some are low skilled and poorly educated and work in low-paid, insecure jobs. Others, however, are highly skilled professionals, who belong at the top of the income ladder. In the case of EU immigrants from Poland, for example, one can find both ends of the socio-economic divide. Likewise, there is hardly any political contestation and party formation along these new ethnic lines. ${ }^{6}$ 
So this new diversity softens some of the traditional concerns about ethnic polarisation and conflict at the national level.

However, there is more reason for concern about social cohesion at the level of neighbourhoods and municipalities. As Western European societies have become classic immigration societies, classic concerns about creating unity within diversity - e pluribus unum - emerge. In the United States for example, Robert Putnam (2007) in his seminal paper has argued that high levels of ethnic diversity are associated with less social trust and engagement. Residents of ethnically very heterogeneous neighbourhoods tend to withdraw socially - they "hunker down" and retreat as turtles tend to do when they feel uncomfortable.

How does this new ethnic diversity affect social cohesion in the Netherlands? Social cohesion is an umbrella term covering many aspects and indicators, such as generalised trust, participation in voluntary work, people's perceptions of their neighbourhood, a sense of safety and registered crime rates (Van der Meer \& Tolsma, 2014; Koopmans et al., 2015). In other words, it is impossible to say how diversity affects social cohesion in general, since social cohesion encompasses so many things. Increasing diversity rates may affect different aspects of social cohesion in different ways.

The relationship between ethnic diversity and social cohesion has been a hotly debated topic in the literature. Different studies operationalise diversity and cohesion in different ways and use different types of data. ${ }^{7}$ Neighbourhoods with a high rate of ethnic diversity generally do not perform worse than other neighbourhoods in terms of generalised trust and the extent to which people are likely to volunteer or care for others. However, they do perform worse in terms of having good relations with one's neighbours who have a different ethnic background. In highly diverse neighbourhoods, residents tend to have less contact with their neighbours. They also tend to have a poor opinion of the people they do talk to, and are more likely to speak disparagingly of their environment. However, it should be pointed out that such diverse neighbourhoods tend to have a relatively large share of residents who are poorly educated and unemployed. Some studies show that this is more likely to affect people's relations with their neighbours than ethnic diversity. Very few studies have been conducted in the Netherlands on the correlation between ethnic diversity and other indicators for social cohesion, such as the safety of a neighbourhood (Glas et al., 2018). 
In our own study, we analysed three aspects of social cohesion, namely neighbourhood cohesion, feeling at home in a place, and experiencing a sense of security when out and about. Our analyses were based on a series of very large datasets. ${ }^{8}$ We observed the following:

- In neighbourhoods with a high degree of ethnic diversity, measured by the $\mathrm{HHI}$, residents perceive the bonds between neighbours as being less cohesive. They also tend to feel less at home, and are more likely to feel unsafe. We found that these indicators for cohesion are more strongly related to the neighbourhood's diversity than to each of the residents' individual characteristics, such as their income or level of education, or the socio-economic status of the neighbourhood. These effects were not limited to native Dutch; we also found these effects for some of the traditional migrant groups.

- The aforementioned correlations are most noticeable in neighbourhoods consisting of people on medium incomes. These people in particular are the ones who will say that their relations with their neighbours deteriorate as the degree of ethnic diversity increases. It is possible that people on lower incomes have more experience of the reality of highly ethnically diverse neighbourhoods, and that people on higher incomes have more choice with regard to where they wish to live. Alternatively, middleincome people may be more likely to feel threatened by their neighbours than high-income or low-income people because they have more to lose.

- In municipalities with a high degree of ethnic diversity, measured by the HHI, people are more likely to be registered as criminal offenders than in municipalities with a lower degree of ethnic diversity. This is an independent effect of diversity - we controlled for a range of variables that influence delinquency according to the literature, such as gender, age, ethnicity, income and level of urbanisation. We also found this effect in richer and less urban municipalities. However, this effect does have an upper limit. Once a municipality hits a certain degree of ethnic diversity, its residents will no longer be more likely to be registered as criminal offenders. For instance, in this regard there is no difference between highly diverse municipalities such as Rotterdam and The Hague and moderately diverse municipalities such as Gorinchem and Helmond. 


\section{$5 \quad$ Challenges for public governance}

Over the last three decades, the Netherlands, which used to be a relatively homogeneous society with a small number of large migrant communities, has morphed into a highly heterogeneous society with a large number of smaller migrant communities. This ethnic heterogeneity will continue to increase in the short and medium term. This transformation can be observed throughout Western Europe. Western European societies have become immigration societies, on a par with Canada, Australia and the US. How does one create some unity amongst these new diversities? How can all these groups live together smoothly and peacefully?

This creates new agendas for public governance and public administration. The wide variety of cultures and mobility among citizens makes living together more complicated. How can national and local governments help these highly heterogeneous communities to coexist more smoothly? Is it possible to reduce the feelings of insecurity and unease among the residents and to reduce the tendency to hunker down? And how can public institutions such as schools, health centres and housing associations cope with so many different languages and cultures? Is it possible to sustain a civil society with such a variety in cultures and residential mobility among citizens? We have identified a series of challenges for public governance and public policy.

\section{Local variety instead of national models}

Organising social cohesion is first and foremost a task for local government. The new migrants settle in the larger cities, but also in rural communities and in the suburbs. Moreover, as we saw, there are wide varieties in settlement patterns and ethnic composition between municipalities. This implies that national models, with a one-size-fits-all nature, will not work everywhere. Social cohesion policies will have to differ across municipalities and neighbourhoods. In some areas literacy or school drop-out will demand attention; in areas with many tertiary educated labour migrants the issue may be high mobility and residential turnover.

\section{Gaining a better understanding of the local migrant communities}

This requires, first of all, that municipal authorities and other local organisations must gain a better understanding of the various migrant communities living in their own municipalities. This is a precondition for drawing up sensible strategies designed to tackle associated issues. Therefore, it is vital that municipal governments obtain knowledge of the various migrant communities 
living in their various neighbourhoods, and that they disseminate this knowledge in a readily accessible manner. They can do so by collecting demographic and ethnographic data on their residents themselves, among other methods.

\section{Equipping organisations}

In recent decades, schools, health centres and other public service providers have gradually managed to cope with the large cohorts of migrants from Turkey, Morocco and the former colonies. Just as these public institutions have learned how to deal with these traditional groups, new migrants from Poland, India, Syria and Eritrea are arriving with new languages, customs and habits. Public institutions will have to find new modi operandi to engage with these new inhabitants. This requires flexibility and high levels of empathy from street level bureaucrats, teachers, police officers and health workers. This also requires a much more rigorous professional schooling in police academies, schools of health and teacher-training colleges on how to deal with super-diversity among citizens, clients and pupils.

\section{Targeting within mainstreaming}

Public and private entities will have to be prepared for ever-changing cultural diversity among their residents, pupils, patients, customers and employees. Municipal authorities, schools, healthcare providers and businesses will have to provide their services and facilities in such a way as always to be able to help new groups of immigrants indiscriminately. This requires a combination of mainstreaming and more targeted, community-specific strategies. Back when the Netherlands had only a few immigrant communities, public institutions were able to obtain expertise, build networks and draw up strategies targeted at specific communities. Now that there are dozens of smaller communities, this is no longer feasible, particularly since many more new groups from different parts of the world keep coming to the country.

On the other hand, it is no use dealing with all communities in the same way, either. Migrants need community-specific strategies, particularly when they first enter the country. Refugees from Eritrea require a different approach from labour migrants from Poland or exchange students from India. This means that targeting is appropriate at the early stages of immigration and settlement, when the characteristics of their country of origin, such as language barriers and cultural distance, are predominant and relevant. However, at later stages of settlement and with second generations, mainstreaming policies are more feasible and appropriate. 


\section{Fair treatment for all}

Traditional integration policies were directed at migrant groups. They were the target of special programmes in schools and on the labour market. The new diversity also requires policies that are directed at society as a whole. The issue of social cohesion and unity affects all groups in society, including the "natives." This is an important finding of Michael Ignatieff (2017: 69) in his work on hyperdiverse neighbourhoods in the United States:

\footnotetext{
"The virtues of interethnic trust, tolerance, and accommodation depend on institutions doing their jobs: police and the courts grinding out a toughand-ready equality before the law, politicians maintaining a reasonably fair distribution of patronage to all groups, real estate and job ladders remaining open to all irrespective of religion or ethnicity."
}

All organisations must treat everyone in a fair and non-discriminatory manner, regardless of whether they are people who have been here for a while or have just migrated with their children. Unfair treatment undermines the trust people of different backgrounds place in each other, makes people feel discriminated against and unsafe, causes tension between communities and undermines organisations' authority. These risks become more prominent as the degree of ethnic diversity increases, because in highly diverse areas communities will constantly compare their own situation to other communities' situations and might feel discriminated against, while other "new" or "old" communities receive preferential treatment.

At the same time, it is equally important that people who have lived in the Netherlands for a long time also feel they are receiving fair treatment. The arrival of sizeable groups of asylum seekers has resulted in the creation of special facilities for their integration. This may be inevitable sometimes, but for reasons of equity and legitimacy education, job-market and housing-related facilities should be open to all citizens and not just to refugees.

\section{Investing in mutual socialisation}

All these ethnic groups would probably be able to coexist more smoothly if they had a better understanding of each other's cultural backgrounds. They can learn more about each other at schools, on the shop floor and out in the various neighbourhoods. Current civic education classes in schools seek to educate pupils on their peers' backgrounds and cultures, but there is still room for improvement in these courses. Knowledge of diverse ethnic 
groups could be integrated more fully into other subjects, such as geography and history.

In addition, a well-thought-out strategy on how to ensure that all migrants are introduced to society will be vital. All newcomers, not just the refugees, but also the highly skilled migrants and the labour migrants from other EU countries, must be given the tools to join in society as soon and as smoothly as possible. It may be useful in this respect to create general public services that can be accessed by all migrants, irrespective of their legal status, plans for the future or length of stay. It is crucial that the municipal authorities, rather than the national ones, be placed in charge of these introductory services. After all, they are best able to decide on the right track for every newcomer, since they know best what kinds of people live in their towns and what individual migrants' personal situations are like.

\section{Public familiarity}

Promoting contacts between the residents of a neighbourhood should be a major focus area. Connectedness strategies do not have to be designed to make people bond and become good friends. All they have to focus on is making neighbours familiar with each other, which is a more realistic plan anyway. The idea behind public familiarity is that a neighbourhood's residents should recognise each other in public spaces, even if they do not actually speak to each other (Blokland \& Nast, 2014). If people who otherwise have nothing to do with each other regularly see each other in public spaces, they will still end up becoming "familiar faces" to each other. This will give them a better feel for who can be trusted and who cannot, which will cause them to feel safer. Furthermore, people are more likely to feel at home in a neighbourhood if they feel known, and if they have a proper understanding of the social codes of its public spaces.

\section{Challenges for the discipline}

What does the rise of this new diversity in society mean for the discipline of public administration and public governance? Firstly, it requires a reconsideration of the curriculum in our bachelor and master programmes. Coping with increasing ethnic heterogeneity and turnover among residents is one of the major challenges in the public domain for the decades to come, and we have to prepare our students for this challenge. After all, many of our students will be the civil servants, policy analysts and street-level bureaucrats who have to manage the increasing variety among citizens and the tensions that come with it. This requires that we 
provide them with the relevant empirical data, theoretical frames and practical skills. Some of these skills may be learned on the spot, as our own institutes are becoming much more heterogeneous due to the rapid influx of students and staff with a migration background. In the Netherlands, many departments of public administration and schools of governance have internationalised rapidly in the past decade and have many students and staff members from other EU or non-EU countries. The influx of students and staff with a more traditional migration background lags behind and varies between institutes. Universities in majority-minority cities, such as Rotterdam and Amsterdam, tend to have much higher percentages than universities in the provincial cities.

Secondly, it should have consequences for our research agendas. The challenges for public governance which we outlined above should also inform our research agenda. We should provide policy-makers and public managers with empirical evidence about what works best in coping with these challenges. This is an agenda across all subfields in our discipline, For HRM the issue is how to deal with increasing heterogeneity in the workplace, how to minimise the transaction costs of an international and diversified staff and how to maximise mutual understanding. For public management the issue is how to deal with the increasing heterogeneity and turnover of clients, how to treat everyone, both immigrants and native groups, in a fair and non-discriminatory manner, and how to socialise all newcomers sufficiently, even those who stay only temporarily. For policy studies, the issue is how to find a balance between targeting and mainstreaming, between effectively attending to the needs of specific groups and preventing stigmatisation or discrimination. For local governance studies, the issue is how to deal with the increasing variety among and within municipalities, and how to maximise social cohesion and public familiarity among residents and minimise feelings of discomfort and insecurity.

\section{From new to commonplace diversity}

Living together in super-diverse societies is a source of social tensions. The books of V.S. Naipaul, Salman Rushdie, John Steinbeck, Octavio Paz, Tom Wolfe and Zadie Smith attest to this. The arrival of new waves of immigrants distorts the delicate balance of power between settled groups and outsiders. This will lead to feelings of discomfort, discrimination and social conflict.

Public policy and public institutions will not be able to make all these tensions disappear. In the short run, it would already be quite an achievement if inhabitants of super-diverse neighbourhoods and municipalities were able to cope with this new diversity. Wessendorf (2010) speaks of "commonplace 
diversity." This implies that citizens do not consider super-diversity as something special, but see it as an everyday reality which is integrated into their daily routines. They pragmatically accept large differences in ethnic origin in public spaces, such as streets, shopping centres and public transport, and even in schools or at work. At the same time, they tend to live together with their ethnic peers in their private lives. Ignatieff calls this "side by side living": in some very diverse neighbourhoods inhabitants manage to live together in the public space, but they also feel the need to live apart in communities in which they can be amongst likeminded others. The challenge for public governance is to maintain a balance in this multitude of LAT relationships.

\section{Notes}

1. This chapter is based on a study we conducted for the Dutch Scientific Council for Government Policy (Jennissen, Engbersen, Bokhorst \& Bovens, 2018).

2. The UK and Sweden also experienced a wave of labour migration in the 1960 s and early 1970s. However, the labour migrants who came to these countries mainly originated from a neighbouring country with a lower level of prosperity, the Irish Republic and Finland respectively (MacLaughlin, 1993; Hammar, 1995).

3. We follow the definition of Statistics Netherlands. A resident with a migration background is defined as a person who lives in the Netherlands and has at least one parent who was born abroad.

4. According to the UN there were only 193 plus 2 (Vatican and Palestine Authority) countries in 2019. However, the country of origin is based on the country in which the migrant or one of his parents was born. Many migrants hail from countries that no longer exist, such as Yugoslavia, Czechoslovakia, the Soviet Union or even Austria-Hungary. This explains the higher number.

5. Source: our own analysis on the basis of data from Statistics Netherlands. Compare also Hartog \& Zorlu (2009).

6. There is some successful party formation among the "traditional" migrant groups, particularly among citizens with a Turkish and Moroccan background, especially in the larger cities, where parties like Denk or Nida have obtained seats on the council. The former also has two seats in Parliament.

7. See, for example, Tolsma \& van der Meer, 2014; Koopmans, Lancee \& Schaeffer, 2015; Abascal and Baldassarri, 2015.

8. We combined the data (Stelsel van Sociaal-statistische Bestanden - SSB) of Statistics Netherlands with the Dutch Police Statistics (Basisvoorziening Handhaving), and the Dutch Safety monitor 2014. The SSB contains data for all 17 million inhabitants of the Netherlands. The Police Statistics contain all persons who have been registered as criminal offenders. Our analysis was limited to all inhabitants between the ages of 12 and 60 $(\mathrm{N}=10.746 .180)$. The Dutch Safety Monitor is an annual national survey regarding safety, quality of life and victimhood. In 2014 over 86,000 people were interviewed in 403 municipalities and 8,798 neighbourhoods in the Netherlands. 


\section{References}

Abascal, M., \& Baldassarri, D. (2015). Love thy neighbor? Ethnoracial diversity and trust reexamined. American Journal of Sociology 121(3): 722-782.

Blokland, T., \& Nast, J. (2014) From public familiarity to comfort zone: The relevance of absent ties for belonging in Berlin's mixed neighbourhoods. International Journal of Urban and Regional Research 38(4): 1142-1159.

Castles, S., H. de Haas, \& Miller, M. (2014). The age of migration: International population movements in the modern world: London: Palgrave Macmillan

Hammar, T. (1995). Labour migration to Sweden: The Finnish case. In R. Cohen (ed.). The Cambridge survey of world migration (pp. 297-301). Cambridge: Cambridge University Press.

Hartog, J., \& Zorlu, A. (2009). Ethnic segregation in the Netherlands. An analysis at neighbourhood level. International Journal of Manpower 30(1-2): 15-25.

Glas, I., Engbersen, G., \& Snel, E. (2018). The street level and beyond: the Impact of Ethnic Diversity on Neighborhood Cohesion and Fear of Crime among Dutch Natives and Non-Natives. Journal of Urban Affairs 41(6): 737-755.

Jennissen, R., van Wissen, L., \& van der Gaag, N. (2006). Searching for similar international migration trends across countries in Europe. Genus 62(2): 37-64.

Jennissen, R., Engbersen, G., Bokhorst, M., \& Bovens, M. (2018). De nieuwe verscheidenheid. Toenemende diversiteit naar herkomst in Nederland. Den Haag: Wetenschappelijke Raad voor het Regeringsbeleid.

Ignatieff, M. (2017). The ordinary virtues: Moral order in a divided world. Cambridge: Harvard University Press.

Mac Laughlin, J. (1993). Ireland: An "emigrant nursery" in the world economy. International Migration 31(1): 149-170.

Koopmans, R., Lancee, B., \& Schaeffer, M. (eds). (2015). Social cohesion and immigration in Europe and North America. Mechanisms, conditions and causality. New York: Routledge.

Putnam, R.D. (2007). E pluribus unum: Diversity and community in the twenty-first century. Scandinavian Political Studies 30(2): 137-174.

van der Meer, T., \& Tolsma, J. (2014). Ethnic diversity and its effects on social capital. Annual Review of Sociology 40: 459-78.

Wessendorf, S. (2010). Commonplace diversity: Social interactions in a super-diverse context. Londen: Palgrave Macmillan.

White, P.E. (1993).The social geography of immigrants in European cities: The geography of arrival. In G. R. King (ed.). The new geography of European migrations (pp. 47-66). London: Belhaven. 


\section{In Search of a Better Understanding of Cultural Diversity in European Public Administration Research and Practice, with a Focus on Religion and Language}

Bogdana Neamtu

\section{$1 \quad$ Introduction}

A couple of years ago, when I first became acquainted with diversity literature, my great puzzlement - coming from a somewhat legal background - was that a significant number of articles seemed to start with a profound quotation, story or metaphor. I quickly understood that the authors were resorting to this writing strategy in order to offer some sort of conceptual clarity to their readers before they became submerged in the complex and sometimes confusing jargon of cultural diversity literature. Following the tradition of respected scholars in cultural diversity, I start my essay with two tales about multiculturalism and cultural diversity. Thus, in section 2, I offer a brief overview of a city and a university in Romania, both described as multicultural or culturally diverse. By briefly comparing the two I try to identify common traits of multicultural organisations/communities. In section 3 , drawing on a variety of sources from literature, I propose a main framework for analysing and understanding cultural diversity in Europe. In section 4, I explore two dimensions of cultural diversity which are currently under-researched, namely language and religion. In that section, I try to suggest additional avenues for future research and propose some secondary frameworks for analysing cultural diversity. Section 5 comprises my conclusions and some suggestions for how European PA research in this field should move forward. 


\section{Starting a conversation about cultural diversity - Two tales, one label}

I currently live in a city (Cluj-Napoca, Romania) and work for an organisation (Babes-Bolyai University, Romania) which both describe themselves as "multicultural" and credit their success to this unique attribute of "multiculturalism" which sets them apart from other communities and organisations, not just in Romania but also in Europe. But what does multiculturalism really mean? How do we recognise a multicultural community/organisation? What is the impact of multiculturalism on how communities and (public) organisations perform? What kind of research questions would these tales spark for a scholar in Public Administration and how are they connected with broader trends in European PA research? In the remaining part of this introductory section, I will reflect upon these two tales of multiculturalism from Romania in an effort to start a meaningful conversation with regard to how culture and diversity are becoming even more diverse in Europe and worldwide, and how PA research and practice should react to these developments.

\subsection{The multicultural city}

Cluj-Napoca (alternative names: Klausenburg in German; Kolozsvár in Hungarian) is currently the second largest city in Romania, after the capital city of Bucharest, with a general population of approximately half a million people (over the entire metropolitan area), of whom around 100,000 are students. The origins of the city date back more than 2,00o years to the Roman period. The ancient city, named Napoca, was one of the main settlements of the Daco-Roman Province. Its economic and cultural importance was maintained during the medieval period when the city developed as one of the seven major fortified cities in Transylvania, described in the German language using the term Siebenburgen. Today, due to its rich historical past, the city has a multi-ethnic and multi-confessional population structure. Out of the entire population, Romanians constitute $80.07 \%$, Hungarians $15.97 \%$, Rroma 1.06\%, Germans 0.16\%, Jewish $0.05 \%$, and other nationalities $2.69 \%$ (Cluj-Napoca city hall official website). The confessional structure is: $69.09 \%$ Orthodox, 10.11\% Reformed, 4.84\% Roman Catholic, 4.73\% Greek Catholic, 2.68\% Pentecostal, 1.1\% Unitarian and $0.32 \%$ Baptist (National Institute for Statistics, Romania, 2011 census).

Multiculturalism in the context of Cluj-Napoca seems to signify more than just a community characterised by ethnic or cultural heterogeneity. Being multicultural implies that cultural diversity is cherished and distinct cultural 
communities are encouraged to coexist. At least at the level of strategic documents (Local development strategy for Cluj-Napoca municipality 2014-2020, p. 261), the city claims that cultural differences are regarded as a source of strength which confers on the city its unique character. The city is envisaged to become a landmark in Europe due to its "dynamic culture, which supports experimentation and initiative." Moreover, "culture is meant to represent a transversal factor in the organization of the community; culture should thus become the driver for social transformation and urban regeneration" (261). The Hungarian community is politically represented by five local councillors. One of the vice-mayors is Hungarian - this represents more an informal tradition than a written legal rule; however it tends to be respected. While significant progress has been made on the road towards managing diversity with regard to the Hungarian community, the situation of the Rroma population, which represents the second largest ethnic minority, is different. It is marginalised and is located both physically and socially on the outskirts of the city. Recently, efforts have been made to integrate this community into the mainstream social and economic life of the general community; however significant challenges continue to exist in this respect. It is difficult to say whether Rroma culture is present in any significant way in the public life of the city.

Cluj's economic and social life is currently booming, while the city is regarded as a model for collaborative governance: the associative sector is very well developed and businesses are regular partners of the city in designing and implementing various public policies. Its multicultural character is specifically invoked as the main factor as to why the city's population is extremely tolerant and welcoming of foreigners. While some surveys legitimate these claims (Eurostat, 2014), most evidence concerning the positive influence of multiculturalism on the city's development is anecdotal.

\subsection{The multicultural university}

In 2010, Angela Merkel, the German Chancellor, described Babes-Bolyai University as follows: "A great example of what we want to achieve in Europe: cooperation between people, cultures and languages, regardless of their origin and confessions" (Babes-Bolyai University website). The University itself, while defining its mission, refers to "multiculturalism, intercultural dialogue and collaboration between different religions."

Babeș-Bolyai University is today the oldest and the largest university in Romania. The institution's roots go back to the Renaissance period when the precursor organisation of Babes-Bolyai University was created in 1581 as a Jesuit college. Its great ethnic and cultural diversity was apparent from the 
very beginning: "the first rector was Polish, the teachers were Italians, Poles, Hungarians and Germans, and the students were to be of different confessions and ethnicities" (Babes-Bolyai University website). Over time, the teaching languages of the university have been Latin, Hungarian, German and - after the First World War - English or other international languages (Babes-Bolyai University website). The history of the university in a way mirrors the cultural, scientific and denominational traditions in Transylvania - where was laid down for the first time in Europe since the sixteenth century the principle of religious tolerance (Babes-Bolyai University website).

The linguistic and cultural diversity is the defining element of this university. Babes-Bolyai University acknowledges the multicultural and multi-ethnic character of the community it is located in and endeavours to play an active role in this respect. In its Charter, the University has set up lines of study in Romanian, Hungarian and German. These lines of study have their own representation within the university's leadership structure (Vice-rectors and the Senate). They also enjoy autonomy of decision at all levels (department, college, university). Institutional arrangements are in place in order to facilitate dialogue among different lines of study (Commission for intercultural dialogue) (Babes-Bolyai University website). "From a linguistic point of view, UBB has one of the most complex systems in Europe: each student can freely choose the language in which he/she will study (English, Hungarian, German or another international language), and the study programmes may be followed in full in the chosen language" (Babes-Bolyai University website).

\subsection{What could potentially be of interest for PA research in light of the two tales?}

My two tales seem to suggest that diversity/multiculturalism is always good and that both the city and the university are currently thriving because of this. Further forays into diversity literature will establish that this is not the case: at the level of both organisations and communities, diversity can lead to conflict, poor integration of minorities, lack of trust among individuals/ groups who are different from each other, etc. I think that this is where PA research could step in and offer scientific evidence about these aspects which at this point, at least in the Romanian context, are merely anecdotal.

The list of questions included here is by no means exhaustive. The purpose is simply to draw attention to issues which could potentially be explored in other jurisdictions in light of my two tales. As already discussed, one area for future research would be related to understanding the effects of values and practices falling under diversity/multiculturalism on the outcomes of organisations and 
communities. Are citizens in a community run on the basis of multicultural values and practices more satisfied with public administration institutions or with their lives compared to those living in a regular community? How do they perceive inclusiveness? Does a multicultural community or a multicultural organisation try to achieve different outcomes and how do citizens perceive this? What kind of methodology could be used to measure the effectiveness of a managerial model focused on diversity/multiculturalism? A second area of research refers to the values that fall under diversity/multiculturalism and how they influence public service norms and ethics in practice. What diversity values are reflected in strategic documents pertaining to organisations/ communities? Are these values significantly different from the ones regular organisations/communities are guided by? Is multiculturalism a source of norms for administrative/organisational practices (empirical research)? A third possible area of interest refers to the influence of multicultural values on the actual functioning (processes) of communities and cities. Do multicultural organisations/communities treat their "clients" differently in the process of service delivery from regular communities/organisations? Are there explicit goals and operational practices in place which incorporate values pertaining to diversity/multiculturalism?

\section{Main framework for analysing cultural diversity}

In the previous section I described a multicultural city and university. While doing my initial assessment of the two case studies, I felt that I was missing a sufficiently comprehensive theoretical framework. Literature usually focuses on one aspect pertaining to this conversation (for example types of strategies or programmes for managing cultural diversity), while leaving aside other considerations which could be relevant. While preparing this contribution I discovered that how one frames the conversation is key when dealing with fuzzy topics. In this section I propose a main framework which is mostly based on the work of van Ewijk (2011). The framework is built around three main questions: What is cultural diversity?; Why do we need cultural diversity?; and How do we achieve it?

\subsection{Defining diversity - the "what' in our discussion}

There seems to be significant theoretical ambiguity and controversy with respect to how one defines diversity. Scholars often do not explicitly state what they understand by diversity, and this is important especially when implicit 
assumptions about what diversity is greatly impact on their findings and policy recommendations (van Ewijk, 2011, p. 681). In a broad sense, diversity refers to differences between people or groups. The characteristics or attributes used to indicate difference need however to be meaningful, meaning that they are likely to influence the identity and the way of life of an individual (van Ewijk, 2011, pp. 684-685). Vertovec (2009) uses the concept of "modes of differentiation" to describe those principles based on which people identify themselves as different from others.

Scholars usually focus either on all-encompassing modes of differentiation such as culture and or/ethnicity (Jones, 1998), which however are sometimes too generic (Thompson, 2008, pp. 543-547) or they opt to address narrower modes of differentiation such as gender, age, class, income, educational background, immigration status, etc. One interesting option in terms of defining diversity, which is relevant with regard to some attributes discussed in section 4, namely language and religion, refers to the binary categorisation of diversity: individual (thereby unique) or collective (members of a group share a specific characteristic which makes them different and affects all members in a similar way) (van Ewijk, 2011, pp. 687-688).

Diversity needs to be understood as a social construction (Campbell, 2000). This means that certain attributes used to differentiate people depend heavily on the context (Triandis, 1995). If context is important, then one huge challenge posed by diversity literature is that it is almost exclusively about the US. Identifying all the differences between US and European contexts is beyond the scope of this essay. However, I would like to mention perhaps three differences that to me are key to understanding diversity in proper context. Firstly, as Wrench (2007) points out, the size of the ethnic population in the US is perhaps five times that in European countries ( $25 \%$ compared to $5 \%-6 \%)$. Secondly, the legal context in the US has long been characterised by strong anti-discrimination legislation and affirmative action, coupled with strong penalties for breaches (van Ewijk, 2011, p. 681). In EU countries, despite increased pressure from European Union anti-discrimination directives, the legal climate is considerably weaker than in the US (Buhrmann, 2017, p. 1636). Thirdly, the most important feature of the European context deals with the existence of nation-states which has meant that training for immigrants and integration into the labour market represented the predominant features of the European diversity policy until the end of the 1990s. This context of nation-states stands in stark contrast to the historic role in absorbing immigrants played by the US (van Ewijk, 2011 p. 683).

While the US-Europe dichotomy is useful and needs to be acknowledged, it is equally important to understand that Europe is not one uniform context 
either. Are there clusters of countries in Europe with a similar context for studying diversity? Is local context more important than national or regional (international) context? In general, the study of European PA is heavily based on clusters of countries (Nordic, Continental, Anglo-Saxon, etc.). The Romanian administrative system, for example, can be described as based on the French one, with some innovations from other systems brought about by years of reform predating our accession into the EU. However, in terms of cultural diversity, the case of Romania cannot be understood by looking at France, Spain or Italy, or by investigating the neighbouring countries of Eastern Europe which were under communist rule after the Second World War until the early 1990s. In my opinion, local context and intra-country differences may be more important in understanding cultural diversity. In Romania, the ethnic minorities, among which the Hungarian is the largest, are concentrated mostly in Transylvania (the central region of the country). Moreover, there is a cluster of two counties where in fact the Hungarian ethnic minority represents the majority of the population (over 70\%). This situation makes intra-country difference more significant than other context-specific factors. On the other hand, specific attributes of diversity, such as gender equality, might exhibit some commonalities in the region of former communist countries, due to the formal claim of communist ideology to support gender equality and equal treatment of workers irrespective of their gender.

\subsection{Motives underlying diversity policies - the "why" in our discussion}

The distinction between "why" and "how" in diversity literature is not always clear, in the sense that it is difficult to separate the means from the ends. Van Ewijk (2011) is, to the best of my knowledge, the only author who proposes an analytical framework which completely separates the two. My proposed framework does not entirely separate the means from the ends but acknowledges that this is something to be considered.

Kelly and Dobin (1998) identify three different sets of values underlying each of the main diversity policy approaches identified in the US and worldwide. Thus, the values underlying the equal employment opportunity (EEO) approach are egalitarianism and meritocracy; those underlying the affirmative action (AA) approach are to remedy past wrongs; and those associated with diversity management are inclusiveness and a respect for difference. But if we refer specifically to Europe, things no longer fit so neatly into these categories. In Europe, AA programmes are not based on ethnicity. Rather, national governments use other proxies for encouraging the selection 
of ethnic students, such as geographical location in certain neighbourhoods or coming from schools which belong to certain priority educational areas (in France) (Sabbagh, 2002, p. 53). This also slightly changes the motivation underlying AA programmes in Europe. In general, AA and EEO policies and programmes are legally mandated (though the moral argument is often used in addition to the legal mandate) while diversity management is driven by practical concerns, where managers are interested in how diversity can support better outcomes and organisational performance.

\subsection{Diversity policy approaches - the "how' in our discussion}

There are very few scholars who have tried to offer a typology or classification of diversity policy approaches (Liff, 1997; van Ewijk, 2011; Wrench, 2007). One distinction that, in my opinion is absolutely crucial is that between equal opportunity approaches (EO) and diversity management approaches (DM). In general, the legal principle underlining the $\mathrm{EO}$ approach is one that stresses the importance of treating people equally irrespective of their sex, ethnic origin, religion, etc. (Dickens, 1994). This approach obviously has its limitations, as it is difficult to strip individuals of attributes such as gender (and the implications gender has, for example, in the case of women with children) for the purpose of organisational decision-making. In response, policies and measures which aim not only to limit discrimination but also to promote equality (Affirmative Action programmes or positive action) have been put in place (Liff, 1997, p.12). This implies that it is often important to recognise social group differences which lead to some groups of individuals being disadvantaged. The role of policies is thus to make sure that such disadvantages or barriers are removed and everybody can compete on an equal basis (Liff, 1997, p. 12). DM approaches are completely opposite to EO approaches with regard to their way of treating differences. The core idea behind DM approaches is to encourage organisations to recognise diversity and value it.

From a historical standpoint, AA and EEO policies predate DM. DM is "the new kid on the block," and literature regards DM as a positive step and superior approach when compared to earlier diversity policy approaches. It is perhaps worth observing the main differences which set DM apart from other diversity policies approaches. Thus, according to Wentling and Palma-Rivas (1997) these differences include the fact that in DM, top management plays a leading role, DM is a strategic element of the business plan, DM is linked to managerial performance evaluations and rewards, DM is long term, and 
it is inclusive of all employees. Moreover, Kersten (2000) argues that DM requires a systemic transformation of the organisation.

It is important to note here that not all the policies discussed require the same degree of organisational change (van Ewijk, 2011, p. 684). The question is whether by implementing diversity policies an organisation would require strategic changes at its core or just minimal ones. Equally important from the perspective of policy-making is the perceived relevance of social group differentiation. Even DM approaches are extremely diverse and they differ significantly in how they treat difference - either as something which is randomly distributed among individuals or as characteristics held as a result of being part of a group (Liff, 1997, p. 13; van Ewijk, 2011, p. 691).

\subsection{Revisiting the two tales in light of the proposed analytical framework}

After describing a potential framework for analysing cultural diversity, I think it is useful briefly to revisit my two tales of multiculturalism from Romania.

In terms of "what," I must admit that I conceptually struggled a bit with multiculturalism versus cultural diversity. Are they similar or somewhat similar? Am I using the wrong tales to start a conversation on cultural diversity? In my paper I describe the two concepts as being similar. In doing so, I draw on various scholars who define multiculturalism as "culturally derived differences found among various cultural communities; and [it] refers to a society within a country, which is characterized by ethnic or cultural heterogeneity" (Dwivedi, 2001, p. 2). I however acknowledge the literature which makes a distinction between diversity and multiculturalism and argues that multiculturalism, as opposed to diversity, usually takes power relations into consideration (Pope-Davis et al., 2003). This will become evident throughout my paper when referring to minorities which sometimes become majorities (inverted power relations) and in relation to the Rroma community in Romania. Multiculturalism is often defined in terms of a strategy or set of policies which have as "their overall goal the promotion of tolerance and respect for group identities, particularly of immigrants and ethnic minorities" (Vertovec, 2010, p. 83). Modood (2013, p. 1) also describes the essence of multiculturalism as "recognition of group differences within the public sphere of laws, policies, and democratic discourses."

My multicultural city and university refer to ethnicity as the main attribute for differentiation. Ethnicity is described in literature as a primary attribute (cannot be changed), while religion and language are secondary (can be changed) (Griggs, 1995). However, in the case of my two tales, ethnicity in my 
opinion incorporates language and religion. My case studies could however be described as selective multiculturalism because the second largest ethnic minority, the Rroma population, is absent. Interestingly enough, this initial multiculturalism, which refers to ethnicity, is now expanded to include other attributes. This is done in light of current patterns of migration to the city (professionals in multi-national companies represent a significant segment of all foreigners) and of the desire of both the city and the university to attract more foreigners in the form of investors, top executives, foreign researchers and international students. This seems to suggest a mutation from ethnicity towards "super-diversity" (Vertovec, 2010).

In terms of describing the strategy used by both the university and the city (the how in the analytical framework), it clearly falls, at least currently, under diversity management. The underlying objectives (the why), as can be depicted both from strategic documents and already implemented policies, are inclusiveness and respect for difference. Though certain legal requirements are in place, the actions of both entities seem to be driven by practical considerations rather than legal ones. The diversity strategies analysed score highly in terms of commitment by both the city and the university to social group equality and perceived relevance of social group differentiation for policy-making. Organisational commitment to equality is included by other authors in their proposed characteristics of cultural diversity. White (1999, pp. 482-483) argues that successful multicultural organisations are those where: a) top management plays a crucial and leading role in making diversity a success; b) diversity is part of the organisation's strategic business objective. In both cases analysed, the organisation's leadership is heavily committed and involved in advancing a multicultural agenda. The role of leadership is observable especially when examining the evolution of the city. From the mid-199os until 2004, Cluj had an ultra-nationalistic mayor, who often used to antagonise the Hungarian community. At that time, though the ethnic and confessional structure of the city's population was relatively the same as today, the city could hardly have been described as multicultural.

At first glance, diversity management seems to be superior to other diversity policy approaches. However, an in-depth understanding of the situation in the multicultural city and university reveals a somewhat different story. In reality, not all ethnic groups are equal. Here is where literature on multiculturalism and power relations actually comes in handy. While the Hungarian and German minorities enjoy a special status, the Rroma community is definitely marginalised. For this community diversity management is not producing any results. In this case, diversity management needs to be supplemented with AA actions. The university, for example, has such policies in place 
with regard to the admission of Rroma students. Cultural diversity can be managed in multiple ways, and AA programmes can co-exist with diversity management ones.

\section{$4 \quad$ Language and religion - two under-researched attributes of diversity in connection to PA}

In section 3, I have proposed a main framework for analysing cultural diversity approaches. In this section, I turn my attention to religion and language as attributes of cultural diversity and I will try to identify additional frameworks for analysis. Interestingly, language and religion are perhaps the most under-researched ones in the literature, both North American and European (Carrizales \& Gaynor, 2013; Sabharwal, Levine \& D’Agostino, 2018; Pitts \& Wise, 2010). I will also try to illustrate possible future research avenues with examples from Romania.

\subsection{Religion}

In my opinion, there are at least two main reasons why religion as an attribute of cultural diversity is under-researched. First of all, this subject seems to be taboo in relation to PA research. While preparing this contribution, I spoke with at least a dozen colleagues of mine, and they were all flabbergasted by the idea that PA research and teaching might touch upon religion and spirituality. As one colleague put it, "connecting religion and PA is the surest way to make sure your research will never be considered scientific and serious." This rejection is due not only to fear of breaching the principle of state-church separation (Garcia-Zamor, 2003) but also to the idea held by both academics in PA and public servants that a professional administration is rather neutral and secular (Houston, Freeman \& Feldman, 2008). I tremendously enjoyed the metaphor of a "naked square" used to describe PA research in connection to religion, stripped of all dialogue that is religious in nature (Houston, Freeman \& Feldman, 2008, p. 428). But practice does not necessarily mirror research; our naked square is not so naked if we think for example of practices in both the public and private sectors which already respond to religious diversity. My university, for example, allows students to take exams on a different date if the scheduled date conflicts with religious practices or holidays. But such policies do not always have to be directed towards valuing diversity. We have European countries where religious symbols reflected in dress codes are prohibited in public spaces (France is perhaps the best-known example). 
However, both policies, irrespective of their underlying goal, are interlinked with religious considerations. A second difficulty comes from defining exactly what religion is. Sometimes, religion is lumped together with culture, or at least discussed as being interconnected with culture (Massam, 1996). At other times, instead of religion, scholars talk about spirituality (Walker, 1998) and acknowledge that although there is a distinction, it is difficult to operationalise it. It also influences whether spirituality is portrayed as being individual (each individual experiences this mode of differentiation in a distinct way), while religion is somewhat collective (all people belonging to a certain denomination exhibit common and meaningful characteristics).

I honestly expected that, at least for the European research on diversity, the connection between religion and PA would be stronger, compared for example with the US. My expectation was due to the fact that European culture, throughout most of its history, has been heavily influenced by Christian belief and has been nearly equivalent to Christian culture (Koch, 1994). Religion in Europe has always been regarded as one of the more dominant forces influencing Western civilisation, concerning the development of philosophy, art, music, science but also social structure and architecture (Koch, 1994). This is not the case, however.

Houston, Freeman \& Feldman (2008), Freeman \& Houston (2010), and Shah, Larbi \& Batley (2007) all offer very plausible reasons why public administrations need to study religion, based on pragmatic considerations. They also propose future research questions taking into account existing gaps in research and methodologies. Firstly, one needs to consider the prominence of religion in the delivery of public services (by faith-based organisations). In many European countries, public-private partnerships between faith-based organisations and municipalities have long been praised as a reflection of New Public Management philosophy. The contracting-out of social care and medical services is a practice which will continue in the future as well. But do we know if faith-based organisations deliver these services more cheaply than the public or the business sector? Or do they provide better quality for the same money? These are very practical questions which influence the quality of services citizens get. And there is, to the best of my knowledge, only one study (Reinikka \& Svensson, 2003) which is unfortunately not related to a European country. Secondly, the current growing religious heterogeneity of populations in Europe, from certain countries, has implications for the internal operations of public action. It is impossible to ignore challenges such as the need for special food in school cafeterias or the requirement to provide special places and to schedule time during official working hours for prayer, or the need to allow for dress codes which reflect religious beliefs. As religious/ 
spiritual diversity increases, the requirements for policies which value this diversity will grow as well. Thirdly, there are some reasons to believe that religious/spiritual values influence the behaviour of public workers, their work ethic and their work performance. There is one empirical study from the US documenting this (Lowery, 2005) and literature on how values associated with Islam promote a strong work ethic (no empirical evidence is available though) (Ball \& Haque 2003). Specific work on European space is needed.

In the remaining part of this section I want to reflect upon one area where religion can have a significant effect in practice but is most often ignored. I refer here to religion viewed as "important for promoting civil society and replenishing a community's supply of social capital” (Houston, Freeman \& Feldman, 2008; Putnam, 2000). My reasons for ending this section with this issue are twofold: all available data pertaining to religious attitudes in my home country (Romania) show that people are still highly religious and that they trust the church as a social institution more than they trust public administration. Under these circumstances, I think that both research and practice need to reflect upon the role of religion and church in public space and in connection with public administration. Church and public administration, though playing separate roles in a secular society, can interact. Secondly, religion and ethnicity seem to be connected with the successful development of certain local communities in Romania, where anecdotal evidence suggests that social capital in the form of intra-community cohesiveness is extremely relevant.

Immediately after the fall of the communist regime, public surveys showed that Romanians mostly trusted the army and the church. Levels of trust of over $85 \%$ were regarded in disbelief by sociologists and considered abnormal for a laïc state. Perhaps it is worth mentioning here that such high levels of trust were mostly due to the fact that the church was perceived during the communist regime as one area of private life left relatively untouched by party interference. In 2018, the situation changed slightly: the church or the clergy had become involved in politics, sexual scandals were uncovered, and citizens are currently mostly dissatisfied with public money being given to churches without transparent procedures. That being said, the level of trust in the church (irrespective of religion) in 2018 was $68 \%$. Firefighters came first with a score of $82 \%$, while public institutions at local level stand at $46 \%$ (CURS Omnibus Survey, May 2018). Many laypeople as well as scholars claim that this trend illustrating declining trust is normal for a laïc society and what is happening in Romania is actually a convergence with other countries in Western Europe. While this may be the case, I am pointing in a different direction: is it wise to simply ignore the social relevance of the church as a community stakeholder 
and just wait for it to fade away? Or could PA institutions use the role of facilitator that the church can play and partner with it in more transparent ways? For example, public surveys show that people are still highly suspicious of NGOs and fund-raising activities. This goes back to the early 1990 os when these organisations were used for questionable purposes. People are more confident about donating money to churches as opposed to NGOs. I really think that this is one area for empirical research with implications for how non-profit-making organisations raise money from citizens.

Social capital at community level and how it is connected to religion and religious beliefs is another area worth investigating. In Romania, one stereotype we usually hear with regard to communities which are inhabited by the Hungarian population is that they are better developed than the rest of the communities; people work better together for community projects, etc. Very few researchers have actually tested such claims, and even fewer have attempted to issue policy recommendations for community development. Dumitru Sandu, a Romania sociologist who focuses on community development and development metrics, investigated whether development differences among regions can be explained in terms of cultural factors, including religion. He claims that when the model includes a variety of factors which could potentially impact on development, the importance of culture and religion is rather small. However, it is clear from his analysis that Hungarian communities tend to be richer and the ones with a majority of Rroma population, poorer (though present, these relationships are not statistically strong) (Sandu, 2011). Religion seems to act like ethnicity, with one difference: it plays a bigger role for cities than for rural communities (Sandu, 2011. p. 17). Sandu explains that religion counts in this analysis either as an explanation for different work ethic values or for different ways in which people relate to one another. This latter variable is further explored by David (2015) who links trust among people in communities with religious affiliation and ethnicity. It is clear that Hungarian Romanians are more likely to trust their neighbours than Romanians (59.1\% versus 40.95\%). Also, Hungarian Romanians are more likely to trust people of a different religion from Romanians (49.5\% versus 18.5\%). Of course, these data do not offer any causal explanation, but they do point towards future research.

\subsection{Language}

When I started to draft this contribution, I very much imagined that religion and language could be discussed together, as the two most under-researched attributes of cultural diversity. And while it is true that they share this feature of being under-researched, there are other aspects which set them apart. For 
example, while religion is vastly under-researched in the majority of social sciences, language has come to the forefront in social psychology and in literature on multiculturalism. Patten (2001, p. 693) argues that while religion can be completely disregarded as a matter which needs to be regulated by the state (what he calls a policy of disengagement, where there is no statesanctioned religion), this can hardly be the case for languages. Even if states do not officially sanction one language as official, practical decisions still need to be made because public authorities need a way to communicate with citizens and one specific or multiple languages still have to be used. Patten also refers to other practical considerations which add up to making language different from religion. He argues that most people speak only one language or multiple languages (the number is finite, however); that it is difficult for individuals to learn a new language; and that it is complicated for public authorities and citizens alike to use translation services which can be expensive and inaccurate (692).

In this contribution I refer to language as a marker of ethnic identity. I am however aware of other scholars who do not necessarily think that language is vital for an ethnic group's identity and that the group can survive even after losing or abandoning its language (Liebkind, 1999, p. 144). Also, some of the case studies and research questions discussed look at language as both an individual attribute and a social group mode of differentiation. In fact language "interweaves the individual's personal identity with his or her collective ethnic identity” (Liebkind, 1999, p. 143).

Studies on organisations and work teams within organisations seem to be one promising area which could deliver more PA research on cultural diversity (including language). The effects of ethnic diversity on work-related outcomes and performance (at both individual and group levels) have been researched thoroughly in the context of private sector organisations (Pitts \& Jarry, 2009). However, very little has been done in the context of public sector organisations (Wise \& Tschirhart, 2000; Buhrmann \& Schonwalder, 2017). Empirical research has produced mixed results (Shore et al., 2009), which need to be understood in the context of significant challenges regarding definition, measurement and examination of the effects of cultural diversity in organisations (Barinaga, 2007).

But no matter how fascinating the literature on organisations is, in a society where multiple languages are spoken on a daily basis and various ethnic groups differentiate themselves from others based on language, a more macro-level perspective is needed. How does a society decide upon the official language or languages? And how does such a decision impact on the language vitality of one ethnic group compared to other ethnic groups in that society? Numerous 
authors pertaining to various areas of social sciences all describe the importance of a certain type of official recognition or formal support offered by governments to one or multiple languages in their societies (Giles \& Johnson, 1987; Rata, 2017). Perhaps the most interesting framing of language policies comes from the work of Patten (2001). In search of a comprehensive framework, he argues that when it comes to their ethnic minorities, all states can apply three distinct language policies: official multilingualism (each language spoken in the community is granted the same recognition); language rationalisation (convergence on a privileged official language); and language maintenance (a policy of selective language recognition) (693-694). According to the same author, each of these three policies has attached its own motivation in terms of equality: official multilingualism offers equality of treatment among different languages; rationalisation ensures equality along non-language dimensions (such as social mobility of individuals); while language maintenance refers to equality in the sense of degree of satisfaction of interests of speakers of different languages (710). This comprehensive framework allows us to analyse the language policies of various European countries, but it also indicates that no matter how comprehensive the framework is, "complications" are bound to occur when using it to understand concrete situations. In the following lines I will briefly reflect upon how this framework could be potentially used to explain the language policy in place in Romania. I also use this national case study to point out how additional research questions linked to more traditional topics in PA research can be derived from here.

Among the countries in Eastern Europe Romania is an interesting example with respect to language rights granted to national minorities. In general, these language rights can be described as extensive, especially compared to the situation encountered in other neighbouring countries. They include the right to study in the minority language, to use the minority language in the relationship with local authorities, to be informed in your own language about important issues at the local level (decisions of Local Councils for example), etc. In this section I am referring only to the use of language rights in connection with public sector organisations. The Romanian Constitution, the framework law on local public administration as well as implementing norms for the law on local public administration all contain the following major provisions: in communities where the percentage of ethnic minorities exceeds $20 \%$ compared to the total population, they can use their language in the relationship with local/county authorities (self-governed bodies) as well as with county branches of the national government (deconcentrated institutions); Decisions of the local/county councils in these communities need to be communicated also in the language of ethnic minorities (the same provisions apply for the agendas of 
the meetings); Local/county council meetings can be held in the language of the minority, provided one-third of councillors belong to the ethnic minority in question (a translation in Romanian needs to be provided); in those communities with over $20 \%$ of ethnic minorities, street signs need to be in the language of the ethnic minorities; irrespective of the percentage of a certain ethnic minority, citizens can petition the local and county authorities in their own language and need to be replied to in both Romanian and the language of the petition. In 2017, a proposal was discussed in the parliament regarding the reduction of the percentage of minority population needed for the granting of language rights from $20 \%$ to $10 \%$. The amendment was finally not adopted but caused friction between the majority in power and the Hungarian political party representing that ethnic minority. There are very few legal provisions which explicitly focus on the recruitment and hiring of civil servants belonging to ethnic minorities. One relevant provision for our discussion deals with the possibility local communities have to hire people who speak Hungarian (mandatory conditions) for positions in the area of public relations where such public servants are expected to interact with ethnic minority citizens.

If we employ Patten's framework, the language policy can be described as official multilingualism motivated by equality of treatment among languages/ individuals. Of course, as mentioned before, there are practical "complications" - not all languages spoken are officially recognised, and even this is subject to a minimum ceiling. But, as Patten argues, these complications and slight departures from equality of treatment among all spoken languages are acceptable as long as the overall objective of this policy is in place. When governments choose one language policy over the others a normative/deontological choice is activated. But while none of these models is necessarily superior to the others, in practice consequences do occur. One concern with multiple official languages is that individuals might become marginalised and thus limited in their social mobility. It is easy to imagine that one person who is schooled mainly in a minority language will experience difficulties finding a job which requires fluency in a different language. Multilingualism can also increase costs for government of translation, the training of civil servants as well as the provision of parallel services in all recognised languages. Other implications are also possible. At least in Romania, 30 years after the choice of the somewhat multilingual model was made, we (including public authorities) know very little about these practical consequences. We do not know how students schooled exclusively in their mother tongue perform in the jobs market (if their social mobility is limited), or how civic participation and deliberation take place in multiethnic communities - which language is used, and which is excluded as a result of this decision. And while the language 
policy is very unlikely to be revisited, it will at least be good to know about practical consequences and how some of them can be addressed.

Interestingly enough, the language policies at the macro-level create implications also at an organisational and individual level (individuals working in those public organisations). This interplay between the macro and micro levels should be investigated in the future. I will only point to several possible research questions. Are recruitment and selection policies of civil servants who need to be proficient in multiple languages transparent? Do unilingual candidates perceive as unfair their exclusion from positions which require knowledge of one minority language? What kind of back-office policies do public organisations have in place to address requests made in languages of ethnic minorities? How do mixed teams of civil servants work and with what effects on individual and group performance? Do the local institutions mirror the population's structure from certain communities in terms of languages spoken? If not, are any policies in place to address it? What outcomes does lack of representation at the level of local bureaucracy cause?

\section{Conclusions}

One of the challenges of drafting this contribution came from the specific requirement of the editors to focus mainly on European PA and practice and how they relate to diversity literature. Quite frankly, while conducting research for this chapter I discovered that cultural diversity has never been an issue in PA in Europe and there is very little systematic research on important attributes of cultural diversity, such as language and religion. Most of the existing literature on this topic comes from the US. In a way, this strong tradition of research on cultural diversity from the US might have acted as a hindrance with respect to the development of a separate European body of similar research.

Why do we even need a separate body of research? In my opinion, this is because cultural diversity research needs to take stock of the diverse historic and demographic variety of Europe. This is a first layer, rooted in history, of the context which is so important when trying to understand diversity. But to simply investigate contextual differences in terms of the US versus Europe dichotomy is rather simplistic. Even within Europe, depending on what attribute or attributes of cultural diversity we are investigating, the context can vary both across national jurisdictions as well as within those jurisdictions. In addition to the first layer of diversity that can be traced back to historical evolutions in Europe, there is also new diversity which is currently unfolding 
in light of recent migration patterns. I will refer to this by proposing the term "super-diversity" for the future. This "super-diversity" will pose even more challenges as long as researchers and practitioners in European PA have not even figured out the old, historic diversity.

Very often when researching this chapter I found that language and religion are lumped together with culture. This is not particularly helpful when one is trying to develop strong research on these topics. In my opinion, they are two distinct attributes of cultural diversity which are too important and also too specific in their own way to be considered under the overarching concept of cultural diversity. Religion is usually avoided as a topic for research in PA because of the assumption that modern, Western states as well as their public administrations are neutral and secular. But this is not always the case. There are very concrete situations in which policies and the daily operation of public organisations are impacted on in one way or another by religion. If religion is sometimes brushed away from serious research preoccupations in the name of a secular and neutral PA, language I believe is more difficult to ignore. Language diversity is highly relevant and present in Europe as numerous European jurisdictions have adopted different policies which recognise and/ or encourage the use of languages pertaining to minority groups. Religion and language as topics for research in connection with Public Administration can be linked to topics which are already addressed in mainstream PA literature such as street level bureaucracy, public employee sorting and socialisation, etc.

A somewhat more mature body of research on cultural diversity at the European level seems to be focused on organisations or individuals within organisations, employing a human resources perspective (for lack of a better term). Of course, compared with similar research from the US, European research is limited. But diversity is not to be examined purely in an organisational context. Within many national European jurisdictions we have communities which exhibit cultural diversity to some degree or other. Are these different perspectives and scales at which diversity can be studied completely disconnected? One way to connect the organisational and the community levels for example is through the study of outcomes. Diversity is credited by most scholars to have some sort of influence on the individual's and/or organisation's performance. But what if we were to try and link diversity within organisations with outcomes outside organisations at a community level? This might suggest that if we have companies with a diverse workforce this might "enhance communities through both economic and social enrichment" (Shore et al., 2009, p. 129).

Clearly, perspectives from other disciplines are necessary if we are to develop a sound body of European PA research on cultural diversity. However, 
identifying these perspectives and integrating them with diversity literature is where the difficulty lies. In my contribution I also somewhat explored literature on multiculturalism. This was due to the fact that this literature was more familiar to me than other works from other connected fields. However, PA needs to develop its own research agenda on this topic.

\section{References}

Babes-Bolyai University website. Mission. Retrieved April 15, 2019, from https://www.ubbcluj. ro/en/despre/prezentare/misiune.

Ball, C., \& Haque, A. (2003). Diversity in Religious Practice: Implications of Islamic Values in the Public Workplace. Public Personnel Management 32(3): 315-330.

Barinaga, E. (2007). “Cultural Diversity” at Work: "National Culture” as a Discourse Organizing an International Project Group. Human Relations 60(2): 315-340.

Buhrmann, A. D., \& Schonwalder, K. (2017). Public Organizations and Diversity: Approaches to an Under-researched Topic. Journal of Ethnic and Migration Studies 43(10): 1635-1643.

Campbell, A. (2000). Cultural Identity as a Social Construct. Intercultural Education 11(1), 31-39.

Carrizales, T., \& Gaynor, T. S. (2013). Diversity in Public Administration Research: A Review of Journal Publications. Public Administration Quarterly 37(3): 306-330.

CURS, Omnibus Survey (May 2018). Trust in Institutions. Retrieved April 25, 2019, from https:// curs.ro/wp content/uploads/2018/05/Omnibus-CURS-mai-2018-1.pdf.

David, D. (2015). Psychology of the Romanian People. The Psychological Profile of Romanians in a Cognitive-Experimental Monograph. Iaşi: Polirom.

Dickens, L. (1994). Wasted Opportunities? Equal Opportunity in Employment. In Keith Sisson (ed.). Personnel Management in Britain (pp. 253-298). Oxford: Blackwell.

Dwivedi, O. P. (2001). The Challenge of Cultural Diversity for Administrative Culture and Good Governance. United Nations Expert Group Meeting on Managing Diversity in the Civil Service, New York, 3-4 May.

Freeman, P. K., \& Houston, D.J. (2010). Belonging, Believing, Behaving. Administration \& Society 42(6): 694-719.

Garcia-Zamor, J.-C. (2003). Workplace Spirituality and Organizational Performance. Public Administration Review 63(3): 355-363.

Giles, H., \& Johnson, P. (1987). Ethnolinguistic Identity Theory: A Social Psychological Approach to Language Maintenance. International Journal of the Sociology of Language 68: 256-269.

Griggs, L. B. (1995). Valuing Diversity: Where From ... Where To?. In L. B. Griggs, \& L.-L. Louw (eds). Valuing Diversity: New Tools for a New Reality (pp. 1-14). New York: McGraw-Hill.

Eurostat (2014). Eurostat Regional Yearbook 2014. Retrieved April 15, 2019, from https:// ec.europa.eu/eurostat/documents/3217494/5785629/KS-HA-14-001-EN.PDF. 
Houston, D. J., Freeman, P. K., \& Feldman, D. L. (2008). How Naked Is the Public Square? Religion, Public Service, and Implications for Public Administration. Public Administration Review 68 (3): 428-444.

Jones, P. (1998). Political Theory and Cultural Diversity. Critical Review of International Social and Political Philosophy 1(1): 28-62.

Kelly, E., \& Dobbin, F. (1998). How Affirmative Action Became Diversity Management. American Behavioral Scientist 47(7): 960-985.

Kersten, A. (2000). Diversity Management: Dialogue, Dialectics and Diversion. Journal of Organizational Change Management 13(3): 235-248.

Koch, C. (1994). The Catholic Church: Journey, Wisdom, and Mission. Winona, MN: St. Mary's Press. Liebkind, K. (1999). Social Psychology. In J. A. Fishman (ed.). Language \& Ethnic Identity (pp. 141-151). New York, Oxford: Oxford University Press.

Liff, S. (1997). Two Routes to Managing Diversity: Individual Differences or Social Group Characteristics. Employee Relations 19(1): 11-26.

Local Development Strategy for Cluj-Napoca Municipality 2014-2020. Cluj-Napoca City Hall. Retrieved April 15, 2019, from https://primariaclujnapoca.ro/strategia-de-dezvoltare-amunicipiului-cluj-napoca-2014-2020/.

Lowery, D. (2005). Self-Reflexivity: A Place for Religion and Spirituality in Public Administration. Public Administration Review 65(3): 324-334.

Massam, K. (1996). Sacred Threads: Catholic Spirituality in Australia, 1922-1962. Sidney, Australia: University of New South Wales Press.

Modood, T. (2013). Multiculturalism. A Civic Idea, $2^{\text {nd }}$ edition, Cambridge: Polity Press.

National Institute for Statistics, Romania (2011). 2011 Census for Population and Households, Final Results. Retrieved April 15, 2019, from http://www.recensamantromania.ro/rezultate-2/. Patten, A. (2001). Political Theory and Language Policy. Political Theory 29(5): 691-715.

Pitts, D.W., \& Jarry, E. M. (2009). Getting to Know You: Ethnic Diversity, Time and Performance in Public Organizations. Public Administration 87(3): 503-518.

Pitts, D.W., \& Wise, L. R. (2010). Workforce Diversity in the New Millennium: Prospects for Research. Review of Public Personnel Administration 30(1): 44-69.

Pope-Davis, D. B., Heesacker, M., Coleman, H. L. K., Ming Liu, W., \& Toporek, R. L. (2003). Handbook of Multicultural Competencies in Counseling and Psychology. Thousand Oaks, London, New Delhi: Sage Publications.

Putnam, R. D. (2000). Bowling Alone: The Collapse and Revival of American Community. New York: Simon \& Schuster.

Rata, E. (2017). Ethnic Revival. In F. M. Moghaddam (ed.). The SAGE Encyclopedia of Political Behavior (pp. 263-266). Thousand Oaks, California: SAGE Publications.

Reinikka, R., \& Svensson, J. (2003). Working for God? Evaluating Service Delivery of Religious Not-for-Profit Health Care Providers in Uganda. World Bank Policy Research Working Paper No. 3058. Retrieved April 25, 2019, from https://ssrn.com/abstract $=636420$. 
Thompson, S. (2008). Multiculturalism without Multiple Cultures?. Social Legal Studies 17(4): 543-547

Triandis, H. C. (1995). The Importance of Context in Diversity Studies. In S.E. Jackson, \& M. N. Ruderman (eds). Diversity in Work Team (pp. 225-233). Washington, D.C.: American Psychological Association.

Sabbagh, D. (2002). Affirmative Action and Science Po. French Politics, Culture \& Society 20(3): 52-64.

Sabharval, M., Levine, H., \& D’Agostino, M. (2018). A Conceptual Content Analysis of 75 Years of Diversity Research in Public Administration. Review of Public Personnel Administration 38(2): 248-267.

Shah, R., Larbi, G., \& Batley, R. (2007). Religion and Public Management Literature Review Working Paper 1-2007. Retrieved April 25, 2019, from https://www.researchgate.net/profile/ Richard_Batley/publication/238712151_Religion_and_Public_Management_Literature_Review/links/5a74703ca6fdcc53fe165f18/Religion-and-Public-Management-LiteratureReview.pdf?origin=publication_detail.

Sandu, D. (2011). Social Disparities in the Regional Development and Policies of Romania. International Review of Social Research 1(1): 1-30.

Shore, L. M., Chung-Herrera, B. G., Dean, M. A., Holcombe Ehrhart, K., Jung, D. I., Randel, A. E., \& Singh, G. (2009). Diversity in Organizations: Where Are we Now and Where are We Going?. Human Resource Management Review 19(2): 117-133.

van Ewijk, A. R. (2011). Diversity and Diversity Policy: Diving into Fundamental Differences. Journal of Organizational Change Management 24(5): 680-694.

Vertovec, S. (2009). Conceiving and Researching Diversity. MMG Working paper 09-01, Max Plank Institute for the Study of Religious and Ethnic Diversity, Gottingen. Retrieved April 15, 2019, from http://www.mmg.mpg.de/fileadmin/user_upload/documents/wp/WP_0901_Vertovec_Diversity.pdf.

Vertovec, S. (2010). Towards Post-Multiculturalism? Changing Communities, Conditions and Contexts of Diversity. International Social Science Journal 61(199): 83-95.

Walker, R. (1998). Preachers on the Payroll. Christian Science Monitor, June 25. Retrieved April 25, 2019 from https://www.csmonitor.com/1998/0625/062598.feat.feat.3.html.

Wentling, R. M., \& Palma-Rivas, N. (1997). Diversity in the Workplace: A Literature Review. Diversity in the Workforce Series report no. 1, MDS-934, National Center for Research in Vocational Education, University of California at Berkley.

White, R. D. (1999). Managing the Diverse Organization: The Imperative for a New Multicultural Paradigm. Public Administration \& Management: An Interactive Journal 4(4): 482-483.

Wise, L. R., \& Tschirhart, M. (2000). Examining Empirical Evidence of Diversity Effects: How Useful is Diversity Research for Public Sector Managers. Public Administration Review 60(5): 386-394.

Wrench, J. (2007). Diversity Management and Discrimination; Immigrants and Ethnic Minorities in the EU. Aldershot: Ashgate Publishing. 
PUBLIC ADMINISTRATION AND PRACTICES 



\title{
Shadowland: The Poorly-Mapped, Underdiscussed Yet Vital Interface Between Public Administration Research and Practice ${ }^{1}$
}

\author{
Christopher Pollitt
}

\section{Introduction}

Why "Shadowland"? Because PA research that is explicitly for practice is a murky arena. Both its full extent and its variety of forms are hard to discern. In these respects it is far less organised and transparent than is PA research aimed at academic publication. Academic publications and their authors are now recorded, measured and compared in a great variety of ways. Journals are classified as A, B or C. Books are classified as refereed and published by academic publishers or as having various lesser statuses. Individual articles have their impact measured by citation scores and a growing range of other numerics. Academic CVs usually give pride of place to a full list of different types of publication. Research for practitioners may get mentioned - especially if it results in an official report or a big grant - but it is usually less prominent and less influential on academic careers. Furthermore practitioner-oriented research is much less discussed - or at least discussed systematically and collectively - within the academic community than is the more academically oriented work. Some of what little discussion there is trades in tired old stereotypes - on the one hand practitioners with impossible deadlines and a politically flexible regard for scientific truths (bemoaned by academics); on the other hand slow, methodologically obsessive and politically naïve academics blundering inconsequentially through the sophisticated, fast-moving world of public policy-making (bemoaned by practitioners). Only recently have early attempts been made to classify and enumerate practitioner "impacts" more closely.

In this chapter I will attempt to document and contextualise this shadowland. Further, I will argue that it is a hugely important province of academic 
PA, and that we (PA academics) can and should do more to shed light into the shadows. Shadowland, I will contend, is both a large territory and a diverse one.

\section{The research/practice interface in the past}

Practitioner-oriented work has always been an important component of the field of academic public administration in North America, the UK and continental Europe (Lynn, 2005; Pollitt, 2016b; Raadschelders, 2011). It remains so today. As the head of one of the leading European centres of PA research put it:

PA research needs to remain connected to PA practice. Most of our students will not become academics after graduation, but are the public administrators and public sector cadres of the future. Their education benefits from research that is informed by or speaks directly to public administration practices. Besides, how in the long run can we legitimize the substantial public spending that goes into academic PA if we are not able to demonstrate that our field maintains an active and productive dialogue with the world of practice? (Bovens, 2010, p. 227).

In his authoritative 2011 review of academic PA Raadschelders identified four main intellectual traditions in the field: scientific knowledge, practical experience, practical wisdom and relativist perspectives (Raadschelders, 2011, pp. 156-173). Thus two of his four key historical and current traditions are practice-oriented. He distinguishes between these two by saying that practical experience is more technocratic, seeking instruments and techniques that will best accomplish particular functions, while practical wisdom is study for the development of practical wisdom and includes ethical and political considerations. Among the former he includes the eighteenth-century German cameralists, French sciences administratives and the work of Taylor and Gulick. Among the latter he locates Von Stein, Waldo and, more recently, Lindblom, Frederickson and Hood.

Looked at from the comparative perspective of national approaches to the study of PA, we find a similar intimacy between the administrative characteristics of a country and the way in which PA has been studied in that country. "In this book we will show that the academic study of public management is also influenced by the particular institutional context of state, politics and administration in the country concerned," writes Kickert (2008:2). 
Alleged global trends such as NPM turn out, on closer inspection, to have been significantly modified and adapted according to national characteristics (Pollitt \& Bouckaert, 2017).

Another aspect of the bond between academic study and practice is the ethical disposition of academics themselves. Many, probably most of them, want to contribute to the public good. This sentiment was perhaps best expressed by an American and a Flemish scholar, writing the preface to their edited collection on public service motivation:

\begin{abstract}
"Our interests in linking 'motivation' - an object of our academic values with 'public service' speaks to our commitments to improving collective action and the status of those seeking to improve the public sphere. We try to approach these commitments with objectivity, but we also have a passion for these commitments as human beings and as citizens" (Perry \& Hondeghem, 2008, p. vii).
\end{abstract}

Before going any further it is important to note that the concept of a "practitioner" has diversified considerably in the past quarter of a century. In many countries, especially the UK and the USA, the provision of large chunks of the public services has been contracted out to for-profit corporations, third sector non-profits and a variety of other organisations. Much effort and many words have also been expended on encouraging more public participation and "co-production" (see, e.g., Alford, 2009). Helping all or any of these may therefore also be a legitimate goal for academic research. Regrettably, this chapter is already bursting at the seams covering the academic (supply) side of the equation, and cannot go far into these "demand side" developments among practitioners.

In short, academic PA has always been closely intertwined with practitioner concerns. There are several reasons for this. One is that the world of practice has, on innumerable occasions, provided the intellectual stimulus for new academic investigations and theorising. The questions and problems that PA academics wrestle with have come as often from practitioners as from within academe. Another is that academic PA has been - and remains - reliant for funding and public support on the perception that part of its output will be relevant to the concerns of citizens and public bodies. And another still is that many PA academics want to help improve practice - they have an ethical commitment to improving the services citizens receive from their governments and public bodies. These considerations are highly salient when we consider the prospects for practitioner-oriented research in the future. 


\section{What kinds of thing can PA academics usefully do for PA practitioners?}

There are many things which academics can usefully do for practitioners, but we are short of information about how extensive each kind of activity is (Pollitt, 2016b, pp. 21-23 and 113-124). Therefore it is easy to give examples of practitioner-related activity (as I will do), but harder to form any reliable impression of how much of that kind of activity takes place. One complication is that what one might term the research content of activities varies a great deal. For example, an academic sitting on a government advisory committee may give advice based on her own research and/or the research of others, but will probably not conduct any new research in order to discharge her advisory responsibilities. In this example, therefore, research is being used, but not created. On the other hand, another academic may be commissioned to analyse the success or failure of a particular administrative reform, and to this end will collect data and carry out interviews in a way that can be said to be original research. Whether they are allowed to publish some or all of their research is another matter, depending on the precise terms of the commission.

In this section I will first set out a list of the main types of for-practitioner activity, and then analyse how each type may or may not be related to research. Subsequently I will focus on those activities that appear most likely to involve active research.

1. Giving informal advice to policy-makers and managers. In so far as policymakers and managers know and trust individual academics they may simply phone them and ask for advice. This is sometimes referred to as the "court jester" or "sparring partner" role - the individual academic is allowed to play with current ideas and suggest novel solutions, but all within the privacy of a closed conversation between confidantes. Although this (ancient) type of for-practitioner activity may occasionally be influential and although the ideas put forward by the academics concerned may be closely informed by research, these exchanges are by their nature "off the record." I was once part of an international group of academics invited to a conference in Ottawa. The deal was, first, that the Canadian government paid; second, that we had a couple of days to discuss some defined academic issues and draft chapters for a book; but, third, that we also had a lunch and some informal meetings with top Canadian officials to whose questions we were expected to respond. These were difficult questions and fascinating discussions. Even more elevated, a colleague of mine was once invited to a private audience with the Belgian King! However, we cannot estimate how much of this kind 
of activity goes on, or how much of it is research-informed, so we cannot for the purposes of this chapter do much more than note its existence as a category of interaction.

2. Serving in a public appointment alongside an academic appointment. In each of the four countries where I have worked as a PA academic many of my colleagues have also had formal public roles. In the UK, for example, some PA academics are governors of schools, some are Justices of the Peace, some serve on the boards of hospitals and some act as advisors to Parliamentary select committees. I was myself once appointed advisor to a European Commissioner on evaluation - which sounded impressive but was in practice a very occasional and informal activity. Knowledge of academic research may be useful in any of these roles, but creating new research is usually not part of them. Thus, when I served as a nonexecutive director of a National Health Service hospital, my arguments at board meetings were sometimes informed by my knowledge of academic research, but I undertook no new research in connection with that appointment. More generally, it is impossible to know how much fresh research is undertaken as a direct result of these public appointments, but my guess is not a great deal.

3. Taking part in the training of public officials. In many countries civil servants and other public officials at various levels receive specific training in management, sometimes in government-created institutions like the famous French ENA, and sometimes in a more contracted-out form (Pollitt and Op de Beeck, 2010). PA academics frequently participate as lecturers, coaches etc. In this they surely draw on research, but it would be unusual to carry out new research simply in order to give a lecture or seminar. Therefore, although training activities are numerous and the numbers of PA academics involved probably large, I would not expect this form of activity directly to produce much new, for-practitioner research. What this engagement often does do, however, is feed fresh practitioner ideas and problems into the minds of their academic trainers. That may well - indirectly - inspire later academic research.

4. Membership of a government-appointed commission or committee inquiring into a specific policy issue, or of a standing advisory committee. For example, a PA academic may be asked to sit on a committee investigating the organisation of social work, or the need to update planning legislation, or the reform of the civil service. This kind of role is more common for academics in some countries than others (e.g. it is probably more common in Sweden or the Netherlands than in Italy or Spain). Here the chances of launching new research are somewhat higher. These commissions may 
have the authority to commission research themselves and any academic members would normally be expected to be closely involved in any such initiative. Furthermore, if commissions publish their reports they may well also publish the supporting research (as the Fulton Committee on the reform of the UK civil service did as far back as the 1960 - Report of the Committee on the Civil Service, 1968). Less research-active, perhaps, are standing advisory committees, although many social scientists may be members of these, and they are conduits for much research-based advice (Barstow et al., 2014, pp. 151-152 and 166-168). Standing advisory committees may be less likely to order substantial new research than one-off committees enquiring into a specific policy problem, but on the other hand they make it easier for medium- and long-term relations of trust to be built up between academics and officials.

5. As participants in formal evaluations of public policies, programmes or projects. Many public programmes, in many countries, have built-in requirements that they should be formally evaluated. For example, evaluations are mandatory for projects financed by the EU structural funds (and a galaxy of consultancies and other evaluation units has grown up around this business). There are even evaluations of evaluations - once I was one of two academics commissioned to look back at the quality of evaluations in an EU programme over a number of years. PA academics led a major, large-scale evaluation of the Norwegian welfare reforms (NAV) of 2005-2012 (Askim et al., 2010). That PA academics are frequently called upon to participate in these exercises is hardly surprising - after all, the purpose of most of these evaluations is to assess the economy, efficiency, effectiveness and/or accessibility of public programmes, which are issues close to the heart of much academic PA. Interestingly, there is a long-running debate within the evaluation and policy analysis communities about how and how far it is possible to make good quality evaluations relevant to practitioners, and what exactly that might mean (see, e.g. Nathan, 1988; Patton, 1997; Pawson, 2013; Walker, 2001; Weiss and Buculvalas, 1980). Certainly, the academic evaluation community has its own theories, associations, conferences and journals, and it is a pity that relatively few PA academics seem actively to participate in these fora. Nevertheless, many evaluations are publicly available on websites, and many do entail genuine new research, even if often on a limited scale. This is, therefore, an activity where we can see PA academics sometimes undertaking research directly for practitioners. In addition to the evaluation reports themselves the academics concerned are sometimes able to negotiate with the commissioning bodies so that they are subsequently 
free to produce academic publications which "piggyback" on the evaluation itself (e.g. Askim et al., 2010; Joss \& Kogan, 1995; Packwood et al., 1998; Pollitt \& Roberts, 1994).

6. When an individual academic or team of academics is specifically commissioned to carry out a defined piece of research for a governmental or public body. Together with 4 and 5 (above) this is the least difficult form of interaction with practitioners in which to track academic PA research. And it clearly creates new, original research. The commission may be very detailed and specific, or it may be quite loose, with only broad statements of the goals and subject matter and the rest left for the academics to decide. Unsurprisingly, such research can be of very variable quality and focus. For example, I was at one point a member of a five-country team, composed mainly of civil servants, looking at the future roles of centres of government (what many academics term "core executives"). It was a stimulating and fascinating project (Ministry of Finance, 2013). The civil servants wanted to find innovative ways of improving government at the centre, very much including the centre's relations with other parts of government. The approach taken was to go to each country and look at projects and procedures that seemed promising. This was research, in the sense that we were gathering, structuring and comparing knowledge, much of it unpublished in the academic literature, at least at that time. And we were able to question some very talented and experienced officials who were working on innovative projects. From a strict social science perspective, however, this was all rather loose. We did not really have a well-defined research question. The selection of countries and subsequently of cases was not made according to any recognisable theoretical or methodological principles. In contrast to this rich but diffuse investigation one might cite, say, a research project commissioned by the Flemish Department of Education and Training in 2013. The problem concerned children with special educational needs, who under existing law were entitled to free transport to the nearest suitable school chosen by the parents. This had led to many unsatisfactorily lengthy commutes. The multi-disciplinary academic team from the KU Leuven which carried out the project was tasked with finding a better system for these children. First it conducted a literature review. Then it carried out exploratory semi-structured interviews with key stakeholders, with questions derived from the literature review. The resulting images of the nature of the problem were put before several expert panels to check their validity. After this, an international comparison of the problem was made - four countries were looked at and then two were chosen for deeper 
investigation and were treated to a similar analysis to that originally used for the Flemish case (Broucker et al., 2014). This was, in short, a careful, multi-method research study of a concrete, closely defined problem.

The importance of practitioner-oriented research can also be assessed by looking at the balance of funding and activity within specific academic units. A number of Europe's leading centres of PA research are heavily dependent on public sector funding for this type of research. In the non-elite universities in the UK this may loom particularly large, because they receive very little of the national flow of "pure" academic research funding. Instead they undertake plenty of smaller, practitioner-oriented projects, often for regional and local authorities and public bodies. Practitioner funding is also highly significant in the USA, both for elite institutions such as the John F. Kennedy School of Government at Harvard and for non-elite institutions and professional schools (Posner, 2009). In Europe my own Institute for Public Governance at KU Leuven may be taken as a more detailed illustration (see table 1).

Table 1 shows that students doing PhDs based on commissioned, forpractitioner research have been an important component of the Institute's activities for the past 15 years and even that, in some years, a majority of our students studying for PhDs have been connected with for-practitioner projects.

The sixfold classification of activities advanced above is far from perfect. It is not theory-driven. The six categories are neither comprehensive nor $100 \%$ mutually exclusive (some activities can blend into others - for example, 5 and 6). Nevertheless, it may serve for a preliminary exploration of our shadowland. It is at least immediately recognisable to most practitioners and academics alike. It is an essentially process-based classification - it enumerates the kinds of processes from which new research can emerge. Academic inputs to the processes would be expert staff, time, money and access. Outputs would be reports, workshops, the briefing of practitioner groups or individuals, and so on. Attempts to measure research "impacts" frequently concentrate on these outputs. Outcomes - usually difficult to measure - would be concrete decisions and reforms directly influenced by the research. Another important type of outcome could be shifts in practitioners' conceptual frames and models and ways of understanding - but such shifts, however consequential, are notoriously difficult to attribute directly to specific pieces of academic research (Weiss and Bucuvalas, 1980). Finally, as a process classification the one used here is subject to cultural limitations: outside western Europe and North America some of the activities described above may be unknown, and other kinds of activity dominate. 
Table 1 Doctoral Research at the Institute for Public Governance, Katholieke Universiteit Leuven, 2002-2016

\begin{tabular}{lccc} 
A. Year & B. Commissioned Ph.Ds & C. General Ph.Ds & D. Ph.Ds completed \\
\hline 2002 & 4 & 3 & 2 \\
2003 & 4 & 3 & 0 \\
2004 & 4 & 6 & 3 \\
2005 & 4 & 5 & 2 \\
2006 & 4 & 5 & 3 \\
2007 & 6 & 8 & 0 \\
2008 & 7 & 10 & 4 \\
2009 & 8 & 9 & 1 \\
2010 & 9 & 8 & 2 \\
2011 & 12 & 8 & 2 \\
2012 & 13 & 6 & 7 \\
2013 & 9 & 9 & 3 \\
2014 & 8 & 9 & 4 \\
2015 & 7 & 14 & 7 \\
2016 & 4 & 21 & \\
\hline
\end{tabular}

Notes:

1. Columns $B$ and $C$ show the number of PhD students studying and resident in the Institute in a given year. Column $D$ shows the number finishing/defended/awarded each year. Thus $B$ and $C$ are, in a sense, "stock" while D is "flow."

2. Even on this micro scale it is not always entirely easy to decide what is commissioned and what is not - there are so many varieties of detailed financial relationship and of degrees of specification as to what work shall be done

3. Behind the rise and fall in the numbers of commissioned $\mathrm{PhDs}$ over the years lie changes in the programmes and instruments of support for practitioner-oriented research offered by governments and public authorities.

There is another, deeper problem. Conceiving, as I do in this paper, that "for practitioner research" is something consciously aimed at practitioners, and usually specified and paid for by them (i.e. commissioned), understates the usefulness of PA research for the world of practice. The reason is simple: even research with the purest academic motives and the purest academic finance can easily turn up practitioner-relevant findings. Without meaning to, so to speak. Alternatively, academics may, in some countries, become political appointees charged with running particular reforms, and may subsequently 
return to academic life, publishing academic materials based on a mixture of their administrative experience and their academic skills. A theoretically and methodologically sophisticated example of this from the USA would be Kelman, 2005 (though this combination is usually not without its problems - see Hill, 2006).

In short the boundary between "for practitioner" and "academic research" is not as clear as we might have supposed (indeed, in some countries civil servants sit as members of the academic research panels which award or withhold grants to what are nominally pure PA research proposals). Regrettably, however, addressing the phenomenon of practitioner-useful findings coming from "pure" academic research cannot be taken any further within the bounds of this chapter. I lack both the space and the information to do it justice. Suffice it to say that estimating its extent and value is probably even more difficult than the present task of casting light into the shadowland of overtly pro-practitioner research. Optimists believe that its value is great, pessimists that it is small.

In sum, there are many ways in which academic research informs practitioners, and at many different levels (including all the activities 1 to 6). New research that bounces straight into the practitioner arena and directly influences identifiable decisions there is very much the exception rather than the rule (Bastow et al., 2014, p. 147; Lakey et al., 2014, p. 39). The paths to influence are complex, diffuse, indirect and sometimes quite lengthy in time (ideas waiting for their time to come). There is no equivalent "hard currency" to the engineers' patenting of new inventions. Occasionally, however, the direct trace of a piece of academic work can be seen in a new procedure, regulation or law (such traces most commonly result from activities $1,4,5$ or 6 ).

There are, therefore, several activities which frequently do generate new for-practitioner research (activities 5 and 6 and, to a lesser extent, 4). The full extent of these is very hard to discern, although Bastow et al. (2014) make a rare, valiant effort to scope the territory. Unfortunately for this chapter, their work is pitched at the level of the social sciences as a whole, and it is not possible to disaggregate PA from this larger basket. Furthermore, even they, for the most part, stop at the auditing of potentially influential contacts, plausibly arguing that it is just not feasible to go further up the causal chain towards influence on specific decisions (Bastow et al., 2014, pp. 52-53). Nevertheless, in many cases one can at least say that more impressionistic and specific assessments of PA chime quite well with the big picture of the social sciences set out in that book. If I were obliged to make a subjective estimate of how much academic PA research was mainly or entirely for practitioners I would put it somewhere between 25 and $50 \%$, but I cannot prove that. 


\section{4 "Relevance" and "impact"}

The recent flurry of interest in the "impact" of social science research in Australia (the EIA), the UK (the pioneering 2014 Research Excellence Framework - REF) and a few other countries has probably been due more to government shove than to any push from within the academic community itself. Nevertheless, alongside a frantic academic scrabbling for evidence of "practitioner relevance," it has resulted in some useful publications (e.g. Barstow et al., 2014; Denicolo, 2014). In this volume impacts are being dealt with in depth by Claire Dunlop, so, important though this aspect is, I will simply sketch a few key points.

In the UK the 2014 REF decreed that one-fifth of the research funds to be allocated by the Higher Education Funding Council for England (HEFCE) would henceforth be related to the "impact" of research. "Impact" was defined as follows:

"an effect on change or benefit to the economy, society, culture, public policy or services, health, the environment or quality of life, beyond academia" (REF, 2011, p. 48).

One does not need to be an academic to see what a vague and catch-all definition this is. A fundamental problem for PA is that even activities 5 and 6 (above) do not usually lead in any straight line to a concrete change or benefit. Rather the research findings enter a maelstrom of other ideas and influences which may - sooner or later, if a window-of-opportunity opens - lead to a decision (which may itself be fully implemented, partly implemented or not implemented at all). So we can count research reports delivered but even a first year PA student would not equate that with any actual "influence" on the on-going practitioner world. Equally, activity 1 may have a significant influence, but leave no audit trail whatsoever.

Other countries are following the UK in developing more structured approaches to assessing the impacts of academic research. For example, in 2017 the EU Parliament began to take a strong interest, as the Committee on Industry, Research and Energy called on "the Commission to provide a broader definition of 'impact', considering both economic and social effects" (Report on the assessment of Horizon 2020 implementation in view of its interim evaluation and Framework Programme 9 proposal, 2017)

Trying to measure impact is, therefore, quite fashionable - albeit within a broader situation where total research funding to the social sciences is usually being reduced. These are, however, early days. Impact measurement is far from 
being as sophisticated, as widespread or as influential on academic careers as the measurement of academic outputs. The trend originates mainly with governments and their funding authorities. The reaction of the PA academic community might be described as one of reluctant acquiescence.

\section{$5 \quad$ Current tensions}

As always, there are many current tensions in the academic world. Here I will identify just two, both of which hold strong implications for the conduct of practitioner-oriented PA research. The first is the way some important parts of PA academia seem to be slipping away from the world of practice. The second is the vigorous recent growth of rivals to universities as organisations producing research of interest to the worlds of policy-making and public management.

It may seem paradoxical to say so, but one current problem for practitioneroriented PA research is that the advancing professionalisation and specialisation of academic PA appears in some ways to be lessening its relevance to practice. Many of the career incentives for budding PA scholars point them towards sophisticated treatments of rather narrow and often very abstract issues (Pollitt, 2017; Posner, 2009, p. 19; Roberts, 2018, passim). These allow them to write the kind of theoretically explicit and methodologically rigorous papers that get published in the top-ranking journals. These papers, even if they can be understood by practitioners (often not), wholly fail to help those who must deal with the broad and messy policy and organisational problems of the day. In a previous publication I tried to count the share of articles published in five top PA journals that could be said to focus on specific contemporary programmes or organisations. Such articles had been fairly common in PA journals during the 1960 s and 1970s, but looking at the two years 2015 and 2016 I found that of a total of 580 main articles only 78 (13\%) were focused on such concrete contemporary concerns. The journals were Governance, International Public Management Journal, Journal of Public Administration Research and Theory, Public Administration, Public Administration Review and Public Management Review (Pollitt, 2017). They seemed more concerned with improving what philosophers' term "type" explanations and conceptualisations rather than "token" explanations or conceptualisations. Most practitioners, however, would probably give priority to the latter (for the type/token distinction see Dowding, 2016, pp. 50-55).

This finding in itself is not conclusive, in that perhaps plenty more practitioner-relevant material is being published elsewhere - in lower status academic journals, or in professional journals, or blogs (there are many more 
outlets than there were 30 or 40 years ago). But even if it is (unproven) that does not alter the influential fact that the "peaks" of academic publication are carrying very little of this kind of material - and it is scaling these peaks that attracts the most attention and career rewards within academia. Arguably, this is a mistaken direction in two ways. First, because of PA's deep roots in and mutual dependence upon the practitioner world, as already described. Second, because the brand of "rigour" that has been espoused seems an unduly restricted one, implicitly or explicitly downgrading the range of available conceptualisations of causality and explanation (for a vigorous yet inclusive treatment of these issues, written by someone who is himself a methodologically rigorous scholar, see Dowding, 2016).

Why has there been this apparent drift into the ivory tower? There have probably been at least four interacting and reinforcing processes. First PA academics have their output measured much more closely than did their forebears on the 1960s and 1970s. Most are subject to scoring systems that give the highest marks for publication in the top refereed journals. Second, units, departments and, indeed, whole universities have become far more competitive with each other than they were 40 years ago. The inhabitants now live a world of league tables, and their rankings are determined more by academic publications than for-practitioner work. REF2014's measurement of impacts may be a sign that this is beginning to change, but in most countries it has not changed much, and even in the UK research publications remain the most important element. Third, academic PA has become more professionalised (Stillman, 2015, p. xiii). Doctoral students are subject to far more formal training, especially in advanced methodologies, and are under greater pressure to publish early - preferably in the high-ranking refereed journals. This is all to the good, of course, but a perhaps unanticipated side-effect of greater professionalisation has been that newly qualified doctoral researchers are sometimes already far removed from the kind of work that practitioners tend to require, or even see it as a second-class kind of work. Fourth, PA, like most social sciences, has become more specialised. Fewer individuals can roam competently over the whole field. Sub-communities using different theories and methods often communicate very little with each other. This perhaps implies less flexibility to deal with practitioners' messy problems and multifaceted needs.

Recently many senior and experienced PA scholars have expressed anxiety about this sliding away from the big issues and from the organisational conundrums of the day (Del Rosso, 2015; Peters et al., 2013; Posner, 2009; Raadschelders and Lee, 2011; Roberts, 2014 and 2018; and Yang, 2015). Radin put the matter sharply in her 2012 John Gaus lecture: 
"Although many tend to look at the worlds of practice and theory as two separate cultures ... a field such as public administration - an applied field - cannot survive without finding ways to bring the insights of both cultures together. Today, however, the worlds have parted, and we seem to be operating in parallel universes" (Radin, 2013, p. 1).

More recently, an Italian academic working in the UK and very familiar with continental European trends, has expressed himself in similar vein:

"Scholars in PA should not content themselves with "zooming in" on specific problems and issues to be investigated with relatively well-specified methods; instead, the big picture should be aimed at - by scholars and practitioners alike" (Ongaro, 2017, p. 214)

An interesting variant of this point is that:

"Rather than synthesize and integrate, writers prefer to analyse and disaggregate: the reverse of what the day-to-day work of public administration is about" (Stillman, 2015, p. xviii)

A second current tension arises from the fact that universities have quite swiftly lost their previous status as the dominant providers to public authorities of research-based new knowledge. "Intermediary institutions include management consultants, think tanks, specialist consultants, survey companies, professions and media companies" (Barstow et al., 2014, p. 25). Management consultancies, in particular, have expanded rapidly and are now major providers of advisory and facilitating services to public authorities, as well as having become major items of public expenditure (National Audit Office, 2006; Sahlin-Andersson \& Engwall, 2002; Saint-Martin, 2000). They are also, incidentally, major employers of PA graduates, and in some countries offer better paid and more glamorous early careers than working in the public services themselves. They frequently employ - and are sometimes run by - ex-civil servants and ex-senior academics. They are able to spend more resources and expertise on securing protective intellectual property rights for their work than is university-based social science (Barstow et al., 2014, pp. 24-26).

Thus, the first tension arises within academia and the second comes as competition from outside. Both reduce the likelihood that PA academics will become heavily engaged with for-practitioner research. Some suggestions about tackling the first are made towards the end of this chapter. The second 
is more intractable - unless a miracle occurs and the material conditions of work in universities, relative to those in private sector business, suddenly alter for the better.

\section{The disconnect between the PA research agenda and current practitioner problems}

Certain international trends and developments seem virtually heavily to influence the future environment in which PA practitioners will work (indeed, they are already influencing it). Each is enormously complex in its panoply of interacting effects. These developments include climate change, demographic change, technological change and the continuance of policies of public sector austerity in much of the western world. Collectively and individually, they affect the work of almost every government agency and department, from agriculture to building and construction, defence, emergency services, energy, finance, foreign policy, health care, the interior, overseas aid, social care, social security and transport. They also require close co-ordination between national and sub-national governments, and serious co-operation internationally. We may call them "megatrends" (Pollitt, 2015, 2016a). If, however, we ask how far recently published PA research addresses these issues and their multifarious consequences for public authorities, the answer has to be "not much." If we take the 580 articles published in the five top journals during 2015 and 2016 (Pollitt, 2017) these are the numbers which have a main focus on these issues:

$$
\begin{aligned}
& \text { Climate change }=2(0.003 \%) \\
& \text { Demographic change }=0(0 \%) \\
& \text { Technological change }=8(0.01 \%) \\
& \text { Austerity }=14(0.024 \%)
\end{aligned}
$$

It is true that PA scholars have occasionally written or part-written articles which have appeared in journals more directly focused on the megatrends themselves - on climate change or austerity, for example. But the number of such articles is not large, and these big topics seem to have aroused more activity from some of our neighbouring disciplines such as economics, geography, political science and sociology. An exception should be made for the topic of digital government, around which a considerable literature has grown (e.g. Borins et al., 2007; Dunleavy et al., 2006) but this is only one part of the vast range of technological changes which will affect governments (Pollitt, 2012). 


\section{The paucity of debate within academia}

In my time I have been to many, many national and international PA conferences. A few have had sessions with practitioners, but hardly ever are there discussions aimed principally at the general principles and approaches that should guide for-practitioner research. This is not to say that the topic never arises. It does, but usually in more specialised, local and functional settings. Thus, in many universities PA academics contributing to the UK's 2014 Research Excellence Framework certainly had plenty of meetings to decide how they could craft their institutional submissions to make their "impact" look good. And academics who are actually working on specific for-practitioner projects most certainly debate among themselves how to maximise the chances that their work will attract the attention and approval of the commissioning authorities. What is missing - or, at the very least, rare - is a general, systematic debate within the academic community about how this kind of research should be done, what priority and recognition it should have, what ethical principles should govern its conduct, and so on. "[T] here is very little written about pracademics" (Posner, 2009, p. 17). Given the very substantial slice of the total academic research effort which for-practitioner research appears to occupy, that seems strange, almost neglectful.

\section{Reflections}

No-one said it would be easy. My own experiences of practitioner-oriented research have often been positive and stimulating, yet overall I would not differ much from Dowding's view that:

"[W]e never get to see how academic policy-oriented solutions would work, because government selects those elements it likes, can afford, or that fit with its ideology: a pick'n'mix approach which often results in a worse policy than existed previously."

And:

"Academics, when they are honest, will always have doubts and see potential problems in any policy reform. Politicians cannot survive on doubts" (Dowding, 2016, p. 169, both quotations). 
I would, however, significantly qualify Dowding's observations by suggesting that, at administrative levels and locations further removed from the policy process (of which there are legion), political sensitivities may be much less, and the scope for recommending and having accepted coherent, evidence-based solutions may be greater.

The title of this section is deliberate - "Reflections," not "Conclusions." So what follows is more personal and more tentative than a set of conclusions might be expected to be. That is partly my choice, but partly also because the shadowland is indeed shadowy: hard data on the extent of practitioneroriented research are very patchy, and evidence-based estimates of its final impacts/influence on concrete decisions rare indeed.

PA is a small academic area within the social sciences. Even its leading units and institutes tend to be modestly sized in staff terms. It lacks a strong professional association at national level (at least in most of the western European and North American countries). It is notoriously divided internally, with no settled consensus view (Pollitt, 2016b, pp.3-15). With all due respect to the International Institute of Administrative Sciences (IIAS) - which is working hard to modernise itself - and to other, more recently created supra-national associations, it also lacks a strong, governmentally- and intergovernmentally-recognised representative body at the international level. It is part of the social science group of disciplines which, when compared with the STEM disciplines (science, technology, engineering and mathematics), is also lacking in these respects, and which receives a very modest share of most governments' expenditures on academic research (Barstow et al., 2014, pp. 273-290).

In short, PA is, from the perspectives of governments, a minnow. If its research is to make a greater impact than hitherto, it will need outside help, allies. But to increase the chances of outside help, it has to change internally as well. That part, at least, is in the hands of our own PA community, though the signs of reform are as yet occasional and local rather than systemic.

What kinds of changes are needed? There can be no settled answer to this, and predictions of intellectual and organisational trends so often turn out to be an embarrassment only a few years later. Nevertheless, I will conclude by hazarding a few suggestions about possible directions of travel (for different, if partly overlapping, reform agendas see Posner, 2009, pp. 24-26 and Roberts, 2018).

Four directions seem particularly promising. One is the direct encouragement of a more structured, collective debate within PA academia about practitioner-oriented research per se. Second, this may require, inter alia, the creation of stronger representational associations at both national and 
international levels. A third is a further movement away from the model of the lone researcher - or even the lone unit or department - towards big teams operating across a range of institutions. A fourth step would be a rapid expansion of interdisciplinary research, not only by co-operation with the other social sciences, but beyond that, so that it would become normal to find PA specialists working alongside STEM and medical academics on teams which address problems such as austerity, climate change, demographic change or technological change. This is a sequence, in the sense that the prospects for each of the second, third and fourth suggestions depend partly (but only partly) on the one before them having been at least partly implemented. That does not, however, have to mean that the actions on the second suggestion cannot be started until the first is, in some sense, complete. All can begin (some have already begun - and there is no time to waste) and they are mutually reinforcing. Taken together they would amount to a considerable cultural change within PA, and such changes are never rapid or easy.

How do these four lines of development help to improve the amount, quality and influence of practitioner-oriented research? Without the first, little can be hoped beyond a continuation of the status quo. No doubt there will be more government "push" for impact information, and it is likely that the techniques used in the UK REF 2014 may spread to other countries. But without collective debate within the PA academic community itself there can be no re-shaping of the categories and criteria of the exercise better to fit the particularities of the field. We have much to learn from each other in any such discussion. It should not be particularly difficult to start - panels could be created at major conferences and leading figures could be persuaded to engage. Among the issues they should address are a) how impact can best be conceptualised and measured in PA, b) how our system of academic rewards and incentives can be re-tuned so as to give better career recognition to high quality for-practitioner research and c) what ethical guidelines should be articulated to provide guidance for those beginning to engage with the often challenging (and powerful) world of practice? We should note, however, that initial progress may be slow. As one top American academic put it to me: "[I] $t$ first requires getting some consensus that this is a problem that needs to be attacked - and we are a long way from that" (Kettl, 2017). Cultural change, as noted above, is usually a hard slog over time.

Second, however, the results of an intra-professional debate need to be forcefully articulated and placed in the public arena. Organisationally, that is the job of a representative national association in each country, and of one or more international associations on the global stage. These could be new associations, or re-vamped and upgraded existing associations. They will 
need both to enter public debate in the media and selectively to target key access points within their respective public sectors. They will need to become competent lobbyists and publicists of success. At an individual level we need to find ways of better honouring and encouraging those valuable individuals who have the experience and the skills to act as "brokers" (Posner, 2009, pp. 16-17) or "interpreters" (Pollitt, 2017) - those who are able to take the complex findings of methodologically sophisticated research and, without undue violence or distortion, render it interesting and intelligible to practitioners.

Third, until recently PA has been rather slow in developing large-scale projects. Small university units and often meagre funding have not helped. An important exception has been EU research competitions, where transnational cooperation between universities has been a requirement. Over the years European universities have learned to play this game better and better, although the percentages of $\mathrm{EU}$ funding that have gone to the social sciences, and within them to PA, have been, respectively, small and very small. Big projects, tackling big questions, conducted by multi-national teams that have the opportunity to access not one but a number of governments are one way of attracting attention and increasing impact (see, e.g. www.cocops.eu).

Finally, there is the argument for reaching beyond the boundaries of PA by seeking multi-disciplinary projects and units. The "megatrends" briefly alluded to earlier virtually demand this kind of approach - extending way beyond the social sciences and into the STEM disciplines. Climate change research, for example, would benefit from PA specialists working alongside scholars from the physical sciences, from technology and from other social sciences such as economics, sociology and international relations. There are a few pioneering institutes and centres which can already show the fruits of this kind of work, but there is surely room for many more (Pollitt, 2015). Hitherto a sojourn in such an environment has not been an obvious priority for promising PA doctoral and post-doctoral researchers; rather it has been an oddity - and that should be changed.

Obviously these are not the only ways in which the PA field can develop. There are many other possibilities, some much less positive (for a few of these, see Pollitt, 2016b, pp. 148-151). Obviously also, none of these developments will occur automatically - each depends on determined effort by a critical mass of PA scholars, which is precisely what we have not yet witnessed.

For-practitioner PA research is not simply financially rewarding for participating institutions, it is often also intellectually fascinating and challenging, and can give engaged scholars a sense of having contributed to public issues and services in a way that will be deeply satisfying. And sometimes it may be profoundly frustrating! A great deal of it is already being undertaken. But we 
know too little of the extent and variety of it, and there are strong arguments for organising ourselves better to cast light into these shadows.

\section{Acknowledgements}

I would like to express my appreciation for the information and comments given to me in writing this chapter by Professors Tony Bovaird (Aston), Geert Bouckaert and Annie Hondeghem (both Leuven), Werner Jann (Potsdam), Don Kettl (University of Maryland) and Mike Mannin (Portsmouth), and by Dr Elke Loeffler (Governance International).

\section{Note}

1. This chapter by Christopher Pollitt, "Shadowland: the poorly mapped, underdiscussed yet vital interface between public administration research and practice," was first published in Andrew Massey (ed.). (2018). A Research Agenda for Public Administration (Chapter 3). Cheltenham and Northampton, MA: Edward Elgar Publishing.

\section{References}

Alford. J. (2009). Engaging public sector clients: from service-delivery to co-production. Basingstoke: Palgrave/Macmillan

Askim, J., Christensen, T., Fimrette, A.-L., \& Lægreid, P. (2010). How to assess administrative reform? Investigating the adoption and preliminary impacts of the Norwegian welfare administration reform. Public Administration 88(1): 232-246.

Barstow, S., Dunleavy, P., \& Tinkler, J. (2014). The impact of the social sciences: how academics and their research makes a difference., London: Sage.

Borins, S., Kernaghan, K., Brown, D., Bontis, N., 6, P., \& Thompson, F. (2007). Digital state at the leading edge. Toronto: University of Toronto Press.

Bovens, M. (2010). The academic advisor: professionalising commissioned research in public administration: Academically sophisticated, irrelevant to practitioners? In G. Bouckaert \& W. Van de Donk (eds). The European Group for Public Administration (1975-2010) Perspectives for the future (pp. 123-126). Brussels: Bruylant.

Broucker, B., Steenberghen, T., Hondeghem, A., Maes, B., and Petry, K. (2014). Leerlingen in Vlaanderen: mogelijkheden en voorwaarden: Eindrapport. Retrieved from http://soc.kuleuven. be/io/onderzoek/project/onderzoeksproject-leerlingenvervoer-vlaanderen-2013-2014. 
Del Rosso, S.J. (2015). Commentary: our three Rs: rigor, relevance and readability. Governance 28(2): 127-130.

Denicolo, P. (ed.). (2014). Achieving impact in research. London: Sage.

Dowding, K. (2016). The philosophy and methods of political science. Basingstoke: Palgrave/ Macmillan.

Dunleavy, P., Margetts, H., Bastow, S., \& Tinkler, J. (2006). Digital era governance: IT corporations, the state and e-government. Oxford, Oxford University Press.

Hill, C.J. (2006). Book review of Kelman, S. (2005). Unleashing change: a study of organizational renewal in government. Washington, DC, Brookings Institution Press, Journal of Policy Ananlysis and Management 25(3): 737-741.

Joss, R., \& Kogan, M. (1995). Advancing quality: Total Quality Management in the National Health Service. Buckingham: Open University Press.

Kelman, S. (2005). Unleashing change: a study of organizational renewal in government. Washington, DC, Brookings Institution Press.

Kettl, D. (2017). Personal communication to the author. 29 September.

Kickert, W. (ed.). (2008). The study of public management in Europe and the USA: a comparative analysis of national distinctiveness. Abingdon: Routledge.

Lakey, J., Rodgers, G., \& Scoble, R. (2014). What are the different characteristics of research impacts?. In P. Denicolo (ed.). Achieving impact in research (pp. 33-46). London: Sage.

Lynn, Jr., L. (2005). Public management: a concise history of the field. In E. Ferlie, L. Lynn Jr. \& C. Pollitt (eds). The Oxford handbook of public management (pp. 27-50). Oxford, Oxford University Press.

Ministry of Finance. (2013). Governments for the future: main report. Helsinki: Ministry of Finance. Retrieved January 4, 2014 from http://www.vm.fi/vm/fi/04_julkaisut_ja_asiakirjat/01_julkaisut/08_muut_julkaisut/20131119Govern/Government_for_the_Future.pdf.

Nathan, R. (1988). Social science in government: uses and misuses. New York: Basic Books.

National Audit Office. (2006). Central government's use of consultants, HC128, Session 2006-7. London: The Stationery Office.

Ongaro, E. (2017). Philosophy and public administration: an introduction. Cheltenham and Northampton, MA: Edward Elgar.

Packwood, T., Pollitt, C., \& Roberts, S. (1998). Good medicine? A case study of Business Process Re-engineering in a hospital. Policy and Politics 26(4): 401-415.

Patton, M. (1997). Utilization-focused evaluation: the new century text. 3rd ed., Thousand Oaks, CA: Sage.

Pawson, R. (2013). The science of evaluation: a realist manifesto. Los Angeles, CA, and London: Sage.

Perry, J.L., \& Hondeghem, A. (eds). (2008). Motivation in public management: the call of public service. Oxford: Oxford University Press.

Peters, G.B., Pierre, J., and Stoker, G. (eds). (2013). The relevance of political science Basingstoke: Macmillan. 
Pollitt, C. (2012). New perspectives on public services: place and technology. Oxford: Oxford University Press.

Pollitt, C. (2015). Wickedness will not wait: climate change and public management research. Public Money and Management 35(3): 81-186.

Pollitt, C. (2016a). Be prepared: an outside-in perspective on the future public sector in Europe. Public Policy and Administration 31(1): 3-28.

Pollitt, C. (2016b). An advanced introduction to public management and administration. Cheltenham and Northampton, MA: Edward Elgar.

Pollitt, C. (2017). Public administration research since 1980: slipping away from the real world?. International Journal of Public Sector Management 30(7): 555-565

Pollitt, C., \& Roberts, S. (1994). Audit or evaluation? A National Audit Office VFM study. Public Administration 72(4): 527-549.

Pollitt, C., \& Op de Beeck, L. (2010). Training top civil servants: a comparative analysis. Leuven, Instituut voor de Overheid, Katholieke Universiteit Leuven.

Pollitt, C., \& Bouckaert, G. (2017). Public management reform: a comparative analysis - into the age of austerity. Oxford: Oxford University Press.

Posner, P.L. (2009). The pracademic: an agenda for re-engaging practitioners and academics. Public Budgeting and Financing 29(1): 12-26.

Raadschelders J.C. (2011). Public administration: the interdisciplinary study of government. Oxford: Oxford University Press.

Raadschelders, J.C., \& Lee, K.-H. (2011). Trends in the study of public administration: empirical and qualitative observations from Public Administration Review, 2000-2009. Public Administration Review 71(1): 19-33.

REF. (2011). REF2014 assessment framework and guidance on submissions REF02.2012. Retrieved from http://www.ref.ac.uk/media/ref/content/pub/assessmentframeworkandguidanceonsubmissions/02_11.pdf.

Report on the assessment of Horizon 2020 implementation in view of its interim evaluation and Framework Programme 9 proposal. (2017). Retrieved September 20, 2017, from http://www. europarl.europa.eu/doceo/document/A-8-2017-0209_EN.pdf

Report of the Committee on the Civil Service. (1968). Cmnd. 3628, London: HMSO.

Roberts, A. (2014). Large forces: what's missing in public administration. Create Space Independent Publishing Platform, Retrieved February 12, 2017 from WWW.LARGEFORCES.NET.

Roberts, A. (2018). The aims of public administration: reviving the classical view. Perspectives on Public Management and Governance 1(1):73-85.

Sahlin-Andersson, K., \& Engwall, L. (2002). The expansion of management knowledge: carriers, flows and sources. Stanford, CA: Stanford University Press.

Saint-Martin, D. (2000). Building the new managerialist state: consultants and the politics of public sector reform in comparative perspective. Oxford: Oxford University Press.

Stillman, R. (2015). Foreword. In M. Guy, \& M. Rubin (eds). Public administration evolving: from foundations to the future (pp. i-xix). New York and London: Routledge/Taylor and Francis. 
Walker, R. (2001). Great expectations: can social science evaluate New Labour's policies?. Evaluation $7(3): 305-330$.

Weiss, C., \& Bucuvalas, M. (1980). Truth tests and utility tests: decision-makers' frames of reference for social science research. American Sociological Review 45(April): 302-313.

Yang, K. (2015). From administration to management. In M. Guy, \& M. Rubin (eds). Public administration: evolving from foundations to the future (pp. 102-122). New York and London: Routledge/Taylor and Francis. 



\title{
2 \\ Forms of Knowledge for the Practice of Public Administration
}

\author{
Edoardo Ongaro
}

In this chapter of the EPPA I book I suggest that knowledge for the practice of public administration comes in three main forms:

- "Enlightening" knowledge: the social-scientific, "theoretical" knowledge generated according to the (highest) standards of the scientific community;

- Problem-orientated knowledge: the kind of knowledge that is mostly produced by practitioners engaged in professional social inquiry and driven by societal problems rather than by the generation of additional knowledge per se, as in discipline-orientated academic knowledge;

- Practice-extrapolation knowledge: a kind of knowledge that is embodied into "practices that work," i.e. knowledge derived by learning from actual experiences of tackling a problem and improving a public service or process, practices typically contrived by practitioners engaged in public services, that are spotted, showcased and analysed for extrapolation and transfer elsewhere.

All three of these forms are significant for the development of the practice of public administration - hereafter PA (intended broadly to encompass the areas of "public policy and administration," "public governance" and "public management") - and my main argument is that these forms of knowledge have to be seen and to be used in a joint way for progress in the practice of PA to occur, in Europe and elsewhere.

I do not discuss the actors of these forms of knowledge: how academics or practitioners navigate more or less successfully the terrain of "academic research for practice-orientated purposes" - the territory that Christopher Pollitt has called "shadowland" (Pollitt, 2019). The nature of this "middle land," the (non-)confidence with which either academics or practitioners get out of their comfort zone and traverse this land, the motivations and inclinations that drive them to do so and how they interact with each other along the way, 
the "logistical tensions that arise when [academics are] trying to make an impact - the different timelines of the academic and 'real world'; clashing incentive structures of different professionals; unequal resource endowment; and, the lack of formal power on the academic side" (Dunlop, 2019; see also Pollitt in this book). At the end of the chapter the role of the EGPA - the European Group for Public Administration - as a learned society in fostering the generation and spread of knowledge about the practice of PA is discussed.

\section{$1 \quad$ Enlightening knowledge}

The starting point of the argument in support of the practical relevance of this first form of knowledge is (deceptively) summed up in the most famous statement "Nothing is more practical than a good theory." According to McCain's review of the usage of this expression (McCain, 2016), "Lewin's Maxim can be found in three forms - 'there is nothing as practical as a good theory' (Lewin, 1943, 1944, 1945), 'there is nothing so practical as a good theory' (Lewin, 1951; Marrow, 1969), and 'there is nothing more practical than a good theory' (generally attributed to Lewin but no canonical source known)," to which prominent management theorist van de Ven (1989) has added his own, slightly more cautious, tweak: "Nothing is quite so practical as a good theory."

The basic idea underpinning the argument is that shedding light on phenomena - understanding the causes of things: rerum cognoscere causas (the expression is drawn from the great Roman Poet Virgil: "Felix, qui potuit rerum cognoscere causas," verse 490 of Book 2 of the Georgics, which may be translated as: "a fortunate life is that of the person who was able to know the causes of things," a reference which has been adopted as its motto by the London School of Economics and Political Science) - is a practically valuable form of knowledge in and by itself, enabling (though not coinciding with) practical reasoning (one of the two main forms of reasoning, according to the ancient Greek Philosopher Aristotle) and the pursuit of value-laden social action (a concept most effectively condensed in the ancient Greek term: praxis). In this line of argument, it is theoretical knowledge that, by definition, sheds light on phenomena.

What, then, is theoretical knowledge? Consistently with the overall thrust of this edited volume which has a different format from "standard" academic pieces and rather aims at indicating broad lines of development of and for PA from European perspectives, we do not dwell here on definitions of theory. Rather, we start from a conventional definition of PA as the interdisciplinary study of government in action. Bauer speaks of "a multidisciplinary endeavour 
with a prime focus on studying government in order to produce insights to improve government practice" (Bauer, 2018). Theoretical knowledge is the knowledge about the causes of things generated in accordance with the scientific standards adopted by the respective disciplines employed to study $\mathrm{PA}$, a list which includes but is not limited to: political science, management, organisation science, law, sociology, economics, and social psychology. It is further integrated by a wider range of disciplines from across the social-natural sciences divide, and notably in the applied fields, thereby including on a more sectoral basis inputs from disciplines ranging from public economics to criminology, from engineering to architecture and urban development, from informatics to medicine, and so on. Hence - the argument runs - the same level of rigour that applies to each of the constituent disciplines in advancing theory applies when such disciplines, often in conjunction, are employed to generate theoretical knowledge of PA (the root word for science in Latin - the verb "scire" - refers to "knowing," and the Greek root word for epistemology denotes "rigour," so scientific-theoretical knowledge of PA consists of knowledge obtained through rigour in investigating the administrative phenomena).

Importantly, in our definition PA is both a science and - also and constitutively - an art, a profession, and, crucially in our view, a form of humanism. The conception of PA as an art and a profession is well consolidated; more distinctive is the notion of PA also as a humanism. PA as "government in action" or the administration of the public institutions and the delivery of public goods, is about wisdom and practical reasoning, it is about the making of value judgments and not just factual judgments, it is about the understanding of things as much as it is about the knowing of things - along all of these dimensions PA, and notably the practice of PA, is a form of humanism. And we could and should add that the very term "explanation" requires qualifications when used in the field PA: in fact "explanation" in PA means identifying the causes of something - the standard notion of explanation in the natural and social sciences alike - but it may also mean "attributing meaning" to something. Possibly this interpretation of PA as both a science and a humanism may better be understood in the sense conveyed by the German language term of Wissenschaft, a field of intellectual inquiry, with an applied thrust and practical implications.

If we agree that PA is (also) a humanism, then we need to adopt definitions of theoretical knowledge as encompassing what theory means also in such disciplines like cultural anthropology (as so aptly illustrated by Christopher Hood's 1998 work which deploys Mary Douglas' grid-group cultural theory to $\mathrm{PA}$, and which is so significantly entitled and subtitled "The Art of the State: 
Culture, Rhetoric and Public Management"), or what theory may mean more broadly in humanities like history (historiography, where theory may mean the drawing of "limited historical generalisations" from in-depth investigations into individual which produce accounts of unique historical episodes), or in human geography (where "contextualised" theories sensitive to spatial and temporal circumstances may be the main currency).Indeed in this conception of PA we should revisit the very foundations of theoretical knowledge into philosophy and philosophical thought (in its articulate branches, from ontology to political philosophy, from epistemology to ethics, which tackle the big questions about reality underpinning and giving sense to any effort to generate knowledge). Indeed, we may qualify PA as an "academic discipline" provided the whole gamut of PA as the interdisciplinary study of government, the art, the profession, and the humanism are considered in an integrated way.

I make the argument for philosophy, as an academic field, to be foundational for PA (Ongaro, 2017). This consideration leads us to another implication of the nature of PA as also a humanism: that the languages utilised to give meaning to PA do matter. In fact, concepts and notions in the humanities and the social sciences may travel much less well when translated from and into the English language than what happens for the natural sciences.

To illustrate, such English-language terms as "governance," "accountability," "management," "leadership," "performance," "agency," "policy" and the like may have nuanced meanings which become problematic to transmit and convey both when translated from English into another language and when used in the original English in the scholarly or especially the public debate of a country where English is not a national language. Analogously, words in other languages, like the Italian azienda or the Croatian uhljeb or the German Regierungspräsidien or the Hungarian jogalkalmazás, may equally not travel that well into English - the relationship of signifiers and signified may entail meanings which are subtly different across languages. This consideration does not necessarily entail any form of radical inter-subjectivism or relativism: linguists like Noam Chomsky remind us of the possibility of distinguishing between superficial structure and deeper structure of a sentence; but it remains a constitutive feature of $\mathrm{PA}$, in its composite nature of a social science, an art, a profession and a humanism, that language plurality matters: this trait demands to be paid substantive attention, and Europe - a region of the world with plenty of national languages each enjoying a rich intellectual history, and all scattered one next to the other in that small hem of the Eurasian landmass which forms the European continent - is a natural laboratory for vetting the implications of language differences for PA. The favourable conditions available in Europe for studying the influence of languages on PA are also 
reinforced by the very compact and highly developed PA scholarly community present in Europe. This PA community which works together is facilitated also by infrastructures like the European Union institutions for the funding of research, which supports research provided it is pan-European in nature, and by the fact of having a natural home like the learned society, the European Group for Public Administration - EGPA - where PA scholars can socialise and arrange ambitious pan-European research. Adopting the language prism for PA entails addressing in an explicit way the issue of the implications of language diversity for the study of PA, and focusing the problem of what is lost in translation: this topic is tackled in a dedicated chapter in Ongaro \& van Thile (2018b), which discusses the issues around how English words like the ones mentioned above travel into the PA scholarly and public debate of 18 European countries.

We have made the argument that theoretical, "academic" knowledge may contribute to the practice of PA by virtue of its enlightening potency. What is, then, the impact of academic knowledge, and how can such impact be gauged? It is a European country, the UK, that has been leading the way worldwide in endeavouring to assess the impact of academic research, that is, academic-generated knowledge. In fact, a major exercise in attempting to measure the impact of knowledge has been carried out in the UK since the inclusion, from the 2014 round of evaluation of the research produced by the UK higher education institutions, in the research assessment of a specific category "impact," which was weighted as $20 \%$ of the entire assessment and influenced the allocation of around $£ 1.6$ billion worth of public funds. This exercise has been considered a major attempt to assess the impact of scientific knowledge (Pollitt, 2016).

Before delving more into what it means to assess the practical impact of research, we should notice that this thrust towards assessing the impact of research is not confined to one, however major, initiative, but is rather part of a growing eco-system: since 2009 researchers in the UK submitting a grant application have had to delineate the expected "pathways to impact" of their research (Australia and Hong Kong introduced a similar requirement in respectively 2014 and 2017).

Within this exercise of evaluation of research, impact is defined in terms of how basic, fundamental research engendered "an effect on, change or benefit to the economy, society, culture, public policy or services, health, the environment or quality of life, beyond academia" (HEFCE, 2011) - a very broad definition, made even more encompassing in the UK's Economic and Social Research Council (ESRC) threefold definition where non-academic impact can be: instrumental: influencing the development of policy, practice or service 
provision, shaping legislation, altering behaviour; conceptual: contributing to the understanding of policy issues, reframing debates; or capacity building: through technical and personal skill development (https://esrc.ukri.org/ research/impact-toolkit/what-is-impact/, accessed 12 April 2019). Importantly, the inclusion of impact in the assessment of research is not implying that all research has impact: the category is that of possibility: research may have an impact, and if and when such is the case such impact can be evaluated.

Claire Dunlop has investigated the evidence about the impact of research in the field of PA, as collectable from the 2014 exercise of evaluation of research in the UK (called REF 2014: the Research Excellence Framework for the period from 2008 to 2013, followed by REF 2021, the Research Excellence Framework covering the period from 2014 to 2021). Within this assessment exercise, PA has had a lion's share within the social scientific field of "Politics and International Relations," with 48 out of 163 , or $23 \%$, of impact case studies in the disciplinary field being classified under the label of "public policy and administration impact case studies": more than any other sub-field within "Politics and International Relations." PA has also had a non-irrelevant presence within business and management studies, with 39 out of 410 , or 9.5\% (see Dunlop, 2018 and 2019, where limitations to this analysis are also discussed: e.g. that some cases have undergone a non-disclosure procedure for issues of confidentiality, and hence are not included in these data, or more importantly that these impact case studies have been self-selected by the universities and units of assessment undergoing an evaluation process, and presented within the context of, at a more technical level, a strict format and auditing procedure, and, at a more sociology-of-knowledge level, within the context of the dominating purpose of receiving recognition and funding for one's own institution, hence with a high stake in the evaluation game).

It is important to emphasise that what has been measured in this assessment exercise is not the academic impact (like the number of citations or other indicators of how successful academically a publication has been), nor scientific dissemination. What is being detected in the REF evaluation of the impact of research is whether and how the outcomes of academic research have had impact on a social or a policy issue.

A number of considerations arise from Dunlop's study. First, impact takes various forms: from contributing to public debate, to engendering a novel practice that has been made possible to contrive as a result of academic research, to setting an issue on the policy agenda... to "stopping a decision"(!), when an impact case pointed to academic knowledge having shed light on possible (likely) negative effects of a policy decision which eventually led to it being turned down. A second and not unexpected consideration is 
that impact takes time to manifest itself (hence reinforcing the argument originally in Weiss, 1977, and developed by Alkin, 2013), and it is unlikely that enlightening knowledge may manifest any meaningful influence on the practice of PA (or any other domain for that matter) over short time spans. A third observation is that in most cases the impact of academic research is about "influencing the elite": the connections between academic and elite (decision-makers') circuits continue to represent the main conduit linking the results of research to the practice of PA: the impact of research in the field of PA on "the general public" remains a rarity.

In terms of which PA themes have found their way in the first systematic exercise to assess the impact of academic research, the analysis conducted by Dunlop (2019) detects that " $[\mathrm{w}]$ hen we look at the universe of 90 case studies over half of them (56\%) are primarily focussed on effectiveness (...) Equity or equality and efficiency themes are each covered in 20 per cent of the cases. The major finding is that only 4 per cent of the public administration case studies address the big legitimacy themes - with three addressing corruption and transparency and one on privacy. Incredibly, we found no studies primarily addressing trust in government or accountability." Whether this finding is of any generalisability, to Europe or beyond, we cannot claim in a warranted way, but it does send a strong message: that the key issue of trust (in PA, and of PA towards citizens), legitimacy (of PA in citizens' eyes), and accountability are domains of inquiry ripe for more research in general and surely for more impactful research in the field of PA, as they seem to be mostly off the radar of academics when they reckon whether their research has had an impact on society.

To sum up on this form of knowledge: we argue that theoretical knowledge can have an impact on PA, and evidence from the UK REF 2014 evaluation of research exercise does add confidence to the claim. We could even argue that the impact agenda is on the rise: with all the caveats that we have recalled here (and more are discussed in Dunlop, 2018 and 2019), the emphasis placed on impact by the 2014 REF has been reinforced in the 2021 REF, in which impact is weighted as $25 \%$ of central government academic funding for research: this may well be a sign that the pendulum - in PA as well as, more generally, in academic research - may be swinging back from an (over-)emphasis on the criterion of "rigour" towards (re-)giving prominence to the criterion of "relevance." The message might be gaining traction that research must also be relevant, that it is of limited value to "know more and more about less and less," that we must be able to generate knowledge that is relevant -possibly also sacrificing a bit of the rigour along the way to gain the prize of being able to make a difference, for the good, to society. We might further qualify the claim by arguing that, logically, relevance in research is not necessarily 
in trade-off with rigour: that relevance and rigour are not by necessity in opposition. But at the very minimum we should not be afraid of arguing that research that is relevant be not displaced by the obsessive search for "rigour only" to which so many scholars are driven by a number of academic rankings and fashionable trends in designing academic career paths that seem to be so dominant in many universities. Ultimately, we should put more emphasis on the adjective "good" when we (rightly) state that "there is nothing more practical than a good theory": "good" does not refer only to the rigorous procedures through which theory has been built and tested, but also to the relevance of the domain of application of the theory itself.

\section{Problem-orientated knowledge}

It has long been debated whether, and the conditions under which, social scientific knowledge is (or should be) structured around "problems" rather than around disciplines and their distinctive research questions (as is the dominant format of organising knowledge in academia).

One horn of the debate concerns the reasons why academia is organised around disciplines, and such reasons range from more functional ones, inherent in the nature of the discipline, to more historical-institutional ones, whereby past choices continue to have a huge influence and shape the way in which the production of knowledge is being organised in academia. The other horn of the debate is the conditions under which knowledge can be supplied, and the extent to which public policy-makers are in demand of it: the giving and taking of advice - in other words, whether there is a supply and demand, a market for problem-orientated knowledge.

Colin and Carole Talbot (2018) make the case for problem-orientated social science (drawing from the works of authors like Lindblom and Cohen). The authors consider problem-orientated knowledge as a development with the potential to provide a more productive framework through which academics and policy-makers can interact than the extant organisation of knowledge in academia and a framework within which the taking of advice by practitioners and policy-makers may be more productive than the way in which it is currently framed, in most instances.

There are two key points to the argument. The first point it that this professional social science is very significant, as a minimum for its sheer size, as I argue using the case of the UK. The second point is that problem-orientated knowledge is based on different assumptions from the discipline-orientated knowledge that is the dominant format in academia. 
Starting from the consideration of size, Talbot and Talbot observe that professional social inquiry is broader than academe:

"[T] here are many more professional practitioners of 'professional social inquiry' (PSI) than merely academics. These non-university practitioners include government officials (at all levels), public service agencies, businesses, voluntary and campaigning organisations, trade unions, political parties, think-tanks, consultancies, market researchers, opinion-pollsters, media organisations and professional groups. The people active in these sorts of organisations and functions are not mere 'translators and mediators' but active producers of social scientific knowledge. Given the UK produces more than 600,000 social science graduates per year it is fairly likely that most of these non-academic PSI producers are themselves academically trained in the social sciences, at least to degree level. We estimate there are about 10,500 academic social scientists in the base disciplines (Economics 3,000; Politics 1,750; Sociology 3,400; Social Psychology 1,500; Anthropology 800). There are about another 25,000 in other social science disciplines that are 'intermediary' or 'applied' (Business Studies; Management Studies; Social Work; Social Policy; Human and Social Geography; Media Studies; Marketing; Accounting; HRM; Tourism; Information Services; Planning; Publicity Studies; Publishing)(Categories and numbers from Bastow et al 2014). This brings the total of academic practitioners of social science to somewhere around 35,000 in the UK. We have estimated that there are perhaps between 350,000 and 500,000 non-academic practitioners of Professional Social Inquiry generating social knowledge. This knowledge may be of variable quality but as a great deal of it is generated for serious purposes and consumes substantial amounts of resources (e.g. in government and business) we can assume that a reasonable proportion is at least as good quality as academic outputs." (Talbot \& Talbot, 2018:1216).

Hence a first consideration is that problem-orientated social science in general (and for public policy and public management in particular) is simply "out there," overlooked if not outright disregarded by academia, but potentially available for tapping.

Most important is the second point: that problem-orientated knowledge is based on different assumptions from discipline-orientated knowledge, for the very fact of being driven by problems rather than the internal organisation of knowledge within a discipline. Governments, businesses and charities start out with social problems or issues they want to tackle, not the consideration of where, in which academic discipline, the problem they are interested in lies, 
which is a central concern for many academic social scientists. The "knowledge production model" for non-academic professional social inquiry is therefore radically different from the academic equivalent in most instances (Talbot \& Talbot, 2018). This framework is in sharp contrast to discipline-orientated knowledge, whereby research is designed to meet the needs of the discipline for generating more knowledge, rather than addressing social issues. Disciplineorientated knowledge is concerned with accumulating knowledge within the discipline, it is focused on more knowledge; problem-orientated knowledge is instead focused on a different kind of knowledge: indeed, whatever knowledge provided it addresses the problem.

One factor driving further the divergence between the way in which academia is organised and problem-orientated knowledge is that performance management systems within higher education institutions are driving towards an extreme emphasis on disciplinary-orientated, academic knowledge, at least in the West and in those non-western countries which are more directly importing their academic models from the US or the UK. Surely in most of Europe they put a premium on discipline-orientated knowledge and generate a disadvantage for those academics engaging in problem-orientated research (although the growth in significance of the evaluation of the impact of research, that we have discussed in the previous section, might be a tensor rather pulling in the direction of emphasising problem-orientated research).

Most fundamentally, the point is that problem-orientated research is based on a different framework - we might call it a "paradigm" - from discipline-orientated research. Problem-orientated research is inherently interdisciplinary: it resorts to whatever discipline may provide knowledge that is usable to address the extant societal issue. And, a second key distinguishing feature, problem-orientated research has a problem focus: it is driven by problems rather than by the generation of additional knowledge per se. To illustrate with a concrete example, for those acquainted with the structure of an academic paper or book, it will be all too familiar that the second section (or thereabouts) of the paper is devoted to the literature review, whose aim is spotting a gap in the literature and arguing that the rest of the paper will successfully fill such gap. This passage of having the literature review section of the paper pursuing the function of demonstrating that the paper adds something new to the very literature that is being reviewed has been rehearsed so many times (especially for those who have reviewed thousands of academic papers, and, as a journal editor and an active external reviewer for some forty journals, the author of this chapter happens to be in such condition) that one may be induced to overlook its significance, but it does reflect an implicit paradigmatic choice of overarching importance: it reinforces the 
claim that research is worth pursuing if it fills a gap in the accumulation of knowledge within the reference discipline. The specific piece of research in the paper might or might not argue explicitly about the societal relevance of the research that is being presented (some will argue thus and others will not), but ultimately the literature review section of the paper conveys a second and deeper message than the specific informative contents it contains: that the function pursued by the research presented in the paper is to contribute to the discipline, to accumulate knowledge within the framework of the discipline. Problem-orientated knowledge in a sense adopts the opposite approach, in the most fundamental sense: since it takes the move from a societal problem, research within the paradigm of problem-orientated knowledge craves for finding already in the literature the answer to its problem (or at least to part of it). For a researcher engaged in discipline-orientated research, finding out through literature review that the research question has already been answered may induce dejection: the thrust is being able to demonstrate that something has not been said, in order to be able to argue that our own research adds something (and hence the publication counts for academic promotion!). The overall thrust of reviewing the literature in problem-orientated research is - or at least should be - the other way round: the best (albeit rare) case is when it is sufficient to review the literature in order to be able to solve the extant societal problem. Only after it has been tested that the answer is not already available in the literature, does the problem-orientated researcher engage in social-scientific inquiry in manners that methods-wise are not that dissimilar to discipline-orientated knowledge. Even if the methods are similar, the direction of travel and overall thrust are radically different.

Other main features of problem-orientated knowledge unearthed by Talbot and Talbot include that problem-orientated research tends to envisage a broad participation of stakeholders, who are not only respondents or the objects of the inquiry, but will tend to have a steering role in the process of generation of research. Problem-orientated research may also tend more towards experimentalism - by this we do not mean experimentation as the favoured research method, rather a general attitude towards trying out "whatever works" if it is deemed to be helpful in addressing the pressing concern that drives the research effort.

In sum, the problem-orientated form of knowledge tends to unfold and develop around a different paradigmatic base from the discipline-, theorybuilding-orientated "enlightening" knowledge considered in the previous section.

In concluding this section, it is worth mentioning action research, on which there is a wide literature reflecting on its very nature and location within the processes of knowledge generation in the social sciences. What 
is the relationship between the problem-orientated research and what goes under the label of "action research"? Action research is part and parcel of - if not outright synonymous with - the kind of research which produces problem-orientated knowledge, with one important qualification. It may be a way explicitly and deliberately to interconnect and "bridge" processes of generation of enlightening knowledge with processes of production of problem-orientated knowledge. This may constitute an especially valuable and laudable contribution, as the two types of knowledge need each other, but the dynamics of their production (including the career pressures and paths of those involved) may actively diverge and make the pursuit of this bridging function both high in demand and short in supply.

\section{Practice-embodied knowledge: Learning from experience and replicating}

At the opposite pole of the enlightening knowledge discussed above, there is what we can label "embodied" knowledge: the kind of knowledge that is incorporated into the "practices that work," the practices that are being adopted by public administrators and managers to tackle extant problems. These practices are very often referred to as "best practices," and there is a consultancy industry that thrives on the sale of these so-called best practices (that generally do not deserve to be qualified as "best," apart from highly specified, very rare instances, as we shall see). The alleged trait of being "best" aside, the key issue here is that practices that work do represent a form of knowledge: a kind of knowledge that is incorporated and lies in a gizmo, a "system that works", rather than manifesting itself in verbal-conceptual forms - as a set of propositions or hypotheses or of an argument or a narrative - as typically occurs for both theoretical (enlightening) knowledge and problem-orientated knowledge.

How is it possible to learn from vicarious experience for replication elsewhere? How is it possible to learn from the often tacit knowledge that has been incorporated into this practice that works "here and now" and use this knowledge to contrive elsewhere a practice that, appropriately adapted to the diverse circumstances, is also able to work and produce similarly positive effects? How can this embodied knowledge - knowledge incorporated into a practice - be leveraged for purposes of bettering the functioning of PA? With a slightly more technical formulation, the question is whether and how analysts in public management can address the problem of improving the performance of public sector organisations in one situation (target site) by 
employing experience acquired elsewhere (source site). We attempt to take stock of what we know about the use of practice-orientated knowledge (Ferlie \& Ongaro, 2015, chapter 8; key scholars who have worked on the question of how to learn from practices include: Eugene Bardach, Michael Barzelay, Stuart Bretschneider and Colin Campbell).

We argue that the research of and into practices that work, which is known under the label of "best practices research," can be deconstructed into two problems:

- The search for excellence problem, which tackles the issue of what is "best" and how to detect it - and if, as is the case, very rarely we can talk about a truly "best" practice, to drop the notion of best and talk about "good" or "adequate" practices - or, put simply, "practices that work under certain circumstances"; and

- The extrapolation problem: how to "extract" what makes the practice produce positive effects under certain circumstances to replicate those effects elsewhere.

\subsection{The search of excellence problem}

The first problem concerns how to find "where excellence lies": how to identify cases that contain practices of excellence, i.e. cases in which the highest possible level of performance is manifested. Bretschneider et al. (2005) have developed a thorough assessment of this problem, and they come out with very stringent conditions: the two joint necessary and sufficient conditions for finding a "best" practice (or better: for finding a case that contains a best practice to be extrapolated) are the completeness of cases considered and the comparability of cases. When pondered appropriately, it arises that meeting these joint conditions is very rare at most (indeed nearly impossible) and, moreover and crucially, these conditions can be satisfied almost exclusively when the performance takes the form of a production function. In fact, Bretschneider et al. "work out a general framework for selecting the proper technical option available to researchers for estimating relationships of inputs to outputs capable of focusing on extreme behaviours, in order to find the best performing case, i.e. for finding the unit where the transformation of inputs into outputs - the production function - is performed at the best level) (...) The focus on the production function is also, in our view, the main limitation in the approach suggested by Bretschneider and colleagues [this entails that] other categories of situations, like the management of complex change processes in the public sector, where performance cannot generally be expressed in the terms of a production function [are not encompassed]" (Ferlie \& Ongaro 2015, pp. 183-184). 
Identifying excellence - a truly "best" practice, a practice that deserves to be qualified as "best" - is an absolute rarity, up to the point that talking of "best" practices should be deemed utterly misleading in most cases. Indeed when consultants tout the solutions they propose as "best" practices they are possibly bordering, if not outright trespassing, on the notion of cheating - and this misleading emphasis on best practices, in the public as in the private sector, has engendered a wide range of nefarious effects: the "best practices industry" should definitely adopt much more restraint if it is to provide a contribution for good - and as a minimum should change its name to "the good practices/adequate practices" industry.

The key question remains to be addressed: after having found a practice that produces positive ("good," "adequate") results, how does one replicate it elsewhere? To answer this question we need to shift our focus from "universalistic" practices to "contextualised" practices, and we do so by specifying the conditions under which the practice works - and more generally recognising that practices are good under certain contextual conditions, and nearly no practice works irrespective of conditioning enabling or hindering - circumstances (on the meanings of "context" and "contextualised" in public management and policy, and a range of theories and perspectives to study context in public management, see the edited work by Christopher Pollitt, 2013). Doing this means addressing the extrapolation problem.

\subsection{The extrapolation problem}

How can "a practice that works" be extracted from one case (source site) and applied elsewhere (target site) to generate similarly successful outcomes? This is the core question of the extrapolation problem, which can also be formulated as follows: how to extract the identified good practice and adapt it to other circumstances to replicate its effects.

It should be noted that "knowledge" here becomes generated in a certain sense only when the practice is analysed, causality is understood, and some form of verbal illustration of how the practice works is developed. This occurs because implicit knowledge becomes explicit/codified/verbal knowledge but more than that, the point is that in a certain sense knowledge is generated when the "embodied practice" is extrapolated, so extrapolation continues and completes the process of knowledge generation that was initiated when the practice was initially contrived. This may reassure the reader who, albeit having patiently followed me all the way here, is still (somewhere in the back of her/his mind) concerned that this "practice-embodied knowledge" 
represented something magic or exoteric: quite the contrary, this knowledge can be likened to engineering knowledge, a problem-solving knowledge which is incorporated into a machine (in our case not exactly a "machine," but a socio-technical practice, because PA problems can never be solved entirely at a technical level and always have a human relations, sociological component). However, unlike engineering, this knowledge is first found in a gimmick, the practice that works, and only later is it codified in more expressible terms, into a project or design that enables the practice to be replicated elsewhere. If it can be likened to engineering, this form of knowledge should more appropriately be likened to reverse engineering. (As said, the other main difference from engineering lies in the fact that practices are socio-technical in nature rather than only technical. To be even more nuanced, also unlike engineering there is in PA practices also an artistic component, which might not be present in an engineering machine - although the author of this chapter, himself an engineer by training before turning to the social sciences and PA in later university degrees, is mindful of the preface to a handbook of construction science where a prominent scholar and engineer, himself the designer of countless civil engineering infrastructures scattered across the globe, warned about the artistic component to any engineering artefact: it is only intuition that can bridge the hiatus between the design of a machine and the real machine; there is always an artistic component to engineering.)

How can the process of extrapolation of a practice for replication elsewhere unfold? The extrapolation protocol worked out in Ferlie and Ongaro can be summarised in a very schematic way in the following steps (for more detail see Ferlie \& Ongaro, 2015:186-197):

- Identify the function to be performed: the first step lies in defining what is the nature of the performance (in both the meanings of the English word performance: doing something, and achieving a level or standard in doing it) that is being sought: for example in defining whether the practice is one that facilitates the management of change (the function to be performed by the practice is then a change function), or whether it is about the delivery of a certain output (the function to be performed by the practice is then a production function), and so forth: what "performance" is required for the effects to be produced;

- Define and analyse the practice: usually this step should be split into two: first, the understanding of how the system operates (what is the nature of the socio-technical system on which the practice intervenes), and then, second, how the practice takes advantage of the way the system operates to produce the given positive effects that are ultimately being sought. Too 
often, the practice is thought "directly" to produce the positive effects: instead, more generally the practice is a way of interacting on a system (which possesses its inner dynamics) in such a way as to beget certain outcomes that eventually steer the system towards producing the desired results;

- Consider all the effects of the practice, including side effects or negative effects that may come with the practice. A bit like in medicine, it is very rare that a practice produces only positive effects without side or outright negative effects, to be stemmed or contained when replicating the practice in the target site;

- Define the context factors: the conditions under which the practice produces its effects - to forecast the impact of the transferred practice under the varied circumstances where it is being replicated.

The gist of this approach is that it relies on a form of knowledge that is different from both the enlightening knowledge and the problem-orientated knowledge (though closer to the latter). It is an embodied knowledge, incorporated into the practice whose process of knowledge generation is more akin to that of reverse engineering.

There is a final and very important qualification: we have so far considered "practices that work," meaning that we have assumed that the effects produced by the practice were ultimately (overall and with the important qualifications outlined above) "positive," i.e. capable of creating, rather than depleting, "public value." It is worth noting that there is a need also to study "practices that do not work," meaning that their effects may be conducive to worsened, rather than improved, situations. These practices too could and should be considered, with a view to "learning from failure" in ways that are not distant from the proposed protocol, although obviously engineered in the opposite way, to try to avoid negative effects to be replicated elsewhere and spread out. This final reflection leads us to reckon a topic which is not discussed directly in this chapter, and this is the question of "who learns," next to the questions of "what is learnt, and how"...

We can now turn to wrapping up on the conjoint usage of the three forms of knowledge we have been discussing. We do so by interrogating what role the leading learned society in Europe can play in each of these three forms of knowledge, for the advancement of the practice of PA. 


\section{Forms of knowledge and the contribution of EGPA}

We may conclude these reflections on the forms of knowledge for the practice of PA for this EPPA I Book by considering what could and ought to be the role of EGPA, as the leading learned society in knowledge generation about PA in Europe, in the production of knowledge in the three forms identified and defined in this chapter.

Looking at the EGPA portfolio of activities (Bouckaert and van de Donk, 2010; Ongaro, 2019), it stands out how the distinctive feature of the organisational model of EGPA lies in the centrality of the Permanent Study Groups, the platforms for the development of research across the sub-fields of PA into which EGPA is organised. They represent major "engines" of the production of research in the field of PA in Europe. Apart from that, already back in the 1980 os and 1990 s and then with reinforced impetus since the early 200os, EGPA has engaged in the development of strategic partnerships with institutions and organisations for the practice of PA in Europe, like EUPAN, the European Public Administration Network which gathers the Departments for Public Administration of most EU Member States and Associate Countries across Europe. In 2014, EGPA has also launched a series of "Policy Papers on European Governance" explicitly aimed at drawing the implications of research generated within the EGPA study groups for the practice of PA. In the mid and second half of the 2010s, the International Institute of Administrative Sciences has further developed its orientation to produce counsel and advice for policy-makers on key global policy issues, and EGPA as the European regional group of IIAS - with its strong research base - has also developed its thrust to engaging into policy advice for the institutions of the EU and the countries of Europe.

Taking all these developments together and interpreting them in the terms of the forms of knowledge discussed in this chapter, we can read (but also critically query and reckon the appropriateness of) the EGPA portfolio of activities as follows:

- The EGPA Permanent Study Groups as aimed at producing enlightening knowledge;

- The EGPA Policy Papers on European Governance and the EGPA partnerships as aimed at producing problem-orientated and practice-embodied knowledge;

- EGPA and IIAS, when engaged in advising policy-makers, as aimed at delivering problem-orientated knowledge. 
The same questions that have been discussed throughout this chapter can be applied to EGPA and its contribution to the three forms of knowledge: what is the "impact" of the theoretical knowledge which EGPA contributes to generating? How can demand-driven/problem-driven knowledge be organised at and for the European level, given the features of European governance? How can EGPA contribute to a critical and constructive development of "good/adequate practices" for the development of PA?

Wrapping up, in this chapter I argue that knowledge for the practice of PA comes in three main forms: enlightening knowledge (theory-centred); problem-orientated knowledge; and practice-embodied knowledge. All three forms of knowledge are required for the development of the practice of PA. This interrogates the extent to which the European PA scholarly community -organised in the national academic systems, in the EU research frameworks, and in European learned societies like EGPA - is apt and adept in its governance forms, in its career paths for scholars, in its professional-epistemic culture to bolster all three forms of knowledge and to integrate them in such a way as to make a difference to the practice of PA.

\section{References}

Bouckaert, G., \& van de Donk, W. (eds). The European Group for Public Administration (1975-2010): Perspectives for the future - Le Groupe Européen pour l'Administration Publique (1975-2010): Perspectives pour le future. Brussels: Bruylant.

Bauer, M. (2018). Public Administration and Political Science. In E. Ongaro, \& S. van Thiel (eds). The Palgrave Handbook of Public Administration and Management in Europe (pp. 1049-1065). Basingstoke and London: Palgrave Macmillan.

Bretschneider, S., Marc-Aurele, F.J. Jr., \& Wu, J. (2005). Best Practices Research: A Methodological Guide for the Perplexed. Journal of Public Administration Research and Theory 15: 307-323.

Dunlop, C. A. (2019). Bracing for Impact: Is Public Administration Ready to be Relevant?. In A. Massey (ed.). A Research Agenda for Public Administration (pp. 79-96). Cheltenham: Edward Elgar.

Dunlop, C A. (2018). The political economy of politics and international studies impact: REF2014 case analysis. British Politics 13(3): 270-294.

Ferlie, E., \& Ongaro, E. (2015). Strategic Management in Public Services Organisations: Concepts, Schools and Contemporary Issues. London and New York: Routledge.

Hood, C. (1998). The Art of the State: Culture, Rhetoric, and Public Management. Oxford: Clarendon Press. 
McCain, K. (2016). Nothing as Practical as a Good Theory? Does Lewin's Maxim still have in the Social Sciences?. Computer Science 52(1). Retrieved from https://doi.org/10.1002/ pra2.2015.145052010077.

Ongaro, E. (2017). Philosophy and Public Administration: An Introduction. Cheltenham and Northampton, MA: Edward Elgar.

Ongaro, E. (ed.). (2019). Public Administration in Europe: The Contribution of EGPA. London: Palgrave.

Ongaro, E., \& van Thiel, S. (eds). (2018a). The Palgrave Handbook of Public Administration and Management in Europe. Basingstoke and London: Palgrave Macmillan.

Ongaro, E., \& van Thiel, S. (2018b). Languages and Public Administration in Europe. In E. Ongaro,\& S. van Thiel (eds). The Palgrave Handbook of Public Administration and Management in Europe (pp. 61-98). London: Palgrave Macmillan.

Pollitt, C. (ed.). (2013). Context in public policy and management: The missing link?. Cheltenham and Northampton, MA: Edward Elgar.

Pollitt, C. (2016). Advanced Introduction to Public Management and Administration. Cheltenham and Northampton, MA: Edward Elgar.

Pollitt C. (2019). Shadowland: The poorly mapped, underdiscussed yet vital interface between public administration research and practice. In A. Massey (ed.). A Research Agenda for Public Administration (pp. 28-46). Cheltenham and Northampton, MA: Edward Elgar.

Talbot, C., \& Talbot, C. (2018). Usable Knowledge: Discipline-Oriented versus Problem-Oriented Social Science in Public Policy. In E. Ongaro, \& S. van Thiel (eds). The Palgrave Handbook of Public Administration and Management in Europe (pp. 1213-1234). Basingstoke and London: Palgrave MacMillan. 



\title{
Do we Practise What we Preach when we Teach (and Research) Public Administration?
}

\author{
Raffaella Saporito
}

"Science further presupposes that what is yielded by scientific work is important in the sense that it is worth being known. In this, obviously, are contained all our problems." Max Weber, Wissenschaft als Beruf, 1917.

\section{$1 \quad$ Introduction}

\subsection{Exploring the borders of the theory-practice gap in PA studies}

In 2018, the new Italian Minister of Public Administration announced a "new" incentive plan for the civil service in order to boost employee motivation and PA performance. The newness of this typically NPM reform is uncertain, since performance-related pay (PRP) for local government employees was introduced in Italy for the first time in 1983, and two years later for the rest of the civil service. Since then, PRP has been revised and reformed several times. Similar patterns can be observed in the rest of European and Western countries, where PRP reforms have kept being introduced since the 1970 s (Lah and Perry, 2008; Proeller et al., 2014). This is a typical example of what we observe within the practice of public administration reforms, which has been so deeply and extensively investigated (Pollit \& Bouckaert 2017; Ongaro 2009).

If we turn our attention to what PA theory says on PRP, we know how huge and consistent the body of knowledge is in demonstrating not only the limited impact of pay for performance on public employee motivation and performance (especially when compared to other sectors), but also the reasons of PRP failure in the public sector and the risks of its persistence 
(Perry et al 2009; Bowman, 2010). This is just an example - among several others - of a short circuit between theory and practice in PA.

The purpose of this example is not meant to establish the rare impact of PA studies on practice - a long list of counter-examples can be made here - but to illustrate that breaks in the learning loop between theory and practice occur, the ways in which they can occur, and what implication this has on the practice of scholars.

In common debate the theory-practice gap is frequently framed as a matter of divergent agendas: scientific reliability of academic research often pushes for the selection of research topics and questions not always relevant in practice; scientific journals and their reviewers are more interested in methodologically solid studies, even if they are not that strong in terms of implications for practice, which is a disincentive for more practice-oriented research. The divergent agendas issue was one of those most cited by participants in the EPPA Seminar on PA Theory and Practice, held in Strasbourg on 5 and 6 April 2018. However, the case of PRP diffusion presented here highlights another aspect of the theory-practice gap: we have a strong coincidence of the PA reforming agenda in the real world with PA research and theory (public management reforms, public personnel policies, public service motivation theory, to provide a list of just the PA research topics involved there), and a remarkable effort to transform this body of academic knowledge into practical implications (Christensen, Paarlberg \& Perry, 2017). Nevertheless, even when theory is relevant to the real world of PA, it does not always inform the practice.

The explanation of why NPM reforms are so persistent, despite the solid body of scientific evidence explaining the highly controversial results (Pollit, 2000), is deeply rooted in PA theory and especially in new institutional theory, which has brought enlightenment to how the logic of appropriateness inspires managerial reforms more than the logic of effectiveness (March and Olsen, 2004; Thoenig, 2003). Yet, what are the implications for the academic practice of such theoretical explanation? This issue constitutes the basis of the present contribution, which aims to offer some thoughts on how to bridge the PA theory-practice gap, starting with the practice of PA scholars themselves, which is not limited to the job of research, but also to teaching and disseminating, as will be illustrated in the next paragraphs.

\subsection{Objectives, structure and approach}

Beyond pure research activities, PA academics are also quite often teachers and sometimes consultants, board members and opinion leaders. According to this perspective, the field of PA practice is also occupied by scholars, and 
this is the locus of this article. The objective of this contribution is to explore how PA studies can inform PA practice, starting with the job of academics in their research, teaching, and other dissemination activities. To this purpose, the methodological approach in this chapter is used to review some of the most common practices of PA scholars in their knowledge production and supply through the lens of some of the most influential PA theories or logics, such as Public Value, Public Governance and New Institutionalism, to explore the coherence of the theory-practice gap within the job of academics.

Thus, this paper is divided into three parts. The first, borrowing some concepts from the Public Value paradigm, aims at exploring what value is created in the process of PA knowledge production in terms of different target beneficiaries and types of PA knowledge: does the difference in terms of target beneficiaries (academics, students and practitioners) affect the type of value created by PA knowledge? Do such differences give rise to different and irreconcilable epistemologies?

In the second part, the focus is narrowed down to a typical field of encounter between scholars and practitioners, which is the executive education of PA. In this part, the practice of teaching PA to executive students is reviewed through the lens of New Institutional Theory and Public Governance: how can we bring PA theory into the practice of teaching in terms of content, approach and methodologies?

The third and concluding part, based upon the previous discussions, offers some tentative recommendations about how we can move and shift teaching and executive education - according to the EPPA purpose - for the next 20 years.

Some premises are required to introduce this chapter.

The first is about how much insight and how many ideas of this work come from discussions with the diverse and passionate group of participants at the EPPA seminars, especially the one held in Strasbourg on 5 and 6 April 2018, which focused on the topic of "PA and Practice," followed by the final EPPA meeting in Lausanne on 4 and 5 September of the same year. Both occasions were an amazing and quite rare opportunity openly to exchange thoughts and opinions in a structured context on this critical topic, combining the perspective of leading scholars in different European schools of public administration, together with consultants and researchers involved in policy-making and advice, and with public executives from all institutional levels, from all over the EU, coming from local, regional and national institutions of many different countries.

The second premise is how the subfield of PA studies, Public Management - where the author comes from - represents at the same time a resource and a limitation. It can be a resource to this debate for its intrinsic practical 
orientation: using and adapting Mintzberg's words to capture this context, management is a combination of art, craft and science (Mintzberg, 2004). So is public management. Even the etymology of "management" derives from the Latin word manus meaning "hand," as a symbol of its strong connection with practice and practical activities. However, Public Management is just one view among several others on PA studies - as discussed in other chapters of this book - and it can limit the perspective on PA practice to the domain of public management practice or - at least - public management reform as a policy field. Thus, this point of view can be challenged or integrated with other perspectives on public administration studies.

The last premise is about the reflexive nature of this contribution, coming from the everyday practice of teaching public executives, designing and delivering action research projects and customised executive education programmes for public administrations in the field of public management. It includes some of the thoughts on how to use PA theory to inform executive education for public managers and why further explorations were needed over recent years, to the point of inspiring the redesign of an Executive Master Programme for Public Managers at SDA Bocconi School of Management, under the direction of the current paper's author (Hiedemann, Nasi and Saporito, 2017). Leveraging on this set of direct experiences and reflexive observation, this contribution aims to share some kind of learning from this. For this reason, even the slightly provocative tone of some of the paragraphs is also full of respect, gratitude and affection for this amazing job we have the privilege to do, which is to create and disseminate knowledge about and for public institutions.

\section{Exploring what (public) value PA knowledge production creates, for whom, and how}

\subsection{Targeting potential PA knowledge beneficiaries}

Whether Public Value is an established PA theory or more a reference for public management practice in search of more theoretical grounds is still under discussion (O’Flynn, 2007; Benington, 2011; Bryson, Crosby \& Bloomberg, 2014; Hartley et al., 2017). For the purpose of this chapter, the notion of public values is conceptualised "as that which is created or added through the activities of public organisations and their managers. The focus is on what is added in value pertinent to societal outcomes" (Hartley et al. 2017, p. 673). From this point of view, based on the pioneering contribution by Moore 
(1994, 1995), a strategic approach is used to redesign public services (Osborne, Radnor \& Nasi, 2013) in order to move them closer to user needs and, thus, more effective and valuable. According to this perspective, which is largely influenced by marketing studies, the key question about public value creation is: for whom is this service creating value? Who will be the direct or indirect beneficiaries of such a service? Without a clear notion of the ideal recipients of any public endeavour (services, projects, programmes), there is no clear reference how to appraise whether the process has created public value or not. For example, the strong tradition of state schools, state universities and almost free (or for a very low fee) education in many European countries is fundamental in guaranteeing a well-established right to education. Yet, we ask if they really create public value. When we look at how educational attainment is distributed in society, we find that family wealth and parental educational attainment still play a crucial role in the schooling experience and success of the children. Paradoxically, we could have a completely free university just populated by the sons and daughters of the richer and more powerful classes, if other obstacles inhibiting access to higher education for the poorest are still there, with costs brushed aside (such as other cultural and social obstacles and many others). If the intention of state universities is to democratise access to higher education, then the public value to be created should be measured in terms of the increase in graduates from the lower classes. According to this logic, a clear definition of the target recipients and their needs is the critical starting point to orient value creation.

Applying the same logic to our context of PA knowledge producers and disseminators leads us to ask what our target is. Who are our ideal beneficiaries? How should we segment the PA knowledge of potential users? These questions can be useful for investigating the coherence of PA knowledge supply in relation to knowledge needs.

Going back to the presentations and commentaries shared in the Strasbourg meeting, the answers to these questions can be summarised as follows: PA knowledge has three typical markets of target beneficiaries - the scholars themselves, students and practitioners.

The first is the academic community. Research findings are meant to become published studies, mostly read by other scholars, with the purpose of building up - through a collaboration process primarily based on academic journals - a stable, reliable, coherent and comprehensive set of descriptions and interpretations around the function and evolution of public institutions. Since the PA field of study is widely fragmented and divided into an increasing number of sub-fields - an indicator of it can be how many new EGPA study groups have been created in the last decade, or how many new academic 
journals have been launched in the field of PA studies - the PA academic community is probably too vast a definition, and it is perhaps more appropriate to talk about several interconnected knowledge communities. However, their mechanisms of functioning are almost the same, thus the academic professional profile - despite some differences among national educational and academic systems - is very similar: research is their primary mission, followed by or in conjunction with teaching undergraduate and/or graduate students, depending on the context. For this reason, PA scholars are at one and the same time PA knowledge producers and beneficiaries.

Moving on to the second segment of PA knowledge beneficiaries, PA students, their profile varies across countries and contexts very much according to a number of variables, such as whether or not Public Administration is a mandatory field of study for those who want to join the civil service; student age and professional seniority; the type of programme (undergraduate course vs. master or executive education programme). This specific target group of PA knowledge beneficiaries can be considered between practice and academia because not all students involved in a PA class are necessarily prospective (or current) public executives, nor is the intellectual development the sole expectation that students have. If $\mathrm{PhD}$ students are probably closer to (or likely to join) the academic community, MPA students are definitely closer to (or likely to join) the practice community. Yet in both cases a good mix of theory and practice is necessary - for PhD students in order to inspire relevant research questions, and for master's students in order to enhance the awareness of public action and its deeper and wider meaning.

The third segment of potential beneficiaries of PA knowledge is the practice community stricto sensu, including all those people involved in the administration of public institutions. Here, on the opposite side of the academic community, the roles and professions within this large group are several and heterogeneous. First, it can include - depending on the definition we want to use - both civil servants and elected or politically appointed representatives. If we focus our attention on European democracies, the separation between politics and administration is still relevant, which is not necessarily the same in other political regimes, for instance, in Central Asian emerging republics. On our continent, when we target the practice community we should be clear if we are including politicians or not. Here the differences can be relevant. Political discourse usually turns our attention to PA issues when we start asking questions like: why are amazing policy plans not effectively implemented, or why do public services cost so much, or why does it take so much time to get things done in public infrastructure. In the political debate, no matter the embraced ideology, PA is still addressed as the "bureaucratic 
burden" to be lifted. Even if the approach to PA is often affected by such stereotypes, politicians are a relevant part of the PA practice community, given the power and interest they can have in the PA reforming agenda. Narrowing the focus down to the non-political community of practice, this group still includes many diverse components. Public managers and, more generically, public officials are probably the most involved in everyday PA practice. Considering the differences in terms of administrative systems, institutional levels, policy fields and contexts, this group is still wide and heterogeneous in terms of attention and interest to PA knowledge. Focusing on Public Management studies, public executives are, at the same time, the typical and ideal object of research and an ideal target as knowledge beneficiaries. Using the lens of Public Governance, since the production of public services more and more involves other players from the non-profit-making or private sector, the PA practice community should also include non-profit managers, social entrepreneurs, impact investors, philanthropists, and a big list of PA stakeholders, funders, providers and, more generically, a large group of private partners of public institutions. All of them can gain some benefit from a better understanding of PA functions, trends and perspectives in this field. Thus, in the "governance" era, when we target the PA practice community we should frame it as a blended group of professionals and intersectoral managers, instead of keeping a line between executives from the public and the private sector.

In other words, in the process of PA knowledge production scholars can address as beneficiaries of their research these three possible groups of recipients. In terms of preferences, according to the Strasbourg seminar results, scholars love to talk to other scholars who are their prominent target audience and the natural recipients of the vast majority of their work. Some of them enjoy sharing their knowledge with students through teaching activities, which is a very typical activity for many PA scholars. Few - according to the Strasbourg seminar results - consider politicians, public executives, social entrepreneurs and private PA partners as an explicit, stable and intentional target of research and dissemination, thus the supply to this group is quite residual. Is this picture a problem or not? Do we want to be more relevant and see scholarly research have a bigger impact on PA practice or not? Going back to the strategic marketing metaphor, are we reaching our ideal target, or not?

During the EPPA seminar discussions, there was a high degree of consensus that there is a potentially strong interest within public institutions and communities in PA research findings, but scholars rarely proactively approach them. In other words, the emerging diagnosis is that the gap may be related more to supply than the potential demand for PA knowledge. A consolidated explanation of this observation offered in Strasbourg seems based 
on rational choice theory: action research is not incentivised enough, since it does not drive academic publications. According to this perspective, the problem is rooted in scholarly practice - there is just too little interest in the practical implications of research. A similar interpretation has been related to the misalignment of academic research questions and practitioner interest and agendas. We are not investigating enough aspects that are interesting for the practice (i.e. making challenges of territorial reforms) while we are collecting evidence on less relevant topics. Here a third tentative explanation is offered: what if different knowledge beneficiaries also require different types of knowledge in terms of layout, places and epistemologies, and not just different topics?

\subsection{PA Knowledge: one or many products?}

Following the Public Value paradigm, we need to better define what kind of value PA knowledge creates for different beneficiaries. To this purpose, the questions to be answered here are: how does PA knowledge differ in terms of layout and format when it is designed for the three different clusters of potential beneficiaries, i.e. academics, practitioners and students? Where can it be accessed, in terms of physical or virtual places? What kind of needs does PA knowledge satisfy and how?

The first difference is the way in which the knowledge format and layout for the three different groups of beneficiaries are used for "consumption." Papers and articles usually presented in academic conferences or published in scientific journals are for the academic community. Students are more often the recipients of books, handbooks and other readings, such as case studies and notes. Turning our attention to policy-makers, public executives and other private PA partners, the knowledge format is less typical and formalised. Some academic journals also explicitly address practitioners, aiming to serve both communities. Quite often handbook prefaces state that they are talking to students, executive students and practitioners. Executive summaries, infographics and other more practitioner-oriented layouts are more frequently used by consulting companies or by other knowledge providers such as applied research centres, but not usually by academics in their practice. In other words, PA knowledge is a product quite well established in terms of layout and format when the recipients are the community of academics or the community of students. Executives are usually more easily addressed by non-academic research, since they are less frequently the recipients of a specific "product line" offered by academic departments. 
The second difference concerning how to access PA knowledge, online journals and academic conferences is respectively the virtual and physical place where scholars meet other scholars to exchange and produce new PA knowledge. Students usually access PA knowledge when they enrol in a PA programme as part of their undergraduate or graduate education. The places where scholars and practitioners can meet are less clearly defined and more casual. Apart from executive programmes or applied research initiatives, the way collaboration between PA scholars and PA executives is organised is less structured, more informal, and often moves from classrooms to boardrooms.

The third difference is about the different needs that the three communities of beneficiaries have regarding PA knowledge. What scholars look for within their community is usually clear evidence to build up rigorous and generalisable explanations and interpretation of PA functions and development. Students - especially in undergraduate programmes - are more interested in descriptive information and illustrations, since they often need to be introduced to the basic function of Public Administration and related theoretical foundations. The most valuable part of PA knowledge for executives and policy-makers is actionable knowledge in terms of solutions to given problems, advice and guidelines. Hence, this leads to the success of best practice and similar approaches to PA knowledge within the practice community. To simplify this, scholars are interested in "why" questions, students in "what" questions, and practitioners in "how" questions. Just as an example, the topic of pay and incentives for performance is addressed by scholars in terms of why they are less effective in the public sector, with related explanations, such as motivational theories. Students need to start with what the features and limits of civil service compensation plans and motivation are, and what the differences from the private sector are, before jumping into deeper issues. Executives - even if they have shown an interest in more theoretical questions -want to know how to design new compensation plans or to motivate their public employees. These different needs give rise to different epistemologies: the first two refer to positive or descriptive theories, while the last refers to normative or prescriptive knowledge. This interpretation of the distance between academia and community of practice has been called the "two cultures" problem, one of the priests of research purity, the other of soldiers of organisational performance, each culture informed by its own norms and frames of reference (March and Sutton, 1997; Datar et al., 2010). Focusing our attention on how PA knowledge can be used in the practice community, new institutional theories explain the power of clear norms, rules, guidelines and other forms of prescription as a source of legitimacy for public action. In that sense, evidence-based PA can be seen as a sort of 
"scientification" of the source of legitimacy of public action and decision making in the public arena.

To conclude, scholars tend barely to focus on how to reach practitioners. Playing with words, we can say that practitioners are not included in academic practice, since this practice is not generally perceived as the business of scholars. As noted in Strasbourg, this gap is not due to a lack of interest in the practice community, but to a scarce supply. Furthermore, the signs of such scarce supply are the shortage of stable and institutionalised places of exchange between academics and practitioners, and the low diffusion of journals, papers or other more usable formats - such as executive reports or infographics - offered by the world of academia for the practice community. In addition, among the list of reasons for this gap that were explored during the Strasbourg seminar - such as small incentives for scholars who want to work on action research projects - the epistemic distance probably deserves to be better investigated. Consulting companies or similar agencies are more willing to carry out research projects aimed at offering benchmarking analysis, costs-benefit analysis, evaluation projects and other types of "actionable knowledge" that can easily be implemented into policy design. In other words, they offer solutions and answers to given questions without any specific theoretical background. Alongside this, PA Theory has clearly explained how much those "evidence-based" solutions play the game of legitimacy in policy-making (Weiss, 2001). If the epistemic gap can be one of the reasons for scarce interaction between scholars and practitioners, how can this be filled? How much space is there in the case of action-research projects to inject some theoretical perspectives, which is typical of academic research? Is it possible to combine critical thinking with prescription, or positive interpretation with normative guidelines?

\section{Back to PA theory to better inform the practice of teaching PA with public executives}

\subsection{Theorising or pushing isomorphism?}

Among the "how" questions coming from the real world of PA practice, "how can we change the administrative system" is the one question that has been among the most persistent and prominent. The ways scholars have been approaching the long list of research questions related to the "change issue" of public administration are countless, but a certain stability can be identified in the theoretical paradigms and explications used to deal with it. Since Max 
Weber's studies, the Public Administration research agenda has been strongly influenced by the institutionalism umbrella paradigm, as over time it has been articulated in a myriad of old and new versions (Peters, 2011). Narrowing the field down to public management reforms in European or Western countries from a comparative and historical perspective (Pollit \& Bouckaert, 2017; Ongaro, 2009; Mele \& Ongaro, 2014), the (new) institutional theories have been useful not only in explaining the dominance of the NPM paradigm, the convergence and hybridisation of different administrative systems, but also the controversial and limited impact of those reforms on performance more often oriented to a formal compliance rather than a measurable managerial impact (Capano, 2003; Anessi-Pessina, Nasi \& Steccolini, 2008; Mele, 2010). The publicness of such organisations makes them more exposed to external legitimacy pressure in the absence of other sources of pressure (i.e. market competition for the private sector) (Frumkin and Galaskiewicz, 2004). After decades of research in this field, the managerial reforming agenda keeps displaying a strong normative and mimetic isomorphic power over public institutions at local, national and international levels in almost every continent, regardless of the impact of performance. In other words, managerial recipes have just been replacing (or more often adding to) red tape, since both - public management and bureaucracy - answer the common need of external legitimacy of public action. Since the diffusion of New Public Management, we have still been observing the perpetual dissemination of a sort of "managerial orthodoxy" for public administrations, as just a set of new management-like formal procedures and prescriptions to comply with and conform to, regardless of the organisational needs it is meant to answer. However, when Public Management becomes an orthodoxy, who are the evangelists?

Years ago, during the redesign of the above-cited Executive Master Programme for Public Managers offered by the SDA Bocconi School of Management, it turned out that the research agenda and results of some faculty members had been under-represented in their teaching, since they did not believe that this kind of knowledge would be interesting to public managers. The effort of providing actionable knowledge and very practical insights to an executive audience - features that have always been considered a strength of the cited executive programme - was driving some of the faculty to a sort of epistemic schizophrenia: the same assumptions and discourse that they were used to challenge through their research were applied to their executive teaching classes to match public manager expectations. The fear of being considered "too theoretical" or "not relevant for the practice" was deeper than the confidence in the implications for practice of their papers 
and research. This observation was an amazing occasion to open an internal debate on what we want to offer our executive students, with regard to what we think they need, which is not necessarily what they ask for. Using this case to reflect on the role of schools and scholars involved in Public Management studies, the question becomes controversial: can we be, at the same time, public management evangelists and their most critical reviewers? Going back to the epistemological issue discussed above, what role do we want to play both in the classrooms and in the boardrooms? How willing are we to raise more questions and doubts, rather than giving quick answers and solutions? How much do we trust in our theories and the value they can offer for the practice? Or are we changing epistemologies once when we change audience?

\subsection{Theorising on governance, but practising government?}

Among the theories of Public Administration, one of the most popular that stands out - and is meant to overcome the New Public Management paradigm - is Governance or Public Governance (Rhodes, 1996; Osborne, 2006), which focuses attention on the plurality of actors involved in the production of public value, the fragmentation of power among different governmental levels, the increasing involvement of non-profit-making organisations in public service delivery, overcoming the diffusion of private-public partnerships, and the formal and informal influence of stakeholders in the policy process. All these aspects call for a new way of explaining the function of public administration. Despite the democratic risks and limits of the informal and negotiating nature of management practice within the governance paradigm, the collaborative exercise and the net metaphor continue to fascinate scholars and practitioners. Yet, how much are the current Public Management programmes (i) intersectoral, (ii) collaborative and (iii) network-based?

(i) Despite the dominant narrative of interdependence across sectors in PA theory, if we turn our attention to executive education and other dissemination initiatives for the practice community, it is still uncertain how much they are open (and attractive), not just to managers of the public sector, but also those from the non-profit-making and private sectors. Here, yet again, differences in terms of institutional contexts and across countries matter and play a major role, but intersectoral initiatives are still a minority within the supply of PA master's or other executive programmes. Conversely, MBA programmes and other general business management programmes have always been attractive for public managers too, and such educational experiences are among the drivers of NPM values dissemination in dominant discourse on public administration. How far can MPA or other PA programmes be 
directed at (or are able to attract) managers from the private or non-profit, not to be able to jump into a public career, but because they recognise the need for new visions and competences to interact and create value with public institutions? If some intersectoral programmes are offered, for instance in the field of public-private partnerships (PPP) or public infrastructure financing and other related topics, they are rarely delivered by PA professors and the paradigm of business is still dominant, which is probably one of the reasons why there is insufficient focus on how to create public value with PPP, instead of just shareholder value (Vecchi et al., 2017).

(ii) How collaborative and co-produced are executive PA programmes in the field of PA, where collaboration and co-production have been recognised as key factors in public value creation? If, on the one hand, we can observe a progressive evolution of the curricula, moving away from the public version of traditional business functions (organisation, ì leadership and HR, budgeting, cost accounting, finance, procurement, etc. ...), to new courses, such as public governance, negotiation, co-production and other collaboration-based topics, how much does this paradigm inform the practice of teaching itself? Educational services - executive programmes included - are a typical coproduced service, since learning is experience-based (Kolb, 1984) and it occurs within the interaction between the teacher and the learner, in an enabling setting. Thus, executive programmes can be amazing laboratories where one can experience co-production and teach it through the practical experience of a well-designed co-produced service. Some insights about how to move from a delivery paradigm to a co-produced approach in the field of executive education for public managers have been offered, in terms of co-designing practices of curricula, learning metrics and evaluation methodologies, and the overall learning experience (Hiedemann, Nasi \& Saporito, 2017). However, a lot of other co-produced practices in executive education can be better formalised and diffused, such as the use of learning journals and self-case studies - together with, or instead of, traditional case studies - in order to have leverage over the reflection of practice as a place of learning, using theories as analytical tools; and in order to enhance peer-to-peer learning, using the classroom as a structured setting to share experiences and ideas.

Finally, (iii) if networks are one of the most popular topics of PA studies, it has not been so influential in training design for public officials and civil servants. There, the assembly line is probably the most appropriate paradigm to understand how training programmes have been run for a long time. Training is not so infrequently conceived as the sum of a linear sequence of courses, each delivered by a different professor or trainer, not necessarily integrating with each other in terms of vision, mindset or frames of reference, like workers 
in an assembly line. If there is any project work, internship or other practical experience, it is added as another workstation through which the manufacturing piece (the trainee) has to pass. This is even more evident in government national schools and similar recruitment and training institutions, built up as the archetype of the French ENA, where in general there are few core faculties, and where teaching hours are bought on the market and provided by different professors, professionals or other senior civil servants. In this model, the trainee is frequently passively exposed to speeches, lectures and lessons, without a clear fil rouge, or any other andragogical methodology, actively and intentionally to help learners to make sense of and create value from such a diverse range of perspectives. The institutional model of government schools has always been more focused on the recruitment side than on educational or training aspects, since these ENA-like schools have been established as the gateway to the civil service elite (Saporito, 2016). The critical part has always been how to access it, and not really what happens after joining the school. Even critics of the ENA model are more focused on how the recruitment process reinforces elitism and corporativism (Rouban, 2014), rather than how effective it is in terms of educational services. Here the network challenge for the education and training of civil servants could be how to organise, integrate and promote collaboration between the plurality of institutions, public and private, local and national, academic and non-academic - such as governmental departments or agencies, academic departments and colleges, research centres, and any other PA knowledge provider or disseminator - to be actively involved in contributing to the development of a new public ruling class.

\section{Concluding remarks and recommendations for teaching PA to executives}

The reasons behind breaks in the learning loop between theory and practice occurring in the public administration field can be researched not just in terms of the behaviours of policymakers or public executives - whose decisions are normatively accused of being insufficiently evidence-based - but also in terms of scholarly behaviour and practice. Using some of the most popular theories or paradigms diffused in public administration theory to observe and interpret PA scholarly practice, some conclusions and many further questions can be offered.

The perceived value of PA knowledge varies within the broad range of beneficiaries. Each of the three clusters of potential knowledge recipients 
(academics, students and practitioners) reaches PA knowledge at a different point, through different formats and layouts, and for different purposes. PA knowledge is predominantly a "product" of scholars for other scholars, aiming at building up a better understanding of PA function and evolution, and academic conferences and journals are the material and immaterial places of interaction. Teaching students, especially undergraduates and/ or graduate students, is another frequent activity in PA scholarly practice, but the type of knowledge offered in this case is not necessarily the same as for the academic community. Students are more interested in a wider, but less profound, description of the state of the art, and even the formats to convey knowledge, such as books, case studies and lessons, are different. Despite the differences, PA knowledge is a quite well-established "product" for both communities and it continues to be reproduced and diffused through institutionalised formats and places. Practitioners, on the other hand, are reached by PA knowledge in sporadic and less formalised ways, and even the places of interaction are less structured and frequently informal. Consulting companies or other knowledge providers on the market more often play a major role, compared to academia, which tries to defend its position, leveraging on the accreditation of the executive education that reinforces the formal value of knowledge, such as credits, degrees, grades and diplomas. However, the greatest difference regarding what kind of value PA theories produce is based around the type of knowledge that is considered valuable by practitioners: best practice, guidelines, tools and other forms of normative knowledge are considered more relevant than explanations, explorations, descriptions and other forms of positive knowledge preferred by scholars in their research and teaching. According to this interpretation, the gap between theory and practice is more than just a misalignment of research interests between scholars and practitioners, and is linked to an epistemic gap between positive vs. normative. How irreconcilable is this gap?

This issue is tremendously most relevant for those scholars involved in executive education programmes, where the interaction with practitioners is structured and formalised. Here the dilemma between positive and normative theory is more superficially framed as being either very analytical but too theoretical, or very practical, but too superficial. Sometimes the risk of this interpretation is to fall into a sort of theoretical schizophrenia, where we offer practitioners the same managerial toolbox that in academic papers we have described as useless or scarcely successful. The evergreen metaphor of the managerial toolbox is a paradigm of what practitioners expect from executive education: clear norms and practical tools. However, if we try to interpret the success of the toolbox metaphor, it is probably due to how it 
openly puts the "problem" of management far from the manager and the set of his/her behaviours, judgments or decisions. Once tools are designed and implemented, things are supposed to be fine, as in a never-ending mechanism of hope (Brunsson \& Olsen, 2018). It is not very often that you listen to a public executive asking to learn what he/she can change in his/her behaviour or decisions in order to improve. Just as an example, public managers usually ask how to motivate public employees, but hardly ever ask what they can change about themselves in order to become better leaders; or they ask how to work on job design to reduce staff turnover, even if team members usually leave because of the boss, not the job, and thus they should be interested in how to change their behaviour. In other words, practitioners tend to consider themselves not to be part of the picture, but more external "shunters." Thus, if a need for knowledge can be observed here, even if not directly expressed by policy-makers or public managers, it is to enhance awareness about their role and the space they have to recognise, interpret and mindfully deal with the context and its challenges, including the isomorphic pressures.

The same conclusions can equally be drawn for scholars in the way they perceive the theory vs. practice gap: what if the problem is not just the scarce interest of practitioners in evidence-based policy or the superficial attraction for best practice and protocols, but the practice of scholars themselves?

Since the purpose of the EPPA project is not just to review the "state of the art" but also to formulate future directions for the next 20 years, what are the implications of these discussions for the practice of teaching PA?

As a sort of summary and a further development of what has been presented up to this point, we can try to identify, by applying the comparative approach, the current or dominant learning model for executive PA programmes in Europe to, better to focus on what it could be like in the future.

The first relevant dimension is about how to define the target beneficiaries, since - as has already been said - the way knowledge is imparted and used is deeply influenced by the characteristics of the users. If, at the present, PA programmes and other initiatives for the practice community tend to look mainly at civil servants, public managers or people within public institutions, the challenge for the future is how to reach a wider social group of PA stakeholders, which also includes private/non-profit entrepreneurs, CSR experts, managers from public utilities and other private industries closer to public interests (like infrastructures or impact investing), consultants for the public interests and all the professionals involved in the "governance" arena. If the creation of public value involves more and more professionals and managers from other sectors, it means that they should be included 
in our concept of PA practice community together with professionals and managers from the public sector.

Table 1 Comparing Learning Models for Teaching PA in the Present and in the Future

\begin{tabular}{|c|c|c|}
\hline Dimensions & Present Learning Model & Future Learning Model \\
\hline $\begin{array}{l}\text { Target } \\
\text { beneficiaries }\end{array}$ & $\begin{array}{l}\text { Public managers and executives } \\
\text { (or prospective public managers } \\
\text { and executives) from public } \\
\text { institutions }\end{array}$ & $\begin{array}{l}\text { Public managers and executives, } \\
\text { non-profit entrepreneurs, CSR } \\
\text { experts, managers from public } \\
\text { utilities and private sectors closer } \\
\text { to public interests (infrastructures, } \\
\text { impact investing), as well as public } \\
\text { interest consultants }\end{array}$ \\
\hline $\begin{array}{l}\text { Learning } \\
\text { objects }\end{array}$ & $\begin{array}{l}\text { Learning logics and tools of } \\
\text { management applied to the public } \\
\text { sector }\end{array}$ & $\begin{array}{l}\text { Empowering individuals, organisa- } \\
\text { tions and public-private networks } \\
\text { to create public value }\end{array}$ \\
\hline Epistemology & $\begin{array}{l}\text { PA knowledge is produced in } \\
\text { academia and transferred to } \\
\text { practitioners }\end{array}$ & $\begin{array}{l}\text { PA knowledge is co-produced } \\
\text { within the process of formally and } \\
\text { informally observing - from outside } \\
\text { and from inside - the functioning } \\
\text { of public institutions }\end{array}$ \\
\hline $\begin{array}{l}\text { Knowledge } \\
\text { dissemination } \\
\text { formats }\end{array}$ & $\begin{array}{l}\text { Formal: books and articles; } \\
\text { conferences; classrooms }\end{array}$ & $\begin{array}{l}\text { Informal: blogs, websites and social } \\
\text { media, infographics, online videos; } \\
\text { high added-value meetings }\end{array}$ \\
\hline Providers & $\begin{array}{l}\text { Local and national PA depart- } \\
\text { ments, schools and universities }\end{array}$ & $\begin{array}{l}\text { European or international networks } \\
\text { of PA knowledge co-producers }\end{array}$ \\
\hline $\begin{array}{l}\text { Performance } \\
\text { measures }\end{array}$ & Credits & $\begin{array}{l}\text { Organisational change and public } \\
\text { value created }\end{array}$ \\
\hline
\end{tabular}

Considering the heterogeneity of potential beneficiaries, the main goal of any executive education and training initiative cannot be limited to disseminating logics and tools of management applied to the public sector: first, for the reasons already given regarding the paradox of considering public management knowledge as an orthodoxy to be preached; second, because the learning objectives should be embedded in the purpose of grouping together public, private and non-profit-making involved in creating public value, which cannot be limited to teaching management. Thus, the main purpose of such executive initiatives should be empowering individuals, organisations and public-private networks to create public value, each from its location and role, and each in coherence with its organisational mission. Even if the learning journey always takes place at the individual level, it should 
also have an impact on organisations and meta-organisations involved in the public value creation process, as will be better explained later on.

The way learning objects are framed is deeply influenced by the underpinning epistemology. If in the current and dominant model PA knowledge continues to be considered more as an "academic product" to be transferred to practitioners, as stated above, PA knowledge can, on the other hand, be considered as co-produced within the process of formally and informally observing the functioning of public institutions. If scholars are in charge of the formal and scientific observation and interpretation, it does not mean that practitioners (both from inside public institutions and from the stakeholders' community) do not have an original and relevant point of view on the evolution and exploration of new arrangements to create public value in a "governance era." This could be the platform for collaboration between scholars and practitioners in the process of knowledge co-creation.

Based on the differences in terms of epistemologies, different dissemination formats can be identified. We keep trying to reach the practitioner audience using traditional and formal tools, such as books and journals for the written discourse, and conferences and other "school-based" settings as a place of meeting and interaction, but how up-to-date, contemporary and coherent are these formats for the proposed epistemology? In a future that has already come, to be influential means being involved in a more informal and collaborative form of written discourse, using blogs, websites and social media, where information is designed in a more user-friendly way through infographics and short videos. Moreover, the digitalisation of the executive education (i.e. MOOC and other online programmes) is spreading out thanks to lower fees, no travel costs, a more flexible learning schedule. Would these electronic places of communication and learning be substitutes for any other form of face-to-face interaction? Here the debate is open. Probably some physical interaction will be in place, if it will offer an irreplaceable value in terms of learning experience: classrooms should be run more like boardrooms, where people are called to participate actively, bringing and sharing their own experiences, negotiating meanings, building mutual trust, and expanding the space for action.

To what extent can individual schools or departments be the sole providers of such a learning experience? Probably we need not just to become more interdepartmental, but we also need to create more collaborative learning initiatives that actively engage different types of institutions, both public and private, according to a partnership and networking mindset. As an example, for teaching "European public management" to public executives, we have been experimenting with quite a successful format, where SDA Bocconi 
partners with ENA, the European Investment Bank (EIB), the European Commission, and the European antenna of the largest Italian association of entrepreneurs. Over the period of a week, Italian public managers travel to the three cities of Strasbourg, Luxembourg and Brussels to visit these institutions and discuss with a select number of representatives a well-structured and agreed agenda of topics. Each institution profits from the experience: ENA can offer their executive students from a similar programme the opportunity to collaborate in small groups with visiting Italian peers; EIB can use the occasion to communicate their investment policies and share opportunities for the Italian public administrations; EU senior executives from the European Commission are willing to meet Italian public managers, not just because policy dissemination is part of their mission, but because it is also an informal occasion to orient the national, regional and local policies, as for the hot topic of how to overcome the limits of accessing and usefully spending European funds; and, finally, lobbyists are willing to engage national institutions on their platforms. At the end of the study tour, participants can take home a wide-reaching perspective on how European institutions work from the inside, and what the role of their national or local institutions could be like within a multi-level governance setting, in addition to the networking opportunity that a similar experience can offer. The main objective of such an experience is to create partnerships where the commitment of each member to the agreed learning goals and defined agenda is strong and well-focused.

Lastly, to better orient the design of a new executive learning experience, we may need new, clear performance measures: the "accreditation" of diplomas and certificates as a result of introducing the European Credit Transfer and Accumulation System (ECTS) and similar systems, can become a bureaucratic trap and risks shifting the focus from looking for a transformative educational experience to accumulating credits. To avoid this trap, it would be worth being bolder in selecting and communicating our educational performance indicators, moving from a more traditional set (i.e. number of face-to-face vs. e-learning hours; numbers of credits; faculty seniority and CVs; impact on career success; traditional exam performance) to more social impact measures: how many innovative ideas have been implemented thanks to the programme; how many new partnerships have been established thanks to the programme's networking opportunities; and, in a few words, what public value has been created, what kind of impact it has had, and for which beneficiaries. 


\section{References}

Anessi-Pessina, E., Nasi, G., \& Steccolini, I. (2008). Accounting reforms: determinants of local governments' choices. Financial Accountability \& Management, 24(3): 321-342.

Benington, J. (2011). From private choice to public value. In J. Benington, \& M. H. Moore (eds). Public value: Theory and practice (pp. 31-49). Basingstoke: Red Globe Press.

Benington, J., \& Moore, M. H. (2011). Public value in complex and changing times. In J. Benington, \& M. H. Moore (eds). Public value: Theory and practice (pp. 1-30). Basingstoke: Red Globe Press.

Bowman, J. S. (2010). The success of failure: the paradox of performance pay. Review of Public Personnel Administration 30(1): 70-88.

Bryson, J. M., Crosby, B. C., \& Bloomberg, L. (2014). Public value governance: Moving beyond traditional public administration and the new public management. Public administration review 74(4): 445-456.

Brunsson, N., \& Olsen, J. P. (2018). The Reforming organization: making sense of administrative change. Abingdon: Routledge.

Capano, G. (2003). Administrative traditions and policy change: when policy paradigms matter. The case of Italian administrative reform during the 1990s. Public administration 81(4), 781-801.

Christensen, R. K., Paarlberg, L., \& Perry, J. L. (2017). Public service motivation research: Lessons for practice. Public Administration Review 77(4): 529-542.

Datar, S. M., Garvin, D. A., Cullen, P. G., \& Cullen, P. (2010). Rethinking the MBA: Business education at a crossroads. Boston, MA/ Harvard Business Press.

Frey, B. S., Homberg, F., \& Osterloh, M. (2013). Organizational control systems and pay-forperformance in the public service. Organization Studies 34(7): 949-972.

Frumkin, P., \& Galaskiewicz, J. (2004). Institutional isomorphism and public sector organizations. Journal of public administration research and theory 14(3): 283-307.

Hartley, J., Alford, J., Knies, E., \& Douglas, S. (2017). Towards an empirical research agenda for public value theory. Public Management Review 19(5): 670-685.

Hiedemann, A. M., Nasi, G., \& Saporito, R. (2017). A public service-dominant logic for the executive education of public managers. Teaching Public Administration 35(1): 66-87.

Lah, T. J., \& Perry, J. L. (2008). The diffusion of the Civil Service Reform Act of 1978 in OECD countries: A tale of two paths to reform. Review of Public Personnel Administration 28(3): 282-299.

March, J. G., \& Olsen, J. P. (2004). The logic of appropriateness. In R. E. Goodin (ed.). The Oxford handbook of political science (pp. 478-497). Oxford: Oxford University Press.

March, J. G., \& Sutton, R. I. (1997). Crossroads: Organizational performance as a dependent variable. Organization science 8(6): 698-706.

Mele, V. (2010). Innovation policy in Italy (1993-2002): Understanding the invention and persistence of a public management reform. Governance 23(2): 251-276.

Mele, V., \& Ongaro, E. (2014). Public sector reform in a context of political instability: Italy 1992-2007. International Public Management Journal 17(1): 111-142. 
Mintzberg, H. (2004). Managers, not MBAs: A hard look at the soft practice of managing and management development. Oakland, CA: Berrett-Koehler Publishers.

Moore, M. (1994). Public value as the focus of strategy. Australian Journal of Public Administration 53(3): 296-303.

Moore, M. H. (1995). Creating public value: Strategic management in government. Cambridge, MA: Harvard University Press.

O’Flynn, J. (2007). From new public management to public value: Paradigmatic change and managerial implications. Australian journal of public administration 66(3): 353-366.

Ongaro, E. (2009). Public management reform and modernization: Trajectories of administrative change in Italy, France, Greece, Portugal and Spain. Cheltenham: Edward Elgar Publishing.

Osborne, S. P. (2006). The New Public Governance? Public Management Review 8(3): 377-387.

Osborne, S. P., Radnor, Z., \& Nasi, G. (2013). A new theory for public service management? Toward a (public) service-dominant approach. The American Review of Public Administration 43(2): 135-158.

Perry, J. L., Engbers, T. A., \& Jun, S. Y. (2009). Back to the Future? Performance-Related Pay, Empirical Research, and the Perils of Persistence. Public Administration Review 69(1): 39.

Peters, B. G. (2011). Institutional theory in political science: The new institutionalism. New York: Bloomsbury Publishing USA.

Pollitt, C. (2000). Is the emperor in his underwear? An analysis of the impacts of public management reform. Public Management an International Journal of Research and Theory 2(2): 181-200.

Pollit, C., \& Bouckaert, G. (2000). Public Management Reform: A Comparative Analysis (pp. 6-23). New York, NY: Oxford University Press.

Proeller, I., Vogel, D., Mussari, R., Casale, D., Turc, E., \& Guenoun, M. (2014) Performance related pay in European local governments. A comparative Analysis of France, Germany, Italy. Conference Paper 2014 Annual conference of the European Group for Public Administration (Speyer, Germany).

Rhodes, R. A. W. (1996). The new governance: governing without government. Political studies 44(4): 652-667.

Rouban, L. (2014). La norme et l'institution: les mutations professionnelles des énarques de 1970 a 2010. Revue francaise d'administration publique 3-4: 719-740.

Saporito, R. (2016). Analisi comparata dei sistemi della selezione della dirigenza delle amministrazioni central. In Barbieri, M., et al., Sistemi di selezione e valutazione dei dirigenti pubblici in Europa. Spunti per la riforma in Italia (pp. 25-92). Milano: Collana OCAP, Egea.

Thoenig, J. C. (2003). Institutional theories and public institutions: Traditions and appropriateness. In G. Peeters, \& J. Pierre (eds). Handbook of public administration (pp. 127-37). London: Sage.

Vecchi, V., Hellowell, M., Della Croce, R., \& Gatti, S. (2017). Government policies to enhance access to credit for infrastructure-based PPPs: an approach to classification and appraisal. Public Money \& Management 37(2): 133-140.

Weiss, C. H. (2001, July). What kind of evidence in evidence-based policy. In Third International, Inter-disciplinary Evidence-Based Policies and Indicator Systems Conference, July 2001. Durham: CEM Centre. 

VI

PUBLIC ADMINISTRATION AND COUNTRY PERSPECTIVES 



\title{
1
}

\section{Public Administration in Estonia: A Search for Identity}

\author{
Külli Sarapuu and Leno Saarniit
}

\section{$1 \quad$ Introduction}

The international debate on the public sector increasingly revolves around change - the need for innovation, creativity, agility and the capability to address new complex challenges. Even if all of today's discussion about digital transformation, artificial intelligence, big data and global megatrends seems to detach us radically from the past, in Central and Eastern Europe (CEE) this demand for innovation and change still evolves against a somewhat different background than in consolidated democracies. The context of CEE has been shaped by recent systemic transformations, overcoming historical legacies and cultural interruptions. Perhaps this is most true of Estonia, which at the beginning of the 1990s had the sharpest break with the preceding system even among the Baltic states by reason of which the first year of independence and breaking out of the centralized soviet structure was named "Year Zero" (Lieven, 1993, p. 316), i.e. starting all over again. Knowing this context and exploring its nuances gives us insight into the shape of Estonian public administration as a field of study today and helps us to make some educated guesses about its nature and challenges in the years to come. Context matters and there are different layers of transformation to grasp with regard to both the past and the future.

CEE's challenges of institutionalising public administration education and research in a situation where state sciences did not exist under the communist rule have been captured elsewhere (for example, Staroňová \& Gajduschek, 2016). Importantly, the challenges were not technical but to a great extent substantial, and affected not only the nature of public administration as a field of study, but the very conceptualisation of the state itself and its core functions (see e.g. Drechsler, 2004; 2005). Consequently, the task of describing the landscape, relevance, and future of public administration in Estonia demands that attention is paid to both sides of the coin - the field and its environment. The snapshots capturing the evolution of public administration 
as a field of study in Estonia (Randma, 2000; Kalev et al., 2008; Lauri, 2019) reveal continuous transformation and adaptation induced and moulded by relentless changes in the context -in the higher education system as well as the Estonian state and public administration. Both the field and the Estonian administrative system have been in search of their identity and the best fit in a turbulent environment. The following chapter attempts to depict public administration as a field of study in Estonia, its nature, context, relationships with actual practice, and potential elements of the future.

\section{The field of public administration}

In Estonia, the evolution of today's public administration programmes started at the beginning of the 1990s. Although the re-establishment of the state and the initial design of its structural elements were strongly influenced by the legacies and the example of the Republic of Estonia which was established in 1918 and occupied by the Soviet Union in 1940, public administration as a field of study had to be built up from scratch after the regaining of independence in 1991. In 1995, the chair of Public Administration was founded at the University of Tartu, after a few years of preparation. The establishment of the chair and the introduction of a corresponding curriculum demanded, among other things, translating the term "public administration," which did not exist in the Estonian language (Randma, 2000, pp. 85-86). The Estonian counterpart "avalik haldus" is essentially a literal translation, but as a novel combination of two common words it was a term that was unknown to society at the time. In Tallinn, a joint public administration programme between Tallinn University of Technology (TalTech) and Tallinn University (formerly Tallinn University of Educational Sciences) was instituted in 1994 with the assistance of a Tempus programme (Kalev et al., 2008, p. 114). A few years later, the curriculum evolved into two separate programmes in the respective universities. The initiative of establishing public administration programmes in the 1990 s was purely academic and not induced or coordinated by the Ministry of Education (Randma, 2000, p. 85). As elsewhere in CEE, the introduction of the curricula and their content was largely dependent on the individuals leading the work, and often required explaining what public administration as a scholarly field was (Randma and Connaughton, 2005).

Unlike other CEE countries, where the dominantly legal approach to public administration education prevailed (Staroňová and Gajduschek, 2016), in Estonia the programmes evolved from a foundation of social science and economics and combined different disciplines from the very beginning 
(Randma, 2000). Furthermore, they often engaged Estonian lecturers who had been educated abroad or foreign lecturers hired with the help of international assistance. As the programmes were novel, interdisciplinary and drew on international experience, they became remarkably popular, attracting high numbers of students and the best high-school graduates. Consequently, in 1998, the Estonian Business School introduced a BA programme in public administration and became the first private institution to offer the degree (Randma, 2000, p. 86). Several other public and private institutions followed suit and started teaching public administration programmes with varying content and quality at a diploma or bachelor level. Such a proliferation of programmes was made possible by the neoliberal approach to the public sector reforms at the beginning of the 1990 s which was also reflected in higher education. As a result, a very fragmented higher education market emerged with a considerable number of autonomous institutions and a competitive atmosphere. The number of institutions providing higher education grew from 21 in 1994 to 49 in 2001 (Estonian Quality Agency for Higher and Vocational Education, 2017). The increase in students of public administration was part of a rapid growth in students in social sciences in general. Notably, the overwhelming majority of them were self-financing as the contribution of the state to social sciences education was modest and in decline.

Nevertheless, this boom in public administration education lasted for less than a decade. By 2008 , only the three original public universities were still teaching it. Other programmes were closed in the mid-2ooos as they failed to obtain accreditation due to problems with the quality of the education and the difficulty of making the curricula profitable (Kalev et al., 2008). Remarkably, even after that, most of the public administration students were still paying for tuition and their studies were not financed by the state. As Kalev et al. (2008) calculated, at the time only about $15 \%$ of all the students in social sciences received state funding and the remaining $85 \%$ had to pay for their education.

In the following decade, public administration education saw some considerable changes yet again. These were induced both by reforms in the higher education system and institutions as well as the changing social scene. First of all, some major changes happened in the higher education system. In the 2009/2010 academic year, the European Credit Transfer and Accumulation System (ECTS) was introduced in Estonia. It happened in parallel with a substantial reform of the higher education quality assurance system whereby assessment of study programme groups was introduced instead of the accreditation of individual programmes. Based on the new system, a positive evaluation of a study programme group in an institution results in the Government of 
the Republic granting a licence to provide education in the respective group. It is complemented by institutional accreditations. In 2009-2012, "transitional evaluations" of study programme groups took place, and since 2012 the evaluation of study quality is completely group-based. Second, in 2011 a momentous reform of higher education financing was initiated, resulting in "free higher education" since the 2013/2014 academic year. Since the reform, all students studying in programmes that are in Estonian, satisfy the requirements of their curriculum, and meet the stringent criteria of full-time study, have received tuition-free higher education. To provide tuition-based programmes in Estonian, the universities need authorisation from the Ministry of Education and Research, and it is considered a big exception. Tuition-based programmes in English are allowed. In order to cover their costs, public universities and state professional institutions of higher education receive performance support from the state budget which consists of baseline funding and performance funding. Performance funding is based on fulfilling performance indicators and adhering to the performance agreement signed with the Ministry of Education and Research. The reform caused significant budgetary tensions in the universities that have only grown in the following years.

Third, the general demographic and societal scene has also been problematic for public administration and social sciences (and humanities) in general. Demographic changes have resulted in contracting numbers of potential students. The number of students admitted to higher education institutions and the size of the student body have been shrinking since 2010 (Estonian Quality Agency for Higher and Vocational Education, 2017). The effects of the diminishing body of potential students have been amplified by more negative attitudes towards public administration studies. On the one hand, the officially declared priorities of the higher education system are emphasising applied higher education, technology and natural sciences. This has resulted in policies oriented towards reducing the numbers of students studying in the "business and administration" group. For example, between 2019 and 2022 TalTech is expected to reduce the number of students admitted to business and administration BA studies by $20 \%$ compared to the intake in 2017 . On the other hand, the trend has also been supported by negative rhetoric in the media, where the problems of the Estonian labour market have been blamed on young people wanting to become "public managers," probably drawing on the image from the beginning of the 2000 s when business management and public administration studies were at the height of their popularity.

Altogether, every single reform in the higher education system and the more recent wave of structural reforms in public universities that has been oriented at consolidation and centralisation have forced public administration 
programmes to reconsider their identity and formulate a rationale for their existence. Every such reform has brought with it changes in the existing curricula or often ended their existence. Consequently, by 2019 the public administration programme, once one of the most popular curricula in Estonia, had contracted drastically and is still taught only in two public universities - TalTech and Tallinn University. There are eight curricula that can be considered public administration programmes, but only three of them, which are taught in TalTech (one BA, one MA, and one $\mathrm{PhD}$ ), can be characterised as "classical," while the remaining five contain a public administration module or are more widely integrated political science programmes (Lauri, 2019). The TalTech public administration programmes are also the only ones that are still categorised as "business and administration" studies in Estonia; the rest of the public administration programmes have moved into the group of "social science" studies. In the University of Tartu - the cradle of the field in Estonia - there is practically no public administration left and the focus is purely on political science and international relations. The changes in Tartu started with the introduction of the Bologna system in 2002, when the distinct public administration curriculum disappeared at BA level, and was dealt the final blow (or one could say the fatal blow) with the move of the core public administration faculty to TalTech in 2006-2007.

While the general trend in the Estonian higher education system is the increasing introduction of English-language public administration programmes, especially at the MA level, in public administration the curricula have disappeared, rather than been turned into English public administration programmes. The English-language public administration programmes that do exist focus on topics that are more easily marketable internationally and have an EU and international relations focus or interdisciplinary ambition (for example, the Technology Governance and Digital Transformation MA programme in TalTech which combines economics, governance, public policy, technology and innovation). The different focal points and contents of the remaining programmes also reveal the different profiles of the institutions teaching them. While Tallinn University has evolved more towards political science, governance and public policy analysis, TalTech is combining classical public administration with economics and innovation studies and is a leading institution in the debate on the digital transformation of states. In CEE comparison, the Estonian public administration programmes are outliers due to the distinctively non-legal character of the curricula and the higher share of analytical courses (Staroňová and Gajduschek, 2016).

Compared to public administration education which has gone through turbulent developments over the past 25 years, public administration research 
was much slower to pick up and reach an internationally acceptable level. In the higher education institutions with their short-lived public administration programmes, decent research never existed, and that was a significant aspect of their problems of assuring high-quality education. In the University of Tartu, once the leading centre of both public administration education and research, any competence largely disappeared together with the move of the core staff to TalTech. In 2019, the TalTech Ragnar Nurkse Department of Innovation and Governance is clearly the strongest and the most internationalised Estonian research institution in the field of public administration and has a very interdisciplinary approach that focuses on governance models and governance capacity, digital governance and digital transformation of societies, science and innovation policies, fiscal governance, different modes of public sector and cross-border collaboration, and governance of small states. The research undertaken in Tallinn University's School of Governance, Law and Society focuses mostly on political science, civic culture, local self-government and public policy analysis, and most notably education policy and migration. There is limited collaboration between the two institutions, both in education and in research. At the same time, inter-disciplinary cooperation in research and policy analysis in general is on the increase, but among institutions and disciplines outside the state sciences, for example IT, life sciences and human geography. This has been driven by financial incentives - the need to bring home research grants in a situation where social sciences and higher education in general are under-financed and research funding is scarce and competitionbased. The impact of project-based financing of public administration study has perhaps been the greatest on doctoral studies where the topics of $\mathrm{PhD}$ dissertations are increasingly shaped by the research projects and the general performance management approach to higher education has directed attention to productivity and outputs rather than curiosity and individual growth.

\section{The context}

This kind of evolution of the field of study in Estonia reflects the turbulent environment of higher education as well as the change in the Estonian state itself. Estonia's development has generally been characterised as a success, and one of the fastest political and economic transformations in CEE, especially by outside observers. Estonia's 2017 human development index (HDI) value was 0.871 , positioning the country in the very high human development category. The 2018 Corruption Perceptions Index of Estonia, published by Transparency International, reached an all-time high of 73 points out of 100 
and set Estonia apart from all the other CEE countries. But perhaps Estonia has gained the most international attention and built a positive image through the quick digitalisation of the state and the transfer of a high number of public and private sector services to electronic platforms (for example, "Estonia, the Digital Republic” in New Yorker (Heller, 2017)).

This perceived success has its roots in the initial transition at the beginning of the 1990s, when the first elections carried out in accordance with the new constitution in September 1992 and the victory of the nationalisticconservative anti-communist Pro Patria Union ensured a sharp break with the preceding system. Vast administrative changes followed, in terms of both institutions and people. The public sector was downsized, many state functions were privatised; the foundations of the organisational structure of the Estonian central government were laid down, and the legal-administrative framework for a merit-based public service was established. Until 2016, Estonian political, economic and social development was characterised by the domination of a neo-liberal worldview with all the governing coalitions being led by liberal right- or centre-oriented parties. Such an ideological disposition can be explained by the domination of New Public Management (NPM) at the time of the initial transition, the communist legacy of distrusting the state, and the Estonian cultural predisposition towards valuing independence and individualism.

Although the NPM-led approach to the state supported extensive reforms of the public sector and the abandonment of Soviet legacies, it had little to contribute to the affirmative conceptualisation of the state and its image as a positive form of organising joint living. The high uncertainty, enormous workload and intensive time pressure of the 1990 introduced a decentralised problem-solving approach that was further supported by the aim of overthrowing the legacy of the centralised and vast soviet public administration. The latter meant that the political elite was very cautious towards all manifestations of central coordination. By the time Estonia started to move towards becoming a member of the European Union, the segmented system had already been institutionalised. Although such a system promoted clear accountability for a number of policy issues and the accumulation of professional knowledge in individual institutions, difficulties emerged in ensuring the coherence of different policies, solving problems that involved several areas of government, and agreeing on joint solutions to deal with "wicked issues." In 2011, the OECD Governance Review on Estonia was published (OECD, 2011). It concluded that Estonia operated a fragmented and decentralised public administration and that "the apparent ambivalence of politicians and administrative leaders regarding reforms seems to reveal 
a lack of shared understanding about the role of the public administration for ensuring Estonia's future" (OECD, 2011, p. 21).

Although the report said little that was new to those familiar with the Estonian administrative system and relied on the information and opinion of local experts, it changed the governance discourse in Estonia and resulted in the Government's formal Action Plan to implement the recommendations of the report. In essence, the Plan and its following formulations became the public administration reform strategy in Estonia, which was mostly financed from the EU structural funds. Consequently, the European Social Fund (ESF) has been the main donor for Estonian public administration development. Transversal activities focusing on the administrative system have been at the centre of "raising administrative capacity" with the ESF's support. The role of the ESF in administrative development and reform has been so central that, in practice, the operational public administration programmes with their requirement of seven-year strategic planning have largely constituted the Estonian public administration reform programme.

What it implies is that until very recently the public administration system was hardly in the sphere of interest of politicians and that the development of public administration has consisted of a number of project-like undertakings. The administrative developments of the last few years that have taken place within the ministries' areas of governance have been dominated by mergers of institutions and measures of standardisation, optimisation and centralisation. This search for efficiency has been the defining characteristic of the reforms. Similarly, the public service system has mostly been approached through the lens of cost-efficiency and cutting back on the number of officials. The democratic aspects of public service and its role in carrying certain values in the state have been over-shadowed by the efficiency concerns. At the same time, Estonia has developed a very open position-based public service system where the responsibility for all the main components of the public service, such as recruitment and selection, performance appraisal, remuneration, training and development, are in the hands of individual organisations and their implementation is inconsistent (Randma-Liiv et al., 2020). In a situation where there is no tenure in the public service, no public service pensions, and public servants can be laid off as easily as in the private sector, the entire system is vulnerable to the application of un-meritocratic practices like politicisation, nepotism and misuse of power by individual managers. Despite this, the latest goals of public administration reform in Estonia still focus largely on efficiency and consolidation. In 2019, the declared political aims of the reform are a more efficient central government and reduction of the administrative burden; maintaining the current ratio of public sector 
personnel in the overall workforce (which in practice means a decrease in public sector employment); and keeping the GDP percentage of public sector costs at the same level (public sector expenditure as less than 40.4\% of GDP) (Ministry of Finance, 2018). Among other means, IT and e-solutions are seen as one of the key levers for increasing efficiency.

\section{$4 \quad$ Links with practice}

Conceptualising the scope and substance of public administration as a field of study is unavoidably related to the national context (Randma-Liiv and Connaughton, 2005). The context also shapes the relations between the field of study and actual practice. In Estonia, the links are influenced by the fragmented nature of the public administration system, the small size of public administration as an academic field, and the small size of the country in general. Only three member states of the European Union - Cyprus, Malta and Luxembourg - are smaller than Estonia with its population of 1.3 million (2017). Although one could expect that in a small state with personalised relationships and limited possibilities for specialisation and in-depth expertise (see Randma-Liiv and Sarapuu, 2019) there would be extensive links between academia and practice to make use of the scarce knowledge, this has not always been the case. Barriers hindering closer cooperation have come from both sides.

First, from the side of the administrative system, its modus operandi has often made it difficult to take advantage of the expertise in universities. The differences in timeframes, expectations and interests of the two sides have perhaps been the most obvious in the case of engaging academia in policy analysis and evaluation, which is often contracted out through procurement procedures and financed from the EU's structural funds. Obtaining know-how through procurement means that there is little room for flexible cooperation. Furthermore, there are formal limitations to consultation during the procurement process. Even in cases where universities have had the competence to provide the expected analysis, procurements have often failed. On the one hand, the deadlines set by administration tend to be too short for academia, both for submitting the tenders and providing the expected outputs. On the other hand, officials' limited competence or time shortages often result in vague or unrealistic calls with contradictory aims, over-regulation of methodological details, unworkable schedules underestimating the time needed for data gathering, writing, and gaining feedback, or reflect the inability of public institutions to use the data that already exist and are available to them. 
The procurement-based approach reflects the widespread projectification characteristic of the Estonian public administration and the fragmentation of analytical activities.

A similar mode of operation and discontinuity has also prevailed in the organisation of civil service training, where the absence of a central civil service training institution and a limited budget have contributed to a patchy system that is not able to cover all the relevant subjects and target groups (except for the group of top civil servants whose training and development have been generously financed). Organisation of horizontal, system-wide training has been dependent on the EU structural funds, and coordination of the training of local government officials has been lacking. With regard to the latter, no understanding of the target-group or long-term strategies exist. Consequently, the training organised by individual public institutions has been heterogeneous and uneven. The central training of civil servants, coordinated by the Ministry of Finance, has been largely procurement-based and project-type in nature, inhibiting the institutionalisation of more stable networks and knowledge exchange.

With regard to the educational background of public officials, systemic demand for public administration education has been largely missing in Estonia. Although public administration graduates have been welcomed and gladly hired by the administrative system, the combination of a decentralised civil service system with market-type higher education regulation has created a situation where respective curricula are not requested from the universities. The development and content of public administration programmes have been fully dependent on the universities. Although programme councils usually include the "representatives of the employers" (i.e. someone from the civil service), the universities normally rely on their own best understanding of the field and international advancements in developing the curricula, rather than the day-to-day demands of practical public administration.

On the other hand, reliance on the practical demands would be accompanied by alternative tensions, represented, most of all, by different perceptions of what should be taught in universities. University curricula of public administration at BA as well as MA level tend to be oriented at the general nature and principles of public policy-making and implementation, trends that influence governance, and analytical skills. The arguments for such an approach derive from changing and turbulent environments where public officials must have critical analysis ability and some core values to anchor their choices. However, public managers expect fresh graduates to be able to "do things" and feel frustrated when they have to be taught how to use document management programmes for sending out official letters or 
which forms have to be filled in for issuing building permits. The demand for academic education to be more skill-based or "technocratic" often manifests itself in discussions on the relevance of social sciences and their funding from the state budget in general.

The usual solution to the trade-off - internships - has so far not been sufficiently functional to overcome the tensions. Characteristically for the decentralised personnel management system of the Estonian civil service, there is no central internship programme and it is up to individual organisations (or even units) whether or not to offer any internship positions. Any information about these positions is available only on institutional websites, quite often simply providing a general e-mail address for contact. Public administration students mostly have to rely on their own initiative to find internship positions. More rarely, institutions publish internship offers that universities can distribute through students' mailing lists. Often, public institutions feel that they do not have the time and human resources to provide needed support for the interns, so they prefer not to take them on at all. More systematic internship opportunities have been institutionalised in the Estonian Academy of Security Sciences which offers specialist education in certain fields such as customs, taxation, prisons and police work. For these fields, internship arrangements with individual government agencies are possible, which is not the case for universities which do not prepare students for working in one specific organisation or policy area.

Second, when looking at academia, several barriers to sharing the expertise and using it to serve society appear too. There are a number of aspects that work against closer cooperation with practitioners. Public administration as a field of study is quite small in Estonia, with few individuals who have to take care of research, teaching and academic administration. Their capabilities are often stretched to the limits. In addition to that, due to the general under-financing of higher education and almost absent baseline funding of research, the budgets of academic institutions are increasingly more dependent on winning competitive research grants. Consequently, due to considerable opportunity costs, academic units and individuals have limited incentives to go after the short-term funding of policy analysis. Large research grants tend to provide more stability than applied studies offering a part-time salary for a couple of people for a restricted time-period.

Difficulties in cooperation also result from different timeframes and interests, not just tight resources. An academic unit that plans its workload months in advance based not only on teaching obligations but also schedules imposed by international research projects, is quite often not able to fit a short-term, strict deadline analytical job into its timeframe. The same applies 
to in-service training projects. Furthermore, the questions asked and topics explored do not necessarily match either. Research staff are selective in undertaking applied studies as the questions asked for making policy decisions are often too narrow or too different from the questions that interest the researchers. The need to satisfy the pressure for international publication and the increased impact factors in combination with growing attention paid to specific methodological solutions do not always combine easily with the practical needs of public institutions. All these factors work against investing one's time in applied activities. Consequently, these have to either be personally intriguing, provide empirical information for further academic research endeavours, bring in additional salary, or rely on academics' sense of mission. Otherwise, the projects often present more of an onus than an opportunity. However, this can lead to further widening of the gap between scientific and applied studies, followed by decreased trust and understanding of each other's perspectives.

Different timeframes, the temporary nature of project-based financing, academic performance management systems, the interests of practical policy analysis, differences in language and aims of study, lack of capabilities - all these factors have proved to be obstacles to cooperation as well as trust. Nevertheless, this does not mean that the relations between academia and practice have been only problematic or non-existent. There are several examples of good cooperation with significant impact. These instances of rewarding cooperation have taken traditional as well as more innovative forms. More traditionally, members of academia have consulted on policy processes, informally as well as formally (e.g. drafting of the new Civil Service Act, analysis of innovation and research policies in Estonia), have conducted in-service training for public officials (from new recruits and future leaders to mid-level managers and top civil servants, on topics as various as strategic and financial management, coordination, mission-based policy design and public service ethics), have served as members of governmental committees, and have conducted policy evaluations. In addition, several innovative ideas and projects have been implemented. For example, the Ragnar Nurkse Department of Innovation and Governance, TalTech, has been coordinating the biggest EU Horizon2020 innovation project "TOOP” (The Once-Only Project) focusing on better exchange of business-related data and documents with and between public administrations in the EU. The Nurkse Department has also implemented the Sohjoa Baltic project in cooperation with the city of Tallinn, resulting in a self-driving minibus being given over to public use.

Another noteworthy example of interaction between academia and civil service is the field of public service ethics, where the expertise existing in 
the universities has contributed to the evolution of ethics-related debate and the introduction of respective institutional structures over a time-period extending over several years or even decades. Academic public administration staff (at the time from the University of Tartu) became involved as early as in 1998, when the drafting of the first public service code of ethics began (Saarniit, 2006). A few years later, when the first anti-corruption strategy was discussed, the idea of a public service ethics council was mooted. This idea was discussed thoroughly in 2009, including during meetings between the Government Office and the (then) Department of Public Administration of TalTech, focusing on the membership, tasks and scope of the proposed council. The ethics council was finally formed in 2013, after the new Civil Service Act came into force. From the beginning, the council has included "external" members from universities (TalTech and the University of Tartu), not only from the field of public administration, but also from philosophy and journalism. The council has adopted a new code of ethics (2015), several good practice guidelines, and consulted on draft regulations on preventing conflicts of interest and given opinions on specific cases of unethical behaviour. Although the council is not as visible as it could be (for example, by being more proactive), it represents a good case of academia-practice cooperation, which is also evident in the civil service ethics training system. Currently, the main ethics trainers include civil servants, representatives of anti-corruption non-profit organisations, as well as members of academia from TalTech.

At their best, similar cooperation endeavours have benefited both sides practitioners have gained analytical and knowledge-based support in everyday work and researchers have obtained empirical data for scientific publications and formulating new research questions. Still, this cooperation of academia and practice has much room for improvement, and it could benefit from having more "pracademics." Although there are several consultancies and one main think-thank, Praxis, that mediate academic knowledge to the administrative system, the merging of academic and civil service careers is still rare. Civil service jobs tend to be full-time positions that do not make it easy to conduct research or teach students. Those who leave academia usually do not return. However, as the Estonian Civil Service Act, which regulates civil servants' auxiliary activities, makes an exception for pedagogical and research activities in educational institutions as a permitted activity (e.g. tax officials teaching tax law in a university), there is a way for the experts of public administration practice to be engaged in academia. In a way, the Estonian legal system recognises the small state issue of limited expertise and provides for the best use of it. Still, combining the two careers is usually sporadic and 
temporary. Public officials teaching in universities tends to be a short-time solution, sensitive to changes in workload, and presents a potential risk to continuity and stability in teaching. Luckily, civil servants are usually open to being involved in academic courses on a more ad hoc basis and happy to accommodate invitations to meet students and share their work experiences.

\section{The future}

In Estonia, public administration as a field of study has gone through considerable changes, in terms of research as well as teaching, in terms of volume as well as substance. The field has evolved in the context of wider societal and economic transformation. Developments in higher education as well as in the administrative system have forced the academic field to define and re-define its identity and to cope with transformative pressures while maintaining its distinctive nature. The pressure to find its unique essence and its place in the scientific and educational landscapes, both in Estonia and internationally, as well as to establish a healthy relationship with the world of practice, can also be expected to be the signature tune for the future.

When looking at tomorrow, the Estonian state and its public administration are facing several demanding questions. For example, how to cope with "global megatrends" in economic, environmental, social and technological spheres as a small state in the European periphery; how to uphold the trust of the people and meet their increasing expectations towards the state while the Estonian society, somewhat controversially, still believes in a lean state as the ideal; how to maintain the earlier success of the digital state without getting locked into unproductive paths and technological solutions; and how to find a good balance between innovation and stability. The country needs a smart, innovative and merit-based public administration that provides a professional and evidence-based perspective on the policy processes during times of political fluctuations and populist calls that are currently increasingly evident and influential in Estonia, as well as elsewhere. All those processes and challenges obtain an additional layer in the context of digitalisation that transforms the role, aims, relationships, problems and solutions of public governance. It changes the functioning of politics and of the media. In the end, the difficult choices land on the desks of individual public officials and politicians who need to be well equipped for making decisions on issues comprising uncertainty, complex alternatives and moral dilemmas.

Public administration as a field of study should have an important role in helping its practitioners to make sense of those complex problems and make 
decisions in the best possible interests of society. The ability of the field to do that depends on its health and strength. If the existing trends continue, then public administration as an academic field in Estonia is on its way towards further consolidation, or perhaps even fading away, at least in the form of "classical public administration." In public administration education, the trend is towards more political science, technology, and management, as well as teaching practical skills. In public administration research, there is a tendency towards analysing different spheres of public policy, their interaction with megatrends, and dissecting the nature, challenges, and impact of digitalisation.

The essence and the functioning of public administration have been highly dependent on the individual people active in the field. Considering the smallness of the field and the state, it can also be expected to be so in the future. Individual professors and programme managers have been those who have conceptualised the field of public administration in the midst of changes, have interpreted the environmental pressures and viable ways forward, and have shaped the face of public administration as a field of study. Several of the professors who assisted with the birth of academic public administration in Estonia are still there in 2019 and have represented the continuity of the field in the otherwise changing higher education landscape. The future will depend on their legacy, descendants and the viability of the field in an increasingly competitive and under-financed academic system where starting a career as a young researcher demands a lot of determination and patience.

With regard to all the significant issues, public administration as a field of study should be there to help the public administration system to find the best responses to all the challenges, questions and dilemmas mentioned above, not only by offering expertise, but also by being critical and providing alternative interpretations of the problems and solutions. This is something that happens increasingly in cooperation with other fields of study or disciplines, all across the academic landscape. The issues related to the future governance of Estonia need attention both in their entirety, but also in their more detailed aspects like the nature of merit-based civil service in the age of populist democracy, the value choices of public officials in the era of digitalisation, the opportunities for collaboration and network-type coordination in designing and implementing policies in complex policy areas, especially those affected by the "megatrends," the challenges, opportunities and ethical aspects of using big data, etc. Public administration as a field of study can support the public administration system and practitioners through educating public officials, participating in providing in-service training, policy analysis and evaluation, and making research and international knowledge available and 
understandable to domestic decision-makers. To succeed in that, the interest and capabilities of the public administration system in making use of the expertise also need to exist.

\section{Acknowledgements}

The writing of this chapter was supported by Estonian Research Council grant PUT1461. The authors are grateful to the civil servants who shared their thoughts on the relevance and needs of public administration education in Estonia.

\section{References}

Drechsler, W. (2004). Governance, Good Governance, and Government: The Case for Estonian Administrative Capacity. Trames 8(58/53): 388-396.

Drechsler, W. (2005). The Re-Emergence of "Weberian” Public Administration after the Fall of New Public Management: The Central and Eastern European Perspective. Halduskultuur 6: 94-108.

Estonian Quality Agency for Higher and Vocational Education. (2017). Higher Education in Estonia 2017. Retrieved August 9, 2019, from http://ekka.archimedes.ee/wp-content/uploads/ Estonian_HE_System_2017.pdf.

Heller, N. (2017). “Estonia, the Digital Republic.” New Yorker, 11 December. Retrieved August 9, 2019, from https://www.newyorker.com/magazine/2017/12/18/estonia-the-digital-republic.

Kalev, L., Jakobson, M.-L., Sillaste, M., Soiver, V., \& Sootla, G. (2008). Privately Funded Public Servants? Public Administration Higher Education in Estonia. In G. Jenei, \& K. Mike (eds). Public Administration and Public Policy Degree Programmes in Europe: The Road from Bologna (pp. 107-152). Bratislava: NISPAcee.

Lauri, T. (2019). Public Administration Education in Estonia. Country Report for the Erasmus+ Strategic Partnership project PAQUALITY.

Lieven, A. (1993). The Baltic Revolution: Estonia, Latvia, Lithuania and the Path to Independence. New Haven, CT: Yale University Press.

Ministry of Finance. (2018). Public Sector Modernisation of Estonia. Retrieved August 6, 2019 from https://www.rahandusministeerium.ee/sites/default/files/riigsektor_inglise_keeles_loplik. pdf.

OECD. (2011). Estonia: Towards a Single Government Approach. Assessment and Recommendations. Paris: OECD.

Randma, T. (2000). Public Administration Education: Estonia. In T. Verheijen, \& J. Nemec (eds). Building Higher Education Programmes in Public Administration in CEE Countries (pp. 81-118). Bratislava: NISPAcee/EPAN. 
Randma-Liiv, T., \& Connaughton, B. (2005). Public administration as a field of study: Divergence or convergence in the light of "Europeanization"?. Trames 9(59/54): 348-360.

Randma-Liiv, T., Pesti, C., \& Sarapuu, K. (2020). Public Service Development in Estonia: from Patronage to Meritocracy. In P. Liverakos (ed.), Public Service Reform in the post-Soviet Union Countries: Challenges and Lessons Learnt. Forthcoming.

Randma-Liiv, T., \& Sarapuu, K. (2019). Public governance in small states: From paradoxes to research agenda. In A. Massey (ed.). A research agenda for public administration (pp. 162-179). Cheltenham: Edward Elgar.

Saarniit, L. (2006). A Public Service Code of Ethics Applied in a Transitional Setting: The Case of Estonia. Public Integrity 8(1): 49-63.

Staroňová, K., and Gajduschek, G. (2016). Public administration education in CEE countries: Institutionalization of a discipline. Policy and Society 35(4): 351-370. 



\title{
2
}

\section{Public Administration in France: The Shadow and Light of a Revival}

\author{
Jean-Michel Eymeri-Douzans
}

Since cultures and contexts matter so much in the structuring of academic disciplines, the present text devoted to presenting public administration studies in France takes as its starting point the fact that public administration is not and never was a unified and stand-alone academic discipline in France. This may sound very paradoxical in the very country where the modern state was invented in the Middle Ages under the Capetian Dynasty, and the neologism "bureaucratie" invented in the mid-170os by the physiocrat Vincent de Gournay. This chapter tries instead to offer an up-to-date sketch and polyphonic approach to the ongoing research devoted by French scholars belonging to various disciplines to the study of administrative institutions, processes and reforms, to outline the state of the art in the study of the state in modern-day France. As all experienced scholars are well aware, the picture of scientific research in a given domain at a given time is not a simple snapshot of the reality in the field, but the product of a conceptual construction of reality by theoretical perspectives which consciously or unconsciously highlight some features, undermine some aspects, and disregards others which are, later, "rediscovered" by new generations of scholars. As a consequence, and in line with the intentions of this volume, this chapter does not address the French State and public administrations, but the position of the study and teaching of the state and public administration in France.

The first part will deal with the problematic history, the second with the recent revival of research on public administration in France, illustrating some of the subjects and methodologies that enjoy the preference of researchers, and indicate the major research questions and perspectives opened up by such an aggiornamento. Then, the third part will consider if and to what extent this research revival has influenced the teaching of Public Administration in France, while the fourth and last part will propose some speculative and provocative thoughts about future possible developments. 


\section{Short history of a paradoxical neglect}

About two decades ago, surveys, collective projects and doctoral researches, articles, books, seminars and conference panels devoted to the study of the administrative apparatus of the state, of its various administrative institutions, and of the personnel who embody them (re-)started flowering in French social sciences, in the disciplines of history, sociology, political science and anthropology (of modernity). This quite recent interest strongly contrasts with the strange form of neglect or oversight that French social sciences, especially history and political science more than sociology, used to show towards the study of public institutions in general, and administrative institutions in particular, from the 1970 s to the 1990 .

This lack of interest was a true paradox in a country where, ever since the "republicanisation" of the regime in the last third of the nineteenth century, the public bureaucracy - not only within central ministries in Paris and in the country, but also the vast web of prefectures and other "deconcentrated" state services in the provinces, plus the public education and public hospitals, the public bodies and agencies, the three levels of regional, provincial and municipal administration, etc. - brought about a huge "army" of public agents, Ia Fonction publique, a world in itself amounting now to 5.2 million persons and a fifth of all those employed in France. It is a true paradox indeed that French social scientists of the 1970s to 1990 os progressively deserted the topic of public and administrative institutions, with the notable exceptions of Michel Crozier, the prominent French scholar of The Bureaucratic phenomenon, and his disciples of the Centre de Sociologie des Organisations (CSO), Jacques Chevallier and his Science administrative team at CURAPP (Amiens) and then CERSA (Paris 2), and a few isolated scholars (Pierre Birnbaum, Jean-Luc Bodiguel, Françoise Dreyfus, Catherine Grémion, Marie-Christine Kessler, Luc Rouban, Jean-Claude Thoenig, ...) who persevered in studying the (top) civil service from various perspectives.

There is no room here for a detailed analysis of the combined causes of this trend. One can just remember that the major influence of Marxism within the French intelligentsia and over two generations of students and researchers, from the end of World War II until the death of Jean-Paul Sartre and the coming to power of the Socialists in 1981 after 25 years of opposition, was probably decisive. The Marxist Zeitgeist, expressed e.g. in the writings of such intellectuals as Leon Althusser and Nikos Poulantzas, tended to deny any real autonomy to the state, the political power and the public bureaucracies, considered "in the latest instance" as mere "superstructures" and "ideological apparatuses" serving the capitalist domination. Consequently, it would have 
been useless, and even erroneous in logical (and political) terms, for young scholars to devote their works to the study of public administration.

An additional factor relates to the structuring of academic disciplines. Political science is, with "sciences de gestion" (management), a latecomer among French academic disciplines. Unlike in other Western countries, political science was almost non-existent before World War II, and it was only in the 1970 s that scholars coming mainly from the Law or Sociology faculties and working on politics succeeded in setting up a new and independent academic discipline. This French political science in statu nasciendi fully enjoyed its new freedom and explored its own topics - political parties, elections, abstention, political socialisation, social movements, etc. - while gladly abandoning the study of public administration, seen as both an "old-fashioned topic" and a "topic-for-jurists," to scholars of administrative law.

Another cultural factor urged French social scientists, historians and political scientists to neglect the study of public administration, if not overtly to look down on its proponents. In fact, since the beginning of the twentieth century, and following the writings of founding fathers such as Alexandre-François Vivien, under the label of "administrative studies" and even "administrative sciences" (in the plural) French higher bureaucrats have published a profusion of books, professional journals, conferences etc., about the state and its public administrations. Compared to other Western countries, France exhibits a remarkable and distinctive feature: the higher civil service, with its famous concours, grandes écoles and grands corps, is at the heart of the social, political and (partially) economic "Elites de la République" (in the sense of Christophe Charle). This elite, which brings together the offspring of the bourgeois and "notable" lineages and the "best and the brightest" carefully chosen from among each generation by a highly selective secondary and higher education system, has always been attracted to the higher civil service, which provided a far better social status and a stronger influence than academia. Hence, according to a long-established tradition, generations of top civil servants have considered themselves fully capable and legitimate to produce their own vision and understanding about public administration. Each generation promoted its own "organic intellectuals" (to borrow from Gramsci) who authored the official discourse about the state... written by the state itself. The sources of this phenomenon go back to the establishment, in 1871, of the Ecole libre des sciences politiques, a selective, autonomous and innovative institution devoted to the multidisciplinary education of politico-administrative elites, in opposition to the Faculties of Law whose curricula were considered too "academic," mono-disciplinary and traditional. Nationalised and re-baptised in 1945 the Institut d'études politiques de Paris, 
best known as Sciences Po, employs high level scholars but is usually under the rule of top civil servants. In addition Sciences Po is intimately related to the Ecole nationale d'administration (ENA), created in the same year to be the national training institute that opens up (or denies) access to the careers of the higher civil service: there are no professors at ENA (which is very different from Speyer ... in that regard), very few university professors are invited to lecture there, and the vast majority of the seminars and courses are delivered by senior ENA alumni, with an explicit mimetic purpose.

Incidentally, this French peculiarity also explains why the French delegates to the International Institute of Administrative Sciences (IIAS) founded in Brussels in 1930 were recruited almost exclusively from among the higher civil servants and why the Institut Français de Sciences Administratives (IFSA) was hosted by the Council of State and dominated by top civil servants from its foundation until its recent dissolution in 2019.

Neither Sciences Po nor the ENA brought about a body of "academics" of administrative science in France. On the contrary, top civil servants, acting in their dual capacity of practitioners and organic intellectuals, have been constantly invited to lecture on administrative matters and have found there two convenient tribunes to promote, publicise, publish and self-legitimise their own endogenous analyses of the dynamics of French public administration, a sort of praxeological, non-critical, cameral "science of government" whose scientific systematic deconstruction is precisely one of the purposes of the current flourishing of researches on public institutions.

\section{A late-coming yet fruitful flourishing of researches}

As mentioned above, the scientific study of public administration is not in France, as it is in some other countries, a (sub-)discipline on its own. Students of public administration can thus be found among political scientists (mainly), sociologists, historians (a few), anthropologists (very few) - and outside social sciences among administrative law specialists and public management scholars. Such fragmentation means that they do not have a common scientific journal, or ritual congress, or learned society. Even grouping together these scholars belonging to diverse disciplines on the stimulating occasion of an EGPA congress held in France, and editing a collective multidisciplinary volume afterwards, as Geert Bouckaert and the author of these lines managed to do in 2010-2013, is something like a tour de force.

However, it is undeniable that a young (or still young) generation of researchers who devote part or all of their work to the study of public 
administration has been producing an impressive advancement of the French state of the art in that very domain during the last two decades. Amongst this highly productive epistemic community, let us quote (in alphabetical order and without being exhaustive), the names of Thomas Alam, Claire Andrieu, Sylvain Barone, Yannick Barthe, Frédéric Barthélémy, Marc Olivier Baruch, Pierre-Yves Baudot, Michel Bauer, Laure Bereni, Bénédicte Bertin-Mourot, Irène Bellier, Daniel Benamouzig, Julien Besançon, Bruno Béthouart, Philippe Bezes, Michel Biard, Didier Bigo, Emilie Biland, Olivier Borraz, Yves Buchet de Neuilly, Nicolas Bué, François Buton, Stéphane Cadiou, Nathalie Carré de Malberg, Marie Cartier, Michel Cattla, Dominique Chagnollaud, Jean-François Chanet, Florian Charvolin, Alain Chatriot, Lionel Chaty, Louise Dangy, Claudine Dardy, Françoise De Barros, Quentin Deluermoz, Fabien Desage, Florence Descamps, Alain Dewerpe, Yves Dezalay, Véronique Dimier, Nicolas Dodier, Anne-Cécile Douillet, Vincent Dubois, Vincent Duclert, Bruno Dumons, Laurence Dumoulin, Patrice Duran, Renaud Epstein, Jean-Michel Eymeri-Douzans, Alain Faure, Eve Fouilleux, Annie Fourcaut, Brigitte Gaït, Natacha Gally, Gabriel Galvez-Behar, Patrick Garcia, Delphine Gardey, Alain Garrigou, Jean-Pierre Gaudin, William Genieys, Didier Georgakakis, Julie Gervais, Jean-Jacques Gleizal, David Guéranger, Jean-Louis Halpérin, Patrick Hassenteufel, Thomas Hélie, Odile Henry, Choukri Hmed, Arnaud-Dominique Houte, Olivier Ihl, Liora Israël, Gilles Jeannot, Jean Joana, Odile Joint-Lambert, Isaac Joseph, Pierre Karila-Cohen, Sarah Kolopp, Pascale Laborier, Sylvain Laurens, Jean Le Bihan, Patrick Le Lidec, Rémy Le Saout, Claire Lemercier, Christian Lequesne, Dominique, Linhart, Dominique Lorrain, Jacques de Maillard, Michel Mangenot, Christelle Manifet, Michel Margairaz, Nicolas Mariot, Guillaume Marrel, Catherine Marry, Gilles Massardier, Pierre Mathiot, Julien Meimon, Hélène Michel, Dominique Monjardet, Igor Moullier, Christine Musselin, Olivier Nay, Gérard Noiriel, Nicolas Offenstadt, Albert Ogien, Romain Pasquier, Renaud Payre, Etienne Pénissat, Gilles Pinson, Frédéric Pierru, Gilles Pollet, Christophe Prochasson, Laure Quennouëlle-Corre, Olivier Quéré, Anne Revillard, Cécile Robert, Violaine Roussel, Guillaume Sacriste, Yasmine Siblot, Alexandre Siné, Alexis Spire, Yves Surel, Gildas Tanguy, Aude Terray, Frédéric Tristram, Bruno Valat, Rachel Vanneuville, Vincent Viet, Antoine Vauchez, Philippe Warin, Jean-Marc Weller... and the rest. The main references to the books and articles of these fellow colleagues, and many others, can be found in the detailed bibliography of the edited book France and its public administrations. A state of the art, which can be used by foreign colleagues as a sort of Who's Who of administrative research in modern-day France. 
As part of the wider spectrum of French social sciences, the study of public institutions in general and administrative ones in particular exhibits, in comparison with its cousins in other countries, several theoretical and methodological specificities. First of all, it is worth underlining that French social sciences at the beginning of the twenty-first century have an outspoken preference for qualitative research. Of course, French sociologists, political scientists and historians are not averse to figures: following Emile Durkheim's example, the use of quantitative data and statistics is frequent and intensive. However, French social scientists are not ontologically quantitativists: most of them treat facts and figures as mere indicators of social processes resulting from a variety of factors. In order to identify these factors better, quantitative data are always combined with an extensive and systematic observation of the object under scrutiny and with long campaigns of (semi-structured) interviews with the relevant actors. The interviews, often quoted in abundance in the final draft of the research, are an essential part of the scientific demonstration (administration de Ia preuve) in the epistemic tradition of the French social sciences, which in turn very rarely use regression analyses. Of course, such an episteme diverges from the "rational choice" paradigm based on over-simplified models of the interplay between "dependent" and "independent" variables, or "principals" and "agents." This is all the more true for the French scholars specialising in the study of public administration, as few of them are fervent users or producers of quantitative data, far preferring qualitative methods of inquiry.

It must be added that current French social sciences put great value on field research. French researchers constantly decry "desk research" and purely theoretical writings addressing overly "abstract" issues, while praising the heuristic virtues of what the French call le terrain, that is the empirical investigation of a given limited research field by means of the fine-grained, systematic and in-depth exploration of one or several cases, exposed in detailed monographs. The prevalence of field research as the only way to produce "true science" has its origins in a generational rupture and a "paradigm shift" that occurred during the 1960s and 1970s. In spite of their divergent theoretical persuasions, "young Turks" (at that time) led by Pierre Bourdieu, Michel Crozier, Michel Foucault and Alain Touraine concertedly conducted a decisive "empirical turn" of French social sciences, to which the current state of the disciplines is still indebted. Without denying the importance of theory, this strong empirical focus postulates that the "job of a social scientist" is not to build abstract models in an ivory tower, but to understand and explain real "social facts" or real "social processes" meticulously observed by the researcher within a specific "situated" context. 
As a consequence of this empirical focus, French researchers dive with great pleasure into the "thickness" of their limited "terrain," ethnographically observing the interactions, interviewing the actors at work and producing their collective biographies ( prosoprographie), exploring all sorts of archives and grey literature, in order to draw in-depth analytical pictures of each case study. This "accumulation of small true facts" (Paul Veyne) may disconcert Anglophone scholars accustomed to publications that usually have the ambition to offer theoretical solutions to hypothetico-deductive "puzzles" posed to "big N" databases. Sometimes, Anglo-American scholars may even question the pertinence of such a method... and ironically ask French scholars, as often heard in international conferences: "so what?!" It is to avoid such intercultural misunderstandings that one has to acknowledge the specific ethos and aesthetic of French social sciences that strive to address wide scientific questions and discuss major theoretical issues only by investigating limited empirical cases. Such a dive into the "terrain" remains the via obligata for almost all French researchers, who afterwards remain very cautious in engaging in some controlled generalisations and macro-conclusions.

This strong preference for field research goes hand in hand with a bottom-up approach (approche par le bas) of institutions, processes and phenomena under study. How do the fellow members of a given group (polity, society or institution) become accustomed to processes designed and implemented from the top, how do they incorporate those schemes (or not) into their daily routines and/or make unanticipated social usages of them? Such scrutiny "from below" is a convenient way to address the concreteness of the social construction of reality by the humans themselves.

This deep constructivist rationale of French social sciences makes researchers very sensitive to the manufactured character of the socio-historic realities they are observing. Even those who do not explicitly take a historiographical stand in their researches never neglect the importance of time and the remains of the past within the prevailing institutions and configurations.

Finally, French social sciences, and Public Administration as part of them, share some common research questions and some major orientations and research programmes that are consistent with the preferred methodologies presented above. Unlike many of their Anglophone colleagues and with the notable exception of Michel Crozier followers, French scholars of Public Administration hardly reason in terms of "organisation." Under the combined influence of Norbert Elias, Michel Foucault, Pierre Bourdieu and Paul Veyne, they tend to apprehend institutions and processes of institutionalisation and to investigate the production and reproduction of administrative institutions over time as the constant rearrangement of predictable practices and 
shared meanings (schemes, beliefs, values) that make two dimensions of any institutional order. Such a perspective, which underlines the importance of socio-historic genesis and historicity, is referred to as "socio-history" in some works or "sociology of institutions" in others. It often bears some similarities with the Anglophone sociological and historical new-institutionalisms. Hence, French scholars of the state and administrative reforms always situate current reforms in the longer run of the life of institutions, carefully looking for elements of innovation and evidence of continuity or resilience.

The ethnographic approach to the way institutions really work also calls for a systematic investigation of the civil servants, not only the top officials, but also the middle or lower ranking agents who embody administrative institutions and who live within, from and for public administrations. That is why French social research is so rich in fine-grained sociographic explorations of civil servants, based on quantitative and qualitative analyses of their social, geographical and educational backgrounds and trajectories, the three major dimensions that the French sociological tradition considers, in a Durkheimian-Marxist vein, to have a decisive impact on the beliefs, values, strategies and behaviours of socialised human beings.

Special attention is also given to the legal status of civil servants, to the rules and procedures of their recruitment and to their evolution in time, as well as to their career paths and scales of remuneration. Current research offers strong empirical evidence that the (very protective) legal status of civil servants, their career prospects (or lack of them), their individual or collective strategies of promotion and the related "esprit de corps" and corporative solidarities have a crucial impact on the way they perform their tasks, and therefore on the way administrative institutions work, and engage in the design, production, implementation and evaluation of public policies at the various layers of territorial government in France.

Researches in recent years have also paid a lot of attention to the interaction between politics and administration, to the cooperation, confrontation and combination between the major players of both sides, political executives and senior bureaucrats, each of the parties of course holding its visions, logics and ambitions. In modern-day France, as in other developed democracies, empirical analyses conducted at the various layers of government have unveiled both the naïveté of the official theory of a bureaucracy strictly compliant to the political will and the hyperbole of the myth of an all-manipulating technocracy. While refuting such Manichean distinctions, current research has shed light on the various forms of contentious cooperation between politicians and bureaucrats, in which top civil servants are not merely protectors of a resilient bureaucratic order but, more and more, co-governors who 
collectively contribute to the technicisation/technocratisation of politics and, symmetrically, to the politicisation of public policies.

All these research perspectives, orientations and methods presented above naturally find a major field of application in the study of state reform and other institutional reforms that France, like other Western democracies, has experienced in recent decades. French scholars have already proven how the (more or less) innovative repertoire of models, precepts, recipes and tools inspired by New Public Management (NPM) that is travelling our globalised world (as compellingly shown by the literature on policy transfers) was belatedly imported into France, and especially "acclimatised" in accordance with the specificities of the French administrative culture and context, by means of endless competitions, "turf wars" and collusions between rival ministries, grand corps and coalitions of actors with diverse, if not divergent, strategies of advocacy, resistance, survival or resilience.

In addition, it is worth underlying that, in those academic marches between social sciences stricto sensu, imperialist economic science and practice-oriented expertise, a new sub-discipline of public management has also emerged in France in the most recent decades. Suffering a rather marginal situation within the sea of French "sciences de gestion" (whose main topics are marketing, private finance and accounting), a few small and attractive islands of public management research have emerged: one is organised round the activities of the scientific journal PMP (Politiques et Management public) led by Patrick Gibert; another is animated by Annie Bartoli at LAREQUOI, Université Versailles Saint-Quentin (UVSQ); yet by far the most dynamic is the radiant IMPGT, founded within Aix-Marseille University by Dean Robert Fouchet and which is the only faculty in France entirely dedicated to public management education and research, the IMPGT team (Olivier Keramidas, Solange Hernandez, Christophe Alaux, Céline Du Boys, Emil Turc, Marcel Guenoun, to quote only a few) constituting the true French pillar of the EGPA-IIAS-EUROMENA community.

Moreover, since the succession of generations has strong effects on the renewal of topics studied in the various disciplines, one might add that, within the faculties of law, young lawyers specialising in droit administratif and droit de la fonction publique are currently producing a huge number of insightful doctrinal reflections, skillful comments on newly issued legislation and regulations, erudite commentaries (notes d'arrêts) on the rulings of the Council of State and other administrative courts. Unfortunately, since positivism tends to dominate the French law faculties, this rich legal gnosis remains essentially self-referenced, with little to no connection with the abovementioned social sciences or public management literature, while social 
scientists in turn hardly read law journals (with few remarkable exceptions like André-Jean Arnaud, Jacques Commaille and Pierre Lascoumes).

To conclude this very succinct presentation of the divided yet lively multidisciplinary field of public administration studies in modern-day France, it is of the utmost importance to underline the following: against the empirically incorrect and scholarly misleading but surprisingly enduring image too often painted in the Anglophone comparative literature of the French public administration as a conservatory of the so-called "Napoleonic model," which is nothing but a myth now, the most up-to-date research done in France reveals the remarkable plasticity of French public institutions. Far from opposing a nostalgic resistance to the managerial ethos and tools (yesterday) or to e-administration, the digital/big data revolution and the march towards a "platform state" (today), the French administrative apparatus and its servants have been succeeding in constantly reinventing themselves by means of waves of reforms... that have become permanent.

\section{A very low impact of PA research on the education and training of future officials and on PA as a practice}

France being a large country with a very complex and highly differentiated society, subdivided into many social "sub-worlds," each of them animated by its autopoiesis, what has been happening in the "research field" has had little or no effect on the "power field" (to phrase it in Pierre Bourdieu's terms). The higher education and then the professional training of the future "state nobility" takes place, mainly at Sciences Po Paris and then at the ENA, in a long-lasting process of "Fabrique des énarques" (as analysed 20 years ago by the author of these lines), combining hyper-selection and pressure to conform. As abovementioned, this socialisation process is essentially mimetic, the role of professors is rather marginal in it, and the future administrative elite definitely has no education to research and very few occasions on which to meet true researchers. It is all the more surprising since, at Sciences Po Paris and at Paris 1 University, the so-called "Prépa ENA" and Public Affairs programmes which train candidates for the highly competitive administrative concours coexist in the same building (but on different floors) as research-oriented Master's programmes, doctoral schools and research centres.

This phenomenon is reinforced by the "programme" of compulsory disciplines to be prepared by the candidates for the administrative concours. With differences between the ENA, the INET, the IRAs and the other specialised schools/training institutes (for judges, public hospital directors, 
police officers, tax administrators, etc.), "the programme" is still very much focused on very classic disciplines, mainly constitutional and administrative law, macro-economics, public finance, international relations and diplomacy, ... with the more recent addition of EU law and European affairs, and a bit of management. Surprisingly for a foreign observer, such disciplines as political science, sociology, public policies and analysis of the policy process, and public administration per se, and especially comparative public administration, are simply not taught at "Prépa ENA." Things could be different for the content of the curriculum to be followed by those happy few within the ENA and other administrative schools, but the many attempts to open their curricula up to less classic disciplines have not been very successful, and have mainly ended up introducing more and more managerial doctrines and techniques, essentially driven by the "skills and competencies development" motto, and not at all knowledge- and research-oriented. To express it amusingly, the ENA alumni and other members of the French administrative elite (amongst whom the author has many friends and even relatives) have no idea of what the "bureau shaping model" or the "three new institutionalisms" may mean, and they have never heard such "big” names (for us) as Guy Peters, R.A.W. Rhodes, Geert Bouckaert and Jim Perry. And if one adds that high level politicians in France are mostly ENA alumni, as President Macron and Prime Minister Edouard Philippe illustrate, and almost never university professors, unlike what can be observed in several Southern or Central Europe EU Member States, e.g. Italy and Romania (with the exception of our colleague Prof. Jean-Michel Blanquer, the current minister for Education), the likelihood of research in PA influencing high-level decision-makers in France seems very low.

As a matter of fact, the situation is not so desperate, since some still limited corrections come from the combination of two variables. Firstly, in parallel with the symbolically and quantitatively dominant "voie royale" of the concours and the tenured status of fonctionnaire, there is now an opportunity to enter and work in public administration at both national and regional/local levels as high-level contractual staff. Around one fifth of public employees are now working under such non-statutory, non-titulaire conditions of employment. Many of them work (temporarily) as special advisors within the ministerial cabinets and other personal staffs of all executives (regional presidents, mayors of large cities, ...). Many of these high level contractual experts also work in a variety of organs bearing various names (SGMAP, DITP, France Stratégie, CNDP at the national level, plus a myriad of "development agencies" at territorial level,...) but whose core mission is, in different ways and means, to provide additional expertise to the policy process, to act as semi-internalised "think tanks" and to bridge the gap with academia, the 
external think tanks, the NGOs, etc. Unsurprisingly, one can find among these politicised special advisors of the executive entourages and among these high level contractual experts many former students of ours, who have been properly taught public administration and public policies, graduated with our Master's degrees after writing lengthy theses, and a few of them even going on to do doctorates. Such young people are aware of the abovementioned scientific state of the art, and marginally contribute to infusing ideas coming from research by calling on their "old" professors to write reports and make recommendations. Unfortunately, the modest influence on policy-making of such expertise rooted in academic knowledge is very often countered - if not annulled! - by two growing powers, in France as elsewhere in the world: the "consultocracy" of the "Fat Four" major consultancy companies who sell their solutions without properly asking the relevant questions... and the "spin doctors" and other communication gurus who assist our governors in the $24 / 7$ storms of media and internet "fake" politics.

\section{What next? Current trends and possible future developments}

As shown above, France, its old and venerable state and its strong politicoadministrative apparatus are a combination of continuities, produced through historicity by a certain culture and trajectory... and, at the same time (the "third-way"-style political discourse of young, bright and determined President Emmanuel Macron insists very much on the "en même temps" simultaneous approach to the complexities of our post-modern polities), France and its public administration, both as a practice and as a domain of academic research, are very much in line with global movements. Therefore, one can expect that future developments will, at the same time, pursue some key inherited features (revealed above) and foster major innovations.

Let us envisage a few of them.

As regards academia per se, the rhythm and quality of French research on public administration and policies will certainly remain very high, given the quality of the people involved and of the new generation of $\mathrm{PhD}$ students. Noblesse oblige! The already increasing involvement of French researchers within European and international research networks and learned societies, such as ECPR, EGPA, ICPP, EU-funded research programmes, and European joint master's programmes, is also expected to continue growing.

As for the influence of such a continued flowering on the education and training of future civil servants, the recent, totally unexpected and much 
debated announcement by President Macron of his will to "suppress" the ENA and/but replace it by something else has opened up an "opportunity window" for institutional redesign and innovation in the content of the future curriculum of the future French Institute of Public Administration. Fierce and foggy "palace wars" are currently being waged, and it is far too early to say what their effective outcome will be.

At the same time, a profound reform (voted in in the summer of 2019) of the General Statute of the Civil Service has opened, in principle, the chance to fill all top management positions within French ministries (secretaries-general, directors-general, their deputies, heads of services, under-directors, directors of projects, heads of bureaux) that have so far been mostly reserved for career civil servants who have graduated from the ENA and other "administrative schools," with "talents" attracted from the private or third sector on the basis of adapted contracts. Justified by the urgent need to gather the very specialists of the digital and big data "revolution" on top of the administrative apparatus of the "Platform State" to be achieved within the two (expected) presidential terms, this innovation has not yet been enforced. It could either be an implementation failure if the top managers or experts from outside the bureaucracy are not attracted (a case observed in the UK under Thatcher and Major, who tried the same personnel policy), or succeed in mixing up the traditional "career system" of the French bureaucracy with a British/Nordiclike "position system" ... if not a US-like "spoils system," since it appears unlikely that incoming ministers will appoint managers and experts from the outside who are not partisan friends, or at least "functionally politicised" (in Renate Mayntz and Hans-Ulrich Derlien's terms) strong supporters of their policy orientations.

If the French higher bureaucracy were to become highly "contractualised" in the future decade, one could expect that the newcomers would come mainly from the digital, high-tech design world, but also that all kinds of high-level experts who often have Master's degrees in public administration and policies (in Paris 1 and the provinces, especially in the Instituts d'études politiques) could find their way towards the higher bureaucracy of the state. A combined aggiornamiento of a rebaptised ENA whose curriculum would be open up to studying the policy process, history of institutions and comparative public administration would ideally contribute to the same process of cross-fertilisation.

However, to really bridge the gap between researchers and practitioners one would recommend the more systematic installation, in each major administration, of "scientific councils" bringing together top bureaucrats, leading academics and foreign experts so as freely to discuss specific 
policy issues, such as the one the author had the honour to chair within the Ministry for Public Administration and the Civil Service some years ago. More ambitiously, the announced transformation of the CESE (Conseil économique, social et environnemental), in a context of increasing demand for more participative democracy, could find inspiration in the Swedish model of systematic "precooking" of draft legislation within commissions that associate researchers and external experts with governmental policy-making. The question is then not on feasibility but on the willingness (or not) of the classic politico-administrative elite still in power in France, in spite of the fanciable political discourse of LaREM on the "renewal of political life," really to share their power with intellectuals and other outsiders promoting "strange" original ideas.

In the end, it is likely that those in power will remain quite cautious about opening the French governing processes to independent researchers who "speak truth to power," since many examples illustrate the political risks for our governors of the intellectual freedom of researchers, e.g. the recent creation of an independent "Observatory of Public Ethics" (OEP) where a socialist MP, René Dosière, has enrolled academics from political science, public administration and policies (including the author of these lines) into an advocacy coalition which politely but firmly supports more constraining legislation against conflicts of interest among higher officials, new rules on the recruitment and role of ministerial cabinets, more transparency in the activities of the Core Executive, and so on and so forth.

L'avenir tranchera... 


\section{References}

Badie, B., \& Déloye, Y. (2007). Le temps de l'Etat: mélanges en l’honneur de Pierre Birnbaum. Paris: Fayard.

Baruch, M. O., \& Duclert, V. (2000). Serviteurs de l'Etat. Une histoire de l'administration française 1875-1945. Paris: La Découverte.

Bezes, P. (2009). Réinventer l'Etat. Les réformes de l'administration française (1962-2008). Paris; PUF. Chevallier, J. (2019). Science administrative. 6th ed., Paris: PUF, Thémis.

Dubois, V. (2016). The Bureaucrat and the Poor: Encounters in French Welfare Offices. London: Routledge.

Dreyfus, F., \& Eymeri-Douzans, J.-M. (eds). (2006). Science politique de l'administration. Une approche comparative. Paris: Economica.

Eymeri-Douzans, J.-M., \& Bouckaert, G. (eds). (2013). La France et ses administrations. Un état des savoirs/France and its public administrations. A state of the art. Brussels: Bruylant.

Ihl, O., Kaluszynski, M., \& Pollet, G. (2003). Les sciences de gouvernement. Paris: Economica. Lagroye, J., \& Offerlé, M. (eds). (2012). Sociologie de l'institution. Paris: Belin.

Payre, R., \& Pollet, G. (2013). Socio-histoire de l'action publique. Paris: La Découverte. 



\title{
Public Administration in Germany: Precarious Present, Promising Future?
}

\author{
Stefan Becker and Michael W. Bauer
}

Public Administration (PA) assumes a precarious existence in Germany. While many scholars work on administrative issues, often with great individual success, the German PA community has never developed into an independent discipline. It has rather remained a loose field of study. Threats of fragmentation and irrelevance loom large, as other established disciplines are more visible in public discourse, attract better students and acquire larger amounts of funding. As a result, the PA community perceives its reputation to be mediocre, attracting only moderate public interest and having little practical influence. Yet, it is not all doom and gloom in German PA. Transformative dynamics are currently materialising in the social sciences and this field of study is no exception. Increasing internationalisation and digitalisation are reshaping the conduct of social sciences. Along with demographic change, these trends can open up new avenues for interdisciplinarity in German PA and thereby provide for a promising future.

This chapter shows how the German PA community can harness these developments to overcome its problematic boundaries and path dependencies to strengthen its collective standing in academia and beyond. It first provides a brief history of PA in Germany, outlining the roots of institutional fragmentation and discussing its negative implications. Weak institutionalisation and heterogeneous scientific orientations currently impair sustained and effective cooperation - neither a core curriculum in teaching, nor a common agenda in research has yet materialised. The chapter then turns to positive developments in recent years and, on this basis, closes with a few plausible scenarios for the future of PA in Germany. This portrayal is informed by results from a recent survey of more than 400 scholars and practitioners who revealed details about their work and shared their view on the community at large (see Bauer \& Becker, 2018 for more information on these data). 


\section{A brief history of Public Administration in Germany}

Academic disciplines emerge in idiosyncratic processes, sometimes with peculiar outcomes. Yet, once disciplines become established, they are often persistent and tend to reproduce themselves. One reason lies in their capacity to absorb new and dissenting paradigms. However, new disciplines occasionally come into being through secession from their mother discipline. In these cases, the relationship between the old and the new usually remains close and contentious. The history of German PA is no exception to this rule. It is also marked by secession and competition, just as in most Western European countries. Knowledge about the state and how it should conduct its affairs was traditionally gathered in a comprehensive "science of the state," which brought together a broad range of expertise that is nowadays divided into public law, macroeconomics, budgeting, management and other fields (Heyen, 1982). Then, with increasing professionalisation and differentiation of the academic sciences, the legal dimension became dominant in the study of public administration, before the advent of modern social sciences led to different national trajectories with individual compromises between the disciplines.

In contrast to other countries, the dominance of legal approaches in studying public administration has never been broken in Germany. This hegemony has its roots in the nineteenth century, when a law degree became the crucial entry ticket for the public service and the more comprehensive approach to educating civil servants became obsolete (Wunder, 1986). The following differentiation of disciplines left issues of public administration firmly in the hands of law schools, and PA in Germany became ever more synonymous with its legal dimension. Even after the Second World War, when a more pluralistic conception of PA spilled over from the United States, the legal dominance persevered. One reason was that the newly established political science departments - often deemed to be the suitable hosts for such a pluralistic PA approach - still needed to consolidate their positions and, for this reason, prioritised other research themes closer to democratic legitimacy. Political science was at first a science of democracy and later developed into an empirical undertaking focused on public institutions and collective decision-making. From this perspective, PA issues were rather technocratic matters not to be bothered with. Several attempts to make PA a central research area in political science, especially at the time when the policy analysis approach became popular in the 1970s, failed to take off (see, e.g., Scharpf, 1973: p. 9-32). The fate of PA in management departments was similar. Even though scholars dominated the reform discourse in the high times of the New Public Management movement, PA never came to play a 
substantial role in management departments and has all but disappeared from their curricula.

A survey of popular topics in German social sciences also reveals the problematic position of public administration issues (see also Jann, 2009). These had their greatest appeal when the political agenda was hopeful about social engineering in the late 1960 os and early 1970 s. At that time, many practitioners and scholars saw social transformation as a promise that only well-designed and effective administrative machinery was able to deliver. With the oil crisis of 1973, the end of the welfare state expansion dreams, and the turn towards lean government concepts under rising austerity, however, German PA developed an agenda of de-bureaucratisation and reform. Major concepts for this undertaking came from think tanks and consultants rather than from the PA community, which remained sceptical about the achievable benefits. As a result, public administration issues lost their appeal and relevance in the empirical social sciences. Only recently, in the 2010s, with the waning ideational supremacy of neo-liberalism and the cautious return of pro-regulation initiatives, have questions of public organisation gathered new interest.

The development of PA in Germany has therefore produced some problematic path dependencies. Traditionally perceived to be a primarily legal matter, PA has neither developed into an independent discipline nor risen to prominence in the relevant disciplines.

\section{The present state of Public Administration in Germany}

The German PA community seems currently to be ill-prepared to seize the opportunity of renewed interest in issues of public organisation. A damaging combination of fragmented institutionalisation and heterogeneous scientific orientations renders its standing precarious. Topical and theoretical convergence on the working level may, however, provide a basis for German PA to work its way out of this impasse.

\subsection{Fragmented institutionalisation}

In Germany, research and teaching pertaining to public administration is widely dispersed (see also Kropp, 2014). A good portion of the formal education is provided by the numerous universities of applied sciences for public administration at federal and state levels. These institutions focus 
exclusively on training civil servants, primarily for entry-level positions in various departments. Most programmes are dual-track, including both academic courses and hands-on training at different levels and in different branches of the public sector. Administrative law typically overshadows other disciplines relevant for public administration in their curricula. It follows that empirical research also remains limited at these institutions. Furthermore, while most faculty members build up expertise in specific areas, their teaching load effectively prevents them from engaging in larger research projects. As a result, many PA students in Germany receive a strong practice-driven, law-focused education with little input from other social sciences. This is all the more true for the second main type of PA education, the public law part of the legal training. After all, many top positions in German administrations still require a law degree (Befähigung zum Richteramt) with obligatory practical training (Referendariat).

The third group of PA students in Germany, non-law students at regular universities, can choose from a more diverse menu which is, however, not easy to navigate. Only a few German universities have established distinct PA profiles in research and teaching, and truly comprehensive programmes are hard to find. More regular are single modules on public administration in larger programmes of political science, law or management. Furthermore, much research is done by individual PA-oriented scholars in the respective departments -quite often productively (see, for instance, Kuhlmann \& Wollmann, 2019; Knill \& Bauer, 2017; Jann, 2009; Benz, 2005; Goetz, 2001). While their outputs have strong visibility in the international research community, they often remain in the shadows of their home institutions. Students generally interested in public administration must therefore invest some time in identifying those programmes and institutions where they can learn about specific administrative issues. Compared to political science, for instance, the institutional landscape of German PA is rather fragmented. Only five institutions represent exceptions to the rule of fragmentation.

First, the German University of Administrative Sciences remains an important node in the German PA community, especially among legal scholars. Its postgraduate teaching focuses on the training of future civil servants in both short-term courses and two-year master's programmes, with emphases on law, economics or the social sciences. In line with its general mission, the research at Speyer University tends to be practice-oriented and Germany-centric, with the occasional project on matters of Europeanisation and internationalisation. Second, the Centre for Policy and Management at the University of Potsdam offers both undergraduate and graduate programmes in the social sciences with a strong emphasis on issues of public administration. Its curricula 
are quite international, with strong theoretical imports from Scandinavia. Accordingly, its main research activities lie in the theory-driven analysis of public organisations. With a research training group on "Wicked Problems, Contested Administrations: Knowledge, Coordination, Strategy” (WIPCAD), Potsdam also had the first exclusively PA-oriented graduate school. Third, a broad curriculum on public administration is also available at the Department of Politics and Public Administration at the University of Konstanz. Its teaching and research tend to be international and strongly policy-oriented, with a heavy focus on quantitative methods. The University of Konstanz is frequently ranked among the best German universities, a fact which is also reflected in this department. Notwithstanding, its relationship with the world of practitioners appears to have worn thinner over the years. Finally, the Hertie School of Governance and the Zeppelin University in Friedrichshafen, two private universities, complete the group of five. The former specialises in postgraduate and executive education, with teaching and research having a strong international orientation, while the latter offers undergraduate and graduate programmes with an explicit focus on public administration issues.

Additional clusters of PA expertise exist regarding practice-oriented research and consulting. The Lorenz von Stein Institute at the University of Kiel enjoys a good reputation in the legal community, especially in the field of digitalisation and electronic government. Furthermore, the Kommunale Gemeinschaftsstelle für Verwaltungsmanagement, an institute of an association of local authorities, is a key participant in debates on administrative reform. In this field the Bertelsmann Foundation is another crucial actor. Lastly, the German Research Institute for Public Administration is an important institution for regulatory impact assessment and evaluation. Together with innumerable individual researchers on public administration at other universities, these clusters make for a strong supply of PA expertise. The fragmented nature of this expertise, however, impairs its impact in both research and practice.

Much of this is rooted in disciplinary differences, which are discussed in the next section. Apart from this supply-side factor, however, fragmentation is also reinforced by extraordinary demand for the persistent dominance of lawyers in the higher echelons of German public administration, which has prevented the establishment of truly comprehensive PA schools. The reasons are twofold: a PA-oriented faculty cannot defend its turf by moving towards strong and consistent labour market demands (as in law), and students are not receptive to specialising in administrative issues with poor employment prospects. While German public administration has become more open towards non-lawyers in recent decades, Germany remains different from other countries, where social scientists and management graduates are more welcomed. 


\subsection{Heterogeneous scientific orientations}

Each scholarly community has its fault lines; conflict and competition exist alongside cooperation. Indeed, differentiation and specialisation can foster scientific progress. German PA is, however, marked by extraordinary boundaries. These range from epistemological positions and research aims to publication strategies. As a result, knowledge on administrative issues is often generated and disseminated in isolation, and German PA fails to realise much potential from interdisciplinary cooperation and dialogue between practice and research. Two main results from our survey illustrate this heterogeneity in scientific orientations.

First, there is a rift regarding research priorities. The German PA community is divided between those who see their prime task in the transfer of insights to the world of practitioners, and those who focus on the production of theoretical knowledge, perhaps hoping that sooner or later their results will find their way to the practitioners. These priorities are not mutually exclusive, but they often entail differences in research designs and questions, method selection and publication outlets. In practice, therefore, scholars often have to face the decision where to invest their time, in either basic or applied research. In the German PA community, this trade-off brings clear disciplinary boundaries to the fore. Political scientists display the strongest preference for basic research and theory development, while students of business administration and legal scholars are at the other end of the spectrum. This mirrors the aforementioned institutional fragmentation and therefore fortifies asymmetries in both research and practical consulting. While the former could benefit from more insights from legal and management research, the latter could do better in terms of political science and sociological input.

Second, German PA scholars perceive the internationalisation of publication habits differently. This long-term process is relevant for all social sciences. PA communities, however, claim to have intimate relationships with their respective domestic systems. Indeed, unique cultures and organisational configurations are the prime contexts, if not the outright objects, of much PA scholarship. Furthermore, practical relevance is often linked to publishing in national journals, as these are more likely to be picked up by practitioners. Accordingly, much research has appeared in national publication outlets, which are now under pressure from international flagship journals. Internationalisation also entails a growing emphasis on benchmarking and rankings, which encourages other priorities for topics and methodological strategies. This development, which amounts to a profound transformation of publishing incentives, most of them highly ambivalent, has polarised the 
German community. Age is a crucial factor in this regard. Younger scholars are, on average, more focused on international publication outlets. Yet again, there are differences among the disciplinary backgrounds, with law scholars being the most ardent supporters of national research and traditional outlets for scientific findings, while social scientists are more open to publishing in international outlets. Neither is necessarily superior, but the results of this division are important, as diverging attention makes interdisciplinary dialogue more difficult.

In both dimensions, the primary disciplinary affiliation does not determine individual outlooks. There are, for instance, political scientists who work on domestic issues and prefer to publish their findings in German-language journals, just as there are legal scholars who engage in cross-country comparisons and present their results in international journals. Perhaps more important than disciplinary boundaries is the general bifurcation between descriptive-normative work on national phenomena on the one hand, and analytical and theory-oriented work on international issues on the other. Again, it is not problematic if scholars specialise in different areas, but the two camps in the German community tend to ignore each other's work. Our survey revealed that members of both camps read different journals with the exception of only a few that the majority regarded as relevant. They also rate the relevance of specific institutions differently. The respective departments in Potsdam and Konstanz, for instance, score more highly in the international, theory-oriented camps, while the German University of Administrative Sciences in Speyer is more popular in the national, descriptive-normative camp. Finally, the two groups differ in their appreciation for leading scholars. These trends go beyond normal academic differentiation. They represent a bifurcation in substantial and social dimensions of German PA that renders productive dialogue difficult.

\subsection{Topical and theoretical convergence}

Beneath institutional fragmentation and differences in scientific orientations, however, scholars often work on similar topics, employing identical theoretical frameworks and integrating insights from other disciplines. There is, therefore, plenty of potential for further cooperation.

Regarding topical convergence, four clusters are currently attracting particular attention across disciplinary boundaries. First, questions related to digitalisation are engaging a growing number in the PA community. Scholars are analysing the possibilities of electronic government and new data sources and how their use has already transformed traditional forms of 
public administration. Especially in the latter sense, digitalisation represents a cross-cutting issue that hardly any scholar or practitioner can ignore. It thus represents a fertile ground for dialogue and cooperation. Second, internalisation remains high on the PA agenda, as the implications of globalisation and Europeanisation for national bureaucracies are still not fully understood, nor are the bureaucratic organisations beyond the nation-state. Linkages between the different administrative levels, i.e. emerging forms of multi-level administration, are also at the core of this research avenue. Third, many scholars conduct research on the relationship between public administration and citizens, which raises a wide array of analytical and normative questions. Studies on traditional and new participation channels, open government initiatives and innovative accountability channels touch upon issues of legality, legitimacy and efficiency, thus rendering this research area almost ideal for interdisciplinary cooperation. Finally, migration and integration pose serious long-term challenges for public administration - both as a matter of regulation and as a transformative force for public administration itself. Accordingly, they attract much scholarly attention. The recent influx of asylum seekers has provided further impetus. Migration and integration touch upon matters of law, politics, management and more. All clusters thus lend themselves to interdisciplinary efforts.

Despite institutional fragmentation and heterogeneity in scientific orientations, such interdisciplinary efforts are not doomed to fail since there is also some common ground in the use of theories. Many scholars find decision theory to be relevant for their work, a sentiment that cuts across all disciplines. The "new" institutionalisms are also relevant for most, although legal scholars have less use for them. With these and many other approaches, such as systems theory, game theory or constructivism, which engage a sizeable minority in all disciplines relevant in the PA community, there is ample potential for cooperation below the level of institutionalised disciplinary integration or leadership. Furthermore, German PA scholars show a great openness towards other disciplines. On average, they name more than two disciplines - apart from their own - as relevant for their work. Political science is the most important discipline, followed by law, sociology, economics and management. In German PA, interdisciplinarity is thus practised on a working level, rather than being institutionalised in formal terms.

The present state of PA in Germany is therefore ambivalent. It is fragmented along disciplinary boundaries and sidelined within the relevant disciplines. As such, it is more a field of study than an academic discipline distinguishable from law, political science or management studies. However, while 
institutional fragmentation and heterogeneity in scientific orientations hold steady, there is potential for cooperation in topical and theoretical terms.

\section{The future of Public Administration in Germany}

Many scholars have concluded that the feeble signs of topical and theoretical convergence may not be enough to bolster the standing of the German PA community or even avert its sliding into irrelevance. In recent years, they have embarked on a soul-searching journey. Admittedly, such exercises come to pass roughly every decade (see, e.g., Benz, 2005 and Bogumil, 2005 for important contributions); so far, however, these ruminations have failed to generate much impact. But the community has, at least, not given up, and ideas for improving its dire situation are proliferating. Some members of the legal community, for instance, seek to overcome the advancing isolation of their subfield by developing a more interdisciplinary decision-making paradigm in administrative law, while some management scholars advocate technology-driven innovation as the post-New Public Management paradigm. Some political scientists, meanwhile, recommend revisiting PA's theoretical foundations or linking its frequently parochial debates to the internationalisation agenda of the broader social sciences.

While providing substantial stimuli for future research, these contributions often merely reinforce disciplinary boundaries. They hardly address the fate of the German PA community as a whole, which can only prosper by overcoming the challenges of problematic boundaries and path dependencies. This section shows that, without realistic prospects of disciplinary integration, the German PA community must strengthen its efforts at the working level. The potential for this endeavour has already been outlined. Broader trends in academic science - internationalisation, digitalisation and demographic change - provide a promising context.

\subsection{Prospects of disciplinary integration}

One important proposal for overcoming PA's precarious standing in Germany is disciplinary independence (see, e.g., Bohne, 2018). According to this reasoning, the permanent need to demarcate itself from other fields of study within the mother disciplines and the concurrent disciplinary competition within the PA community are detrimental. Establishing an independent discipline, effectively integrating the various approaches in studying public administration, would instead help to bolster the standing of PA in Germany. 
At first glance, this sounds plausible; however, the most significant problem with this approach is its lack of popularity in the community itself.

Reservations about developing PA into an independent discipline already start at the problem definition phase. Most German PA scholars agree that the current state of interdisciplinarity is in dire need of improvement. Two out of five argue outright that the rather multidisciplinary character of German PA has negative consequences in terms of standing, funding and teaching, but the majority fails to see it as the major factor. Furthermore, even the critical assessments tend to translate into calls for reform, not a revolution in the sense of establishing a new discipline. Accordingly, only a minority in the German PA community calls for such efforts. Two main rationales lie behind this reservation, which cuts across all disciplinary boundaries. On the one hand, academic turfs are defended. Many scholars fear that an independent discipline would lead to their own discipline losing influence in public administration issues. On the other hand, most German PA scholars argue that scientific progress is achieved at disciplinary frontiers. An independent discipline, no matter how careful the integration, might impede such creative multidisciplinarity. Disciplinary integration is, therefore, no realistic option for German PA.

Another proposal to bolster PA's standing in Germany is to install a lead discipline. This solution would leave the multidisciplinary character of the field intact, while one specific discipline would provide orientation in terms of topics, research questions and methods. Stronger agenda-setting would solve collective action problems without ultimate hierarchy. While this proposal meets concerns about the stifling of scientific progress by an independent discipline, it fails to dispel anxieties about the potential loss of influence for specific disciplines. The views in the German PA community about potential lead disciplines are telling. Most scholars state that such leadership would be welcome; however they overwhelmingly name their own discipline as being the most suitable to fulfil this function. Political scientists are the most resolute group in this regard, but scholars from other disciplines are also reluctant to grant another discipline leadership functions. The results of our survey indicate that researchers from political science are the most prominent representatives of PA in Germany. This could support the case for a stronger social science-based PA, but this leadership would still have little following in other disciplines. The proposal for a lead discipline is, therefore, also without realistic prospects.

In this light, more debates on the disciplinary integration of German PA currently appear futile. At best, they lead to nothing, and at worst they incite conflict between the disciplines. At any rate, such reforms will not materialise 
in the near future. Instead, the German PA community will continue to be plagued by institutional fragmentation and heterogeneity in scientific orientations. Its long-term standing, therefore, will depend on how fruitful the topical and theoretical convergence at the working level will become. Against the background of broader developments in the academic social sciences, there are some reasons to be cautiously optimistic.

\subsection{Broader developments in the academic social sciences}

Internationalisation and digitalisation are more than just fashionable research topics; they are responsible for transforming the academic social sciences and reshaping how research is conducted and communicated. In this regard, both developments can also affect German PA in that they offer new links and opportunities for interdisciplinary cooperation.

While opinions differ starkly on the internationalisation of the social sciences, almost everyone in the German PA community accepts its transformative impact. These days, tenure committees and grant commissions are placing increasing emphasis on the internationality of an applicant's publication habits, research projects and professional networks. The impact of this internationalisation - in all its facets - on the quality and relevance of the social sciences is not relevant for this chapter. What matters here is that the internationalisation trend is disrupting the current equilibrium of German PA. On the one hand, young scholars must meet the standards of international journals. This effectively reduces the incentives to engage in descriptive studies of individual German bureaucracies and raises the rewards for doing rigorous, comparative work. Admittedly, standards and peer review processes still vary, but this internationalisation trend will reduce the parochialism in some strands of the German PA community. This, by the way, does not necessitate moving away from the research objects, as some critics argue; it is rather about approaching them more systematically. Practical relevance must therefore not suffer either and could, by contrast, benefit from more rigorous work, as long as it also resonates in the national community. On the other hand, internationalisation brings the German community into closer contact with established independent PA disciplines. In light of the considerations above, this is unlikely to foster identical developments in Germany, but the community can benefit from being even more strongly confronted with different approaches to the study of public administration.

Digitalisation can be another catalyst for the release of German PA from its current equilibrium. In terms of scientific communication, it amplifies the effects of internationalisation. The dissemination of research results has 
become quicker and more versatile. A scholar no longer has to wait months for a printed journal. Findings are first published online, placed in additional repositories, and reported in blogs and social media outlets. Again, the overall impact of this development is ambivalent, but as dissemination structures evolve, German PA scholars are increasingly drawn from their national echo chambers. The opportunities and the demands of this trend may bring them into closer contact with other approaches to the study of public administration. In the end, scientific orientations in the German community may converge. New data sources may have a similar effect. Research on public administration has traditionally been tedious. Information was hard to obtain, as secrecy reigned supreme. Research confined to individual bureaucracies was sometimes simply a matter of practical constraints. Digitalisation, along with higher transparency, is now creating new possibilities. Public organisations are increasingly opening their archives, while constantly producing transactional data. Statistical analysis of large data sets - from surveys, official databases and budgets - has become easier. As the black boxes of public administration become more and more illuminated, comparative research, both international and rigorous, is slowly becoming the mainstream of German PA.

The positive traits of internationalisation and digitalisation can provide the impetus for German PA to further converge on a working level. The fact that younger scholars are more open to both developments than their older counterparts will increase the likelihood of convergence, since German PA is currently undergoing profound demographic change. However, neither internationalisation nor digitalisation should necessarily lead to more constructive convergence; they could also produce a debilitating mainstream or increase divisions between the relevant disciplines. In the end, the fate of German PA as a common project depends on mutual disciplinary toleration and the constructive use of differences.

\subsection{Three scenarios for the future}

Despite its institutional fragmentation and heterogeneity in scientific orientations, German PA is still alive. It is, however, only moderately kicking. Its standing, as most of its members would agree, is precarious. Considering the circumstances described above, three scenarios for the medium term are currently plausible: drifting apart, muddling through or growing together.

Drifting apart appears to be the least likely scenario. After all, German PA has endured disciplinary fragmentation and differentiation for most of its existence. Why would it drift apart now? Because, as a loosely coupled system, it would not need much to break. The German PA community is 
of only moderate size. As reported, it currently has problems attracting the best and brightest students. Career opportunities simply look brighter in the other fields of their mother disciplines. And it does not need much more than one generation to rip the feeble fabric of the German PA community. However, with ever growing student numbers at German universities, public administration programmes are also booming. From this pool it should be possible to recruit an adequate number of promising young scholars. But this alone is hardly enough, because what is taught in these programmes also matters. If the current trend towards even more specialisation continues, future graduates may enter the multidisciplinary field with a narrow view of public administration issues. This could impede constructive dialogue and, therefore, contribute to the community drifting apart.

Muddling through is a more realistic scenario, however. The decade-long resilience of the German PA community suggests that it will endure in the future. Given the renewed attention to issues of public organisation, there should be enough demand to keep study programmes attractive and grantgivers interested. As the civil service is still becoming more open to graduates of disciplines other than law, the situation of the few centres of PA teaching and research should remain stable. Yet, big improvements in the community's overall standing should not be expected. The challenges of multidisciplinarity remain. Scholars working on issues of public administration will have to defend their turf within their mother disciplines and come to scientific understandings with their counterparts in other disciplines. As the field's history in Germany shows, this is hard work. Yet given the convergence in topical and theoretical orientations, the prospects of succeeding in keeping the community alive appear to be good.

Could German PA accomplish more than muddling through? Considering all its limitations, expectations for a promising future and for the community to grow closer together are currently low. Even below the level of disciplinary integration, there is no "German school," no unifying paradigm, in sight which could guide and foster the community. Scientific pluralism can be productive, but a lack of coherence can also be detrimental in today's academia. The heterogeneity in scientific orientations and the fragmented publication habits hardly bode well for the community at large. However, the transformative force of internationalisation and digitalisation, together with the topical and theoretical convergence among German scholars, may lead the way out of this impasse. An emerging research agenda based on behavioural approaches, studied with experimental and quasi-experimental methods, is, for instance, one avenue where PA scholars of all disciplines may come together. But this will work only when the strengths and weaknesses of such approaches - and 
many others - are acknowledged by the community at large. It will neither save research on public administration, nor will it be its downfall. Innovations always come with turf wars, but mutual toleration and the productive use of differences could keep such struggles to a minimum for the benefit of the community at large.

\section{Concluding remarks}

When comparing PA in other European countries, the German community is found to share some of the problems. The field is under pressure, but it has the resources and ideas to reassert itself (Bertels, Bouckaert and Jann, 2016, p. 24). Given the peculiarities of the German community, however, we should caution against excessive optimism. For a stable or even promising future, German PA still has to face the double integration challenge of multidisciplinarity. Respective scholars must be embedded in their mother disciplines and nonetheless come to collective understandings in the broader PA community. This chapter has shown that, currently, there is much heterogeneity in German PA - not only in institutional, but also in substantive terms. Its fate depends on how the latter diversity will be approached. Given the current bifurcation of German $\mathrm{PA}$, it can go either way. If the internationalised and theory-driven faction drifts further apart from the more practical and country-oriented faction, the collective standing of the community will suffer. Both sides will have to make efforts to find some middle ground.

\section{References}

Bauer, M. W., \& Becker, S. (2017). Verwaltungswissenschaft in Deutschland: Relevanz und Reputation im Urteil der Fachvertreterinnen und Fachvertreter. der moderne staat 10(1): 31-48. https://doi.org/10.3224/dms.v10i1.02.

Bauer, M. W., \& Becker, S. (2018). Public Administration in Germany: Problems and Potential of a Fragmented Community. International Journal of Public Administration. Online first. https:// doi.org/10.1080/01900692.2018.1560318.

Bertels, J., Bouckaert, G., and Jann, W. (2016). European Perspectives for Public Administration (EPPA). Utrecht: Paper presented at EGPA Annual Conference.

Benz, A. (2005). Public Administrative Science in Germany: Problems and Prospects of a Composite Discipline. Public Administration 83(3): 659-668. https://doi.org/10.1111/j.00333298.2005.00468.x. 
Bogumil, J. (2005). On the Relationship between Political Science and Administrative Science in Germany. Public Administration 83(3): 669-684. https://doi.org/10.1111/j.00333298.2005.00469.x.

Bohne, E. (2018). Verwaltungswissenschaft: Eine interdisziplinäre Einführung in die Grundlagen. Wiesbaden: Springer-Verlag.

Goetz, K. H. (2001). Making sense of post-communist central administration: modernization, Europeanization or Latinization? Journal of European Public Policy 8(6): 1032-1051. https:// doi.org/10.1080/13501760110098332.

Heyen, E. V. (1982). Geschichte der Verwaltungsrechtswissenschaft in Europa: Stand und Probleme der Forschung. Frankfurt am Main: Klostermann.

Jann, W. (2009). Praktische Fragen und theoretische Antworten. 50 Jahre Policy-Analyse und Verwaltungsforschung. Politische Vierteljahresschrift 50(3): 476-505. https://doi.org/10.1007/ s11615-009-0145-2.

Knill, C., \& Bauer, M.W. (2016). Policy-making by international public administrations: concepts, causes and consequences. Journal of European Public Policy 23(7): 949-959. https://doi.org/ 10.1080/13501763.2016.1168979.

Kropp, S. (2014). Breit aufgestellt und doch verstreut. Politikwissenschaftliche Verwaltungsforschung und universitäre Ausbildung in Deutschland. Österreichische Zeitschrift für Politikwissenschaft 43(4): 367-383. https://doi.org/10.15203/ozp.262.vol43iss4.

Kuhlmann, S., \& Wollmann, H. (2019). Introduction to Comparative Public Administration. Administrative Systems and Reforms in Europe. $2^{\text {nd }}$ ed., Cheltenham: Edward Elgar.

Scharpf, F. W. (1973). Planung als Politischer Prozess: Aufsätze zur Theorie der planenden Demokratie. Frankfurt: Suhrkamp.

Wunder, B. (1986). Geschichte der Bürokratie in Deutschland. Frankfurt am Main: Suhrkamp. 



\title{
4 \\ Public Administration in Hungary: Emerging Dynamics in an Illiberal Democracy
}

\author{
György Hajnal
}

\section{$1 \quad$ Introduction}

Over the past decade, phases of both gradual evolution and abrupt - and possibly even disruptive - changes can be detected as patterns characterising the development of the field of Hungarian Public Administration (PA) as a field of study and research. The general aim of this essay is to give a somewhat sketchy, broad-brushed and inevitably subjective overview of these developments ranging, time-wise, from the systemic change of 1989-1990 to the present day, and on this basis to speculate about its possible future(s).

The starting point of this journey, the systemic change of 1989-1990, is marked by the sudden emergence of a Public Administration/Management and Public Policy scholarship inspired by, and similar to, contemporary Western approaches. Whereas this new strand of scholarship remained, quantitatively, definitely inferior to the still dominant (public) law-based approach inherited from the recent (and even from the more distant) past, the next two decades were characterised, in general, by a modest and incremental development of the field. The end-point of the journey portrayed by this study is yet another more disruptive change impacting on the field from the early 2010 onwards: the "nationalisation" that is, a governmental take-over, of much of the public administration field, and the attempt at creating a disciplinary and institutional home for a state-centred and openly pro-government "science of the state."

Earlier work on the above topic area, partly done by the author of the current study, described and analysed different aspects of the above development path: Hajnal (1999) and Hajnal and Jenei (2007) traced the historical roots of PA in Hungary and of the development of university education from the pre-modern ages through the post-transition decades. Hajnal (2016) focused 
on the process and the effect exerted by Hungary's post-2010, macro-level illiberal turn on the disciplinary/topical composition and focus as well as on the institutional landscape of university education (for other, internationally comparative regional overviews of the PA field covering Hungary see, for example, Bauer, 2005; Hajnal, 2003; Nemec, Spacek, Suwaj \& Modrzejewski, 2012; and Staronova \& Gajduschek, 2012).

The more specific aim of the present study is closely connected to most of these studies. However it is different and, in some respects, goes beyond them. Firstly, its focus is broader so as to include the entirety of the scholarly field; that is, the primary focus is not on university programmes and teaching, but on the whole academic field, extending beyond university education to important research institutes and activities of the field. Secondly, the temporal focus is different from the time perspective taken in earlier studies. It encompasses the past three decades, allowing for a comparison of how the two influential turning points framing Hungary's post-transition PA development affected the field. These two turning points are the effect of the liberal democratic turn, having happened at the time of the systemic change on the one hand, and the constitutional, institutional and political transformation of the post-2010 era, sometimes labelled as a transformation from a liberal to an illiberal democracy, on the other. Thirdly and finally, the aim of this study involves an aim beyond the received views on possible ambitions of PA scholarship: speculating about, and possibly sketching, some of the possible scenarios of the field's future development.

I will pursue the above aims in the following structure. Section 2 outlines the first change of paradigm in Hungarian PA scholarship by outlining the emerging new approach to PA research and teaching rooted in multidisciplinary social science in the early 1990s. Section 3 describes the field's development from the early 1990 os to 2010 . Section 4 portrays how the macro level, landslide political and institutional changes in the broadest realm of governance affected PA in the 2010s. Finally, Section 5 discusses the presentday dynamics of the field and sketches out some possible scenarios for the future.

The basis of the overview is twofold. Firstly, I rely on earlier published work or work in progress (partly already referred to above) describing various aspects of the above development path. Secondly, I rely on some interim results of an ongoing joint research effort aimed at improving the quality of PA education in Central and Eastern Europe, ${ }^{1}$ as well as on some primary data on graduate programme enrolment. 


\section{The early 1990s: the emergence of a new paradigm}

In order to allow for a real appreciation of the disciplinary and institutional innovation I will soon describe, it is reasonable to sketch very briefly the historical antecedents of PA in Hungary. From the mid-18oos onwards, Hungarian PA followed the continental development path. The multidisciplinary study of PA - in the German-Austrian tradition, dominating the field's development in Hungary, often referred to as Kameralistik or Polizeywissenschaft - gave way, from the second half of the 1800 s onwards, to a law-dominated approach to the topic. With some notable but isolated exceptions, such as Zoltán Magyary's scholarship in the 1930 attempting to transplant US-inspired administrative science in Hungarian academic and practice soil, this approach, which was almost exclusively comprised of (public) law, remained dominant, at least until the late 1960 .

However, the practical needs of administrative functions, especially at the local level, increasingly demanded the inclusion of disciplinary components other than the prevailing administrative law and the dominant focus on learning the details of the laws and regulations of the day. A training institute of a predominantly applied nature was established and subsequently developed into a sort of university of applied science (Államigazgatási Föiskola/College of Public Administration). This college tried to strike a certain balance between predominantly legal subjects on the one hand, and more diverse social science, managerial and technical components on the other. However, the main bulk of the curriculum continued to be law or law-dominated components. Moreover, the college trained future civil servants mostly for clerical positions only; high-prestige and managerial positions continued to be occupied mainly by law graduates (and, to a lesser extent, by graduates of economics and other technical sciences).

In the meantime, nevertheless, PA research and scholarship continued to be predominantly tied to the law faculties of a few well-established universities in Budapest and in some of the larger cities in the country (e.g. Pécs, Szeged, Debrecen and Miskolc). Notably, the Hungarian Institute of Public Administration (Magyar Közigazgatási Intézet) was established in the early 1970s, based on a pre-existing entity specialising in local (council) administration and closely tied to the College of Public Administration. In a similar vein to the college it pursued legally dominated PA research, which nevertheless involved multidisciplinary and, in particular, empirical social science components. A notable difference, however, was its closer ties to the practice of administration, and the more applied nature of its approach. Until its abolition in 2007, the institute operated as an increasingly multidisciplinary background institution 
of central government ministries in charge of public administration reform, having participated in the preparation of numerous pieces of legislation and other government decisions related to civil service training and, more generally, public administration reform. In brief, Hungary's pre-transition development patch largely resembled that of most former communist countries, in that it lacked a separate academic identity and was conceived partly as a sub-topic within legal sciences and partly as a vocational, non-academic field of training.

A qualitatively genuine element of the field's development was the establishment in 1992 of the Department of Public Service (Közszolgálati Tanszék) at the (then) Budapest University of Economic Sciences. Staffed (mostly) by a disciplinarily diverse group of former practitioners from various central government agencies, the department launched the first university-level programme in Public Policy and Management (note that until then only college programmes equivalent to BA level existed in PA). The research and teaching profile of the department was built up in close collaboration with leading US, Belgian, Dutch, UK and other universities, donor agencies and international PA academic organisations, and strongly resembled public policy programmes customarily offered in these settings. Hereinafter, somewhat simplistically, I will refer to this new strand of PA scholarship instituted by the Department of Public Service as Public Policy and Management, PPM.

The unique features of this new strand of scholarship were threefold. Firstly, it explicitly aimed at departing from the predominantly law-based, deductive and non-empirical/speculative, as well as from the applied, non-academic, technical character of PA studies characteristic of most of the preceding decades. Instead, it intended to join the mainstream international practice of a truly scientific, interdisciplinary and multidisciplinary PA grounded in and understood as empirical social science. This tradition had significant preliminaries in Hungary from the 1930s onwards but remained, throughout the subsequent decades, a marginal and neglected component of PA. In terms of its disciplinary orientation, to put it more precisely, this new paradigm constituted a departure from the pre-existing - and, even if sometimes trying to depart, but still continually predominantly legal - approach to PA in several respects:

- It abandoned the largely unidisciplinary perspective of earlier decades. Instead of being dominated by one Leitdisziplin (Bauer, 2005), it consciously tried to apply to its subject area a much more balanced mix consisting of several disciplinary components.

- Moreover, the mix of disciplinary components - using the corresponding terms used by Bauer (2005) and Hajnal (2003) - were such that the emerging model can be located between "public" and "political science" on the one hand, and "corporate" or "management-oriented" on the other. 
Secondly, it intentionally tried to strike a balance between theoretical and academic approaches on the one hand, and practical-analytical applications on the other. Nevertheless, it explicitly pursued the aim of becoming an academic field in its own right. Finally, yet another distinguishing feature of the new approach taken by the Department of Public Service was that, in contrast to the traditionally inward-looking stance of the PA community, it explicitly and successfully attempted to create close ties and engage in deep and long-term research and teaching collaboration with a broad range of international academic actors. For the programme curriculum and research profile this also meant an increasingly international and comparative focus.

\section{From the early 1990s to 2010: consolidation and gradual development}

The next historical epoch in the development of the Hungarian PA field was characterised by a consolidation and incremental development of the field. Until the so-called Bologna reforms (implemented in 2006), the PA education landscape was mainly populated by the College of Public Administration on the one hand, and the more economics and methodology-oriented Department of Public Service at the Budapest University of Economic Sciences on the other.

In 2000, the former Budapest University of Economic Sciences merged with the College of Public Administration to become the Faculty of Public Administration of the re-named Budapest University of Economic Sciences and Public Administration (BUESPA). This change however did not affect the pre-existing patterns of PA research and teaching. Whereas in terms of volume the law-based strand of PA teaching was thus dominant throughout this epoch, the PPM-oriented scholarship was more at ease with joining the international PA research and teaching community. In particular, the Department of Public Service at BUESPA (in 2004 yet again renamed as Corvinus University of Budapest) was very active in this regard. It hosted several of the most important international academic PA events, co-founded prestigious European teaching and research networks, and its faculty presided over or participated in the steering of the most important regional and international academic associations in the field.

The Central European University (CEU) has gradually built up a strong profile in public policy research and education. Partly building on its track record in policy research and education at its Department of Political Science, in 2000 it founded its Centre for Policy Studies (and in 2006 its Department of Public Policy), and in 2004 it launched its first master's level programme 
in public policy. The CEU, which enjoys the dual institutional-legal identity of an American-Hungarian privately funded university, has gradually built up a very strong and internationally highly visible academic profile.

The Bologna reforms had a huge impact on the PA education field. Most importantly, the new separate bachelor's and master's level programmes opened the field up to a broad range of higher education institutions. Consequently, both the law-based and the PPM-based versions of the PA programmes at bachelor's and master's level were being offered by a rapidly increasing number of higher education institutions, in addition to the two faculties at Corvinus University. In particular, an increasing number of law faculties started to offer a bachelor's level programme reminiscent of the one that had been run by the College of Public Administration for many years. In 2010, close to $90 \%$ of PA students were enrolled in this law-based type of PA programme. The remaining $10 \%$ were enrolled in PPM-type programmes offered by about ten universities, the largest being at the Department of Public Service at the Corvinus University of Budapest (Hajnal, 2016, p. 215). Overall, the traditional law-based approach to PA continued to dominate the field after the Bologna reforms.

As regards PA-related scholarship, legal scholars working at different universities - in particular, the ELTE University in Budapest and the Universities of Pécs, Debrecen and Szeged - produced a sizeable body of PA-related scholarship during this period. While most of this scholarship pursued the traditional public/administrative and constitutional law dominated research agenda (and thus continued to be mono-disciplinary, deductive and analytical), efforts were made to involve social scientists (economists, regional planners, sociologists etc.) in their research. Much of the internationally most visible research at the BUES/BUESPA (Corvinus since 2004) was partly produced, nevertheless, by renowned international guest professors hosted by the university.

In terms of non-university-based PA scholarship several entities deserve attention. The Hungarian Institute of Public Administration - in the dual role of civil service training centre and public administration think-tank of the government - continued to offer a home to a small but significant interdisciplinary community of PA researchers. It produced intellectually independent and internationally visible research outputs, at the same time exerting visible influence on, and enjoying high prestige in, the practice field of public administration. In the early and mid-1990s, the institute produced important pieces of legislation and policy in diverse fields of administrative reform. In the years preceding the EU accession until the mid-20oos, its practical impact became somewhat more limited, but it still remained a highly 
visible player in civil service training and public administration research. However, the role and scope of policy advice expected by public administration reform decision makers constantly and significantly shrank from the mid-20oos onwards. Business-inspired, New Public Management-style reforms and reformers increasingly questioned the legitimacy of and need for specific public administration expertise and research in informing the government's public administration reform agenda. The institute was finally abolished in 2007, and remnants of this research capacity were then merged with another government background institution named ECOSTAT. ${ }^{2}$ This entity was reshuffled and stripped of its scientific research capacity after the 2010 change in government, reflecting the sharp acceleration of the - since the mid-20oos already strong - tendency to devalue expertise and scientific evidence in governmental (and, in particular, administrative reform) practice.

Another notable institutional actor of the PA community was the Centre for Regional Studies (Regionális Kutatások Központja/RKK) founded in 1984, which has since operated as a member of the research network of research centres of the Hungarian Academy of Sciences. The institute was predominantly active in the field of regional and local development and administration studies, and produced important research and developed important international research connections (partly as a member of FP6 and seven consortia) in these fields. The centre's interdisciplinary staff of researchers contributed to the foundation of the regulatory and institutional system of Hungarian regional policy and to the improvement of the local government system, devoted to the principle of regional decentralisation. Another member of this network of research institutes, the (then) Institute of State and Legal Sciences (Állam-és Jogtudományi Intézet), conducted mainly public law-based research (having been considered, throughout the preceding decades, as mainstream PA). In the late 199os, the Institute for State and Legal Sciences was re-named the Institute for Legal Studies; however its actual research portfolio has not changed much.

From a comparative perspective, the above development path of the 1990-2010 epoch seems to feature a pattern of increasing divergence from most other Central and Eastern European developments. The majority of post-communist countries made a sharp shift towards a managerial PA in the early years of transition, and most of those remaining, such as Romania and Slovenia, gradually departed from their law-based approach over the years that followed and converged towards either a (public) management or a social/ political science-oriented model (Bauer, 2005; Hajnal, 2003; see also Hajnal, 2015). However, Hungary remained, along with Germany (Hajnal, 2016), a diehard member of the ever shrinking "legal club" in Central and Eastern Europe. 


\section{$4 \quad$ Public administration in the illiberal era}

At the 2010 national elections, the FIDESZ-KDNP formation led by Viktor Orbán achieved a landslide victory resulting in a two-thirds supermajority in the unicameral legislature. This enabled the governing forces to transform basic, including constitutional, foundations of the state without any negotiation with either the opposition or any other societal actors. As a result, a lengthy and radical series of constitutional institutional reforms began immediately after the election victory, followed by two additional election victories in 2014 and 2018.

Albeit not presenting an entirely linear development path, and especially not one with clear ex ante stated objectives and principles, the emerging system of governance increasingly took a shape distinctly different from those of established Western liberal democracies. Up to now, this has frequently been called, by scholars and in the official rhetoric of the governing political forces, illiberal democracy, or simply illiberalism (Bogaards, 2018, p. 2). In one sentence, thus strongly over-simplified, this means the utmost centralisation of power - political, administrative and economic, formal and informal alike - in the hands of the prime minister, and minimising constraints, checks and balances limiting it either in functional terms or in time. It is not my intention, however, to review here the process and the result of this huge transformation. Instead, I focus on outlining the blatant effects it exerted, among many other fields of societal and policy practice, and on the landscape of PA science and education.

As part of their much broader effort completely to transform the Hungarian politico-administrative landscape, soon after their election victory, the governing forces started to prepare an entirely new institutional framework for civil service education and training. At the centre of this framework one finds a newly created, all-encompassing institution, the National University of Public Service (NUPS). The new university was founded out of three pre-existing institutions: the national military academy, the national police academy and the former Faculty of Public Administration at the Corvinus University of Budapest.

The new institution, by design, had several important features sharply distinguishing it from either its domestic or its international peers.

Firstly, it integrated civilian PA education with military and police officer training. This was not only a structural issue; the core idea of the new university was the substantive integration of the three main - recently called - corps of public service: the military, the police and the civilian. The idea of merging civilian and military/police education into a single higher education 
institution is, from an international as well as from a Hungarian historical perspective, highly unusual. The official rhetoric justified this move by emphasising the necessity to create a socially and culturally distinct patriotic cadre of professionals devoted to serving the government, to strengthen horizontal links between the three "corps" of public service (military, police and civilian), and to control and strengthen the patriotic and moral value base of future public servants.

Secondly, as a result of successive steps, NUPS was granted a monopoly in the traditional, law-based (and dominant) type of PA education. Other higher education institutions offering this type of programme were gradually banned by successive changes in government policy and legislation from continuing their operation in this education field. Subsequently, this monopoly was extended by the introduction of a new type of university programme, the so-called MA in State Science. (The initial idea extended to legislatively requiring all public administrators in managerial positions to complete this programme; however, this idea was later abandoned.)

Thirdly, NUPS was granted key and monopolistic functions within the entire civil service training system. According to the applicable legislation, NUPS is the sole entity developing, authorising and providing general-scope in-service training measures for public administrators at central, territorial and local levels alike. It was (and continues to be) the sole beneficiary of a broad range of European Union-funded (ESF) public administration capacity-building measures.

Fourthly and finally, the governance arrangements of NUPS are markedly different from those of the regular public higher education institutions. From its inception, NUPS was clearly a high-priority "pet project" of the government and the Prime Minister personally. Symbolically, this was made clear, among other indications, by NUPS's founding ceremony being held in the Houses of Parliament in the presence of the Prime Minister and all the cabinet ministers - a highly unusual act for higher education institutions. Legally speaking, NUPS was removed from the institutional framework for regular higher education institutions and placed directly under a distinct legal and supervisory regime; it was positioned directly below its three supervisory ministries, the Ministry of Public Administration and Justice, the Defence Ministry and the Ministry of the Interior. ${ }^{3}$

The second item of the above list of NUPS's unique features deserves additional attention since it has directly and fundamentally affected the institutional landscape of not only the Hungarian PA community (wherever its boundaries exactly lie), but also that of the much broader realm of law 
schools and their programmes. In the official classification of higher education programmes, the former category of "State and Legal Sciences" was abolished and a separate category for "State Sciences" was created, the "Legislative Sciences" category was reserved for NUPS, so that law faculties became formally banned from launching these programmes.

In 2016, a new unified (five-year BA plus MA) "State Science" programme launched by NUPS made a half-hearted move towards interdisciplinarity by introducing more emphatic elements in public finance and other social science subjects, for example. Interestingly, the programme's name (as well as that of the programme category) translates literally as "State Science" (Államtudomány), and appears in official English translations as "Public Governance."4 Still, not only the content, but also the self-identity of the new approach were explicitly grounded in the early twentieth-century Germanic tradition of Staatswissenschaft (see Cs. Kiss, 2017). Despite repeated attempts at outlining a coherent and genuine disciplinary contour for the new, would-be discipline (see Cs. Kiss, 2017; Kaiser, 2016), the fundamentally law-based character of research and training remained largely intact.

In sum, within a few years after its founding, NUPS had taken over, mostly through direct legislative measures, close to $100 \%$ of the PA higher education scene. Content-wise this training nevertheless remained remarkably similar to the established tradition of law-based PA studies, having dominated the field in the preceding decades. A solid majority of curricular components continued to be various subfields of administrative and public law, frequently emphasising the knowledge of specific pieces of legislation and under-emphasising analytical and generic skills. Published evidence regarding the content of PA training and education programmes offered by NUPS is unequivocal regarding its main findings. Three different authors (Gajduschek, 2012; Gellén, 2014; Hajnal, 2015) strongly maintain that the content of NUPS programmes underwent minimal change. Certain changes on a university level (that is, for the entirety of the military, police and civilian programmes), such as the introduction of some joint modules taught at the civilian, military and policy faculties, took place. Nonetheless, the conclusion of these works was unanimous in that these changes have not affected the fundamentally unchanged legal character of the civilian PA programmes.

Whereas NUPS, building on its top-level political support, successfully monopolised most of the PA higher education field within a matter of two years after its creation, the other traditional PA higher education entity operating at the Corvinus University of Budapest chose - amid severely constraining legislative, financial and higher education market conditions - another way forward. Building on its close cooperation with Corvinus University's other 
research units specialising in various subfields (such as health economics and public performance management), it was among the first universities in Central and Eastern Europe to acquire the EAPAA international accreditation. This helped the programme to sustain its appeal to Hungarian and, increasingly, to international students as well. The programme, run by the former Department of Public Service, renamed the Department of Public Policy and Management, further extended its international research and teaching cooperation network with substantial domestic and regional standards.

In a process parallel to the above, PPM-type programmes at both the bachelor's and master's levels evaporated, so that by the second half of the 2o10s only one master's programme continued to operate, that being the one offered by the Corvinus University of Budapest.

International private universities, such as the English-language CEU and the German-language Andrássy University, continued to offer such programmes and conduct-related research in these fields. In particular, on the initiative of its founder, George Soros, CEU in 2012 launched the School of Public Policy. The school, which subsequently merged with the Department of Public Policy and after a short period of parallel operation integrated its master's and doctoral programmes, has a global outreach and ambition in that it aims to address global problems in its teaching curricula. It recruits primarily foreign students while employing a diverse international faculty.

In terms of non-university-based PA scholarship, the Institute for Political Science, which was integrated into a larger Centre for Social Sciences of the Hungarian Academy of Sciences in 2012, deserves special mention as a central actor. In 2010, the inner structure of the institute was changed, so that a separate Department of Public Policy and Governance was called into existence. A declared objective of the Institute was, and continues to be, to become a leading centre of political science, understood as including public policy research in Hungary. The institute became a member (and, in some cases, the leader) of prestigious European and international research networks and initiatives in public policy research. The former Institute for Legal Studies became another entity within the new Centre for Social Sciences. As a result of conscious and enduring efforts, interdisciplinary PA research within the centre became increasingly significant and visible in the 2010 .

As another consequence of the large-scale restructuring of the research network of the Hungarian Academy of Science in 2012, the former Centre for Regional Studies was also merged with two other institutes, namely the Institute of Economics and the Institute of World Economics. Since then, it has operated under the new name of the Institute for Regional Studies as part 
of the Centre for Economic and Regional Studies. Although the number of researchers has decreased and two regional units ceased to exist, PA research activity has remained on the agenda of the new entity. The main research topics have continued to focus on territorial administration, regional development policy and institutional system development.

The 2010 saw Hungary continuing on its route diverging from the increasingly multidisciplinary and social science informed varieties of other Central and Eastern European countries' PA science, a route it had already embarked on in the 1990 os. However, in addition to its increasingly outlying disciplinary character, yet another specificity characterising the country's PA field appeared. The institutional landscape - at the centre of which one finds a single, politically tightly controlled academic entity absorbing most of the field's resources and monopolising, among many other functions, close to $100 \%$ of PA higher education - can be regarded as unique, not only in the Central and Eastern European, but, most probably, also from a global perspective.

\section{Present-day developments and future prospects}

The years 2010-2015, as the previous section showed, were characterised by exceedingly intense dynamics in the field of PA. As a result of landslide changes in government policy on PA education, by the second half of the $2010 \mathrm{~s}$ decades-long traditions had been broken and disciplinary and institutional arrangements fundamentally re-shaped.

In the field of PA higher education - as was the case in many other policy areas and social and economic fields - the emerging new arrangement was one dominated by a monopoly directly controlled by the government: the NUPS. In addition to the small foreign-language programmes run by international higher education institutions, the only additional programme to survive was the one run by Corvinus University of Budapest. Until about 2017, it seemed that the government's resolution to monopolise training and education for the civil service in NUPS was ever increasing, and consequently the fundamental legal character of university education remained largely untouched.

Interestingly, however, this thrust to promote (i) the fundamentally law-based and (ii) a government-controlled kind of PA seems, at the time of writing, somewhat questionable. Recent changes in the applicable legislation have substantially softened - practically ended - the compulsory nature of some flagship NUPS degrees in the civil service. Instead, a different move can be observed: from 2018 NUPS started to offer, in addition to the 
ones already running, its own PPM-type master's programme, an MSc in Public Policy and Management, a programme it (or its predecessors) had never run.

The figure below illustrates the developments in Hungary's PA higher education market, focusing only on master's programmes (including the unified five-year State Science programme).

Figure 1 Enrolment for different types of master's PA programmes run by Corvinus, NUPS and other universities

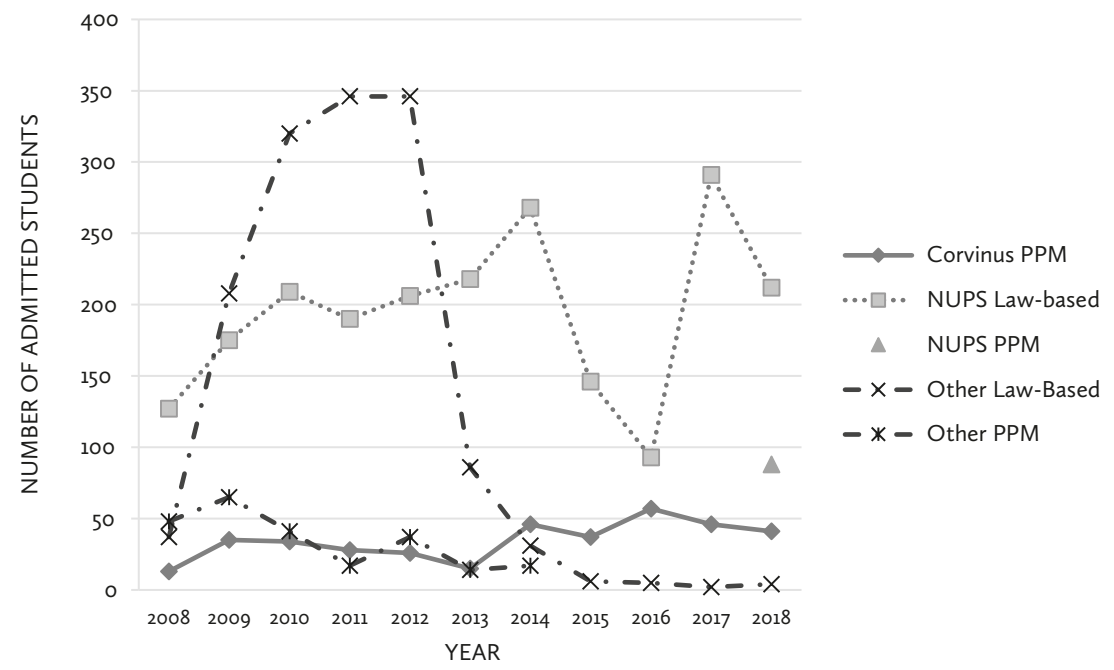

Source: www.felvi.hu, compiled by the author

Another major change happening at the time of writing is the fundamental institutional transformation of Corvinus University of Budapest. The government is transforming the university into a new type of entity, the first of its kind in Hungary: a semi-private university placed under the oversight of a (formally) autonomous oversight body and funded by a sizeable government endowment. The stated objective of the institutional innovation is to help Corvinus gain higher than ever international visibility and recognition, and to turn it into a competitive university increasingly appealing for Hungarian as well as international students and scholars.

A qualitatively unique element of the above transformation process of the PA field is the high-profile conflict between the Central European University and the Orbán government, triggered by an openly politically motivated legislative change, as a result of which many of CEU's activities are threatened with illegality. The final outcome of this conflict on the PA/public policy 
research and education capacity and activities of CEU in Hungary is not known at the time of writing.

As yet another instance of the Orbán government's conflicts with the academic sector, another controversy started in 2018 when the government started to target the network of research institutes of the Hungarian Academy of Sciences. The network comprises 15 research centres covering virtually all disciplines (two of them being the Centre for Social Sciences and the Centre for Economic and Regional Studies already mentioned above). The government intends to gain more control over the network and has explicitly accused the Hungarian Academy of Sciences of being "too political," pointing to social science research results which set a critical tone concerning government policies, or are ideologically not in line with the government's stance. The outcomes of this conflict and its effect on the prospects of PA/ public policy research conducted by the research institutes of the Academy are, at the time of writing, still uncertain.

In the view of the above, it may seem that most of the dynamics in the PA field in recent years - indeed in the past one and half decades since the Bologna reform - have originated from (changes in) government policies. One may ask nevertheless what effect other actors, such as the EU or the academic institutions themselves, have exerted on the above dynamics. There is no systematic and publicly available evidence of how such EU policies, most importantly the Erasmus scheme, have affected the field. On the basis of anecdotal evidence, two observations deserve mention.

Firstly, the supply of English-language courses, let alone programmes, in the field has been very modest over the observed period. The only full English-language programme continues to be the one taught at the Corvinus Department of Public Policy and Management. Whereas other entities - the NUPS from the mid-2o10s onwards - did offer some courses in the English language, the modest overall supply of such courses clearly constrains the flow of incoming students. Likewise, international faculty exchange is similarly limited by the scarcity of English-language programmes/courses in Hungary on the one hand, and the (relative) lack of Hungarian faculty able and willing to teach in English and in an international environment on the other. However, the Erasmus scheme has certainly added a great impetus to the internationalisation ambitions of universities and programmes and created the conditions for exploring and creating more in-depth academic relations.

This leads to the second observation relating to the more indirect effects on PA higher education. More ambitious forms of international academic relationships started to materialise from the early 2010 on. Whereas ad hoc faculty exchange had taken place, albeit infrequently, in a number of 
institutions and programmes, the more significant types of benefits were basically limited to Corvinus' PUMA programme. It concluded double- and triple-degree agreements with several prestigious PA education institutions both in the old and in the new EU Member States, and hosts renowned international scholars on a regular basis as guest professors.

Nevertheless, overall it is safe to assume that the Erasmus programme has created an environment in which international actors, standards and relationships are becoming increasingly important, even if such effects are constrained by the varying levels of academic capacity of individual higher education institutions or programmes.

As the above overview suggests, change is happening to almost all the features of the PA discipline at a consistantly high rate. Moreover, most of these changes are triggered by unexpected and unpredictable, even disruptive, shifts in government policy - much more so than one had expected before the onset of Hungary's current, post-2010 shift towards "strong-state-inspired," illiberal governance. Illustrative of these expectations is the list of main contextual factors put forward by Bouckaert (2010) who, albeit on a broader European scale, pointed to Europeanisation, public sector reforms, globalisation and marketisation as the main determinants of dynamics within the PA field.

As a result of successive large-scale shifts in domestic policy, key institutional actors changed. All the law faculties of the largest universities in the country, for example, became "outsiders," whereas gigantic new entities, such as the NUPS, were created within a matter of months. Funding from the EU and the government is reallocated among this constantly changing set of players with ease. Political, governmental influence on academic issues - such as what constitute legitimate fields of scientific inquiry or teaching, or what is or is not a separate discipline - has entered the realm not only of possible futures, but of everyday reality too. Moreover, these and other frequently highly politicised and highly conflicting changes are happening at the very moment of writing too.

So what does all this suggest in relation to the future - or, rather, the possible futures - of the field of PA in Hungary?

Substance-wise - that is, regarding the fundamental disciplinary perspectives, theoretical frameworks and methodical approaches used in the study of PA - the most probable future scenario seems to be a gradual, troubled and imperfect, but still actual convergence with the international mainstream. In other words, despite renewed attempts at preserving or even reinstalling (as in the case of Staatswissenschaft) the (public) law-based disciplinary approach dominant in earlier historical epochs, an increasingly multidisciplinary, inductive-empirical social science field is likely to appear on (or beyond) the 
horizon. This, however, by no means implies that law as a discipline becomes obsolete in studying PA phenomena, and especially not that a law-oriented approach to practising public administration as a profession would not continue to be a decisive feature of the field. However, the main bulk of what is considered PA can be expected increasingly to exhibit features of Western, mainstream PA. Whether this future model will resemble the - yet again using Bauer's (2005) and Hajnal's (2003) corresponding typologies - "public/ political science oriented" or the "corporate/management oriented" ideal type or, rather, something in between, remains to be seen though.

On the other hand, however, the above convergence with mainstream PA has its limits. Firstly, the politico-administrative system in which Hungarian governance takes place seems to diverge, in certain substantive respects, from the Western mainstream. (One may argue, however, that the very orthodoxy of mainstream Western liberal democratic governance is increasingly under pressure and in the process of fragmentation - but discussing this line of argument would be beyond the scope of the present essay.) Secondly, the institutional capacity of Hungarian PA is likely to remain below the levels of many of its international peers. Closing this gap in institutional and human resource capacity would, even under optimal conditions characterised by strong and sustained government support (which is definitely not the case these days), take a historically long period of time.

This leads us to a second key dimension of the futures of Hungarian PA: the institutional landscape of the discipline. Which ones will be the institutions educating the main bulk of future PA professionals, and producing the most influential and visible body of research? And what will be the role of PA as a discipline (as opposed to other disciplines, for example law) in these education and research activities?

Focusing mainly on the educational perspective, in seeking answers to the above questions some important - and possibly longer-term - contextual conditions have to be considered:

- Firstly, despite a continued radical anti-NPM and, at least rhetorically, Neo-Weberian spirited public administration reform agenda, many recent (and not so recent; see Hajnal and Boda, 2019) changes in administrative policy follow an NPM-style, "anti-bureaucratic," downsizing and deregulation-oriented pattern. Consequently, salary and staff freezes as well as large-scale layoffs happen at regular intervals, accompanied by a markedly bureaucracy-hostile rhetoric on the part of the government. This pattern substantially lessens the appeal of public administration as an employer for future administrators and does not help the prestige of $\mathrm{PA}$ as a discipline either. 
- Secondly, at a macro level the Hungarian economy is being hit by chronic workforce shortages (triggered by diverse factors ranging from macroeconomic developments to mass emigration, and to the prevailing lower end, labour-intensive economic sectors). This pattern further dampens the prospects of public administration as an appealing and prospering field of education.

- Finally, there are positive signs of development patterns towards an elite-oriented education. Senior mid-career administrators as well as international students (the latter frequently aided by Hungarian government scholarships) increasingly tend to be interested in well-targeted, well-designed education programmes. Such programmes might offer insights into the specifics of certain policy fields or policy instruments, or into the specifics of a country (and a region) frequently seen as home to non-liberal democratic forms of governance - being much more the rule than the exception in diverse continents and countries.

The above external conditions seem to imply the gradual emergence of an institutional landscape populated by a much smaller number of institutions than was the case, say, a decade ago. Whereas most of the higher education institutions active in PA have (frequently involuntarily) left the scene, the remaining few have, almost inevitably, a stronger position, and enjoy a rather monopolistic position in the Hungarian PA higher education "market."

Thus, for some of the prospective key institutional players the outlook, at the moment of writing, is relatively promising. Others are, however, threatened by government policies in their very existence and operations. This is the case with two centres of the Hungarian Academy of Sciences (the Centre for Regional and Economic Studies and the Centre for Social Sciences) and, notoriously, with the Central European University. One of the big questions is whether the freedom of academia, and academic research and teaching, can be preserved amidst strengthening signs of governmental actors' intention to interfere. While this is a burning issue for the entire academic sector, it is particularly so when it comes to Public Administration and Public Policy - fields that have the tightest connections to what the government does. At another, more operational level, an additional question for the future is where the fortunes of government policy will lead NUPS - whether it will continue to play its central role in training future as well as current public servants, and whether its recent - albeit on a small-scale, but novel - endeavour in the area of a non-law-based, PPM-type education programme will gain impetus or remain a small island. 


\section{Acknowledgements}

Inputs, comments and suggestions by the following individuals are hereby gratefully acknowledged: Zsolt Boda (Centre for Social Sciences of the Hungarian Academy of Sciences); György Gajduschek (Corvinus University of Budapest and Centre for Social Sciences of the Hungarian Academy of Sciences); Tamás Horváth (University of Debrecen); Krisztián Kádár (Corvinus University of Budapest); Éva Kovács (National University of Public Service); Gergő Medve-Bálint (Centre for Social Sciences of the Hungarian Academy of Sciences and Corvinus University of Budapest); Éva Perger (Centre for Regional and Economic Studies of the Hungarian Academy of Sciences); Zoltán Szente (National University of Public Service and Centre for Social Sciences of the Hungarian Academy of Sciences).

\section{Notes}

1. PAQUALITY: Public Administration Education Quality Enhancement, Erasmus+ Strategic Partnership KA203, Slovakia, 09/2018-08/2021.

2. Ecostat Government Institute for Strategic Research of Economy and Society, Department of Public Administration.

3. The composition of the oversight body has since changed several times; at the time of writing the sole supervisory function is performed by the head of the prime minister's office.

4. Government Decree 282/2016. (IX. 21.), Annex 3. (retrieved June 20, 2019 from https: //net.jogtar.hu/jogszabaly?docid=A1600282.KOR\&timeshift=20160929\&txtrefer er=00000001.txt).

\section{References}

Bauer, M. W. (2005). Damit ist ein Staat zu machen. Verwaltungsstudien in Ostmitteleuropa, Osteuropa. Zeitschrift für Gegenwartsfragen des Ostens 55(11): 55-66.

Bogaards, M. (2018). De-democratization in Hungary: diffusely defective democracy. Democratization 25(8): 1481-1499.

Bouckaert, G. (2010). Research in public administration for the future. Society and Economy $32(1): 3-15$.

Cs. Kiss, L. (2017). Államelméleti helyzetkép [The state of affairs in state sciences]. Államtudományi Mühelytanulmányok. National University of Public Service, 2017/6.

Gajduschek, G. (2012). A magyar közigazgatás és közigazgatás-tudomány jogias jellegéről [On the legal character of Hungarian Public Administration]. Politikatudományi Szemle 21(4): 21-49. 
Gellén, M. (2014). Public administration education in a continental European legalistic setting: the Hungarian case. Teaching Public Administration 32(2): 111-126.

Hajnal, G. (1999). Evaluation of academic programs in the field of public administration in CEE countries. Country Report - Hungary. Thematic Network in Public Administration Annual Conference. 2-3 July 1999, Leiden, the Netherlands.

Hajnal, G. (2003). Diversity and convergence: A quantitative analysis of European Public Administration education programs. Journal of Public Affairs 9(4): 245-258.

Hajnal, G. (2016). Illiberal or simply unorthodox? Public Administration education in Hungary: A comparative perspective. Teaching Public Administration 34(2): 205-224.

Hajnal, G. (2015). Public administration education in Europe: Continuity or reorientation. Teaching Public Administration 33(2): 95-114.

Hajnal, G., \& Boda, Z. (2019). "Though this be madness, yet there is method in it": The anatomy of illiberal transformation of government bureaucracy. The case of Hungary. Democratic backsliding and public administration, Robert Schuman Centre and School of Transnational Governance, 31 January-1 February 2019, Florence.

Hajnal, G., \& Jenei, G. (2007). The study of public management in Hungary. Management and the transition to democratic Rechtsstaat. In W.J. M. Kickert (ed.), The Study of Public Management in Europe and the United States. A Comparative Analysis of National Distinctiveness (pp. 208-232). London: Routledge.

Kaiser, T. (2016). Az államkutatások helye a társadalomtudományok rendszerében [The place of state reseach in the system of social sciences]. Államtudományi Mühelytanulmányok, National University of Public Service, 2017/1.

Nemec, J., Spacek, D., Suwaj, P., \& Modrzejewski, A. (2012). Public management as a university discipline in new European Union member states. Public Management Review 14(8): 1087-1108.

Staranova, K., \& Gajduschek, G. (2016). Public Administration Education in CEE Countries: Institutionalization of a Discipline. Policy and Society 35(4): 351-370. 



\title{
5 \\ Public Administration in Italy
}

\author{
Denita Cepiku, Marco Meneguzzo
}

\section{Introduction: Italy and its public administration}

Italy is a Mediterranean country considered to belong to the Napoleonic state tradition.

The Italian public administration is considered to be part of a distinctive southern European model of democracy and a distinctive model of bureaucracy (Sotiropoulos, 2004). According to Kickert (2007), it is characterised by formalism and legalism, historically introduced as a counterbalance against political interference. Both are considered major reasons for the rigidity and inefficiency of its bureaucracy. The vast majority of civil servants are still administrative lawyers.

Considering that unification occurred only in 1861, the Italian public administration is relatively young when compared to the administrative systems of other European countries such as France, Spain and the United Kingdom. Still, performance is disproportionately heterogeneous across regions, particularly when considering central, northern and southern Italy.

The Italian public administration is the result of cross-fertilisation of different administrative cultures, including the Rechtsstaat tradition (Austro-Hungarian derived, Lombardia and Veneto), bureaucratic Napoleonic traditions (Piemonte and Sardinia), and weak-state models more similar to the Vatican State and the kingdom of the Two Sicilies (south Italy). These administrative cultures embody vastly differing visions of institutional and organisational models, and a diversity of relationships between public administration, citizens/civil society and other social and economic factors (Meneguzzo, 2007).

Selection and training of civil servants is usually carried out by the administration itself, a factor which has brought about the isolation of bureaucracy. A changing trend has taken place over the past five years, with the National School of Administration relying more on universities to deliver postgraduate master's programmes to civil servants by accrediting programmes and funding full scholarships. 
Administrative elites, such as those developed in France and the United Kingdom (Cassese, 1983), are all but non-existent, and the closeness of career paths does not allow for the development of an education system similar to the ENA in France. Needless to say, Italy does not have established public administration education traditions such as the French grands corps or the English "Oxbridge."

Since the early 1990s, the Italian public administration has been living in a period of permanent reform that has affected almost every area of public management (Cepiku \&Meneguzzo, 2011). Such reforms are based on a managerial frame of reference and focus on effectiveness and efficiency, which is contradictory to the legal frame of reference in terms of legal accountability. As management reforms have to be formulated in juridical language in order to become legally enacted, the legalistic monopoly remained unbroken (Kickert, 2005). A survey of the Italian Department of Public Administration in 2003 reported that as many as $99 \%$ of the 1,588 senior civil servants interviewed felt they lacked managerial competencies.

The main public administration reform areas from 1990 to date include: transparency, access to information and fighting against corruption, territorial decentralisation, civil service reform, privatisations and contracting out, e-government, managerial controls and performance management, the creation of independent administrative authorities and executive agencies, government-citizen relations (service charter, customer satisfaction and quality management), administrative simplification, spending reviews and reform of the public expenditure management and accounting systems, among others.

Two main considerations can be seen at a glance: the high political turnover and the rich variety of themes. A consequence can be easily grasped: the combination of political instability and the prevalence of legalism, among other features, has led to a serious implementation gap. There is a recurrence of reform themes in political agendas that is not motivated by a change in reform contents due to some policy evaluation, but rather in a reiteration of normative obligations (Cepiku, 2017). These include: performance management, political-administrative relations, administrative simplification, transparency, e-government and accounting, among others.

Today, the role of the Public Administration and public management academic community remains weak, although it has improved, in designing and guiding public sector reforms. This is true both at the central level, where reforms are designed, and at the individual agency level, where they are implemented. 


\section{The state of the field of Public Administration in Italy}

The distinctive national characteristics of the study of public administration in Italy can be understood by considering: the traditional dominance of the administrative law approach, notwithstanding the coexistence of other competing disciplines, in the study of public administration; and the more recent influence of the Anglo-American managerial approach, New Public Management ideas, and the Italian managerial theory economia aziendale.

The Italian public administration is still dominated by lawyers and is obsessed with legalism; thus, it is no wonder that the study of administration continues to be dominated by public law. The major part of the competitions and tests for accessing public sector positions, in any rank or policy area, entail a good dose of public and administrative law.

One of the most important public competitions for access to management positions in the public sector (corso-concorso) organised by the National School of Government is based on a first written exam including questions on administrative law, constitutional law and EU and international law; a second written exam concerns political economy, public management and economics.

The fairly recent development of public management research in Italy could be considered the result of the administrative reforms that started in the early 1990s (Kickert, 2007; Meneguzzo, 2007). A previous hesitant initiative in the 1970 s could be mentioned aimed at introducing scientific management principles, which encountered obstacles related to a formalistic and juridical culture. Public management is only gradually gaining some terrain, both in practice and in academia.

A vicious circle has been in place with reforms in design and implementation conducted by either administrative law experts or business administrations experts (the latter lacking a thorough knowledge of public administration specifics and often acting as consultants to individual agencies implementing reforms), leading to no or negative and unforeseen results, which in turn have weakened the confidence of politicians and practitioners in management theories. Other disciplines such as political sciences, sociology and public economics have hardly been involved.

The Italian public management academic contribution is interdisciplinary and distinctively characterised by the so-called economia aziendale approach, a kind of institutional economic theory of organisations. Although the development of economia aziendale dates back to the first decades of the twentieth century, it was thoroughly applied to public administrations only in the past three decades. A possible translation of economia aziendale may be business management or business administration, even if neither of these 
terms satisfactorily captures its meaning, which corresponds to the German Betriebswirtschaftslehre. It is different from the analytical, model-based and econometric focus of the Anglo-American approach and also from the French sciences de gestion, since these studies are centred around the capitalist enterprise archetype, focused on individual profitability objectives. Other differences concern the research method (inductive/deductive in the former, mainly inductive in the latter) (Borgonovi, 1992).

The application of the economia aziendale paradigm to public administrations in Italy gained more space and interest during the initial phase of the $\mathrm{New}$ Public Management wave. It is still at the heart of many reforms applied to the public sector. Two main critiques of this approach have been the excessive focus on accounting and a failure to include politics and, more generally, adapt concepts and tools to public administration specificities.

\subsection{Public Administration education}

A statistical analysis has explored Public Administration education in Italian universities, with the aim of verifying whether the administrative law approach to university-based Public Administration teaching is still prevalent in Italy (Cepiku, 2013).

Information was collected between June and July 2007 from 204 faculties of 72 universities and 2,198 education programmes at different levels were registered and classified. Overall the evidence suggests that the legal cluster, including but not limited to administrative law, is not the prevalent approach in teaching Public Administration in Italy. The public cluster - mainly political sciences and a public economy approach - is widespread at Italian universities. Furthermore, there is a strong, yet more recent, development of the managerial approach. In general, an insufficient supply of PA programmes has been registered in southern regions, which also put a greater emphasis on doctoral education.

Cotta (1996) offers an interesting historical analysis of the Italian faculties of political sciences. They are depicted as multifaceted units covering a variety of disciplinary areas: the main ones are sociology, contemporary history, constitutional and international law, economics, political philosophy and history of political ideas, statistics and political science stricto sensu. Faculties of political science began to spread in other universities particularly after World War II, but quite often the first step was to create a programme of political science under the umbrella of a more well-established law faculty. In time, such programmes have generally managed to become institutions autonomous from the faculties of law. 
Political science as a specific and autonomous discipline began to gain a role in the university curricula in the 1960s, although in the Italian academic tradition the disciplines of political philosophy, history of political ideas and state theory (the German Staatslehre) had existed long before the appearance of political science (Cotta, 1996).

The number of Public Administration programmes taught at the faculties of economics is higher than that taught at the faculties of law and political science. This result is quite interesting, but it does not take into account the disciplinary character of the programmes. In other words, over $37 \%$ of Public Administration programmes taught at the faculties of economics also include courses and programmes in administrative or public law.

A description of Public Administration programmes in terms of their disciplinary character brings about a more balanced picture, with law-oriented programmes counting for $31 \%$, economics for $17.1 \%$, management $12.6 \%$, political science for $9.3 \%$ and sociology for $1.9 \%$. Law-oriented programmes concentrate on undergraduate studies, while professional and mid-career training is quite under-developed in this disciplinary area. This may be explained by the fact that Italian civil servants graduate mainly in law and want to develop other kinds of competences.

Finally, if we consider the weight of $\mathrm{PhD}$ programmes as an indicator predicting the development of the disciplinary area, we can see that economics and law are well in the forefront, followed by political science and management.

Law is the predominant disciplinary area (31.1\%), but not the main cluster, which is the public one with $41 \%$. This can be easily explained by considering the high homogeneity of the legal cluster (matching with law) and the high fragmentation of the other two clusters, especially the public one, split into several different disciplines. While undergraduate programmes are prominent in the legal cluster, in the public cluster there is a strong relevance of $\mathrm{PhD}$ programmes, and in the corporate cluster the emphasis is on professional training.

The disciplinary approach adopted in teaching issues such as accounting and administrative science merits a further analysis. We would expect accounting (public, regional or national) to be taught mainly at the faculties of economics and to adopt a managerial approach. However, 70\% of the programmes in accounting are characterised by a legal approach.

Programmes entitled "Scienza dell'amministrazione" include only undergraduate courses and one bachelor's degree at the faculty of political sciences of the University of Palermo. $80 \%$ of the courses pertain to the political science disciplinary area (therefore to the public cluster) and are mainly taught at the faculties of political science. Nonetheless, nearly $20 \%$ of the courses are taught according to the legalistic approach. 


\subsection{Main research themes}

The following tables illustrate the main topics addressed by articles published in the most important Italian academic journal on public management, Azienda Pubblica. 362 articles from 1996 to 2006 and 232 articles from 2007 to 2018 were analysed. The two timeframes represent two different waves of public administration reforms in Italy. These were classified according to the areas of research (table 1), government levels addressed and research strategies.

In the first decade, public administration reform, innovation, planning and control, and accounting systems are the issues that emerge most often in academic/scientific articles (114 articles). There are surprisingly few articles addressing institutional decentralisation and devolution (12), processes which have been of great interest in Italian public administration since 2000. Also infrequent are articles referring to e-government and interdisciplinary issues, with only ten articles in ten years on administrative theories.

Other research issues more in line with the current trends of public management and the progressive evolution towards public governance are emerging: these are human resources management and leadership development, government-citizen relationships, public and institutional communication, public accountability and social/environmental responsibility, inter-institutional networks and strategic management.

Data from the last decade (2007-2018) show the stable presence of accounting research and the emergence of new themes such as performance management, presumably following a major reform approved in 2009. Attention to some topics has sensibly weakened: these include marketing and quality management, privatisation and liberalisation, leadership and human resources management.

The analysis of publications on public management and administration in Italy offers other interesting information on the levels of government researched and the methodology adopted. In the first decade, regional and local administrations were the most analysed organisations (161 articles). The attention given to the local level of government is easily understandable when one considers the higher innovation rate of these administrations. This is the direct effect of greater managerial autonomy, the direct election of mayors and close contact with citizens. Furthermore, the type of activities included in local governmental functions - service delivery as opposed to the policy-making activities characterising ministries and regions - may explain the viability of applying managerial principles (economia aziendale and New Public Management) and techniques. Such greater attention to these levels of government is confirmed in the following decade. New entrants are 
universities, which have been affected by major performance, financial and human resources management reforms. Attention to healthcare organisations has also increased. International comparisons or best practice analysis of foreign public administrations now attracts less attention.

Table 1 Main themes addressed in Azienda Pubblica articles

\begin{tabular}{|c|c|c|}
\hline \multirow[t]{2}{*}{ Theme } & \multicolumn{2}{|c|}{ Number of articles } \\
\hline & $1996-2006$ & $2007-2018$ \\
\hline Accounting* & 40 & 41 \\
\hline Leadership and human resources management & 40 & 6 \\
\hline Planning and managerial controls & 39 & 8 \\
\hline Other issues $* *$ & 36 & 29 \\
\hline Public administration reform and innovation & 35 & 21 \\
\hline $\begin{array}{l}\text { Marketing, quality, customer relationship } \\
\text { management }\end{array}$ & 25 & 4 \\
\hline $\begin{array}{l}\text { Local public services, privatisation, } \\
\text { liberalisation }\end{array}$ & 22 & 1 \\
\hline $\begin{array}{l}\text { Accountability and social, environmental } \\
\text { reporting } * * * *\end{array}$ & 19 & 21 \\
\hline Inter-institutional networks & 15 & 11 \\
\hline Strategic management & 15 & 15 \\
\hline Devolution, decentralisation & 12 & 2 \\
\hline Financial management & 12 & 6 \\
\hline Organisational change & 12 & 3 \\
\hline Performance management & 10 & 35 \\
\hline Administrative theories & 10 & 1 \\
\hline Government-business-non-profit relations & 8 & 9 \\
\hline e-Government & 7 & 13 \\
\hline Public value & 3 & 6 \\
\hline Public procurement & 3 & o \\
\hline
\end{tabular}

* Two special issues on accounting included.

** Special issue on corruption included

*** Special issue on accountability and social reporting included.

Source: Cepiku (2006) for 1996-2006 data; original data for 2007-2018. 
Regarding the levels of government researched, local governments were the most frequent objects of analysis in both periods. Interestingly, attention to foreign best practices has significantly weakened in recent years (from 30 articles in 1996-2006 to only 3 in 2007-2018). While regions were extensively investigated in the first period, universities and healthcare organisations are more often analysed in the second period.

A final feature of Italian research on public management is represented by the predominance of qualitative research in the first decade ( 324 articles out of 362 ). In the second decade there was some improvement, with fewer articles on generic literature reviews and more articles employing methods such as action research and simulations. The case study method remains equally important.

\subsection{Academia-practice relations}

The Public Administration and management field of study is considered to fare better than other academic disciplines in terms of theory-practice relations. This is in part attributed to its multidisciplinary and interdisciplinary nature as well as, as far as the Italian tradition of economia aziendale is concerned, the inductive-deductive research approach.

Two empirical surveys have investigated such a link.

In Cepiku (2011), elite interviewing was undertaken involving 26 senior practitioners and 37 academics; 15 and 23 semi-structured interviews respectively were obtained. The key informants came from all over Italy and the practitioners were from both central and local government.

Is there a rigour-relevance gap in Italian public management? Almost all of the key informants believed there was a significant gap between research and practice in public management in Italy. Just two scholars out of 23 did not see a gap, and one of them said that the relevance of public management research for practitioners was higher than the relevance of private management studies for businesses.

Practitioners were also asked to distinguish between the different types of research they had access to. Most said that a more serious gap existed with reference to research carried out by management consulting firms and Italian universities. Research carried out by professional associations and national/ regional innovation units was also perceived as barely useful.

The practitioners said that the academic research they accessed through international journals was the most useful.

Why is there a gap? Most of the key informants thought that the gap was a result of the papers in academic journals being of little practical relevance 
and also the inadequacy of research methods. This, they thought, was due to the lack of dialogue and movement between the two sectors. As one practitioner stated: "Both worlds are excessively inward-oriented and this discourages collaboration between researchers and public managers, apart from consultancy activities."

Other perceived determinants, especially by academics, include poor accessibility and comprehensibility of scholarly publications to practitioners; the absence of an evidence-based culture among practitioners; and the poor autonomy of public managers from politicians.

Researchers have little knowledge of the public sector context and especially of political-administrative relations. In a senior practitioner's words, "An in-depth acquaintance of management scholars with the real functioning mechanisms in public administrations (political-administrative relations, the characteristics of the public employment etc.) would help raise the credibility of their research and their engagement in reform programmes."

Where is the gap? The key informants were asked to identify the areas in which the gap is most evident. Academics and practitioners were more in agreement on management topics (performance management, financial management, quality and customer relationship management, strategic management, human resources management), and less so on interdisciplinary issues (trust, ethics and integrity, workforce ageing).

Academics were seen to have a strong orientation towards issues firmly rooted in a discipline such as strategic planning, financial management, human resources management and marketing. Very few articles were published on issues that practitioners considered highly relevant - for example network management, public sector workforce ageing, ethics and trust.

The strong preference for the case study method in the academic study of public management, although still regarded as rather low in status by some methodologists, represents a research method that is ideally suited to creating managerially-relevant knowledge (Pollitt, 2008). Evidence at the international level shows that only $53.8 \%$ of public management research is qualitative (Pitts, 2007); this compares to $91.3 \%$ in Italy.

It is worth noting that the ability to conduct quantitative studies in Italy is hampered by the lack of comparable data over different administrations and over extended periods of time. One of the academics interviewed commented, "Lack of empirical evidence is one of the main weaknesses of public management research in Italy. The collaboration between scholars and public managers could contribute to both orient research on more relevant issues and give researchers access to qualitative and quantitative data." 


\subsection{Attitudes towards solutions for bridging the gap}

During the interviews, the attitudes of the two groups to the main approaches suggested by the literature $\left(\mathrm{M}_{2} \mathrm{~K}\right.$ based on a convergence of roles or coproduction based on complementarity) were investigated.

A shared and prevailing preference for co-production - maintaining the distinctive characteristics of the two groups and on a complementarity of roles - was found. Sixteen out of 21 academics and nine out of 11 practitioners thought that coproduction was the solution: "[ $t$ ]erritorial based and institutional partnerships should be established, though safeguarding the necessary autonomy of scientific research." The co-production approach was also considered as something that needed to go beyond treating public managers simply as users of research, or relating to them purely for funding or data gathering.

Furthermore, in the words of one academic, "The involvement of public managers ... serves as a means of empirical testing of the theories...coproduction does not only contribute to create practical knowledge but also to strengthen the rigour of theoretical models." In a similar vein, "co-production helps scholars to develop theories that are grounded in reality."

One practitioner highlighted the need to "institutionalise" partnerships, first of all by acknowledging the time and commitment that involvement in research activities requires.

Several respondents from both groups called for strengthening trust as a fundamental condition for any partnership between researchers and public managers.

Italian practitioners are increasingly becoming interested in pursuing high-level education, but several said they were unhappy with the role played by the National School of Government (SSPA).

In sum, the gap was clearly reported as a "lost before translation" gap and manifested itself in two ways:

- Low relevance of current or published research.

- Insufficient academic research on high relevance issues - academics focus more on rigour.

The problem was found in both cases to be one of knowledge production rather than knowledge transfer.

A more recent survey, conducted by Cepiku, Ferrante and Lovergine (2019), aimed at highlighting the views of Italian public managers on the relevance, use and impact of academic research in their day-to-day jobs. A questionnaire was sent to 561 public managers who had accessed public employment through 
the National School of Government and 211 individuals (37.61\%) replied. Most of the respondents ( $72 \%)$ worked in central public administrations.

Public managers who entered public administrations through the National School of Government exam and subsequent training are considered to be younger, with higher education levels, better knowledge of foreign languages and more international experience compared to the average public manager. They share the same centre-southern geographical origins and legal background, are strong supporters of reforms but often frustrated by a diffused resistance to change and innovation (Mattarella, 2009).

The findings show that public managers access academic research through professional journals (44.1\%) and newspapers (40.3\%). Preferences for different academic disciplines considering their relevance to practice include: law (90\%), management (67.8\%), administrative sciences (55.5\%) and economics (47.9\%).

Almost $87 \%$ of the interviewees highlighted the relevance of academic research in their work, and $96.6 \%$ considered (in total or in part) academic research a source of conceptual frameworks to use currently in their work. 93.5\% of the respondents recognised the contribution of academic research to providing a common language.

$56.3 \%$ of the respondents resorted to academic research more than once a year, while $11.5 \%$ declared they never used it.

$40.8 \%$ of the interviewees considered the results of academic research to be as important as the competencies they possess.

The case study method was considered the most useful for producing relevant results for practitioners $(65.4 \%)$.

$61.6 \%$ of the public managers collaborated with academicians as experts and consultants in policy design, while $38.4 \%$ interacted with them during training and education activities.

In summary, the findings supported the literature stream that views the practice-academia missing link as a problem of knowledge-production rather than one of knowledge-transfer. In fact, more than half of the respondents did not have an access problem, but many fewer used academic research. Moreover, they preferred qualitative methods, as compared with academic disciplines that have become increasingly specialised and quantitative. 


\section{Efforts within the Public Administration community to reflect on the future of the field}

Two important venues have recently hosted a debate on the future of the Public Administration field of studies.

The first was an important event entitled "Lo stato dell'arte della ricerca italiana sulla Pubblica amministrazione in una prospettiva europea" ("The state of the art of Italian research on Public Administration from a European perspective"), which took place in November 2017 and was promoted by the National School of Government in collaboration with the EGPA-IIAS.

It gathered scholars from all academic areas interested in Public Administration including the Italian society of political science (SISP), the Italian academy of management (AIDEA) and the Italian association of management engineering (AIIG). It also gathered disciplinary streams such as public management, law, public policies and sociology of organisations.

The law group included streams different from administrative law such as administrative history and administrative science. Administrative law focused on topics such as public procurement and administrative transparency.

Public management scholars debated themes like the evolution of managerial reforms in different types of public administration in the past four decades and the "New Public Management by law" that features Italian reforms (Ongaro, 2009). Other key issues discussed included performance management, change management by organisation theory scholars and public network management.

The political science approach - organised through academic associations such as the Italian Political Science Association (AISP) and the Italian Association of Evaluation (AIV) - focused on key public policies and on European governance and better regulation.

A fourth disciplinary approach is represented by management engineering, which has been increasingly recognised in Italy, which also hosted the annual 2017 EGPA conference at the Polytechnic of Milan. The focus was on the use of ICTs by public administrations, e-Government and the design and management of public works and infrastructure.

Therefore, the future of academic research builds on four well-balanced pillars, of which three are represented in EGPA permanent strategic groups (Ongaro, 2019). In particular, two permanent strategic groups - i.e. Public network policy and management and Financial Management and public sector accounting - are characterised by a strong coordinating role of Italian scholars. Significant Italian participation is also observed in the groups on Sociology of the State: reform and resilience and on Justice and Court administration. 
Beside disciplinary specificities, some themes are characterised by a strong multi- and interdisciplinary nature. Among these, it is worth mentioning transparency, accountability and government-citizens relations, fighting corruption and the topic of public governance, which is developed through the collaboration of the public management, political science and public policies approaches.

The second event was the eighth Azienda Pubblica workshop, held in Venice in June 2018, on the theme "Managing innovation in the public sector. Theory and practice." The key theme related to the challenges of innovation and change in the public sector. Most of the papers focused on accounting; key issues were accounting harmonisation, accrual accounting and measurement. Public expenditure management and spending reviews were also a recurring topic. Other issues included public value, the need for a renewed élite and public leadership, managerial controls, corruption prevention, coproduction, networks and public-private partnerships.

The main levels of government analysed were regions, hospitals, universities and government-owned enterprises.

\section{Elements of futures of Public Administration}

It is important to mention that Italian academia in general is undergoing a severe brain drain due to the cuts in research funding. For instance, researchers of Italian nationality are highly competitive in winning ERC projects (second only to German scholars), but rarely choose to develop their research in Italian institutions. The latter also fail to attract foreign researchers. The data of the past ten years are dramatic: university students have decreased by $20 \%$, professors and administrative staff by $18 \%$, and overall resources (already below the EU average) by $21 \%$ (less 6.6 billion euros from the state). In ten years, $\mathrm{PhD}$ positions have been reduced by $40 \%$ : in Italy there are $0.5 \mathrm{PhD}$ students for every 1,000 inhabitants compared to 1.7 in the United Kingdom and 2.5 in Germany.

These data explain the decreasing interest of Italian scholars, including those working on public administration, in national journals and, more generally, research published in Italian.

As regards national publication outlets, the main national academic journal remains Azienda Pubblica, founded in 1988 by a group of scholars from the Bocconi University. It hosts mainly public management research, but also research from other approaches as well as from practitioners. Administrative law outlets include Amministrare, Giornale di Diritto Amministrativo, 
Rivista di Diritto amministrativo and the Rivista trimestrale di Scienza della Amministrazione.

The attractiveness of national academic journals was discussed in September 2018 in Verona in a round table entitled "The future of national scientific journals: addressing the internationalisation challenge," organised by the Italian Society of Accounting and Business Administration Scholars (SIDREA). The driver for internationalisation has been emphasised by the classification of academic journals by the National Agency for the Evaluation of University and Research and by the AIDEA. Both are strongly inspired by the Anglo-Saxon model of A-ranking journals. As far as management journals - including public management - are concerned, all journals are in the English language. Thus, Italian scholars have no incentive to publish in Italian. Moreover, the availability of A-ranking journals that have Public Administration as part of their aims and scope is quite limited compared to other disciplines which are part of the economic sciences area.

A paradoxical effect has been that the choice of research topics by Italian public management scholars is often based on the expectation of what could more probably be published in an A-ranking journal. In other terms, supply guides demand (Mussari, 2018).

As a consequence of the brain drain and of the increased national competition, the presence of Italian scholars in international journals, books and conferences has grown steadily, hand in hand with the number of expatriates in the United Kingdom, France, Austria, Sweden, Switzerland, Germany and Spain.

The international contamination of research topics has resulted in more comparative research and in the introduction of new themes such as, for example, public service motivation.

A weak international influence is exercised by Italian administrative law scholars on the administrative law schools in Latin America (Brazil and Argentina), and inside networks such as the IIAS and the Centro Latinoamericano de Administración para el Desarrollo (CLAD).

A third internationalisation trend is supported by the EU fund on interregional collaboration (Interreg) but also by the Instrument for Pre-Accession Assistance (IPA) and the European Neighbourhood and Partnership Instrument (ENPI), which contribute to comparisons and debate between Italian, Swiss, Austrian, French and, to a minor extent, Tunisian and Slovenian scholars on topics of public governance and management.

A final consideration refers to the education role of the Public Administration academic community. The low attractiveness of the public employee, due to the turn-over freeze and the public opinion perception of civil servants, 
makes undergraduate programmes struggle with a low number of students. The reverse is true of postgraduate and professional training programmes in which public management disciplines thrive.

In sum, some resilience can be observed: the brain drain, along with academic career reform, has led to greater internationalisation; the disciplinary fragmentation is slowly evolving towards multidisciplinary efforts; disconnected specialised research triggers research on grand questions such as sustainable development goals, digital government, non-profit-making management and social innovation.

\section{References}

Borgonovi, E. (1992). Continuity and Renewal in the Study of Public Administration. Economia aziendale 6(5): 407-22.

Cassese, S. (1983). Il sistema amministrativo italiano / The Italian administrative system. Bologna: Il Mulino.

Cepiku, D. (2013). Embracing disciplinary diversity: public administration education in Italy. Croatian Comparative Public Administration/Hrvatska i komparativna javna uprava: časopis za teoriju i praksu javne uprave 13(1): 71-104.

Cepiku, D. (2017). Report on Public Administration Reform Trends and Reform Dynamics in Italy. Support for developing better country knowledge on public administration and institutional capacity building (EUPACK). Retrieved from https://ec.europa.eu/social/Bl obServlet?docId=19208\&langId=en.

Cepiku, D., Ferrante, A., \& Lovergine, S. (2019), Dirigenti pubblici e ricerca accademica: rilevanza, uso e impatto/Public managers views on the relevance, use and impact of academic research. Forthcoming in Azienda Pubblica.

Cepiku, D. (2011). Two ships passing in the night? Practice and academia in public management. Public Money \& Management 31(2): 131-138.

Cotta, M. (1996). Political Science in Italy. In J.-L. Quermonne (ed.). Political Science in Europe: Education, Co-operation, Prospects: Report on the State of the Discipline in Europe. Paris: Thematic Network Political Science.

Kickert, W.J. M. (2005). Distinctiveness in the study of public management in Europe: a historicalinstitutional analysis of France, Germany and Italy. Public Management Review 7(4): 537-563. doi:10.1080/14719030500362470.

Kickert, W. J. M. (2007). The Study of Public Management in Europe and the US: A Competitive Analysis of National Distinctiveness. London and New York: Routledge/Taylor and Francis. doi:10.4324/9780203936177. 
Mattarella, B. G. (2009). La dirigenza dello Stato e il ruolo della Scuola superiore della pubblica amministrazione/State leadership and the role of the national school of government, Roma: Scuola Superiore della Pubblica Administrazione.

Meneguzzo, M. (2007). The study of public management in Italy. Management and the dominance of public law. In W.J. M. Kickert (ed.). The study of public management in Europe and the US: a competitive analysis of national distinctiveness. London: Routledge. 167-188.

Mussari, R. (2018). Editoriale. Azienda Pubblica 1: 7-12.

Ongaro, E. (2009). Public management reform and modernisation: Trajectories of administrative change in Italy, France, Greece, Portugal and Spain. Cheltenham: Edward Elgar.

Ongaro, E. (2019). Public Administration in Europe. Governance and Public Management. Basingstoke: Palgrave Macmillan. https://doi.org/10.1007/978-3-319-92856-2.

Pollitt, C. (2008). Public management from an international perspective. Speech on the occasion of the 10th anniversary of the KULeuven Public Management Institute. http://citeseerx.ist. psu.edu/viewdoc/download?doi=10.1.1.619.922\&rep=rep1\&type=pdf.

Sotiropoulos, D. A. (2004). Southern European public bureaucracies in comparative perspective. West European Politics 27(3): 405-422. doi:10.1080/0140238042000228077. 


\section{6}

\section{Public Administration in the Netherlands: State of the Field}

Philip Marcel Karré, Martijn van der Steen, Zeger van der Wal, and Thomas Schillemans

\section{Introduction}

This chapter looks at the state of the field of public administration in the Netherlands. We paint a picture of the current state of Dutch public administration, and discuss where it might go from here. We do so based on our book on these questions (Karré, Schillemans, Van der Steen \& Van der Wal, 2017) which developed from a series of articles published in Bestuurskunde, the Dutch journal of public administration. In this series, each individual author approached the state and future of the field of public administration in a personal and rather unique way. Martijn van der Steen organised a survey among public administration professionals, following a discussion on national television about the societal benefit (or, better, lack thereof) of social sciences. Zeger van der Wal analysed the challenges public managers face in the twenty-first century and developed ways of dealing with them. And Thomas Schillemans developed a road map for where our field of study should go in the future. In the following paragraphs we present short summaries of each of these contributions. In order to put them into context, we start with a short elaboration on the state of the field of public administration by Philip Marcel Karré.

\section{State of the field: public administration between academia and practice (Philip Marcel Karré)}

Public administration has left its mark on Dutch society: public administration scholars play an important role in consulting government and public organisations. Many public managers, as well as ministers of the current and former governments, are alumni of public administration programmes. 
Dutch public administration scholars are highly productive and have a high standing in the international scientific community.

But there are also those who doubt public administration's scientific character, mainly due to the field's close links with administrative practice. An example of this are the words uttered by Jacques van Doorn, former professor of sociology and the very first dean of the faculty of social sciences at Erasmus University Rotterdam, when he learned that the university was planning to introduce public administration to his faculty in the 1980 s. Van Doorn experienced this as a personal defeat and left in disgust. To him, public administration was "purely practical, with some theoretical concepts thrown in here and there. A great field of study, for sure, but it does not belong at university" (Dicke, 2003, p. 25).

This prejudice is nothing new. Gerrit van Poelje, a lawyer and civil servant, who became the first professor of public administration, had to deal with similar sentiments. In 1928, Van Poelje accepted a position at the institution that later developed into Erasmus University Rotterdam. However, public administration did not yet exist as an independent field of study. Van Poelje's position was at an institution which, at that time, mainly focussed on promoting commerce at the behest of Rotterdam's business tycoons. Knowledge about public administration was seen as an essential tool in this, but not as a goal or valuable field of study in itself. Hence Van Poelje was not given a full professorship with the corresponding institutional and societal status. $\mathrm{He}$ left after five years to work once again as a civil servant. Yet, Van Poelje had managed to establish the field of public administration in the Netherlands. The prize awarded by the Dutch Association of Public Administration (Vereniging voor Bestuurskunde) still bears his name. His spirit and ideas are still felt to this day, e.g. his call that public administration should be an applied science in close connection with administrative practice.

As an independent and institutionalised field of study, public administration has a history of only about 45 years in the Netherlands. In 1974, Vereniging voor Bestuurskunde was established, and in 1976 the very first stand-alone educational programme at the University of Twente. Since then, public administration has seen a steady rise, growing and further developing as an academic field in sync with the development of the Dutch welfare state and the subsequent discussions about its reform, and the ever shifting relationship between state, market and society as ways of dealing with public issues. It is no longer merely seen as an important tool to foster business but as a grown-up and fully developed field of study that can help us make sense of a complex, uncertain and highly dynamic world.

There are now courses and educational programmes in public administration, both at research universities as well as at universities of applied sciences. 
Civil servants and other public sector professionals can also choose from several post-experience programmes. Research in public administration is still closely linked to administrative practice, as demanded by Van Poelje, but is also undergoing a process of "scientification" with the development and application of more rigorous research strategies and methods. For some, this is an important step to develop public administration into a "real" science instead of mere "consultancy," while others fear that straying away from practice will make our discipline become irrelevant for tackling real-life social problems. This discussion is not unique to public administration in the Netherlands (political scientists and sociologists are debating the very same issues) but is perhaps indicative of a discipline that is still developing and, after 40 years, sees itself at a kind of crossroads where new choices have to be made between societal relevance and methodological rigour.

In the aforementioned series in Bestuurskunde, Gadellaa, Curry and Van der Walle (2015) and Braun, Fenger, 't Hart, Van der Veer and Verheij (2015) describe how the field of public administration in the Netherlands presents itself today. The modern founding fathers, who helped to establish public administration as an independent field of study in the 1970s, i.e. Roel in ' $t$ Veld and Henk Brasz, were of the same opinion as Van Poelje that it should be science for policy. This was an odd choice at a time when the world of science was steeped in activism and serious, yet often also rather pompous, debates were raging on the role of science in criticising and changing society's status $q u .^{2}$ The modern founding fathers of the field of public administration in the Netherlands saw it as their mission to support the professional development of administrative practice. In order to achieve this, it was deemed necessary to establish a close and pragmatic relationship.

In later years, the field of public administration in the Netherlands has distanced itself somewhat from administrative practice amidst discussions that a more critical approach was needed. This process was accelerated by a further "scientification" and internationalisation of the field. As the field of public administration grew up, it sought closer connections with its peers abroad. Nowadays, the overall majority of research in Dutch public administration is published in English and scholars aim to publish in international journals, which are often not read by practitioners. This leads to the paradoxical situation that while public administration scholars mostly do empirical research, its results do not naturally reach those who could use it most. By doing so, we seem to be wandering off the road set out by Van Poelje with his focus on integrating science and practice.

Dutch public administration, just like its counterparts in other countries, is a multidisciplinary field based on insights from a diverse range of fields, e.g. 
law, economics, sociology, political sciences, psychology and communication sciences. Just like scholars of historical science, who use several auxiliary sciences (e.g. epigraphy, genealogy and heraldry) to help them in their research, scholars of public administration avail themselves of insights from these other disciplines to understand what is happening during collective efforts to tackle public issues. Public administration is understood to be more than just the sum of these other academic disciplines though. It is seen as an independent field of research and not as part of, let us say, management sciences. A majority of scholars in the field have a degree in public administration, a survey found on which Gadellaa, Curry and Van der Walle based their article. They do not come from other disciplines but often use insights from those disciplines in their research.

In order to establish how this research has developed over recent years, I have undertaken a small comparison of the content and methodological approach used in the $\mathrm{PhD}$ theses that won the prestigious Van Poelje prize awarded every year by the Vereniging voor Bestuurskunde for the best dissertation in the field of public administration (Karré, 2017). I found indicative for developments in the field as a whole, that four distinct yet interconnected developments had taken place, which he refers to as reticulation, refinement, dilation and diversity:

Reticulation refers to the fact that while public administration in the Netherlands always has been a multidisciplinary field of study, researchers now go far beyond the traditional auxiliary disciplines in order to enrich our understanding of how public issues can be dealt with. Behavioural economics in particular is now often used, but Dutch public administration scholars also do not shy away from writing philosophical monographs.

A second trend concerns a refinement of theories and methods used. In general, public administration research conducted in the Netherlands always was of high quality, but we now see an increased number of $\mathrm{PhD}$ theses which score excellent on all quality aspects of the Van Poelje prize.

Dilation refers to the development that scholars from other disciplines increasingly discover the field of public administration and help to develop it further by adding insights from their own disciplines, often research using mixed methods. Also, public administration is no longer "only" the study of government but also of governance, adopting a broader definition of the field than its original founding fathers' focus on science for policy. Public administration now is more science for society, though this new focus comes with its own challenges (see the following section).

Diversity, finally, refers to the variety of research that can be found in the field of public administration in the Netherlands today. There are philosophical monographs and historical analyses, next to quantitative and qualitative 
studies. What is evident is that public administration scholars in the Netherlands do not adhere to one single philosophy or school of thought, but are as diverse in their work as the social reality they study in their research.

\section{What Dutch public administration professionals say about their field (Martijn van der Steen)}

In a popular news programme in the Netherlands there was recently a discussion about how government policy could help migrants find jobs. One of the guests on the programme was an academic scholar, who was asked by the anchor of the show to explain to viewers how government policy might help. The scholar started by explaining how complex the issue was, and stressed the "wickedness" of the problem. He was asked if the government was aware of the size of the problem and the urgency of the need to act; he sighed deeply and explained that it was actually not all that clear what the size of the problem really was and that there was much debate about the actual urgency of the issue. In fact, he continued, it was not really clear what the problem was exactly, and if it was really a problem. The anchor of the show looked at the academic for a while and asked him, with a troubled look, "What good are you to us?"

It was not the anchor's intent to offend his academic guest. He was merely expressing that the intention in inviting an academic was to shed light on the issue and help the audience understand it better. What he got in return was an academic who did a good job in explaining that it was all much more complicated than that and the issue itself was not clear at all. "Still confused, but at a higher level," but that was not the intention of the show.

At the same time this exchange is recognisable for many public administration scholars and professionals. One of the features of the discipline is that it explains that issues are often more complicated, that easy solutions do not exist, and that clear and simple definitions often cover only part of the problem. That draws attention to the news anchor's question; what good is public administration anyway?

We thought that it would be interesting to ask public administration professionals - academics and practitioners - how they would answer that question. Not necessarily to investigate whether or not the discipline is useful - we are quite sure it is - but to learn more about what professionals themselves see as the heart and use of the discipline. We selected an audience of over 80 active contributors to a popular Public Administration Blog website. Some contributors are academics, others are practitioners. We asked them to answer the question of the "use" of public administration in four different contexts; 
- The context of a popular television programme; what is the use of public administration for the broader public?

- The context of a conversation with a Director-General of a Ministry, about an important policy issue; what is the use of public administration in the context of a practitioner who is desperate for a solution, or even a quick fix?

- The context of a reflection in private; what does public administration mean for me personally; what is the use of it for me, or what is my "use" as public administration professional?

- The context of the future; what is the future of public administration and can it remain useful?

We will briefly discuss the patterns in the answers here.

\subsection{A public administration professional in a television programme}

The respondents stress that there is great value in the ability to show the complexity of issues. Most respondents feel that public administration can provide a broad public with a good understanding of the issue; not by providing them with clear-cut black and white explanations, but by showing the different shades of grey and the various sides of the issue. They also feel that a good public administration professional is able to do that in a way that is understandable for a broader audience.

Respondents argue that public administration contributes to society in an "indirect way"; public administration professionals help by helping government to govern society better. It is good for society that public administration reflects on what government does. Good government is a crucial enabler of a strong society, and good public administration is an enabler of good government. That is why most respondents think that public administration is highly relevant and "present" in the daily lives of ordinary people. Because the consequences of government intervention are all around them, the consequences of public administration are too.

Most respondents also agree that all this is and will remain hard to explain in the context of a fast-paced news programme. Some even argue that perhaps public administration scholars should avoid such contexts; they do not think that the kinds of theories and knowledge that public administration produces are suitable for 30 -second soundbites. However, most respondents say that they consider the understanding of the ambiguity and complexity of issues to be the heart of public administration, and that they should at least try to make that case to the broader public. 


\subsection{A public administration professional at the Ministry}

Most respondents find it much easier to explain the use of public administration insights to the Director General. Again, they all choose to stress the complexity of the issue and stress the absence of quick-fixes; they argue that they are doing the DG a favour by explaining how "impossible" the proposed solution is.

In fact, most respondents feel that this is really a crucial matter for the field; public administration should always keep its autonomous position when offering advice to government. That is a core value for all respondents; even though the DG will probably reward a clear answer and a seemingly "ready to use" solution, public administration professionals should never give up their professional autonomy.

Respondents also quite agree about the nature of their advice. They would probably start by looking for the deeper underlying public value(s) behind the perceived problem; they all also stress the importance of extending the number of stakeholders and look at patterns in the dynamics between actors. Respondents all stress that they would probably not advise on the "content" of the solution, but rather lay out a process that allows a solution to emerge. Public administration also provides insights into how such processes work, how they can be managed, and offers very practical tools and instruments for doing so. In order to help, respondents argue, public administration first makes the issues more complex, and then helps to navigate the complexity.

\subsection{A public administration professional at home}

In this question, respondents take a more personal approach; what is their personal drive to be active in the field of public administration? Many respondents see public administration as a mission, not just as a profession. They want to contribute to society and feel that their work as public administration professionals is the way to do it.

What is very interesting in the respondents' answers is that they make a clear distinction between the political and a more technical way to contribute society. They do not have a political agenda, but want to help the government to govern better; almost no matter what the exact political direction of the government is. To them, public administration is not a political project, but a technical one to help democratically elected politicians govern and protect the rule of law.

At the same time, this is exactly the main critique of several others; they argue that public administration has become overly technical and has lost 
its activism and its political commitment. This is becoming a more urgent issue with the rise of populist and at times outspoken "anti-government" political parties.

\subsection{Public administration professionals about the future}

Respondents are positive about the future of public administration, but they see important challenges ahead. They are worried that government cannot keep pace with societal dynamics. They are worried about the adaptive capacity of government and see that as an important issue for public administration.

Respondents stress that public administration should be careful not to lose itself in hypes. For example, bottom-up networks and self-organisation are "real" and deserve scholarly and practical attention, but they should also be reviewed critically and in context. Respondents also see the importance of rebalancing traditional values of good governance and the rule of law with the dynamics of networks, new technologies and situational logics in the approach to problems. Respondents see it as their mission to find new ways to balance these different values.

\section{Public administration and the public manager of the twenty-first century (Zeger van der Wal)}

The operating environment of the public servants and organisations we study, teach and consult for is dynamic and changes constantly. ${ }^{1} \mathrm{~A}$ popular saying these days is that our objects of study increasingly operate in a "VUCA world" (Johansen, 2007), characterised by volatility, uncertainty, complexity and ambiguity. These are the working conditions that public administrations in The Netherlands very much recognise as the everyday context of their work; and one that is becoming ever more important to them.

Clearly, this operating environment poses various challenges for public administration professionals; it is the context for the public administration of the (very near) future. However, emerging developments also provide exciting opportunities for achieving unprecedented levels of public service excellence, together with citizens and vanguards of change from other sectors. In order to turn challenges into opportunities, twenty-first-century public servants need to acquire and display a variety of skillsets and mindsets, all of which affect our field as well in terms of the research and teaching we conduct, and how that will continue to be perceived as relevant. 
At the same time, the nature of what makes public servants and organisations "public" implies that changes and reforms may by definition be less radical and drastic than in corporate environments. Indeed, despite decades of discussing new types of public management and public managers, one key aspect will always differentiate them from business managers. This key aspect is their additional onus of upholding public values and interests, and safeguarding institutional integrity without overstepping the politician's comfort zone.

Moreover, much of the discourse on private sector-oriented and crosssectoral network management seems to ignore how legal and constitutional responsibilities and mandates of public servants have remained in place. Indeed, many of the responsibilities and qualities of public servants are institutional rather than transformational.

Still, in recent years, a dazzling number of recent scholarly articles and books as well as consultancy reports and government documents have discussed the future public sector workforce. According to such writings, "new style" public servants should be entrepreneurial and locally minded, display interpersonal skills and commercial savvy, master collaboration and communication, and lead and manage change, deliver projects and programmes, redesign services and deliver them digitally. They should have the ability to operate in increasingly cross-sectoral, international and co-producing networks in which citizens manage alongside public servants rather than being managed by them.

Others suggest that in an era of networked governance public managers should retreat from business-like skills and approaches to return to six classical qualities or administrative "crafts." The six crafts he puts forward are counselling, stewardship, prudence, judgment, diplomacy, and political nous, referred to by others as political savvy, political antennas or "political astuteness."

Dutch colleagues of the Netherlands School of Public Administration (NSOB) portray this hybrid reality of public servants as "sedimentation" to indicate that effective public servants combine the various repertoires corresponding to the three key paradigms in our field - Traditional, 'Weberian' Public Administration, New Public Management and New Public Governance that emphasises horizontal collaboration - in complementary ways. In their prioritisation, such public managers are mindful of when repertoires come into or out of fashion, depending on context, key events and the government of the day. As an example, in the aftermath of the global financial crisis in 2008, many governments re-emphasised the importance of a "strong state" 
while pointing to the shortcomings of markets, with some even suggesting undoing prior privatisations.

Various authors have addressed the "multiplicity" and "ambiguity" of public sector settings before. However, the magnitude and interrelatedness of twenty-first-century trends and drivers mean that new demands and dilemmas will be:

- The norm rather than the exception;

- Mutually reinforcing and exacerbating (with decreasing predictability);

- Affecting all types of public managers rather than just those at the very top; and

- Affecting the nature and practice and not just the content of (public administration) work.

Box 1 lists a number of commonly observed consequences and challenges produced by global megatrends and what they mean for the study of public administration.

\section{Box 1 Global megatrends: consequences for public actors and educational programmes}

1. Stakeholder dynamics, a multiplication of - more ambiguous - interests that must be taken into consideration;

2. Collaborative modes of working in co-producing stakeholder networks requiring power sharing, use of new media and open innovation;

3. Power shifts away from traditional to new authorities, and more frequent and sudden authority shifts from one leader or constituency to another;

4. Increased legitimacy and performance requirements towards an increasingly assertive, savvy and scrutinising array of stakeholder networks

5. New working practices, the emergence of new types of work, working and workers due to technological revolutions, changing attitudes towards work and new generations of employees;

6. Pressures for smarter organising and budgeting due to scarcity of talent and natural resources and the use of advanced technology in an era of low growth and austerity;

7. Ethnicisation, a demand for the highest ethical standards from organisations and their leaders.

Do we need drastically to reform MPA, MPP, MPM and Executive Education programmes, or is the way in which they currently take shape sufficient for producing twenty-first-century skills, competences and values? In my view, we should aim to 
further update and upgrade existing frames, tools and assumptions as times progress, and make the learning we offer more experiential and experimental.

For instance, the readings and frameworks we use all propagate "collaboration" but do we actually teach future managers how to collaborate, beyond the mandated group work so dreaded by most students? Similarly, do we optimally utilise opportunities for students to mix with their future counterparts from other sectors, by offering exciting combined modules, projects and internships with programmes in business administration, law, social work, economics, computer science, marketing and engineering?

Do we pay sufficient attention to "skills" in general - sometimes looked down upon in academic environments - let alone specific new skills such as designing social media campaigns, video editing, prototyping policies and services derived through open innovation, and big data analytics? No school or programme can do everything, but given the rapidly changing environment of public managers and of higher education itself, public management education also needs to become twentyfirst century proof.

What are the key scholarly implications of this dynamic and constantly changing operating environment, in terms of what we should teach and research?

Comparative global studies of senior civil servant training note that expectations towards senior public servants have risen in recent years. Public sectors are increasingly concerned with formulating key competences and designing various types of training. A degree from an elite institution is no longer sufficient for one to rise through the ranks as it was one or two decades ago. To become twenty-first-century public servants, employees need to pursue exchanges with other organisations, sectors, and networks, and upgrade both generic and specific skills and competences based on frequent, critical assessments. To meet such dynamic lifelong learning demands, public management education has to evolve as suggested in Box 1.

Indeed, while training is important, experience is king. Inspired by such studies, I suggest that aspiring twenty-first-century public servants take the following into account:

1. The amount of time spent in the field or in a specific agency remains key (with the average time spent in the same function, role or agency likely continuously to decrease);

2. While experience may be a good teacher in itself, this is not so much the case in dysfunctional systems, creating serious issues for HR managers in such systems; 
3. (Reverse-)mentoring provides hands-on opportunities to experience how systems operate, and to identify skills gaps and training and development needs of individuals and teams;

4. Rotational opportunities and experiences - including (overseas) study trips, "secondments" to the political, private sector or non-profit domain, and participation in peer networks and long-term experiential training programmes - all widen the views of (aspiring) public managers, challenge current assumptions and provide exposure to potential collaborators, competitors or adversaries in other sectors and countries;

5. Critical, transparent and high-quality feedback and appraisal systems that combine qualitative and quantitative assessment, and include individual and collective exercises and indicators, produce more competent and conscious managers.

To conclude, relevant research into twenty-first-century public professionals and organisations is likely to have the following features:

1. It is interdisciplinary and multidisciplinary, and much like in the early days of the field constantly aiming for links with other relevant fields (some of which, however, were considered much less relevant in the early days of the field), such as management studies, computer science, media studies, environmental sciences, marketing, design studies and behavioural psychology and economics;

2. It happens "on the ground," with critical distance and rigour but at the same time being closely related to administrative and managerial practices that matter, and in doing so creating meaningful feedback loops that produce new questions for academic research while improving and inspiring practice at the same time; and

3. It is international and comparative in nature, as there is still much to gain from gaining insight into how actors in other, sometimes politically and historically very different regions and jurisdictions, and despite significant progress in the internationalisation of our field in recent years (including the Anglo-Saxon top journals), much research is still fairly parochial and both academia and practice would benefit from more comparative insights into how public actors in vastly different contexts deal with the rather universal trends and challenges discussed in this chapter. 


\section{Public administration in The Netherlands: where to go from here? (Thomas Schillemans)}

Now, where does Dutch public administration stand? To answer this question, we rely on Burawoy's (2005) analysis of the state of sociological research and apply his analytical perspective to Dutch public administration.

In his paper, Burawoy distinguishes two crucial dimensions with relevance for any social scientific discipline. The first distinction refers to the type of research that is developed. The work of many scholars can be understood as instrumental, positive science, where scholars build on each other's work and aim to make progress within generally accepted theoretical, conceptual and empirical parameters. Instrumental, positive science aims to push the known frontiers further. Others engage in more reflective, critical or dialogical research, in which common assumptions, theoretical foundations and popular myths are addressed. In an almost Kuhnian sense, this type of research challenges the status quo in the field in a disruptive way. This distinction between instrumental and reflective knowledge aligns with Argyris and Schön's (1978) distinction between single loop (aka instrumental, positive science) and double loop (aka reflective science) learning.

The second distinction refers to the type of audience a discipline addresses with its publications and other types of output. On the one hand, some scholars principally relate to and write for others in the discipline and engage with problems identified and defined by other scholars. Some others, however, focus on other publics such as political leaders, civil servants, other practitioners in the field or the general public. In this approach, scholars engage with and focus on the problems identified as salient, and the understanding of those problems by people outside the disciplinary field.

If we combine these two distinctions, a neat $2^{*} 2$ emerges with four versions of public administration, as is visualised in the table below: Academic Public Administration, Applied Public Administration, Reflective Public Administration, Public Administration for the Public. 
Table 1 Four Public Administrations

Type of audience

\begin{tabular}{|c|c|c|c|}
\hline & \\
\hline & & Internal & External \\
\hline \multirow[b]{2}{*}{$\begin{array}{l}\text { Type of } \\
\text { knowledge }\end{array}$} & Instrumental & $\begin{array}{l}\text { Academic Public Administra- } \\
\text { tion: Instrumental, positive } \\
\text { social science }\end{array}$ & $\begin{array}{l}\text { Applied Public Administra- } \\
\text { tion: Research for policy } \\
\text { practices }\end{array}$ \\
\hline & Reflective & $\begin{array}{l}\text { Reflective Public Administra- } \\
\text { tion: Challenging assump- } \\
\text { tions, foundations and myths } \\
\text { in the field }\end{array}$ & $\begin{array}{l}\text { Public administration for the } \\
\text { public: Engaging with the } \\
\text { public and public debate }\end{array}$ \\
\hline
\end{tabular}

\subsection{Academic Public Administration: a forceful field moving further}

In comparative perspective, Dutch public administration is relatively institutionalised and "forceful," as it was described more than ten years ago (Noordegraaf et al., 2006). Dutch public administration has a relatively long history: Van Poelje was appointed the first professor in public administration as early as in 1928 and the first academic journal in Dutch is more than 70 years old (Reussing, 2016). Now, almost all Dutch universities have public administration (sub-) departments and various teaching programmes. The number of students studying public administration is also relatively high, which is in turn reflected in the fact that twice as many Dutch PA scholars studied public administration themselves, compared to their colleagues in other European countries (Gaadellaa et al., 2015).

Dutch public administration is also quite visible and strong in the international academic field. Two thirds of the EGPA study groups for instance have co-chairs affiliated to Dutch universities. Five Dutch public administration departments are ranked among the best 25 PA departments according to the Shanghai ranking of 2018. And if one browses through the tables of contents of the various top journals, many publications from Dutch universities are featured.

In the past decade or so since the Noordegraaf et al. (2006) publication in the field, Dutch public administration has more forcefully advanced in this top left corner of the disciplinary $2^{*} 2$. Dutch public administration has been going through a process of academisation (Braun et al., 2015) of which the scholars in the field are acutely aware. There have been various debates, surveys and interview studies in recent years on the state of the field in which 
this deepening of academic public administration always surfaces as the main issue (Karré et al., 2017).

The sunny side of this academisation process is the increased methodological quality and rigour of the field in combination with a more truly international theoretical approach. The evolution of $\mathrm{PhD}$ theses over the years for instance clearly demonstrates that (younger) scholars engaging in technically more advanced research are writing almost exclusively in English and oriented towards the international (top) journals. Dutch scholars play important roles in advancing sub-fields characterised by methodologically demanding research, such as behavioural public administration and public sector HRM and personnel. This contributes to academic research in a field which is theoretically more integrated than ever and is methodologically almost uniquely diverse (Hood, 2011).

At the same time, however, there is some reason for concern. Does the academisation process not come at the expense of the relevance of this research? And is the focus on top publications and glamorous research grants not distracting from important critical reflection and engagement with the wider public? In short, is the expansive academic public administration not straddling the other three forms of scholarship?

\subsection{Applied Public Administration: awareness of relevance}

As mentioned, in surveys of public administration scholars and debates and interviews, the tensions between the rigour of academic public administration and the relevance of research for the real world of public administration always surface. The founding fathers of the discipline such as Van Poelje in the Netherlands and Wilson in the US already stressed the "applied" nature of the field (Gadellaa et al., 2015) and the importance of "discovering" what governments can do and how they can do this efficiently (Wilson, 1887). The advancements in academic research are now sometimes seen to threaten the focus on practical and applied knowledge for policy-makers. A survey amongst public administration scholar for instance found that Dutch scholars were less focused on applied research and policy practice than their colleagues in other European countries (Gadellaa et al., 2015). These signs suggest that the relevance of the "technically" expanding public administration community may be at issue.

But looks can be misleading. If one compares the numbers of students, staff, universities where public administration is studied and relationships to policy-makers to the scan made more than ten years ago (Noordegraaf et al., 2006), there are no signs of decline; even the opposite. One important trend 
is the further disciplinary collaboration with other disciplines and also with representatives from policy practice. This also implies a shift towards more societally defined challenges and issues in various universities. There is also a rich body of applied public administration research. This was noted by the research review of Dutch public administration research in 2014:

"Dutch researchers continue to undertake research in an engaged way, working with a range of stakeholders to define problems and develop solutions. The societal impact of the research (...) spanned local, regional, national and international spheres. Policy-makers, public managers, professionals and citizens benefit from the public value created with and through the research." (Hartley et al., 2014: 5).

So far, thus, the advancements of academic public administration do raise concerns and critical thoughts about the relevance of applied public administration research, yet "on the ground" there are few real indications of loss. We would contend that this widespread mental awareness of the relevance of relevance is a good sign and is probably really helpful in safeguarding this constitutional value of the field. However, other constitutive values may also be challenged, and this suggests that there is more reason for concern related to the reflective and public character of public administration.

\subsection{Reflective and public: where do we stand?}

From the early days of the discipline, public administration has aimed to separate politics from administration, leaving the former to others while focusing on the latter. The contention was that political decisions need to be made in the democratic arena and that the true task of public administration research and practice is then to "discover" how such decisions can be translated effectively and efficiently into policies. Leaving the criticism of the politics-administration divide aside, it is important to note that this disciplinary self-understanding is based on at least two premises. First of all the premise that policy decisions are democratic, constitutional and legally sound, and need no further normative consideration by the public administration scholar herself. Once the dust settles on the political dispute and a decision has been taken, the public administration can go about its business and deliver that policy in a neutral way. Secondly the premise was that it is accepted and expected by politicians and the wider audience that these politically defined tasks are enacted by public administrations, thus, by bureaucrats and governmental organisations. 
But times are changing, and so is the academic field of public administration itself. The internationalisation of academic research takes insights and research from Dutch (and other) public administration scholars to countries where the decision-making is not democratic at all. The rise of autocratic politicians challenges democratic and constitutional principles in established democracies. The rise of populism challenges the in principle technocratic ideal of neutral policy implementation by public administration experts and bureaucrats. And in the heated public discussions these days on politics and policies, the capacity of governments effectively to address issues is strongly questioned. The current practices in public administration may be at odds with popular perceptions of governance (Stoker, 2019).

The foundational premises of the field thus seem to be affected by internal and external developments, yet, at least in the Dutch public administration, the field is relatively quiet in addressing this in academic and public settings as most of the energies are spent on the nexus of rigorous and relevant research. There are relatively few reflective or public-public administration scholars. While in the past, scholars like Michel Foucault and James C. Scott have forced public administration to reconsider its foundations; it would seem relevant in the current politically turbulent times of globalisation to reflect more self-critically on the foundations of the field. Simultaneously, as Burawoy (2005) pleaded for a public sociology engaging with the social issues identified by the public, and as Flinders (2012) rose to the defence of politics, a truly public version of public administration is still to be developed. At least in the Netherlands, the relatively forceful field of public administration scholars has been relatively quiet in the public arena.

\section{Notes}

1. Parts of this chapter are edited and updated paragraphs from Van der Wal, Z (2017). The $21^{\text {st }}$ Century Public Manager. London: Macmillan.

2. With the Daudt-affair in Amsterdam in 1969 as its low point, when Marxist students managed to practically oust a professor, Hans Daudt, from the university because he did not fit their idea of an academic joining activists on the barricades fighting against the capitalist exploitation of the working man - Daudt rather thought it to be important to be able to assess critically what was going on, instead of becoming immersed in activism of one sort or another. 


\section{References}

Argyris, C., Schön, D. A. (1978). Organisational learning. A theory of action perspective. Reading, MA: Addison-Wesley.

Braun, C., Fenger, M., 't Hart, P., van der Veer, J., \& Verheij, T. (2015). Quo vadis, Nederlandse Bestuurskunde?. Bestuurskunde 24(4): 82-92.

Burawoy, M. (2005). For public sociology. American Sociological Review 70(1): 4-28.

Dicke, M. (2003). 40 jaar FSW: De Rotterdamse formule in de praktijk. Rotterdam: Stad en Bedrijf/ Erasmus Universiteit Rotterdam.

Flinders, M. (2012). Defending politics: Why democracy matters in the 21st century. Oxford: Oxford University Press.

Gadellaa, S., Curry, D., \& van de Walle, S. (2015). Hoe bestuurskundig is de bestuurskunde? Bestuurskunde 24(3): 67-79.

Hartley, J., et al. (2014), Research Review Public Administration 2008-2013. Retrieved from https:// www.uu.nl/files/ubdobfinal-report-public-administrationpdf.

Hood, C. (2011). Public management research on the road from consilience to experimentation?. Public Management Review 13(2): 321-326.

Karré, P.M., Schillemans, T., van der Steen, M., \& van der Wal, Z. (2017). Toekomst van de Bestuurskunde. The Hague: Boom.

Karré, P.M. (2017). Lessen van prijswinnaars: een duiding van excellent bestuurskundig onderzoek. In P.M. Karré, T. Schillemans, M. van der Steen, \& Z. van der Wal (eds). Toekomst van de Bestuurskunde. The Hague: Boom. 51-58.

Noordegraaf, M., Brandsen, T., \& Huitema, D. (2006). Fragmented but forceful: Dutch administrative sciences and their institutional evolution. Public Administration 84(4): 989-1006.

Reussing, R. (2016). Bestuurswetenschappen 70 jaar: de mannen (en een vrouw) van het eerste uur. Bestuurswetenschappen 70(4): 5-17.

Stoker, G. (2019). Can the governance paradigm survive the rise of populism?. Policy \& Politics 47(1): 3-18.

Wilson, W. (1887). The study of administration. Political Science Quarterly 2(2): 197-222. 


\section{7 \\ Public Administration Research in Norway: An Organisational and Institutional Approach to Political Organisations ${ }^{1}$}

Per Lægreid

\section{Introduction: Government capacity and good government}

Over the past 40 years, the central public administration in Norway has been characterised by both stability and change, reflecting to some degree different reform trajectories such as New Public Management (NPM) and New Public Governance (Christensen et al., 2018). The boundaries between the public and private sectors, between the national, sub-national and supra-national levels and between policy areas have become more blurred. To grasp this complexity and dynamic development simple context-free instrumental rationality has to be supplemented by institutional approaches (Olsen, 2010).

Since the 1990s, more attention has been paid to the importance of governance capacity, the quality of government and a well-performing administrative apparatus to understand why some countries are more successful than others in looking after their citizens' welfare and ensuring a high standard of living. Fukuyama suggested that the world should look to the Nordic countries in order to build prosperous, well-governed and liberal democracies. In his view, the Nordic combination of a strong state, well-functioning rule of law and a responsible democracy is a useful recipe for good government. This attention to governance capacity and the related "institutional turn" in public administration research has highlighted the need to "bring the bureaucracy back in" and to apply an organisation theory-based institutional approach (March and Olsen, 1989). Politics is not only about deliberation and decisionmaking but also about putting decisions into practice. Thus, administrative capacity regarding specialisation, coordination, regulation, analysis and delivery is crucial. In representative democracies, good government depends 
not only on the input side of the political process but also on the output side; here the quality of the administrative apparatus has a major role to play.

The Norwegian approach to the study of public administration underlines two main reasons why it is important. First, because it is a major political actor and not simply a neutral managerial, technical and logistical tool. Organising entails mobilising biases; in other words, how we organise the administrative apparatus affects which problems and solutions receive attention and are prioritised, and which are neglected or rejected (Egeberg \& Trondal, 2018). The way the public administration is organised and how it recruits its personnel has significant impact on the quality of the government's governance of society. Thus, the organisation of the administration is a potentially powerful political instrument able to influence the content of public policy. Second, the capacity and competence of the administrative apparatus are crucial for the implementation of public policy and have implications for the living conditions and wellbeing of citizens. The quality and impartiality of the administration and the absence of corruption are core components of good government.

In this chapter, I will examine three substantial trends in Public Administration research from a Norwegian perspective, focusing especially on administrative reforms and the central government level. First, I will describe the theoretical development; second, I will address the development towards more time series and comparative data; and third, I will discuss the trend towards effects and impact studies and the vision of public administration as an architectonic discipline. Finally, I describe the links and relevance to practice and teaching in the field and draw some conclusions.

\section{Theoretical development: the institutional turn and meso-level theories}

Norwegian Public Administration research has been hugely inspired by the longstanding collaboration between Johan P. Olsen and James G. March who have changed the way we think about organisations, institutions and democratic governance. They developed theories of bounded rationality and also challenged the legal-constitutional legacy in Public Administration (PA) research. In the 1970s they introduced the Garbage Can Model in the study of loosely coupled organisations, non-routine situations and decision-making under ambiguity. In the 198 os they launched the New Institutionalism, painting a picture of public organisations integrated into a complex and dynamic political and societal network of organised interests and stakeholders and focussing on the potential for democratic governance 
by combining environmental features, purposeful actors and historically developed institutions. Later on, Norwegian PA research also developed stronger connections with Nordic and European scholars, not least through the ARENA, Centre for European Studies, at the University of Oslo and through several European comparative projects.

Norwegian research on public administration is mainly based on organisation and institutional theory and on democratic theory (Christensen \& Lægreid, 2017; Olsen, 2016). It paints a picture of public administration as a component of complex political and societal networks of organised interests and clients. It is a story of the complex and dynamic interplay between competing logics, loyalties and influences, demanding more elaborate models of decision-making and change than assumed by the Weberian ideal model.

A main argument is that patterns of governance cannot be adequately understood without including organisational factors, and a core question is what difference organisational factors play in the governance processes (Egeberg \& Trondal, 2018). The public bureaucracies are seen not only as an instrument but also as partly autonomous institutions that do not adapt in a simple and straightforward way to new steering signals or to changing environmental pressure. The ideal model of bureaucracy is supplemented by empirical studies of living institutions and how they work in practice. The internal management focus is supplemented by a broader political and normative focus; and the emphasis on rule-following, processes and procedures is supplemented by a results and performance orientation. Theories of decision-making under ambiguous circumstances and institutional constraints where the logic of appropriateness supplements a logic of consequentiality, complement the theoretical tradition based on bounded rationality (March \& Olsen, 1989).

This approach goes beyond the generic macro-oriented theories that try to arrive at universally valid insights for all types of formal organisation in the public sector. Instead, it applies meso-level theories that take diverse contextual constraints into consideration (Pollitt, 2013) and argue that we have to go beyond "catch all" theories that can be applied at all times, everywhere and in all situations. For example, the level of citizens' trust in government institutions and the level of corruption in a given country will make a big difference to how the public administration works.

A broad organisation theory-based institutional approach to public administration contrasts with ideas like a "generic" public administration, convergence of administrative arrangements and simple models of administrative reforms (Olsen, 2010). Global prescriptions for administrative reforms have been responded to differently depending on national and sector-specific institutional arrangements and historical traditions resulting in organisational 
principles derived from multiple contextual factors working together in a complex mix (Olsen, 2016). There are, however, also ambitions to contribute to a general organisational approach to public governance by focusing on dimensions that cut across governance structures and processes and might allow for generalisation across time and space (Egeberg \& Trondal, 2018).

Norwegian PA research often combines organisation theory with more general political science theory. Bounded rationality is a central concept. The historical-institutional context has been brought back in, and neo-institutional theories have increasingly been used, in particular from the 1990 on on. Especially during turbulent and unsettled times or periods of transition, unruly and wicked problems emerge that make it necessary to supplement the simple principal-agent way of thinking with institutional approaches in order to grasp developments. The discretion enjoyed by public sector organisations with regard not only to administrative and technical issues but also policy issues has increased, and they have become political actors that not only serve their political principals but also have multiple relationships to society. We cannot assume that they have sufficient capability, cognitive capacity or power to act as rational actors.

There is no generally accepted empirical administrative theory that specifies under what conditions one set of factors has greater explanatory power than another or how their mutual influence can be understood. Norwegian PA researchers embrace the growing claim that context matters, but there is still no good understanding of how different contexts matter and we still have some way to go to uncover the complexity of contexts as a missing link between the general and the particular (Pollitt, 2013). We face the challenge of explaining how hybrid organisations based on mixed political orders and partly competing organisational principles can be contextualised and understood (Olsen, 2010). We have to include the context in our models at the same time as we have to acknowledge that institutions have their own explanatory power and are not dominated by environmental determinism. Rather than decontextualised generic theories, we need middle-range theories that account for cultural trajectories, polity features and environmental constraints (Christensen \& Lægreid, 2016).

In contrast to the simple diffusion or copying of administrative reforms across countries, the story of administrative reform in Norway is more about translating, editing and adapting to the historical-institutional tradition (Røvik, 2011). We may question how fruitful a stage or phases approach is whereby one set of reforms replaces previous reforms over time. It might be more useful to look at the different reform trends as complementary or supplementary perspectives. The argument is that PA is a compound system 
where one has to find a balance between partly competing goals and values (Olsen, 2010). There are permanent tensions between hierarchies, markets and networks and between loyalty to political signals from the government of today, to Rechtstaat values, to due process, to stakeholder interests and to efficiency and service quality. These constitute trade-offs that the public administration has to live with, yielding a picture of a complex and dynamic public administration. The implication is that good governance is more than majority rule and that it is necessary to pay heed to several competing concerns and values.

An organisational approach to administrative reforms is preoccupied with how different ways of organising the reform processes may influence the reform trajectories (Egeberg \& Trondal, 2018). Reform processes are seen as decision-making processes, which allocate resources, attention, actors, identities, roles, resources and capabilities. Organisational characteristics and administrative structures create possibilities for reform while at the same time they place restrictions on it. Adding to this the role of professions is also important. Professional bureaucrats are essential trendsetters and the bureaucracy is often more important than political parties as political workshops defining what is true, possible and valuable.

What we have witnessed in recent decades is not a successive replacement of one model by another, but rather a process of adaptation, layering and co-existence of different doctrines in line with the theory of gradual institutional change. This has been labelled a transformative approach, because it underlines the need to go beyond single factor explanations and to examine how political design is enabled and constrained by environmental factors, administrative traditions and polity features (Christensen \& Lægreid, 2016). The multi-level governance approach has likewise been challenged by a multilevel administrative system approach designed to grasp the organisational dimension of turbulent change processes.

This theoretical development might provide material for an interesting debate about whether PA is a separate discipline or a sub-discipline of Political Science. In contrast to the continental European and US tradition, Political Science and Public Administration are not seen as separate disciplines in Norway but are integrated into one discipline. Maybe the Norwegian take on the study of Public Administration as a mixture of political science and organization theory with a substantial empirical orientation is a fruitful combination that furthers an understanding of public organisations as important political actors in a democratic governance setting. These features were typical in Public Administration research in the US until 1960, but were later lost. Norwegian PA research is more process-oriented than American 
research, applies qualitative approaches and focuses more on how ideas about organising are shaped, diffused and translated. Many scholars come from a constructivist tradition, but most of them take a moderate stand and avoid a purely subjectivist position.

One might claim that Norwegian public management scholarship has been remote enough to evade the "paradigm police" and connected enough to influence the more vulnerable elements of non-Nordic research communities. One special feature of Scandinavian organisation theory is its interest in the practice of organising - an interest that is facilitated by the openness and transparency of organisations and the easy access to them, especially in the public sector. Scandinavian institutionalism revolves round the concepts of appropriateness, translation, loose coupling and decision-making under ambiguity and uncertainty. Global and local approaches are combined, there are strong disciplinary roots combined with interdisciplinary openness combining paradigmatic approaches and empirical pluralism. A main focus is on how public sector organisations work in practice from a behavioural perspective rather than how they work on paper or are supposed to work in line with a formal-legal and normative tradition.

Norwegian organisational studies pay a lot of attention to the public sector as a whole. Their focus is less on internal management, ideas about cost cutting and efficiency in individual organisations and organisational strategies, and more on the institutional environment. An interest in democratic governance and the organisational basis for politics is a special characteristic of the Norwegian approach (Christensen \& Lægreid, 2017). A main question is how different institutions affect citizens' living conditions and contribute to a legitimate governance system (Olsen, 2010). Administrative institutions and their staff affect policy design and the implementation of decisions, and thus influence whose values, interests and world views are attended to. While the study of organisational design is dominated by empirical analyses, it also includes normative and prescriptive analyses. An organisational perspective on decision-making in public organisations addresses formal structure, but also looks at demography and organisational locus (Egeberg \& Trondal, 2018).

The special mixture of political science and organisation theory has given Norwegian public administration research a relatively strong theoretical profile. A core argument in the Norwegian research community is that political processes and the content of public policy cannot be fully understood without considering the structure and practice of public administration. Norwegian public administration research shows continuity through its focus on the political features and the democratic context of the civil service, on formal structure, bounded rationality and internal processes. It has also been 
characterised by a development from an intra-organisational approach to an inter-organisational one and by a greater interest in comparative studies and the internationalisation of public administration.

A main lesson from Norwegian PA research is that public administration faces increasingly complex and dynamic environmental and internal conditions, reflected in multi-functional organisational forms. Compound administrative reforms are multi-dimensional and represent combinations of competing, inconsistent and contradictory organisational principles and structures that co-exist and balance interests and values. It is not a question of hierarchy, market or networks but of how the mixtures of these forms of coordination change in different reform movements and how the trade-off between them alters. In a composite public administration system democratic governance is about organising to cope with lasting tensions and shifting balances of power (Olsen, 2010). There is no best practice, quick fix or panacea and the chances of successful reform increase when the historical-institutional context is taken into account. Normally the reforms have to pass a compatibility test, and if they avoid a cultural clash they are more likely to be successful. Criticism of de-contextualised theories such as rational choice has gradually expanded.

The Norwegian lesson is that administrative reforms must be matched carefully with the needs, traditions and resources of each political system. Reforms that do not consider the historical-institutional context tend to result in new reforms rather than increasing performance. The argument is that global myths and prescriptions for administrative reforms are interpreted, translated and responded to differently depending on national and sectorspecific institutional arrangements and historical traditions. In recent years enthusiasm for some of the NPM-related reforms has gradually waned. There has been a rediscovery of the historical-institutional context and the NeoWeberian state. The lesson is that there is a need to consider the domestic administrative and institutional context when designing and implementing administrative reforms.

The study of Norwegian public administration and political organisations can be summed up in the following propositions (see Olsen, 2018).

- Organisations and institutions matter.

- The organisational basis of government institutions matters.

- Living institutions matter. Beyond the formal-legal institutions.

- Attentions, identities and appropriateness matter. Beyond the decisionmaking framework and rational choice.

- Normative principles matter. Democracy, the sovereign people and the sovereign individual. 
- Organisational principles matter: Democracy beyond simple principalagent relations.

- Connections and networks matter. Organised interest built into governmental organisations.

- Europeanisation and internationalisation matter. Beyond the state as the dominant policy entity.

- History, culture and context matter. But history is ambiguous.

- Complexity and dynamics matter. Organisations are complex and dynamic systems. Outcome is difficult to predict.

\section{Longitudinal, comparative and panel data}

In recent decades, $\mathrm{PA}$ researchers have put greater emphasis on large empirical studies. The Norwegian public sector is rather unique in the easy access to data that it provides to PA researchers, which is clearly a major advantage for the latter. It also says something about the status of this research in a high-trust society. One important empirical focus has been real-life studies of public administration organisations and institutions and of administrative reforms and policies. These have shown that reform processes in Norway are often characterised by compromise and an apolitical rhetoric, yielding incremental and uncertain results. The search for unifying compromises indicates that system effects might often trump substantive policy effects.

What the research on public administration reforms needs more than anything else is reliable data that go beyond single reforms, individual organisations, cross-sectional data and one-country studies. Over the past decades, there has been a significant contribution to the development and production of databases and data sets for public management research in Norway. I will mention just a few.

First, the Norwegian State Administrative Data Base, a dataset comprising all formal structural changes in the Norwegian state administration over the past 70 years covering ministries, central agencies, state-owned companies and foundations. The database provides unique and systematic information about structural changes in the government administration, such as the establishment of new organisations, organisational deaths, the merging and splitting of organisations and moving organisations vertically and horizontally.

Second, the Norwegian Civil Service survey, which includes responses from civil servants in ministries and central agencies collected every tenth year since 1976, provides unique longitudinal data on the tasks, role perceptions and behavioural patterns of civil servants in ministries and central agencies 
as well as on the degree of Europeanisation and attitudes to administrative reforms.

Third, the Municipal Organisational Data Base Surveys, which have been conducted every fourth year since 1996, mainly cover political and administrative structures and working methods at local and regional levels. These datasets are internationally unique. ${ }^{2}$ They are invariably used in government-commissioned research as well as by Norwegian political and administrative actors in Norway addressing reorganisation issues.

Fourth, the development of international cross-country datasets on administrative reforms, agencification, autonomy, control, accountability, proliferation, coordination, management and performance of government agencies throughout Europe. Examples are Comparative Public Organisation Data Base for Research and Analysis (COBRA), ${ }^{3}$ the Cost Action on Comparative Research into Current Trends in Public Sector Organisations (CRIPO), ${ }^{4}$ the project on Coordination for Cohesion in the Public Sector of the Future (COCOPS $)^{5}$ and the permanent study group in EGPA on Governance of Public Sector Organisations. The development and maintenance of international cutting-edge datasets has provided invaluable knowledge and experience for the study of public administration and governance in Norway and has enabled PA researchers to participate regularly in international collaborative research.

Fifth, a national infrastructure of coordinated online panels for social science research on democracy and governance is planned in Bergen. It will enable coordinated data-collection in online panels that cover the entire core of the structure of democratic governance. The plan is to establish three new online panels (public administrators, judges and journalists) and expand two existing panels (the citizens' panel and the electorate panel) as part of the Digital Social Science Core Facility (DIGSSCORE) at the University of Bergen. The Panel of Public Administrators emerges from a 40-year long tradition of surveying central government officials in Norway. It will recruit from a base of public administrators at all levels of government - national, regional, local. This infrastructure will make it possible to analyse across sub-populations, to do panel studies and longitudinal studies. It can also contribute to the behavioural turn in PA research focusing on different types of experimental research.

The realisation that it is impossible to understand the development of the public administration from an internal, domestic point of view alone has led to a greater interest in comparative studies between countries. The same goes for the need - occasioned by the fact that different countries have different starting points and are in different phases of reform - to track changes over 
time using longitudinal data. What the discipline probably needs more than anything else is good comparative data, both over time and across countries.

\section{From processes and administrative behaviour to performance and effects}

What should an organisation theory for studying public administration and public policy explain, i.e. what are the dependent variables? The focus in Norway has been on processes and on public decision-making behaviour. What pattern of influence is there between different actors in agenda-setting, assessment of alternatives, formal decisions and implementation? Less emphasis has been placed on the effects of the different reforms and forms of organisation. Over time, however, there has been an enhanced interest in the societal outcome, effects and impact. Public administration is an integral part of the political-administrative system and the focus is therefore on the dynamic relationship between political and administrative actors in a democratic context (March \& Olsen, 1989). When addressing effects and implications, the Norwegian approach has gone beyond the narrower focus on decision-making and also addressed the broader concept of performance, including the effects on political steering and control, power relations, accountability, trust and legitimacy.

One concern is what empirical support there is for the assumed or expected effects of the different administrative reforms. The paradox is that reforms like NPM build their legitimacy on the assumption that they will produce effects, such as more efficiency and effectiveness and a more economic use of resources, yet at the same time there is little systematic, reliable or generalised knowledge about these kinds of effects. The jury is still out on the degree to which administrative reforms work in practice; for example has New Public Management led to a "government that works better and costs less" as promised. One of the big flaws in NPM was probably the claim that a clear dividing line existed between policy-making and formulation on the one hand and policy implementation on the other, reflecting the old debate about the separation between politics and administration. Unfortunately, there is very little evidence on the effects of NPM. New Public Management has been around for 30 years, yet there have been few comparative evaluations of it. Instead, NPM scholars have spent too much time studying the reform process and examining the forces driving the reforms while merely speculating about their impact on efficiency and service quality. 
Effects are often assumed or promised, but there are few systematic and reliable studies of whether they actually happen. Evidence of efficiency gains has been patchy and incomplete and systematic quantitative empirical investigations over time have been lacking, so there is little hard evidence of whether NPM's main goal of cost reduction and improved efficiency has actually been achieved. There is therefore a great need for good, reliable data over time on the effects of NPM reforms. The Achilles heel in administrative reforms is that the reform actors have limited understanding of the consequences and implications of their own reform initiatives.

Reform agents often face the problem of over-selling; because to get a reform accepted they often have to promise more than they can deliver. They are also puzzled by the implementation problem versus the model problem. When the results fail to materialise, their answer is often that one has to try harder. Their solution is to take a more sophisticated approach, to train political and administrative executives better or to replace them. Another strategy is to ask if there is something wrong with the model - maybe the model should be adjusted to make it fit administrative reality better. This is also linked to the fact that NPM reforms are a rather loose collection of tools and measures, a shopping basket from which reform agents can choose partly competing reform elements. We have to remember that administrative reforms are often a political exercise not informed by a coherent theory.

A third concern is the efficiency problem versus the expectation problem. Often dissatisfaction with public sector organisations has more to do with unrealistically high expectations among users, clients and citizens about what public sector organisations are capable of rather than with poor efficiency. So maybe we need a policy to lower expectations rather than to increase efficiency. A good administrative apparatus requires not only governance capacity but also governance legitimacy.

We also have to focus on the relationship between efficiency and other important aspects of performance in the public sector such as fairness, impartiality and predictability. One implication is that there is a need to go beyond the narrow concept of performance linked to economy and efficiency and to include the broader democratic implications for power relations, trust and legitimacy.

An important implication is that public sector organisations cannot just copy private sector management tools and organisational forms and expect successful implementation and results. After all, public sector organisations differ significantly from private sector organisations in that they are more multifunctional, they have political executives as leaders and many of them do not operate in a market. So the policy advice is that administrative 
reforms need to be adapted to local contexts, implying that holistic models have clear limitations. One strength of Norwegian administrative reforms is that they have been adjusted to the Norwegian tradition of openness and stakeholder involvement even if this has also changed since the heyday of corporatism.

One lesson is that most governments fail to learn sufficiently from previous administrative reforms in their country or in other countries, the reasons being that their impacts are often ambiguous, and politicians are generally more interested in launching new reforms than learning from previous ones. Another lesson is that when means-end knowledge of reforms is poor, a cautious, experimental and incremental reform style is more promising than big bang reform. In Norway, the former reform style is more common than the latter.

\section{Towards an "architectonic discipline"?}

To what extent PA can live up to the vision of being an architectonic and applied discipline has been a core question in the Norwegian approach. Successful deliberate institutional design requires realism with regard to analytical capacity and knowledge of cause-effect relations as well as to power relations, resources and action capabilities (Olsen, 2018).

Morten Egeberg has developed a prescriptive design model in which the criteria for selecting explanatory factors are that they, in addition to being relevant for understanding variations in decision-making behaviour, must also be manipulative and operational. He has emphasised three main groups of such factors, which might be seen as toolkits for organisational design: formal organisational structure, organisational locus and organisational demography (Egeberg \& Trondal, 2018).

Numerous analyses based on the data from the Norwegian administrative surveys from 1976 to 2016 show systematically that structural features are most important for understanding variations in civil servants' work related to their attitudes and decision-making behaviour. Organisational boundaries and constraints matter for the bureaucrats' perceptions and behaviour at work, meaning that organising implies mobilising bias. Organisational affiliations, positions and tasks are important to understand the civil servants' perceptions and decision-making behaviour: where you stand depends on where you sit. The only demographic variable that has a significant and stable effect is professional background. These findings have implications for organisational design. Knowledge of how organisational factors might be deliberately changed and 
how they can influence governance processes is an important precondition for institutional design. The argument is that organisational factors can be seen as design instruments which can make a difference to public governance.

That being said, often the evidence-based knowledge about specific effects of different organisational arrangements is uncertain and the powers of reform agents are limited (Lægreid, 2018). Different organisational forms matter and affect the way public organisations operate and work in practice. However, usually there is no one-to-one relationship between organisational design and performance. Organisational structure cannot determine particular policy outcomes, but they might create biases in governance processes, making some choices more likely than others. The provable connections indicate a direction for development more than a precise statement about the strength or extent of changes in decision content when formal structures change.

The impacts of design factors are especially uncertain when we move from effect on decision-making behaviour further out in the effect chain towards societal impacts. While Egeberg mainly focuses on effects on public governance such as vertical and horizontal processes and meta-governance like reorganising, Olsen has a broader agenda on how the dynamics of institutional change and political institutions might affect democratic governance and political consequences beyond the policy process. Thus, it is important also to address the broader effect on democracy and legitimacy.

One has to accept that governance through institution building is challenging. Organisations are complex and dynamic systems, interactions between the parts might produce an outcome that is difficult to predict, and experiential learning is difficult because the future may be uncertain and ambiguous. From a democratic perspective institutional design should be about how citizens can decide how to be organised politically and about how it can enhance democratic identities among citizens. Even if there are many normative theories of political design, there is little agreement among them. Thus, there are no objective criteria for good design because goals and values will vary and the dynamic relationship between political and administrative actors in a democratic context has to be taken into consideration in organisational tool design.

\section{$6 \quad$ Links and relevance to practice and teaching}

Norwegian PA research has strong relevance and links to practice. One example of this is that Norway has been a frontrunner in Power Studies, 
starting with the first power study in the 1970 s with a special focus on public administration, followed by a second one in the 1990 s. These research programmes were launched by the government and the parliament. The first power study has been characterised as the big leap forward in Norwegian political science. It brought new theory-informed empirical knowledge about how the public administration works in practice and paved the way for subsequent databases about the structure and practice of public administration which have been useful not only for research but also for practitioners and policy-makers.

Second, by introducing new concepts and approaches to the study of the political-administrative system it influenced the political and administrative leaders' vocabulary, image and understanding of public administration more generally. Third, PA scholars have been involved in studying the processes and effect of a wide range of administrative reform policies and administrative reforms such as municipal organisations in the aftermath of the new Municipal Act in 1992, the Hospital Reform (2001), the Labour and Welfare Administration (the NAV Reform) in 2005, the Collaboration Reform (2012), the Municipal Reform (2014) and the Police Reform (2015). Fourth, PA scholars have been used as experts in different public commissions on administrative reforms and administrative policies.

Fifth, PA in Norway has also been successful in teaching, which can be seen as a result of good theoretical informed and practical and empirically inspired research. In contrast to many other European countries the study and teaching of public administration has not been singled out as an individual speciality, academically and organisationally independent of political science, democratic theory and organisational theory. The field of Public Administration in Norway has over time had a practical aim, and the research questions have grown out of specific societal problems and not merely focussed on internal management issues but also on public organisations as political organisations and institutions. Political knowledge is to a great extent pragmatic, based on experiences that citizens, politicians and bureaucrats have had when they have faced problems and conflicts. Public administration research and teaching in Norway has been a success because it has been able to combine interests for contemporary problems with interest for general issues that different political-administrative systems face.

This knowledge has been disseminated through several popular textbooks that have been widely used at Norwegian Universities and University Colleges over the years (Christensen et al., 2002, 2005). Public Administration teaching in Norway is attracting a lot of students and it produced a whole new generation of political scientists, of whom many have become civil servants 
who have had a great impact on the way civil service works in practice. Since 1976 the percentage of social scientists in Norwegian ministries has increased from $4 \%$ to $30 \%$ and in central agencies from $4 \%$ in 1986 to $20 \%$ in 2016 . Most of these are political scientists and many have majored in Public Administration. They also have a strong position in regional and local governments.

\section{Conclusion}

One main development in PA research in Norway over recent decades has been increased internationalisation. In contrast to 30 years ago, most publications are in English, and there are much stronger international networks among scholars, more participation in international conferences and also more joint international research projects and publications. Overall, this has strengthened the quality of research in the field both theoretically and empirically as well as in terms of substance. This development also makes it more difficult to distinguish a specifically Norwegian approach from the broader international one because over the years it has become more integrated into international PA research. There are still some distinguishing features, however. Norwegian research has gone beyond formal-legal categories and studied the actual practice of "living" organisations and institutions. Single factor explanations face considerable problems when their claims are confronted with empirical data. The same goes for principal-agent models, especially in transitional periods and unsettled situations. What we see in diverse empirical realities is much in contrast to the ideas of "global recipies" and simple models of administrative reforms.

Another feature of the Norwegian approach is its strong empirical orientation. The empirical focus has, however, been more on processes and decision-making than on effects and implications. There is a need for stronger evidence-based policy-making in the field of administrative studies in both Norway and elsewhere. So far, the knowledge base regarding the effects and implications of administrative reforms is rather inconclusive. Reliable data are lacking, and systematic analyses of effects are scarce. To decrease the gap between citizens' demands on the one hand and what public administration can deliver on the other, one can either improve the performance of the public administration, reduce expectations or a combination of the two. So far, most effort has been on the performance side, but perhaps there should also be an increased focus on the demand side. One might ask if reform failure is more political than bureaucratic which might be the case if the political 
institutions failed to provide clear, consistent and realistic goals and rarely allocated adequate resources to deal with the scope of the problem.

The challenge for future research in Norway is a need for more systematic comparative studies over time and across countries, administrative levels and policy areas. There is also a need for more and better studies able to analyse the dynamics of reform and change by focusing on the effects of structural reforms using a broad concept of performance. Reforms and organisational tools do work sometimes under specific conditions. Rather than an idealistic and over-optimistic approach, a more humble and modest path might be more appropriate.

In my view PA research in Norway should stick to its roots and search for theories-based empirical knowledge about living public administration, governmental institutions and democratic governance. Rather than focussing on management, administration, organisations and organising in general it should address public and political organisations. A political organisation approach should analyse the mixed order of the political-administrative system and the tensions between different governance levels such as the local, the regional, the national and the international, as well as between policy areas and between different institutional spheres such as public administration, parliament, government, courts, corporatist arrangements, media, markets and civil society (Olsen, 2018).

Public administration faces challenges regarding how to handle big transboundary "wicked issues" in which the problem structure does not overlap with the organisational structure. A critique of PA research has been that it has been too preoccupied with internal management issues and administrative techniques of public administration and neglected the larger forces at work (Roberts, 2014). There is also a need for PA to go back to its roots and address the big issues that contemporary political-instrumental systems have to deal with. We have to go beyond internal management problems and address the big wicked issues that society is facing in our time and examine the "megatrends" such as digitalisation, climate change, demography, migration, internationalisation, societal security and financial stress to explain the path taken by public administration reforms. In Norway, some ongoing comparative projects are trying to address the problems of coordination, capacity and legitimacy in wicked policy areas, such as internal security, climate change, immigration and crime. ${ }^{6}$

We also have to go beyond routine situations and business as usual and address the conditions for democratic governance in transitional periods and unsettled situations. Management matters, but how we organise also has political implications. There is a need for long-term commitments and good 
access to reliable data to make progress in theoretically informed empirical studies of public organisations in democratic contexts.

\section{Notes}

1. This chapter is a revised, updated and expanded version of Lægreid (2017).

2. For more information on this database and the following please see: www.nsd.uib.no/ polsys/.

3. http://soc.kuleuven.be/io/cost/about/cobra.htm.

4. http://www.cost.eu/COST_Actions/isch/IS0601.

5. http://www.cocops.eu/.

6. For example, the GOVCAP project (Organising for Internal Security and Crisis Management. Building Governance Capacity and Legitimacy), the COCAL project (Coordination, Capacity and Legitimacy: Organising for Climate Change, Immigration, and the Police) and the TROPICO project (Transforming into Open, Innovative and Collaborative Governments).

\section{References}

Christensen, T., Larsen, H. O., Lægreid, P., Roness, P. G., \& Egeberg, M. (2002). Forvaltning og Politikk (Administration and Politics). 5th ed. 2014 by T. Christensen, M. Egeberg and J. Aars. Oslo: Universitetsforlaget.

Christensen, T., Lægreid, P., Roness, P. G., \& Røvik, K. A. (2005). Organisasjonsteorifor Offentlig Sektor (Organization Theory and Public Sector). 3rd ed. 2015. Oslo: Universitetsforlaget.

Christensen, T., Lægreid, P., Roness, P. G., \& Røvik, K. A. (2020). Organization Theory and the Public Sector. 2nd ed. 2020. London: Routledge. First edition 2007.

Christensen, T., Egeberg, M., Lægreid, P., \& Trondal, J. (2018). Sentralforvaltningen. Stabilitet og endring gjennom 40 år. (Ministries and central agencies. Stability and change over 40 years). Oslo: Universitetsforlaget.

Christensen, T., \& Lægreid, P. (2016). A transformative perspective. In S. van de Walle, \& S. Groeneveld (eds). Theories and Practices of Public Sector Reforms. London: Routledge.

Christensen, T., \& Lægreid, P. (2017). An Organizational Approach to Public Administration. In E. Ongaro, \& S. Van Thiel (eds). The Palgrave Handbook of Public Administration and Public Management in Europe. Volume 2, Chapter 55 (pp. 1087-1104). Basingstoke: Palgrave Macmillan.

Egeberg, M., \& Trondal, J. (2018). An Organizational Approach to Public Governance. Oxford: Oxford University Press.

Lægreid, P. (2017). Getting to Norway - thirty years of public management research: theoretical, empirical and practical challenges. International Journal of Public Sector Management 30(6/7): $584-594$. 
Lægreid, P. (2018). Designing Organizational Tools. In M. Howlett, \& I. Mukherjee (eds). Routledge Handbook of Policy Design (pp. 274-287). London: Routledge.

March, J. G., \& Olsen, J. P. (1989). Rediscovering Institutions. New York: The Free Press.

Olsen, J. P. (2010). Governing through Institution Building. Oxford: Oxford University Press.

Olsen, J. P. (2016). An institutional approach. In S. van de Walle, \& S. Groeneveld (eds). Theories and Practices of Public Sector Reforms. London: Routledge.

Olsen, J. P. (2018). The Bergen approach to public administration and political organization. Norsk Statsvitenskapelig Tidsskrift 34(4): 188-206.

Pollitt, C. (ed.). (2013). Context in Public Policy and Management. The Missing Link? Cheltenham: Edward Elgar.

Røvik, K. A. (2011). From Fashion to Virus: An Alternative Theory of Organizations' Handling of Management Ideas. Organizational Studies 32(5): 631-653. 


\title{
8
}

\section{Public Administration in Portugal}

\author{
Filipe Teles
}

Public Administration sciences in Portugal reflect its administrative culture and history. In a country where the national government centralises a wide range of functions, despite some decentralisation initiatives in recent years (Teles, 2016), the long tradition of strong and concentrated administration (Tavares, 2019) has had a significant impact on several aspects of training, research and knowledge development in the field of Public Administration. The enduring influence of this tradition should not be undermined and, therefore, a historical contextualisation and an in-depth knowledge of the state apparatus is needed to understand public administration and administrative practice in Portugal (Tavares \& Alves, 2006).

As in other significantly centralised countries in Europe, Portugal has to deal with a sense of distance between citizens and administration. This is reinforced by the systematic institutionalisation of a hegemonic paradigm which is based on the prevalence of administrative law particularly focused on formal issues. This has had an impact both on the way the study of public administration is concentrated on its formal and procedural aspects, as well as on the incidence of law prescriptions in the processes of reform. This concern for structuring the internal organisation of public administration and its relationship with citizens in terms of top-down determination through legal instruments should not be considered without having in view one of the pillars of parliamentary and representative democracy, where models of delegation are preferred over total control of public administration from the political sphere. This desirable separation, in particular if one considers the democratic context of recent decades in Portugal, cannot be dissociated from models of delegation and with the concern to reduce the gap between administration and citizens.

In fact, from the early 1980 s, under the influence of a set of reforms inspired by new public management, Portugal has witnessed a growing concern with the modernisation and efficiency of public administration, particularly through reforms aimed at reducing its cost and size. This has occurred in parallel with 
the search for the promotion of service and process quality, as well as a relevant growth of the state's responsibilities over a set of policy areas, as expected in a post-dictatorship country. In such a complex and - sometimes - contradictory context, several reforms in recent years have promoted market logic in the management of public services and fostered the creation of agencies with greater administrative and financial autonomy, as a way of ensuring a rational, quasi-entrepreneurial public management approach.

A simple overview of the Portuguese context clearly points to this struggle between an enduring legalistic and centralised tradition and the need to promote reforms that challenge the distance between citizens and administration as well as being able to facilitate the provision of new services with a concern for efficiency, quality and decentralisation. The democratisation of the country, together with its fast modernisation and European integration, needed special attention from the disciplines of administrative sciences and their study of the Portuguese context.

The best way to understand the essence of Portuguese public administration is to acknowledge the influence of the French model, with its strong legalistic tradition, which shaped its organisation and relation with citizens through administrative law (Tavares \& Alves, 2006). Hence, this chapter will describe, in parallel, both the evolution of Portuguese public administration and the training and research practices on the topic, with a particular emphasis on the implications of recent changes and reforms on the future of public administration.

\section{Origins and consolidation}

"Administration sciences" in Portugal had their origins in the Cameralism tradition of the eighteenth century (Bilhim, Ramos \& Pereira, 2015). Its focus on the need for strong control over a centralised economy was clearly adequate to the model of administration of the country's absolutist monarchy and highly useful in strengthening the powers of the influential and authoritative Marquis of Pombal, the eighteenth-century statesman and Secretary of State for Internal Affairs of the Kingdom, under the rule of King José I. His reforms aimed at consolidating the crown's control over the economy on the continent and in the colonies, where a strong and centralised administration was a useful tool. In the nineteenth century, the success of the liberal reforms and belief in the separation of powers led to a central role of administrative law, which acquired a hegemonic position in the studies devoted to public administration. At the same time, the old classical school, born in France in the first quarter of the nineteenth century and then spread throughout 
Europe, namely Portugal, was consolidating a paradigm that saw Public Administration sciences as associated with the legal sciences and, in particular, with administrative law. For Bonnin, one of the main French authors of this - then, progressive - perspective, to acquire knowledge of all matters of public administration it would be sufficient to master administrative law. There is clear evidence that Bonnin's influence did not take long to have an impact in Portugal. In fact, the critical role that Bonnin played in the administrative reforms of Mouzinho da Silveira, promulgated in 1832, very much influenced by and with clear similarities to the French law of 1802 , which was the basis of the Napoleonic administrative organisation, is acknowledged.

However, if on the one hand there was an almost immediate impact of the French administrative tradition in Portugal, through the political action of the liberal reforms during the first half of the nineteenth century, its influence on university studies took a little longer. Only in 1853, at the Law School of the University of Coimbra, was a course included as a way to teach the principles of administration and administrative law, following Bonnin's "Principes d'administration publique." Later, in 1861, Justino de Freitas published the noteworthy "Instituições de Direito Administrativo Português [Institutions of Portuguese Administrative Law],” and Lobo D’Ávila, in 1874, presented his "Estudos de Administração [Studies of Administration]." Although the last contains issues that could be included in a manual of Public Administration sciences, they were, in fact, administrative law handbooks. In the book "Curso de Ciência da Administração e Direito Administrativo [Course in Administration Science and Administrative Law]," published in the early twentieth century, the author Guimarães Pedrosa seems to promote a first attempt to avoid the strictly normative and legal perspective of the theme, although his sciences of administration still appear as a subsidiary of administrative law. In addition, one of the most influential and notable authors of the twentieth century in Portugal was Marcelo Caetano. Head of government after Salazar, in the authoritarian regime, he was also, and essentially, a devotee of administrative law.

The first sign of transition and evolution from the national status quo resulted from the creation of the Colonial School in 1906, within the Lisbon Society of Geography, where the training of overseas public officials eventually justified the themes of public administration being also considered with a non-juridical approach (Araújo, 2006). However, only at the beginning of the second half of the twentieth century did the Salazar regime begin to consider the need to train top public officials using a similar approach to the one in place since 1906 for the colonial administration of the overseas provinces. Nevertheless, for several reasons, but above all given the traditional role and rule of administrative 
law, and the long-lasting power of law schools in the context of Portuguese universities (which ultimately reinforced the legalistic tradition in Portuguese public administration that has lasted until today), this did not allow for the creation of an autonomous degree in Public Administration.

One of the most interesting examples of this evolution within the study of Public Administration in Portugal results from the reform of the "Instituto Superior de Ciências Sociais e Políticas Ultramarinas [Higher Institute for Overseas Social and Political Sciences]," under the guidance of Adriano Moreira, who had been Overseas Minister in Salazar's government. When the institute merged with the Technical University of Lisbon in 1961, faculty members were expected to be trained abroad, and special attention was paid to management sciences. For the first time, a different paradigm was being implemented, which was clearly influenced by an Anglo-Saxon tradition, rather than the dominant continental French perspective. Nevertheless, this was only possible though a three-year bachelor's degree in Overseas Administration, which was seen as complementary to the areas of social sciences, like anthropology and ethnographic studies.

This rupture, which followed the Columbia University model as in other colonial empires, created a short window of opportunity to develop the sciences of administration outside the national legalistic paradigm. This did not last long, since one of Marcelo Caetano's first decisions when he took over as head of government after Salazar was to replace Adriano Moreira.

\section{The democratic period and the reforms}

The institutionalisation of bureaucracy in Portugal allowed for a long period of stability of Salazar's authoritarian regime. Together with the highly centralised state and its legalistic tradition (Salazar being a law scholar himself), public administration helped the regime to steer and control policies and service delivery. In fact, as suggested by Oliveira Rocha (1991), corporations and other corporate structures, constitutionally relevant to the political regime before democracy, never really existed as powerful agents in the Portuguese political scene, since Salazar failed to institutionalise them, precisely because of the highly centralised administrative system. Interestingly, these same features of Portuguese administrative culture can explain its stability during the transition to democracy and the continuity of a large part of its elite.

The Carnation Revolution in 1974 and the country's transition to democracy came to question the corporative model of the previous regime, but the political change was clearly insufficient to transform the organisational culture. 
Despite this initial continuity in the process of transition between regimes, there were obvious changes in the democratic period that significantly altered the organisation and size of public administration in Portugal. In this period, the growth in the state's functions came to generate new opportunities and sources of power for the bureaucratic elite, whose importance grew in the face of governmental instability and the fragility of the political system during the first decade of democracy. In fact, with ten governments in ten years, the first decade after the revolution was characterised by significant governmental instability. The central role of political and regime issues and the stability of the social and economic order of the country did not allow for any special attention from policy-makers to public administration. Thus, in addition to its discredit as a consequence of the role played in the stability of the previous regime, Portuguese public administration had relatively low levels of formal educational training. It was only during the process of accession to the European Economic Community that the country began a trajectory that was aimed at ensuring its professionalisation. Besides the needed institutional reforms, these efforts were also seen in the establishment of specialised training for public officials and top-level bureaucrats. The foundation of the National Institute of Administration in 1979 and the creation of the Centre for Municipal Studies and Training (CEFA) in 1980 were important elements to boost administrative reform during this period. It was also in 1979 that the first statute of the public administration officialy was approved and published, which reflected a special concern with a new relationship between the political sphere and the parties in government and public administration managers. This statute repealed the lifetime nomination procedure that was in place and was inherited from the previous political regime.

It is important to acknowledge the fact that the number of public administration officials more than doubled between 1968 and 1979. The expansion of the functions of the state, particularly in the sectors of education, health and social security, greatly contributed to this growth. The number of civil servants grew steadily grew steadily until 2011. In parallel, this increase also reflects the way the public sector included trained human resources that came to be in surplus due to the decolonisation process. Finally, the government's nationalisation programme of major private economic and industrial agents should not be disregarded, since it played an important role in expanding the functions of the state over the economy, also contributing to increasing public employment.

If the size of Portuguese public administration has undergone considerable growth during the democratic period, it is no less true that its structure has suffered important adjustments. As claimed above, the country was not immune to new public management reforms. This modernisation process in 
Portugal was fundamentally grounded on a trend that argued for the relevance of an extensive delegation of competences towards more autonomous public agencies. Although this was not totally new in the Portuguese administrative structure - there were 22 public institutes in 1974 - their number increased considerably, until in 2002 there were 440 public institutes (Tavares, 2019). In addition, in 1989, a constitutional reform put an end to the irreversibility principle of nationalisation, which allowed for a process of privatisation.

These reforms, which were already included in public discourse during the authoritarian regime at the end of the 1960s, with Marcelo Caetano's government, suggested an idea of state modernisation based on the need to promote economic development and, after the democratic transition, the need to enable public administration to fulfil welfare state roles. A Secretariat for Administrative Reform was created in November 1969 to suggest new organisational structures, new statutes for public administration employees and new ways of delivering public services. However, none of these reforms were implemented before 1974 .

It was only in 1986, the year in which Portugal became a full member of the - then - European Economic Community, that the topic of State and public administration reform was placed high on the political agenda, with the establishment of the Secretary of State for Administrative Modernisation. As regards its policy agenda and strategy, one should take into consideration the role of the Office for Studies and Coordination of the Administrative Reform, operational since 1982, under the Minister for Justice and Administrative Reform. Its focus was on delivering advice on several relevant aspects, such as administrative simplification, the training of top-level officials and public servants' careers. The mission of this new Secretary of State was highly demanding and aimed at changing public administration's organisational aspects, processes and practices, which were rooted in decades of administrative culture. However, the course of the reform over the following years can be characterised more as a set of small scale and sectoral changes, with specific policy measures, rather than a mobilising strategic orientation.

The enactment of the Administrative Procedure Code in 1992 represents an important instrument that consolidated the governmental initiatives of the period, since it provided new accountability mechanisms and new citizens' rights as regards public administration, introducing transparency in reaction to a culture of unpredictability. Nevertheless, it is still evidence of a legalist tradition, motivated by a significant degree of wishful thinking associated with top-down enforcement of legislation. It was also during this period that, together with privatisation, the first experiences of contracting out began, though in some cases with unsatisfactory results, especially in services 
provided by a monopolistic market, as was the paradigmatic case of meals in hospital canteens, with poorer quality for the same price (Tavares, 2019).

As with the new public management-inspired reforms, Portugal was also influenced by the quality movement in public administration. One could see in this behaviour a culture of adherence to international trends, especially in the case of reforms originating in Europe's most advanced administrations. Several initiatives emphasising quality as a central feature of public administration modernisation were implemented, and they included a National Quality Council (1992) a Charter for Quality of Public Services (1993), a quality competition in public services and advanced training in quality management for senior public managers. Quality management standards, which in essence go far beyond the bureaucratisation of services, were not valued by reason of their content and desirable impact, but suffered from the same hindrances that new public management measures had to face: the fulfilment of top-down defined procedures, which had to obey a set of rules and norms that were predefined, in accordance with the resilient legalist tradition.

This commitment of the Portuguese government to the implementation of quality management tools is even clearer with the approval in 2000, in Porto, of the Common Assessment Framework, aimed at promoting self-assessment of public services, by the Quality Steering Group of which Portugal was a member. It included the European Foundation for Quality Management, the European Commission, the European Institute of Public Administration, the Speyer Academy, together with Austria, Finland and Germany. Even though it quickly became more of an ephemeral and short-term tool, like other trends of reform in Portuguese public administration, amongst other services, the Social Security Institute for Financial Management was one of the enthusiasts for using the CAF model (Tavares, 2019).

This "trend adherence" culture is also evident in the way some international reports and reference works were cited and widely used as an explicit inspiration to many subsequent governments seeking modernisation and administration restructuring. Amongst other examples, this was true of Reinventing Government (1992) by David Osborne and Ted Gabler, and of the Gore Report (1994).

This first step in Portuguese public administration reform was the result of a relatively stable period of governance, between 1986 and 2001, with different governments' political agendas on the theme being supported by a large and enduring majority in parliament, from both the Social Democrats and the Socialists. These reform agendas (e.g. citizen-centred services, mission driven, focus on outcomes, delegation of competencies, and partnerships with the private and third sectors) were often influenced by the principles of new public 
management, and by the major international public administration trends, presented as rational and efficiency-driven reforms rather than ideologically influenced ones. However, these measures faced two main problems. On the one hand, there was no clear distinction between the different policy initiatives, which often resulted in ephemeral and contradictory effects. For example, service outsourcing and privatisation allowed for some cost reduction, but were unable to maintain the expected quality standards. Naturally this came in clear contradiction of the quality management discourse in terms of relevant reform measures. On the other hand, while the efficiency rhetoric was presented in direct association with administrative simplification, problems related to market monopolies, corporative interests and a growth of more than 100,000 employees in the public sector, resulted in clear contradictions (Tavares, 2019).

The following years were characterised, again, by a collection of stand-alone policy measures and partial reforms rather than a strategic implementation of a modernisation agenda. The most emblematic and controversial measure was the adoption of a new integrated system of performance assessment for public administration (SIADAP), influenced by the principles of management by objectives. This had the merit of substantially altering - or at least trying to - the focus of a process-centred and legalistic framework to a results-oriented public administration. To this reform one should add a programme of administrative and legal simplification (SIMPLEX), which recently had its second generation of policy measures (SIMPLEX+), and the government's reform aimed at restructuring public administration's central services (PRACE). In essence, these followed an integrated and reformist logic, taking advantage of the existing knowledge and international best practices, particularly in Western Europe, and were followed up by a newly established Agency for Administrative Modernisation, in close collaboration with a Secretary of State for Administrative Modernisation. Evidently, the recent financial crisis and the subsequent Troika involvement through the assistance programme placed significant pressure on national authorities to accelerate public sector reforms. The bail-out process explains to some extent the recent pace of reforms (Teles, 2014).

The more recent initiatives, though still emerging and quite embryonic, are related to a closer collaboration with civil society's initiatives and partnerships with the third sector, in an attempt to follow policy co-creation and co-delivery strategies.

Despite the fact that the implementation of change, and above all the institutionalisation of new practices in the public sector, particularly in a context where it results from a long tradition of centralisation and of legalist 
culture, tends to be rather slow, these 45 years of democracy have witnessed significant advances in administrative simplification, modernisation, citizen proximity, the public service's efficiency improvements and accountability. Unfortunately, despite the fact that most of the policy measures, service professionalisation and simplification strategies have been implemented, their impacts have never been comprehensively evaluated, which reveals a culture that tends to avoid scrutiny and continues to keep the political and administrative spheres within close ties.

\section{Teaching and research: the recent years}

Democratic transition, with its subsequent academic freedom and the growth in number and quality of public universities in Portugal, has allowed for the fast adaptation and internationalisation of teaching and research in the administrative sciences. This would have allowed for a rapid adjustment of this scientific field to European standards if it had not been for both the long tradition of being considered as a subfield of Administrative Law, to which we have extensively referred, and the insipient and underfunded higher education system during the first years of democracy. Nevertheless, in 1980 the science of administration began its emancipation of administrative law as the bachelor's degree in management and Public Administration was established, in the context of the reform of the ISCSP (the Higher Institute for Social and Political Sciences, which had already lost its "Overseas" label). Also in the 1980 s the young University of Minho launched a degree in regional and local Public Administration, which was later restructured in the following decade, adopting the designation of just Public Administration, as ISCSP did in 2008, in the framework of the Bologna reform (Rocha, 2006).

The School of Economics and Management of the University of Minho with its Bachelor's, Master's and PhD degrees in the field of Public Administration was clearly focused on internationalisation, encouraging its faculty members to obtain doctoral degrees abroad, and promoting international research networks, in particular with the US.

Different paths, but all giving higher visibility and autonomy to this disciplinary approach, were followed in subsequent years by other Portuguese universities. That was the case of the - already referred to - ISCSP. Even though it was not able to adapt with the flexibility that all these reforms required, since it was actually the oldest and most pioneering school in the field, it was later able to introduce a master's degree in management and public administration and a doctorate programme in social sciences, with 
a specialisation in the field (later a $\mathrm{PhD}$ in Public Administration). The last 20 years have been, in fact, the most relevant in this field, regarding the way several universities answered to the need to offer degrees, train public administrators and create research centres on the topic: at the University of Aveiro, with a clear focus on an interdisciplinary approach to Public Administration, with bachelor's and master's degrees in Public Administration and a $\mathrm{PhD}$ in Public Policies; at ISCTE - University Institute of Lisbon, initially within the department of Sociology, and now with a special focus on training top-level public managers; and at the Faculty of Law of the University of Coimbra, there is clear evidence of change in the academic landscape. There are also, in the higher education sector, some polytechnic institutes with degree courses in specific fields within administration, such as Municipal Public Management.

At the same time, in the field of training staff and managers - a perspective of vocational training and development - the National Institute of Administration (INA) has played an important role since the 1980 os (Tavares \& Alves, 2006).

Each of these institutions has particular and distinguishable features: teaching and research at the School of Economics and Management of the University of Minho are obviously conditioned by the main scientific fields in the title of the school where it is incorporated; and in the case of the University of Aveiro, within the Department of Social, Political and Territorial Sciences, governance, public policies and particularly regional and local governments obtain special attention, with clear impact on the research agenda being developed at the Governance, Competitiveness and Public Policies Research Centre. Though relying on a small group of faculty members and researchers, both were able to establish significant and extensive international networks. These specialisations can be translated into specific scientific fields that reflect institutional histories or departmental focuses: ISCSP, political science; ISCTE, sociology; University of Coimbra, law. Nevertheless, through following different routes, the research and teaching of Public Administration in Portugal has evolved significantly in recent years.

\section{$4 \quad$ Looking ahead}

Despite all the significant advances in administrative simplification, and even with the more recent implementation of the digital government agenda, administrative modernisation has rarely been seen as having a clear path with a strategic plan, although there have been moments of greater coherence. The best moments were reflected in the adoption and implementation of 
dozens of fragmented measures, geared towards micro aspects of public management, with considerable impact on the quality of services provided to citizens (Tavares, 2019). This has occurred in the majority of government scenarios but, even in these cases, administrative reform was always seen as falling short of expectations. The trend to emphasise simplification and service amalgamation may have hidden several real problems with the administrative culture, human resources and processes. However, it is fair to emphasise the role in this process of the Agency for Administrative Modernisation, since it has been the main coordinating body for modernisation over the last ten years. There has been a clear commitment from political logic and technical rationality to provide greater legitimacy to the reform processes but, as in all such commitments, compromises and concessions often do not allow for the full implementation of policy choices.

Public administration is becoming more complex and demanding. As an immediate consequence, Public Administration sciences in Portugal are asked to play a relevant role and require fast adaptation. As claimed by Tavares \& Alves (2006), the daily life of the Portuguese public administration is still very much subject to administrative law, but it is possible to trace the history of the contribution of different sub-fields since the 1960s: economic planning, technological development, social and political sciences, systems management and management sciences.

Another relevant feature to take into account is the fact that most of the academic programmes in Public Administration were precisely - and explicitly - aimed at improving the quality of human resources. This focus on training, both at the graduate level and with long-life learning courses, is understandable given the rate of reforms, the growth of employment in the public sector and the inherited administrative tradition. However, in most cases this has been pursued to the detriment of research.

This context requires a shift to a more pluralistic and interdisciplinary approach within the study of Public Administration. Critical aspects such as policy assessment, strategic management, decentralisation mechanisms, governance, information management and budgeting, public procurement, digitalisation and citizen engagement are placed high on the agenda of the next year's challenges for Portuguese public administration. These do not result from a divergent approach when compared to other international trends, which means that the available knowledge and research on the topic will certainly contribute to the modernisation of public administration in the country. In addition, there is a need to intensify the internationalisation of research and teaching. This path has already been followed by some of the universities' training programmes and research centres (as is the case of the Research Unit 
on Governance, Competitiveness and Public Policies, and the Department of Social, Political and Territorial Sciences at the University of Aveiro; and of the Research Centre in Political Science, with its two research groups on Public Policy and Management and on Governance and Democracy, at the University of Minho), but, nonetheless, it still requires further development. Evidently, a balance is needed between this path of internationalisation and the deep and detailed knowledge of the contextual and cultural aspects of national public administration, at the central, regional and local levels. Evidently, given the fact that this academic field has a longer history of research and knowledge dissemination in other countries, there could be a temptation to emulate and produce a "foreign view" over national embedded problems. The risk of reductionism, redundancy and divorce in relation to the daily life of public administration in Portugal should be avoided.

As explained above, the teaching of Public Administration in Portugal has clearly followed two paths, inspired by different paradigms: the Anglo-Saxon Administrative Sciences which find shelter in Public Administration Science as an autonomous scientific area, and the Continental Europe approach, which considers it as an evolution of Administrative Law. In parallel to professional training, the teaching of Public Administration in universities has been gradually incorporating as core areas courses in Administrative Sciences and Public Management in parallel to Political Science, Management, Law and Economics, particularly after the Bologna process. Evidently, the weight of these areas varies according to each university's profile and the influence of its faculty's training background.

The adjustment of the study plans of the different degrees in Public Administration after the Bologna declaration and subsequent process, together with the integration of Portuguese academics in European research networks in this field and the inescapable influence of the Europeanisation of public policies and of public administration trends as new public management, helps to explain the growing relevance of courses in public management and administrative sciences in training. This recent, and gradual, change in the profile of training and research in Public Administration is leading the country to a detachment from its historical path which was dominated by Administrative Law.

More than a competition between European and US influence on training and reforms in Portugal, the main cleavage is still between traditional and contemporary perspectives. A recent example of this is the contracts with researchers suggested by the parliamentary ad hoc commission (in 2019) created to present paths of reforms regarding the decentralisation process in Portugal, which were, almost exclusively, established by Administrative 
Law professors. This is also evidence that reinforces a political culture that attributes reliability and authority to law, rather than to administrative sciences. Evidence can also be found in the relative low level of importance given to academia and research in this field during the reform processes, with important impacts on public administration in Portugal, as a consequence of the crisis and the Memorandum of Understanding signed with the Troika (EU, IMF, ECB) in 2011.

It is admissible to consider that there are two significant conflicts within Public Administration research and teaching in Portugal. The first is the consequence of a clear generation cleavage between a formalist and legalist group of scholars, publishing mostly in Portuguese and with an important influence on decision making, and a younger group, trained abroad, with relevant international networks and publishing in English-language journals. The second results from the clear division between the traditional approach to Public Administration as a specific branch of law (i.e. administrative law) and the multidisciplinary approach with a focus on administrative sciences as an autonomous field of knowledge. These two cleavages coincide in separate groups, which means that the most influential at the national level is still embedded in a traditional and legalist perspective, while the more internationalised one is developing research within the agenda of associated scholars in Europe. This has often resulted in a tension between policy formulation and research. However, the training of civil servants and the changing culture of Public Administration research in Portuguese universities are leading to a growing relevance of the most internationalised units and research groups, which will certainly lead to a more relevant role of academia in designing and supporting reforms and to a greater impact on agenda setting, and in its implementation and assessment.

\section{References}

Araújo, J. F. (2006). A Gestão Pública no Contexto da Ciência da Administração Pública. In A. Tavares (ed.), Estudo e Ensino da Administração Pública em Portugal (pp. 55-82). Lisbon: Escolar Editora.

Bilhim, J., Ramos, R., \& Pereira, L. M. (2015). Paradigmas Administrativos, ética e intervenção do Estado na Economia: o Caso de Portugal. Revista Digital de Derecho Administrativo 14: 91-125. Rocha, J. A. de Oliveira. (1991). Princípios de Gestão Pública. Lisbon: Editorial Presença. Rocha, J. A. de Oliveira. (2006). A Licenciatura em Administração Pública: uma História Exemplar. In A. Tavares (ed.), Estudo e Ensino da Administração Pública em Portugal. Lisbon: Escolar Editora. 13-31. 
Tavares, A. (2019). Administração Pública Portuguesa. Lisbon: Fundação Francisco Manuel dos Santos.

Tavares, L. Valadares, \& Alves, A. Azevedo. (2006). The Future of Portuguese Public Administration and a New Agenda for Public Administration Sciences in the 21st century. Public Administration 84(2): 389-406.

Teles, F. (2014). Local Government and the Bailout: reform singularities in Portugal. European Urban and Regional Studies 23(3): 455-467.

Teles, F. (2016). Local governance and Inter-municipal Cooperation. Basingstoke: Palgrave. 
VII

LESSONS AND NEXT STEPS 



\title{
Lessons and Next Steps
}

\author{
Geert Bouckaert and Werner Jann
}

We are at the end of our book and our surveys of the state and the future of PA in Europe in the next 20 years, but we are not at the end of our journey. Quite the opposite; we are at the beginning. It is obvious that the academic field of Public Administration needs to evolve with the realities and practices of public administration, and that it should try better to understand, explain and ultimately influence those realities - hopefully for the better. It is less obvious to say how that should happen in concrete terms: how we should prepare and develop our research and the teaching of our academic field of Public Administration for the future of the public sector. In ideal terms our research and teaching are academic evidence to its theories and methods; it is considered relevant by practice; and it is ahead of problems and anticipating possible solutions and trajectories. A contemporary Public Administration is critical to its contemporaneous practices. This requires an organised reflection of what and how we conduct our research and organise our teaching.

\section{$1 \quad$ European Perspectives (plural) for Public Administration}

The strategy of EPPA I has been to look for common challenges and possible answers across the European public administration space. We hope we made clear in the introduction that our project is not about coherence, but about diversity. It is not about predicting the next big theme or "fad" of PA, but about starting a process of reflection about our field.

We have used the Minnowbrook perspective as an inspiration, not as a blueprint, and even when we refer to the Minnowbrook conclusions (2018) and to the recent NAPA's Grand Challenges, this book is still about Europe. We are interested in how PA communities in Europe could and should prepare themselves and anticipate research and teaching to be open to new questions and challenges, to be able to cope and understand them, and eventually 
influence how we can better deal with futures, and yet unknown developments and challenges. From this point of ambition, we looked at the meta level of PA challenges and strategies for research and teaching. This resulted in our four coherent dimensions for driving research and teaching: a focus on futures, on disciplines, on cultures, and on practice. We are not claiming that these four dimensions are the only ones relevant for PA in Europe for the next 20 years, but our survey and especially our seminars have confirmed that "futures," "disciplines," "culture" and "practice" are important and necessary starting points and concerns for the next 20 years.

It should also be clear from the beginning that the purpose of the project and the book is not comparative. We did not start out to compare countries or approaches, but we aimed at bringing out commonalities and differences. Our focus is not to look at what the public sector will look like in 20 years, even if we refer to significant and dramatic changes and shifts in the public sphere (climate, demography, ICT, politics...). Given our awareness of these changes, our focus is on the fact that PA should not continue on the path of the last 20 years, but needs to discuss and find a strategy for the next 20 . If there is a comparative result at all, it is that these four strategies (futures, disciplines, cultures, practice) should be a part of that strategy, and will allow us to challenge the unknown and be ahead of possible future realities.

Based on the four seminars the contributions in this volume sharpen these concerns and sketch current, but especially desirable, essential and necessary developments and orientations. Again, the main aim is not comparison and coherence, but to highlight and learn from diversity.

Concerning "futures" Paul Joyce argues convincingly that much more attention needs to be paid to the future by academics in both their research and their teaching, and that appropriate techniques and skills useful for the delivery of long-term strategic visions and goals should, amongst others, include trend analysis, scenario planning and policy experimentation. Geert Bouckaert and Meelis Kitsing discuss in more detail the probably most controversial but also most useful of these approaches, i.e. the use of utopias (and dystopias) and of scenarios as thought experiments. Instead of emphasising one prediction or forecast on the basis of previous developments and current trends, they argue, it would be wise to think teleologically about it in terms of alternative scenarios.

As regards "disciplines" Thurid Hustedt, Tiina Randma-Liiv and Riin Savi give an overview of the many disciplines concerned with public administrations and their quite often problematic relationships, which furthermore differ greatly between European regions. But PA is much more than "the chameleon moving through the ivory tower." Quoting Christopher Pollitt 
they stress that what unifies public administration is its subject - the state, the public sector and the public realm - not its aims, theories or methods. We are a field, not a discipline. They confirm that PA needs all these different disciplines, but that overall the field is shaped by structures of academic organisation, conferences, journals and academic communities that tend to create silos of specialisation and hinder research across fields. So the proper institutionalisation of PA teaching and research should be one of our main concerns. Martin Burgi and Philippe Bezes take up these concerns and discuss the problematic relations between PA and two of its most important, but for some time sidelined disciplines, public law and sociology. They strongly argue that the potential for cooperation between Public Administration, administrative law and sociology is far from exhausted, and that these two disciplines offer important and indispensable empirical and theoretical foundations of PA, which we can ignore only to the great detriment of our field.

Concerning "cultures" Salvador Parado shows, using Spain as an example, how democratisation, the territorialisation of power, the expansion of the welfare state and the diversity of society and religion through substantial population inflows change the cultural and institutional basis of public administrations. Unfortunately, PA scholars have not dealt sufficiently with the challenges that these pressures are exerting at all governmental and administrative levels, and on organisations. Based on a comprehensive study in the Netherlands, Mark Bovens and his co-authors illustrate the transformation of society from the increasing ethnic heterogeneity, which they call "super-diversity," and discuss the consequences for public governance and administration. The importance of religion and language is taken up by Bogdana Neamtu, again from her specific perspective of her country and university in Cluj-Napoca, Romania, which has a long tradition of the co-existence of different languages and religions. Cultural diversity obviously has a long history in Europe, and she stresses that PA research needs to take stock of this diverse historic and demographic variety. Perspectives from other disciplines are necessary if we are to develop a sound body of European PA research on cultural diversity, but PA first of all needs to develop its own research agenda on these topics.

Finally, as regards the relationship with "practice" we have re-printed a seminal paper from the late Christopher Pollitt. He argues for a more structured, collective debate within PA academia about practitioner-oriented research, the creation of stronger representational associations at both national and international levels, a further movement away from the model of the lone researcher - or even the lone department - towards big teams operating across a range of institutions, and a rapid expansion of interdisciplinary 
research, not just by co-operation with other social sciences. It ought to become normal to find PA specialists working with colleagues from the natural sciences on teams which address problems such as climate and demographic or technological change. Edoardo Ongaro makes the important distinction between enlightening, problem-orientated and practice-extrapolation knowledge and argues that all three are significant and necessary for the development of the practical relevance of PA. He argues, like Pollitt, that our associations, like EGPA, have an important role to play in developing the use of these different forms of knowledge. Raffaella Saporito starts from the breaks in the learning loop between theory and practice, the well-known theory-practice gap, and argues that the problem is not just the scarce interest of practitioners in evidence-based policy or their superficial attraction for best practice and protocols, but the practice of scholars themselves. There is an obvious heterogeneity of potential beneficiaries of PA research, and furthermore the epistemic gap between positive and normative theories, which have to be addressed from our side.

But we did not look only for diversity and common denominators across the European public administration space; we also emphasised (and we had already learned in the cross-cutting chapters) that Europe is extremely diverse in its legal, historical and cultural contexts. There is not just one European Perspective for Public Administration; there is a plurality of perspectives, which could even be called, in some cases, perspectives in Europe, which we share with other parts of the world. It is interesting to read that nearly all of the recent Minnowbrook conclusions (see chapter one) also apply to the European context. That makes a dialogue useful and possible. But we have to go beyond Minnowbrook and define our own agenda.

The PA diversity and plurality in Europe are grounded in the diversity of Europe. This book, therefore, also looks into the diversity and path-dependent perspectives in a range of countries, as a complement to the common perspectives. The legal frameworks, the languages, the cultures and all the other elements discussed in the first part of this book heavily influence these diverse perspectives, and it is indeed amazing how different we are.

In the case of Norway, a non-EU country that has one of the highest EU-compliance scores, empirical research is influencing policies, as part of a high-trust society. It being a small country with an open approach, internationalisation of its research is important. Research has been heavily influenced by organisation theory and focuses increasingly on political and democratic contexts, and on transboundary wicked issues.

Dutch Public Administration research is also international, but is also aware that local relevance should be taken into account. After a divergence 
from political science, there is an awareness that a more converging strategy will be useful to integrate changing political processes in a democratic system.

In Germany, the disciplinary silos are not matching well with the multidisciplinary needs. Here the "bifurcation" of German PA between internationalised and theory-driven, and the more practical and country-oriented factions need to find common ground.

In France, PA is not a proper and autonomous field of its own, but is part of a wider spectrum of French social sciences, with their own methodological traditions of sociological qualitative and grassroots-driven micro studies. Sociology remains an important academic discipline for PA, even if law has dominated the practice.

In Portugal, PA has had to adjust its research to changing political contexts, to European requirements and to crisis-driven reforms. Even if law is very dominant in the public sphere, PA is considered to be relevant, especially in the field of human resources. As such, Portuguese PA has been influenced by an Anglo-Saxon and a continental European PA tradition. The PA future in Portugal will be influenced by a generational change which will reduce the influence of the formalist and legalist group of scholars. That may push PA out of law departments to allow for more disciplinary inputs.

Italian PA has suffered from significant national budget cuts, which triggered a reduction in research capacity and an increase in the migration of Italian scholars to foreign universities. Paradoxically, this has resulted in more comparative research including Italian cases and a broadening of topics being researched. This is a sign of the resilience of Italian PA.

In the Estonian case, the awareness that a small country needs a smart, innovative and merit-based public sector even more has strongly influenced PA research. Combined with a clear digital agenda, which was also picked up by research, Estonian PA is at the front line and very international.

Finally, the Hungarian PA community, which developed only after the demise of communism, is coping with the "illiberal" democratic system changes within a European context. This could lead to a diverging trend because of institutional redesigns.

So, even in our limited and unrepresentative survey of the state of PA in different European countries there is much more diversity than expected. Our firm belief is that we should take this not as a weakness, but as a strength of PA in and for Europe. PA will become stronger if it acknowledges and uses the multitude of disciplines, cultures, futures and practices in European PA. For this broad agenda we need associations like EGPA and projects like EPPA. But we also have to think about alternatives for how we conduct and organise our research and teaching in the next 20 years. 


\section{Seven possible approaches to redefining research and teaching futures for PA}

But how do we prepare for this future? We have observed that research and teaching in the field of PA have been too reactive and not sufficiently proactive, anticipating or even guiding possible futures and realities. If or when this is the case, the question arises how we define strategies that are ahead of possible realities, so that we can influence them by supporting desirable and refraining from undesirable possible futures.

Looking at the collective experience amassed in this book, we could define seven approaches for determining possible trends in Public Administration. Obviously, there is, on the one hand, an assumption that trends are there and should be discovered. There is, on the other hand, another assumption that states that trends are to be invented and created in a self-fulfilling or self-denying way. All these trends need to be confirmed by realities to come.

A Contingency Approach: a first way to define futures is to rely on a classical contingency approach. Based on facts, it is possible to extrapolate and to assume that a certain trend which is announced and detected will proceed and continue. In this sense, PA is still reacting to external trends which it is following, sometimes in an early, sometimes in a later stage, since research calls certainly do not always anticipate our possible futures.

It is clear that in the European case, the contingencies of the changing European context have influenced our research and teaching strategies in PA. The fall of the Berlin Wall in 1989 resulted in a whole portfolio of "transition governance" for Central and Eastern European countries. The expansion of the EU, adding ten countries in 2004 plus two in 2007 , plus one in 2013, probably minus one in 2020, has redefined and added "convergence governance" as a new topic in research and teaching. The Balkan wars have certainly added "post-conflict governance," and even a "failed state" agenda, to our research and teaching. The EU's deepening strategy with Schengen in 1985, the Single Market in 1993 and the security portfolio has emphasised the topics of "governance of co-ordination" for policies and agencies. The Global Economic Crises (GEC) in 2008 has further enhanced and expanded an existing agenda. From establishing the "Euro" (1999-2002), through the Lisbon Treaty (2009), to the Greek crisis (2011-2012), a whole "macro governance" agenda studying institutional stability was added. Obviously, the European agenda has also embraced the SDGs, especially with the Paris Agreement in 2015 on climate change. This has resulted in a "wicked problems governance" agenda, including migration, climate and all 17 SDGs. Finally, the European agenda to protect 
privacy by means of the General Data Protection Regulation (GDPR) has triggered and pushed the agenda of "cloud and big data governance" with all its related issues, such as, for example, block-chain, and algorithms.

This contingency approach has been partly supported by the ERC research calls, the $\mathrm{H}_{2} \mathrm{O}_{2} \mathrm{O}$ and the next generation of national and European research programmes. It is clear that this approach of looking for contingencies could be more detailed. We could assume, with the shifts in our political systems, that even more fundamental and systemic contingencies may become very pressing and urgent, such as governance with or without democracy, or governance with or without government, or governance of fragile/failed states with/without a rule of law. It is also obvious that increasingly "non-western governance" is emerging as an alternative and effective model, for example Chinese governance models. It will be essential for Europe to develop its own governance perspectives, but also to start a dialogue with Asian, Latin-American, African and MENA Perspectives for Public Administration. Obviously, we should begin and continue to have a dialogue between EPPA and US-based Minnowbrook discussions.

A Normative Approach: we have argued in this book that our current PA teaching and research are running too far behind the facts, that they are too much dominated by a single discipline (which could be law, or economics, or management, or politics, ...), that we have too much causality and not enough teleological thinking, that we aim too much for general mechanisms and not enough for cultural features, and, finally, that we have too little relevance for practice, even if we are academically sound. If these assumptions are correct, we will have to change.

Therefore, a normative approach to future trends tells us that PA should do the opposite of what is happening, and be ahead of facts, combine existing and new disciplines, be more teleological, include more culture, and have more relevance. Launching this EPPA I has as an ambition to push European researchers to include more futures, more disciplines, more culture (defined as ethnicity, languages and religion), and be more relevant.

A Strategic Approach: when research means addressing known questions and generating scientifically known answers, we define our history of PA. When research means addressing known questions which generate unknown answers, we talk about most of our current research programmes. However, a strategic approach is about starting to raise unknown questions which trigger an intuition regarding what to develop as answers, such as, for example, big, open and cloudy data. Finally, there is the double strategy of unknown questions and answers, which really pushes us out of our comfort zone as 
researchers, but also out of our predictable career and publication trajectories. The unknown questions with unknown answers push us to the real wicked PA issues or to non-Western PA topics. It will be our responsibility to develop strategies to cover these topics in an academic way, since they may have the highest relevance for our futures.

A Disciplinary Approach: historically, there has been an ever continuing debate on the various disciplines constituting the academic field of PA. The emphasis or most dominant discipline is often defined by historical and cultural features of a country and its tradition. However, we observe that the changing way the public sphere is interacting with society, and the technological shifts propelling our way of life and our production functions impact immensely on the need for PA. It certainly implies that a monodisciplinary approach, with its vertical deepening, is necessary but certainly not sufficient. It needs to be combined with the widening of a horizontal disciplinary approach. It requires a rebalancing of the existing disciplines (law, politics, economics, management, sociology). It also requires new disciplines such as social psychology, anthropology, robotics/engineering or geomatics. Behavioural PA is presented as a new type of thinking although it has been around since WW II, with Herbert Simon as its main proponent. Within EPPA, we have developed the debate on this discipline as a special topic.

An Epistemological Approach: academic quality is possible for all types of epistemological stands. However, some epistemological approaches are more accepted and popular, even if their relevance is under-developed. There are clear trends for the future in our epistemological approaches. It will be important for our main research institutes to have a critical mass of methodologies and a fair mix of all methods responding to different epistèmes. When moving towards a full range of epistemological approaches with their related methods and techniques, about five schools are emerging:

- A mechanistic/physicalistic method responds not just to the first decades of the twentieth century, with its scientific management, with the machine models and the engineer-driven authors. The mathematics of the systems were mostly algebraic with closed systems without context. It allowed for replications of practices. There were some degrees of development with operations research technologies which allowed a distant environment, unclear objectives and satisficing rather than optimising objective functions.

- There is the scientific orthodoxy, with a cause-effect modelling, allowing for hypotheses to be tested and conclusions to be generalised. Realities are transformed into data populations, with probabilities for statistical 
testing in surveys. Dependent and independent variables are definable and defined. Context is recycled and corrected by concepts such as "outliers," "noise" and error rates.

- Critical realism describes and explains mechanisms using case studies which could be very similar or very different. Contexts are made explicit. Thick descriptions allow for frameworks, modelling, trends, patterns.

- Radical constructivism relies on intersubjective and consensual interpretations. Realities can be defined as stories which are socially constructed as meanings. Language analysis is combined with participatory processes.

- Within the Chaos Paradigm we are in a field where everything is context, since the border lines are absent. This is the field of the known unknown and, even more, of the unknown unknown. It is the field of unmanageable wickedness. It is hoped that Monte-Carlo methodologies of simulations and random thinking could be helpful.

It will be important to combine all these methods, rather than reduce our vision on realities by selecting and focusing on one epistème, with its related and necessarily restricted approaches.

A Relevance Approach: this pragmatic approach refers to the supply and demand of PA knowledge. The most convenient zone is where some supply meets some demand. Here we can only move to have a closer and more intense interaction of supply and demand, where supply becomes an answer to the demand which raises a question. The other three zones are problematic. A first mismatch is the supply of knowledge by the PA community for which there is no demand. It triggers the reaction of "so what?". We can have some of this, but not too much. And the key question is whether this research is just irrelevant or ahead of its time. A second frustration zone is where we have a clear question and an obvious societal problem but no supply of PA research. It is clear that some of the big questions, of the current paradigms, of for example big data related problems, are in this zone. Finally, and as a worst case scenario, there is a zone where we have no demand and no supply. This "terra incognita" is about a societal meteorite that will fall on us, and we are totally unaware of it; nobody saw this coming (for example, major parts of populations that feel abandoned, major migrations, ...).

A Self-Contained Approach: finally, a research strategy could just be very insular and myopic. It is just blindly or routinely driven by the existing data and the existing methods. It becomes an academic vortex which disconnects itself from reality and relevance. Most of this research output is "empty." 


\section{$3 \quad$ Next Steps}

This EPPA I project ended with the 2018 concluding seminar on the occasion of the EGPA conference at IDHEAP, UNIL, in Lausanne, where EPPA was handed over to EGPA as one of its strategic projects. It has been our ambition to show and map the diversity within Europe. This diversity is far from demonstrating incoherence. We explicitly emphasise the plural, "European Perspectives," to cope with future challenges which are partly common, and partly contingent and path-dependent. As a complement to these common perspectives, we wanted to illustrate this diversity. In the end, we found much more diversity than expected. Our firm belief is that we should take this not as a weakness, but as a strength of PA in and for Europe. PA will become stronger if it acknowledges and uses the multitude of disciplines, cultures, futures and practices in European PA.

Of course, the questions we raise and the tentative answers we discuss are just a beginning, not the end. Our aim has never been to summarise a debate but to start a new one, and therefore our determination and goal have always been to repeat this exercise regularly, for example every 20 years, obviously inspired by the long-lasting success of the Minnowbrook initiatives, however critically one may look at some of their results and recommendations. So we are aiming for EPPA II in 2038, and EPPA III in 2058. The idea is to keep it alive by having a dialogue with the US-based Minnowbrook reflections, which we assume will continue with Minnowbrook IV in 2028 and Minnowbrook $\mathrm{V}$ in 2048. Ultimately, a broader dialogue of EPPA in Europe with other regional perspectives should be an ambition, i.e. with Asian Perspectives (APPA), MENA Perspectives (MENAPPA), African Perspectives (AFPPA) and Latin-American Perspectives (LAPPA).

\section{Seven points to remember}

It is impossible to summarise the EPPA I initiative, which proved to be more productive and fascinating than we had ever expected in our wildest dreams, in a few sentences. But still, we want to conclude with seven points to remember:

1. PA needs to keep an eye on the future and anticipate possible futures in research and teaching so as to be ahead of events.

2. PA needs to consolidate disciplines better and actively create platforms to integrate classical disciplines, including sociology, law and public policy expertise, but also new disciplines and fields of expertise such as 
ICT and behavioural psychology. Institutionalisation of cooperation is of the utmost importance.

3. PA needs to be more serious and effective in taking culture into account. This is not just about the relevance of context, but much more about the impact of languages, religions, ethnicities and the increasing high diversity of populations.

4. PA needs to connect to practice better by focusing on different forms of knowledge, on different normative needs, a professional way of communicating, and by having scientists in residence at administrations.

5. PA needs periodically to take stock of its research and teaching, of its relevance and faults, to attract and keep its capacity for change.

6. PA should look for common denominators in Europe, but should at the same time appreciate the European diversities and make use of them to improve trends and directions within and across countries.

7. Europe needs more dialogue within its European PA space, but also outside that space with other regions of the world. This of course includes Minnowbrook, but even more the rest of the world.

EPPA I has been a first, feeble step in these directions, but we strongly argue and hope that EGPA will keep EPPA alive within its PA community. 

The public sector in our society has over the past two decades undergone substantial changes, as has the academic field studying Public Administration (PA). In the next twenty years major shifts are further expected to occur in the way futures are anticipated and different cultures are integrated. Practice will be handled in a relevant way, and more disciplines will be engaging in the field of Public Administration.

The prominent scholars contributing to this book put forward research strategies and focus on priorities in the field of Public Administration. The volume will also give guidance on how to redesign teaching programmes in the field. This book will provide useful insights to compare and contrast European PA with PA in Europe, and with developments in other parts of the world.

Geert Bouckaert is professor at the KU Leuven Public Governance Institute, and former president of the European Group for Public Administration, and of the International Institute of Administrative Sciences.

Werner Jann is professor emeritus of Political Science, Administration and Organization at the University of Potsdam and former President of the European Group for Public Administration.

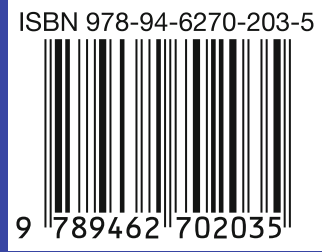

